\title{
Afghanistan Opium Poppy Database 1995
}

UNDCP/R:OSWA

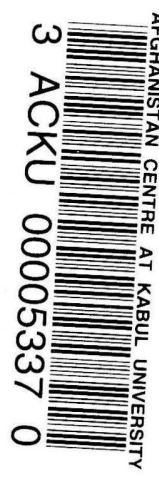


Opium Production Survey by village in Badakhshan province, 1995.

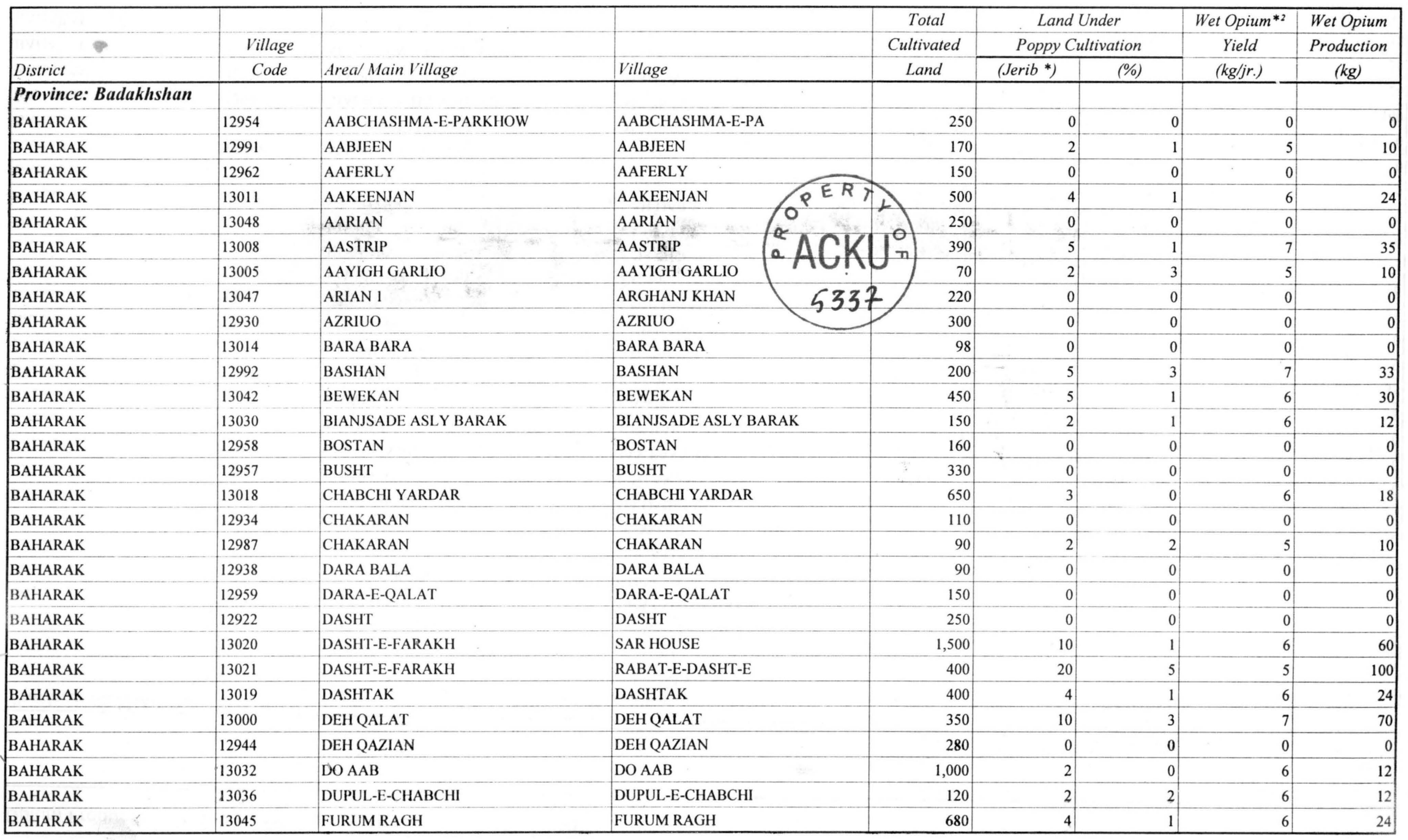

$* 1$ Jerib $=1 / 5 \mathrm{Ha}$

*2 Dry Opium $=$ Wet Opium - $30 \%$ moisture 
Opium Production Survey by village in Badakhshan province, 1995.

\begin{tabular}{|c|c|c|c|c|c|c|c|c|}
\hline \multirow[b]{3}{*}{ District } & \multirow{3}{*}{$\begin{array}{c}\text { Village } \\
\text { Code }\end{array}$} & \multirow{3}{*}{ Areal Main Village } & \multirow[b]{3}{*}{ Village } & \multirow{3}{*}{$\begin{array}{c}\text { Total } \\
\text { Cultivated } \\
\text { Land } \\
\end{array}$} & \multirow{2}{*}{\multicolumn{2}{|c|}{$\begin{array}{c}\text { Land Under } \\
\text { Poppy Cultivation }\end{array}$}} & \multirow{3}{*}{$\begin{array}{c}\text { Wet Opium }{ }^{* 2} \\
\text { Yield } \\
(\mathrm{kg} / \mathrm{jr} .)\end{array}$} & \multirow{3}{*}{$\begin{array}{c}\text { Wet Opium } \\
\text { Production } \\
(\mathrm{kg})\end{array}$} \\
\hline & & & & & & & & \\
\hline & & & & & $\left(\right.$ Jerib $\left.^{*}\right)$ & $(\%)$ & & \\
\hline \multicolumn{9}{|c|}{ Province: Badakhshan } \\
\hline BAHARAK & 12995 & GHACHAN & GHACHAN & 240 & 3 & 1 & 5 & 15 \\
\hline BAHARAK & 12990 & GHAINO & GHAINO & 100 & 0 & 0 & 0 & 0 \\
\hline BAHARAK & 12956 & GHAZALI-E-BALA & GHAZALI-E-BALA & 280 & 0 & 0 & 0 & 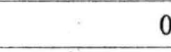 \\
\hline BAHARAK & 12952 & GHAZALIO-E-PAYAN & GHAZALIO-E-PAYA & 220 & 0 & 0 & 0 & 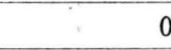 \\
\hline BAHARAK & 12963 & GHOZ MURGH & GHOZ MURGH & 80 & 0 & 0 & 0 & 0 \\
\hline BAHARAK & 12942 & GHOZIO & GHOZIO & 500 & 0 & 0 & 0 & 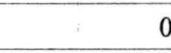 \\
\hline BAHARAK & 13046 & GUMAAB & GUMAAB & 250 & 0 & 0 & 0 & 0 \\
\hline BAHARAK & 13017 & HATIMBIGEE & HATIMBIGEE & 400 & 5 & 1 & 6 & 30 \\
\hline BAHARAK & 12983 & ISHTAKAN & ISHTAKAN & 200 & 0 & 0 & 0 & 0 \\
\hline BAHARAK & 12973 & JAY PARYAN & JAY PARYAN & 130 & 0 & 0 & 0 & 0 \\
\hline BAHARAK & 12961 & JOY BAR & JOY BAR & 120 & 0 & 0 & 0 & 0 \\
\hline BAHARAK & 13452 & KAWIK & KAWIK & 150 & 3 & 2 & 6 & 18 \\
\hline BAHARAK & 12999 & KAZDEH & KAZDEH & 355 & 5 & 1 & 7 & 35 \\
\hline BAHARAK & 13004 & KHASH BEEN & KHASH BEEN & 70 & 2 & 2 & 6 & 9 \\
\hline BAHARAK & 12984 & KHISRAW & KHISRAW & 120 & 0 & 0 & 0 & 0 \\
\hline BAHARAK & 13012 & KHUSH DARIO & KHUSH DARIO & 150 & 6 & 4 & 6 & 36 \\
\hline BAHARAK & 13026 & KOH-E-DARAZ & KOH-E-DARAZ & 200 & 1 & 1 & 6 & 6 \\
\hline BAHARAK & 12970 & KORKHO & KORKHO & 150 & 1 & 0 & 6 & 3 \\
\hline BAHARAK & 13013 & KOUCH & KOUCH & 110 & 0 & 0 & 0 & 0 \\
\hline BAHARAK & 12968 & LAB DARA & LAB DARA & 200 & 0 & 0 & 0 & 0 \\
\hline BAHARAK & 12946 & MADRABA & MADRABA & 160 & 0 & 0 & 0 & 0 \\
\hline BAHARAK & 13039 & MADRASA 2 RBABI & MADRASA 2 RBABI & 220 & 0 & 0 & 0 & 0 \\
\hline BAHARAK & 12976 & MADRASA MALANAB & MADRASA MALANAB & 200 & 0 & 0 & 0 & 0 \\
\hline BAHARAK & 12998 & MADRASA TEERGHARAN & MADRASA TEERGHA & 400 & 20 & 5 & 7 & 140 \\
\hline BAHARAK & 12986 & MADRASA-E-CHAKARAN & MADRASA-E-CHAKA & 160 & 4 & 3 & 6 & 24 \\
\hline BAHARAK & 13022 & MADRASA-E-DASHT FARA & MADRASA-E-DASHT & 3,000 & 10 & 0 & 5 & 50 \\
\hline BAHARAK & 13037 & MADRASA-E-KHAIR & MADRASA-E-KHAIR & 250 & 3 & 1 & 6 & 18 \\
\hline BAHARAK & 13054 & MADRASA-E-PAYAN SHAH & MADRASA-E-PAYAN SHAH & 800 & 5 & 1 & 5 & 25 \\
\hline BAHARAK & 13041 & MADRASA-E-RABAB & MADRASA-E-RABAB & 320 & 2 & 1 & 6 & 12 \\
\hline
\end{tabular}


Opium Production Survey by village in Badakhshan province, 1995.

\begin{tabular}{|c|c|c|c|c|c|c|c|c|}
\hline \multirow[b]{3}{*}{ District } & \multirow{3}{*}{$\begin{array}{l}\text { Village } \\
\text { Code }\end{array}$} & \multirow[b]{3}{*}{ Areal Main Village } & \multirow[b]{3}{*}{ Village } & \multirow{3}{*}{$\begin{array}{c}\text { Total } \\
\text { Cultivated } \\
\text { Land } \\
\end{array}$} & \multirow{2}{*}{\multicolumn{2}{|c|}{$\begin{array}{c}\text { Land Under } \\
\text { Poppy Cultivation }\end{array}$}} & \multirow{3}{*}{$\begin{array}{c}\text { Wet Opium*2 } \\
\text { Yield } \\
(\mathrm{kg} / \mathrm{jr} .)\end{array}$} & \multirow{3}{*}{$\frac{\text { Wet Opium }}{\text { Production }}$} \\
\hline & & & & & & & & \\
\hline & & & & & $\left(\right.$ (Jerih $\left.{ }^{*}\right)$ & $(\%)$ & & \\
\hline \multicolumn{9}{|c|}{ Province: Badakhshan } \\
\hline BAHARAK & 12971 & MAGHAYIB BALA & MAGHAYIB BALA & 200 & 0 & 0 & 0 & 0 \\
\hline BAHARAK & 12966 & MAGHAYIB SUFLA & MAGHAYIB SUFLA & 100 & 0 & 0 & 0 & 0 \\
\hline BAHARAK & 12988 & MAHALA-E-CHAKARAN & MAHALA CHAKARAN & 100 & 2 & 2 & 5 & 8 \\
\hline BAHARAK & 13034 & MARKAZ-E-CHАBCH & MARKAZ-E-CHABCH & 500 & 1 & 0 & 6. & 6 \\
\hline BAHARAK & 13033 & MASJID LAB NAHR & MASJID LAB NAHR & 400 & 0 & 0 & 0 & 0 \\
\hline BAHARAK & 13038 & MASJID-E-PAYAN & MASJID-E-PAYAN & 300 & 0 & 0 & 0 & 0 \\
\hline BAHARAK & 13023 & MAZAR & MAZAR & 150 & 2 & 1 & 6 & 9 \\
\hline BAHARAK & 12941 & MAZAR & MAZAR & 400 & 0 & 0 & 0 & 0 \\
\hline BAHARAK & 13053 & MIYAN DEH & MIYAN DEH & 1.200 & 30 & 3 & 6 & 180 \\
\hline BAHARAK & 12955 & MIYAN DEH YARHO & MIYAN DEH YARHO & 100 & 0 & 0 & 0 & 0 \\
\hline BAHARAK & 12985 & MOHERKAN-E-CHAKARAN & MOHERKAN-E-CHAKARAN & 180 & 4 & 2 & 6 & 24 \\
\hline BAHARAK & 12969 & MOHMOODDAN & MOHMOODDAN & 160 & 1 & 1 & 6 & 6 \\
\hline BAHARAK & 13044 & NAW ABAD RURUM RAGH & NAW ABAD RURUM RAGH & 210 & 0 & 0 & 0 & 0 \\
\hline BAHARAK & 12978 & NAWABAD MALANAB & NAWABAD MALANAB & 200) & 0 & 0 & 0 & 0 \\
\hline BAHARAK & 12947 & PALIL & PALIL & 80 & 0 & 0 & 0 & 0 \\
\hline BAHARAK & 12948 & PANJANGIO & PANJANGIO DEH P & 290 & 0 & 0 & 0 & 0 \\
\hline BAHARAK & 12953 & PARKHAWAB ARGHNDAB & ARKHAWAB ARGHANDAB & 150 & 0 & 0 & 0 & 0 \\
\hline BAHARAK & 12979 & PASIRA & PASIRA & 200 & 5 & 3 & 6 & 30 \\
\hline BAHARAK & 12981 & PASS BAGH & PASS BAGH & 180 & 7 & 4 & 5 & 35 \\
\hline BAHARAK & 12964 & PASS-E-KHUM & PASS-E-KHUM & 100 & 0 & 0 & 0 & 0 \\
\hline BAHARAK & 13006 & PEASH & PEASH & 190 & 3 & 2 & 6 & 18 \\
\hline BAHARAK & 12974 & PETOW & PETOW & 120 & 1 & 1 & 6 & 6 \\
\hline BAHARAK & 12967 & PEYOY & PEYOY & 170 & 0 & 0 & 0 & 0 \\
\hline BAHARAK & 13043 & QALA-E-BAZAR & QALA-E-BAZAR & 150 & 1 & 1 & 6 & 6 \\
\hline BAHARAK & 13035 & QULDASHT & QULDASHT & 150 & 2 & 1 & 6 & 12 \\
\hline BAHARAK & 13040 & RABABI & MADRASA-E-RABAB & 240 & 5 & 2 & 6 & 30 \\
\hline BAHARAK & 13453 & RABAT CHELTAN & RABAT CHELTAN & 60 & 1 & 2 & 6 & 6 \\
\hline BAHARAK & 12945 & RAGHNAKAN & RAGHNAKAN & 120 & 0 & 0 & 0 & 0 \\
\hline BAHARAK & 12935 & RIZWAN & RIZWAN & 140 & 0 & 0 & 0 & 0 \\
\hline
\end{tabular}

- 1 Jerib $=1 / 5 \mathrm{Ha}$

" 2 Dry Opium $=$ Wet Opium $-30 \%$ moisture 
Opium Production Survey by village in Badakhshan province, 1995.

\begin{tabular}{|c|c|c|c|c|c|c|c|c|}
\hline \multirow[b]{3}{*}{ District } & \multirow{3}{*}{$\begin{array}{c}\text { Village } \\
\text { Code }\end{array}$} & \multirow[b]{3}{*}{ Area/ Main Village } & \multirow[b]{3}{*}{ Village } & \multirow{3}{*}{$\begin{array}{c}\text { Total } \\
\text { Cultivated } \\
\text { Land }\end{array}$} & \multirow{2}{*}{\multicolumn{2}{|c|}{$\begin{array}{c}\text { Land Under } \\
\text { Poppy Cultivation }\end{array}$}} & \multirow{3}{*}{$\begin{array}{c}\text { Wet Opium }{ }^{* 2} \\
\text { Yield } \\
(\mathrm{kg} / \mathrm{jr} .)\end{array}$} & \multirow{3}{*}{$\begin{array}{c}\text { Wet Opium } \\
\text { Production } \\
(\mathrm{kg}) \\
\end{array}$} \\
\hline & & & & & & & & \\
\hline & & & & & $\left(\right.$ Jerib $\left.^{*}\right)$ & $(\%)$ & & \\
\hline \multicolumn{9}{|c|}{ Province: Badakhshan } \\
\hline BAHARAK & 13007 & RUKHSHAN & RUKHSHAN & 210 & 5 & 2 & 5 & 25 \\
\hline BAHARAK & 13002 & SAFID DARA & SAFID DARA & 195 & 6 & 3 & 6 & 36 \\
\hline BAHARAK & 12927 & SAGHI & SAGHI & 200 & 0 & 0 & 0 & 0 \\
\hline BAHARAK & 12975 & SAKHA & SAKHA & 320 & 0 & 0 & 0 & 0 \\
\hline BAHARAK & 12924 & SANG AAB & SANG AAB & 260 & 0 & 0 & 0 & 0 \\
\hline BAHARAK & 12939 & SANGAIYAN & SANGAIYAN & 120 & 0 & 0 & 0 & 0 \\
\hline BAHARAK & 13028 & SAR SHAHIR & SAR SHAHIR & 200 & 4 & 2 & 6 & 24 \\
\hline BAHARAK & 13016 & SAR-E-PULBARDAR & SAR-E-PULBARDAR & 310 & 5 & 2 & 5 & 25 \\
\hline BAHARAK & 12929 & SARASAK & SARASAK & 110 & 0 & 0 & 0 & 0 \\
\hline BAHARAK & 13027 & SARDIA-E-BAHARAK & SARDIA-E-BAHARAK & 170 & 2 & 1 & 6 & 12 \\
\hline BAHARAK & 12931 & SEELA & SEELA & 130 & 0 & 0 & 0 & 0 \\
\hline BAHARAK & 13009 & SHAHGAN & SHAHGAN & 640 & 4 & 1 & 5 & 20 \\
\hline BAHARAK & 12936 & SHAKH YARAK & SHAKH YARAK & 110 & 0 & 0 & 0 & 0 \\
\hline BAHARAK & 13025 & SHASHPUL-E-FARHAD & SHASHPUL-E-FARHAD & 1,200 & 5 & 0 & 6 & 30 \\
\hline BAHARAK & 12933 & SHER KANI & SHER KANI & 160 & 0 & 0 & 0 & 0 \\
\hline BAHARAK & 12923 & SOCHIO & SOCHIO & 160 & 0 & 0 & 0 & 0 \\
\hline BAHARAK & 13003 & SUFIAN & SUFIAN & 250 & 8 & 3 & 6 & 48 \\
\hline BAHARAK & 13050 & TAKH SAAB & TAKH SAAB & 220 & 0 & 0 & 0 & 0 \\
\hline BAHARAK & 13010 & TARANG & TARANG & 300 & 12 & 4 & 5 & 60 \\
\hline BAHARAK & 14011 & TARWAZA & TARWAZA (AWACH) & 80 & 0 & 0 & 0 & 0 \\
\hline BAHARAK & 13001 & TEERGHARAN(PAYANDEH) & TEERGHARAN & 350 & 15 & 4 & 6 & 90 \\
\hline BAHARAK & 12937 & WAAYEM & WAAYEM & 120 & 0 & 0 & 0 & 0 \\
\hline BAHARAK & 13029 & WAKH SHER & DEH CHASHMA & 100 & 0 & 0 & 0 & 0 \\
\hline BAHARAK & 13015 & WAKHAL & WAKHAL & 280 & 7 & 3 & 5 & 35 \\
\hline BAHARAK & 13031 & WAKSHER & DEH BALA & 70 & 0 & 0 & 0 & 0 \\
\hline BAHARAK & 13052 & WALAR & WALAR & 700 & 6 & 1 & 5 & 30 \\
\hline BAHARAK & 13051 & WALAR SAHIR & WALAR SAHIR & 260 & 0 & 0 & 0 & 0 \\
\hline BAHARAK & 12932 & WANAR & WANAR & 200 & 0 & 0 & 0 & 0 \\
\hline BAHARAK & 13049 & WARNAIL & WARNAIL & 270 & 0 & 0 & 0 & 0 \\
\hline
\end{tabular}

${ }^{*}$ I Jerib $=1 / 5 \mathrm{Ha}$

* ${ }^{2}$ Dry Opium $=$ Wet Opium $-30 \%$ moisture 
Opium Production Survey by village in Badakhshan province, 1995.

\begin{tabular}{|c|c|c|c|c|c|c|c|c|}
\hline & & & & Total & \multicolumn{2}{|c|}{ Land Under } & Wet Opium*2 & Wet Opium \\
\hline & Village & & & Cultivated & \multicolumn{2}{|c|}{ Poppy Cultivation } & Yield & Production \\
\hline District & Code & Area/ Main Village & Village & Land & $\left(\right.$ Jerib $\left.^{*}\right)$ & $(\%)$ & $(\mathrm{kg} / \mathrm{jr})$. & $(\mathrm{kg})$ \\
\hline \multicolumn{9}{|c|}{ Province: Badakhshan } \\
\hline BAHARAK & 12960 & WENJ & WENJ & 130 & 0 & 0 & 0 & 0 \\
\hline BAHARAK & 12982 & WOSHKAN & WOSHKAN & 80 & 5 & 6 & 6 & 30 \\
\hline BAHARAK & 12977 & WWEJAINJ & WWEJAINJ & 100 & 0 & 0 & 0 & 0 \\
\hline BAHARAK & 12951 & YABAB-E- KALAN & YABAB-E- KALAN & 120 & 0 & 0 & 0 & 0 \\
\hline BAHARAK & 12950 & YABAB-E-MAGHAIB & YABAB-E-MAGHAIB & 100 & 0 & 0 & 0 & 0 \\
\hline BAHARAK & 12949 & YAJIK-E-PARKHOW & YAJIK-E-PARKHOW & 400 & 0 & 0 & 0 & 0 \\
\hline BAHARAK & 12928 & YAKHCHIO DEH BALA & YAKHCHIO DEH BA & 220 & 0 & 0 & 0 & 0 \\
\hline BAHARAK & 12926 & YAKHCHIO DEH PAYAN & YAKHCHIO DEH PA & 330 & 0 & 0 & 0 & 0 \\
\hline BAHARAK & 12989 & YAKHSHERA & YAKHSHERA & 95 & 2 & 2 & 6 & 12 \\
\hline BAHARAK & 12965 & YAREEM & YAREEM & 150 & 0 & 0 & 0 & 0 \\
\hline BAHARAK & 12921 & YASECH & YASECH & 200 & 0 & 0 & 0 & 0 \\
\hline BAHARAK & 12997 & YASHISTAN & YASHISTAN & 130 & 3 & 2 & 6 & 18 \\
\hline BAHARAK & 12996 & YAWAR & YAWAR & 120 & 3 & 3 & 6 & 18 \\
\hline BAHARAK & 12925 & YOUYOUNAK & YOUYOUNAK & 100 & 0 & 0 & 0 & 0 \\
\hline BAHARAK & 12994 & YUMAL & YUMAL & 900 & 10 & 1 & 7 & 70 \\
\hline BAHARAK & 12980 & ZIORANGE & ZIORANGE & 420 & 0 & 0 & 0 & 0 \\
\hline BAHARAK & 12993 & $\mathrm{ZOO}$ & $\mathrm{ZOO}$ & 190 & 5 & 3 & 5 & 25 \\
\hline FAIZABAD & 12720 & ABSITI & ABSITI & 220 & 20 & 9 & 5 & 100 \\
\hline FAIZABAD & 12711 & AFAQI & AFAQI & 320 & 8 & 3 & 6 & 48 \\
\hline FAIZABAD & 13404 & AFAQI MAIDAH & AFAQI MAIDAH & 110 & 7 & 6 & 6 & 42 \\
\hline FAIZABAD & 12549 & ALANI & ALANI & 1,500 & 115 & 8 & 5 & 575 \\
\hline FAIZABAD & 12608 & ALIMANGO & MASJED-E-BALA & 250 & 6 & 2 & 6 & 36 \\
\hline FAIZABAD & 12609 & ALIMANGO & ALIMANGO & 270 & 10 & 4 & 6 & 60 \\
\hline FAIZABAD & 12707 & ALOCHAHBOLAGH & ALOCHAHBOLAGH & 75 & 0 & 0 & 0 & 0 \\
\hline FAIZABAD & 12607 & AQBURYA & M. JAME AQBURYA & 1,000 & 70 & 7 & 10 & 700 \\
\hline FAIZABAD & 12614 & AQBURYA & AQBURYA & 900 & 80 & 9 & 10 & 800 \\
\hline FAIZABAD & 12821 & ARGHANJ KHAW & KHAM BEW & 300 & 0 & 0 & 0 & 0 \\
\hline FAIZABAD & 12817 & ARGHANJ KHULA & BAI NAW & 160 & 0 & 0 & 0 & 0 \\
\hline FAIZABAD & 12813 & ARGHANJ KHWA & KHAN AGHA & 170 & 0 & 0 & 0 & 0 \\
\hline
\end{tabular}


Opium Production Survey by village in Badakhshan province, 1995.

\begin{tabular}{|c|c|c|c|c|c|c|c|c|}
\hline \multirow[b]{3}{*}{ District } & \multirow{3}{*}{$\begin{array}{c}\text { Village } \\
\text { Code }\end{array}$} & \multirow[b]{3}{*}{ Area/ Main Village } & \multirow[b]{3}{*}{ Village } & \multirow{3}{*}{$\begin{array}{c}\text { Total } \\
\text { Cultivated } \\
\text { Land }\end{array}$} & \multirow{2}{*}{\multicolumn{2}{|c|}{$\begin{array}{c}\text { Land Under } \\
\text { Poppy Cultivation }\end{array}$}} & \multirow{3}{*}{$\begin{array}{c}\text { Wet Opium }^{* 2} \\
\text { Yield } \\
(\mathrm{kg} / \mathrm{jr} .)\end{array}$} & \multirow{3}{*}{$\begin{array}{c}\text { Wet Opium } \\
\frac{\text { Production }}{(\mathrm{kg})}\end{array}$} \\
\hline & & & & & & & & \\
\hline & & & & & $\left(\right.$ Jerib $\left.^{*}\right)$ & $(\%)$ & & \\
\hline \multicolumn{9}{|c|}{ Province: Badakhshan } \\
\hline FAIZABAD & 12816 & ARGHANJ KHWA & MASJED PAYAN & 180 & 0 & 0 & 0 & 0 \\
\hline FAIZABAD & 12820 & ARGHANJ KHWA & GHALA DARAH & 300 & 0 & 0 & 0 & 0 \\
\hline FAIZABAD & 12819 & ARGHANJ KHWA & KULAN & 180 & 0 & 0 & 0 & 0 \\
\hline FAIZABAD & 12814 & ARGHANJ KHWA & TOSOM DARAH & 200 & 0 & 0 & 0 & 0 \\
\hline FAIZABAD & 12810 & ARGHANJ KHWA & TAGABAK & 220 & 0 & 0 & 0 & 0 \\
\hline FAIZABAD & 12818 & ARGHANJ KHWA & NAW ABAD & 250 & 0 & 0 & 0 & 0 \\
\hline FAIZABAD & 12666 & ARGHUND & YANTAGHLAGH & 110 & 6 & 5 & 4 & 24 \\
\hline FAIZABAD & 13403 & ARGHUND & KHUJA CHESHMAH & 90 & 4 & 4 & 5 & 20 \\
\hline FAIZABAD & 12859 & AROSHIA, GOZARDARA & AORSHIA & 775 & 0 & 0 & 0 & 0 \\
\hline FAIZABAD & 12870 & ARQA QESHLAQ & ARQA QESHLAQ & 300 & 0 & 0 & 0 & 0 \\
\hline FAIZABAD & 14008 & ASHKASHAN & ASHKASHAN BALA & 110 & 0 & 0 & 0 & 0 \\
\hline FAIZABAD & 12811 & ASS & ASS & 250 & 1 & 0 & 8 & 4 \\
\hline FAIZABAD & 12679 & ATEN JELAW & ATEN JELAW & 1,000 & 0 & 0 & 0 & 0 \\
\hline FAIZABAD & 12653 & AUTRANCHI AAB BARIK & AAB BARIK BALA & 600 & 15 & 3 & 4 & 60 \\
\hline FAIZABAD & 13463 & AUTRANCHI AB BURIK & MASJID JAME & 400 & 10 & 3 & 4 & 40 \\
\hline FAIZABAD & 12648 & AUTRANCHI-E-PAYAN & AUTRANCHI PAYAN & 550 & 12 & 2 & 4 & 48 \\
\hline FAIZABAD & 13410 & AUTRINCHI HESARI & AUTRINCHI HESAR & 750 & 0 & 0 & 0 & 0 \\
\hline FAIZABAD & 13456 & AUZONGOL & MASJED-E-MORAD & 200 & 6 & 3 & 4 & 24 \\
\hline FAIZABAD & 13457 & AUZONGOL & MASJED NOWABAD & 150 & 4 & 3 & 4 & 16 \\
\hline FAIZABAD & 12675 & AUZONQUL & AUZONQUL & 250 & 8 & 3 & 3 & 24 \\
\hline FAIZABAD & 13436 & AYLAQ SANGI & AYLAQ SANGI & 230 & 60 & 26 & 7 & 420 \\
\hline FAIZABAD & 12598 & BAGH MUBARAK & BAGH MUBARAK & 200 & 80 & 40 & 8 & 640 \\
\hline FAIZABAD & 12900 & BAGH-E-BALEEN & BALJAB & 170 & 0 & 0 & 0 & 0 \\
\hline FAIZABAD & 12899 & BAGH-E-BALEEN & GOZAR-E-MASJED & 200 & 0 & 0 & 0 & 0 \\
\hline FAIZABAD & 12901 & BAGH-E-BALEEN & MASJED-E-ZARAB & 80 & 0 & 0 & 0 & 0 \\
\hline FAIZABAD & 12502 & BAGH-E-SUFI & HAZAR BAGH SUFI & 470 & 150 & 32 & 6 & 900 \\
\hline FAIZABAD & 12907 & BAGHAK & BAGHAK & 185 & 0 & 0 & 0 & 0 \\
\hline FAIZABAD & 12722 & BAGHI SHAH & BAGHI SHAH & 250 & 40 & 16 & 4 & 160 \\
\hline FAIZABAD & 12617 & BAKHT-E-SHAH & CHANGA & 100 & 50 & 50 & 10 & 500 \\
\hline
\end{tabular}


Opium Production Survey by village in Badakhshan province, 1995.

\begin{tabular}{|c|c|c|c|c|c|c|c|c|}
\hline \multirow[b]{3}{*}{ District } & \multirow{3}{*}{$\begin{array}{l}\text { Village } \\
\text { Code }\end{array}$} & \multirow[b]{3}{*}{ Area/ Main Village } & \multirow[b]{3}{*}{ Village } & \multirow{3}{*}{$\begin{array}{c}\text { Total } \\
\text { Cultivated } \\
\text { Land } \\
\end{array}$} & \multirow{2}{*}{\multicolumn{2}{|c|}{$\begin{array}{c}\text { Land Under } \\
\text { Poppy Cultivation }\end{array}$}} & \multirow{3}{*}{$\begin{array}{c}\text { Wet Opium }{ }^{* 2} \\
\text { Yield } \\
(\mathrm{kg} / \mathrm{jr} .)\end{array}$} & \multirow{3}{*}{\begin{tabular}{|c|} 
Wet Opium \\
Production \\
$(\mathrm{kg})$ \\
\end{tabular}} \\
\hline & & & & & & & & \\
\hline & & & & & $\left(\right.$ Jerib $\left.{ }^{*}\right)$ & $(\%)$ & & \\
\hline \multicolumn{9}{|c|}{ Province: Badakhshan } \\
\hline FAIZABAD & 12615 & BAKHTISHA & BAKHTISHA & 480 & 130 & 27 & 11 & 1,430 \\
\hline FAIZABAD & 12855 & BAL DARAH & BAL DARAH & 504 & 0 & 0 & 0 & 0 \\
\hline FAIZABAD & 12903 & BAR BAGH & BAR BAGH & 310 & 0 & 0 & 0 & 0 \\
\hline FAIZABAD & 14013 & BARLAS CHENAR & MASJED HAWAR & 154 & 10 & 6 & 5. & 50 \\
\hline FAIZABAD & 12652 & BARLAS CHENAR & BARLAS CHENAR & 260 & 15 & 6 & 5 & 75 \\
\hline FAIZABAD & 12651 & BARLAS CHENAR & MASJED-E-HAIDAR & 100 & 10 & 10 & 4. & 40 \\
\hline FAIZABAD & 12650 & BARLAS CHENAR & MASJED SHASHKAL & 155 & 10 & 6 & 5 & 50 \\
\hline FAIZABAD & 12641 & BARLAS SHAMAR & BARLAS SHAMAR & 410 & 10 & 2 & 5 & 50 \\
\hline FAIZABAD & 12647 & BARLAS SHAMAR & MASJED-E-JAME & 250 & 6 & 2 & 5 & 30 \\
\hline FAIZABAD & 12623 & BARLAS WAKHEYAN & BARLAS WAKHEYAN & 330 & 70 & 21 & 10 & 700 \\
\hline FAIZABAD & 14005 & BATASH & KOHNA BATASH & 170 & 20 & 12 & 7 & 140 \\
\hline FAIZABAD & 14006 & BATASH & BATASH & 240 & 80 & 33 & 7 & 560 \\
\hline FAIZABAD & 13437 & BATASH & MASJID JAME & 226 & 50 & 22 & 8 & 400 \\
\hline FAIZABAD & 12732 & BAY MALASI & BAY MALASI & 240 & 50 & 21 & 9 & 450 \\
\hline FAIZABAD & 13439 & BAY MALASI & M.H.M.SEDIQ & 200 & 30 & 15 & 4 & 120 \\
\hline FAIZABAD & 12892 & BAYD-E-KALAN & MASJED-E-JAME & 145 & 0 & 0 & 0 & 0 \\
\hline FAIZABAD & 12894 & BED KALAN & MASJID HJ.EBRAH & 150 & 0 & 0 & 0 & 0 \\
\hline FAIZABAD & 12895 & BED KALAN & MASJID A. WALI & 160 & 0 & 0 & 0 & 0 \\
\hline FAIZABAD & 12890 & BED KALAN & MASJID DASHT & 155 & 0 & 0 & 0 & 0 \\
\hline FAIZABAD & 12856 & BEKHWAH & BEKHWAH SOFLA & 1,002 & 0 & 0 & 0 & 0 \\
\hline FAIZABAD & 12857 & BEKHWAH & BEKHWAH OLYA & 754 & 0 & 0 & 0 & 0 \\
\hline FAIZABAD & 12581 & BELAKI & BELAKI & 150 & 6 & 4 & 4 & 24 \\
\hline FAIZABAD & 12808 & BOCHI & BOCHI & 70 & 0 & 0 & 0 & 0 \\
\hline FAIZABAD & 13461 & BOIMI QARAH & MASJED-E-BALA & 420 & 6 & 1 & 5 & 30 \\
\hline FAIZABAD & 13400 & BOINIQARA & BOINIQARA & 80 & 0 & 0 & 0 & 0 \\
\hline FAIZABAD & 12667 & BOINIQARAH & BOINIQARAH & 630 & 8 & 1 & 6 & 48 \\
\hline FAIZABAD & 12832 & BROKAN & BROKAN & 1,040 & 0 & 0 & 0 & 0 \\
\hline FAIZABAD & 12867 & CHAH ABOUK & CHAH ABOUK & 1,500 & 7 & 0 & 4 & 28 \\
\hline FAIZABAD & 12781 & CHAHOSH DARAH & CHAHOSH DARAH & 210 & 0 & 0 & 0 & 0 \\
\hline
\end{tabular}

$* 1 \mathrm{Jerib}=1 / 5 \mathrm{Ha}$

${ }^{*}{ }^{2}$ Dry Opium $=$ Wet Opium $-30 \%$ moisture 
Opium Production Survey by village in Badakhshan province, 1995.

\begin{tabular}{|c|c|c|c|c|c|c|c|c|}
\hline \multirow[b]{3}{*}{ District } & \multirow{3}{*}{$\begin{array}{c}\text { Village } \\
\text { Code }\end{array}$} & \multirow[b]{3}{*}{ Area/ Main Village } & \multirow[b]{3}{*}{ Village } & \multirow{3}{*}{$\begin{array}{c}\text { Total } \\
\text { Cultivated } \\
\text { Land } \\
\end{array}$} & \multicolumn{2}{|c|}{ Land Under } & \multirow{3}{*}{\begin{tabular}{|c|} 
Wet Opium $^{* 2}$ \\
Yield \\
$(\mathrm{kg} / \mathrm{jr})$. \\
\end{tabular}} & \multirow{3}{*}{$\begin{array}{c}\text { Wet Opium } \\
\text { Production } \\
(\mathrm{kg})\end{array}$} \\
\hline & & & & & \multicolumn{2}{|c|}{ Poppy Cultivation } & & \\
\hline & & & & & $\left(\right.$ Jerib $\left.^{*}\right)$ & $(\%)$ & & \\
\hline \multicolumn{9}{|c|}{ Province: Badakhshan } \\
\hline FAIZABAD & 12773 & CHAHOSH DARAH BALA & CHAHOSH DARAH & 300 & 0 & 0 & 0 & 0 \\
\hline FAIZABAD & 12630 & CHAK ABDUL & CHAK ABDUL & 190 & 40 & 21 & 5 & 200 \\
\hline FAIZABAD & 12595 & CHAK HA & CHAK HA & 150 & 50 & 33 & 5 & 250 \\
\hline FAIZABAD & 12583 & CHAPAH & MASJED SARKOTAL & 3,000 & 120 & 4 & 6 & 720 \\
\hline FAIZABAD & 12585 & CHAPAH & CHAPAH & 4,100 & 160 & 4 & 6 & 960 \\
\hline FAIZABAD & 13390 & CHAQEL QESHLAQ & CHAQEL QESHLAQ & 160 & 40 & 25 & 7 & 280 \\
\hline FAIZABAD & 12658 & CHAR GOW & KHAM SALEH & 250 & 20 & 8 & 6 & 120 \\
\hline FAIZABAD & 12664 & CHARDARAH & CHARDARAH & 220 & 15 & 7 & 5 & 75 \\
\hline FAIZABAD & 12590 & CHATA & QASHQAR BEGI & 147 & 2 & 1 & 6 & 9 \\
\hline FAIZABAD & 12589 & CHATA & MASJID DEH BALA & 180 & 5 & 3 & 8 & 40 \\
\hline FAIZABAD & 12592 & CHATA & DEH MIANA & 230 & 3 & 1 & 7 & 21 \\
\hline FAIZABAD & 12593 & CHATA & MASJED-E-RAHIM SHAH & 150 & 0 & 0 & 0 & 0 \\
\hline FAIZABAD & 12591 & CHATA & MASJED CHARMGAR & 86 & 0 & 0 & 0 & 0 \\
\hline FAIZABAD & 12636 & CHATRAQ & CHATRAQ & 100 & 15 & 15 & 5 & 75 \\
\hline FAIZABAD & 12663 & CHEL KAPAH & CHEL KAPAH & 60 & 5 & 8 & 6 & 30 \\
\hline FAIZABAD & 12582 & CHESHMA-E-QALANDER & CHESHMA QALANDE & 200 & 50 & 25 & 5 & 250 \\
\hline FAIZABAD & 13364 & CHESHMA-E-QALANDER & MASJED-E-KARIM & 170 & 40 & 24 & 7 & 280 \\
\hline FAIZABAD & 12569 & CHESHMA-E-QALANDER & MASJED-E-JAME & 350 & 100 & 29 & 6 & 600 \\
\hline FAIZABAD & 12882 & DAHAN DARA & KOHNA QESHLAQ & 30 & 0 & 0 & 0 & 0 \\
\hline FAIZABAD & 13434 & DAHAN DARA & BALA HESAR & 35 & 0 & 0 & 0 & 0 \\
\hline FAIZABAD & 12881 & DAHAN DARA & NAW ABAD & 65 & 0 & 0 & 0 & 0 \\
\hline FAIZABAD & 12633 & DANISHMANDAN & DANISHMANDAN & 300 & 10 & 3 & 7 & 70 \\
\hline FAIZABAD & 12584 & DARA KHATAK & DARA KHATAK & 180 & 40 & 22 & 6 & 240 \\
\hline FAIZABAD & 12571 & DARA-E-CHEREK & SANG-E-QALAT & 200 & 30 & 15 & 6 & 180 \\
\hline FAIZABAD & 12576 & DARA-E-CHEREK & DARA-E-CHEREK & 200 & 50 & 25 & 6 & 300 \\
\hline FAIZABAD & 12534 & DARA-E-MAHMOOD & DARA-E-MAHMOOD & 160 & 18 & 11 & 6 & 108 \\
\hline FAIZABAD & 12656 & DARAH JANI & DARAH JANI & 115 & 20 & 17 & 7 & 140 \\
\hline FAIZABAD & 12871 & DARAH RAST & MASJED PAYAN & 82 & 0 & 0 & 0 & 0 \\
\hline FAIZABAD & 12868 & DARAH RAST & MASJED BALA & 25 & 0 & 0 & 0 & 0 \\
\hline
\end{tabular}

* $1 \mathrm{Jerib}=1 / 5 \mathrm{Ha}$

* 2 Dry Opium $=$ Wet Opium - $30 \%$ moisture 
Opium Production Survey by village in Badakhshan province, 1995.

\begin{tabular}{|c|c|c|c|c|c|c|c|c|}
\hline \multirow[b]{3}{*}{ District } & \multirow{3}{*}{$\begin{array}{l}\text { Village } \\
\text { Code }\end{array}$} & \multirow[b]{3}{*}{ Area/ Main Village } & \multirow[b]{3}{*}{ Village } & \multirow{3}{*}{$\begin{array}{c}\text { Total } \\
\text { Cultivated } \\
\text { Land }\end{array}$} & \multirow{2}{*}{\multicolumn{2}{|c|}{$\begin{array}{c}\text { Land Under } \\
\text { Poppy Cultivation }\end{array}$}} & \multirow{3}{*}{\begin{tabular}{|c|} 
Wet Opium $^{* 2}$ \\
Yield \\
(kg/jr.) \\
\end{tabular}} & \multirow{3}{*}{$\begin{array}{c}\text { Wet Opium } \\
\text { Production } \\
(\mathrm{kg})\end{array}$} \\
\hline & & & & & & & & \\
\hline & & & & & $\left(\right.$ Jerib $\left.^{*}\right)$ & $(\%)$ & & \\
\hline \multicolumn{9}{|c|}{ Province: Badakhshan } \\
\hline FAIZABAD & 13372 & DARAYEM & HAJI PAHLAWAN & 800 & 160 & 20 & 6 & 960 \\
\hline FAIZABAD & 12620 & DARKHAN & DARKHAN & 300 & 150 & 50 & 10 & 1,500 \\
\hline FAIZABAD & 12812 & DASHT PING & DASHT PING & 250 & 50 & 20 & 7 & 350 \\
\hline FAIZABAD & 12566 & DEH BASI & DEH BASI ULYA & 120 & 30 & 25 & 6 & 180 \\
\hline FAIZABAD & 12567 & DEH BASI & DEH BASI SUFLA & 200 & 40 & 20 & 5 & 200 \\
\hline FAIZABAD & 12559 & DEH BAZAR & SAYEDHA DEHBAZA & 580 & 200 & 34 & 7 & 1,400 \\
\hline FAIZABAD & 13370 & DEH BAZAR & AFGHANHA & 20 & 5 & 25 & 6 & 30 \\
\hline FAIZABAD & 12568 & DEH BAZAR & DEH BAZAR & 200 & 130 & 65 & 6 & 780 \\
\hline FAIZABAD & 12558 & DEH BAZAR & NOW ABAD & 280 & 160 & 57 & 7 & 1,120 \\
\hline FAIZABAD & 13371 & DEH BAZAR & ASAD ABAD & 20 & 10 & 50 & 5 & 50 \\
\hline FAIZABAD & 12619 & DEH DEHI & DEH DEHI & 250 & 160 & 64 & 12 & 1,920 \\
\hline FAIZABAD & 12565 & DEH MULAIAN & DEH MULAIAN & 750 & 150 & 20 & 6 & 900 \\
\hline FAIZABAD & 12564 & DEH MULAIAN & DARA-E-CHARMAGHZ & 70 & 20 & 29 & 6 & 120 \\
\hline FAIZABAD & 12862 & DEH ZINDAN & DEH ZINDAN & 663 & 0 & 0 & 0 & 0 \\
\hline FAIZABAD & 12618 & DEHMAGAS & TAJIK & 150 & 60 & 40 & 12 & 720 \\
\hline FAIZABAD & 12616 & DEHMAGAS & DEHMAGAS & 450 & 130 & 29 & 12 & 1,560 \\
\hline FAIZABAD & 12640 & DEHQAN KHANAH & DEHQAN KHANAH & 350 & 200 & 57 & 30 & 6,000 \\
\hline FAIZABAD & 12516 & DOAAB & PASS SHAKH & 210 & 90 & 43 & 5 & 450 \\
\hline FAIZABAD & 12518 & DOAAB & DOAAB & 200 & 80 & 40 & 6 & 480 \\
\hline FAIZABAD & 12520 & DOAAB & SAR-E-TAL & 150 & 50 & 33 & 5 & 250 \\
\hline FAIZABAD & 13398 & DOGHALAT & KHUJA MOULI & 50 & 0 & 0 & 0 & 0 \\
\hline FAIZABAD & 12677 & DOGHALAT & MASJED-E-AAQ KOTAL & 160 & 3 & 2 & 5 & 15 \\
\hline FAIZABAD & 13402 & DOGHALAT & DOULAT ABAD & 27 & 0 & 0 & 0 & 0 \\
\hline FAIZABAD & 12680 & DOGHALAT KALAN & BOIM CHESIIMAII & 150 & 0 & 0 & 0 & 0 \\
\hline FAIZABAD & 12678 & DOGHALAT KAI.AN & MASJID QABRESTA & 50 & 0) & 0 & 0 & 0 \\
\hline FAIZABAD & 12672 & DOGHALAT KAL.AN & CHAQEL MASJED & 150 & 0 & 0 & 0 & 0 \\
\hline FAIZABAD & 12676 & DOGHALAT KALAN & MASJED-E-BAYHA & 70 & 0 & 0 & 0 & 0 \\
\hline FAIZABAD & 14000 & DOGHALAT KALAN & DOGHALAT KALAN & 300 & 4 & 1 & 6 & 24 \\
\hline FAIZABAD & 13399 & DOGHALAT KHUJA MOULI & DOGHALAT & 150 & 0 & 0 & 0 & 0 \\
\hline
\end{tabular}

* I Jerib $=1 / 5 \mathrm{Ha}$

* 2 Dry Opium $=$ Wet Opium $-30 \%$ moisture 
Opium Production Survey by village in Badakhshan province, 1995.

\begin{tabular}{|c|c|c|c|c|c|c|c|c|}
\hline \multirow{2}{*}{ District } & \multirow{2}{*}{$\begin{array}{c}\text { Village } \\
\text { Code }\end{array}$} & \multirow[b]{2}{*}{ Area/ Main Village } & \multirow[b]{2}{*}{ Village } & \multirow{2}{*}{$\begin{array}{c}\text { Total } \\
\text { Cultivated } \\
\text { Land }\end{array}$} & \multicolumn{2}{|c|}{$\begin{array}{c}\text { Land Under } \\
\text { Poppy Cultivation }\end{array}$} & \multirow{2}{*}{$\begin{array}{c}\text { Wet Opium*2 } \\
\text { Yield } \\
(\mathrm{kg} / \mathrm{jr} .)\end{array}$} & \multirow{2}{*}{$\begin{array}{c}\text { Wet Opium } \\
\text { Production } \\
(\mathrm{kg})\end{array}$} \\
\hline & & & & & $\left(\right.$ Jerib $\left.^{*}\right)$ & $(\%)$ & & \\
\hline \multicolumn{9}{|c|}{ Province: Badakhshan } \\
\hline FAIZABAD & 12624 & EARGITAL & EARGITAL & 300 & 70 & 23 & 5 & 350 \\
\hline FAIZABAD & 13369 & ESHAN DARA BAGH & MASJED SARIHAUS & 50 & 7 & 14 & 5 & 35 \\
\hline FAIZABAD & 12561 & ESHAN DARA BAGH & ESHAN DARA BAGH & 90 & 15 & 17 & 6 & 90 \\
\hline FAIZABAD & 12560 & ESHAN DARA BAGH & MASJED MADRASA & 40 & 8 & 20 & 7. & 56 \\
\hline FAIZABAD & 13368 & ESHAN DARA BAGH & RUIA DASHT & 25 & 10 & 40 & 7 & 70 \\
\hline FAIZABAD & 12622 & ESHANA & ESHANA & 270 & 120 & 44 & 12 & 1,440 \\
\hline FAIZABAD & 12866 & FARGHAMBOL & FARGHAMBOL & 32 & 0 & 0 & 0 & 0 \\
\hline FAIZABAD & 12691 & GAJI & GAJI & 350 & 0 & 0 & 0 & 0 \\
\hline FAIZABAD & 12643 & GANJA CHESHMA & MASJED-E-JAME & 1,100 & 80 & 7 & 8 & 640 \\
\hline FAIZABAD & 12644 & GANJA CHESHMAH & MASJED QAYOMBAY & 700 & 40 & 6 & 7 & 280 \\
\hline FAIZABAD & 12642 & GANJA CHESHMAH & MASJED TURSANBY & 900 & 50 & 6 & 8 & 400 \\
\hline FAIZABAD & 12645 & GANJA CHESIIMAH & MASJED WAZIRBAY & 900 & 70 & 8 & 8 & 560 \\
\hline FAIZABAD & 12686 & GARAB & GARAB & 150 & 0 & 0 & 0 & 0 \\
\hline FAIZABAD & 12833 & GAZANAK & GAZANAK & 1,200 & 0 & 0 & 0 & 0 \\
\hline FAIZABAD & 12573 & GAZKEEL & GAZKEEL & 150 & 30 & 20 & 5 & 150 \\
\hline FAIZABAD & 12627 & GHAZAK DARAH & MASJID-E-JAME & 415 & 110 & 27 & 6 & 660 \\
\hline FAIZABAD & 12828 & GHOOZ MARGH & GHOOZ MARGH & 787 & 0 & 0 & 0 & 0 \\
\hline FAIZABAD & 12626 & GHUZAK DARAH & JUWAZOK & 288 & 88 & 31 & 6 & 528 \\
\hline FAIZABAD & 12629 & GHUZAK DARAH & GHUZAK DARAH & 140 & 40 & 29 & 6 & 240 \\
\hline FAIZABAD & 12587 & GULAKI & GULAKI & 550 & 60 & 11 & 5 & 300 \\
\hline FAIZABAD & 12869 & GULAKI & GULAKI BALA & 1,500 & 3 & 0 & 3 & 9 \\
\hline FAIZABAD & 12765 & GULAKI & GULAKI PAYAN & 3,000 & 0 & 0 & 0 & 0 \\
\hline FAIZABAD & 13388 & HAFEZ MUGHOL & MASJED-E-PAYAN & 270 & 70 & 26 & 9 & 630 \\
\hline FAIZABAD & 12661 & HAFEZ MUGHOL & HAFEZ MUGHOL & 340 & 90 & 26 & 8 & 720 \\
\hline FAIZABAD & 12660 & HAFEZ MUGOI & MADRASAH & 200 & 60 & 30 & 8 & 480 \\
\hline FAIZABAD & 12579 & HAJI PAHLAWAN & DOGHALAT & 160 & 18 & 11 & 6 & 108 \\
\hline FAIZABAD & 12545 & HAJI PAHLAWAN & MAHAJERHA & 300 & 50 & 17 & 6 & 300 \\
\hline FAIZABAD & 12702 & HALQAJAR & HALQAJAR & 220 & 7. & 3 & 4 & 28 \\
\hline FAIZABAD & 13412 & HASAN BEGI & HASAN BEGI & 200 & 2 & 1 & 9 & 18 \\
\hline
\end{tabular}


Opium Production Survey by village in Badakhshan province, 1995.

\begin{tabular}{|c|c|c|c|c|c|c|c|c|}
\hline \multirow[b]{3}{*}{ District } & \multirow{3}{*}{$\begin{array}{l}\text { Village } \\
\text { Code }\end{array}$} & \multirow[b]{3}{*}{ Area/ Main Village } & \multirow[b]{3}{*}{ Village } & \multirow{3}{*}{\begin{tabular}{|c|} 
Total \\
Cultivated \\
Land \\
\end{tabular}} & \multirow{2}{*}{\multicolumn{2}{|c|}{$\begin{array}{c}\text { Land Under } \\
\text { Poppy Cultivation }\end{array}$}} & \multirow{3}{*}{$\begin{array}{c}\text { Wet Opium }^{* 2} \\
\text { Yield } \\
(\mathrm{kg} / \mathrm{jr} .)\end{array}$} & \multirow{3}{*}{$\begin{array}{c}\text { Wet Opium } \\
\text { Production } \\
(\mathrm{kg})\end{array}$} \\
\hline & & & & & & & & \\
\hline & & & & & $\left(\right.$ Jerib $\left.^{*}\right)$ & $(\%)$ & & \\
\hline \multicolumn{9}{|c|}{ Province: Badakhshan } \\
\hline FAIZABAD & 12897 & HASAN-E-DEWANAH & HASAN-E-DEWANAH & 900 & 0 & 0 & 0 & 0 \\
\hline FAIZABAD & 12848 & HAWZAKAN & HAWZAKAN & 1,036 & 0 & 0 & 0 & 0 \\
\hline FAIZABAD & 12563 & HAZARA DARABAGH & HAZARA DARABAGH & 50 & 10 & 20 & 8 & 80 \\
\hline FAIZABAD & 12649 & HAZARA-E-GAWI & HAZARA-E-GAWI & 270 & 20 & 7 & 4 & 80 \\
\hline FAIZABAD & 12508 & HAZARA-E-KHAIRBEK & HAZARA-E-KHAIRB & 100 & 35 & 35 & 8 & 280 \\
\hline FAIZABAD & 12689 & HAZARAH BEDAK & HAZARAH BEDAK & 90 & 0 & 0 & 0 & 0 \\
\hline FAIZABAD & 12714 & HAZARAH DIWANAH & HAZARAH DIWANAH & 110 & 0 & 0 & 0 & 0 \\
\hline FAIZABAD & 12662 & HAZARAH MISHI & HAZARAH MISHI & 150 & 16 & 11 & 5 & 80 \\
\hline FAIZABAD & 12688 & HAZARAH QADAM & HAZARAH QADAM & 450 & 0 & 0 & 0 & 0 \\
\hline FAIZABAD & 13455 & HOWZI & MASJED-E-RAHMAT & 144 & 0 & 0 & 0 & 0 \\
\hline FAIZABAD & 12671 & HOWZI & HOWZI & 200 & 0 & 0 & 0 & 0 \\
\hline FAIZABAD & 12721 & IATARCHI & IATARCHI & 260 & 10 & 4 & 7 & 70 \\
\hline FAIZABAD & 13464 & IATARCHI PAYAN & IATARCHI PAYAN & 120 & 5 & 4 & 6 & 30 \\
\hline FAIZABAD & 12726 & ISHAK KITI & ISHAK KITI & 410 & 100 & 24 & 8 & 800 \\
\hline FAIZABAD & 12634 & JAMURAD & JAMURAD & 150 & 3 & 2 & 5 & 13 \\
\hline FAIZABAD & 13405 & JAMURAD & MASJED-E-JAME & 90 & 2 & 2 & 4 & 8 \\
\hline FAIZABAD & 12510 & JANGAL BEED & JANGAL BEED & 170 & 46 & 27 & 7 & 322 \\
\hline FAIZABAD & 12729 & JATA & JATA & 200 & 40 & 20 & 7 & 280 \\
\hline FAIZABAD & 12746 & JAW ZOON & DEW DARAH & 80 & 0 & 0 & 0 & 0 \\
\hline FAIZABAD & 12739 & JAW ZOON & PARANA KHAM & 130 & 0 & 0 & 0 & 0 \\
\hline FAIZABAD & 12815 & JAW ZOON & YASHI & 90 & 0 & 0 & 0 & 0 \\
\hline FAIZABAD & 12740 & JAWZON & DEH SHAHANAH & 60 & 0 & 0 & 0 & 0 \\
\hline FAIZABAD & 12741 & JAWZON & RABAT & 65 & 0 & 0 & 0 & 0 \\
\hline FAIZABAD & 12742 & JAWZON & CHASHMA-E-BEED & 113 & 0 & 0 & 0 & 0 \\
\hline FAIZABAD & 12756 & JAWZON & MEDREEL & 90 & 0 & 0 & 0 & 0 \\
\hline FAIZABAD & 13445 & JAWZON & NAW ABAD & 50 & 0 & 0 & 0 & 0 \\
\hline FAIZABAD & 12738 & JAWZON & GAZAN & 100 & 0 & 0 & 0 & 0 \\
\hline FAIZABAD & 13422 & JAWZOON & ELGAK & 60 & 0 & 0 & 0 & 0 \\
\hline FAIZABAD & 13421 & JAWZOON & DEH MISHIN & 100 & 0 & 0 & 0 & 0 \\
\hline
\end{tabular}

* $1 \mathrm{Jerib}=1 / 5 \mathrm{Ha}$

${ }^{*}$ Dry Opium $=$ Wet Opium $-30 \%$ moisture 
Opium Production Survey by village in Badakhshan province, 1995.

\begin{tabular}{|c|c|c|c|c|c|c|c|c|}
\hline \multirow[b]{3}{*}{ District } & \multirow{3}{*}{$\begin{array}{l}\text { Village } \\
\text { Code }\end{array}$} & \multirow[b]{3}{*}{ Area/ Main Village } & \multirow[b]{3}{*}{ Village } & \multirow{3}{*}{$\begin{array}{c}\text { Total } \\
\text { Cultivated } \\
\text { Land }\end{array}$} & \multirow{2}{*}{\multicolumn{2}{|c|}{$\begin{array}{c}\text { Land Under } \\
\text { Poppy Cultivation }\end{array}$}} & \multirow{3}{*}{$\begin{array}{c}\text { Wet Opium }^{* 2} \\
\text { Yield } \\
(\mathrm{kg} / \mathrm{jr} .) \\
\end{array}$} & \multirow{3}{*}{$\begin{array}{c}\text { Wet Opium } \\
\text { Production } \\
(\mathrm{kg})\end{array}$} \\
\hline & & & & & & & & \\
\hline & & & & & $\left(\right.$ Jerib $\left.^{*}\right)$ & $(\%)$ & & \\
\hline \multicolumn{9}{|c|}{ Province: Badakhshan } \\
\hline FAIZABAD & 13420 & JAWZOON & MALMONEGE & 60 & 0 & 0 & 0 & 0 \\
\hline FAIZABAD & 12743 & JAWZOON & GUL DARAH & 90 & 0 & 0 & 0 & 0 \\
\hline FAIZABAD & 12754 & JAWZOON & BAZGERAN & 50 & 0 & 0 & 0 & 0 \\
\hline FAIZABAD & 12744 & JAWZOON & KOLGA-E-PAYAN & 80 & 0 & 0 & 0 & 0 \\
\hline FAIZABAD & 12745 & JAWZOON & KOLGA-E-BALA & 100 & 0 & 0 & 0 & 0 \\
\hline FAIZABAD & 12749 & JAWZOON & CHAP DARAH & 40 & 0 & 0 & 0 & 0 \\
\hline FAIZABAD & 12747 & JAWZOON & SAR DARAH & 100 & 0 & 0 & 0 & 0 \\
\hline FAIZABAD & 12504 & KAFTAR KHANA & KAFTAR KHANA UL & 80 & 30 & 38 & 7 & 210 \\
\hline FAIZABAD & 12513 & KAFTAR KHANA & KAFTAR KHANA SU & 130 & 50 & 38 & 8 & 400 \\
\hline FAIZABAD & 12503 & KAFTAR KHANA-E-TAJEK & KAFTAR KHANA & 210 & 40 & 19 & 7 & 280 \\
\hline FAIZABAD & 12710 & KAKAN & KAKAN & 315 & 20 & 6 & 5 & 100 \\
\hline FAIZABAD & 13459 & KAKAN & MASJED-E-BABA & 240 & 15 & 6 & 4 & 60 \\
\hline FAIZABAD & 13460 & KAKAN & MASJED M. AHMAD & 210 & 12 & 6 & 5 & 60 \\
\hline FAIZABAD & 12863 & KANDILAN & KANDILAN & 2,000 & 0 & 0 & 0 & 0 \\
\hline FAIZABAD & 12577 & KARSANG & KARSANG & 130 & 30 & 23 & 5 & 150 \\
\hline FAIZABAD & 13386 & KASHANDARA OLYA & KASHANDARA OLYA & 104 & 5 & 5 & 1 & 5 \\
\hline FAIZABAD & 13394 & KATA DARAH & KATA DARAH & 60 & 2 & 3 & 4 & 8 \\
\hline FAIZABAD & 12586 & KATAH QESHLAQ & KATAH QESHLAQ & 350 & 50 & 14 & 6 & 300 \\
\hline FAIZABAD & 14002 & KATAH QESHLAQ & KATAH QESHLAQ & 1,050 & 50 & 5 & 6 & 300 \\
\hline FAIZABAD & 12708 & KAZER & KAZER & 110 & 0 & 0 & 0 & 0 \\
\hline FAIZABAD & 14007 & KAZER NISHIR & KAZER NISHIR & 185 & 60 & 32 & 8 & 480 \\
\hline FAIZABAD & 13435 & KAZER PETAW & KAZER PETAW & 310 & 100 & 32 & 8 & 800 \\
\hline FAIZABAD & 14014 & KESHEN DARAH & KESHEN DARAH SU & 72 & 0 & 0 & 0 & 0 \\
\hline FAIZABAD & 12396 & KESHTGAH & MASJED JAME & 340 & 0 & 0 & 0 & 0 \\
\hline FAIZABAD & 12898 & KESHTGAH & GOZAR PANJSHER & 150 & 0 & 0 & 0 & 0 \\
\hline FAIZABAD & 12507 & KHAIRBEK & KHAIRBEK & 180 & 80 & 44 & 7 & 560 \\
\hline FAIZABAD & 13391 & KHAKSARI & PSWTA KHOR & 170 & 30 & 18 & 5 & 150 \\
\hline FAIZABAD & 12685 & KHAKSARI & KHAKSARI & 700 & 40 & 6 & 8 & 320 \\
\hline FAIZABAD & 12540 & KHAM MIRAHZAR & KHAM MIRAHZAR & 250 & 80 & 32 & 7 & 560 \\
\hline
\end{tabular}


Opium Production Survey by village in Badakhshan province, 1995.

\begin{tabular}{|c|c|c|c|c|c|c|c|c|}
\hline & & & & Total & \multicolumn{2}{|c|}{ Land Under } & Wet Opium*2 & Wet Opium \\
\hline & Village & & & Cultivated & \multicolumn{2}{|c|}{ Poppy Cultivation } & Yield & Production \\
\hline District & Code & Area/ Main Village & Village & Land & $\left(\right.$ Jerib $\left.^{*}\right)$ & $(\%)$ & $(\mathrm{kg} / \mathrm{jr})$. & $(\mathrm{kg})$ \\
\hline \multicolumn{9}{|c|}{ Province: Badakhshan } \\
\hline FAIZABAD & 12822 & KHAM-I-AWAL & KHAM-I-AWAL & 300 & 0 & 0 & 0 & 0 \\
\hline FAIZABAD & 12734 & KHANAQA & KHANAQA & 50 & 0 & 0 & 0 & 0 \\
\hline FAIZABAD & 13374 & KHANDAN SHAHER & DAHANA ABI GAZK & 170 & 40 & 24 & 5 & 200 \\
\hline FAIZABAD & 12580 & KHANDAN SHAHER & KHANDAN SHAHER & 400 & 50 & 13 & 5 & 250 \\
\hline FAIZABAD & 13373 & KHANDAN SHAHER & KHANDAN SHAHER & 100 & 55 & 55 & 4 & 220 \\
\hline FAIZABAD & 12535 & KHASPAK & MASJID-E-JAME & 160 & 10 & 6 & 4 & 40 \\
\hline FAIZABAD & 12533 & KHASPAK & GUZAR-E-MULABAY & 80 & 13 & 16 & 5 & 65 \\
\hline FAIZABAD & 12572 & KHAWAJA-E-BAGH & KHAWAJA-E-BAGH & 450 & 80 & 18 & 7 & 560 \\
\hline FAIZABAD & 12731 & KHAWJAH ASHTAL & KHAWJAH ASHTAL & 550 & 35 & 6 & 6 & 210 \\
\hline FAIZABAD & 12703 & KHUREK & KHUREK & 120 & 20 & 17 & 6 & 120 \\
\hline FAIZABAD & 13458 & KOHAKI & KOHAKI & 300 & 25 & 8 & 6 & 150 \\
\hline FAIZABAD & 12804 & KOHNA QESHLAQ & KOHNA QESHLAQ & 204 & 0 & 0 & 0 & 0 \\
\hline FAIZABAD & 12874 & KOHNAH QESHLAQ & GOZAR BALA & 65 & 0 & 0 & 0 & 0 \\
\hline FAIZABAD & 12872 & KOHNAH QESHLAQ & GOZAR-E-PAYAN & 55 & 0 & 0 & 0 & 0 \\
\hline FAIZABAD & 12578 & KOLABI & KOLABI & 1,500 & 200 & 13 & 6 & 1,200 \\
\hline FAIZABAD & 12824 & KOOL DARA & SANGCHIL ZYARAT & 215 & 0 & 0 & 0 & 0 \\
\hline FAIZABAD & 12827 & KOOL DARA & MASJED DEHPAYAN & 420 & 0 & 0 & 0 & 0 \\
\hline FAIZABAD & 12654 & KORCHESHMAH & KORCHESHMAH & 250 & 0 & 0 & 0 & 0 \\
\hline FAIZABAD & 13438 & KOREC & MASJID JAME & 155 & 25 & 16 & 4 & 100 \\
\hline FAIZABAD & 13397 & KUMORKHOR & MASJED NEYAZBY & 200 & 0 & 0 & 0 & 0 \\
\hline FAIZABAD & 12673 & KUMORKHUR & KUMORKHUR & 250 & 0 & 0 & 0 & 0 \\
\hline FAIZABAD & 12529 & KUR GYAN & KUR GYAN & 180 & 30 & 17 & 5 & 150 \\
\hline FAIZABAD & 12730 & KURI & KURI & 160 & 20 & 13 & 5 & 100 \\
\hline FAIZABAD & 13396 & LAL ABAH & LAL ABAH & 100 & 20 & 20 & 5 & 100 \\
\hline FAIZABAD & 14010 & LANGAR & SANG AAB & 100 & 0 & 0 & 0 & 0 \\
\hline FAIZABAD & 12891 & LANGAR & SANG AAB-E-BALA & 60 & 0 & 0 & 0 & 0 \\
\hline FAIZABAD & 12889 & LANGAR & LANGAR & 150 & 0 & 0 & 0 & 0 \\
\hline FAIZABAD & 12546 & LANGAR & MADRASAH & 550 & 50 & 9 & 6 & 300 \\
\hline FAIZABAD & 12562 & LANGAR & LANGAR & 2,500 & 200 & 8 & 7 & 1,400 \\
\hline
\end{tabular}

* 1 Jerib $=1 / 5 \mathrm{Ha}$

* 2 Dry Opium $=$ Wet Opium $-30 \%$ moisture 
Opium Production Survey by village in Badakhshan province, 1995.

\begin{tabular}{|c|c|c|c|c|c|c|c|c|}
\hline \multirow[b]{3}{*}{ District } & \multirow{3}{*}{$\begin{array}{c}\text { Village } \\
\text { Code }\end{array}$} & \multirow[b]{3}{*}{ Areal Main Village } & \multirow[b]{3}{*}{ Village } & \multirow{3}{*}{\begin{tabular}{|c|} 
Total \\
Cultivated \\
Land \\
\end{tabular}} & \multicolumn{2}{|c|}{ Land Under } & \multirow{3}{*}{$\begin{array}{c}\text { Wet Opium }^{* 2} \\
\text { Yield } \\
(\mathrm{kg} / \mathrm{jr} .)\end{array}$} & \multirow{3}{*}{\begin{tabular}{|l} 
Wet Opium \\
Production \\
$(\mathrm{kg})$
\end{tabular}} \\
\hline & & & & & \multicolumn{2}{|c|}{ Poppy Cultivation } & & \\
\hline & & & & & $\left(\right.$ Jerib $\left.^{*}\right)$ & $(\%)$ & & \\
\hline \multicolumn{9}{|c|}{ Province: Badakhshan } \\
\hline FAIZABAD & 12800 & LAYABAH & LAYABAH & 140 & 0 & 0 & 0 & 0 \\
\hline FAIZABAD & 12853 & LOL DARAH/GULESTANOK & LOL DARAH & 375 & 0 & 0 & 0 & 0 \\
\hline FAIZABAD & 12668 & MADRASA-E-ARGHUND & MADRASA ARGHOND & 350 & 5 & 1 & 6 & 30 \\
\hline FAIZABAD & 13385 & MAGHZAR & MAGHZAR & 23 & 0 & 0 & 0 & 0 \\
\hline FAIZABAD & 12725 & MAIDA DAGHLAT & MAIDA DAGHLAT & 170 & 40 & 24 & 4 & 160 \\
\hline FAIZABAD & 12575 & MALANGA & MALANGA & 145 & 45 & 31 & 6 & 270 \\
\hline FAIZABAD & 12613 & MALWAYDARAH & MALWAYDARAH & 360 & 8 & 2 & 6 & 48 \\
\hline FAIZABAD & 12669 & MASJED JAME ARGHAND & MASJED JAME & 160 & 7 & 4 & 5 & 35 \\
\hline FAIZABAD & 12570 & MASJID AHMAD & MASJID AHMAD & 100 & 20 & 20 & 5 & 100 \\
\hline FAIZABAD & 13462 & MASJID JAME ARGHUND & MASJID SHEBAR & 130 & 6 & 5 & 5 & 30 \\
\hline FAIZABAD & 12551 & MASJID QUTAN & MASJID QUTAN & 360 & 30 & 8 & 7 & 210 \\
\hline FAIZABAD & 12574 & MASJID-E-JAME & MASJID-E-JAME & 250 & 50 & 20 & 6 & 300 \\
\hline FAIZABAD & 12544 & MIR BAQI & MIR BAQI & 680 & 100 & 15 & 6 & 600 \\
\hline FAIZABAD & 12858 & MIR SHIKARAN & MIR SHIKARAN & 103 & 0 & 0 & 0 & 0 \\
\hline FAIZABAD & 12657 & MORCHAK & MORCHAK & 100 & 10 & 10 & 5 & 50 \\
\hline FAIZABAD & 12522 & MUGHAL TEY & MUGHAL TEY & 160 & 50 & 31 & 8 & 400 \\
\hline FAIZABAD & 13367 & MUNJI & AAB SHAKH-E-BUZ & 200 & 80 & 40 & 5 & 400 \\
\hline FAIZABAD & 12537 & MUNJI & DEH PAS-E-MUNJI & 180 & 40 & 22 & 4 & 160 \\
\hline FAIZABAD & 13366 & MUNJI & MASJED-E-TEGHAH & 140 & 60 & 43 & 5 & 300 \\
\hline FAIZABAD & 12527 & MUNJI & DEH BALA & 220 & 90 & 41 & 6 & 540 \\
\hline FAIZABAD & 12521 & MUNJI & MUNJI & 160 & 50 & 31 & 5 & 250 \\
\hline FAIZABAD & 12847 & NALAND & NALAND & 300 & 0 & 0 & 0 & 0 \\
\hline FAIZABAD & 12875 & NAR DARAH & NAR DARAH PAYAN & 50 & 0 & 0 & 0 & 0 \\
\hline FAIZABAD & 12873 & NAR DARAH & NAR DARAH BALA & 140 & 0 & 0 & 0 & 0 \\
\hline FAIZABAD & 12880 & NAR DARAH & KHAS AABAH & 36 & 0 & 0 & 0 & 0 \\
\hline FAIZABAD & 12692 & NARMANGAB & NARMANGAB & 400 & 0 & 0 & 0 & 0 \\
\hline FAIZABAD & 12849 & NASREEN & NASREEN SOFLA & 135 & 0 & 0 & 0 & 0 \\
\hline FAIZABAD & 12851 & NASREEN & NASREEN OLYA & 370 & 0 & 0 & 0 & 0 \\
\hline FAIZABAD & 12517 & NAW ABAD & NAW ABAD & 100 & 30 & 30 & 5 & 150 \\
\hline
\end{tabular}

* I Jerib $=1 / 5 \mathrm{Ha}$

* ${ }^{2}$ Dry Opium $=$ Wet Opium $-30 \%$ moisture 
Opium Production Survey by village in Badakhshan province, 1995.

\begin{tabular}{|c|c|c|c|c|c|c|c|c|}
\hline \multirow[b]{3}{*}{ District } & \multirow{3}{*}{$\begin{array}{l}\text { Village } \\
\text { Code } \\
\end{array}$} & \multirow[b]{3}{*}{ Area/ Main Village } & \multirow[b]{3}{*}{ Village } & \multirow{3}{*}{\begin{tabular}{|c|} 
Total \\
Cultivated \\
Land \\
\end{tabular}} & \multirow{2}{*}{\multicolumn{2}{|c|}{$\begin{array}{c}\text { Land Under } \\
\text { Poppy Cultivation }\end{array}$}} & \multirow{3}{*}{$\begin{array}{c}\text { Wet Opium }^{* 2} \\
\text { Yield } \\
(\mathrm{kg} / \mathrm{jr} .)\end{array}$} & \multirow{3}{*}{$\begin{array}{c}\text { Wet Opium } \\
\text { Production } \\
(\mathrm{kg})\end{array}$} \\
\hline & & & & & & & & \\
\hline & & & & & $\left(\right.$ Jerib $\left.^{*}\right)$ & $(\%)$ & & \\
\hline \multicolumn{9}{|c|}{ Province: Badakhshan } \\
\hline FAIZABAD & 13377 & NEEM TALAH & MASJID ROID AAB & 300 & 55 & 18 & 6 & 330 \\
\hline FAIZABAD & 13376 & NEEM TALAH & MASJIE DEH MIANA & 350 & 50 & 14 & 5 & 250 \\
\hline FAIZABAD & 13375 & NEEM TALAH & MASJID DEH BALA & 300 & 50 & 17 & 5 & 250 \\
\hline FAIZABAD & 12530 & NEEM TALAH & MASJID DASTGEER & 300 & 50 & 17 & 7 & 350 \\
\hline FAIZABAD & 12505 & NEEM TALAH & MASJID NAMATULA & 250 & 100 & 40 & 4 & 400 \\
\hline FAIZABAD & 12528 & NEEM TALAH & MASJID-E-JAME & 400 & 100 & 25 & 7 & 700 \\
\hline FAIZABAD & 12506 & NEEMTALLAH & MASJID MABAIN & 310 & 80 & 26 & 6 & 480 \\
\hline FAIZABAD & 14003 & NOW ABAD & NOW ABAD & 250 & 65 & 26 & 7 & 455 \\
\hline FAIZABAD & 13365 & NOW ABAD & MASJED-E-GHULAM & 210 & 40 & 19 & 6 & 240 \\
\hline FAIZABAD & 12840 & PAHAN DARAH & PAHAN DARAH SUFLA & 56 & 0 & 0 & 0 & 0 \\
\hline FAIZABAD & 12850 & PAHEN DARAH & PAHEN DARAH ULY & 155 & 0 & 0 & 0 & 0 \\
\hline FAIZABAD & 13429 & PALANG DARAH & PALANG DARAH & 90 & 0 & 0 & 0 & 0 \\
\hline FAIZABAD & 12762 & PASI BAND & ZRANGAN DARALS & 250 & 0 & 0 & 0 & 0 \\
\hline FAIZABAD & 13440 & PASI BAND & DEGA & 280 & 0 & 0 & 0 & 0 \\
\hline FAIZABAD & 12753 & PASI BAND & KARAKSHAHK & 40 & 0 & 0 & 0 & 0 \\
\hline FAIZABAD & 12757 & PASI BAND & DEH TAGAB & 50 & 0 & 0 & 0 & 0 \\
\hline FAIZABAD & 13406 & PASI BAND & KHAM SARBANAN & 100 & 0 & 0 & 0 & 0 \\
\hline FAIZABAD & 12594 & PASI BAND & DASHTAK & 30 & 0 & 0 & 0 & 0 \\
\hline FAIZABAD & 12771 & PASI BAND & DARGAK & 100 & 0 & 0 & 0 & 0 \\
\hline FAIZABAD & 13415 & PASI BAND & PASI BAND & 50 & 0 & 0 & 0 & 0 \\
\hline FAIZABAD & 12776 & PASI BAND & KHAM-I-MIR & 200 & 0 & 0 & 0 & 0 \\
\hline FAIZABAD & 12783 & PASI BAND & KAJI KHAM BALA & 100 & 0 & 0 & 0 & 0 \\
\hline FAIZABAD & 13419 & PASI BAND & ERT & 100 & 0 & 0 & 0 & 0 \\
\hline FAIZABAD & 13418 & PASI BAND & SELAGI DUSHT & 60 & 0 & 0 & 0 & 0 \\
\hline FAIZABAD & 13417 & PASI BAND & ANGORA-E-KALAN & 100 & 0 & 0 & 0 & 0 \\
\hline FAIZABAD & 12780 & PASI BAND & KHAMBEL & 150 & 0 & 0 & 0 & 0 \\
\hline FAIZABAD & 13409 & PASI BAND & RAGH DARAH & 40 & 0 & 0 & 0 & 0 \\
\hline FAIZABAD & 12782 & PASI BAND & KHAM HAFIZ & 300 & 0 & 0 & 0 & 0 \\
\hline FAIZABAD & 12779 & PASI BAND & KHAIR ABAD & 60 & 0 & 0 & 0 & 0 \\
\hline
\end{tabular}


Opium Production Survey by village in Badakhshan province, 1995.

\begin{tabular}{|c|c|c|c|c|c|c|c|c|}
\hline \multirow[b]{3}{*}{ District } & \multirow{3}{*}{$\begin{array}{l}\text { Village } \\
\text { Code }\end{array}$} & \multirow[b]{3}{*}{ Area/ Main Village } & \multirow[b]{3}{*}{ Village } & \multirow{3}{*}{$\begin{array}{c}\text { Total } \\
\text { Cultivated } \\
\text { Land } \\
\end{array}$} & \multirow{2}{*}{\multicolumn{2}{|c|}{$\begin{array}{c}\text { Land Under } \\
\text { Poppy Cultivation }\end{array}$}} & \multirow{3}{*}{$\begin{array}{c}{\text { Wet } \text { Opium }^{* 2}} \\
\text { Yield } \\
(\mathrm{kg} / \mathrm{jr} .) \\
\end{array}$} & \multirow{3}{*}{$\begin{array}{c}\text { Wet Opium } \\
\text { Production } \\
\text { (kg) }\end{array}$} \\
\hline & & & & & & & & \\
\hline & & & & & $\left(\right.$ Jerib $\left.^{*}\right)$ & $(\%)$ & & \\
\hline \multicolumn{9}{|c|}{ Province: Badakhshan } \\
\hline FAIZABAD & 12777 & PASI BAND & KAJI KHAM PAYAN & 80 & 1 & 1 & 5 & 5 \\
\hline FAIZABAD & 13416 & PASI BAND & DO ABA-E-YAFTAL & 200 & 2 & 1 & 7 & 14 \\
\hline FAIZABAD & 12766 & PASI BAND & DEH BALA & 180 & 1 & 1 & 6 & 6 \\
\hline FAIZABAD & 13428 & PASI BAND & NAGHZ DARAH & 200 & 0 & 0 & 0 & 0 \\
\hline FAIZABAD & 12752 & PASI BAND & SAR-E-QUL-E-PAS & 50 & 0 & 0 & 0 & 0 \\
\hline FAIZABAD & 12751 & PASI BAND & PASHEAYAL & 105 & 0 & 0 & 0 & 0 \\
\hline FAIZABAD & 13430 & PASI BAND & ANIW & 200 & 0 & 0 & 0 & 0 \\
\hline FAIZABAD & 12748 & PASI BAND & ZAKAK & 180 & 0 & 0 & 0 & 0 \\
\hline FAIZABAD & 13425 & PASI BAND & LANGAR & 66 & 0 & 0 & 0 & 0 \\
\hline FAIZABAD & 13424 & PASI BAND & PUNITUN & 85 & 0 & 0 & 0 & 0 \\
\hline FAIZABAD & 12761 & PASI BAND & ABI DARAH & 250 & 0 & 0 & 0 & 0 \\
\hline FAIZABAD & 12767 & PASI BAND & DEH BLAND & 110 & 0 & 0 & 0 & 0 \\
\hline FAIZABAD & 13407 & PASI BAND & SENJIT & 100 & 0 & 0 & 0 & 0 \\
\hline FAIZABAD & 13408 & PASI BAND & MARGAN DARAH & 60 & 0 & 0 & 0 & 0 \\
\hline FAIZABAD & 13427 & PASI BAND & COLGI DARAH & 170 & 0 & 0 & 0 & 0 \\
\hline FAIZABAD & 13426 & PASI BAND & AL DARAH & 100 & 0 & 0 & 0 & 0 \\
\hline FAIZABAD & 14009 & PASI BAND & ZARDALAW & 100 & 0 & 0 & 0 & 0 \\
\hline FAIZABAD & 12760 & PASI BAND & SHAH KALAN & 200 & 0 & 0 & 0 & 0 \\
\hline FAIZABAD & 12759 & PASI BAND & SHAH KHURD & 150 & 0 & 0 & 0 & 0 \\
\hline FAIZABAD & 12770 & PASI BAND & SHAHID DARAH & 90 & 0 & 0 & 0 & 0 \\
\hline FAIZABAD & 12775 & PASI BAND & MUZUNG & 120 & 0 & 0 & 0 & 0 \\
\hline FAIZABAD & 12768 & PASI BAND & DAREL & 200 & 0 & 0 & 0 & 0 \\
\hline FAIZABAD & 12631 & PASI JAR & PASI JAR & 110 & 12 & 11 & 7 & 84 \\
\hline FAIZABAD & 12888 & PASTA KALAN & PASTA KALAN & 0 & 0 & $\cdot$ & 0 & 0 \\
\hline FAIZABAD & 12836 & PAZLA KHWAH & PAZLA KHWAH & 135 & 0 & 0 & 0 & 0 \\
\hline FAIZABAD & 12723 & PECHIQ AND ANGRO & PECHIQ AND ANGR & 400 & 50 & 13 & 7 & 350 \\
\hline FAIZABAD & 12555 & PENGANI & MASJID QURBAN & 220 & 20 & 9 & 6 & 120 \\
\hline FAIZABAD & 12550 & PENGANI & MASJID ABDULLAH & 450 & 30 & 7 & 6 & 180 \\
\hline FAIZABAD & 12554 & PENGANI & MASDJID A. AZIZ & 100 & 20 & 20 & 6 & 120 \\
\hline
\end{tabular}


Opium Production Survey by village in Badakhshan province, 1995.

\begin{tabular}{|c|c|c|c|c|c|c|c|c|}
\hline \multirow[b]{3}{*}{ District } & \multirow{3}{*}{$\begin{array}{l}\text { Village } \\
\text { Code }\end{array}$} & \multirow[b]{3}{*}{ Area/ Main Village } & \multirow[b]{3}{*}{ Village } & \multirow{3}{*}{$\begin{array}{c}\text { Total } \\
\text { Cultivated } \\
\text { Land }\end{array}$} & \multirow{2}{*}{\multicolumn{2}{|c|}{$\begin{array}{c}\text { Land Under } \\
\text { Poppy Cultivation }\end{array}$}} & \multirow{3}{*}{\begin{tabular}{|c|} 
Wet Opium $^{* 2}$ \\
Yield \\
$(\mathrm{kg} / \mathrm{jr})$. \\
\end{tabular}} & \multirow{3}{*}{$\begin{array}{c}\text { Wet Opium } \\
\text { Production } \\
(\mathrm{kg}) \\
\end{array}$} \\
\hline & & & & & & & & \\
\hline & & & & & $\left(\right.$ Jerib $\left.^{*}\right)$ & $(\%)$ & & \\
\hline \multicolumn{9}{|c|}{ Province: Badakhshan } \\
\hline FAIZABAD & 12552 & PENGANI & MASJID JAME & 500 & 40 & 8 & 6 & 240 \\
\hline FAIZABAD & 12556 & PENGANI & MASJED-E-MULA SARDAR & 280 & 30 & 11 & 5 & 150 \\
\hline FAIZABAD & 12553 & PENGANI & MASJED MADRASA & 400 & 60 & 15 & 5 & 300 \\
\hline FAIZABAD & 13401 & PESHKA & PESHKA & 50 & 0 & 0 & 0 & 0 \\
\hline FAIZABAD & 12665 & PETAW & PETAW & 370 & 15 & 4 & 4 & 60 \\
\hline FAIZABAD & 12512 & PETAWAK & PETAWAK & 130 & 60 & 46 & 7. & 420 \\
\hline FAIZABAD & 12511 & PETAWAK & PETAWAK SUFLA & 280 & 60 & 21 & 6 & 360 \\
\hline FAIZABAD & 13432 & PULAR LIKWEEK & PULAR LIKWEEK & 80 & 10 & 13 & 4 & 40 \\
\hline FAIZABAD & 12621 & QAILAH DARAH & QAILAH DARAH & 510 & 15 & 3 & 8 & 120 \\
\hline FAIZABAD & 12696 & QARA KAMA & QARA KAMA & 190 & 2 & 1 & 4 & 8 \\
\hline FAIZABAD & 13395 & QARA KAMAR & NAW ABAD & 55 & 0 & 0 & 0 & 0 \\
\hline FAIZABAD & 12700 & QARA QUZI & QARA QUZI & 430 & 20 & 5 & 9 & 180 \\
\hline FAIZABAD & 12864 & QARACHAH & QARACHA & 510 & 2 & 0 & 4 & 8 \\
\hline FAIZABAD & 12655 & QARAH MUGHUL & QARAH MUGHUL & 210 & 30 & 14 & 5 & 150 \\
\hline FAIZABAD & 12638 & QARAH MUGHUL & MASJID JAME & 0 & 0 & $\cdots$ & 0 & 0 \\
\hline FAIZABAD & 13392 & QARGHAH & QARGHAH & 130 & 10 & 8 & 10 & 100 \\
\hline FAIZABAD & 12547 & QARLOQ QROQ & QARLOQ QROQ & 4,000 & 570 & 14 & 7 & 3,990 \\
\hline FAIZABAD & 12543 & QARLOQ QROQ & MOMEN ABAD & 280 & 70 & 25 & 6 & 420 \\
\hline FAIZABAD & 12557 & QARLOQ ZER KOTEL & QARLOQ ZER KOTE & 0 & 0 & 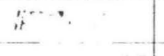 & 0 & 0 \\
\hline FAIZABAD & 12646 & QASH QULAQ & QASH QULAQ & 260 & 40 & 15 & 4 & 160 \\
\hline FAIZABAD & 12681 & QATAR KHERMAN & QATAR KHERMAN & 220 & 0 & 0 & 0 & 0 \\
\hline FAIZABAD & 12860 & QAWIZ & QAWIZ & 106 & 1 & 0 & 5 & 3 \\
\hline FAIZABAD & 12588 & QAZI QESHLAQ & QAZI QESHLAQ & 400 & 30 & 8 & 7 & 210 \\
\hline FAIZABAD & 12699 & QOCHI & QOCHI & 300 & 0 & 0 & 0 & 0 \\
\hline FAIZABAD & 12887 & QODUQ & MASJED PAYAN & 125 & 0 & 0 & 0 & 0 \\
\hline FAIZABAD & 12877 & QODUQ & MASJED JAMEE & 120 & 0 & 0 & 0 & 0 \\
\hline FAIZABAD & 12519 & QOWAT ALI & QOWAT ALI & 750 & 40 & 5 & 6 & 240 \\
\hline FAIZABAD & 12911 & RABATAK & RABATAK & 1,860 & 0 & 0 & 0 & 0 \\
\hline FAIZABAD & 12809 & RAZAN-E-SOM DARA & RAZAN-E-SOM DARA & 240 & 80 & 33 & 6 & 480 \\
\hline
\end{tabular}

$*$ I Jerib $=1 / 5 \mathrm{Ha}$

${ }^{*}$ Dry Opium $=$ Wet Opium $-30 \%$ moisture 
Opium Production Survey by village in Badakhshan province, 1995.

\begin{tabular}{|c|c|c|c|c|c|c|c|c|}
\hline \multirow[b]{3}{*}{ District } & \multirow{3}{*}{$\begin{array}{l}\text { Village } \\
\text { Code } \\
\end{array}$} & \multirow[b]{3}{*}{ Area/ Main Village } & \multirow[b]{3}{*}{ Village } & \multirow{3}{*}{$\begin{array}{c}\text { Total } \\
\text { Cultivated } \\
\text { Land } \\
\end{array}$} & \multirow{2}{*}{\multicolumn{2}{|c|}{$\begin{array}{c}\text { Land Under } \\
\text { Poppy Cultivation }\end{array}$}} & \multirow{3}{*}{$\begin{array}{c}\text { Wet Opium }^{* 2} \\
\text { Yield } \\
(\mathrm{kg} / \mathrm{jr} .)\end{array}$} & \multirow{3}{*}{$\begin{array}{c}\text { Wet Opium } \\
\text { Production } \\
(\mathrm{kg})\end{array}$} \\
\hline & & & & & & & & \\
\hline & & & & & $\left(\right.$ Jerib $\left.^{*}\right)$ & $(\%)$ & & \\
\hline \multicolumn{9}{|c|}{ Province: Badakhshan } \\
\hline FAIZABAD & 12542 & RESHDARA $Z$ & RESHDARAZ SOFLA & 250 & 90 & 36 & 7 & 630 \\
\hline FAIZABAD & 12541 & RESHDAR $\_\mathrm{Z}$ & RESHDARAZ OLYA & 300 & 100 & 33 & 6 & 600 \\
\hline FAIZABAD & 12600 & ROBAT & ROBAT & 200 & 80 & 40 & 9 & 720 \\
\hline FAIZABAD & 12695 & SABZABAHAR & SABZABAHAR & 520 & 0 & 0 & 0 & 0 \\
\hline FAIZABAD & 12376 & SAFEDAG PELBAND & SAFEDAG PELBAND & 102 & 0 . & 0 & 0 & 0 \\
\hline FAIZABAD & 12917 & SAFEDARAL MANSOOR & SAFEDARAL MANSOOR & 105 & 8 & 8 & 4 & 32 \\
\hline FAIZABAD & 12709 & SAMATI & SAMATI & 250 & 0 & 0 & 0 & 0 \\
\hline FAIZABAD & 12886 & SAR KHAK & NAW ABAD & 135 & 1 & 1 & 5 & 5 \\
\hline FAIZABAD & 13411 & SAR-E-TAL & SAR-E-TAL & 70 & 1 & 1 & 9 & 9 \\
\hline FAIZABAD & 12683 & SARIDARAH & SARIDARAH & 100 & 0 & 0 & 0 & 0 \\
\hline FAIZABAD & 12834 & SAROON & SARBAND & 450 & 0 & 0 & 0 & 0 \\
\hline FAIZABAD & 12852 & SEMEEN JAB & SEMEEN JAB & 1,510 & 0 & 0 & 0 & 0 \\
\hline FAIZABAD & 12693 & SHAH WAZIR & SHAH WAZIR & 70 & 0 & 0 & 0 & 0 \\
\hline FAIZABAD & 12612 & SHAHMARI & SHAHMARI & 180 & 80 & 44 & 10 & 800 \\
\hline FAIZABAD & 12610 & SHAHMARI & M. JAME SHAMASI & 140 & 50 & 36 & 10 & 500 \\
\hline FAIZABAD & 12611 & SHAHMARI & NOW ABAD & 70 & 40 & 57 & 10 & 400 \\
\hline FAIZABAD & 12344 & SHAKARLAB & DEH BALA & 430 & 0 & 0 & 0 & 0 \\
\hline FAIZABAD & 12842 & SHAKARLAB & SHAKARLAB BALA & 3,100 & 0 & 0 & 0 & 0 \\
\hline FAIZABAD & 12838 & SHAKARLAB & SHAHIL & 210 & 0 & 0 & 0 & 0 \\
\hline FAIZABAD & 12343 & SHAKARLAB & SARI SANG & 160 & 0 & 0 & 0 & 0 \\
\hline FAIZABAD & 12346 & SHAKARLAB & DEH PAYAN & 310 & 0 & 0 & 0 & 0 \\
\hline FAIZABAD & 12345 & SHAKARLAB & SOKHTA KOH & 385 & 0 & 0 & 0 & 0 \\
\hline FAIZABAD & 12839 & SHAKARLAB & WAJKIL YONS PAHEN DA & 300 & 0 & 0 & 0 & 0 \\
\hline FAIZABAD & 12532 & SHAKH GULLAN & SHAKH GULLAN & 80 & 25 & 31 & 7 & 175 \\
\hline FAIZABAD & 12902 & SHALKHAH ZAAR & SHALKHAH ZAAR & 700 & 0 & 0 & 0 & 0 \\
\hline FAIZABAD & 13393 & SHATAK & KHUSHDARAH & 240 & 3 & 1 & 5 & 15 \\
\hline FAIZABAD & 12674 & SHATAK & TALDARAH & 50 & 2 & 4 & 5 & 10 \\
\hline FAIZABAD & 13454 & SHATAK & MADRASA SHATAK & 140 & 25 & 18 & 6 & 150 \\
\hline FAIZABAD & 12684 & SHATAK & SHATAK & 210 & 40 & 19 & 6 & 240 \\
\hline
\end{tabular}


Opium Production Survey by village in Badakhshan province, 1995.

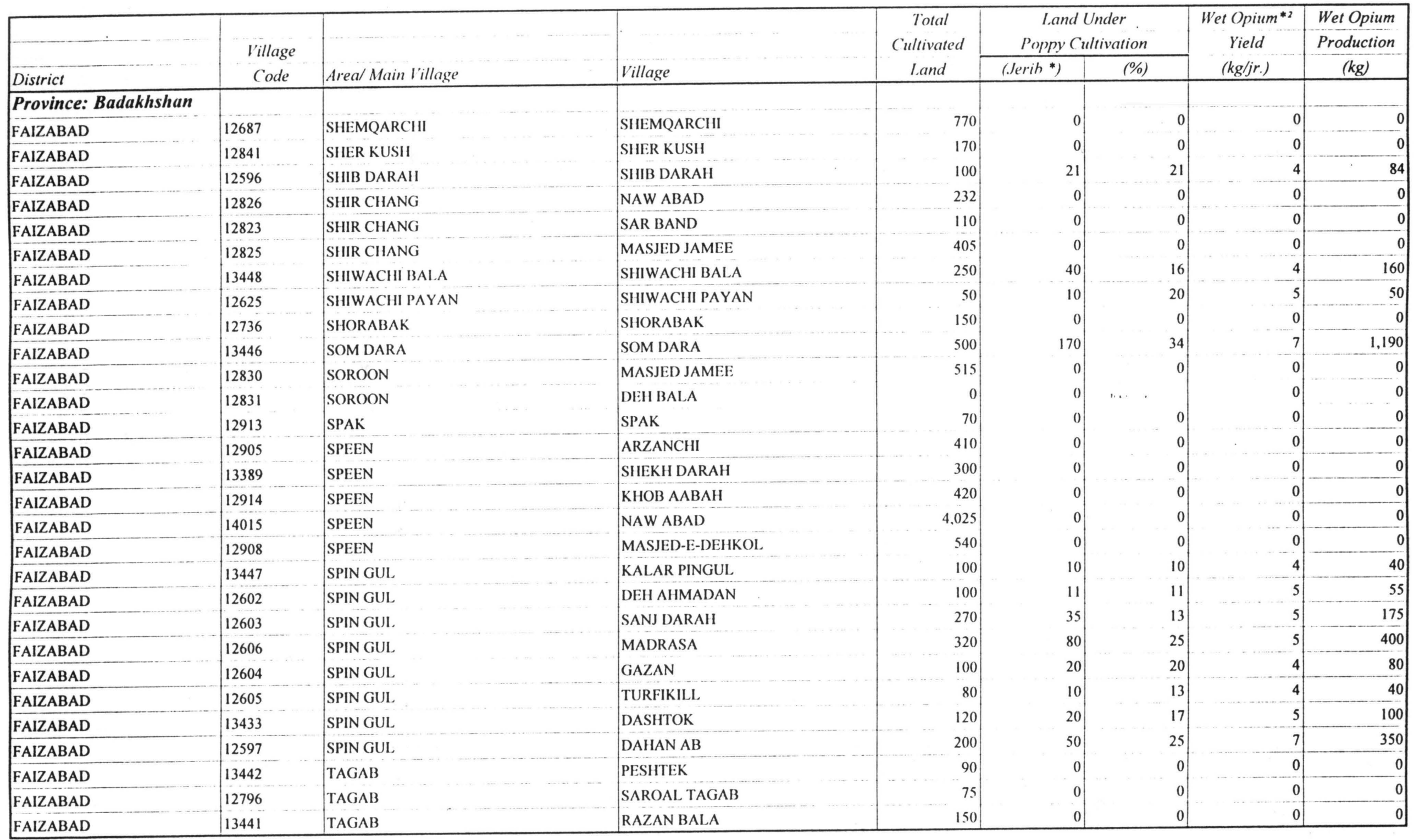

* 1 Jerib $=1 / 5 \mathrm{Ha}$

* ${ }^{2}$ Dry Opium $=$ Wet Opium $-30 \%$ moisture 
Opium Production Survey by village in Badakhshan province, 1995.

\begin{tabular}{|c|c|c|c|c|c|c|c|c|}
\hline \multirow[b]{3}{*}{ District } & \multirow{3}{*}{$\begin{array}{c}\text { Village } \\
\text { Code }\end{array}$} & \multirow{3}{*}{ Area/ Main Village } & \multirow[b]{3}{*}{ Village } & \multirow{3}{*}{$\begin{array}{c}\text { Total } \\
\text { Cultivated } \\
\text { Land } \\
\end{array}$} & \multirow{2}{*}{\multicolumn{2}{|c|}{$\begin{array}{c}\text { Land Under } \\
\text { Poppy Cultivation }\end{array}$}} & \multirow{3}{*}{$\begin{array}{c}\text { Wet Opium }{ }^{* 2} \\
\text { Yield } \\
\text { (kg/jr. }) \\
\end{array}$} & \multirow{3}{*}{$\frac{\frac{\text { Wet Opium }}{\text { Production }}}{\text { (kg) }}$} \\
\hline & & & & & & & & \\
\hline & & & & & $\left.(. J e r i b)^{*}\right)$ & (\%) & & \\
\hline \multicolumn{9}{|c|}{ Province: Badakhshan } \\
\hline FAIZABAD & 12791 & TAGAB & NARWAN & 30 & 0 & 0 & 0 & 이 \\
\hline FAIZABAD & 12785 & TAGAB & GAZ DARAH & 100 & 0 & 0 & 0 & 0 \\
\hline FAIZABAD & 13431 & TAGAB & SHIN DARAII & 75 & 0 & 0 & 0 & 0 \\
\hline FAIZABAD & 12764 & TAGAB & KHUMAR DARAH & 250 & 0 & 0 & 0 & 0 \\
\hline FAIZABAD & 13444 & TAGAB & ABRI DARAH & 160 & 0 & 0 & 0 & 0 \\
\hline FAIZABAD & 12793 & TAGAB & KOLOKH KAN & 70 & 0 & 0 & 0 & 0 \\
\hline FAIZABAD & 13443 & TAGAB & GHONGA & 150 & 0 & 0 & 0 & 0 \\
\hline FAIZABAD & 12792 & TAGAB & SUMCHAN & 200 & 0 & 0 & 0 & 0 \\
\hline FAIZABAD & 12788 & TAGAB & DAARAH-E-KALAN & 70 & 0 & 0 & 0 & 0 \\
\hline FAIZABAD & 12805 & TAGAB & SAR GALLAHA & 200 & 0 & 0 & 0 & 0 \\
\hline FAIZABAD & 13451 & TAGAB & OGAR-I-PAYAN & 80 & 2 & 3 & 6 & 12 \\
\hline FAIZABAD & 12787 & TAGAB & SHAHZAMIN & 50 & 0 & 0 & 0 & 0 \\
\hline FAIZABAD & 12755 & TAGAB & BAGHI GERDAK & 35 & 0 & 0 & 0 & 0 \\
\hline FAIZABAD & 14004 & TAGAB & KARSAID & 40 & 0 & 0 & 0 & 0 \\
\hline FAIZABAD & 13414 & TAGAB & TALBAZANG BALA & 70 & 0 & 0 & 0 & 0 \\
\hline FAIZABAD & 12784 & TAGAB & SYA SHAKH & 104 & 0 & 0 & 0 & 0 \\
\hline FAIZABAD & 12802 & TAGAB & TALBAZANG PAYAN & 50 & 1 & 2 & 8 & 8 \\
\hline FAIZABAD & 13413 & TAGAB & DEH MIANA & 100 & 3 & 3 & 9 & 27 \\
\hline FAIZABAD & 12789 & TAGAB & DEH KALAN & 300 & 2 & 1 & 7. & 14 \\
\hline FAIZABAD & 12798 & TAGAB & RAZAN PAYAN & 130 & 0 & 0 & 0 & 0 \\
\hline FAIZABAD & 12807 & TAGAB & WARSAK & 300 & 0 & 0 & 0 & 0 \\
\hline FAIZABAD & 12797 & TAGAB & GURK KUSHAN & 80 & 0 & 0 & 0 & 0 \\
\hline FAIZABAD & 12801 & TAGAB & CHOCOLCH & 90 & 2 & 2 & 5 & 10 \\
\hline FAIZABAD & 13380 & TAGAB & OGAR-E-BALA & 170 & 1 & 1 & 5. & 5 \\
\hline FAIZABAD & 13423 & TAGAB & SHAR-E-ZARAN & 60 & 0 & 0 & 0 & 0 \\
\hline FAIZABAD & 12790 & TAGAB & TAWJAHAD PARAH & 270 & 0 & 0 & 0 . & 0 \\
\hline FAIZABAD & 12794 & TAGAB & TAGH DARAH & 300 & 0 & 0 & 0 & 0 \\
\hline FAIZABAD & 13387 & TAGHARCHEK & MASJED-E-MULABU & 125 & 0 & 0 & 0 . & 0 \\
\hline FAIZABAD & 12682 & TAGHARCHEK & TAGHARCHEK & 150 & 0 & 0 & 0 & 0 \\
\hline
\end{tabular}


Opium Production Survey by village in Badakhshan province, 1995.

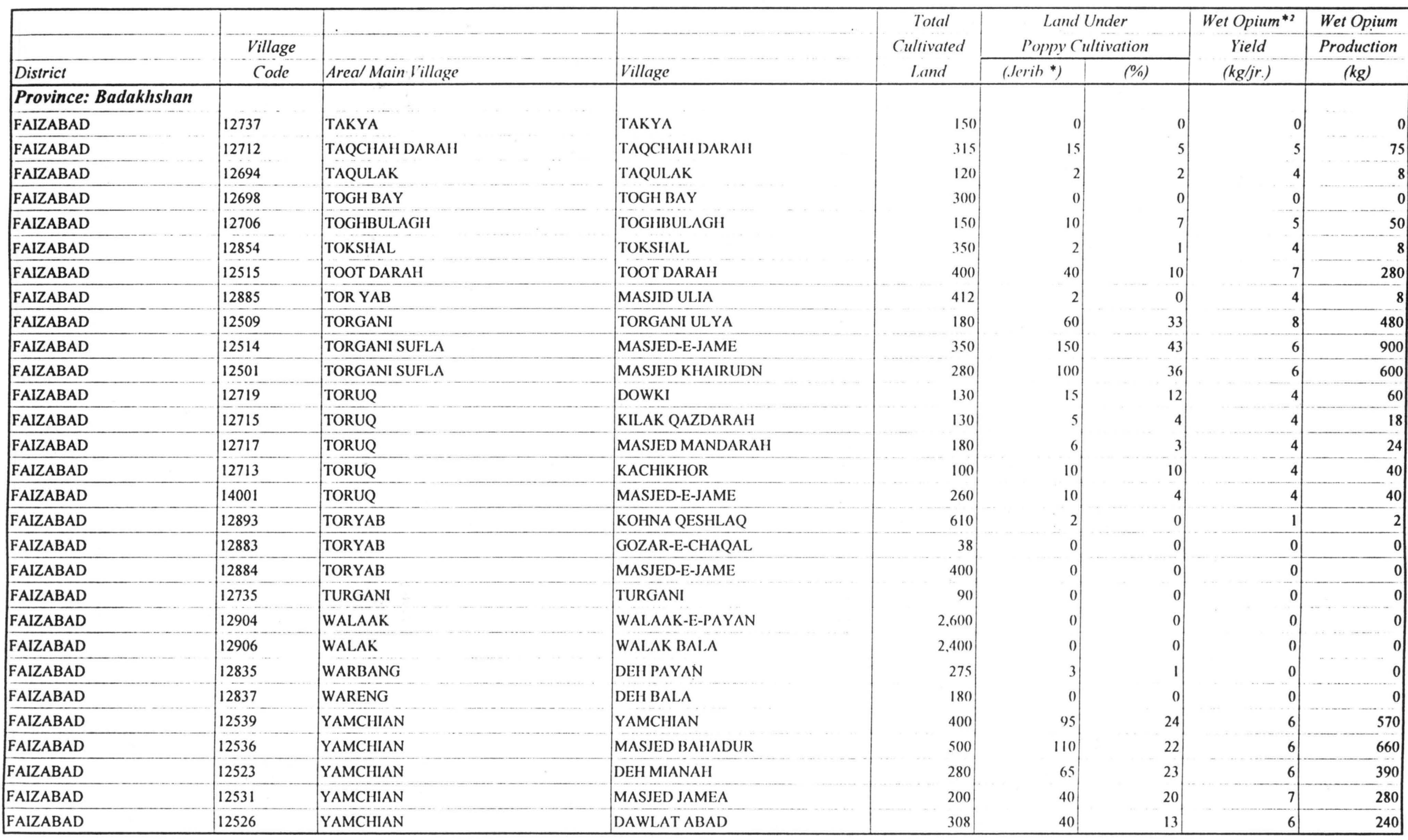

* 1 Jerib $=1 / 5 \mathrm{Ha}$

*2 Dry Opium $=$ Wet Opium $-30 \%$ moisture 
Opium Production Survey by village in Badakhshan province, 1995.

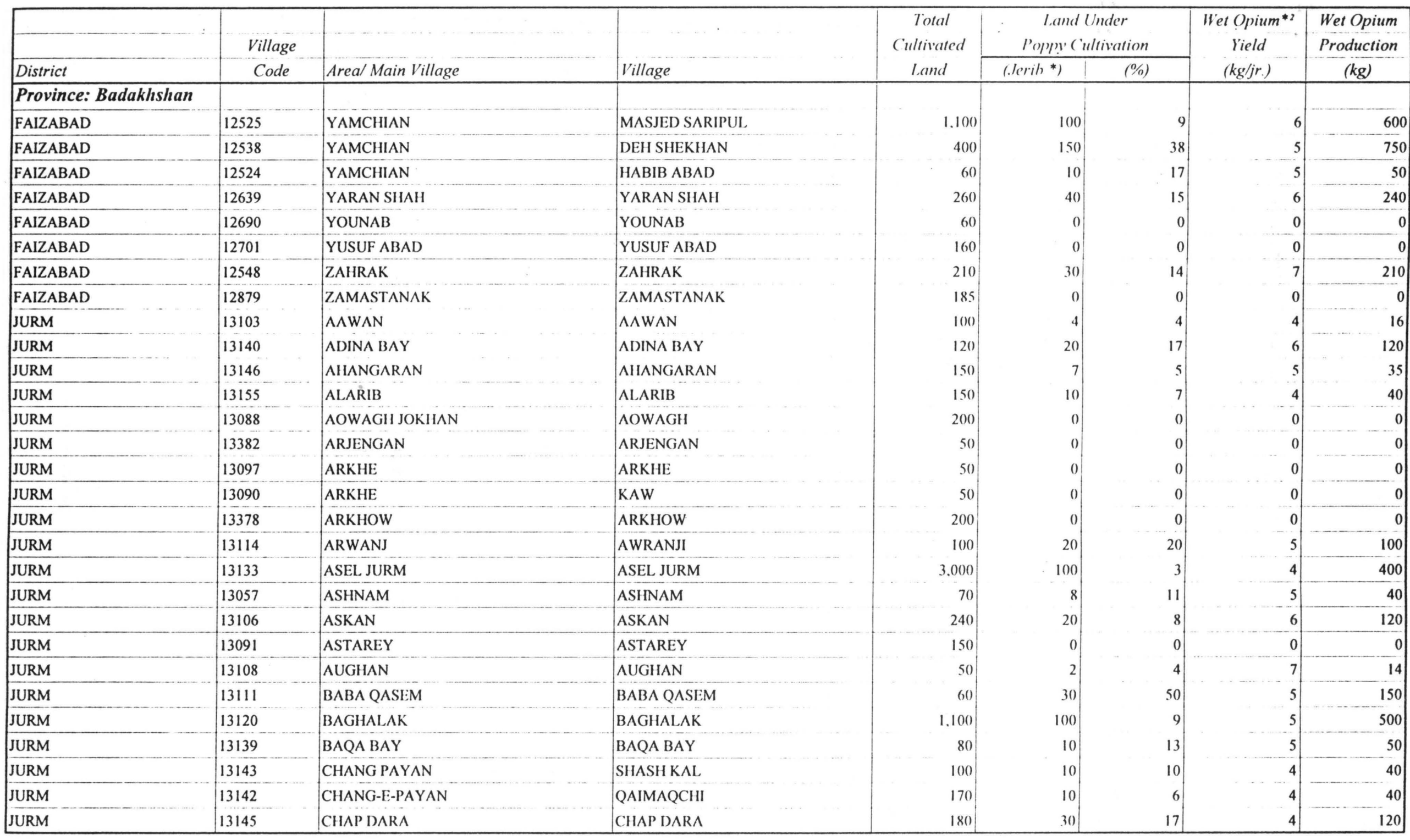

- I Jerib $=1 / 5 \mathrm{Ha}$

* 2 Dry Opium $=$ Wet Opium $-30 \%$ moisture 
Opium Production Survey by village in Badakhshan province, 1995.

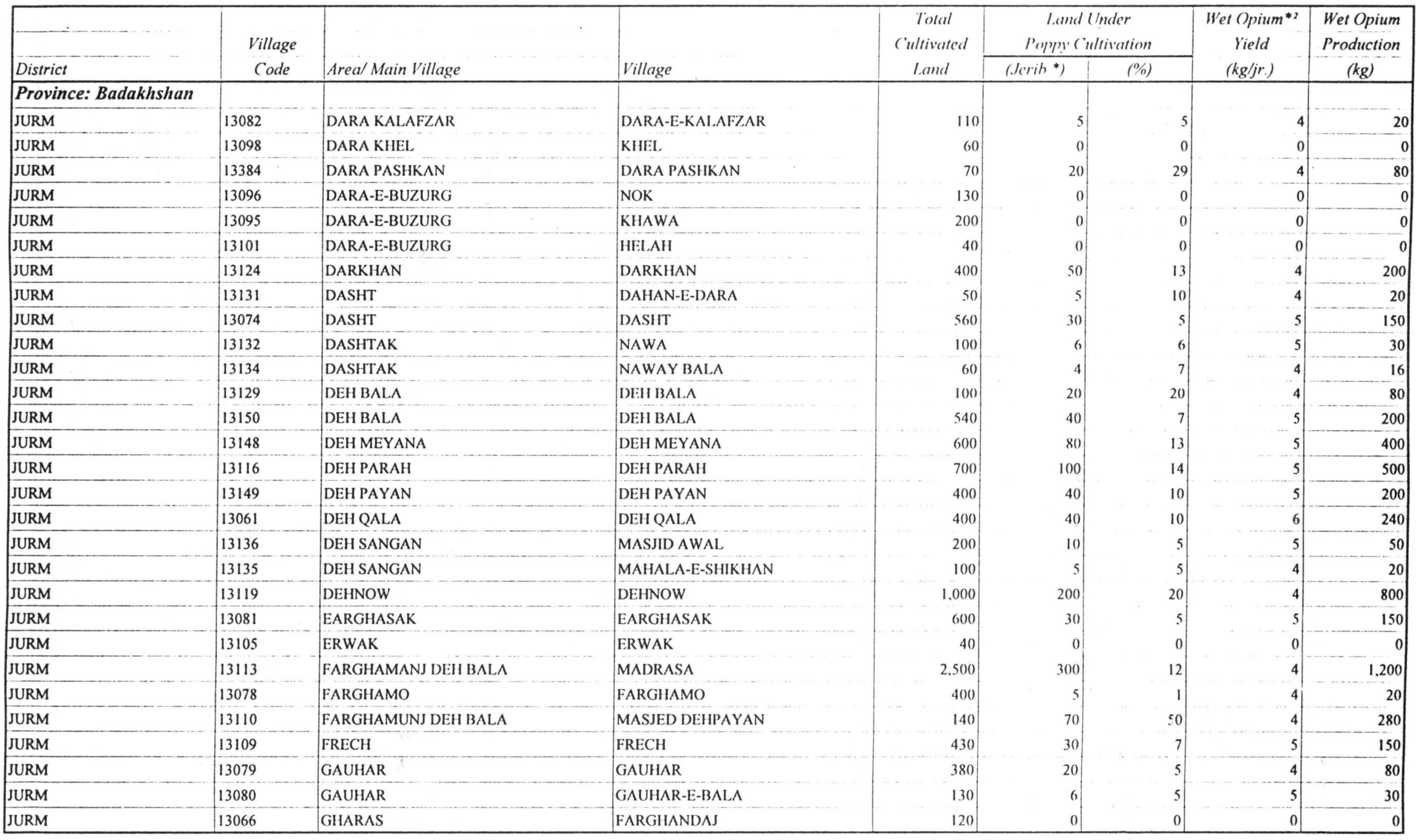


Opium Production Survey by village in Badakhshan province, 1995.

\begin{tabular}{|c|c|c|c|c|c|c|c|c|}
\hline \multirow[b]{3}{*}{ District } & \multirow{3}{*}{$\begin{array}{c}\text { Village } \\
\text { Code }\end{array}$} & \multirow[b]{3}{*}{ Areal Main Village } & \multirow[b]{3}{*}{ Village } & \multirow{3}{*}{$\begin{array}{c}\text { Total } \\
\text { Cultivated } \\
\text { Land } \\
\end{array}$} & \multirow{2}{*}{\multicolumn{2}{|c|}{$\begin{array}{c}\text { Land Under } \\
\text { Poppy Cultivation }\end{array}$}} & \multirow{3}{*}{$\begin{array}{c}\text { Wet Opium }{ }^{* 2} \\
\text { Yield } \\
(\mathrm{kg} / \mathrm{jr} .)\end{array}$} & \multirow{3}{*}{$\begin{array}{c}\text { Wet Opium } \\
\text { Production } \\
(\mathrm{kg}) \\
\end{array}$} \\
\hline & & & & & & & & \\
\hline & & & & & $\left(\right.$ Jerib $\left.^{*}\right)$ & $(\%)$ & & \\
\hline \multicolumn{9}{|c|}{ Province: Badakhshan } \\
\hline JURM & 13070 & GHARAS & KHALGA & 80 & 0 & 0 & 0 & 0 \\
\hline JURM & 13071 & GHARAS & SANOWRY & 100 & 0 & 0 & 0 & 0 \\
\hline JURM & 13073 & GHARAS TAKHT DEH & SARAWASH & 60 & 0 & 0 & 0 & 0 \\
\hline JURM & 13379 & GHARMEY & KHASEP & 50 & 0 & 0 & 0 & 0 \\
\hline JURM & 13068 & GHARMI & MADRASA & 160 & 8 & 5 & 5 & 40 \\
\hline JURM & 13065 & GHARMI & DEH BALA & 90 & 4 & 4 & 4 & 16 \\
\hline JURM & 13147 & HESARAK & HESARAK & 200 & 10 & 5 & 4 & 40 \\
\hline JURM & 13381 & JO KHAN & CHARMAGHZISTAN & 50 & 0 & 0 & 0 & 0 \\
\hline JURM & 13118 & KAJGARAN & KAJGARAN & 500 & 100 & 20 & 4 & 400 \\
\hline JURM & 13086 & KALAFZAR & KALAFZAR & 200 & 5 & 3 & 4 & 20 \\
\hline JURM & 13112 & KAMANGARAN & KAMANGARAN & 350 & 50 & 14 & 4 & 200 \\
\hline JURM & 13154 & KATEEB & KATEEB & 1,200 & 120 & 10 & 5 & 600 \\
\hline JURM & 13093 & KHAERANG & KHAERANG & 150 & 0 & 0 & 0 & 0 \\
\hline JURM & 13072 & KHASH & KHASH & 180 & 0 & 0 & 0 & 0 \\
\hline JURM & 13102 & KHASTAK KHOOL & KHOOL & 200 & 5 & 3 & 5 & 25 \\
\hline JURM & 13087 & KHORI & KHORI & 350 & 20 & 6 & 5 & 100 \\
\hline JURM & 13100 & KOJAGAK & KOJAGAK & 500 & 30 & 6 & 4 & 120 \\
\hline JURM & 13383 & LANGAR H.SAYEED & LANGAR H.SAYEED & 120 & 5 & 4 & 4 & 20 \\
\hline JURM & 13076 & LARKEY & LARKEY & 530 & 30 & 6 & 4 & 120 \\
\hline JURM & 13094 & LERAK ZO & LERAK ZO & 170 & 0 & 0 & 0 & 0 \\
\hline JURM & 13130 & MADRASA & MADRAȘA & 400 & 40 & 10 & 4 & 160 \\
\hline JURM & 13104 & MAGHZAR-E-KHOOL & MAGHZAR-E-KHOOL & 80 & 8 & 10 & 7 & 56 \\
\hline JURM & 13123 & MASJED BALA & MASJED BALA & 1,100 & 50 & 5 & 3. & 150 \\
\hline JURM & 13085 & MASJED MADRASA & MASJED MADRASA & 900 & 30 & 3 & 5 & 150 \\
\hline JURM & 13084 & MASJED PAYAN & MASJED PAYAN & 1,600 & 60 & 4 & 4 & 240 \\
\hline JURM & 13122 & MASJED-E-MADRASA & MASJED MADRASA & 200 & 10 & 5 & 4 & 40 \\
\hline JURM & 13121 & MASJED-E-SAR CHOWK & MASJED SARCHOWK & 1,000 & 50 & 5 & 5 & 250 \\
\hline JURM & 13115 & MISYANI & MISYANI & 500 & 100 & 20 & 4 & 400 \\
\hline JURM & 13127 & MUGHLA ZAMEER & MUGHLA ZAMEER & 60 & 20 & 33 & 4 & 80 \\
\hline
\end{tabular}

* 1 Jerib $=1 / 5 \mathrm{Ha}$

Source: UNDCP Opium Production Survey, 1995 
Opium Production Survey by village in Badakhshan province, 1995.

\begin{tabular}{|c|c|c|c|c|c|c|c|c|}
\hline \multirow[b]{3}{*}{ District } & \multirow{3}{*}{$\begin{array}{l}\text { Village } \\
\text { Code }\end{array}$} & \multirow[b]{3}{*}{ Area/ Main Village } & \multirow[b]{3}{*}{ Village } & \multirow{3}{*}{\begin{tabular}{c|} 
Total \\
Cultivated \\
Land \\
\end{tabular}} & \multirow{2}{*}{\multicolumn{2}{|c|}{$\begin{array}{c}\text { Land Under } \\
\text { Poppy Cultivation }\end{array}$}} & \multirow{3}{*}{$\begin{array}{c}\text { Wet Opium }{ }^{* 2} \\
\text { Yield } \\
(\mathrm{kg} / \mathrm{jr} .)\end{array}$} & \multirow{3}{*}{$\begin{array}{c}\text { Wet Opium } \\
\text { Production } \\
(\mathrm{kg})\end{array}$} \\
\hline & & & & & & & & \\
\hline & & & & & $\left(\right.$ Jerib $\left.^{*}\right)$ & $(\%)$ & & \\
\hline \multicolumn{9}{|c|}{ Province: Badakhshan } \\
\hline JURM & 13056 & MULLA KHEL & MULLA KHEL & 400 & 20 & 5 & 6 & 120 \\
\hline JURM & 13075 & NASHE & NASHE & 560 & 20 & 4 & 5 & 100 \\
\hline JURM & 13141 & NAWAI JURM & NAWAI JURM & 200 & 13 & 7 & 4 & 52 \\
\hline JURM & 13099 & PALAR & PALAR & 1,000 & 30 & 3 & 5 & 150 \\
\hline JURM & 13151 & QALA-E-GUNBAD & QALA-E-GUNBAD & 100 & 10 & 10 & 6 & 60 \\
\hline JURM & 13138 & SANGLAKH & SANGLAKH & 300 & 30 & 10 & 4 & 120 \\
\hline JURM & 13064 & SAR SHAKH & SAR SHAKH & 100 & 5 & 5 & 4 & 20 \\
\hline JURM & 13144 & SAR-E-HOUZ & SAR-E-HOUZ & 350 & 15 & 4 & 5 & 75 \\
\hline JURM & 13092 & SARAAB & SARAAB & 140 & 0 & 0 & 0 & 0 \\
\hline JURM & 13137 & SARI'TAL & SARI TAL & 240 & 20 & 8 & 5 & 100 \\
\hline JURM & 13117 & SARLOLA & SARLOLA & 600 & 100 & 17 & 6 & 600 \\
\hline JURM & 13449 & SARSEEL & SARSEEL & 60 & 3 & 5 & 4 & 12 \\
\hline JURM & 13062 & SENAH & SENAH & 250 & 5 & 2 & 6 & 30 \\
\hline JURM & 13125 & SHAHRAN & SHAHRAN & 150 & 10 & 7 & 2 & 20 \\
\hline JURM & 13055 & SHIF CHAN & SHIF CHAN & 200 & 20 & 10 & 7 & 140 \\
\hline JURM & 13063 & SHIRIN QESHLAQ & SHIRIN QESHLAQ & 30 & 0 & 0 & 0 & 0 \\
\hline JURM & 13060 & SOUCH-E-BALA & SOUCH-E-BALA & 300 & 12 & 4 & 5 & 60 \\
\hline JURM & 13058 & SOUCH-E-PAYAN & SOUCH-E-PAYAN & 400 & 10 & 3 & 4 & 40 \\
\hline JURM & 13083 & SPOJMAY & SPOJMAY & 250 & 10 & 4 & 5 & 50 \\
\hline JURM & 13067 & TAHT DEH & WACH BEE & 60 & 0 & 0 & 0 & 0 \\
\hline JURM & 13069 & TAHT DEH & SAFASH & 80 & 0 & 0 & 0 & 0 \\
\hline JURM & 13128 & TAJEKAN & TAJEKAN & 500 & 80 & 16 & 4 & 320 \\
\hline JURM & 13107 & TEEGARAN & TEERGARAN & 60 & 2 & 3 & 6 & 12 \\
\hline JURM & 13077 & WAJAN & WAJAN & 400 & 20 & 5 & 5 & 100 \\
\hline JURM & 13089 & WANI & WANI & 80 & 0 & 0 & 0 & 0 \\
\hline JURM & 13153 & YABAB & YABAB & 250 & 30 & 12 & 5 & 150 \\
\hline JURM & 13126 & YOUZ NAMAT & YOUZ NAMAT & 300 & 60 & 20 & 4 & 240 \\
\hline KESHEM & 13302 & AAB SHER AHMAD & AAB-E-SHER AHMAD & 1,800 & 0 & 0 & 0 & 0 \\
\hline KESHEM & 13202 & AAT & AAT & 500 & 1 & 0 & 6 & 6 \\
\hline
\end{tabular}

* 1 Jerib $=1 / 5 \mathrm{Ha}$

* 2 Dry Opium $=$ Wet Opium $-30 \%$ moisture 
Opium Production Survey by village in Badakhshan province, 1995.

\begin{tabular}{|c|c|c|c|c|c|c|c|c|}
\hline \multirow[b]{3}{*}{ District } & \multirow{3}{*}{$\begin{array}{l}\text { Village } \\
\text { Code }\end{array}$} & \multirow[b]{3}{*}{ Areal Main Village } & \multirow[b]{3}{*}{ Village } & \multirow{3}{*}{$\begin{array}{c}\text { Total } \\
\text { Cultivated } \\
\text { Land } \\
\end{array}$} & \multirow{2}{*}{\multicolumn{2}{|c|}{$\begin{array}{c}\text { Land Under } \\
\text { Poppy Cultivation }\end{array}$}} & \multirow{3}{*}{\begin{tabular}{|c|} 
Wet Opium \\
Yield \\
(kg/jr. $)$
\end{tabular}} & \multirow{3}{*}{$\begin{array}{c}\text { Wet Opium } \\
\text { Production } \\
(\mathrm{kg})\end{array}$} \\
\hline & & & & & & & & \\
\hline & & & & & $\left(\right.$ Jerib $\left.^{*}\right)$ & $(\%)$ & & \\
\hline \multicolumn{9}{|c|}{ Province: Badakhshan } \\
\hline KESHEM & 13308 & ARGHAND KAN & MASJID JAMEA & 1,250 & 0 & 0 & 0 & 0 \\
\hline KESHEM & 13307 & ARGHANDKAN & M.QESHLAQ BALA & 700 & 0 & 0 & 0 & 0 \\
\hline KESHEM & 13156 & ASHUQAN & ASHUQAN & 1,480 & 0 & 0 & 0 & 0 \\
\hline KESHEM & 13167 & AUJIL & M. SANG KATAH & 450 & 0 & 0 & 0 & 0 \\
\hline KESHEM & 13171 & AUJIL & M.AUJIL ULIA & 700 & 0 & 0 & 0 & 0 \\
\hline KESHEM & 13170 & AUJIL & M. AUJIL WASAT & 500 & 0 & 0 & 0 & 0 \\
\hline KESHEM & 13280 & BALA HESAR & BALA HESAR & 880 & 0 & 0 & 0 & 0 \\
\hline KESHEM & 12236 & BALA TASHKAL & BALA TEASHKAN & 700 & 0 & 0 & 0 & 0 \\
\hline KESHEM & 13276 & BULBUL DARA & BULBUL DARA & 650 & 0 & 0 & 0 & 0 \\
\hline KESHEM & 13246 & CHAQOUL QESHLAQ & CHAQOUL QESHLAQ & 1,200 & 0 & 0 & 0 & 0 \\
\hline KESHEM & 13277 & CHARMAGHZ DARA & CHARMAGHZ DARA & 1,300 & 0 & 0 & 0 & 0 \\
\hline KESHEM & 13227 & DALARAN & KHOSHKDARA BALA & 1,800 & 0 & 0 & 0 & 0 \\
\hline KESHEM & 13239 & DARA BOLAQ & BABA DARWESH & 800 & 0 & 0 & 0 & 0 \\
\hline KESHEM & 13243 & DARA-E-JEAM & TARNAB & 600 & 0 & 0 & 0 & 0 \\
\hline KESHEM & 13242 & DARA-E-JEAM & ZARDKHAK & 1,200 & 0 & 0 & 0 & 0 \\
\hline KESHEM & 13236 & DARA-E-JEAM & KAJ DARA & 850 & 0 & 0 & 0 & 0 \\
\hline KESHEM & 13237 & DARA-E-JEAM & TALAKWA GHIRAT & 550 & 0 & 0 & 0 & 0 \\
\hline KESHEM & 13313 & DARA-E-QAQ & KOHNAN QSHLAQ & 1,400 & 0 & 0 & 0 & 0 \\
\hline KESHEM & 13314 & DARA-E-QAQ & NAW ABAD & 1,500 & 0 & 0 & 0 & 0 \\
\hline KESHEM & 13178 & DEH KALAN & MALA-E-HAZARA & 450 & 0 & 0 & 0 & 0 \\
\hline KESHEM & 13309 & DEH SAEYDAN & ZAGHM DARA & 1,100 & 0 & 0 & 0 & 0 \\
\hline KESHEM & 13301 & DEH SAYDAN & MARGHZAR & 1,400 & 0 & 0 & 0 & 0 \\
\hline KESHEM & 13304 & DEH SAYDAN & 300695 & 1,600 & 0 & 0 & 0 & 0 \\
\hline KESHEM & 13285 & DEH WASTALAN & NAW ABAD & 1,450 & 0 & 0 & 0 & 0 \\
\hline KESHEM & 13284 & DEH WASTAYAN & DARA-E-CHASHMAK & 1,500 & 0 & 0 & 0 & 0 \\
\hline KESHEM & 13283 & DEH WASTAYAN BALA & MASJID JAMEA & 1,600 & 0 & 0 & 0 & 0 \\
\hline KESHEM & 13290 & ELMICH & ELMICH & 1,000 & 0 & 0 & 0 & 0 \\
\hline KESHEM & 13303 & FAIZANI & FAIZANI & 1,200 & 0 & 0 & 0 & 0 \\
\hline KESHEM & 13216 & FARAJ GHANI & M. SAR-E-JAR & 1,400 & 0 & 0 & 0 & 0 \\
\hline
\end{tabular}


Opium Production Survey by village in Badakhshan province, 1995.

\begin{tabular}{|c|c|c|c|c|c|c|c|c|}
\hline & \multirow[b]{2}{*}{ Village } & \multirow[b]{3}{*}{ Areal Main Village } & \multirow[b]{3}{*}{ Village } & \multirow{3}{*}{$\begin{array}{c}\text { Total } \\
\text { Cultivated } \\
\text { Land } \\
\end{array}$} & \multirow{2}{*}{\multicolumn{2}{|c|}{$\begin{array}{c}\text { Land Under } \\
\text { Poppy Cultivation }\end{array}$}} & \multirow{3}{*}{$\begin{array}{c}\text { Wet Opium }{ }^{* 2} \\
\text { Yield } \\
\text { (kg/jr.) }\end{array}$} & \multirow{3}{*}{$\begin{array}{c}\text { Wet Opium } \\
\frac{\text { Production }}{(\mathrm{kg})} \\
\end{array}$} \\
\hline & & & & & & & & \\
\hline District & Code & & & & $\left(\right.$ (.erih $\left.{ }^{*}\right)$ & $(\%)$ & & \\
\hline \multicolumn{9}{|c|}{ Province: Badakhshan } \\
\hline KESHEM & 13251 & FARAJGHANI & MASJED MERJAN & 700 & 0 & 0 & 0 & 0 \\
\hline KESHEM & 13214 & FARMAN QULI & DASHT SHAMEL & 500 & 0 & 0 & 0 & 0 \\
\hline KESHEM & 13215 & FARMAN QULI & SAR QESHLAQ & 1.100 & () & 0 & 0 & 0 \\
\hline KESHEM & 13201 & FARMAN QULI & LIZH DEH & $9(0)$ & 0) & 0 & 0 & 0 \\
\hline KESHEM & 13213 & FARMANQULI & PAYAN QESHLAQ & 580 & 0 & 0 & 0 & 0 \\
\hline KESHEM & 13330 & GANDOM QOOL & MASJID WAKILII & 1.300 & 0 & 0 & 0 & 0 \\
\hline KESHEM & 13321 & GANDOM QOOL & WAIIDAT ABAD & 1.300 & 0) & 0 & 0 & 0 \\
\hline KESHEM & 13329 & GANDOM QOOL & M.KOORI A.KHALQ & 1.100 & 0 & 0 & 0 & 0 \\
\hline KESHEM & 13326 & GANDOM QOOL & M.ABDUL RAHIM & 1,113 & 0 & 0) & 0 & 0 \\
\hline KESHEM & 13327 & GANDOM QOOL & M.JAM-A-MASJID & 1,305 & 0 & 0 & 0 & 0 \\
\hline KESHEM & 13325 & GANDOM QOOL & MAULAWI QAZI & 1.100 & 0 & 0 & 0 & 0 \\
\hline KESHEM & 13328 & GANDOM QOOL & MASJID MAKHDOM & 1,600 & 0 & 0 & 0 & 0 \\
\hline KESHEM & 13331 & GANDOM QOOL & MASJID ABDUL SAMAD & 1,000 & 0 & 0 & 0 & 0 \\
\hline KESHEM & 13311 & GAZ DARA & GAZ DARA & 1,800 & 0 & 0 & 0 & 0 \\
\hline KESHEM & 13344 & GHELAWAK & GHELAWAK & 1,400 & 0 & 0 & 0 & 0 \\
\hline KESHEM & 13161 & GHELLAN & GHELLAN & 440 & 0 & 0 & 0 & 0 \\
\hline KESHEM & 13293 & GHORI SANG & KASHO & 1,000 & 0 & 0 & 0 & 0 \\
\hline KESHEM & 13240 & GOUNG SHAHAR & GOUNG SHAHAR & 800 & 0 & 0 & 0 & 0 \\
\hline KESHEM & 13264 & GUNBAD & GUNBAD & 600 & 0 & 0 & 0 & 0 \\
\hline KESHEM & 13271 & HAFT CHENAR & HAFT CHENAR & 700 & 0 & 0 & 0 & 0 \\
\hline KESHEM & 13339 & JANAZADARA & JANAZSDARA & 900 & 0 & 0 & 0 & 0 \\
\hline KESHEM & 13210 & JAR SHAH BABA & M. MIRIIA & 670 & 0 & 0 & 0 & 0 \\
\hline KESHEM & 13211 & JAR SHAH BABA & M. DUST NOWROOZ & 800 & 0 & 0 & 0 & 0 \\
\hline KESHEM & 13222 & KANGOUR CHI & MASJID JAMEA & 1.000 & 0 & 0 & 0 & 0 \\
\hline KESHEM & 13225 & KANGOUR CHI & MASJID SANG KAIAN & 1.300 & 0 & 0 & 0 & 0 \\
\hline KESHEM & 13230 & KANGOUR CHI & MASJID NAGARAN & 1.600 & () & 0 & 0 & 0 \\
\hline KESHEM & 13229 & KANGOURCHI & MASJID SAR DARG & 1.500 & 0 & 0 & 0 & 0 \\
\hline KESHEM & 13159 & KARAST DEH & WAWECII & 1.150 & 0 & 0 & 0 & \\
\hline KESHEM & 13194 & KAREST DEII & M. WASAT & 600 & 0 & 0 & 0 & 0 \\
\hline
\end{tabular}


Opium Production Survey by village in Badakhshan province, 1995.

\begin{tabular}{|c|c|c|c|c|c|c|c|c|}
\hline \multirow[b]{2}{*}{ District } & \multirow{2}{*}{$\begin{array}{l}\text { Village } \\
\text { Code }\end{array}$} & \multirow[b]{2}{*}{ Area/ Main Village } & \multirow[b]{2}{*}{ Village } & \multirow{2}{*}{$\begin{array}{c}\text { Total } \\
\text { Cultivated } \\
\text { Land }\end{array}$} & \multicolumn{2}{|c|}{$\begin{array}{c}\text { Land Under } \\
\text { Poppy ('ultivation }\end{array}$} & \multirow{2}{*}{$\begin{array}{c}\text { Wet Opium }^{* 2} \\
\text { Yield } \\
\text { (kg/jr.) }\end{array}$} & \multirow{2}{*}{$\begin{array}{l}\text { Wet Opium } \\
\frac{\text { Production }}{(\mathrm{kg})}\end{array}$} \\
\hline & & & & & (.Jerih *) & (\%) & & \\
\hline \multicolumn{9}{|c|}{ Province: Badakhshan } \\
\hline KESHEM & 13209 & KATOU & SHAK DARA & 1.300 & 0 & 0 & 0 & 0 \\
\hline KESHEM & 13203 & KATOU & KHELE ASP & 1,200 & 0 & 0 & 0 & 0 \\
\hline KESHEM & 13207 & KATOU & KHOROUK & 1,200 & 0 & 0 & 0 & 0 \\
\hline KESHEM & 13208 & KATOU & RABAT & 1,000 & 0 & 0 & 0 & 0 \\
\hline KESHEM & 13204 & KATOU & AURGHANJ & 900 & 0 & 0 & 0 & 0 \\
\hline KESHEM & 13205 & KATOU BALA & CHAKORAN & 700 & 0 & 0 & 0 & 0 \\
\hline KESHEM & 13206 & KATOU BALA & $\mathrm{DO} A \mathrm{BI}$ & 645 & 0 & 0 & 0 & 0 \\
\hline KESHEM & 13175 & KHAMBAK & M. DEH BALA & 600 & 0 & 0 & 0 & 0 \\
\hline KESHEM & 13176 & KHAMBAK & DUSHTAK & 500 & 0 & 0 & 0 & 0 \\
\hline KESHEM & 13163 & KHAMBAK & RABAT & 740 & 0 & 0 & 0 & 0 \\
\hline KESHEM & 13179 & KHAMBAK & ASIABAK & 1,000 & 0 & 0 & 0 & 0 \\
\hline KESHEM & 13173 & KHAMBAK & DEH KALAN & 1,000 & 0 & 0 & 0 & 0 \\
\hline KESHEM & 13168 & KHAMBAK & AFGHAN DARA & 1,300 & 0 & 0 & 0 & 0 \\
\hline KESHEM & 13177 & KHAMBAK & M. DEWALSANG & 620 & 0 & 0 & 0 & 0 \\
\hline KESHEM & 13169 & KHAMBAK & PLAHRAN & 1.300 & 0 & 0 & 0 & 0 \\
\hline KESHEM & 13172 & KHAMBAK & DARA-E-MIR & 900 & 0 & 0 & 0 & 0 \\
\hline KESHEM & 13174 & KHAMBAK BALA & M. JAMEA & 1.400 & 0 & 0 & 0 & 0 \\
\hline KESHEM & 13260 & KHAMBAK NOW SFULA & MASJED-E-BALA & 560 & 0 & 0 & 0 & 0 \\
\hline KESHEM & 13281 & KHAMBAK NOW SUFIA & MADRASA-E-JAME & 1.000 & 0 & 0 & 0 & 0 \\
\hline KESHEM & 13305 & KHANA QA & KHANA QA & 900 & 0 & 0 & 0 & 0 \\
\hline KESHEM & 13199 & KHOWARK & KHOWARK & 680 & 0 & 0 & 0 & 0 \\
\hline KESHEM & 13320 & KHWAJA AFGHANI & KHWAJA AFGHANI & 1,800 & 1 & 0 & 8 & 4 \\
\hline KESHEM & 14012 & KOREST DEH & MASJID SAR DOWA & 720 & 0 & 0 & 0 & 0 \\
\hline KESHEM & 13196 & KOURI & DARA-E-SAMB & 1,400 & 0 & 0 & 0 & 0 \\
\hline KESHEM & 13197 & KOURI & DUSHT-E-BALA & 1,200 & 0 & 0 & 0 & 0 \\
\hline KESHEM & 13212 & KOURI & ASIA WANI & 1,000 & 0 & 0 & 0 & 0 \\
\hline KESHEM & 13190 & KREST DEH & MASJID SAR TAL & 625 & 0 & 0 & 0 & 0 \\
\hline KESHEM & 13258 & M. JAMEA TAJARI & M. JAMEA TAJARI & 1.500 & 0 & 0 & 0 & 0 \\
\hline KESHEM & 13279 & MADRASA-E-KALAN & MADRASA-E-KALAN & 1.000 & 0 & 0 & 0 & 0 \\
\hline
\end{tabular}


Opium Production Survey by village in Badakhshan province, 1995.

\begin{tabular}{|c|c|c|c|c|c|c|c|c|}
\hline \multirow[b]{3}{*}{ District } & \multirow{3}{*}{$\begin{array}{l}\text { Village } \\
\text { Code }\end{array}$} & \multirow[b]{3}{*}{ Area/ Main Village } & \multirow[b]{3}{*}{ Village } & \multirow{3}{*}{$\begin{array}{c}\text { Total } \\
\text { Cultivated } \\
\text { Land } \\
\end{array}$} & \multirow{2}{*}{\multicolumn{2}{|c|}{$\begin{array}{c}\text { Land Under } \\
\text { Poppy Cultivation }\end{array}$}} & \multirow{3}{*}{$\begin{array}{c}\text { Wet Opium }^{* 2} \\
\text { Yield } \\
(\mathrm{kg} / \mathrm{jr} .)\end{array}$} & \multirow{3}{*}{$\begin{array}{c}\text { Wet Opium } \\
\text { Production } \\
(\mathrm{kg}) \\
\end{array}$} \\
\hline & & & & & & & & \\
\hline & & & & & $\left(\right.$ Jerib $\left.^{*}\right)$ & $(\%)$ & & \\
\hline \multicolumn{9}{|c|}{ Province: Badakhshan } \\
\hline KESHEM & 13300 & MALGANI & M.JAMES MASJID & 1,500 & 0 & 0 & 0 & 0 \\
\hline KESHEM & 13249 & MASHHAD & M.WAKIL SHERDEL & 763 & 0 & 0 & 0 & 0 \\
\hline KESHEM & 13244 & MASHHAD & M. SARANWAL & 350 & 0 & 0 & 0 & 0 \\
\hline KESHEM & 13261 & MASHHAD & M.JAMES SHAR & 1,100 & 0 & 0 & 0 & 0 \\
\hline KESHEM & 13257 & MASJ.HAJI QARA & MASJ.HAJI QARA & 600 & 0 & 0 & 0 & 0 \\
\hline KESHEM & 13262 & MASJED MAKHDOMA & MASJED MAKHDOMA & 630 & 1 & 0 & 8 & 8 \\
\hline KESHEM & 13245 & MASJED SAR TIPA & MASJED SAR TIPA & 150 & 0 & 0 & 0 & 0 \\
\hline KESHEM & 13274 & MASJED-E-JAME & MASJED-E-JAME & 1.700 & 0 & 0 & 0 & 0 \\
\hline KESHEM & 13250 & MASJID A. AZIZ & MASJID A: AZIZ & 700 & 0 & 0 & 0 & 0 \\
\hline KESHEM & 13270 & MASJID AESHAN S & MASJID AESHAN S & 800 & 0 & 0 & 0 & 0 \\
\hline KESHEM & 13269 & MASJID ARBABGIR & MASJID ARBABGIR & 1,100 & 0 & 0 & 0 & 0 \\
\hline KESHEM & 13265 & MASJID ATABAY & MASJID ATABAY & 1,200 & 0 & 0 & 0 & 0 \\
\hline KESHEM & 13193 & MASJID BALA & MASJID BALA & 1,000 & 0 & 0 & 0 & 0 \\
\hline KESHEM & 13157 & MASJID CHOGOL & MASJID CHOGOL & 900 & 0 & 0 & 0 & 0 \\
\hline KESHEM & 13341 & MASJID DARA & MASJID DARA & 1,200 & 4 & 0 & 6 & 24 \\
\hline KESHEM & 13323 & MASJID HJ.DAULA & MASJID HJ.DAULA & 1,200 & 0 & 0 & 0 & 0 \\
\hline KESHEM & 13267 & MASJID HJ.TIMAR & MASJID HJ.TIMAR & 1,000 & 0 & 0 & 0 & 0 \\
\hline KESHEM & 13292 & MASJID JAMEA & MASJID JAMEA & 1,200 & 0 & 0 & 0 & 0 \\
\hline KESHEM & 13191 & MASJID JAMEA & MASJID JAMEA & 700 & 0 & 0 & 0 & 0 \\
\hline KESHEM & 13324 & MASJID JAMEA & MASJID JAMEA & 2,000 & 0 & 0 & 0 & 0 \\
\hline KESHEM & 13192 & MASJID MIAN DEH & MASJID MIAN DEH & 650 & 0 & 0 & 0 & 0 \\
\hline KESHEM & 13278 & MASJID ML.ASHOR & MASJID ML.ASHOR & 470 & 0 & 0 & 0 & 0 \\
\hline KESHEM & 13268 & MASJID ML.JOURA & MASJID ML.JOURA & 1,200 & 0 & 0 & 0 & 0 \\
\hline KESHEM & 13322 & MASJID ML.ZARIF & MASJID ML.ZARIF & 850 & 0 & 0 & 0 & 0 \\
\hline KESHEM & 13164 & MIRKAN & MASJID PAYAN & 1,600 & 0 & 0 & 0 & 0 \\
\hline KESHEM & 13165 & MIRKAN & M. BALA & 2,000 & 0 & 0 & 0 & 0 \\
\hline KESHEM & 13166 & MIRKAN & M. JAMEA & 1,200 & 0 & 0 & 0 & 0 \\
\hline KESHEM & 13200 & MIYAN SHAHR & MASJID QASHQAR & 700 & 0 & 0 & 0 & 0 \\
\hline KESHEM & 13184 & MIYAN SHAHR & CHEQEL QESHLAQ & 900 & 0 & 0 & 0 & 0 \\
\hline
\end{tabular}

* 1 Jerib $=1 / 5 \mathrm{Ha}$

* 2 Dry Opium $=$ Wet Opium $-30 \%$ moisture 
Opium Production Survey by village in Badakhshan province, 1995.

\begin{tabular}{|c|c|c|c|c|c|c|c|c|}
\hline \multirow[b]{3}{*}{ District } & \multirow{3}{*}{$\begin{array}{c}\text { Village } \\
\text { Code } \\
\end{array}$} & \multirow[b]{3}{*}{ Area/ Main Village } & \multirow[b]{3}{*}{ Village } & \multirow{3}{*}{$\begin{array}{c}\text { Total } \\
\text { Cultivated } \\
\text { Land } \\
\end{array}$} & \multirow{2}{*}{\multicolumn{2}{|c|}{$\begin{array}{c}\text { Land Under } \\
\text { Poppy Cultivation }\end{array}$}} & \multirow{3}{*}{$\begin{array}{c}\text { Wet }_{\text {Opium }}^{* 2} \\
\text { Yield } \\
(\mathrm{kg} / \mathrm{jr} .)\end{array}$} & \multirow{3}{*}{$\begin{array}{c}\text { Wet Opium } \\
\text { Production } \\
\text { (kg) }\end{array}$} \\
\hline & & & & & & & & \\
\hline & & & & & $\left(\right.$ Jerib $\left.^{*}\right)$ & $(\%)$ & & \\
\hline Province: Badakhshan & & & & & & . & & \\
\hline KESHEM & 13183 & MIYAN SHAHR & MASJID JAMEA & 1,200 & 0 & 0 & 0 & 0 \\
\hline KESHEM & 13195 & MIYAN SHAHR & BAGH-E-TARK & 380 & 0 & 0 & 0 & 0 \\
\hline KESHEM & 13275 & ML. SECANDAR & ML. SECANDAR & 558 & 0 & 0 & 0 & 5 \\
\hline KESHEM & 13343 & MUZAFRI & MASJID DAMULLA & 1,100 & 0 & 0 & 0 & 0 \\
\hline KESHEM & 13335 & MUZAFRI & MAIDAN MUZFRI & 1,200 & 0 & 0 & 0 & 0 \\
\hline KESHEM & 13342 & MUZAFRI & M.HAJI AKHUND & 700 & 0 & 0 & 0 & 0 \\
\hline KESHEM & 13217 & NAAIB HA & NAAIB HA & 1,000 & 0 & 0 & 0 & 0 \\
\hline KESHEM & 13218 & NABHAI GHARBI & NABHAI GHARBI & 1,150 & 4 & 0 & 5 & 20 \\
\hline KESHEM & 13220 & NAMAZGA & SAR SEBAKHSHA & 930 & 0 & 0 & 0 & 0 \\
\hline KESHEM & 13223 & NAMAZGA & NAZIR BOY & 780 & 0 & 0 & 0 & 0 \\
\hline KESHEM & 13221 & NAMAZGA & M. JAMEA & 2,300 & 0 & 0 & 0 & 0 \\
\hline KESHEM & 13259 & NAW ABAD & NAW ABAD & 840 & 0 & 0 & 0 & 0 \\
\hline KESHEM & 13272 & NOW CHAY & NOW CHAY & 1,100 & 0 & 0 & 0 & 0 \\
\hline KESHEM & 13224 & PALARAN & KHOSHKDARA LAB & 850 & 0 & 0 & 0 & 0 \\
\hline KESHEM & 13186 & PALARAN & PALARAN & 600 & 0 & 0 & 0 & 0 \\
\hline KESHEM & 13234 & PALARAN & KHOSHK DARA PAYANI & 1,200 & 0 & 0 & 0 & 0 \\
\hline KESHEM & 13185 & PALARAN & KHWAJA BAGH & 800 & 0 & 0 & 0 & 0 \\
\hline KESHEM & 13336 & PASTA KHOR & M.JAMEA & 1,600 & 0 & 0 & 0 & 0 \\
\hline KESHEM & 13337 & PASTA KHOR & M.PAYAN & 900 & 0 & 0 & 0 & 0 \\
\hline KESHEM & 13252 & QARA BOLAQ & QARA BOLAQ & 1,100 & 0 & 0 & 0 & 0 \\
\hline KESHEM & 13306 & QEERMASHI & M.JAMEA & 1,400 & 0 & 0 & 0 & 0 \\
\hline KESHEM & 13188 & QESHLAQ EASHAN & QESHLAQ EASHAN & 280 & 0 & 0 & 0 & 0 \\
\hline KESHEM & 13160 & SABZ DARA & MAGHZAR & 2,500 & 0 & 0 & 0 & 0 \\
\hline KESHEM & 13162 & SABZDARA NEEM DASTA & SABZDARA & 1,300 & 0 & 0 & 0 & 0 \\
\hline KESHEM & 13332 & SAFID REAG & SAFID REAG & 1,400 & 1 & 0 & 7 & 7 \\
\hline KESHEM & 13282 & SAMARQANDI & SAMARQANDI & 800 & 0 & 0 & 0 & 0 \\
\hline KESHEM & 13182 & SANG AAB & MASJID KELKAK & 900 & 0 & 0 & 0 & 0 \\
\hline KESHEM & 13180 & SANG AAB & SANG AAB & 1,200 & 0 & 0 & 0 & 0 \\
\hline KESHEM & 13198 & SANG AAB ULIA & MASJID JAMEA & 1,160 & 0 & 0 & 0 & 0 \\
\hline
\end{tabular}


Opium Production Survey by village in Badakhshan province, 1995.

\begin{tabular}{|c|c|c|c|c|c|c|c|c|}
\hline \multirow[b]{3}{*}{ District } & \multirow{3}{*}{$\begin{array}{l}\text { Village } \\
\text { Code }\end{array}$} & \multirow[b]{3}{*}{ Areal Main Village } & \multirow[b]{3}{*}{ Village } & \multirow{3}{*}{$\begin{array}{c}\text { Total } \\
\text { Cultivated } \\
\text { Land } \\
\end{array}$} & \multirow{2}{*}{\multicolumn{2}{|c|}{$\begin{array}{c}\text { Land Under } \\
\text { Poppy Cultivation }\end{array}$}} & \multirow{3}{*}{$\frac{\text { Wet Opium }^{* 2}}{\text { Yield }}$} & \multirow{3}{*}{$\begin{array}{c}\text { Wet Opium } \\
\text { Production } \\
(\mathrm{kg}) \\
\end{array}$} \\
\hline & & & & & & & & \\
\hline & & & & & $\left(\right.$ Jerib $\left.^{*}\right)$ & $(\%)$ & & \\
\hline \multicolumn{9}{|c|}{ Province: Badakhshan } \\
\hline KESHEM & 13340 & SANG KALAN & SANG KALAN & 1,000 & 2 & 0 & 7 & 13 \\
\hline KESHEM & 13231 & SAR SHERQI & M. HAJI MAJID & 530 & 0 & 0 & 0 & 0 \\
\hline KESHEM & 13232 & SARI GHARBI MAS & SARI GHARBI MAS & 3,600 & 0 & 0 & 0 & 0 \\
\hline KESHEM & 13219 & SARI SHARQI MASHHAD & M. SARA KHAN & 840 & 0 & 0 & 0 & 0 \\
\hline KESHEM & 13333 & SARPESHANI & SARPESHANI & 1,100 & 3 & 0 & 4 & 12 \\
\hline KESHEM & 13158 & SHAHEED KAN & M. BALA & 860 & 0 & 0 & 0 & 0 \\
\hline KESHEM & 13235 & SHAKESTAGAN & SHAKESTAGAN & 1,100 & 0 & 0 & 0 & 0 \\
\hline KESHEM & 13273 & SHOLASH DARA & SHOLASH DARA & 800 & 0 & 0 & 0 & 0 \\
\hline KESHEM & 13263 & SOFIHA & SOFIHA & 700 & 0 & 0 & 0 & 0 \\
\hline KESHEM & 13295 & SOSAGAN & KHOLIAN & 1,000 & 0 & 0 & 0 & 0 \\
\hline KESHEM & 13297 & SOSAGAN & RODGAH & 700 & 0 & 0 & 0 & 0 \\
\hline KESHEM & 13296 & SOSAGAN & DEH MIRI & 1,000 & 0 & 0 & 0 & 0 \\
\hline KESHEM & 13294 & SOSGAN & MASJID JAMEA & 800 & 0 & 0 & 0 & 0 \\
\hline KESHEM & 13181 & SUNG AAB & SSAAR -E-TANGI & 1,200 & 0 & 0 & 0 & 0 \\
\hline KESHEM & 13241 & SUNG AAB & SHUHADA & 1,800 & 0 & 0 & 0 & 0 \\
\hline KESHEM & 13266 & TAJARI & TAJARI & 750 & 0 & 0 & 0 & 0 \\
\hline KESHEM & 13254 & TAKYAH & CHAQAL WASAR AS & 850 & 0 & 0 & 0 & 0 \\
\hline KESHEM & 13253 & TAKYAH & SHAKHDAN & 1,200 & 0 & 0 & 0 & 0 \\
\hline KESHEM & 13248 & TAKYAH & SAR-E-JAR & 1,600 & 0 & 0 & 0 & 0 \\
\hline KESHEM & 13247 & TAKYAH & HAZARA HA & 800 & 0 & 0 & 0 & 0 \\
\hline KESHEM & 13312 & TEASHKAM & MASJID SHARQI & 600 & 0 & 0 & 0 & 0 \\
\hline KESHEM & 13310 & TEASHKAN & BAZAR-E-TEASHKA & 1,300 & 0 & 0 & 0 & 0 \\
\hline KESHEM & 13338 & TOTAK & TOTAK & 130 & 0 & 0 & 0 & 0 \\
\hline KESHEM & 13299 & TOUGHAK & TOUGHAK & 350 & 0 & 0 & 0 & 0 \\
\hline KESHEM & 13288 & WAJIB & SHUTARGARDAN & 740 & 0 & 0 & 0 & 0 \\
\hline KESHEM & 13256 & WAKHSHI & MAKHDOM HA & 675 & 0 & 0 & 0 & 0 \\
\hline KESHEM & 13287 & YARSAZ & YARSAZ & 1,000 & 0 & 0 & 0 & 0 \\
\hline KESHEM & 13315 & YAWAL & YAWAL PAYAN & 500 & 0 & 0 & 0 & 0 \\
\hline KESHEM & 13318 & YAWAL BALA & MASJID BALA & 500 & 0 & 0 & 0 & 0 \\
\hline
\end{tabular}

* I Jerib $=1 / 5 \mathrm{Ha}$

${ }^{*}$ Dry Opium $=$ Wet Opium $-30 \%$ moisture
Source: UNDCP Opium Production Survey, 1995 File: BDK1.XLS - 9/4/95 
Opium Production Survey by village in Badakhshan province, 1995.

\begin{tabular}{|c|c|c|c|c|c|c|c|c|}
\hline \multirow[b]{3}{*}{ District } & \multirow{3}{*}{$\begin{array}{l}\text { Village } \\
\text { Code }\end{array}$} & \multirow[b]{3}{*}{ Area/ Main Village } & \multirow[b]{3}{*}{ Village } & \multirow{3}{*}{$\begin{array}{c}\text { Total } \\
\text { Cultivated } \\
\text { Land } \\
\end{array}$} & \multirow{2}{*}{\multicolumn{2}{|c|}{$\begin{array}{c}\text { Land Under } \\
\text { Poppy Cultivation }\end{array}$}} & \multirow{3}{*}{$\begin{array}{c}\text { Wet }^{\text {Opium }}{ }^{* 2} \\
\text { Yield } \\
(\mathrm{kg} / \mathrm{jr} .)\end{array}$} & \multirow{3}{*}{$\begin{array}{c}\text { Wet Opium. } \\
\text { Production } \\
(\mathrm{kg}) \\
\end{array}$} \\
\hline & & & & & & & & \\
\hline & & & & & $\left(\right.$ Jerib $\left.^{*}\right)$ & $(\%)$ & & \\
\hline \multicolumn{9}{|c|}{ Province: Badakhshan } \\
\hline KESHEM & 13316 & YAWAL BALA & MASJID JAMEA & 880 & 0 & 0 & 0 & 0 \\
\hline KESHEM & 13319 & YAWAL BALA & MASJID NIYAZI & 450 & 0 & 0 & 0 & 0 \\
\hline KESHEM & 13317 & YAWAL BALA & CHASHM DARAZ & 1,100 & 0 & 0 & 0 & 0 \\
\hline ZEBAK & 13361 & AID KHUARD & AID KHUARD & 90 & 1. & 1 & 5 & 5 \\
\hline ZEBAK & 13024 & CHABCHI MAGHZAR & CHABCHI MAGHZAR & 300 & 1. & 0 & 6 & 6 \\
\hline ZEBAK & 13450 & DAND & DAND & 140 & 1 & 1 & 5 & 5 \\
\hline ZEBAK & 13359 & DASHT KHAN & DASHT KHAN & 90 & 1 & 1 & 5 & 5 \\
\hline ZEBAK & 13349 & DASHT RABAT & DASHT RABAT & 300 & 1 & 0 & 6 & 6 \\
\hline ZEBAK & 13355 & DEHGUL & DEHGUL & 100 & 0 & 0 & 0 & 0 \\
\hline ZEBAK & 13348 & ESKATOL & ESKATOL & 130 & 2 & 1 & 6 & 9 \\
\hline ZEBAK & 13346 & FAROOQ & FAROOQ & 140 & 0 & 0 & 0 & 0 \\
\hline ZEBAK & 13362 & GHARIB & GHARIB & 170 & 1 & 1 & 6 & 6 \\
\hline ZEBAK & 13351 & GUL KANA & GUL KANA & 150 & 1 & 1 & 6 & 6 \\
\hline ZEBAK & 13358 & KAZDAN & KAZDAN & 250 & 1 & 0 & 5 & 5 \\
\hline ZEBAK & 13353 & KHAL KHAN & KHAL KHAN & 160 & 1 & 1 & 5 & 5 \\
\hline ZEBAK & 13352 & KULALHA & KULALHA & 200 & 1 & 1 & 6 & 6 \\
\hline ZEBAK & 13354 & MARKAZ-E-ZEBAK & MARKAZ-E-ZEBAK & 550 & 2 & 0 & 6 & 12 \\
\hline ZEBAK & 13356 & NOWABAD SHINGOK & NOWABAD SHINGOK & 260 & 3 & 1 & 5 & 13 \\
\hline ZEBAK & 13360 & RAZ RAK & RAZ RAK & 280 & 1 & 0 & 6 & 6 \\
\hline ZEBAK & 13345 & SANGLUCH & SANGLUCH & 400 & 1 & 0 & 6 & 6 \\
\hline ZEBAK & 13347 & TAKYA KHUBAN & TAKYA KHUBAN & 130 & 0 & 0 & 0 & 0 \\
\hline ZEBAK & 13357 & ZAR KHAN & ZAR KHAN & 300 & 1 & 0 & 5 & 5 \\
\hline
\end{tabular}


Opium Production Survey by village in Farah province, 1995.

\begin{tabular}{|c|c|c|c|c|c|c|c|c|}
\hline & Village & & & Total & Land & & Wet Opium *2 & Wet Opium \\
\hline \multirow[t]{2}{*}{ District } & \multirow[t]{2}{*}{ Code } & \multirow[t]{2}{*}{ Area/ Main Village } & \multirow[t]{2}{*}{ Village } & \multirow{2}{*}{$\begin{array}{c}\text { Cultivated } \\
\left.\text { Land (Jerib }{ }^{*}\right)\end{array}$} & \multicolumn{2}{|c|}{ Poppy Cultivation } & \multirow{2}{*}{$\begin{array}{l}\text { Yield } \\
(\mathrm{kg} / \mathrm{jr} .)\end{array}$} & \multirow{2}{*}{$\begin{array}{c}\text { Production } \\
\text { (kg) }\end{array}$} \\
\hline & & & & & (jerih) & $(\%)$ & & \\
\hline \multicolumn{9}{|c|}{ Province: Farah } \\
\hline BAKWA & 47 & ABDUL RAZAQ KHAN & ABDUL RAZAQ KHAN & 0 & 0 & 0 & 0 & 0 \\
\hline BAKWA & 63 & ASLI CHAI M.NAWAB & CHAI A.QADER & 0 & 0 & 0 & 0 & 0 \\
\hline BAKWA & 37 & BAKHTAYAR & BAKHTAYAR & 0 & 0 & 0 & 0 & 0 \\
\hline BAKWA & 36 & BARGHANA & BARGHANA & 0 & 0 & 0 & 0 & 0 \\
\hline BAKWA & 54 & BAZ MOHD & BAZ MOHD & 0 & 0 & 0 & 0 & 0 \\
\hline BAKWA & 53 & CHAHI MULLAH AGHA & CHAHI MULLAH AGHA & 0 & 0 & 0 & 0 & 0 \\
\hline BAKWA & 52 & CHAHI NAMAT & CHAHI NAMAT & 0 & 0 & 0 & 0 & 0 \\
\hline BAKWA & 55 & CHAHI SAFIULLAH & CHAHI SAFIULLAH & 0 & 0 & 0 & 0 & 0 \\
\hline BAKWA & 31 & CHOCHA & СНОСНA & 0 & 0 & 0 & 0 & 0 \\
\hline BAKWA & 29 & DASTAK & DASTAK & 0 & 0 & 0 & 0 & 0 \\
\hline BAKWA & 34 & DAWALLAK & DAWALLAK & 0 & 0 & 0 & 0 & 0 \\
\hline BAKWA & 20 & FOLADBIG & FOLADBIG & 0 & 0 & 0 & 0 & 0 \\
\hline BAKWA & 18 & GHAZY ABAD & GHAZY ABAD & 0 & 0 & 0 & 0 & 0 \\
\hline BAKWA & 13 & GOY SAFID & GOY SAFID & 0 & 0 & 0 & 0 & 0 \\
\hline BAKWA & 25 & GOY SAFID & KOCHNI JOYSAFID & 0 & 0 & 0 & 0 & 0 \\
\hline BAKWA & 21 & GURZ & GURZ & 0 & 0 & 0 & 0 & 0 \\
\hline BAKWA & 69 & HAJI AMIR JOHN & HAJI AMIR JOHN & 0 & 0 & 0 & 0 & 0 \\
\hline BAKWA & 68 & HAJI GARNAIL & HAJI GARNAIL & 0 & 0 & 0 & 0 & 0 \\
\hline BAKWA & 23 & HUSSIANABAD & HUSSIANABAD & 0 & 0 & 0 & 0 & 0 \\
\hline BAKWA & 30 & JANG & JANG & 0 & 0 & 0 & 0 & 0 \\
\hline BAKWA & 19 & KEERTA & KEERTA & 0 & 0 & 0 & 0 & 0 \\
\hline BAKWA & 64 & KEN KAREZ & KEN KAREZ & 0 & 0 & 0 & 0 & 0 \\
\hline BAKWA & 41 & KHUDA RAAM & KHUDA RAAM & 0 & 0 & 0 & 0 & 0 \\
\hline BAKWA & 60 & KHUDA RAM & KHUDA RAM & 0 & 0 & 0 & 0 & 0 \\
\hline BAKWA & 38 & LERBAGEE & LERBAGEE & 0 & 0 & 0 & 0 & 0 \\
\hline BAKWA & 51 & MAMOR TANK KUBULY & MAMOR TANK KUBULY & 0 & 0 & 0 & 0 & 0 \\
\hline BAKWA & 15 & MARJA & MARJA & 0 & 0 & 0 & 0 & 0 \\
\hline BAKWA & 43 & MOHD GUL & MOHD GUL & 0 & 0 & 0 & 0 & 0 \\
\hline BAKWA & 45 & MOHD KHAN & MOHD КНАN & 0 & 0 & 0 & 0 & 0 \\
\hline
\end{tabular}


Opium Production Survey by village in Farah province, 1995.

\begin{tabular}{|c|c|c|c|c|c|c|c|c|}
\hline & Village & & & Total & Land & & Wet Opium $^{* 2}$ & Wet Opium \\
\hline \multirow[t]{2}{*}{ District } & \multirow[t]{2}{*}{ Code } & \multirow[t]{2}{*}{ Area/ Main Village } & \multirow[t]{2}{*}{ Village } & \multirow{2}{*}{$\begin{array}{c}\text { Cultivated } \\
\left.\text { Land (Jerib }{ }^{*}\right)\end{array}$} & \multicolumn{2}{|c|}{ Poppy Cultivation } & \multirow{2}{*}{$\begin{array}{l}\text { Yield } \\
(\mathrm{kg} / \mathrm{jr} .) \\
\end{array}$} & \multirow{2}{*}{$\begin{array}{c}\text { Production } \\
(\mathrm{kg})\end{array}$} \\
\hline & & & & & (jerib) & $(\%)$ & & \\
\hline \multicolumn{9}{|c|}{ Province: Farah } \\
\hline BAKWA & 42 & MOHD RAHIM & MOHD RAHIM & 0 & 0 & 0 & 0 & 0 \\
\hline BAKWA & 16 & MUGHAL ABAD & MUGHAL ABAD & 0 & 0 & 0 & 0 & 0 \\
\hline BAKWA & 40 & MULLAH HATAM & MULLAH HATAM & 0 & 0 & 0 & 0 & 0 \\
\hline BAKWA & 50 & NAZAR GAH & MULLAH SHADI & 0 & 0 & 0 & 0 & 0 \\
\hline BAKWA & 22 & NAZAR GAH & NAZAR GAH & 0 & 0 & 0 & 0 & 0 \\
\hline BAKWA & 62 & QASEEM ABAD & CHAI NOOR MOHD & 0 & 0 & 0 & 0 & 0 \\
\hline BAKWA & 48 & QASEEM ABAD & HAJI RAQAM KHAN & 0 & 0 & 0 & 0 & 0 \\
\hline BAKWA & 49 & QASEEM ABAD & KHUDI DAD & 0 & 0 & 0 & 0 & 0 \\
\hline BAKWA & 61 & QASEEM ABAD & MULLAH NIK M.AK & 0 & 0 & 0 & 0 & 0 \\
\hline BAKWA & 14 & SAGHEE & SAGHEE & 0 & 0 & 0 & 0 & 0 \\
\hline BAKWA & 66 & SAY SIR CHA & ABDULRASHED & 0 & 0 & 0 & 0 & 0 \\
\hline BAKWA & 65 & SAY SIR CHA & HAJI MULLAH & 0 & 0 & 0 & 0 & 0 \\
\hline BAKWA & 26 & SEIA QALA & HAJI A.KARIM & 0 & 0 & 0 & 0 & 0 \\
\hline BAKWA & 27 & SEIA QALA & QALA HANGARAN & 30 & 2 & 5 & 0 & 0 \\
\hline BAKWA & 28 & SEIA QALA & SEIA QALA & 0 & 0 & 0 & 0 & 0 \\
\hline BAKWA & 46 & SHAKEY NASIR & SHAKEY NASIR & 0 & 0 & 0 & 0 & 0 \\
\hline BAKWA & 39 & SHAMS ABAD & SHAMS ABAD & 250 & 3 & 1 & 0 & 0 \\
\hline BAKWA & 17 & SHAND & SHAND & 0 & 0 & 0 & 0 & 0 \\
\hline BAKWA & 44 & SHER MOHD & SHER MOHD & 0 & 0 & 0 & 0 & 0 \\
\hline BAKWA & 57 & SHIR SURKH & CHI H.NAIB & 0 & 0 & 0 & 0 & 0 \\
\hline BAKWA & 58 & SHIR SURKH & GH.DASTAGER & 0 & 0 & 0 & 0 & 0 \\
\hline BAKWA & 67 & SHIR SURKH & MOHD UMAR & 0 & 0 & 0 & 0 & 0 \\
\hline BAKWA & 59 & SHIR SURKH & SUFI AZIZ & 0 & 0 & 0 & 0 & 0 \\
\hline BAKWA & 56 & SHIR SURKH & YAQUB KHAN & 0 & 0 & 0 & 0 & 0 \\
\hline BAKWA & 33 & SHOR AQA & SHOR AQA & 0 & 0 & 0 & 0 & 0 \\
\hline BAKWA & 24 & SIA QALA & HAJI GULU JOHN & 0 & 0 & 0 & 0 & 0 \\
\hline BAKWA & 32 & SPINKAY & SPINKAY & 0 & 0 & 0 & 0 & 0 \\
\hline BAKWA & 35 & SULTAN BAKWA & SULTAN BAKWA & 0 & 0 & 0 & 0 & 0 \\
\hline BALA BULOK & 7 & CALL KALA & CALL KALA & 0 & 0 & 0 & 0 & 0 \\
\hline
\end{tabular}

* Jerib (jr.) = 1/5 ha

${ }^{*}{ }^{2}$ Dry Opium $=$ Wet Opium - $30 \%$ moisture 
Opium Production Survey by village in Farah province, 1995.

\begin{tabular}{|c|c|c|c|c|c|c|c|c|}
\hline & Village & & & Total & Land 1 & & Wet Opium * 2 & Wet Opium \\
\hline \multirow[t]{2}{*}{ District } & \multirow[t]{2}{*}{ Code } & \multirow[t]{2}{*}{ Area/ Main Village } & \multirow[t]{2}{*}{ Village } & \multirow{2}{*}{$\begin{array}{c}\text { Cultivated } \\
\left.\text { Land (Jerib }{ }^{*}\right)\end{array}$} & \multicolumn{2}{|c|}{ Poppy Cultivation } & \multirow{2}{*}{$\begin{array}{l}\text { Yield } \\
(\mathrm{kg} / \mathrm{jr} .)\end{array}$} & \multirow{2}{*}{$\begin{array}{l}\text { Production } \\
\text { (kg) }\end{array}$} \\
\hline & & & & & (jerib) & $(\%)$ & & \\
\hline \multicolumn{9}{|c|}{ Province: Farah } \\
\hline BALA BULOK & 1 & DEHZAK & DEHZAK & 0 & 0 & 0 & 0 & 0 \\
\hline BALA BULOK & 5 & GANJ ABAD & GANJ ABAD & 0 & 0 & 0 & 0 & 0 \\
\hline BALA BULOK & 6 & GARANI & GARANI & 0 & 0 & 0 & 0 & 0 \\
\hline BALA BULOK & 12 & KANSAK & KANSAK & 0 & 0 & 0 & 0 & 0 \\
\hline BALA BULOK & 2 & KHUJA JAFAR & KHUJA JAFAR & 320 & 3 & 1 & 0 & 0 \\
\hline BALA BULOK & 11 & NALAK & NALAK & 0 & 0 & 0 & 0 & 0 \\
\hline BALA BULOK & 10 & PULL GANLE & PULL GANLE & 0 & 0 & 0 & 0 & 0 \\
\hline BALA BULOK & 9 & SEIA JANGAL & SEIA JANGAL & 0 & 0 & 0 & 0 & 0 \\
\hline BALA BULOK & 4 & SHEKH LALA & SHEKH LALA & 0 & 0 & 0 & 0 & 0 \\
\hline BALA BULOK & 3 & SHEWAN & SHEWAN & 4,000 & 38 & 1 & 0 & 0 \\
\hline BALA BULOK & 8 & TAQSERAT & TAQSERAT & 0 & 0 & 0 & 0 & 0 \\
\hline
\end{tabular}


Opium Production Survey by village in Helmand province, 1995.

\begin{tabular}{|c|c|c|c|c|c|c|c|c|}
\hline & \multirow{3}{*}{$\begin{array}{l}\text { Village } \\
\text { Code }\end{array}$} & \multirow{3}{*}{ Area/Main Village } & \multirow{3}{*}{ Village } & \multirow{3}{*}{$\begin{array}{c}\text { Total } \\
\text { Cultivated } \\
\text { land } \\
\end{array}$} & \multirow{2}{*}{\multicolumn{2}{|c|}{$\begin{array}{c}\text { Land Under } \\
\text { Poppy cultivation }\end{array}$}} & \multirow{3}{*}{$\begin{array}{c}\text { Wet Opium } \\
\text { Yield } \\
(\mathrm{kg} / \mathrm{jr} .)\end{array}$} & \multirow{3}{*}{$\begin{array}{c}\text { Wet Opium } \\
\text { Production } \\
(\mathrm{kg}) \\
\end{array}$} \\
\hline \multirow[t]{2}{*}{ District } & & & & & & & & \\
\hline & & & & & $\left(\right.$ jerib $\left.^{*}\right)$ & (\%) & & \\
\hline \multicolumn{9}{|c|}{ Province: Helmand } \\
\hline BAGHRAN & 1671 & ADAM KHAN & ADAM KHAN & 25 & 12 & 48 & 12 & 144 \\
\hline BAGHRAN & 1661 & ALI SHANA & ALI SHANA & 40 & 15 & 38 & 13 & 195 \\
\hline BAGHRAN & 1659 & ANAR KAS, CHOGHAKAI & ANAR KAS & 25 & 10 & 40 & 12 & 120 \\
\hline BAGHRAN & 1004 & ANARAK & ANARAK & 60 & 20 & 33 & 11 & 220 \\
\hline BAGHRAN & 1153 & ANARAK & ANARAK & 20 & 8 & 40 & 12 & 96 \\
\hline BAGHRAN & 1006 & ANJUMAN & ANJUMAN & 50 & 20 & 40 & 12 & 240 \\
\hline BAGHRAN & 1654 & ARGHAMAN & ARGHAMAN & 100 & 30 & 30 & 12 & 360 \\
\hline BAGHRAN & 1005 & ARNI & ARNI & 40 & 16 & 40 & 12 & 192 \\
\hline BAGHRAN & 1007 & ARNI & ARNI & 40 & 16 & 40 & 12 & 192 \\
\hline BAGHRAN & 1008 & ASMANIAN & ASMANIAN & 25 & 12 & 48 & 12 & 144 \\
\hline BAGHRAN & 1009 & ATANA & ATANA & 150 & 50 & 33 & 12 & 600 \\
\hline BAGHRAN & 1649 & AWILA & AWILA & 80 & 40 & 50 & 12 & 480 \\
\hline BAGHRAN & 1011 & BABA ZANGEE & BABA ZANGEE & 150 & 50 & 33 & 12 & 600 \\
\hline BAGHRAN & 1652 & BADAMAK & BADAMAK & 80 & 35 & 44 & 13 & 455 \\
\hline BAGHRAN & 1123 & BADI & BADI & 50 & 20 & 40 & 12 & 240 \\
\hline BAGHRAN & 1016 & BAGH JOY & BAGH JOY & 75 & 30 & 40 & 11 & 330 \\
\hline BAGHRAN & 1014 & BAGHAL & BAGHAL & 150 & 60 & 40 & 13 & 780 \\
\hline BAGHRAN & 1667 & BAGHELA & BAGHELA & 30 & 12 & 40 & 12 & 144 \\
\hline BAGHRAN & 1013 & BAGHRAN KHOLA & BAGHRAN KHOLA & 260 & 120 & 46 & 13 & 1,560 \\
\hline BAGHRAN & 1012 & BAJBAND & BAJBAND & 170 & 50 & 29 & 13 & 650 \\
\hline BAGHRAN & 1041 & BALOCHI & BALOCHI & 60 & 30 & 50 & 12 & 360 \\
\hline BAGHRAN & 1047 & BARAS KILY & BARAS KILY & 100 & 35 & 35 & 11 & 385 \\
\hline BAGHRAN & 1040 & BARI QOL & BARI QOL & 180 & 80 & 44 & 11 & 880 \\
\hline BAGHRAN & 1668 & BASHILANG & SAR BASHILANG & 180 & 80 & 44 & 13 & 1,040 \\
\hline BAGHRAN & 1669 & BASHILANG & MIYAN BASHILANG & 100 & 40 & 40 & 13 & 520 \\
\hline BAGHRAN & 1670 & BASHILANG & PAI BASHILANG & 100 & 35 & 35 & 13 & 455 \\
\hline BAGHRAN & 1048 & BAT KHARA & BAT KHARA & 150 & 75 & 50 & 11 & 825 \\
\hline BAGHRAN & 1023 & BATAL & BATAL & 25 & 8 & 32 & 11 & 88 \\
\hline BAGHRAN & 1043 & BAZIGARA PASAW & BAZIGARA PASAW & 50 & 25 & 50 & 12 & 300 \\
\hline
\end{tabular}

* $1 \mathrm{Jerib}=1 / 5 \mathrm{Ha}$

*2 Dry Opium = Wet Opium - $30 \%$ moisture 
Opium Production Survey by village in Helmand province, 1995.

\begin{tabular}{|c|c|c|c|c|c|c|c|c|}
\hline & \multirow{3}{*}{$\begin{array}{l}\text { Village } \\
\text { Code }\end{array}$} & \multirow{3}{*}{ Area/Main Village } & \multirow{3}{*}{ Village } & \multirow{3}{*}{$\begin{array}{c}\text { Total } \\
\text { Cultivated } \\
\text { land }\end{array}$} & \multirow{2}{*}{\multicolumn{2}{|c|}{$\begin{array}{c}\text { Land Under } \\
\text { Poppy cultivation }\end{array}$}} & \multirow{3}{*}{$\begin{array}{c}\text { Wet Opium *2 } \\
\text { Yield } \\
(\mathrm{kg} / \mathrm{jr} .) \\
\end{array}$} & \multirow{3}{*}{$\begin{array}{c}\text { Wet Opium } \\
\text { Production } \\
(\mathrm{kg})\end{array}$} \\
\hline \multirow[t]{2}{*}{ District } & & & & & & & & \\
\hline & & & & & $($ jerib *) & $(\%)$ & & \\
\hline \multicolumn{9}{|c|}{ Province: Helmand } \\
\hline BAGHRAN & 1044 & BELAND JOY & BELAND JOY & 160 & 60 & 38 & 11 & 660 \\
\hline BAGHRAN & 1045 & BERENJAK & BERENJAK & 100 & 40 & 40 & 12 & 480 \\
\hline BAGHRAN & 1039 & BIBI GIRAN & BIBI GIRAN & 80 & 30 & 38 & 12 & 360 \\
\hline BAGHRAN & 1042 & BURGHANA JOEY & BURGHANA JOEY & 350 & 100 & 29 & 11 & 1,100 \\
\hline BAGHRAN & 1046 & BURJ & BURJ & 100 & 50 & 50 & 11 & 550 \\
\hline BAGHRAN & 1147 & CHACHAR & CHACHAR & 100 & 40 & 40 & 10 & 400 \\
\hline BAGHRAN & 1118 & CHAGHAL GHONDA & CHAGHAL GHONDA & 50 & 20 & 40 & 12 & 240 \\
\hline BAGHRAN & 1050 & CHAMAN & CHAMAN/GORA & 70 & 35 & 50 & 12 & 420 \\
\hline BAGHRAN & 1030 & CHANAR & CHANAR & 200 & 100 & 50 & 11 & 1,100 \\
\hline BAGHRAN & 1343 & CHANGAL SARAJUDDEN & BUZ SHIKASTA & 40 & 14 & 35 & 11 & 154 \\
\hline BAGHRAN & 1647 & CHARDO & CHARDO & 80 & 30 & 38 & 12 & 360 \\
\hline BAGHRAN & 1049 & CHARTO & CHARTO & 25 & 10 & 40 & 11 & 110 \\
\hline BAGHRAN & 1052 & CHASHMAGAK & CHASHMAGAK & 60 & 25 & 42 & 13 & 325 \\
\hline BAGHRAN & 1658 & CHEHEL GAZI & CHEHEL GAZI & 50 & 18 & 36 & 12 & 216 \\
\hline BAGHRAN & 1053 & CHEHELGAZI & CHEHELGAZI & 50 & 22 & 44 & 10 & 220 \\
\hline BAGHRAN & 1116 & CHEHL MURDAH & CHEL MURDAH & 35 & 17 & 49 & 12 & 204 \\
\hline BAGHRAN & 1189 & CHENARAK & CHENARAK & 30 & 8 & 27 & 9 & 72 \\
\hline BAGHRAN & 1051 & CHESHMA SAID & CHESHMA SAID & 50 & 20 & 40 & 13 & 260 \\
\hline BAGHRAN & 1054 & CHIA & CHIA & 85 & 40 & 47 & 11 & 440 \\
\hline BAGHRAN & 1173 & CHINAR & CHINAR & 125 & 50 & 40 & 12 & 600 \\
\hline BAGHRAN & 1080 & CHINARAN & CHINARAN & 250 & 60 & 24 & 11 & 660 \\
\hline BAGHRAN & 1055 & DAHAN BURS & DAHAN BURS & 50 & 20 & 40 & 12 & 240 \\
\hline BAGHRAN & 1334 & DARA & KALIZA,SHAJOY & 120 & 60 & 50 & 12 & 720 \\
\hline BAGHRAN & 1191 & DARA SHAH & DARA SHAH & 250 & 120 & 48 & 12 & 1,440 \\
\hline BAGHRAN & 1339 & DAROGHA & DAROGHA & 200 & 55 & 28 & 12 & 660 \\
\hline BAGHRAN & 1062 & DARWESHANAK & DARWESHANAK & 100 & 40 & 40 & 11 & 440 \\
\hline BAGHRAN & 1061 & DASHT & DASHT & 100 & 30 & 30 & 12 & 360 \\
\hline BAGHRAN & 1063 & DASHTAK & DASHTAK & 25 & 13 & 52 & 10 & 130 \\
\hline BAGHRAN & 1176 & DASHTI & KARA & 150 & 70 & 47 & 12 & 840 \\
\hline
\end{tabular}

* 1 Jerib $=1 / 5 \mathrm{Ha}$

* 2 Dry Opium $=$ Wet Opium - $30 \%$ moisture
Page 237
Source: UNDCP Opium Production Survey, 1995 File: HLD3.XLS - 07/08/95 
Opium Production Survey by village in Helmand province, 1995.

\begin{tabular}{|c|c|c|c|c|c|c|c|c|}
\hline & Village & & & Total & Land 1 & & Wet Opium * 2 & Wet Opium \\
\hline \multirow[t]{2}{*}{ District } & \multirow[t]{2}{*}{ Code } & \multirow[t]{2}{*}{ Area/Main Village } & \multirow[t]{2}{*}{ Village } & \multirow{2}{*}{$\begin{array}{c}\text { Cultivated } \\
\text { land }\end{array}$} & \multicolumn{2}{|c|}{ Poppy cultivation } & \multirow{2}{*}{$\begin{array}{c}\text { Yield } \\
(\mathrm{kg} / \mathrm{jr} .)\end{array}$} & \multirow{2}{*}{$\begin{array}{c}\text { Production } \\
(\mathrm{kg})\end{array}$} \\
\hline & & & & & $\left(\right.$ jerib $\left.{ }^{*}\right)$ & $(\%)$ & & \\
\hline \multicolumn{9}{|l|}{ Province: Helmand } \\
\hline BAGHRAN & 1027 & DEH YAK & DEH YAK & 250 & 120 & 48 & 13 & 1,560 \\
\hline BAGHRAN & 1640 & DEWANA QUL & DEWANA QUL & 20 & 3 & 15 & 12 & 36 \\
\hline BAGHRAN. & 1065 & DISANG & DISANG . & 60 & 20 & 33 & 11 & 220 \\
\hline BAGHRAN & 1060 & DORWADE & DO RODEE & 20 & 10 & 50 & 12 & 120 \\
\hline BAGHRAN & 1069 & GARM AB & MALIK MOHD & 250 & 125 & 50 & 12 & 1,500 \\
\hline BAGHRAN & 1070 & GARM AB & ISRAIEL & 275 & 140 & 51 & 12 & 1,680 \\
\hline BAGHRAN & 1075 & GAZAK & GAZAK & 350 & 150 & 43 & 13 & 1,950 \\
\hline BAGHRAN & 1015 & GERDI BAGH GODARA & BAGH GODARA & 120 & 40 & 33 & 12 & 480 \\
\hline BAGHRAN & 1198 & GHALACHA & GHALACHA & 100 & 50 & 50 & 12 & 600 \\
\hline BAGHRAN & 1338 & GHAMBAR JOI & GHAMBAR JOI & 300 & 100 & 33 & 12 & 1,200 \\
\hline BAGHRAN & 1076 & GHAMJO & GHAMJO & 20 & 8 & 40 & 13 & 104 \\
\hline BAGHRAN & 1079 & GHARGHARAW & GHARGHARAW & 25 & 10 & 40 & 12 & 120 \\
\hline BAGHRAN & 1078 & GHARIBAK & GHARIBAK & 50 & 25 & 50 & 11 & 275 \\
\hline BAGHRAN & 1336 & GHICH NAWA & GHICH NAWA & 100 & 30 & 30 & 10 & 300 \\
\hline BAGHRAN & 1196 & GIMIAN & GIMIAN & 140 & 70 & 50 & 12 & 840 \\
\hline BAGHRAN & 1190 & GOMBATA & GOMBATA & 100 & 50 & 50 & 12 & 600 \\
\hline BAGHRAN & 1071 & GONAY & GONAY & 300 & 100 & 33 & 12 & 1,200 \\
\hline BAGHRAN & 1086 & GONGACH & GONGACH & 100 & 40 & 40 & 10 & 400 \\
\hline BAGHRAN & 1020 & GUL KHANA & GUL KHANA & 175 & 60 & 34 & 13 & 780 \\
\hline BAGHRAN & 1085 & GULDOOM & GULDOOM & 100 & 40 & 40 & 12 & 480 \\
\hline BAGHRAN & 1126 & GULO SHELA & GULO SHELA & 35 & 10 & 29 & 12 & 120 \\
\hline BAGHRAN & 1022 & HASAD & HASAD & 350 & 160 & 46 & 14 & 2,240 \\
\hline BAGHRAN & 1087 & HAZAR BUZ & HAZAR BUZ & 62 & 20 & 32 & 11 & 220 \\
\hline BAGHRAN & 1089 & HAZAR DARAKHT & HAZAR DARAKHT & 175 & 90 & 51 & 12 & 1,080 \\
\hline BAGHRAN & 1059 & HOKOMAT BAGHRAN & HOKOMAT BAGHRAN & 80 & 40 & 50 & 11 & 440 \\
\hline BAGHRAN & 1192 & ISLAM GHARA & ISLAM GHARA & 50 & 25 & 50 & 11 & 275 \\
\hline BAGHRAN & 1092 & JARANTI & JARANTI & 50 & 20 & 40 & 11 & 220 \\
\hline BAGHRAN & 1098 & JOOZ KAREZ & JOOZ KAREZ & 80 & 30 & 38 & 12 & 360 \\
\hline BAGHRAN & 1093 & JOSHAH & JOSHAH & 80 & 30 & 38 & 12 & 360 \\
\hline
\end{tabular}

* $1 \mathrm{Jerib}=1 / 5 \mathrm{Ha}$

* 2 Dry Opium = Wet Opium - $30 \%$ moisture
Page 338
Source: UNDCP Opium Production Survey, 1995 File: HLD3.XLS - 07/08/95 
Opium Production Survey by village in Helmand province, 1995.

\begin{tabular}{|c|c|c|c|c|c|c|c|c|}
\hline \multirow{3}{*}{ District } & \multirow{3}{*}{$\begin{array}{l}\text { Village } \\
\text { Code }\end{array}$} & \multirow{3}{*}{ Area/Main Village } & \multirow{3}{*}{ Village } & \multirow{3}{*}{$\begin{array}{c}\text { Total } \\
\text { Cultivated } \\
\text { land } \\
\end{array}$} & \multirow{2}{*}{\multicolumn{2}{|c|}{$\begin{array}{c}\text { Land Under } \\
\text { Poppy cultivation }\end{array}$}} & \multirow{3}{*}{$\begin{array}{c}\text { Wet Opium }^{* 2} \\
\text { Yield } \\
(\mathrm{kg} / \mathrm{jr} .)\end{array}$} & \multirow{3}{*}{$\begin{array}{c}\text { Wet Opium } \\
\text { Production } \\
(\mathrm{kg})\end{array}$} \\
\hline & & & & & & & & \\
\hline & & & & & $\left(\right.$ jerib $\left.{ }^{*}\right)$ & $(\%)$ & & \\
\hline \multicolumn{9}{|c|}{ Province: Helmand } \\
\hline BAGHRAN & 1337 & KADAL JOY & KADAL JOY & 200 & 60 & 30 & 12 & 720 \\
\hline BAGHRAN & 1665 & KAJKARKA & KAJKARKA & 20 & 10 & 50 & 13 & 130 \\
\hline BAGHRAN & 1188 & KAJKO & KAJKO & 70 & 35 & 50 & 11 & 385 \\
\hline BAGHRAN & 1036 & KALAK & KALAK & 200 & 90 & 45 & 13 & 1,170 \\
\hline BAGHRAN & 1095 & KALAN JOY & KALAN JOY & 50 & 25 & 50 & 10 & 250 \\
\hline BAGHRAN & 1094 & KALANA & KALANA & 60 & 25 & 42 & 12 & 300 \\
\hline BAGHRAN & 1096 & KALATA & MOLA NAW KHAN & 250 & 110 & 44 & 12 & 1,320 \\
\hline BAGHRAN & 1097 & KALATA & ABDUL RASHID & 250 & 125 & 50 & 12 & 1,500 \\
\hline BAGHRAN & 1021 & KAPAR KOH & KAPAR KOH & 275 & 120 & 44 & 12 & 1,440 \\
\hline BAGHRAN & 1627 & KAREZ & KAREZ & 40 & 25 & 63 & 12 & 300 \\
\hline BAGHRAN & 1082 & KAREZ ADAM KHAN & KAREZ ADAM KHAN & 25 & 13 & 52 & 12 & 156 \\
\hline BAGHRAN & 1099 & KAREZ HOSAIN & KAREZ HOSAIN & 15 & 8 & 53 & 11 & 88 \\
\hline BAGHRAN & 1633 & KAREZOONA & TAKTO & 25 & 14 & 56 & 11 & 154 \\
\hline BAGHRAN & 1103 & KARGHO & KARGHO & 60 & 18 & 30 & 13 & 234 \\
\hline BAGHRAN & 1657 & KESHATA GHABALTO & KESHATA GHABALTO & 70 & 20 & 29 & 12 & 240 \\
\hline BAGHRAN & 1110 & KHANAJAK MAZAR & SHADI JAN & 360 & 180 & 50 & 14 & 2,520 \\
\hline BAGHRAN & 1737 & KHANAN & KHANAN & 470 & 180 & 38 & 11 & 1,980 \\
\hline BAGHRAN & 1106 & KHANDAN & KHANDAN & 20 & 10 & 50 & 12 & 120 \\
\hline BAGHRAN & 1179 & KHANJAK MAZAR & KHANJAK MAZAR & 100 & 50 & 50 & 12 & 600 \\
\hline BAGHRAN & 1109 & KHANJAK MAZAR & HAJI MOHD DEEN & 400 & 200 & 50 & 14 & 2,800 \\
\hline BAGHRAN & 1111 & KHANJAKAK & KHANJAKAK & 200 & 90 & 45 & 11 & 990 \\
\hline BAGHRAN & 1083 & KHARA SANG & KHARA SANG/TARKA & 55 & 22 & 40 & 12 & 264 \\
\hline BAGHRAN & 1636 & KHARBIDA & KHARBIDA & 25 & 12 & 48 & 11 & 132 \\
\hline BAGHRAN & 1108 & KHASA & KHASA GRZAW & 100 & 30 & 30 & 12 & 360 \\
\hline BAGHRAN & 1663 & KHAZANA/KALKAI & KHAZAN/KALKAI & 35 & 15 & 43 & 12 & 180 \\
\hline BAGHRAN & 1631 & KHENJKAK & KAREZ KHENJKAK & 25 & 12 & 48 & 11 & 132 \\
\hline BAGHRAN & 1342 & KHERS ABB & KHERS ABB & 25 & 13 & 52 & 11 & 143 \\
\hline BAGHRAN & 1114 & KHOBIAN & KHOBIAN & 50 & 20 & 40 & 11 & 220 \\
\hline BAGHRAN & 1113 & KHOJA GORAH & KHOJA GORAH & 30 & 15 & 50 & 12 & 180 \\
\hline
\end{tabular}


Opium Production Survey by village in Helmand province, 1995.

\begin{tabular}{|c|c|c|c|c|c|c|c|c|}
\hline & \multirow{3}{*}{$\begin{array}{l}\text { Village } \\
\text { Code }\end{array}$} & \multirow{3}{*}{ Area/Main Village } & \multirow{3}{*}{ Village } & \multirow{3}{*}{$\begin{array}{c}\text { Total } \\
\text { Cultivated } \\
\text { land } \\
\end{array}$} & \multirow{2}{*}{\multicolumn{2}{|c|}{$\begin{array}{c}\text { Land Under } \\
\text { Poppy cultivation }\end{array}$}} & \multirow{3}{*}{$\begin{array}{c}\text { Wet Opium }^{* 2} \\
\text { Yield } \\
(\mathrm{kg} / \mathrm{jr} .)\end{array}$} & \multirow{3}{*}{$\begin{array}{c}\text { Wet Opium } \\
\text { Production } \\
(\mathrm{kg})\end{array}$} \\
\hline \multirow[t]{2}{*}{ District } & & & & & & & & \\
\hline & & & & & $\left(\right.$ jerib $\left.^{*}\right)$ & (\%) & & \\
\hline \multicolumn{9}{|c|}{ Province: Helmand } \\
\hline BAGHRAN & 1152 & KHOJA RANGAL & NAGTOO & 40 & 15 & 38 & 12 & 180 \\
\hline BAGHRAN & 1090 & KHOJA ZARGUL & JAGHATO & 50 & 12 & 24 & 12 & 144 \\
\hline BAGHRAN & 1026 & KHOSHKAK & KHOSHKAK & 40 & 20 & 50 & 10 & 200 \\
\hline BAGHRAN & 1632 & KHUAJA MESAL & KHUJA MESAL & 25 & 13 & 52 & 12 & 156 \\
\hline BAGHRAN & 1664 & KOCHANAI KHODSAR & KOCHNAI KHODSAR & 75 & 25 & 33 & 12 & 300 \\
\hline BAGHRAN & 1195 & KONJAK & KONJAK & 125 & 65 & 52 & 13 & 845 \\
\hline BAGHRAN & 1100 & KONJEN & MULA MOHD.MIR & 250 & 120 & 48 & 12 & 1,440 \\
\hline BAGHRAN & 1101 & KONJEN & KONJEN & 250 & 110 & 44 & 12 & 1,320 \\
\hline BAGHRAN & 1648 & KOOR & KOOR & 80 & 30 & 38 & 12 & 360 \\
\hline BAGHRAN & 1128 & LABA & LABA & 40 & 15 & 38 & 11 & 165 \\
\hline BAGHRAN & 1169 & LALA KHAN KALAY & LALA KHAN KALAY & 70 & 7 & 10 & 11 & 77 \\
\hline BAGHRAN & 1033 & LARONAI & LARONAI & 100 & 50 & 50 & 12 & 600 \\
\hline BAGHRAN & 1017 & LIWANIAN & LIWANIAN & 240 & 110 & 46 & 13 & 1,430 \\
\hline BAGHRAN & 1117 & LODARA & LODARA & 20 & 10 & 50 & 13 & 130 \\
\hline BAGHRAN & 1129 & LOKA & LOKA & 120 & 50 & 42 & 12 & 600 \\
\hline BAGHRAN & 1131 & LOWAR GHABLTO & LOWAR GHABLTO & 70 & 30 & 43 & 12 & 360 \\
\hline BAGHRAN & 1197 & LOWAR PAIGAW & LOWAR PAIGAW & 110 & 60 & 55 & 12 & 720 \\
\hline BAGHRAN & 1133 & MALANG & MALANG, KAPIR & 50 & 20 & 40 & 13 & 260 \\
\hline BAGHRAN & 1037 & MANO & MANO & 350 & 160 & 46 & 12 & 1,920 \\
\hline BAGHRAN & 1058 & MARGHA & MARGHA & 20 & 10 & 50 & 11 & 110 \\
\hline BAGHRAN & 1134 & MAYAGAN & MAYAGAN & 190 & 95 & 50 & 11 & 1,045 \\
\hline BAGHRAN & 1646 & MAYAN SHALMEN & SEDEQA & 20 & 10 & 50 & 11 & 110 \\
\hline BAGHRAN & 1105 & MEYAM SHALMEN & CHAGHAN & 50 & 25 & 50 & 12 & 300 \\
\hline BAGHRAN & 1643 & MEYAN SHALMEN & NOBAR & 40 & 20 & 50 & 11 & 220 \\
\hline BAGHRAN & 1645 & MEYAN SHALMEN & ANAR JOY & 75 & 35 & 47 & 12 & 420 \\
\hline BAGHRAN & 1121 & MIAN KALA & MIAN KALA & 50 & 20 & 40 & 13 & 260 \\
\hline BAGHRAN & 1163 & MIAN SHALMIN & SAWADKA & 15 & 6 & 40 & 12 & 72 \\
\hline BAGHRAN & 1644 & MIAN SHALMIN & GHULAM JOY & 60 & 35 & 58 & 11 & 385 \\
\hline BAGHRAN & 1072 & MIR ASHKAR & MIR ASHKAR & 55 & 25 & 45 & 11 & 275 \\
\hline
\end{tabular}

* 1 Jerib $=1 / 5 \mathrm{Ha}$

* 2 Dry Opium = Wet Opium - $30 \%$ moisture
Page 540
Source: UNDCP Opium Production Survey, 1995 File: HLD3.XLS - 07/08/95 
Opium Production Survey by village in Helmand province, 1995.

\begin{tabular}{|c|c|c|c|c|c|c|c|c|}
\hline \multirow{3}{*}{ District } & Village & \multirow{3}{*}{ Area/Main Village } & \multirow{3}{*}{ Village } & \multirow{3}{*}{$\begin{array}{c}\text { Total } \\
\text { Cultivated } \\
\text { land } \\
\end{array}$} & \multirow{2}{*}{\multicolumn{2}{|c|}{$\begin{array}{c}\text { Land Under } \\
\text { Poppy cultivation }\end{array}$}} & \multirow{3}{*}{$\begin{array}{c}\text { Wet Opium }^{* 2} \\
\text { Yield } \\
(\mathrm{kg} / \mathrm{jr} .) \\
\end{array}$} & \multirow{3}{*}{$\begin{array}{c}\text { Wet Opium } \\
\text { Production } \\
(\mathrm{kg}) \\
\end{array}$} \\
\hline & Code & & & & & & & \\
\hline & & & & & $\left(\right.$ jerib $\left.{ }^{*}\right)$ & $(\%)$ & & \\
\hline \multicolumn{9}{|c|}{ Province: Helmand } \\
\hline BAGHRAN & 1639 & MIRGHA SHOOM & MIRGHA SHOOM & 20 & 4 & 20 & 12 & 48 \\
\hline BAGHRAN & 1135 & MIYAN SHALMIN & LOEY KAREZ & 320 & 120 & 38 & 12 & 1,440 \\
\hline BAGHRAN & 1137 & MONDE KAKA & MONDE KAKA KALY & 25 & 12 & 48 & 12 & 144 \\
\hline BAGHRAN & 1119 & MORGHAB & MORGHAB/SANG SH & 50 & 20 & 40 & 13 & 260 \\
\hline BAGHRAN & 1138 & MOTIKA & MOTIKA & 75 & 35 & 47 & 11 & 385 \\
\hline BAGHRAN & 1139 & NAGANG & NAGANG & 50 & 25 & 50 & 10 & 250 \\
\hline BAGHRAN & 1140 & NAMAZGAH & NAMAZGAH & 300 & 80 & 27 & 11 & 880 \\
\hline BAGHRAN & 1641 & NARIKAN & NARIKAN & 110 & 50 & 45 & 11 & 550 \\
\hline BAGHRAN & 1650 & NARTOOT & NAR TOOT & 200 & 70 & 35 & 12 & 840 \\
\hline BAGHRAN & 1141 & NATEA & NATEA & 100 & 50 & 50 & 11 & 550 \\
\hline BAGHRAN & 1223 & NATEA & SEYA CHOWLA & 25 & 10 & 40 & 12 & 120 \\
\hline BAGHRAN & 1142 & NAWA DEDAD & NAWA BEDAD & 250 & 65 & 26 & 10 & 650 \\
\hline BAGHRAN & 1150 & NAWA MESH & JAGH & 250 & 90 & 36 & 12 & 1,080 \\
\hline BAGHRAN & 1151 & NAWA MESH & SURKH JOY & 250 & 70 & 28 & 11 & 770 \\
\hline BAGHRAN & 6706 & NAWA MESH & NAWA MESH & 650 & 170 & 26 & 11 & 1,870 \\
\hline BAGHRAN & 1018 & OKARCHI & OKARCHI & 250 & 100 & 40 & 13 & 1,300 \\
\hline BAGHRAN & 1154 & ORGSOON & ORGSOON & 150 & 75 & 50 & 12 & 900 \\
\hline BAGHRAN & 1066 & PAEY SHALMEN & DO SANG, REGI & 100 & 50 & 50 & 11 & 550 \\
\hline BAGHRAN & 1161 & PAEY SHILMEN & PAEY SHILMEN & 100 & 50 & 50 & 11 & 550 \\
\hline BAGHRAN & 1160 & PAI JANJI & PAI JANJI & 90 & 40 & 44 & 11 & 440 \\
\hline BAGHRAN & 1193 & PAIGAW & KSHTA PAIGAW & 50 & 25 & 50 & 13 & 325 \\
\hline BAGHRAN & 1122 & PAIKALA & PAIKALA & 75 & 30 & 40 & 13 & 390 \\
\hline BAGHRAN & 1164 & PAISANG & PAISANG & 200 & 90 & 45 & 13 & 1,170 \\
\hline BAGHRAN & 1165 & PALARZARA & PALARZARA & 25 & 8 & 32 & 11 & 88 \\
\hline BAGHRAN & 1157 & PAR KOND & HAJI LALA JAN & 325 & 160 & 49 & 13 & 2,080 \\
\hline BAGHRAN & 1156 & PAR KOND & HAJI MOHD HASAN & 300 & 150 & 50 & 13 & 1,950 \\
\hline BAGHRAN & 1043 & PASAW & PASAW/BAZGIRA & 75 & 30 & 40 & 13 & 390 \\
\hline BAGHRAN & 1088 & PAY HAZAR NAW & PAY HAZAR NAW & 50 & 20 & 40 & 11 & 220 \\
\hline BAGHRAN & 1656 & PIK & PIK NAW ABAD & 100 & 40 & 40 & 12 & 480 \\
\hline
\end{tabular}

* 1 Jerib $=1 / 5 \mathrm{Ha}$

* 2 Dry Opium = Wet Opium - 30\% moisture
Source: UNDCP Opium Production Survey, 1995 File: HLD3.XLS - 07/08/95 
Opium Production Survey by village in Helmand province, 1995.

\begin{tabular}{|c|c|c|c|c|c|c|c|c|}
\hline \multirow{3}{*}{ District } & \multirow{3}{*}{$\begin{array}{l}\text { Village } \\
\text { Code }\end{array}$} & \multirow{3}{*}{ Area/Main Village } & \multirow{3}{*}{ Village } & \multirow{3}{*}{$\begin{array}{c}\text { Total } \\
\text { Cultivated } \\
\text { land } \\
\end{array}$} & \multirow{2}{*}{\multicolumn{2}{|c|}{$\begin{array}{c}\text { Land Under } \\
\text { Poppy cultivation }\end{array}$}} & \multirow{3}{*}{$\begin{array}{c}\text { Wet Opium }^{* 2} \\
\text { Yield } \\
(\mathrm{kg} / \mathrm{jr} .)\end{array}$} & \multirow{3}{*}{$\begin{array}{c}\text { Wet Opium } \\
\text { Production } \\
\text { (kg) } \\
\end{array}$} \\
\hline & & & & & & & & \\
\hline & & & & & $\left(\right.$ jerib $\left.^{*}\right)$ & $(\%)$ & & \\
\hline \multicolumn{9}{|c|}{ Province: Helmand } \\
\hline BAGHRAN & 1194 & PIRGHOL & PIRGHOL & 130 & 70 & 54 & 12 & 840 \\
\hline BAGHRAN & 1091 & PUSHTAH & PUSHTAH & 25 & 10 & 40 & 12 & 120 \\
\hline BAGHRAN & 1115 & QADIRAK & QADIRAK & 80 & 20 & 25 & 13 & 260 \\
\hline BAGHRAN & 1167 & QARA BAGH & MULLA MOHD SHAH & 80 & 9 & 11 & 11 & 99 \\
\hline BAGHRAN & 1182 & QOLBA & QOLBA & 80 & 35 & 44 & 12 & 420 \\
\hline BAGHRAN & 1170 & RAHMAT JOY & RAHMAT JOY & 20 & 10 & 50 & 11 & 110 \\
\hline BAGHRAN & 1651 & RANGAN & RANGAN & 140 & 70 & 50 & 12 & 840 \\
\hline BAGHRAN & 1175 & RASHTI & KHALAMAT & 110 & 50 & 45 & 11 & 550 \\
\hline BAGHRAN & 1172 & RASHTI & KHUSHK AAB & 60 & 30 & 50 & 11 & 330 \\
\hline BAGHRAN & 1032 & RIAH & RIAH KAMANKA & 110 & 60 & 55 & 13 & 780 \\
\hline BAGHRAN & 1180 & ROAD & SANGSHAN & 125 & 50 & 40 & 11 & 550 \\
\hline BAGHRAN & 1181 & ROAD & ANAR JOY & 150 & 50 & 33 & 12 & 600 \\
\hline BAGHRAN & 1159 & ROOD & PAIGAW & 50 & 20 & 40 & 12 & 240 \\
\hline BAGHRAN & 1184 & SABZI QUL & SABZI QUL & 100 & 40 & 40 & 10 & 400 \\
\hline BAGHRAN & 1222 & SACHO ULIA & SACHO ULIA & 175 & 80 & 46 & 12 & 960 \\
\hline BAGHRAN & 1185 & SAD MANI & SAD MANI & 120 & 40 & 33 & 12 & 480 \\
\hline BAGHRAN & 1662 & SAHRAKA/BINBA & SAHRAKA/BINBA & 40 & 18 & 45 & 11 & 198 \\
\hline BAGHRAN & 1672 & SAHRBAT, LAKHTOGHI & SHARBAT, LAKHTO & 50 & 20 & 40 & 12 & 240 \\
\hline BAGHRAN & 1200 & SALBIN & SALBIN & 40 & 10 & 25 & 9 & 90 \\
\hline BAGHRAN & 1174 & SANGINAK WA KAJOY & SANGINAK/KAJOY & 100 & 60 & 60 & 11 & 660 \\
\hline BAGHRAN & 1186 & SANGOWANA & H. MOHTADIN & 150 & 80 & 53 & 13 & 1,040 \\
\hline BAGHRAN & 1187 & SANGWANA & YASIN HOKA & 160 & 80 & 50 & 13 & 1,040 \\
\hline BAGHRAN & 1202 & SAR GHOCH & SAR GHOCH & 100 & 50 & 50 & 11 & 550 \\
\hline BAGHRAN & 1203 & SAR JANJI & SAR JANJI & 100 & 50 & 50 & 12 & 600 \\
\hline BAGHRAN & 1124 & SAR KALA & SAR KALA & 100 & 40 & 40 & 12 & 480 \\
\hline BAGHRAN & 1205 & SAR SHALMIN & SAR SHALMIN & 220 & 110 & 50 & 12 & 1,320 \\
\hline BAGHRAN & 1132 & SAR ZARGHAIN & SAR ZARGHAIN & 20 & 10 & 50 & 12 & 120 \\
\hline BAGHRAN & 1031 & SARI BAZ & SARI BAZ & 300 & 120 & 40 & 12 & 1,440 \\
\hline BAGHRAN & 1207 & SAYED BAHAUDEEN & SAIFULLAH & 280 & 150 & 54 & 13 & 1,950 \\
\hline
\end{tabular}

* $1 \mathrm{Jerib}=1 / 5 \mathrm{Ha}$

${ }^{*}$ Dry Opium $=$ Wet Opium $-30 \%$ moisture
Page 742
Source: UNDCP Opium Production Survey, 1995 File: HLD3.XLS - 07/08/95 
Opium Production Survey by village in Helmand province, 1995.

\begin{tabular}{|c|c|c|c|c|c|c|c|c|}
\hline & \multirow{3}{*}{ Code } & 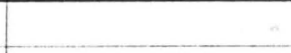 & & Total & Land & & Wet Opium *2 & Wet Opium \\
\hline \multirow[t]{2}{*}{ District } & & \multirow[t]{2}{*}{ Area/Main Village } & \multirow[t]{2}{*}{ Village } & \multirow{2}{*}{$\begin{array}{c}\text { Cultivated } \\
\text { land }\end{array}$} & \multicolumn{2}{|c|}{ Poppy cultivation } & \multirow{2}{*}{$\begin{array}{c}\text { Yield } \\
(\mathrm{kg} / \mathrm{jr} .) \\
\end{array}$} & \multirow{2}{*}{$\begin{array}{c}\text { Production } \\
(\mathrm{kg})\end{array}$} \\
\hline & & & & & $\left(\right.$ jerib $\left.^{*}\right)$ & $(\%)$ & & \\
\hline \multicolumn{9}{|c|}{ Province: Helmand } \\
\hline BAGHRAN & 1206 & SAYED BAHUDEEN & NASEEM KAKAK & 350 & 180 & 51 & 13 & 2,340 \\
\hline BAGHRAN & 1634 & SAYEDAN & SAYEDAN & 7 & 3 & 43 & 11 & 33 \\
\hline BAGHRAN & 1220 & SHAH HAIDAR & SHAH HAIDAR & 15 & 8 & 53 & 11. & 88 \\
\hline BAGHRAN & 1028 & SHAHGHAMBER & SHAHGHAMBER & 200 & 100 & 50 & 13 & 1,300 \\
\hline BAGHRAN & 1213 & SHAHJOY & SHAHJOY & 100 & 45 & 45 & 11 & 495 \\
\hline BAGHRAN & 1201 & SHAKINAG & SHAKINAG LIWA & 25 & 6 & 24 & 8 & 48 \\
\hline BAGHRAN & 1229 & SHALA BASHLAN & TAKAL GHO & 50 & 15 & 30 & 11 & 165 \\
\hline BAGHRAN & 1209 & SHALIGAR & SHALIGAR & 25 & 12 & 48 & 11 & 132 \\
\hline BAGHRAN & 1215 & SHANDDRA & HAJI DASTGIR & 320 & 150 & 47 & 14 & 2,100 \\
\hline BAGHRAN & 1125 & SHANGHARAH & SHANGHARAH & 25 & 12 & 48 & 11 & 132 \\
\hline BAGHRAN & 1025 & SHARI YAK & JAR SHARI YAK & 100 & 50 & 50 & 13 & 650 \\
\hline BAGHRAN & 1219 & SHARI YAK & HAJI JAMAL & 300 & 100 & 33 & 13 & 1,300 \\
\hline BAGHRAN & 1212 & SHARI YAK & HAJI ESMATULLAH & 300 & 120 & 40 & 13 & 1,560 \\
\hline BAGHRAN & 1208 & SHELA BASHLAN & SEDIQA & 55 & 15 & 27 & 12 & 180 \\
\hline BAGHRAN & 1216 & SHENDARA & OTAKE & 350 & 160 & 46 & 13 & 2,080 \\
\hline BAGHRAN & 1210 & SHINA & SHINA & 60 & 30 & 50 & 12 & 360 \\
\hline BAGHRAN & 1635 & SHINA & SHINA & 25 & 12 & 48 & 11 & 132 \\
\hline BAGHRAN & 1104 & SHINDARA & JAR MOHD & 120 & 50 & 42 & 13 & 650 \\
\hline BAGHRAN & 1660 & SHINKAI KOTAL & SHINKAI KOTAL & 25 & 10 & 40 & 11 & 110 \\
\hline BAGHRAN & 1675 & SHINYAH & SHINYAH & 50 & 25 & 50 & 12 & 300 \\
\hline BAGHRAN & 1673 & SHIRAW & SHIRAW & 60 & 22 & 37 & 12 & 264 \\
\hline BAGHRAN & 1218 & SHIRIN HADA & SHIRIN HADA & 25 & 12 & 48 & 11 & 132 \\
\hline BAGHRAN & 1029 & SHWI & SHIWI & 300 & 150 & 50 & 12 & 1,800 \\
\hline BAGHRAN & 1155 & SIA SANG & SIA SANG & 20 & 10 & 50 & 12 & 120 \\
\hline BAGHRAN & 1002 & SIACHO & SIACHO & 90 & 40 & 44 & 11 & 440 \\
\hline BAGHRAN & 1221 & SOKHTA & SOKHTA & 120 & 40 & 33 & 12 & 480 \\
\hline BAGHRAN & 1120 & SOOM & SOOM, KIWASH & 75 & 30 & 40 & 13 & 390 \\
\hline BAGHRAN & 1217 & SORKH KOTAL & SORKH KOTAL & 60 & 18 & 30 & 12 & 216 \\
\hline BAGHRAN & 1038 & SORNI & SORNI & 250 & 120 & 48 & 13 & 1,560 \\
\hline
\end{tabular}


Opium Production Survey by village in Helmand province, 1995.

\begin{tabular}{|c|c|c|c|c|c|c|c|c|}
\hline \multirow{3}{*}{ District } & \multirow{3}{*}{$\begin{array}{l}\text { Village } \\
\text { Code }\end{array}$} & \multirow{3}{*}{ Area/Main Village } & \multirow{3}{*}{ Village } & \multirow{3}{*}{$\begin{array}{c}\text { Total } \\
\text { Cultivated } \\
\text { land } \\
\end{array}$} & \multirow{2}{*}{\multicolumn{2}{|c|}{$\begin{array}{c}\text { Land Under } \\
\text { Poppy cultivation }\end{array}$}} & \multirow{3}{*}{$\begin{array}{c}\text { Wet Opium }^{* 2} \\
\text { Yield } \\
(\mathrm{kg} / \mathrm{jr} .)\end{array}$} & \multirow{3}{*}{$\begin{array}{c}\text { Wet Opium } \\
\text { Production } \\
(\mathrm{kg}) \\
\end{array}$} \\
\hline & & & & & & & & \\
\hline & & & & & $\left(\right.$ jerib $\left.^{*}\right)$ & $(\%)$ & & \\
\hline \multicolumn{9}{|c|}{ Province: Helmand } \\
\hline BAGHRAN & 1199 & SRAH POZAH & SRAH POZAH & 20 & 6 & 30 & 9 & 54 \\
\hline BAGHRAN & 1149 & SRAY BAGH & SRAY BAGH & 100 & 35 & 35 & 11 & 385 \\
\hline BAGHRAN & 1642 & SYA CHO & SYA CHO & 200 & 80 & 40 & 12 & 960 \\
\hline BAGHRAN & 1630 & TAAJO KHAN & KAREZ TAAJO KHA & 25 & 0 & 0 & 0 & 0 \\
\hline BAGHRAN & 1224 & TACHARAI & TACHARAI/AMRTOT & 200 & 60 & 30 & 13 & 780 \\
\hline BAGHRAN & 1628 & TACHENA & TACHENA & 13 & 6 & 46 & 11 & 66 \\
\hline BAGHRAN & 1340 & TAK & TAK & 220 & 70 & 32 & 11 & 770 \\
\hline BAGHRAN & 1629 & TAKHAM JOYE & TAKHAM JOYE & 100 & 60 & 60 & 11 & 660 \\
\hline BAGHRAN & 1081 & TAKHUM & TAKHUM & 320 & 100 & 31 & 11 & 1,100 \\
\hline BAGHRAN & 1230 & TALI & TALI & 80 & 30 & 38 & 12 & 360 \\
\hline BAGHRAN & 1034 & TANGI & TANGI & 120 & 60 & 50 & 12 & 720 \\
\hline BAGHRAN & 1638 & TANGI & MAIN TANGI & 15 & 3 & 20 & 12 & 36 \\
\hline BAGHRAN & 1228 & TANGI & TANGI & 20 & 4 & 20 & 11 & 44 \\
\hline BAGHRAN & 1127 & TANGI/SHAHO & TANGI/SHAHO & 25 & 10 & 40 & 13 & 130 \\
\hline BAGHRAN & 1637 & TANGY & SIR TANGI & 15 & 3 & 20 & 11 & 33 \\
\hline BAGHRAN & 1225 & TASINA & TASINA & 25 & 12 & 48 & 11 & 132 \\
\hline BAGHRAN & 1655 & TOLI & TOLI & 20 & 10 & 50 & 11 & 110 \\
\hline BAGHRAN & 1035 & UNAI & UNAI & 350 & 150 & 43 & 12 & 1,800 \\
\hline BAGHRAN & 1231 & WALA & WALA & 20 & 10 & 50 & 11 & 110 \\
\hline BAGHRAN & 1168 & WARZA & TARYAK & 50 & 25 & 50 & 11 & 275 \\
\hline BAGHRAN & 1348 & WARZA & GHARIAN/SIA ZAMINA & 100 & 40 & 40 & 11 & 440 \\
\hline BAGHRAN & 1232 & WORZAH & WORZAH & 60 & 25 & 42 & 11 & 275 \\
\hline BAGHRAN & 1019 & YASEEN DEWANA & YASEEN DEWANA & 250 & 100 & 40 & 13 & 1,300 \\
\hline BAGHRAN & 1233 & ZARAWAN & ZAHER & 200 & 100 & 50 & 12 & 1,200 \\
\hline BAGHRAN & 1162 & ZARGHAIN & PAI ZARGHAIN & 25 & 10 & 40 & 12 & 120 \\
\hline BAGHRAN & 1234 & ZAROWAN & MORGHAKAI & 100 & 40 & 40 & 12 & 480 \\
\hline BAGHRAN & 1674 & ZEYARAT WALA WATANGI & ZEYARAT WALA & 50 & 20 & 40 & 13 & 260 \\
\hline BAGHRAN & 1235 & ZOBAR & KAJA DAGH & 65 & 20 & 31 & 12 & 240 \\
\hline BAGHRAN & 1236 & ZOBAR & NAFAS ZOBAR & 80 & 30 & 38 & 13 & 390 \\
\hline
\end{tabular}


Opium Production Survey by village in Helmand province, 1995.

\begin{tabular}{|c|c|c|c|c|c|c|c|c|}
\hline & Village & & & Total & Land $\mathrm{C}$ & & Wet Opium $^{* 2}$ & Wet Opium \\
\hline \multirow[t]{2}{*}{ District } & \multirow[t]{2}{*}{ Code } & \multirow[t]{2}{*}{ Area/Main Village } & \multirow[t]{2}{*}{ Village } & \multirow{2}{*}{$\begin{array}{c}\text { Cultivated } \\
\text { land } \\
\end{array}$} & \multicolumn{2}{|c|}{ Poppy cultivation } & \multirow{2}{*}{$\begin{array}{c}\text { Yield } \\
(\mathrm{kg} / \mathrm{jr} .)\end{array}$} & \multirow{2}{*}{$\begin{array}{c}\text { Production } \\
(\mathrm{kg})\end{array}$} \\
\hline & & & & & $($ jerib *) & $(\%)$ & & \\
\hline \multicolumn{9}{|c|}{ Province: Helmand } \\
\hline BAGHRAN & 1074 & ZORANGI & ZORANGI & 50 & 20 & 40 & 12 & 240 \\
\hline BAGHRAN & 1347 & & ZIARAT,ZAMINKA & 30 & 12 & 40 & 11 & 132 \\
\hline BAGHRAN & 1346 & & HAZAR JAOZ & 25 & 10 & 40 & 11 & 110 \\
\hline BAGHRAN & 344 & & HGARBOR KANA & 40 & 16 & 40 & 11 & 176 \\
\hline BAGHRAN & 1341 & & CHAHAR DARAKHT & 150 & 55 & 37 & 11 & 605 \\
\hline BAGHRAN & 1130 & & LAJEE & 100 & 40 & 40 & 11 & 440 \\
\hline BUST & 1509 & A.SAMAD \&A.ALI KELY & A.SAMAD\&A.ALI KELY & 400 & 40 & 10 & 18 & 720 \\
\hline BUST & 1543 & ALI SOOR & ALI SOOR & 585 & 100 & 17 & 18 & 1,800 \\
\hline BUST & 1529 & ASTOZAI & ASTOZAI & 800 & 280 & 35 & 18 & 5,040 \\
\hline BUST & 1536 & BABAGHEE FASEL & BABAGHEE FASEL & 443 & 80 & 18 & 22 & 1,760 \\
\hline BUST & 1504 & BAREESI KALAY & BAREESI KALAY & 50 & 0 & 0 & 0 & 0 \\
\hline BUST & 1515 & BASHARAN ULIA & BASHARAN ULIA & 450 & 100 & 22 & 20 & 2,000 \\
\hline BUST & 1524 & BRENG & BRENG & 1,010 & 540 & 53 & 18 & 9,720 \\
\hline BUST & 1518 & BULAN FORM & BULAN FORM & 610 & 50 & 8 & 18 & 900 \\
\hline BUST & 1527 & CHAR BAGH KELY & CHAR BAGH KELY & 380 & 150 & 39 & 19 & 2,850 \\
\hline BUST & 1535 & DEH AFGHAN & DEH AFGHAN & 1,500 & 0 & 0 & 0 & 0 \\
\hline BUST & 1502 & DEWAR KELY & DEWAR KELY & 250 & 2. & 1 & 18 & 27 \\
\hline BUST & 1534 & FASL-E-BOLAN & FASL-E-BOLAN & 135 & 42 & 31 & 22 & 924 \\
\hline BUST & 1508 & GULIKHAR SAYDAN & GULIKHAR SAYDAN & 150 & 0 & 0 & 0 & 0 \\
\hline BUST & 1511 & HAJI GHAFOOR KHAN & GHAFOOR KHAN & 1,200 & 220 & 18 & 18 & 3,960 \\
\hline BUST & 1517 & HAJI KHOSHDIL KELY & HAJI KHOSHDIL KELY & 770 & 120 & 16 & 18 & 2,160 \\
\hline BUST & 1500 & HAYAT KHAN MALZAI & HAYAT KHAN MALZAI & 130 & 2 & 1. & 18 & 27 \\
\hline BUST & 1525 & IBRAHIMZAI KELY & IBRAHIMZAI KELY & 200 & 150 & 75 & 18 & 2,700 \\
\hline BUST & 1514 & KAKARANO KELY & KAKARANO KELY & 720 & 130 & 18 & 18 & 2,340 \\
\hline BUST & 1513 & KARGARO KELY & KARGARO KELY & 134 & 20 & 15 & 18 & 360 \\
\hline BUST & 1516 & KHANANO KELY & KHANANO KELY & 1,400 & 150 & 11 & 18 & 2,700 \\
\hline BUST & 1531 & KHANKHEL & KHANKHEL & 130 & 100 & 77 & 19 & 1,900 \\
\hline BUST & 1533 & KHARI KELY & KHARI KELY & 500 & 47. & 9 & 18 & 846 \\
\hline BUST & 1540 & KHUSHKAWA & KHUSHKAWA & 400 & 40 & 10 & 18 & 720 \\
\hline
\end{tabular}

* $1 \mathrm{Jerib}=1 / 5 \mathrm{Ha}$

* ${ }^{2}$ Dry Opium $=$ Wet Opium $-30 \%$ moisture
Page $10 \quad 45$
Source: UNDCP Opium Production Survey, 1995 File: HLD3.XLS - 07/08/95 
Opium Production Survey by village in Helmand province, 1995.

\begin{tabular}{|c|c|c|c|c|c|c|c|c|}
\hline \multirow{3}{*}{ District } & \multirow{3}{*}{$\begin{array}{l}\text { Village } \\
\text { Code }\end{array}$} & \multirow{3}{*}{ Area/Main Village } & \multirow{3}{*}{ Village } & \multirow{3}{*}{$\begin{array}{c}\text { Total } \\
\text { Cultivated } \\
\text { land } \\
\end{array}$} & \multirow{2}{*}{\multicolumn{2}{|c|}{$\begin{array}{c}\text { Land Under } \\
\text { Poppy cultivation }\end{array}$}} & \multirow{3}{*}{$\begin{array}{c}\text { Wet Opium *2 } \\
\text { Yield } \\
(\mathrm{kg} / \mathrm{jr} .)\end{array}$} & \multirow{3}{*}{$\begin{array}{c}\text { Wet Opium } \\
\text { Production } \\
(\mathrm{kg})\end{array}$} \\
\hline & & & & & & & & \\
\hline & & & & & $\left(\right.$ jerib $\left.^{*}\right)$ & $(\%)$ & & \\
\hline \multicolumn{9}{|c|}{ Province: Helmand } \\
\hline BUST & 1503 & LACHMI & LACHMANI & 0 & 0 & \#DIV/0! & 0 & 0 \\
\hline BUST & 1537 & LOWARA & LOWARA & 210 & 2 & 1 & 18 & 36 \\
\hline BUST & 1542 & MALIK KHERO & MALIK KHERO & 825 & 20 & 2 & 18 & 360 \\
\hline BUST & 1507 & MOHD. IBRAHIM KELY & MOHD.IBRAHIM & 35 & 0 & 0 & 0 & 0 \\
\hline BUST & 1510 & MOHD.AMIN KELY & MOHD. AMIN KELY & 1,500 & 120 & 8 & 18 & 2,160 \\
\hline BUST & 1538 & NADER KHAN & NADER KHAN & 500 & 80 & 16 & 22 & 1,760 \\
\hline BUST & 1545 & PANJAB & PANJAB & 40 & 20 & 50 & 18 & 360 \\
\hline BUST & 1501 & QALA-E- KHARI & QALA-E-KHARI & 450 & 0 & 0 & 0 & 0 \\
\hline BUST & 1520 & QARGHCHA & QAGHCHA & 850 & 130 & 15 & 18 & 2,340 \\
\hline BUST & 1539 & RADAR & RADAR & 335 & 68 & 20 & 22 & 1,496 \\
\hline BUST & 1522 & RAHIM KHALIL & SAEYD ALI KELY & 780 & 150 & 19 & 18 & 2,700 \\
\hline BUST & 1523 & RAHIM KHALIL & HAJI BAHLOL KELY & 1,000 & 220 & 22 & 18 & 3,960 \\
\hline BUST & 1521 & RAHIM KHEL & HAJI KUL EKHTYAR & 110 & 40 & 36 & 18 & 720 \\
\hline BUST & 1519 & RAHIM KHLIL & CHARDA KELY & 700 & 250 & 36 & 18 & 4,500 \\
\hline BUST & 1528 & RASTO ZAI & MOHD. GHOUS & 800 & 180 & 23 & 18 & 3,240 \\
\hline BUST & 1547 & SAHIB ZADA KHEL & SAHIB ZADA KHEL & 200 & 30 & 15 & 18 & 540 \\
\hline BUST & 1506 & SAKHIDAD KILI & SAKHIDAD & 30 & 0 & 0 & 0 & 0 \\
\hline BUST & 1505 & SAKHTA KELY & SAKHTA KELY & 120 & 1 & 1 & 18 & 18 \\
\hline BUST & 1548 & SAWA GADAR & SAWA GADAR & 200 & 50 & 25 & 18 & 900 \\
\hline BUST & 1546 & SOOR GUDAR & & 200 & 50 & 25 & 18 & 900 \\
\hline BUST & 1541 & SPINA KOTA(BABAGEE F & SPINA KOTA(BABA & 350 & 100 & 29 & 22 & 2,200 \\
\hline BUST & 1526 & SULAIMAN SHAH ZAI & SULAIMAN SHAH & 2,000 & 330 & 17 & 18 & 5,940 \\
\hline BUST & 1530 & WALIZAI & WALIZAI & 2,550 & 220 & 9 & 18 & 3,960 \\
\hline BUST & 1544 & ZARIFSHAH KHAN KALAY & ZARIFSHAH KHAN KALAY & 280 & 0 & 0 & 0 & 0 \\
\hline GARMSER & 1460 & ABDUL WAHED KHAN & ABDUL WAHED KHN & 300 & 40 & 13 & 14 & 560 \\
\hline GARMSER & 1496 & ABDUL ZAHER & ABDUL ZAHER & 800 & 2 & 0 & 14 & 28 \\
\hline GARMSER & 1499 & ABDUL ZAHER & ABDUL ZAHER & 300 & 10 & 3 & 14 & 140 \\
\hline GARMSER & 1466 & AHGA MIR KHAN KELAY & AGHA MIR KHAN & 280 & 30 & 11 & 12 & 360 \\
\hline GARMSER & 1426 & ALI MOHD KHAN & ALI MOHD KHAN & 130 & 30 & 23 & 12 & 360 \\
\hline
\end{tabular}

* 1 Jerib $=1 / 5 \mathrm{Ha}$

${ }^{*}{ }_{2}$ Dry Opium $=$ Wet Opium $-30 \%$ moisture
Page 1146
Source: UNDCP Opium Production Survey, 1995 File: HLD3.XLS - 07/08/95 
Opium Production Survey by village in Helmand province, 1995.

\begin{tabular}{|c|c|c|c|c|c|c|c|c|}
\hline & \multirow{3}{*}{$\begin{array}{l}\text { Village } \\
\text { Code }\end{array}$} & \multirow{3}{*}{ Area/Main Village } & \multirow{3}{*}{ Village } & \multirow{3}{*}{$\begin{array}{c}\text { Total } \\
\text { Cultivated } \\
\text { land }\end{array}$} & \multirow{2}{*}{\multicolumn{2}{|c|}{$\begin{array}{c}\text { Land Under } \\
\text { Poppy cultivation }\end{array}$}} & \multirow{3}{*}{$\begin{array}{c}\text { Wet Opium }{ }^{* 2} \\
\text { Yield } \\
(\mathrm{kg} / \mathrm{jr} .)\end{array}$} & \multirow{3}{*}{$\begin{array}{c}\text { Wet Opium } \\
\text { Production } \\
(\mathrm{kg})\end{array}$} \\
\hline \multirow{2}{*}{ District } & & & & & & & & \\
\hline & & & & & $($ jerib *) & $(\%)$ & & \\
\hline \multicolumn{9}{|c|}{ Province: Helmand } \\
\hline GARMSER & 1495 & AMANULLAH & AMANULLAH & 300 & 11 & 4 & 16 & 176 \\
\hline GARMSER & 1448 & AMANULLAH KHAN KELY & AMANULLAH KHAN & 400 & 40 & 10 & 20 & 800 \\
\hline GARMSER & 1458 & AMER QAYUM KELAY & AMER QAYUM KELAY & 1,000 & 300 & 30 & 18 & 5,400 \\
\hline GARMSER & 1491 & BAQI KHAN & BAQI KHAN & 2,500 & 70 & 3 & 13 & 910 \\
\hline GARMSER & 1465 & GHUL.HAYDER KHAN KLY & GHUL HAYDER KHAN KLY & 490 & 40 & 8 & 18 & 720 \\
\hline GARMSER & 1490 & GORGAK & GORGAK & 700 & 20 & 3 & 12 & 240 \\
\hline GARMSER & 1455 & GUL SAR KELAY & GUL SAR KELAY & 800 & 100 & 13 & 12 & 1,200 \\
\hline GARMSER & 1483 & HABIBULLAH UZBAK & HABIBULLAH UZBA & 100 & 36 & 36 & 13 & 468 \\
\hline GARMSER & 1468 & HAJI ABDUL HAMEED & ABDUL HAMEED & 60 & 10 & 17 & 11 & 110 \\
\hline GARMSER & 1481 & HAJI ABDUL ZAHER & ABDUL ZAHER KHN & 300 & 50 & 17 & 20 & 1,000 \\
\hline GARMSER & 1428 & HAJI ASADULLAH KHAN & H.ASADULLAH KHA & 300 & 20 & 7 & 12 & 240 \\
\hline GARMSER & 1431 & HAJI FAIZ MOHD.KHAN & HAJI FAIZ MOHD.KHAN & 500 & 15 & 3 & 13 & 195 \\
\hline GARMSER & 1446 & HAJI GHULAM HAIDAR & GHULAM HAIDAR & 430 & 30 & 7 & 14 & 420 \\
\hline GARMSER & 1445 & HAJI HGIASULLAH KHAN & HAJI GHIASULLAH KHAN & 280 & 20 & 7 & 20 & 400 \\
\hline GARMSER & 1424 & HAJI MOHD. UMAR KHAN & MOHD. UMAR KHAN & 200 & 15 & 8 & 12 & 180 \\
\hline GARMSER & 1472 & HAJI MOHD.ANWAR KHAN & MOHD. ANWAR KHN & 150 & 30 & 20 & 12 & 360 \\
\hline GARMSER & 1441 & HAJI MOHD.NADER KHAN & HAJI MOHD.NADER KHAN & 500 & 30 & 6 & 18 & 540 \\
\hline GARMSER & 1480 & HAJI MOHD.NAEEM KHAN & MOHD.NAEEM KHAN & 550 & 50 & 9 & 18 & 900 \\
\hline GARMSER & 1497 & HAJI RAHO KHAN & HAJI RAHO KHAN & 300 & 3 & 1 & 12 & 36 \\
\hline GARMSER & 1470 & HAJI WAKIL MOHD KHAN & WAKIL MOHD.KHAN & 370 & 100 & 27 & 18 & 1,800 \\
\hline GARMSER & 1452 & HAJI ZOQAM KHAN & HAJI ZOQAM KHAN KELY & 350 & 50 & 14 & 9 & 450 \\
\hline GARMSER & 1420 & HAJI.S.OMARKHAN KLEY & HAJI.S.OMAR KHAN & 640 & 64 & 10 & 20 & 1,280 \\
\hline GARMSER & 1487 & HASAN KHAIL & HASAN KHAIL & 300 & 10 & 3 & 20 & 200 \\
\hline GARMSER & 1436 & HERATIAN KELY & HERATIAN KELY & 400 & 49 & 12 & 12 & 588 \\
\hline GARMSER & 1449 & HJ.AWRANG KHAN KLEY & HJ.AWRANGKHAN & 1,500 & 109 & 7 & 20 & 2,180 \\
\hline GARMSER & 1477 & HJ.DOST MOHD.KHAN KL & HJ.DOST.MOHD & 60 & 3 & 5 & 13 & 39 \\
\hline GARMSER & 1440 & HJ.GHULAMJAN KLEY & HJ.GHULAMJAN & 1,000 & 50 & 5 & 10 & 500 \\
\hline GARMSER & 1429 & HJ.GUL.MOHD.KHAN.KLY & HJ.GUL.MOHD.KHAN & 300 & 30 & 10 & 18 & 540 \\
\hline GARMSER & 1771 & HJ.KAMAL KHAN KELAY & HJ.KAMAL KHAN & 300 & 30 & 10 & 12 & 360 \\
\hline
\end{tabular}

* $1 \mathrm{Jerib}=1 / 5 \mathrm{Ha}$

* ${ }_{2}$ Dry Opium $=$ Wet Opium $-30 \%$ moisture 
Opium Production Survey by village in Helmand province, 1995.

\begin{tabular}{|c|c|c|c|c|c|c|c|c|}
\hline & Village & & & \multirow{3}{*}{$\begin{array}{c}\text { Total } \\
\text { Cultivated } \\
\text { land } \\
\end{array}$} & \multirow{2}{*}{\multicolumn{2}{|c|}{$\begin{array}{c}\text { Land Under } \\
\text { Poppy cultivation }\end{array}$}} & \multirow{3}{*}{$\begin{array}{c}\text { Wet }^{\text {Opium }}{ }^{* 2} \\
\text { Yield } \\
(\mathrm{kg} / \mathrm{jr} .) \\
\end{array}$} & \multirow{3}{*}{$\begin{array}{c}\text { Wet Opium } \\
\text { Production } \\
(\mathrm{kg}) \\
\end{array}$} \\
\hline \multirow[t]{2}{*}{ District } & Code & Area/Main Village & Village & & & & & \\
\hline & & & & & $($ jerib *) & $(\%)$ & & \\
\hline \multicolumn{9}{|c|}{ Province: Helmand } \\
\hline GARMSER & 1451 & HJ.MOHD ALAM KLEY & HJ.MOHD ALAM KL & 2,500 & 100 & 4 & 8 & 800 \\
\hline GARMSER & 1469 & HJ.MULLA HABIBULLAH & HJ.MULLAH HABIB & 220 & 50 & 23 & 14 & 700 \\
\hline GARMSER & 1479 & HJ.RAHO KHAN KELAY & HJ.RAHO KHAN KELAY & 300 & 60 & 20 & 13 & 780 \\
\hline GARMSER & 1447 & KATORAI & KATORAI & 500 & 40 & 8 & 16 & 640 \\
\hline GARMSER & 1476 & LALO AKA KELAY & LALO AKA KELAY & 400 & 30 & 8 & 18 & 540 \\
\hline GARMSER & 1494 & MOHAMMAD WALI & MOHAMMAD WALI & 300 & 7 & 2 & 14 & 98 \\
\hline GARMSER & 1425 & MOHD RAHIM KHAN & MOHD RAHIM KHAN & 150 & 15 & 10 & 9 & 135 \\
\hline GARMSER & 1463 & MOHD. ANWAR KHAN & MOHD. ANWAR KHN & 500 & 50 & 10 & 18 & 900 \\
\hline GARMSER & 1427 & MOHD. JAN KHAN KELY & MOHD. JAN KHAN & 530 & 50 & 9 & 18 & 900 \\
\hline GARMSER & 1461 & MOHD.KARIM KHAN & MOHD.KARIM KHAN & 270 & 30 & 11 & 15 & 450 \\
\hline GARMSER & 1478 & MOHD.NABI KHAN KELAY & MOHD.NABI KHAN & 600 & 200 & 33 & 13 & 2,600 \\
\hline GARMSER & 1489 & MOQAM KHAN & MOQAM KHAN & 52 & 6 & 12 & 16 & 96 \\
\hline GARMSER & 1467 & MUDER GHULAM MOHD KL & MUDER GHULAM.M & 50 & 20 & 40 & 18 & 360 \\
\hline GARMSER & 1421 & MULAYAN KELY & MULAYAN KELY & 250 & 20 & 8 & 22 & 440 \\
\hline GARMSER & 1450 & MULLAH HABIBULLAH & ML.HABIBULLAH & 300 & 21 & 7 & 10 & 210 \\
\hline GARMSER & 1423 & NAMATULLAH KHAN & NAMATULLAH KHAN & 513 & 20 & 4 & 20 & 400 \\
\hline GARMSER & 1484 & NAQEL MOHD ALI KHAN & NAQEL MOHD ALI KHAN & 220 & 50 & 23 & 20 & 1,000 \\
\hline GARMSER & 1485 & NAQEL SHAD MIR KHAN & NAQEL SHAD MIR & 330 & 74 & 22 & 14 & 1,036 \\
\hline GARMSER & 1492 & NAQELIN DORZI & NAQELIN DORZI & 2,000 & 100 & 5 & 17 & 1,700 \\
\hline GARMSER & 1488 & NAQELIN HAZARA & NAQELIN HAZARA & 110 & 29 & 26 & 15 & 435 \\
\hline GARMSER & 1422 & NAQELIN LOUGARI & NAQELIN LOUGARI & 70 & 5 & 7. & 12 & 60 \\
\hline GARMSER & 1430 & NAQELIN-E-HERATI & NAQELIN HERATI & 160 & 45 & 28 & 9 & 405 \\
\hline GARMSER & 1437 & NAQELIN-E-LOUGARI & NAQELIN LOUGARI & 350 & 15 & 4 & 13 & 195 \\
\hline GARMSER & 1473 & NAQILIN DERAHOTI & NAQILIN DERAHOT & 960 & 60 & 6 & 22 & 1,320 \\
\hline GARMSER & 1475 & NAQILIN-E-WARDAKI & NAQILIN WARDAKI & 460 & 60 & 13 & 18 & 1,080 \\
\hline GARMSER & 1482 & NAQLEN ACHEKZAIE & NAQLEN ACHEKZAI & 200 & 20 & 10 & 13 & 260 \\
\hline GARMSER & 1433 & NAQLEN ASEKZAI KLEY & NAQLEN ASEKZAI KLEY & 300 & 90 & 30 & 22 & 1,980 \\
\hline GARMSER & 1438 & NAQLEN HAZARHA & NAQLEN HAZARHA & 300 & 0 & 0 & 0 & 0 \\
\hline GARMSER & 1434 & NAQLEN LAGHMANI & NAQLEN LAGHMANI & 250 & 70 & 28 & 15 & 1,050 \\
\hline
\end{tabular}

* 1 Jerib $=1 / 5 \mathrm{Ha}$

* ${ }^{2}$ Dry Opium $=$ Wet Opium $-30 \%$ moisture
Source: UNDCP Opium Production Survey, 1995 File: HLD3.XLS - 07/08/95 
Opium Production Survey by village in Helmand province, 1995.

\begin{tabular}{|c|c|c|c|c|c|c|c|c|}
\hline & Village & \multirow{3}{*}{ Area/Main Village } & \multirow{3}{*}{ Village } & \multirow{3}{*}{$\begin{array}{c}\text { Total } \\
\text { Cultivated } \\
\text { land } \\
\end{array}$} & \multirow{2}{*}{\multicolumn{2}{|c|}{$\begin{array}{c}\text { Land Under } \\
\text { Poppy cultivation }\end{array}$}} & \multirow{3}{*}{$\begin{array}{c}\text { Wet Opium }{ }^{* 2} \\
\text { Yield } \\
(\mathrm{kg} / \mathrm{jr} .) \\
\end{array}$} & \multirow{3}{*}{$\begin{array}{c}\text { Wet Opium } \\
\text { Production } \\
\text { (kg) }\end{array}$} \\
\hline \multirow[t]{2}{*}{ District } & \multirow[t]{2}{*}{ Code } & & & & & & & \\
\hline & & & & & $\left(\right.$ jerib $\left.^{*}\right)$ & $(\%)$ & & \\
\hline \multicolumn{9}{|c|}{ Province: Helmand } \\
\hline GARMSER & 1474 & NAQLEN LAGHMANI & NAQLEN LAGHMANI & 540 & 51 & 9 & 18 & 918 \\
\hline GARMSER & 1443 & NEEM PAS KELY & NEEM PAS KELY & 100 & 10 & 10 & 12 & 120 \\
\hline GARMSER & 1442 & SALAMKHAN KLEY & SALAMKHAN KLEY & 320 & 50 & 16 & 14 & 700 \\
\hline GARMSER & 1486 & SAYEDAN & SAYEDAN & 30 & 3 & 10 & 10 & 30 \\
\hline GARMSER & 1493 & SHAH WALI KHAN & SHAH WALI KHAN & 370 & 20 & 5 & 14 & 280 \\
\hline GARMSER & 1453 & SHRIF KHAN KELAY & SHARIF KHAN KELAY & 3,000 & 150 & 5 & 8 & 1,200 \\
\hline GARMSER & 1444 & SOKHTA KLY.NAQLEN HZ & SOKHTA.KLY.HZRA & 130 & 2 & 2 & 14 & 28 \\
\hline GARMSER & 1464 & SUNGIN KHAN KELAY & SUNGIN KHAN & 200 & 18 & 9 & 17 & 306 \\
\hline GARMSER & 1439 & TOBE & TOBE & 800 & 30 & 4 & 18 & 540 \\
\hline GARMSER & 1456 & WAKIL BABRAY KELAY & WAKIL BABRAY KELAY & 300 & 40 & 13 & 16 & 640 \\
\hline GARMSER & 1432 & WAKIL KAMAL KLEY & WAKIL KAMAL & 550 & 100 & 18 & 15 & 1,500 \\
\hline GARMSER & 1435 & WAKIL MASTAN KELY & WAKIL MASTAN KELY & 150 & 20 & 13 & 13 & 260 \\
\hline GARMSER & 1457 & WAKIL ZAHER & WAKIL ZAHER & 600 & 200 & 33 & 14 & 2,800 \\
\hline GARMSER & 1462 & WAKIL.HJ.MOHD.OMAR & HJ.MOHD.OMAR KHAN.KL & 350 & 20 & 6 & 20 & 400 \\
\hline GARMSER & 1459 & WAZIR KHAN KELAY & WAZIR KHAN KELAY & 250 & 30 & 12 & 14 & 420 \\
\hline GARMSER & 1498 & YAR MOHAMMAD & YAR MOHAMMAD & 450 & 8 & 2 & 16 & 128 \\
\hline GARMSER & 1454 & ZARIF KHAN KLEY & ZARIFKHAN KLEY & 400 & 40 & 10 & 10 & 400 \\
\hline KAJAKI & 840 & ABDAR & ABDAR & 250 & 180 & 72. & 12 & 2,160 \\
\hline KAJAKI & 845 & ABDUL MALIK & ABDUL MALIK & 300 & 200 & 67 & 16 & 3,200 \\
\hline KAJAKI & 842 & AGA MOHD & AGA MOHD & 200 & 130 & 65 & 18 & 2,340 \\
\hline KAJAKI & 838 & AHANGARAN & AHANGARAN & 20 & 8 & 40 & 6 & 48 \\
\hline KAJAKI & 846 & AKHOND KHEL & AKHOND KHEL & 200 & 150 & 75 & 14 & 2,100 \\
\hline KAJAKI & 921 & AKHOND ZADA KHEL & AKHOND ZADA KHL & 150 & 50 & 33 & 14 & 700 \\
\hline KAJAKI & 841 & ALBELAGH & ALBELAGH & 300 & 140 & 47 & 9 & 1,260 \\
\hline KAJAKI & 844 & ALI ABAD & ALI ABAD & 300 & 150 & 50 & 18 & 2,700 \\
\hline KAJAKI & 1570 & ALI ABAD & HAJI MALIM & 300 & 200 & 67 & 17 & 3,400 \\
\hline KAJAKI & 837 & ANARAK & ANARAK & 15 & 8 & 53 & 12 & 96 \\
\hline KAJAKI & 850 & ANJARAN & ANJARAN & 200 & 100 & 50 & 10 & 1,000 \\
\hline KAJAKI & 1371 & ARAB ZAI & ARAB ZAI & 250 & 100 & 40 & 17 & 1,700 \\
\hline
\end{tabular}


Opium Production Survey by village in Helmand province, 1995.

\begin{tabular}{|c|c|c|c|c|c|c|c|c|}
\hline \multirow{3}{*}{ District } & \multirow{3}{*}{$\begin{array}{l}\text { Village } \\
\text { Code }\end{array}$} & \multirow{3}{*}{ Area/Main Village } & \multirow{3}{*}{ Village } & \multirow{3}{*}{$\begin{array}{c}\text { Total } \\
\text { Cultivated } \\
\text { land } \\
\end{array}$} & \multirow{2}{*}{\multicolumn{2}{|c|}{$\begin{array}{c}\text { Land Under } \\
\text { Poppy cultivation }\end{array}$}} & \multirow{3}{*}{$\begin{array}{c}\text { Wet Opium }^{* 2} \\
\text { Yield } \\
(\mathrm{kg} / \mathrm{jr} .) \\
\end{array}$} & \multirow{3}{*}{$\begin{array}{l}\text { Wet Opium } \\
\text { Production } \\
(\mathrm{kg}) \\
\end{array}$} \\
\hline & & & & & & & & \\
\hline & & & & & $\left(\right.$ jerib $\left.^{*}\right)$ & $(\%)$ & & \\
\hline \multicolumn{9}{|c|}{ Province: Helmand } \\
\hline KAJAKI & 1372 & ARAB ZAI & KHUMARI ZAI & 120 & 60 & 50 & 24 & 1,440 \\
\hline KAJAKI & 1373 & ARAB ZAI & JAMLIAN & 250 & 150 & 60 & 24 & 3,600 \\
\hline KAJAKI & 1376 & ARAB ZAI & MOGHDOZAI & 300 & 90 & 30 & 7 & 630 \\
\hline KAJAKI & 848 & ATAL & ATAL & 45 & 20 & 44 & 12 & 240 \\
\hline KAJAKI & 839 & AZAN & PATAKZAY & 150 & 100 & 67 & 16 & 1,600 \\
\hline KAJAKI & 843 & AZAN & IBRAHIM ZAY & 180 & 100 & 56 & 16 & 1,600 \\
\hline KAJAKI & 1583 & AZAN MIRZAI & AZAN MIRZAI & 130 & 70 & 54 & 14 & 980 \\
\hline KAJAKI & 855 & BAGAL KHEL & BAGAL KHEL & 100 & 50 & 50 & 12. & 600 \\
\hline KAJAKI & 854 & BAGHAK & BAGHAK & 300 & 200 & 67 & 10 & 2,000 \\
\hline KAJAKI & 857 & BAJAGHAR & LWAL BAJAGHAR & 200 & 100 & 50 & 14. & 1,400 \\
\hline KAJAKI & 1378 & BALEKCHO & BALEKCHO & 50 & 20 & 40 & 10 & 200 \\
\hline KAJAKI & 852 & BANONSH & BANOSH & 400 & 200 & 50 & 16 & 3,200 \\
\hline KAJAKI & 854 & BESHAM & BESHAM & 200 & 80 & 40 & 12 & 960 \\
\hline KAJAKI & 853 & BIBANAK & BIBANAK & 450 & 250 & 56 & 23 & 5,750 \\
\hline KAJAKI & 851 & BIBIKA & BIBIKA & 15 & 8 & 53 & 10 & 80 \\
\hline KAJAKI & 856 & BIWAR & BIWAR & 500 & 300 & 60 & 12 & 3,600 \\
\hline KAJAKI & 865 & CHACHA & CHACHA & 200 & 130 & 65 & 10 & 1,300 \\
\hline KAJAKI & 863 & CHAHAR DAH & CHAHARDAH & 100 & 50 & 50 & 15 & 750 \\
\hline KAJAKI & 960 & CHAR BAGH & CHAKIZAI & 600 & 400 & 67 & 16 & 6,400 \\
\hline KAJAKI & 961 & CHAR BAGH & PIRZAI & 600 & 400 & 67 & 16 & 6,400 \\
\hline KAJAKI & 864 & CHAR BAGH KUNJAK & CHARBAGH KUNJAK & 500 & 350 & 70 & 12 & 4,200 \\
\hline KAJAKI & 945 & CHARDAR & CHARDAR & 150 & 120 & 80 & 12 & 1,440 \\
\hline KAJAKI & 860 & CHEHEL KAZEE & CHEHEL GAZEE & 200 & 140 & 70 & 17 & 2,380 \\
\hline KAJAKI & 899 & CHELCHAGH & CHELCHAGH & 200 & 130 & 65 & 18 & 2,340 \\
\hline KAJAKI & 1390 & CHELTAN & CHELTAN & 150 & 100 & 67 & 12 & 1,200 \\
\hline KAJAKI & 862 & CHENA & WABAR GAJWI & 240 & 150 & 63 & 12 & 1,800 \\
\hline KAJAKI & 861 & CHENGAI & CHENGAI & 25 & 25 & 100 & 30 & 750 \\
\hline KAJAKI & 1367 & CHINGZAI & CHINGZAI & 70 & 50 & 71 & 9 & 450 \\
\hline KAJAKI & 868 & DAROZAI & DAROZAI & 60 & 30 & 50 & 16 & 480 \\
\hline
\end{tabular}

* 1 Jerib $=1 / 5 \mathrm{Ha}$

* ${ }^{2}$ Dry Opium $=$ Wet Opium $-30 \%$ moisture
Page $15 \quad 50$
Source: UNDCP Opium Production Survey, 1995 File: HLD3.XLS - 07/08/95 
Opium Production Survey by village in Helmand province, 1995.

\begin{tabular}{|c|c|c|c|c|c|c|c|c|}
\hline \multirow{3}{*}{ District } & \multirow{3}{*}{$\begin{array}{l}\text { Village } \\
\text { Code }\end{array}$} & \multirow{3}{*}{ Area/Main Village } & \multirow{3}{*}{ Village } & \multirow{3}{*}{$\begin{array}{c}\text { Total } \\
\text { Cultivated } \\
\text { land } \\
\end{array}$} & \multirow{2}{*}{\multicolumn{2}{|c|}{$\begin{array}{c}\text { Land Under } \\
\text { Poppy cultivation }\end{array}$}} & \multirow{3}{*}{$\begin{array}{c}\text { Wet } \text { Opium }^{* 2} \\
\text { Yield } \\
(\mathrm{kg} / \mathrm{jr} .)\end{array}$} & \multirow{3}{*}{$\begin{array}{l}\text { Wet Opium } \\
\text { Production } \\
(\mathrm{kg})\end{array}$} \\
\hline & & & & & & & & \\
\hline & & & & & $($ jerib *) & $(\%)$ & & \\
\hline \multicolumn{9}{|c|}{ Province: Helmand } \\
\hline KAJAKI & 924 & DAWRAN KAREZ & DAWRAN KAREZ & 200 & 100 & 50 & 14 & 1,400 \\
\hline KAJAKI & 871 & DAYAK & DAYAK & 100 & 50 & 50 & 16 & 800 \\
\hline KAJAKI & 867 & DEH BABA & DEH BABA & 1,500 & 600 & 40 & 16 & 9,600 \\
\hline KAJAKI & 1575 & DEH KHWAJA & DEH KHWAJA & 70 & 30 & 43 & 18 & 540 \\
\hline KAJAKI & 866 & DEH MOSHK & DEH MOSHK & 150 & 100 & 67 & 15 & 1,500 \\
\hline KAJAKI & 1576 & DEH YAK & DEH YAK & 79 & 10 & 13 & 13 & 130 \\
\hline KAJAKI & 1386 & DEWALAK & DEWALAK & 50 & 25 & 50 & 7 & 175 \\
\hline KAJAKI & 1581 & DONGYAN & DONGYAN & 130 & 50 & 38 & 17 & 850 \\
\hline KAJAKI & 874 & GANDOMREZ OLYA & GANDOMREZ OLYA & 200 & 100 & 50 & 9 & 900 \\
\hline KAJAKI & 873 & GANDOMREZ SOFLA & GANDOMREZ SOFLA & 270 & 150 & 56 & 13 & 1,950 \\
\hline KAJAKI & 1366 & GAREZAI & GAREZAI & 200 & 34 & 17 & 10 & 340 \\
\hline KAJAKI & 878 & GHAWAND & GHAWAND & 400 & 250 & 63 & 10 & 2,500 \\
\hline KAJAKI & 1369 & GHAZNI KHAIL & GHAZNI KHAIL & 90 & 10 & 11 & 6 & 60 \\
\hline KAJAKI & 877 & GHOLMISH & GHOLMISH & 100 & 55 & 55 & 8 & 440 \\
\hline KAJAKI & 872 & GHULAM HASAN & GHULAM HASAN & 200 & 120 & 60 & 16 & 1,920 \\
\hline KAJAKI & 880 & HAIDAR NAKHJI & HAIDAR NAKHJI & 240 & 100 & 42. & 9 & 900 \\
\hline KAJAKI & 879 & HAJI BORA & HAJI BORA & 300 & 230 & 77 & 18 & 4,140 \\
\hline KAJAKI & 870 & HAJI KHEL & HAJI KHEL & 100 & 35 & 35 & 24 & 840 \\
\hline KAJAKI & 897 & HOKOMAT KHAN KAREZ & HOKOMAT KAREZ & 400 & 200 & 50 & 12 & 2,400 \\
\hline KAJAKI & 917 & HYDIL KAREZ & HYDIL KAREZ & 290 & 180 & 62 & 14 & 2,520 \\
\hline KAJAKI & 881 & JARCHI & JARCHI & 200 & 100 & 50 & 10 & 1,000 \\
\hline KAJAKI & 884 & JARYAH & JARYAH & 100 & 50 & 50 & 12 & 600 \\
\hline KAJAKI & 882 & JOLA & JOLA & 150 & 90 & 60 & 11 & 990 \\
\hline KAJAKI & 930 & KAJBID & KAJBID & 15 & 6 & 40 & 12 & 72 \\
\hline KAJAKI & 1380 & KAJOY & KAJOY & 140 & 90 & 64 & 11 & 990 \\
\hline KAJAKI & 914 & KAKA KHEL & KAKA KHEL & 150 & 90 & 60 & 11 & 990 \\
\hline KAJAKI & 1568 & KAKI & KAKI & 150 & 80 & 53 & 10 & 800 \\
\hline KAJAKI & 964 & KALAI GUL & KHALAWAK & 320 & 145 & 45 & 10 & 1,450 \\
\hline KAJAKI & 1382 & KALIZAI & KALIZAI & 200 & 200 & 100 & 10 & 2,000 \\
\hline
\end{tabular}

* $1 \mathrm{Jerib}=1 / 5 \mathrm{Ha}$

${ }^{*}{ }^{2}$ Dry Opium $=$ Wet Opium $-30 \%$ moisture 
Opium Production Survey by village in Helmand province, 1995.

\begin{tabular}{|c|c|c|c|c|c|c|c|c|}
\hline & Village & & & Total & Land $L$ & & Wet Opium *2 & Wet Opium \\
\hline \multirow[t]{2}{*}{ District } & \multirow{2}{*}{ Code } & \multirow[t]{2}{*}{ Area/Main Village } & \multirow[t]{2}{*}{ Village } & \multirow{2}{*}{$\begin{array}{c}\text { Cultivated } \\
\text { land }\end{array}$} & \multicolumn{2}{|c|}{ Poppy cultivation } & \multirow{2}{*}{$\begin{array}{c}\text { Yield } \\
\text { (kg/jr.) }\end{array}$} & \multirow{2}{*}{$\begin{array}{c}\text { Production } \\
(\mathrm{kg})\end{array}$} \\
\hline & & & & & $\left(\right.$ jerib $\left.^{*}\right)$ & $(\%)$ & & \\
\hline \multicolumn{9}{|c|}{ Province: Helmand } \\
\hline KAJAKI & 938 & KANJAK & QASEM LOR & 150 & 90 & 60 & 11 & 990 \\
\hline KAJAKI & 911 & KANZAI & KANZAI & 90 & 30 & 33 & 9 & 270 \\
\hline KAJAKI & 904 & KAREZ GORGAK & KAREZ GORGAK & 400 & 250 & 63 & 24 & 6,000 \\
\hline KAJAKI & 886 & KAREZ NAW GHARBI & KAREZ NAW GHARBI & 200 & 150 & 75 & 12 & 1,800 \\
\hline KAJAKI & 1566 & KAREZ NAW KUNJAK & KAREZNAW KUNJAK & 250 & 150 & 60 & 9 & 1,350 \\
\hline KAJAKI & 922 & KAREZ SAFID & KAREZ SAFID & 400 & 200 & 50 & 16 & 3,200 \\
\hline KAJAKI & 1571 & KAREZ SHAHR & KAREZ SHAHR & 100 & 50 & 50 & 15 & 750 \\
\hline KAJAKI & 895 & KAREZ SORKH & KAREZ SORKH & 500 & 300 & 60 & 20 & 6,000 \\
\hline KAJAKI & 898 & KAREZ TAJOY & KAREZ TAJOY & 80 & 50 & 63 & 10 & 500 \\
\hline KAJAKI & 1389 & KAREZ-E- NAW SHARQ & KAREZ NAW SHARQ & 100 & 50 & 50 & 11 & 550 \\
\hline KAJAKI & 887 & KAREZAK & KAREZAK & 70 & 50 & 71 & 10 & 500 \\
\hline KAJAKI & 1579 & KAREZAK & KAREZAK & 180 & 100 & 56 & 14 & 1,400 \\
\hline KAJAKI & 940 & KAREZONA & KAREZONA & 30 & 20 & 67 & 10 & 200 \\
\hline KAJAKI & 934 & KAREZOY & KAREZOY & 200 & 100 & 50 & 17 & 1,700 \\
\hline KAJAKI & 1368 & KESITWAN & KESITWAN & 50 & 7 & 14 & 9 & 63 \\
\hline KAJAKI & 1377 & KHANANO KELI & KHANANO KELI & 20 & 10 & 50 & 24 & 240 \\
\hline KAJAKI & 907 & KHANDAQ KAREZ & KHANDAQ KAREZ & 500 & 300 & 60 & 17 & 5,100 \\
\hline KAJAKI & 885 & KHINJAKAK & KHINJAKAK & 250 & 120 & 48 & 10 & 1,200 \\
\hline KAJAKI & 891 & KHISHTE POKHTA & KHISHTE POKHTA & 150 & 90 & 60 & 17 & 1,530 \\
\hline KAJAKI & 890 & KHOGANI & KHOGANI & 250 & 180 & 72 & 9 & 1,620 \\
\hline KAJAKI & 901 & KHOJA GHARIB & KHOJA GHARIB & 100 & 50 & 50 & 18 & 900 \\
\hline KAJAKI & 1567 & KHOJA KHALIGH & KHOJA KHALIGH & 500 & 350 & 70 & 20 & 7,000 \\
\hline KAJAKI & 915 & KHOJA PAKSAR & KHOJA PAKSAR & 300 & 150 & 50 & 14 & 2,100 \\
\hline KAJAKI & 918 & KHOL ABAD & KHOL ABAD & 90 & 10 & 11 & 7 & 70 \\
\hline KAJAKI & 932 & KHOMBALAGH & BARIGAW & 200 & 100 & 50 & 14 & 1,400 \\
\hline KAJAKI & 896 & KHOWA CHAI & KHOWA CHAI & 500 & 250 & 50 & 16 & 4,000 \\
\hline KAJAKI & 935 & KHUJA AZIZ & KHUJA AZIZ & 800 & 200 & 25 & 11 & 2,200 \\
\hline KAJAKI & 888 & KISHMISH KHAN & KISHMISH KHAN & 250 & 180 & 72 & 12 & 2,160 \\
\hline KAJAKI & 902 & КОСНАК & КОСНАK & 100 & 50 & 50 & 10 & 500 \\
\hline
\end{tabular}

* 1 Jerib $=1 / 5 \mathrm{Ha}$

* 2 Dry Opium $=$ Wet Opium - $30 \%$ moisture 
Opium Production Survey by village in Helmand province, 1995.

\begin{tabular}{|c|c|c|c|c|c|c|c|c|}
\hline \multirow{3}{*}{ District } & \multirow{3}{*}{$\begin{array}{l}\text { Village } \\
\text { Code }\end{array}$} & \multirow{3}{*}{ Area/Main Village } & \multirow{3}{*}{ Village } & \multirow{3}{*}{$\begin{array}{c}\text { Total } \\
\text { Cultivated } \\
\text { land } \\
\end{array}$} & \multirow{2}{*}{\multicolumn{2}{|c|}{$\begin{array}{c}\text { Land Under } \\
\text { Poppy cultivation }\end{array}$}} & \multirow{3}{*}{$\begin{array}{c}\text { Wet Opium *2 } \\
\text { Yield } \\
(\mathrm{kg} / \mathrm{jr} .) \\
\end{array}$} & \multirow{3}{*}{$\begin{array}{l}\text { Wet Opium } \\
\text { Production } \\
(\mathrm{kg}) \\
\end{array}$} \\
\hline & & & & & & & & \\
\hline & & & & & $\left(\right.$ jerib $\left.^{*}\right)$ & $(\%)$ & & \\
\hline \multicolumn{9}{|c|}{ Province: Helmand } \\
\hline KAJAKI & 1384 & KOH-E- LALA & KOH-E- LALA & 60 & 40 & 67 & 10 & 400 \\
\hline KAJAKI & 1582 & KOKACHEL & KOKACHEL & 300 & 100 & 33 & 14 & 1,400 \\
\hline KAJAKI & 1388 & KOLO & KOLO & 200 & 120 & 60. & 12 & 1,440 \\
\hline KAJAKI & 919 & KOTI OLYA & KOTI OLYA & 500 & 170 & 34 & 11 & 1,870 \\
\hline KAJAKI & 936 & KOTI SUFLA & KOTI SUFLA & 400 & 200 & 50 & 12 & 2,400 \\
\hline KAJAKI & 903 & KSHATA BAJGHAR & KSHATA BAJGHAR & 200 & 100 & 50 & 20 & 2,000 \\
\hline KAJAKI & 937 & KSHATA WAZDAN & KSHATA WAZDAN & 50 & 20 & 40 & 12 & 240 \\
\hline KAJAKI & 942 & LABJAK & KAREZ BIDAK & 35 & 15 & 43 & 13 & 195 \\
\hline KAJAKI & 941 & LABJOY & LABJOY & 130 & 50 & 38 & 5 & 250 \\
\hline KAJAKI & 927 & LAHOR KHEL & LAHOR KHEL & 130 & 25 & 19 & 19 & 475 \\
\hline KAJAKI & 900 & LANDAI KAREZ & LANDAI KAREZ & 110 & 50 & 45 & 10 & 500 \\
\hline KAJAKI & 943 & LOWAR WAZON & LOWAR WAZON & 80 & 30 & 38 & 12 & 360 \\
\hline KAJAKI & 948 & MACHI KHEL & MACHI KHEL & 150 & 50 & 33 & 10 & 500 \\
\hline KAJAKI & 951 & MAIDANI & MAIDANI & 130 & 50 & 38 & 9 & 450 \\
\hline KAJAKI & 928 & MAKTAB KOHNA & MAKTAB KOHNA & 150 & 60 & 40 & 19 & 1,140 \\
\hline KAJAKI & 963 & MALEK SHAH & MALEK SHAH & 20 & 10 & 50 & 13 & 130 \\
\hline KAJAKI & 1577 & MAMANZAI & MAMANZAI & 150 & 130 & 87 & 16 & 2,080 \\
\hline KAJAKI & 926 & MAMON ZAI & MAMON ZAI & 30 & 5 & 17. & 11 & 55 \\
\hline KAJAKI & 929 & MANDA & MANDA & 120 & 30 & 25 & 17 & 510 \\
\hline KAJAKI & 949 & MARKHOR SOFLA & MARKHOR SOFLA & 80 & 20 & 25 & 13 & 260 \\
\hline KAJAKI & 1381 & MARKHOR ULYA & MARKHOR ULYA & 100 & 50 & 50 & 12 & 600 \\
\hline KAJAKI & 1578 & MAZAR & MAZAR & 50 & 40 & 80 & 10 & 400 \\
\hline KAJAKI & 946 & MAZARE HOWLIA & MARZARE HOWLIA & 500 & 250 & 50 & 13 & 3,250 \\
\hline KAJAKI & 950 & MAZDOORAK & MAZDOORAK & 200 & 100 & 50 & 11 & 1,100 \\
\hline KAJAKI & 947 & MAZDORAK & MAZDORAK & 120 & 70 & 58 & 11 & 770 \\
\hline KAJAKI & 923 & MEIANROADY & MEIANROADY & 200 & 50 & 25 & 5 & 250 \\
\hline KAJAKI & 892 & MIN ABAD KAREZ & MIN ABAD KAREZ & 100 & 30 & 30 & 16 & 480 \\
\hline KAJAKI & 944 & MIRZAI & MIRZAI & 200 & 100 & 50 & 12 & 1,200 \\
\hline KAJAKI & 979 & $\mathrm{MOCHI}$ & MOCHI & 100 & 30 & 30 & 16 & 480 \\
\hline
\end{tabular}

* 1 Jerib $=1 / 5 \mathrm{Ha}$

*2 Dry Opium $=$ Wet Opium $-30 \%$ moisture 
Opium Production Survey by village in Helmand province, 1995.

\begin{tabular}{|c|c|c|c|c|c|c|c|c|}
\hline \multirow{3}{*}{ District } & Village & \multirow{3}{*}{ Area/Main Village } & \multirow{3}{*}{ Village } & \multirow{3}{*}{$\begin{array}{c}\text { Total } \\
\text { Cultivated } \\
\text { land } \\
\end{array}$} & \multirow{2}{*}{\multicolumn{2}{|c|}{$\begin{array}{c}\text { Land Under } \\
\text { Poppy cultivation }\end{array}$}} & \multirow{3}{*}{$\begin{array}{c}\text { Wet Opium }^{* 2} \\
\text { Yield } \\
(\mathrm{kg} / \mathrm{jr} .) \\
\end{array}$} & \multirow{3}{*}{$\begin{array}{l}\text { Wet Opium } \\
\text { Production } \\
\quad(\mathrm{kg}) \\
\end{array}$} \\
\hline & \multirow[t]{2}{*}{ Code } & & & & & & & \\
\hline & & & & & $\left(\right.$ jerib $\left.^{*}\right)$ & $(\%)$ & & \\
\hline \multicolumn{9}{|c|}{ Province: Helmand } \\
\hline KAJAKI & 913 & MOGHRI KAREZ & MOGHRI KAREZ & 130 & 100 & 77 & 17 & 1,700 \\
\hline KAJAKI & 1385 & MURCH & MURCH & 10 & 5 & 50 & 10 & 50 \\
\hline KAJAKI & 955 & NAICHA SUFLA & NAICHA SUFLA & 250 & 150 & 60 & 11 & 1,650 \\
\hline KAJAKI & 956 & NAICHA ULYA & NAICHA ULYA & 300 & 100 & 33 & 11 & 1,100 \\
\hline KAJAKI & 953 & NALAN & NALAN & 170 & 70 & 41 & 8 & 560 \\
\hline KAJAKI & 1379 & NANO KHAIL & NANO KHAIL & 90 & 70 & 78 & 16 & 1,120 \\
\hline KAJAKI & 954 & NASWARYAN & NASWARYAN & 200 & 130 & 65 & 17 & 2,210 \\
\hline KAJAKI & 959 & NAW ABAD & NAW ABAD & 150 & 90 & 60 & 10 & 900 \\
\hline KAJAKI & 1596 & NAWAI KAREZ & NAWAI KAREZ & 100 & 50 & 50 & 9 & 450 \\
\hline KAJAKI & 952 & NICHA KALAN & NICHA KALAN & 250 & 100 & 40 & 10 & 1,000 \\
\hline KAJAKI & 925 & PAEAN KELE & PAEAN KELE & 70 & 25 & 36 & 16 & 400 \\
\hline KAJAKI & 1580 & PAI & PAI & 200 & 100 & 50 & 10 & 1,000 \\
\hline KAJAKI & 962 & PAI SANG & PAI SANG & 250 & 25 & 10 & 10 & 250 \\
\hline KAJAKI & 1370 & PAIGAW & PAIGAW & 200 & 100 & 50 & 20 & 2,000 \\
\hline KAJAKI & 912 & QAMBARYAN KAREZ & QAMBARYAN KAREZ & 150 & 70 & 47 & 18 & 1,260 \\
\hline KAJAKI & 905 & QANDAGHA KAREZ & QANDAGHA & 450 & 250 & 56 & 20 & 5,000 \\
\hline KAJAKI & 910 & QAZI KHEL & QAZI KHEL & 180 & 70 & 39 & 8 & 560 \\
\hline KAJAKI & 876 & QESRAK & QESRAK & 300 & 170 & 57 & 16 & 2,720 \\
\hline KAJAKI & 875 & QICHIZAR & QICHIZAR & 280 & 120 & 43 & 10 & 1,200 \\
\hline KAJAKI & 889 & QUL WALAT & BAKHTARI & 200 & 100 & 50 & 10 & 1,000 \\
\hline KAJAKI & 931 & RABAT & RABAT & 400 & 280 & 70 & 12 & 3,360 \\
\hline KAJAKI & 908 & RAHIMULLAH & AKHTAR AKA & 100 & 50 & 50 & 10 & 500 \\
\hline KAJAKI & 920 & REGI & REGI & 40 & 10 & 25 & 7 & 70 \\
\hline KAJAKI & 999 & ROSHAN ABAD & HAJI SADO KHAN & 100 & 50 & 50 & 10 & 500 \\
\hline KAJAKI & 998 & ROSHAN ABAD & HAJI ASHRAF & 100 & 50 & 50 & 9 & 450 \\
\hline KAJAKI & 965 & ROSHAN ABAD & HAJI MULA BARAN & 100 & 60 & 60 & 9 & 540 \\
\hline KAJAKI & 966 & ROSHAN ABAD & HAJI NEDA MOHD & 100 & 50 & 50 & 9 & 450 \\
\hline KAJAKI & 977 & SABZIKAR & SABZIKAR & 300 & 50 & 17 & 20 & 1,000 \\
\hline KAJAKI & 988 & SADAT & SADAT & 170 & 90 & 53 & 8 & 720 \\
\hline
\end{tabular}

* 1 Jerib $=1 / 5 \mathrm{Ha}$

* 2 Dry Opium $=$ Wet Opium $-30 \%$ moisture 
Opium Production Survey by village in Helmand province, 1995.

\begin{tabular}{|c|c|c|c|c|c|c|c|c|}
\hline \multirow{3}{*}{ District } & \multirow{3}{*}{$\begin{array}{l}\text { Village } \\
\text { Code }\end{array}$} & \multirow{3}{*}{ Area/Main Village } & \multirow{3}{*}{ Village } & \multirow{3}{*}{$\begin{array}{c}\text { Total } \\
\text { Cultivated } \\
\text { land } \\
\end{array}$} & \multirow{2}{*}{\multicolumn{2}{|c|}{$\begin{array}{c}\text { Land Under } \\
\text { Poppy cultivation }\end{array}$}} & \multirow{3}{*}{$\begin{array}{c}\text { Wet Opium }{ }^{* 2} \\
\text { Yield } \\
(\mathrm{kg} / \mathrm{jr} .) \\
\end{array}$} & \multirow{3}{*}{$\begin{array}{l}\text { Wet Opium } \\
\text { Production } \\
(\mathrm{kg})\end{array}$} \\
\hline & & & & & & & & \\
\hline & & & & & $\left(\right.$ jerib $\left.^{*}\right)$ & (\%) & & \\
\hline \multicolumn{9}{|c|}{ Province: Helmand } \\
\hline KAJAKI & 983 & SANGINAK & SANGINAK & 20 & 10 & 50 & 11 & 110 \\
\hline KAJAKI & 984 & SAPIDA & SAPIDA & 400 & 200 & 50 & 9 & 1,800 \\
\hline KAJAKI & 1573 & SAR CHASHMA & SAR CHASHMA & 330 & 130 & 39. & 16 & 2,080 \\
\hline KAJAKI & 972 & SARGINAK & SARGINAK & 20 & 10 & 50 & 10 & 100 \\
\hline KAJAKI & 909 & SAYEDAN & SAYEDAN & 400 & 250 & 63 & 18 & 4,500 \\
\hline KAJAKI & 987 & SEKANDAR ABAD & SEKANDAR ABAD & 80 & 50 & 63 & 9 & 450 \\
\hline KAJAKI & 968 & SHADIKA & SHADIKA & 50 & 40 & 80 & 4. & 160 \\
\hline KAJAKI & 971 & SHADIKA & SHADIKA & 500 & 300 & 60 & 12 & 3,600 \\
\hline KAJAKI & 980 & SHAH KAREZ & SHAH KAREZ & 600 & 350 & 58 & 12 & 4,200 \\
\hline KAJAKI & 1584 & SHAH MALI, ZANGAL & SHAHMALI,ZANGAL & 300 & 100 & 33 & 7 & 700 \\
\hline KAJAKI & 982 & SHAH PESAND & SHAH PESAND & 450 & 250 & 56 & 20 & 5,000 \\
\hline KAJAKI & 1378 & SHAHBAZ KHAIL & SHAHBAZ KHAIL & 35 & 10 & 29 & 3 & 30 \\
\hline KAJAKI & 976 & SHAHZADA & SHAHZADA & 185 & 19 & 10 & 10 & 190 \\
\hline KAJAKI & 978 & SHEKH ZAI KAREZ & SHEKHZAI KAREZ & 150 & 80 & 53 & 16 & 1,280 \\
\hline KAJAKI & 973 & SHER AHMAD & SHER AHMAD & 300 & 200 & 67 & 12 & 2,400 \\
\hline KAJAKI & 973 & SHER MULA & SHER MULA & 200 & 100 & 50 & 11 & 1,100 \\
\hline KAJAKI & 975 & SHERI/HAZAR GAZI & SHERI/HAZAR GAZ & 200 & 100 & 50 & 12 & 1,200 \\
\hline KAJAKI & 970 & SIA GHOL & SIA GHOL & 150 & 90 & 60 & 9 & 810 \\
\hline KAJAKI & 985 & SIA KHOLA & SIA KHOLA & 20 & 8 & 40 & 12 & 96 \\
\hline KAJAKI & 981 & SOOR KAREZ & SOOR KAREZ & 100 & 25 & 25 & 18 & 450 \\
\hline KAJAKI & 967 & SULTAN RABAT & SULTAN RABAT & 300 & 150 & 50 & 8 & 1,200 \\
\hline KAJAKI & 990 & TAJOYAK & TAJOYAK & 130 & 100 & 77 & 16 & 1,600 \\
\hline KAJAKI & 992 & TAKHAWI & TAGHAWI & 240 & 140 & 58 & 25 & 3,500 \\
\hline KAJAKI & 991 & TALRAQ & TALRAQ & 300 & 150 & 50 & 16 & 2,400 \\
\hline KAJAKI & 1572 & TARGHI SOFLA & TARGHI SOFLA & 300 & 150 & 50 & 18 & 2,700 \\
\hline KAJAKI & 993 & TARGHOI OLYA & TARGHI OLYA & 300 & 100 & 33 & 17 & 1,700 \\
\hline KAJAKI & 994 & TARKAK & TARKAK & 150 & 50 & 33 & 9 & 450 \\
\hline KAJAKI & 894 & WALANG & WALANG & 90 & 45 & 50 & 17 & 765 \\
\hline KAJAKI & 906 & YAR BABA & YAR BABA & 50 & 30 & 60 & 11 & 330 \\
\hline
\end{tabular}

* 1 Jerib $=1 / 5 \mathrm{Ha}$

* ${ }^{2}$ Dry Opium $=$ Wet Opium $-30 \%$ moisture
Page $20 \quad 55$
Source: UNDCP Opium Production Survey, 1995 File: HLD3.XLS - 07/08/95 
Opium Production Survey by village in Helmand province, 1995.

\begin{tabular}{|c|c|c|c|c|c|c|c|c|}
\hline \multirow{3}{*}{ District } & Village & \multirow{3}{*}{ Area/Main Village } & \multirow{3}{*}{ Village } & \multirow{3}{*}{$\begin{array}{c}\text { Total } \\
\text { Cultivated } \\
\text { land } \\
\end{array}$} & \multirow{2}{*}{\multicolumn{2}{|c|}{$\begin{array}{c}\text { Land Under } \\
\text { Poppy cultivation }\end{array}$}} & \multirow{3}{*}{$\begin{array}{c}\text { Wet Opium }{ }^{* 2} \\
\text { Yield } \\
(\mathrm{kg} / \mathrm{jr} .)\end{array}$} & \multirow{3}{*}{$\begin{array}{c}\text { Wet Opium } \\
\text { Production } \\
(\mathrm{kg}) \\
\end{array}$} \\
\hline & Code & & & & & & & \\
\hline & & & & & $\left(\right.$ jerib $\left.^{*}\right)$ & $(\%)$ & & \\
\hline \multicolumn{9}{|c|}{ Province: Helmand } \\
\hline KAJAKI & 996 & ZAMENDAWAR & AGHUL & 300 & 150 & 50 & 11 & 1,650 \\
\hline KAJAKI & 995 & ZAMENDAWAR & SAFED KHOLA & 90 & 40 & 44 & 12 & 480 \\
\hline KAJAKI & 1383 & ZERANDA & ZERANDA & 40 & 20 & 50 & 12. & 240 \\
\hline KAJAKI & 997 & ZERKHAR & ZERKHAR & 200 & 150 & 75 & 11 & 1,650 \\
\hline KAJAKI & 939 & ZOBAIR KAREZ & ZOBAIR KAREZ & 200 & 50 & 25 & 12 & 600 \\
\hline MUSA QALA & 696 & ABDUL JABAR KHAN & ABDUL JABAR & 140 & 120 & 86 & 17 & 2,040 \\
\hline MUSA QALA & 789 & ABDUL MOHAMMAD & ABDUL MOHAMMAD & 200 & 180 & 90 & 17 & 3,060 \\
\hline MUSA QALA & 779 & ABDUL RAHMAN KAREZ & ABDUL RAHMAN/KA & 300 & 150 & 50 & 20 & 3,000 \\
\hline MUSA QALA & 1324 & AHMAD KHAZI & AHMAD KHAZI & 537 & 400 & 74 & 17 & 6,800 \\
\hline MUSA QALA & 1601 & AHMAD SHAH KAREZ & AHMAD SHAH KA & 40 & 35 & 88 & 20 & 683 \\
\hline MUSA QALA & 1608 & AKHUNDZADA KHEL & AKHUNDZADA KHEL & 100 & 30 & 30 & 18 & 540 \\
\hline MUSA QALA & 1609 & ASHRAF KECH & ASHRAF KECH & 250 & 80 & 32 & 20 & 1,600 \\
\hline MUSA QALA & 1313 & ASMANIAN & ASMANIAN & 150 & 50 & 33 & 8 & 400 \\
\hline MUSA QALA & 770 & BAHADUR KAREZ & BAHADUR KAREZ & 250 & 150 & 60 & 18 & 2,700 \\
\hline MUSA QALA & 700 & BAHEER & BAHEER & 100 & 50 & 50 & 20 & 1,000 \\
\hline MUSA QALA & 699 & BEDAK & BEDAK & 100 & 60 & 60 & 19 & 1,140 \\
\hline MUSA QALA & 707 & CHAGHALI & CHAGHALI & 190 & 170 & 89 & 17 & 2,890 \\
\hline MUSA QALA & 764 & CHAHEL & CHAHEL & 200 & 130 & 65 & 13 & 1,690 \\
\hline MUSA QALA & 701 & CHAKAW & CHAKAW & 36 & 20 & 56 & 16 & 320 \\
\hline MUSA QALA & 706 & CHANAKZAI & CHANAKZAI & 140 & 100 & 71 & 18 & 1,800 \\
\hline MUSA QALA & 703 & CHARDEH & CHARDEH & 200 & 160 & 80 & 16 & 2,560 \\
\hline MUSA QALA & 708 & CHARSAIE & CHARSHIE & 110 & 15 & 14 & 9 & 135 \\
\hline MUSA QALA & 704 & CHENA SUFLA & CHENA SUFLA & 300 & 250 & 83 & 17 & 4,250 \\
\hline MUSA QALA & 705 & CHENA ULIA & CHENA ULIA & 70 & 60 & 86 & 17 & 1,020 \\
\hline MUSA QALA & 712 & DAGYAN & DAGYAN & 160 & 90 & 56 & 19 & 1,710 \\
\hline MUSA QALA & 717 & DAND & DAND & 300 & 210 & 70 & 17 & 3,570 \\
\hline MUSA QALA & 711 & DARA KHAIL & DARA KHAIL & 150 & 90 & 60 & 19 & 1,710 \\
\hline MUSA QALA & 714 & DAROZ ULIA & DAROZ ULIA & 600 & 400 & 67 & 14 & 5,400 \\
\hline MUSA QALA & 715 & DEH GHOUCHAK & DEH GHOUCHAK & 400 & 250 & 63 & 17 & 4,250 \\
\hline
\end{tabular}

* 1 Jerib $=1 / 5 \mathrm{Ha}$

* ${ }^{2}$ Dry Opium $=$ Wet Opium $-30 \%$ moisture 
Opium Production Survey by village in Helmand province, 1995.

\begin{tabular}{|c|c|c|c|c|c|c|c|c|}
\hline \multirow{3}{*}{ District } & Village & \multirow{3}{*}{ Area/Main Village } & \multirow{3}{*}{ Village } & \multirow{3}{*}{$\begin{array}{c}\text { Total } \\
\text { Cultivated } \\
\text { land } \\
\end{array}$} & \multirow{2}{*}{\multicolumn{2}{|c|}{$\begin{array}{c}\text { Land Under } \\
\text { Poppy cultivation }\end{array}$}} & \multirow{3}{*}{$\begin{array}{c}\text { Wet }^{\text {Opium }}{ }^{* 2} \\
\text { Yield } \\
(\mathrm{kg} / \mathrm{jr} .) \\
\end{array}$} & \multirow{3}{*}{$\begin{array}{l}\text { Wet Opium } \\
\text { Production } \\
\text { (kg) }\end{array}$} \\
\hline & \multirow[t]{2}{*}{ Code } & & & & & & & \\
\hline & & & & & $\left(\right.$ jerib $\left.^{*}\right)$ & (\%) & & \\
\hline \multicolumn{9}{|c|}{ Province: Helmand } \\
\hline MUSA QALA & 719 & DOGHAK & DOGHAK & 500 & 300 & 60 & 14 & 4,200 \\
\hline MUSA QALA & 787 & EKHLAS AKA KELY & EKHLAS AKA KELY & 200 & 130 & 65 & 17 & 2,210 \\
\hline MUSA QALA & 1602 & GERDEY JANGAL & GERDEY JANGAL & 80 & 20 & 25 & 15 & 300 \\
\hline MUSA QALA & 723 & GHONDE KELY & GHONDE KELY & 600 & 400 & 67 & 13 & 5,200 \\
\hline MUSA QALA & 720 & GHORI KAREZ & GHORI KAREZ & 300 & 150 & 50 & 19 & 2,850 \\
\hline MUSA QALA & 815 & GHRATULAH & GHRATULAH & 500 & 330 & 66 & 17 & 5,610 \\
\hline MUSA QALA & 710 & GHULAM DASTAGEER & GHULAM DASTAGEER & 130 & 70 & 54 & 23 & 1,610 \\
\hline MUSA QALA & 726 & GHULJEE KAS & GHOLJEE KAS & 50 & 20 & 40 & 13 & 260 \\
\hline MUSA QALA & 1606 & GORAY NIKA & GORAY NIKA & 120 & 70 & 58 & 18 & 1,260 \\
\hline MUSA QALA & 709 & GURJAT KAREZ & GURJAT KAREZ & 125 & 60 & 48 & 13 & 780 \\
\hline MUSA QALA & 729 & H. MOHD ESSA & H. MOHD ESSA & 280 & 250 & 89 & 18 & 4,375 \\
\hline MUSA QALA & 761 & H.NOOR M.AGA & H.NOOR M.AGA & 500 & 300 & 60 & 20 & 6,000 \\
\hline MUSA QALA & 1318 & HAJI ABDUL SATAR & HAJI ABDUL SATAR & 250 & 190 & 76 & 18 & 3,420 \\
\hline MUSA QALA & 728 & HAJI AHMAD AKA KELY & HAJI SAMAD AKA & 300 & 150 & 50 & 17 & 2,550 \\
\hline MUSA QALA & 785 & HAJI AKHTER MOHD & AKHTER MOHD & 200 & 180 & 90 & 17 & 3,060 \\
\hline MUSA QALA & 788 & HAJI AMIR JAN AKA & HAJI AMIR JAN & 300 & 140 & 47 & 17 & 2,380 \\
\hline MUSA QALA & 743 & HAJI AMIR KHAN AKA & HAJI AMIR KHAN AKA & 300 & 210 & 70 & 18 & 3,780 \\
\hline MUSA QALA & 731 & HAJI ATA. MOHD & HAJI ATA. MOHD & 500 & 350 & 70 & 17 & 5,950 \\
\hline MUSA QALA & 772 & HAJI BUZORG & HAJI BUZORG & 200 & 180 & 90 & 19 & 3,420 \\
\hline MUSA QALA & 755 & HAJI FAIZULLAH AKA & HAJI FAIZULLAH & 150 & 40 & 27 & 18 & 720 \\
\hline MUSA QALA & 740 & HAJI G.RASUL & HAJI G.RASUL & 140 & 70 & 50 & 14 & 945 \\
\hline MUSA QALA & 746 & HAJI GHANI AKA & HAJI GHANI AKA & 500 & 390 & 78 & 18 & 7,020 \\
\hline MUSA QALA & 1320 & HAJI GHULAM GHAWS & HAJI GH. GHAWS & 260 & 180 & 69 & 18 & 3,240 \\
\hline MUSA QALA & 730 & HAJI GHULAM. MOHD & HAJI GHULAM. MOHD & 500 & 400 & 80 & 17 & 6,800 \\
\hline MUSA QALA & 733 & HAJI GUL MOHD & HAJI GUL MOHD & 500 & 420 & 84 & 17 & 7,140 \\
\hline MUSA QALA & 1610 & HAJI JAMAL & HJAI JAMAL & 380 & 250 & 66 & 13 & 3,250 \\
\hline MUSA QALA & 750 & HAJI KHAYRULLAH & HAJI KHAYRULLAH & 400 & 250 & 63 & 17 & 4,250 \\
\hline MUSA QALA & 741 & HAJI MALEM & HAJI MALEM & 400 & 200 & 50 & 13 & 2,600 \\
\hline MUSA QALA & 758 & HAJI MALEM & HAJI MALEM & 300 & 200 & 67 & 17 & 3,400 \\
\hline
\end{tabular}

* $1 \mathrm{Jerib}=1 / 5 \mathrm{Ha}$

${ }^{*}{ }^{2}$ Dry Opium $=$ Wet Opium $-30 \%$ moisture

Page $22 \quad 57$


Opium Production Survey by village in Helmand province, 1995.

\begin{tabular}{|c|c|c|c|c|c|c|c|c|}
\hline \multirow{3}{*}{ District } & \multirow{3}{*}{$\begin{array}{l}\text { Village } \\
\text { Code }\end{array}$} & \multirow{3}{*}{ Area/Main Village } & \multirow{3}{*}{ Village } & \multirow{3}{*}{$\begin{array}{c}\text { Total } \\
\text { Cultivated } \\
\text { land } \\
\end{array}$} & \multirow{2}{*}{\multicolumn{2}{|c|}{$\begin{array}{c}\text { Land Under } \\
\text { Poppy cultivation }\end{array}$}} & \multirow{3}{*}{$\begin{array}{c}\text { Wet Opium *2 } \\
\text { Yield } \\
(\mathrm{kg} / \mathrm{jr} .) \\
\end{array}$} & \multirow{3}{*}{$\begin{array}{c}\text { Wet Opium } \\
\text { Production } \\
\text { (kg) }\end{array}$} \\
\hline & & & & & & & & \\
\hline & & & & & $\left(\right.$ jerib $\left.^{*}\right)$ & $(\%)$ & & \\
\hline \multicolumn{9}{|c|}{ Province: Helmand } \\
\hline MUSA QALA & 744 & HAJI MANAF AKA & HAJI MANAF AKA & 350 & 230 & 66 & 17 & 3,910 \\
\hline MUSA QALA & 1319 & HAJI MOHD JOMA & HAJI MOHD JOMA & 300 & 250 & 83 & 18 & 4,500 \\
\hline MUSA QALA & 732 & HAJI MUSAM AKA & HAJI MUSAM AKA & 500 & 400 & 80 & 18 & 7,200 \\
\hline MUSA QALA & 745 & HAJI NURI & HAJI NURI & 400 & 210 & 53 & 17 & 3,570 \\
\hline MUSA QALA & 742 & HAJI QASEM AKA & HAJI QASEM AKA & 180 & 120 & 67 & 18 & 2,160 \\
\hline MUSA QALA & 1326 & HAJI QULI & HAJI QULI & 600 & 500 & 83 & 19 & 9,500 \\
\hline MUSA QALA & 739 & HAJI RAHMATULLAH & HAJI RAHMATULAH & 157 & 110 & 70 & 22 & 2,420 \\
\hline MUSA QALA & 734 & HAJI RUSTAM KELY & HAJI RUSTAM & 300 & 250 & 83 & 14 & 3,500 \\
\hline MUSA QALA & 807 & HAJI SAYED NABI AKA & HAJI SAYED NABI & 300 & 200 & 67 & 13 & 2,600 \\
\hline MUSA QALA & 753 & HAJI SHERIN AKA & HAJI SHERIN AKA & 300 & 280 & 93 & 18 & 5,040 \\
\hline MUSA QALA & 762 & HAJI WALI KHAN AKA & HAJI WALI KHAN & 300 & 150 & 50 & 20 & 3,000 \\
\hline MUSA QALA & 735 & HAJI WAZIR & HAJI WAZIR & 400 & 250 & 63 & 13 & 3,250 \\
\hline MUSA QALA & 751 & HAJI ZAHER AKA & HAJI ZAHER AKA & 400 & 230 & 58 & 14 & 3,220 \\
\hline MUSA QALA & 736 & HAJI. MOHD SHARIF & HAJI.MOHD SHARI & 450 & 350 & 78 & 13 & 4,550 \\
\hline MUSA QALA & 1607 & HAJI.IBRAHIM AKA & HAJI IBRAHIM AK & 300 & 100 & 33 & 18 & 1,800 \\
\hline MUSA QALA & 689 & HALA & HALA & 100 & 60 & 60 & 18 & 1,080 \\
\hline MUSA QALA & 808 & ISMAIEEL JAN AKA & ISMAIEEL JAN & 400 & 300 & 75 & 17 & 5,100 \\
\hline MUSA QALA & 749 & JAMAL KHAN & JAMAL KHAN & 200 & 150 & 75 & 8 & 1,200 \\
\hline MUSA QALA & 763 & JOGH RAGH & JOGH RAGH & 150 & 30 & 20 & 8 & 240 \\
\hline MUSA QALA & 795 & JOI BAWARI & JOI BAWARI & 280 & 200 & 71 & 17 & 3,400 \\
\hline MUSA QALA & 737 & JUZ GHARAI & HAJI MOHD.AFZAL & 400 & 300 & 75 & 19 & 5,700 \\
\hline MUSA QALA & 702 & KALAWAL & KALAWAL & 50 & 40 & 80 & 13 & 520 \\
\hline MUSA QALA & 1332 & KANDER & KANDER & 100 & 90 & 90 & 17 & 1,530 \\
\hline MUSA QALA & 777 & KAREZ & KAREZ & 400 & 350 & 88 & 17 & 5,950 \\
\hline MUSA QALA & 713 & KAREZ HAZAR MISH & KAREZ HAZARMISH & 500 & 250 & 50 & 17 & 4,250 \\
\hline MUSA QALA & 774 & KAREZ KHUSHK & KAREZ KHUSHK & 600 & 500 & 83 & 22 & 11,000 \\
\hline MUSA QALA & 766 & KAREZ NEDA MOHD & KAREZ NEDA MOHD & 336 & 168 & 50 & 17 & 2,856 \\
\hline MUSA QALA & 1600 & KAREZ-E ESSA KHAN & KAREZ-E ESSA KH & 300 & 270 & 90 & 18. & 4,860 \\
\hline MUSA QALA & 780 & KAREZ-E- GHULAM & KAREZ-E- GHULAM & 150 & 100 & 67 & 19 & 1,900 \\
\hline
\end{tabular}

* I Jerib $=1 / 5 \mathrm{Ha}$

* ${ }^{2}$ Dry Opium $=$ Wet Opium - $30 \%$ moisture 
Opium Production Survey by village in Helmand province, 1995.

\begin{tabular}{|c|c|c|c|c|c|c|c|c|}
\hline \multirow{3}{*}{ District } & \multirow{3}{*}{$\begin{array}{l}\text { Village } \\
\text { Code }\end{array}$} & \multirow{3}{*}{ Area/Main Village } & \multirow{3}{*}{ Village } & \multirow{3}{*}{$\begin{array}{c}\text { Total } \\
\text { Cultivated } \\
\text { land } \\
\end{array}$} & \multirow{2}{*}{\multicolumn{2}{|c|}{$\begin{array}{c}\text { Land Under } \\
\text { Poppy cultivation }\end{array}$}} & \multirow{3}{*}{$\begin{array}{c}\text { Wet Opium }{ }^{* 2} \\
\text { Yield } \\
(\mathrm{kg} / \mathrm{jr} .)\end{array}$} & \multirow{3}{*}{$\begin{array}{c}\text { Wet Opium } \\
\text { Production } \\
(\mathrm{kg}) \\
\end{array}$} \\
\hline & & & & & & & & \\
\hline & & & & & $($ jerib *) & $(\%)$ & & \\
\hline \multicolumn{9}{|c|}{ Province: Helmand } \\
\hline MUSA QALA & 776 & KAREZ-E-NAW & KARIZ-E-NAW & 250 & 140 & 56 & 11 & 1,540 \\
\hline MUSA QALA & 765 & KARGHAK/DEH SOFIAN & KARGHAK/DEH SOFIAN & 150 & 100 & 67 & 18 & 1,800 \\
\hline MUSA QALA & 775 & KHARWARE . & KHARWARE & 650 & 500 & 77 & 20 & 10,000 \\
\hline MUSA QALA & 769 & KHOSH KAREZ & KHOSH KAREZ & 200 & 160 & 80 & 17 & 2,720 \\
\hline MUSA QALA & 1611 & KSHATA JALJAI & KSHATA JALJAI & 300 & 200 & 67 & 20 & 4,000 \\
\hline MUSA QALA & 1604 & KUNJAK & KUNJAK & 60 & 20 & 33 & 20 & 400 \\
\hline MUSA QALA & 1603 & KUS ANDER & KUS ANDER & 150 & 70 & 47 & 20 & 1,400 \\
\hline MUSA QALA & 784 & LANDAI KAREZ & LANDAI KAREZ & 250 & 150 & 60 & 18 & 2,700 \\
\hline MUSA QALA & 783 & LASHKARAK & LASHKARAK & 190 & 150 & 79 & 22 & 3,300 \\
\hline MUSA QALA & 792 & MASH HADYAN & MASH HADYAN & 300 & 250 & 83 & 17 & 4,250 \\
\hline MUSA QALA & 786 & MASHOZAI & MASHOZAI & 600 & 500 & 83 & 22 & 11,000 \\
\hline MUSA QALA & 793 & MAZDOOR ABAD JUNOBI & MAZDOOR ABAD & 600 & 450 & 75 & 17 & 7,650 \\
\hline MUSA QALA & 794 & MAZDOOR ABAD SHAMALI & MAZDOOR ABAD & 400 & 300 & 75 & 18 & 5,400 \\
\hline MUSA QALA & 805 & MIAN KILA & M. HUSAIN AKA & 200 & 130 & 65 & 17 & 2,210 \\
\hline MUSA QALA & 796 & MIRAJAN AKA & MIRAJAN AKA & 150 & 130 & 87 & 18 & 2,340 \\
\hline MUSA QALA & 759 & MOHD. AKA KELY & MOHD. AKA KELY & 350 & 340 & 97 & 17 & 5,780 \\
\hline MUSA QALA & 727 & MULLAH ZAHER AKA & MULLAH ZAHER AKA & 320 & 200 & 63 & 17 & 3,400 \\
\hline MUSA QALA & 798 & NAJIBULLAH & NAJIBULLAH & 280 & 180 & 64 & 18 & 3,240 \\
\hline MUSA QALA & 799 & NUR MOHD KELY & NUR MOHD KELY & 280 & 210 & 75 & 17 & 3,570 \\
\hline MUSA QALA & 797 & NUR ZO REGEE & NUR ZO REGI & 150 & 90 & 60 & 17 & 1,530 \\
\hline MUSA QALA & 800 & PATLEK & PATLEK & 300 & 200 & 67 & 17 & 3,400 \\
\hline MUSA QALA & 768 & QERAN KAREZ & QERAN KAREZ & 350 & 300 & 86 & 13 & 3,900 \\
\hline MUSA QALA & 823 & SAHIBZADA KHEL & SAHIBZADA KHEL & 80 & 40 & 50 & 18 & 720 \\
\hline MUSA QALA & 811 & SAMANDCHAY & SAMANDCHE & 400 & 180 & 45 & 18 & 3,240 \\
\hline MUSA QALA & 814 & SANDALA & SANDALA & 350 & 200 & 57 & 18 & 3,600 \\
\hline MUSA QALA & 808 & SANGAR & SANGAR & 400 & 300 & 75 & 17 & 5,100 \\
\hline MUSA QALA & 821 & SAR BESHAH & SAR BESHAH & 200 & 120 & 60 & 20 & 2,400 \\
\hline MUSA QALA & 778 & SAR KAREZ & SARKAREZ & 250 & 180 & 72 & 13 & 2,340 \\
\hline MUSA QALA & 803 & SARDAR KAS & SARDAR KAS & 250 & 80 & 32 & 20 & 1,600 \\
\hline
\end{tabular}


Opium Production Survey by village in Helmand province, 1995.

\begin{tabular}{|c|c|c|c|c|c|c|c|c|}
\hline \multirow{3}{*}{ District } & Village & \multirow{3}{*}{ Area/Main Village } & \multirow{3}{*}{ Village } & \multirow{3}{*}{$\begin{array}{c}\text { Total } \\
\text { Cultivated } \\
\text { land } \\
\end{array}$} & \multirow{2}{*}{\multicolumn{2}{|c|}{$\begin{array}{c}\text { Land Under } \\
\text { Poppy cultivation }\end{array}$}} & \multirow{3}{*}{$\begin{array}{c}\text { Wet Opium *2 } \\
\text { Yield } \\
(\mathrm{kg} / \mathrm{jr} .) \\
\end{array}$} & \multirow{3}{*}{$\begin{array}{c}\text { Wet Opium } \\
\text { Production } \\
\text { (kg) }\end{array}$} \\
\hline & \multirow[t]{2}{*}{ Code } & & & & & & & \\
\hline & & & & & $\left(\right.$ jerib $\left.^{*}\right)$ & $(\%)$ & & \\
\hline \multicolumn{9}{|c|}{ Province: Helmand } \\
\hline MUSA QALA & 810 & SARHANA & SARHANA & 500 & 250 & 50 & 18 & 4,500 \\
\hline MUSA QALA & 813 & SEEAB & SEEAB & 600 & 500 & 83 & 18 & 9,000 \\
\hline MUSA QALA & 826 & SHABAN & SHABAN & 230 & 170 & 74 & 20 . & 3,400 \\
\hline MUSA QALA & 822 & SHAHIDAN & SHAHEDAN & 120 & 80 & 67 & 10 & 800 \\
\hline MUSA QALA & 1605 & SHARBAT KAS & SHARBAT KAS & 250 & 90 & 36 & 20 & 1,800 \\
\hline MUSA QALA & 817 & SHEL-E-SHOR & SHEL-E-SHOR & 80 & 40 & 50. & 18 & 720 \\
\hline MUSA QALA & 828 & SHER GHAZNI & SHER GHAZNI & 300 & 170 & 57 & 22 & 3,740 \\
\hline MUSA QALA & 806 & SIA CHOW & SIA CHOW & 400 & 200 & 50 & 18 & 3,600 \\
\hline MUSA QALA & 318 & SOKHTA & SOKHTA & 100 & 50 & 50 & 9 & 450 \\
\hline MUSA QALA & 809 & SORAKYAN & SORAKYAN & 150 & 120 & 80 & 17 & 2,040 \\
\hline MUSA QALA & 820 & SUFAN & SUFAN & 100 & 20 & 20 & 9 & 180 \\
\hline MUSA QALA & 312 & SULTAN RUBAT & SULTAN RUBAT & 900 & 600 & 67 & 20 & 11,700 \\
\hline MUSA QALA & 824 & SURPAAN & SURPAN & 120 & 60 & 50 & 18 & 1,080 \\
\hline MUSA QALA & 722 & TOGHI & TOGHI & 250 & 200 & 80 & 17 & 3,400 \\
\hline MUSA QALA & 829 & USMA KAS & USMAN KAS & 120 & 70 & 58 & 13 & 910 \\
\hline MUSA QALA & 628 & VASKA & ATA M.KALAY & 200 & 100 & 50 & 18 & 1,800 \\
\hline MUSA QALA & 831 & WALA KHOR & WALA KHOR & 200 & 40 & 20 & 10 & 400 \\
\hline MUSA QALA & 760 & WALI MOHD AKHUNDZADA & WALI MOHD AKHUNDZADA & 350 & 300 & 86 & 17 & 5,100 \\
\hline MUSA QALA & 832 & YAK LING & YAK LING & 40 & 25 & 63 & 18 & 450 \\
\hline MUSA QALA & 804 & YAKA KHAK & YAKA KHAK & 130 & 100 & 77 & 18 & 1,800 \\
\hline MUSA QALA & 754 & ZAHER KHAN & ZAHER KHAN & 200 & 130 & 65 & 17 & 2,210 \\
\hline MUSA QALA & 834 & ZARA KALA & ZARA KALA & 250 & 130 & 52 & 17 & 2,210 \\
\hline MUSA QALA & 836 & ZARIF KHAN KELY & ZARIF KHAN KELY & 400 & 280 & 70 & 17 & 4,760 \\
\hline MUSA QALA & 835 & ZULM ABAD & ZULM ABAD & 750 & 500 & 67 & 17 & 8,500 \\
\hline NAD-E ALI & 169 & ABDUL ALIM & ABDUL ALIM & 780 & 120 & 15 & 14 & 1,620 \\
\hline NAD-E ALI & 31 & ABDUL GHAFOOR & ABDUL GHAFOOR & 600 & 150 & 25 & 13 & 1,950 \\
\hline NAD-E ALI & 35 & ABDUL HAKIM & ABDUL HAKIM & 520 & 120 & 23 & 13 & 1,560 \\
\hline NAD-E ALI & 93 & ABDUL KHALEQ & ABDUL KHALEQ & 350 & 50 & 14 & 14 & 675 \\
\hline NAD-E ALI & 3 & ABDUL WAHAB & ABDUL WAHAB & 400 & 200 & 50 & 14 & 2,700 \\
\hline
\end{tabular}


Opium Production Survey by village in Helmand province, 1995.

\begin{tabular}{|c|c|c|c|c|c|c|c|c|}
\hline & Village & & & \multirow{3}{*}{$\begin{array}{c}\text { Total } \\
\text { Cultivated } \\
\text { land } \\
\end{array}$} & \multirow{2}{*}{\multicolumn{2}{|c|}{$\begin{array}{c}\text { Land Under } \\
\text { Poppy cultivation }\end{array}$}} & \multirow{3}{*}{$\begin{array}{c}\text { Wet Opium *2 } \\
\text { Yield } \\
(\mathrm{kg} / \mathrm{jr} .)\end{array}$} & \multirow{3}{*}{$\begin{array}{c}\text { Wet Opium } \\
\text { Production } \\
(\mathrm{kg}) \\
\end{array}$} \\
\hline \multirow[t]{2}{*}{ District } & Code & Area/Main Village & Village & & & & & \\
\hline & & & & & $\left(\right.$ jerib $\left.^{*}\right)$ & $(\%)$ & & \\
\hline \multicolumn{9}{|c|}{ Province: Helmand } \\
\hline NAD-E ALI & 67 & ABDUL WAHED AKHUND & ABDUL WAHED AKH & 390 & 200 & 51 & 14 & 2,700 \\
\hline NAD-E ALI & 1253 & ABDULLA JAN & ABDULLA JAN & 500 & 125 & 25 & 17 & 2,125 \\
\hline NAD-E ALI & 34 & ABDULLAH JOHN & ABDULLAH JOHN & 640 & 100 & 16 & 13 & 1,300 \\
\hline NAD-E ALI & 108 & ACHAKZAYE & ACHEKZAYE & 600 & 100 & 17 & 14 & 1,350 \\
\hline NAD-E ALI & 1241 & ACHAKZO & ACHAKZO & 660 & 400 & 61 & 20 & 8,000 \\
\hline NAD-E ALI & 5 & BALOSAN & BALOSAN & 100 & 30 & 30 & 15 & 450 \\
\hline NAD-E ALI & 1244 & BALOSAN & MAMOOR KARIM & 1,000 & 200 & 20 & 13 & 2,600 \\
\hline NAD-E ALI & 1240 & BAYAN ALI KILY & BAYAN ALI KILY & 820 & 250 & 30 & 18 & 4,500 \\
\hline NAD-E ALI & 155 & BLOCK $10 . C 2$ & BLOCK 10.C2 & 500 & 8 & 2 & 14 & 108 \\
\hline NAD-E ALI & 152 & BLOCK 10.D & BLOCK 10.D & 280 & 20 & 7 & 14 & 270 \\
\hline NAD-E ALI & 63 & BLOCK 11 B1 & BLOCK 11 B1 & 1,500 & 110 & 7 & 14 & 1,485 \\
\hline NAD-E ALI & 103 & BLOCK 11.A1 & BLOCK 11.A1 & 200 & 6 & 3. & 9 & 54 \\
\hline NAD-E ALI & 106 & BLOCK 11.A2 & BLOCK 11.A2 & 1,200 & 100 & 8 & 14 & 1,350 \\
\hline NAD-E ALI & 104 & BLOCK 11.B2 & BLOCK 11.B2 & 600 & 10 & 2 & 14 & 135 \\
\hline NAD-E ALI & 166 & BLOCK 1A & KHODAI NAZAR & 900 & 150 & 17 & 13 & 1,950 \\
\hline NAD-E ALI & 165 & BLOCK IB & BLOCK 1B & 1,000 & 250 & 25 & 14 & 3,500 \\
\hline NAD-E ALI & 164 & BLOCK 1C & BLOCK 1C & 1,500 & 300 & 20 & 13 & 3,900 \\
\hline NAD-E ALI & 161 & BLOCK 2-C & BLOCK 2-C & 1,200 & 100 & 8 & 13 & 1,300 \\
\hline NAD-E ALI & 162 & BLOCK 2B & BLOCK 2B & 1,800 & 500 & 28 & 14 & 6,750 \\
\hline NAD-E ALI & 160 & BLOCK 3A & BLOCK 3A & 1,000 & 300 & 30 & 16 & 4,800 \\
\hline NAD-E ALI & 159 & BLOCK 3B & BLOCK 3B & 1,100 & 130 & 12 & 16 & 2,080 \\
\hline NAD-E ALI & 117 & BLOCK 4-A & BLOCK 4-A & 500 & 25 & 5 & 14 & 338 \\
\hline NAD-E ALI & 119 & BLOCK 4-B1 & BLOCK 4-B1 & 900 & 300 & 33 & 13 & 3,900 \\
\hline NAD-E ALI & 120 & BLOCK 4-C & BLOCK 4-C & 300 & 150 & 50 & 13 & 1,950 \\
\hline NAD-E ALI & 121 & BLOCK 4-D & BLOCK 4-D & 200 & 50 & 25 & 10 & 500 \\
\hline NAD-E ALI & 118 & BLOCK 4B2 & BLOCK 4B2 & 500 & 100 & 20 & 15 & 1,500 \\
\hline NAD-E ALI & 122 & BLOCK 4E & BLOCK 4E & 400 & 90 & 23 & 14 & 1,215 \\
\hline NAD-E ALI & 123 & BLOCK 5-A & BLOCK 5-A & 900 & 100 & 11 & 14 & 1,400 \\
\hline NAD-E ALI & 125 & BLOCK 5-D & BLOCK 5-D & 1,200 & 100 & 8 & 14 & 1,400 \\
\hline
\end{tabular}

*1 Jerib $=1 / 5 \mathrm{Ha}$

* 2 Dry Opium $=$ Wet Opium $-30 \%$ moisture
Page 26
Source: UNDCP Opium Production Survey, 1995 File: HLD3.XLS - 07/08/95 
Opium Production Survey by village in Helmand province, 1995.

\begin{tabular}{|c|c|c|c|c|c|c|c|c|}
\hline \multirow{3}{*}{ District } & \multirow{3}{*}{$\begin{array}{l}\text { Village } \\
\text { Code } \\
\end{array}$} & \multirow{3}{*}{ Area/Main Village } & \multirow{3}{*}{ Village } & \multirow{3}{*}{$\begin{array}{c}\text { Total } \\
\text { Cultivated } \\
\text { land } \\
\end{array}$} & \multirow{2}{*}{\multicolumn{2}{|c|}{$\begin{array}{c}\text { Land Under } \\
\text { Poppy cultivation }\end{array}$}} & \multirow{3}{*}{$\begin{array}{c}\text { Wet Opium *2 } \\
\text { Yield } \\
(\mathrm{kg} / \mathrm{jr} .) \\
\end{array}$} & \multirow{3}{*}{$\begin{array}{c}\text { Wet Opium } \\
\text { Production } \\
\text { (kg) }\end{array}$} \\
\hline & & & & & & & & \\
\hline & & & & & $($ jerib *) & $(\%)$ & & \\
\hline \multicolumn{9}{|c|}{ Province: Helmand } \\
\hline NAD-E ALI & 126 & BLOCK 5-E & BLOCK 5-E & 1,300 & 204 & 16 & 14 & 2,754 \\
\hline NAD-E ALI & 127 & BLOCK 5-F & BLOCK 5-F & 1,000 & 200 & 20 & 13 & 2,600 \\
\hline NAD-E ALI & 128 & BLOCK 6-A & BLOCK 6-A & 1,000 & 100 & 10 & 14 & 1,400 \\
\hline NAD-E ALI & 129 & BLOCK 6-B & BLOCK 6-B & 400 & 30 & 8 & 13 & 375 \\
\hline NAD-E ALI & 130 & BLOCK 6-C & BLOCK 6-C & 420 & 100 & 24 & 14 & 1,350 \\
\hline NAD-E ALI & 131 & BLOCK 6-D & BLOCK 6-D & 400 & 15 & 4 & 14 & 203 \\
\hline NAD-E ALI & 132 & BLOCK 6-E & BLOCK 6-E & 1,381 & 288 & 21 & 14 & 3,888 \\
\hline NAD-E ALI & 133 & BLOCK 6-F & BLOCK 6-F & 600 & 120 & 20 & 14 & 1,620 \\
\hline NAD-E ALI & 134 & BLOCK 7 & BLOCK 7 & 300 & 100 & 33. & 14 & 1,350 \\
\hline NAD-E ALI & 145 & BLOCK 7 F1 & BLOCK 7F1 & 450 & 200 & 44 & 14 & 2,700 \\
\hline NAD-E ALI & 135 & BLOCK 7B & BLOCK 7B & 650 & 80 & 12 & 14 & 1,080 \\
\hline NAD-E ALI & 136 & BLOCK 7B2 & BLOCK 7B2 & 650 & 80 & 12. & 14 & 1,080 \\
\hline NAD-E ALI & 137 & BLOCK 7C & BLOCK 7C & 700 & 100 & 14 & 14 & 1,350 \\
\hline NAD-E ALI & 139 & BLOCK 7D & BLOCK 7D & 400 & 150 & 38 & 14 & 2,025 \\
\hline NAD-E ALI & 142 & BLOCK 7E & BLOCK 7E & 1,100 & 100 & 9 & 14 & 1,350 \\
\hline NAD-E ALI & 144 & BLOCK 7E2 & BLOCK 7E2 & 500 & 100 & 20 & 14 & 1,350 \\
\hline NAD-E ALI & 1592 & BLOCK 8 EF & BLOCK 8 EF & 700 & 40 & 6 & 14 & 540 \\
\hline NAD-E ALI & 1591 & BLOCK 8 B & BLOCK 8 B & 1,200 & 200 & 17 & 14 & 2,700 \\
\hline NAD-E ALI & 157 & BLOCK 8A & BLOCK 8A & 1,200 & 200 & 17 & 11 & 2,200 \\
\hline NAD-E ALI & 146 & BLOCK 8C & BLOCK 8C & 1,200 & 250 & 21 & 14 & 3,375 \\
\hline NAD-E ALI & 147 & BLOCK 8D & BLOCK 8D & 1,000 & 250 & 25 & 14 & 3,375 \\
\hline NAD-E ALI & 148 & BLOCK 9A & BLOCK 9A & 500 & 125 & 25 & 14 & 1,688 \\
\hline NAD-E ALI & 149 & BLOCK 9B & BLOCK 9B & 510 & 80 & 16 & 13 & 1,040 \\
\hline NAD-E ALI & 150 & BLOCK 9C & BLOCK 9C & 500 & 130 & 26 & 13 & 1,690 \\
\hline NAD-E ALI & 1237 & BLOCK A-1 & WAKIL S. AHMAD & 900 & 200 & 22 & 10 & 2,000 \\
\hline NAD-E ALI & 163 & BLOCK A-2 & H. BESMELLAH & 774 & 74 & 10 & 11 & 814 \\
\hline NAD-E ALI & 1238 & BLOCK B-C 5 & HAJI MAWLADAD & 900 & 160 & 18 & 12 & 1,920 \\
\hline NAD-E ALI & 124 & BLOCK B-C-5 & WAKIL BAZ MOHD & 900 & 150 & 17 & 14 & 2,025 \\
\hline NAD-E ALI & 158 & BLOCK C3 & BLOCK C3 & 994 & 350 & 35 & 14 & 4,900 \\
\hline
\end{tabular}

* 1 Jerib $=1 / 5 \mathrm{Ha}$

* 2 Dry Opium $=$ Wet Opium - $30 \%$ moisture
Page $27 \quad 62$
Source: UNDCP Opium Production Survey, 1995 File: HLD3.XLS - 07/08/95 
Opium Production Survey by village in Helmand province, 1995.

\begin{tabular}{|c|c|c|c|c|c|c|c|c|}
\hline \multirow{3}{*}{ District } & \multirow{3}{*}{$\begin{array}{l}\text { Village } \\
\text { Code }\end{array}$} & \multirow{3}{*}{ Area/Main Village } & \multirow{3}{*}{ Village } & \multirow{3}{*}{$\begin{array}{c}\text { Total } \\
\text { Cultivated } \\
\text { land }\end{array}$} & \multirow{2}{*}{\multicolumn{2}{|c|}{$\begin{array}{c}\text { Land Under } \\
\text { Poppy cultivation }\end{array}$}} & \multirow{3}{*}{$\begin{array}{c}\text { Wet } \text { Opium }^{* 2} \\
\text { Yield } \\
(\mathrm{kg} / \mathrm{jr} .) \\
\end{array}$} & \multirow{3}{*}{$\begin{array}{c}\text { Wet Opium } \\
\text { Production } \\
(\mathrm{kg}) \\
\end{array}$} \\
\hline & & & & & & & & \\
\hline & & & & & $\left(\right.$ jerib $\left.^{*}\right)$ & $(\%)$ & & \\
\hline \multicolumn{9}{|c|}{ Province: Helmand } \\
\hline NAD-E ALI & 94 & BOKHARAYAN & BOKHARAYAN & 1,191 & 600 & 50 & 18 & 10,800 \\
\hline NAD-E ALI & 45 & DAHANA & ABDUL SAMAD & 1,000 & 300 & 30 & 14 & 4,050 \\
\hline NAD-E ALI & 41 & DAHANA 57 . & KHARWARYAN & 200. & 100 & 50 & 12 & 1,200 \\
\hline NAD-E ALI & 42 & DAHANA 57 & WARDAGAN & 230 & 0 & 0 & 0 & 0 \\
\hline NAD-E ALI & 44 & DAHANA 57 & SARKATEB & 630 & 125 & 20 & 14 & 1,688 \\
\hline NAD-E ALI & 40 & DAHANA 57 & BESMELLAH & 200 & 50 & 25 & 14 & 675 \\
\hline NAD-E ALI & 43 & DAHANA 57 & SULAIMAN SHAH & 340 & 40 & 12 & 14 & 540 \\
\hline NAD-E ALI & 110 & DAHANA 60 & HAJI HAIDAR & 60 & 10 & 17 & 14 & 135 \\
\hline NAD-E ALI & 46 & DAHNA 60 & WALI JAN & 1,100 & 250 & 23 & 14 & 3,375 \\
\hline NAD-E ALI & 48 & DAHNA 62 & HAJI KHALIL & 600 & 240 & 40 & 14 & 3,240 \\
\hline NAD-E ALI & 53 & DAHNA 66 & ABDUL GHAFOOR & 510 & 100 & 20 & 13 & 1,300 \\
\hline NAD-E ALI & 50 & DAHNA 66 & ABDUL QAYOM & 500 & 50 & 10 & 14 & 675 \\
\hline NAD-E ALI & 49 & DAHNA 66 & MURAD KHAN & 2,000 & 400 & 20 & 14 & 5,400 \\
\hline NAD-E ALI & 51 & DAHNA 69 & WAKEEL FAZUDEEN & 200 & 70 & 35 & 14 & 945 \\
\hline NAD-E ALI & 39 & DANA 57 & HAJI HAMEED KHAN & 500 & 100 & 20 & 14 & 1,350 \\
\hline NAD-E ALI & 47 & DANA 60 & WAKEEL IKHLAS & 1,170 & 250 & 21 & 14 & 3,375 \\
\hline NAD-E ALI & 107 & DANA 66 & WAKIL HANOLLAH & 750 & 50 & 7 & 14 & 675 \\
\hline NAD-E ALI & 109 & DANA 66 & H.WAKIL WAZIR & 500 & 100 & 20 & 14 & 1,350 \\
\hline NAD-E ALI & 1597 & DANA 66 & MOHAMMAD SUDIQ & 1,900 & 230 & 12 & 14 & 3,105 \\
\hline NAD-E ALI & 168 & DOST MOHD & DOST MOHD & 700 & 100 & 14 & 14 & 1,350 \\
\hline NAD-E ALI & 111 & GHULAM MOHD & GHULAM MOHD & 300 & 20 & 7 & 14 & 270 \\
\hline NAD-E ALI & 1243 & GHULAM SAKHI KILY & GHULAM SAKHI & 1,000 & 256 & 26 & 20 & 5,120 \\
\hline NAD-E ALI & 11 & GOUHAR KHAN KELY & GOUHAR KHAN KEL & 150 & 65 & 43 & 18 & 1,170 \\
\hline NAD-E ALI & 87 & H. A. KARIM & H. A. KARIM & 500 & 170 & 34 & 14 & 2,295 \\
\hline NAD-E ALI & 71 & H.WAKIL SAFI & H.AKHTER MOHD & 1,350 & 300 & 22 & 18 & 5,400 \\
\hline NAD-E ALI & 68 & HABIBULLAH KILY & HABIBULLAH & 450 & 200 & 44 & 20 & 4,000 \\
\hline NAD-E ALI & 22 & HAJI A.KARIM & HAJI A.KARIM & 1,000 & 300 & 30 & 14 & 4,050 \\
\hline NAD-E ALI & 1242 & HAJI ABDUL ALIM & HAJI ABDUL ALIM & 500 & 320 & 64 & 21 & 6,720 \\
\hline NAD-E ALI & 77 & HAJI ABDULLAH & HAJI ABDULLAH & 120 & 50 & 42 & 12 & 600 \\
\hline
\end{tabular}

* $1 \mathrm{Jerib}=1 / 5 \mathrm{Ha}$

${ }^{*}{ }_{2}$ Dry Opium $=$ Wet Opium $-30 \%$ moisture
Page $28 \quad 63$
Source: UNDCP Opium Production Survey, 1995 File: HLD3.XLS - 07/08/95 
Opium Production Survey by village in Helmand province, 1995.

\begin{tabular}{|c|c|c|c|c|c|c|c|c|}
\hline & \multirow{3}{*}{$\begin{array}{l}\text { Village } \\
\text { Code }\end{array}$} & \multirow{3}{*}{ Area/Main Village } & \multirow{3}{*}{ Village } & \multirow{3}{*}{$\begin{array}{c}\text { Total } \\
\text { Cultivated } \\
\text { land } \\
\end{array}$} & \multirow{2}{*}{\multicolumn{2}{|c|}{$\begin{array}{c}\text { Land Under } \\
\text { Poppy cultivation }\end{array}$}} & \multirow{3}{*}{$\begin{array}{c}\text { Wet Opium }{ }^{* 2} \\
\text { Yield } \\
(\mathrm{kg} / \mathrm{jr} .) \\
\end{array}$} & \multirow{3}{*}{$\begin{array}{c}\text { Wet Opium } \\
\text { Production } \\
(\mathrm{kg}) \\
\end{array}$} \\
\hline \multirow{2}{*}{ District } & & & & & & & & \\
\hline & & & & & $($ jerib *) & $(\%)$ & & \\
\hline \multicolumn{9}{|c|}{ Province: Helmand } \\
\hline NAD-E ALI & 57 & HAJI DANGAR KHAN & HI. DANGAR KHAN & 700 & 380 & 54 & 20 & 7,600 \\
\hline NAD-E ALI & 70 & HAJI FATEH KHAN & HAJI FATEH KHAN & 675 & 236 & 35 & 20 & 4,720 \\
\hline NAD-E ALI & 54 & HAJI GHULAM HASAN & HAJI GH. HASAN & 720 & 200 & 28 & 22 & 4,400 \\
\hline NAD-E ALI & 29 & HAJI GHULAM HUSAIN & HAJI GHULAM HUS & 520 & 200 & 38 & 13 & 2,600 \\
\hline NAD-E ALI & 167 & HAJI JALAT KHAN & HAJI JALAT KHAN & 1,300 & 127 & 10 & 14 & 1,715 \\
\hline NAD-E ALI & 30 & HAJI KHIAR MOHD & HAJI KHAIR MOHD & 600 & 130 & 22 & 14 & 1,755 \\
\hline NAD-E ALI & 91 & HAJI KHODAI NAZAR & H. KHODAI NAZAR & 500 & 30 & 6 & 14 & 405 \\
\hline NAD-E ALI & 115 & HAJI MOLA WAZIR & HAJI MOLA WAZIR & 300 & 200 & 67 & 14 & 2,700 \\
\hline NAD-E ALI & 6 & HAJI NOOR AHMAD & HAJI NOOR AHMAD & 1,100 & 200 & 18 & 14 & 2,700 \\
\hline NAD-E ALI & 15 & HAJI NOOR MOHD & MOHD RAZA & 250 & 50 & 20 & 14 & 700 \\
\hline NAD-E ALI & 13 & HAJI NOOR MOHD JAN & HAJI NOOR MOHD & 500 & 250 & 50 & 18 & 4,500 \\
\hline NAD-E ALI & 14 & HAJI NOOR MOHD KHAN & HAJI NOOR MOHD & 600 & 100 & 17 & 17 & 1,700 \\
\hline NAD-E ALI & 65 & HAJI RANG & HAJI RANG & 1,300 & 300 & 23 & 14 & 4,050 \\
\hline NAD-E ALI & 21 & HAJI SARKATEB & HAJI SARKATEB & 500 & 150 & 30 & 14 & 2,025 \\
\hline NAD-E ALI & 19 & HAJI TALEB & HAJI TALEB & 660 & 300 & 45 & 14 & 4,050 \\
\hline NAD-E ALI & 18 & HAJI TALEB & HAJI TALEB & 700 & 180 & 26 & 14 & 2,430 \\
\hline NAD-E ALI & 69 & HAJI WAKIL SAFAR & HAJI WAKIL & 1,170 & 200 & 17 & 15 & 2,900 \\
\hline NAD-E ALI & 28 & HAJI WALI MOHD & HAJI WALI MOHD & 700 & 150 & 21 & 14 & 2,025 \\
\hline NAD-E ALI & 27 & HAJI YAHYA KHAN & HAJI YAHYA KHAN & 608 & 150 & 25 & 14 & 2,025 \\
\hline NAD-E ALI & 17 & HAZRATSHAH & HAZRATSHAH & 300 & 70 & 23 & 14 & 945 \\
\hline NAD-E ALI & 81 & HJ.ALAWODEN & HJ. ALAWODEN & 500 & 200 & 40 & 14 & 2,700 \\
\hline NAD-E ALI & 9 & KAKALAN EKLY & KAKALAN KELY & 900 & 200 & 22 & 17 & 3,400 \\
\hline NAD-E ALI & 1247 & KAKARAN & GUL MOHD KILY & 600 & 400 & 67 & 18 & 7,200 \\
\hline NAD-E ALI & 7 & KAKARAN & ABDUL QADIR & 600 & 420 & 70 & 22 & 9,240 \\
\hline NAD-E ALI & 1245 & KAKARAN & ABDUL ALIM & 1,000 & 600 & 60 & 25 & 15,000 \\
\hline NAD-E ALI & 1595 & KALO ZO & QARYA-E- JADID & 1,000 & 100 & 10 & 14 & 1,350 \\
\hline NAD-E ALI & 82 & KHAYR MOHAMMAD RAKA & KHAY MOHAMMAD R & 300 & 200 & 67 & 14 & 2,700 \\
\hline NAD-E ALI & 12 & KHOSHAL KELY & MULAH FAZLO & 500 & 100 & 20 & 13 & 1,300 \\
\hline NAD-E ALI & 1250 & LAL JAN & LAL JAN & 700 & 100 & 14 & 15 & 1,500 \\
\hline
\end{tabular}

* $1 \mathrm{Jerib}=1 / 5 \mathrm{Ha}$

* 2 Dry Opium $=$ Wet Opium $-30 \%$ moisture 
Opium Production Survey by village in Helmand province, 1995.

\begin{tabular}{|c|c|c|c|c|c|c|c|c|}
\hline \multirow{3}{*}{ District } & \multirow{3}{*}{$\begin{array}{l}\text { Village } \\
\text { Code }\end{array}$} & \multirow{3}{*}{ Area/Main Village } & \multirow{3}{*}{ Village } & \multirow{3}{*}{$\begin{array}{c}\text { Total } \\
\text { Cultivated } \\
\text { land }\end{array}$} & \multirow{2}{*}{\multicolumn{2}{|c|}{$\begin{array}{c}\text { Land Under } \\
\text { Poppy cultivation }\end{array}$}} & \multirow{3}{*}{$\begin{array}{c}\text { Wet }^{\text {Opium }}{ }^{* 2} \\
\text { Yield } \\
(\mathrm{kg} / \mathrm{jr} .)\end{array}$} & \multirow{3}{*}{$\begin{array}{c}\text { Wet Opium } \\
\text { Production } \\
(\mathrm{kg})\end{array}$} \\
\hline & & & & & & & & \\
\hline & & & & & $\left(\right.$ jerib $\left.{ }^{*}\right)$ & $(\%)$ & & \\
\hline \multicolumn{9}{|c|}{ Province: Helmand } \\
\hline NAD-E ALI & 74 & LALA JAN KELY & LALA JAN KELY & 150 & 35 & 23 & 12 & 420 \\
\hline NAD-E ALI & 66 & MALANG KAKA & MALANG KAKA & 454 & 162 & 36 & 18 & 2,916 \\
\hline NAD-E ALI & 58 & MALANG KHUDAIDAD & MALANG KHUDAIDA & 1,350 & 270 & 20 & 18 & 4,860 \\
\hline NAD-E ALI & 153 & MARJA & MARJA & 420 & 20 & 5 & 14 & 270 \\
\hline NAD-E ALI & 73 & MAWEN ALAH DAD & MAWEN ALAH DAD & 700 & 90 & 13 & 20 & 1,800 \\
\hline NAD-E ALI & 32 & MIR AB HABIBULLAH & MIR AB HABIBULA & 640 & 100 & 16 & 13 & 1,300 \\
\hline NAD-E ALI & 1598 & MIR HAMZA KHAN & MIR HAMZA KHAN & 390 & 100 & 26 & 14 & 1,350 \\
\hline NAD-E ALI & 1248 & MIRZA WALI MOHD KHAN & MIRZA WALI MOHD & 320 & 160 & 50 & 20 & 3,200 \\
\hline NAD-E ALI & 1593 & MOHAMMAD ALI & MOHAMMAD ALI & 400 & 170 & 43 & 14 & 2,295 \\
\hline NAD-E ALI & 83 & MOHAMMAD AMAN & MOHAMMAD AMAN & 450 & 300 & 67 & 14 & 4,050 \\
\hline NAD-E ALI & 1594 & MOHAMMAD AYUB & MOHAMMAD AFZAL & 600 & 200 & 33 & 14 & 2,700 \\
\hline NAD-E ALI & 1255 & MOHD HASHIM KILY & MOHD HASHIM & 700 & 60 & 9 & 17 & 1,020 \\
\hline NAD-E ALI & 36 & MOHD WAZIR & MOHD WAZIR & 500 & 150 & 30 & 13 & 1,950 \\
\hline NAD-E ALI & 37 & MOHD YAQOOB & MOHD YAQOOB & 500 & 100 & 20 & 14 & 1,350 \\
\hline NAD-E ALI & 55 & MULA ABDUL HAKIM & MULA ABD.HAKIM & 300 & 15 & 5 & 14 & 203 \\
\hline NAD-E ALI & 98 & MULA KHIAL & MULA KHIAL & 1,200 & 500 & 42 & 18 & 9,000 \\
\hline NAD-E ALI & 1599 & MULA MUSA JAN & MULA MUSA JAN & 700 & 300 & 43 & 14 & 4,050 \\
\hline NAD-E ALI & 1256 & MULLA KHALIL AKHUND & MULLA KHALIL AKHUND & 600 & 200 & 33 & 18 & 3,600 \\
\hline NAD-E ALI & 1254 & MULLA KHAN AGHA & MULLA KHAN AGHA & 600 & 300 & 50 & 22 & 6,600 \\
\hline NAD-E ALI & 90 & MULLAH ABDUL AHAD & MULLAH A. AHAD & 200 & 55 & 28 & 14 & 743 \\
\hline NAD-E ALI & 78 & MULLAH JABAR & MULLAH JABAR & 350 & 100 & 29 & 14 & 1,350 \\
\hline NAD-E ALI & 1257 & NADIR ALI KILY & NADIR ALI KILY & 894 & 30 & 3 & 14 & 420 \\
\hline NAD-E ALI & 1249 & NEWI ZO KILY & NEWI ZO KILY & 0 & 0 & \#DIV/0! & 0 & 0 \\
\hline NAD-E ALI & 56 & NOORDIN & NOORDIN & 1,310 & 600 & 46 & 13 & 7,500 \\
\hline NAD-E ALI & 75 & NOORDIN & NOORDIN & 100 & 20 & 20 & 9 & 180 \\
\hline NAD-E ALI & 72 & QARI SAHIB & QARI SAHIB & 1,500 & 200 & 13 & 14 & 2,700 \\
\hline NAD-E ALI & 1252 & RAHMAT YAR KILY & RAHMAT YAR & 395 & 197 & 50 & 18 & 3,546 \\
\hline NAD-E ALI & 85 & SAID HASAN & SAID HASAN & 650 & 200 & 31 & 14 & 2,700 \\
\hline NAD-E ALI & 8 & SARAJUDIN & SARAJUDIN & 350 & 100 & 29 & 19 & 1,900 \\
\hline
\end{tabular}

* 1 Jerib $=1 / 5 \mathrm{Ha}$

* ${ }^{2}$ Dry Opium = Wet Opium - $30 \%$ moisture
Page $30 \quad 6 h$
Source: UNDCP Opium Production Survey, 1995 File: HLD3.XLS - 07/08/95 
Opium Production Survey by village in Helmand province, 1995.

\begin{tabular}{|c|c|c|c|c|c|c|c|c|}
\hline & Village & & & Total & Land $L$ & & Wet Opium * 2 & Wet Opium \\
\hline \multirow[t]{2}{*}{ District } & \multirow{2}{*}{ Code } & \multirow[t]{2}{*}{ Area/Main Village } & \multirow[t]{2}{*}{ Village } & \multirow{2}{*}{$\begin{array}{c}\text { Cultivated } \\
\text { land }\end{array}$} & \multicolumn{2}{|c|}{ Poppy cultivation } & \multirow{2}{*}{$\begin{array}{c}\text { Yield } \\
(\mathrm{kg} / \mathrm{jr} .) \\
\end{array}$} & \multirow{2}{*}{$\begin{array}{c}\text { Production } \\
(\mathrm{kg})\end{array}$} \\
\hline & & & & & $\left(\right.$ jerib $\left.^{*}\right)$ & $(\%)$ & & \\
\hline \multicolumn{9}{|c|}{ Province: Helmand } \\
\hline NAD-E ALI & 1 & SAYED ABAD & SAYED ABAD & 50 & 0 & 0 & 0 & 0 \\
\hline NAD-E ALI & 23 & SAYED MOHD KHAN & SAYED MOHD KHAN & 600 & 300 & 50 & 20 & 6,000 \\
\hline NAD-E ALI & 20 & SAYED SAMIULLAH & SAYED SAMIULLAH & 380 & 100 & 26 & 14 & 1,350 \\
\hline NAD-E ALI & 38 & SHAH MOHD & SHAH MOHD & 600 & 150 & 25 & 13 & 1,950 \\
\hline NAD-E ALI & 113 & SHAH NAZAR KHAN & SHAH NAZAR KHAN & 150 & 50 & 33 & 14 & 675 \\
\hline NAD-E ALI & 97 & SHAH NAZAR KHAN KELY & SHAH NAZAR KHAN & 1,450 & 200 & 14 & 13 & 2,600 \\
\hline NAD-E ALI & 10 & SHAHZADA & SHAHZADA & 300 & 150 & 50 & 14 & 2,025 \\
\hline NAD-E ALI & 101 & SHEEN GHAZAK & SHEEN GHAZAK & 1,640 & 400 & 24 & 14 & 5,400 \\
\hline NAD-E ALI & 112 & SHEEN GHAZAK & MOLA AHMAD AKH. & 1,000 & 20 & 2 & 14 & 270 \\
\hline NAD-E ALI & 33 & SHER MOHAMMAD & SHER MOHAMMAD & 640 & 200 & 31 & 12 & 2,400 \\
\hline NAD-E ALI & 16 & SULTAN HASSAN & SULTAN HASSAN & 750 & 250 & 33 & 18 & 4,500 \\
\hline NAD-E ALI & 24 & TARJUMAN & TARJUMAN & 100 & 50 & 50 & 14 & 675 \\
\hline NAD-E ALI & 26 & TAZA GUL & TAZA GUL & 700 & 250 & 36 & 14 & 3,375 \\
\hline NAD-E ALI & 59 & WAKEEL KHODAIDAD & WAKEL KHODAIDAD & 800 & 400 & 50 & 18 & 7,200 \\
\hline NAD-E ALI & 95 & WAKEEL SAILANI & WAKEEL SAILANI & 1,400 & 700 & 50 & 20 & 14,000 \\
\hline NAD-E ALI & 92 & WAKIL ABDUL QAYUM & WAKIL ABDUL QAYUM & 360 & 70 & 19 & 14 & 945 \\
\hline NAD-E ALI & 80 & WAKIL DAWRAN KELY & WAKIL DAWRAN & 426 & 150 & 35 & 20 & 3,000 \\
\hline NAD-E ALI & 61 & WAKIL KHUDI DAD & HOWLIA & 800 & 400 & 50 & 22 & 8,800 \\
\hline NAD-E ALI & 62 & WAKIL KUDAIDAD KELY & HAJI FATEH ABAD & 800 & 200 & 25 & 21 & 4,200 \\
\hline NAD-E ALI & 86 & WAKIL LAL MOHAMMAD & WAKIL LAL MOHAMMAD & 600 & 200 & 33 & 14 & 2,700 \\
\hline NAD-E ALI & 1239 & WAKIL MULLAH SULTAN & WAKIL M. SULTAN & 880 & 220 & 25 & 12 & 2,640 \\
\hline NAD-E ALI & 96 & WAKIL SAYEDJOHN & WAKIL SAYEDJOHN & 920 & 300 & 33 & 14 & 4,050 \\
\hline NAD-E ALI & 79 & WAKIL SHINKAI & WAKIL SHINKAI & 800 & 300 & 38 & 20 & 6,000 \\
\hline NAD-E ALI & 84 & WAKIL ZAHER & WAKIL ZAHER & 130 & 30 & 23 & 14 & 405 \\
\hline NAD-E ALI & 89 & ZAHER BALOCH & ZAHER BALOCH & 400 & 200 & 50 & 14 & 2,700 \\
\hline NAHR-E SARAJ & 558 & ABAZAN & MOHD. NABI & 150 & 0 & 0 & 0 & 0 \\
\hline NAHR-E SARAJ & 559 & ABAZAN & TAWAKAL & 140 & 0 & 0 & 0 & 0 \\
\hline NAHR-E SARAJ & 561 & ABAZAN & ML GHULAM ALI & 140 & 0 & 0 & 0 & 0 \\
\hline NAHR-E SARAJ & 560 & ABBAZAN & BAHAWODDIN & 150 & 0 & 0 & 0 & 0 \\
\hline
\end{tabular}

* I Jerib $=1 / 5 \mathrm{Ha}$

* 2 Dry Opium $=$ Wet Opium - $30 \%$ moisture
Page $31 \quad 66$
Source: UNDCP Opium Production Survey, 1995 File: HLD3.XLS - 07/08/95 
Opium Production Survey by village in Helmand province, 1995.

\begin{tabular}{|c|c|c|c|c|c|c|c|c|}
\hline \multirow{3}{*}{ District } & \multirow{3}{*}{$\begin{array}{l}\text { Village } \\
\text { Code }\end{array}$} & \multirow{3}{*}{ Area/Main Village } & \multirow{3}{*}{ Village } & \multirow{3}{*}{$\begin{array}{c}\text { Total } \\
\text { Cultivated } \\
\text { land } \\
\end{array}$} & \multirow{2}{*}{\multicolumn{2}{|c|}{$\begin{array}{c}\text { Land Under } \\
\text { Poppy cultivation }\end{array}$}} & \multirow{3}{*}{$\begin{array}{c}\text { Wet Opium *2 } \\
\text { Yield } \\
(\mathrm{kg} / \mathrm{jr} .) \\
\end{array}$} & \multirow{3}{*}{$\begin{array}{c}\text { Wet Opium } \\
\text { Production } \\
(\mathrm{kg})\end{array}$} \\
\hline & & & & & & & & \\
\hline & & & & & $(j e r i b *)$ & $(\%)$ & & \\
\hline \multicolumn{9}{|c|}{ Province: Helmand } \\
\hline NAHR-E SARAJ & 562 & ABBAZAN & SHEKH DAD & 140 & 2 & 1 & 9 & 18 \\
\hline NAHR-E SARAJ & 564 & ABDUL RASHED KAREZ & ABDUL RASHED KAREZ & 150 & 10 & 7 & 18 & 180 \\
\hline NAHR-E SARAJ & 1618 & ABDUL SALAM SHAKH & A. SALAM SHAKH & 1,020 . & 100 & 10 & 9 & 900 \\
\hline NAHR-E SARAJ & 565 & ADINZAI & ENZAI & 1,100 & 550 & 50 & 14 & 7,425 \\
\hline NAHR-E SARAJ & 566 & ADINZAI KELY & ABDUL SALAM & 1,300 & 700 & 54 & 14 & 9,800 \\
\hline NAHR-E SARAJ & 1617 & AFGHANI KAREZ & AWGHANI KAREZ & 280 & 100 & 36 & 14 & 1,350 \\
\hline NAHR-E SARAJ & 557 & AGHLAGH KAREZ & AGHLAGH KAREZ & 450 & 100 & 22 & 14 & 1,350 \\
\hline NAHR-E SARAJ & 647 & AHMAD ZAI & AHMAD ZAI & 865 & 432 & 50 & 14 & 5,832 \\
\hline NAHR-E SARAJ & 570 & ALOKOZO SHAKH & ALOKOZO SHAKH & 800 & 70 & 9 & 9 & 630 \\
\hline NAHR-E SARAJ & 1616 & AMANULLAH KAREZ & AMANULLAH KAREZ & 120 & 40 & 33. & 13 & 520 \\
\hline NAHR-E SARAJ & 569 & ANEEZI & ENZAY & 1,100 & 550 & 50 & 14 & 7,425 \\
\hline NAHR-E SARAJ & 847 & ARABZAEE & ARABZAEE & 300 & 100 & 33 & 6 & 600 \\
\hline NAHR-E SARAJ & 1394 & ARIAN ZAI KELY & ARIAN ZAI MALIK & 400 & 100 & 25 & 18 & 1,800 \\
\hline NAHR-E SARAJ & 1405 & ATA KHAN & ATA KHAN & 500 & 166 & 33 & 14 & 2,241 \\
\hline NAHR-E SARAJ & 597 & BAD WAN & HAJI MOHD SHAH & 284 & 142 & 50 & 9 & 1,278 \\
\hline NAHR-E SARAJ & 650 & BADAWAN & HAJI AMANULLAH & 496 & 150 & 30 & 9 & 1,350 \\
\hline NAHR-E SARAJ & 571 & BAGHONA KALAY & BAGHO KELE & 1,000 & 333 & 33 & 9 & 2,997 \\
\hline NAHR-E SARAJ & 598 & BAHAR KHAN KHOGYANI & BAHAR KHOGYANI & 561 & 300 & 53 & 14 & 4,050 \\
\hline NAHR-E SARAJ & 611 & BAHEER & BAHEER KALAY & 550 & 200 & 36 & 14 & 2,700 \\
\hline NAHR-E SARAJ & 1620 & BALA KHANA WAZIRAN & BALA KHANA WAZI & 1,200 & 0 & 0 & 0 & 0 \\
\hline NAHR-E SARAJ & 572 & BALUCHANO SHAKH & BALUCHANO SHAKH & 1,500 & 90 & 6 & 9 & 810 \\
\hline NAHR-E SARAJ & 671 & BAND BARQ & SARDAR NEMATULA & 1,400 & 60 & 4. & 9 & 540 \\
\hline NAHR-E SARAJ & 577 & BARAK ZO KELY & BARAK ZO KELY & 260 & 50 & 19 & 14 & 675 \\
\hline NAHR-E SARAJ & 577 & BAREKZO KALAY & BAREKZO KALAY & 600 & 0 & 0 & 0 & 0 \\
\hline NAHR-E SARAJ & 600 & BAYANZAI & BAYANZAI & 532 & 300 & 56 & 18 & 5,400 \\
\hline NAHR-E SARAJ & 654 & CHAGHARY KELE & CHAGARY KELE & 244 & 81 & 33 & 81 & 6,561 \\
\hline NAHR-E SARAJ & 580 & CHAKHANDAZ & CHARKHANDAZ & 798 & 196 & 25 & 14 & 2,646 \\
\hline NAHR-E SARAJ & 599 & CHAWKARAK & CHAWKARAK & 517 & 300 & 58 & 18 & 5,400 \\
\hline NAHR-E SARAJ & 583 & DAGIAN & DAGIAN & 400 & 133 & 33 & 14 & 1,796 \\
\hline
\end{tabular}

* 1 Jerib $=1 / 5 \mathrm{Ha}$

* 2 Dry Opium $=$ Wet Opium $-30 \%$ moisture
Page 32
Source: UNDCP Opium Production Survey, 1995 File: HLD3.XLS - 07/08/95 
Opium Production Survey by village in Helmand province, 1995.

\begin{tabular}{|c|c|c|c|c|c|c|c|c|}
\hline \multirow{3}{*}{ District } & Village & \multirow{3}{*}{ Area/Main Village } & \multirow{3}{*}{ Village } & \multirow{3}{*}{$\begin{array}{c}\text { Total } \\
\text { Cultivated } \\
\text { land } \\
\end{array}$} & \multirow{2}{*}{\multicolumn{2}{|c|}{$\begin{array}{c}\text { Land Under } \\
\text { Poppy cultivation }\end{array}$}} & \multirow{3}{*}{$\begin{array}{c}\text { Wet Opium }^{* 2} \\
\text { Yield } \\
(\mathrm{kg} / \mathrm{jr} .)\end{array}$} & \multirow{3}{*}{$\begin{array}{c}\text { Wet Opium } \\
\text { Production } \\
(\mathrm{kg}) \\
\end{array}$} \\
\hline & \multirow[t]{2}{*}{ Code } & & & & & & & \\
\hline & & & & & $\left(\right.$ jerib $\left.^{*}\right)$ & (\%) & & \\
\hline \multicolumn{9}{|c|}{ Province: Helmand } \\
\hline NAHR-E SARAJ & 587 & DAH MAZANG & DEH MAZANG & 380 & 50 & 13 & 9 & 450 \\
\hline NAHR-E SARAJ & 1622 & DAHANA-E-ABBAZAN & DAHANA ABAZAN & 600 & 0 & 0 & 0 & 0 \\
\hline NAHR-E SARAJ & 582. & DAKTAR JAY & DAKTAR & 1,100 & 0 & 0 & 0. & 0 \\
\hline NAHR-E SARAJ & 684 & DARBAND & DARBAND & 1,500 & 40 & 3 & 9 & 360 \\
\hline NAHR-E SARAJ & 569 & ENZAY & ENZAY & 1,100 & 550 & 50 & 14 & 7,425 \\
\hline NAHR-E SARAJ & 591 & FAQEER PAYAN & FAQEEE PAYAN & 140 & 15 & 11 & 9 & 135 \\
\hline NAHR-E SARAJ & 593 & GAWBAND GHUNDY & GOBAND GHOUNDI & 2,400 & 70 & 3 & 9 & 630 \\
\hline NAHR-E SARAJ & 1409 & GHULBA & GHULBA & 233 & 77 & 33 & 14 & 1,040 \\
\hline NAHR-E SARAJ & 1396 & GOMBATE & GOMBATE & 286 & 95 & 33 & 18 & 1,710 \\
\hline NAHR-E SARAJ & 596 & GORE MAGHOL & AKHUNZANDAKHEL & 800 & 100 & 13 & 14 & 1,350 \\
\hline NAHR-E SARAJ & 1404 & HAFT RANG & HAFT RANG & 668 & 160 & 24 & 9 & 1,440 \\
\hline NAHR-E SARAJ & 601 & HAIDAR ZAI KELY & HAIADAR ZAI & 649 & 216 & 33 & 9 & 1,944 \\
\hline NAHR-E SARAJ & 679 & HAIDER ABAD & TAMBA & 155 & 90 & 58 & 18 & 1,620 \\
\hline NAHR-E SARAJ & 590 & HAJI ABDULAH & HAJI ABDULAH & 696 & 100 & 14 & 14 & 1,350 \\
\hline NAHR-E SARAJ & 625 & HAJI AZIZ & HAJI AZIZ & 500 & 200 & 40 & 9 & 1,800 \\
\hline NAHR-E SARAJ & 669 & HAJI FAZIL AKHUNDZAD & HAJI FAZIL AKON & 616 & 205 & 33 & 14 & 2,768 \\
\hline NAHR-E SARAJ & 589 & HAJI G.NAQSHBAND & HAJI G.NAQISH B & 284 & 44 & 15 & 18 & 792 \\
\hline NAHR-E SARAJ & 648 & HAJI GHULAM HAIDER & H.GHULAM HAIDER & 10 & 6 & 60 & 9 & 54 \\
\hline NAHR-E SARAJ & 592 & HAJI JAMAL KHAN & HAJI JAMAL KAHN & 222 & 74 & 33 & 14 & 999 \\
\hline NAHR-E SARAJ & 632 & HAJI MAMOR KELY & HAJI MAMOOR & 162 & 30 & 19 & 13 & 390 \\
\hline NAHR-E SARAJ & 673 & HAJI MOHD AYUB & HAJI MOHD AYUB & 295 & 98 & 33 & 14 & 1,323 \\
\hline NAHR-E SARAJ & 1416 & HAJI MOHD SEDIQ & HAJI MULA SEDDIQ & 420 & 190 & 45 & 12 & 2,280 \\
\hline NAHR-E SARAJ & 685 & HAJI NAMATULLAH & HAJI NAMATULLAH & 800 & 50 & 6 & 14 & 675 \\
\hline NAHR-E SARAJ & 688 & HAJI QADIR KHAN & HAJI QADIR KHAN & 550 & 80 & 15 & 14 & 1,080 \\
\hline NAHR-E SARAJ & 606 & HAJI TUR KHAN & HAJI TUR KHAN & 500 & 0 & 0 & 0 & 0 \\
\hline NAHR-E SARAJ & 659 & HAJIAN MADENZAI & HAJIAN MADINZAI & 750 & 400 & 53 & 9 & 3,600 \\
\hline NAHR-E SARAJ & 605 & HAZARA GAN & HAZARA GAN & 200 & 20 & 10 & 9 & 180 \\
\hline NAHR-E SARAJ & 607 & JAN MOHD SHAKH & JAN MOHD SHAKH & 600 & 30 & 5 & 9 & 270 \\
\hline NAHR-E SARAJ & 608 & JANUBI NURZAI & NURZAI JANUBI & 200 & 25 & 13 & 18 & 450 \\
\hline
\end{tabular}

* I Jerib $=1 / 5 \mathrm{Ha}$

${ }^{*}$ Dry Opium $=$ Wet Opium - $30 \%$ moisture 
Opium Production Survey by village in Helmand province, 1995.

\begin{tabular}{|c|c|c|c|c|c|c|c|c|}
\hline \multirow{3}{*}{ District } & \multirow{3}{*}{$\begin{array}{c}\text { Village } \\
\text { Code }\end{array}$} & \multirow{3}{*}{ Area/Main Village } & \multirow{3}{*}{ Village } & \multirow{3}{*}{$\begin{array}{c}\text { Total } \\
\text { Cultivated } \\
\text { land }\end{array}$} & \multirow{2}{*}{\multicolumn{2}{|c|}{$\begin{array}{c}\text { Land Under } \\
\text { Poppy cultivation }\end{array}$}} & \multirow{3}{*}{$\begin{array}{c}\text { Wet }^{\text {Opium }}{ }^{* 2} \\
\text { Yield } \\
(\mathrm{kg} / \mathrm{jr} .)\end{array}$} & \multirow{3}{*}{$\begin{array}{c}\text { Wet Opium } \\
\text { Production } \\
(\mathrm{kg})\end{array}$} \\
\hline & & & & & & & & \\
\hline & & & & & $\left(\right.$ jerib $\left.^{*}\right)$ & $(\%)$ & & \\
\hline \multicolumn{9}{|c|}{ Province: Helmand } \\
\hline NAHR-E SARAJ & 613 & KAKARAN KELY & KAKALAN KELY & 614 & 150 & 24 & 9 & 1,350 \\
\hline NAHR-E SARAJ & 610 & KAKARAN SAYED GUL & KAKARAN & 50 & 0 & 0 & 0 & 0 \\
\hline NAHR-E SARAJ & 603 & KAKOZI & KAKOZI & 400 & 250 & 63 & 18 & 4,500 \\
\hline NAHR-E SARAJ & 612 & KAREEM KAREZ & KAREEM KAREZ & 180 & 60 & 33 & 14 & 810 \\
\hline NAHR-E SARAJ & 1532 & KAREZ-E-KOKA & KAREZ KOKA & 60 & 30 & 50 & 14 & 405 \\
\hline NAHR-E SARAJ & 1615 & KHAKASH KAREZ & KHAKASH KAREZ & 180 & 40 & 22 & 14 & 540 \\
\hline NAHR-E SARAJ & 1408 & KHALIFA KHEL & KHALIFA KHEL & 351 & 117 & 33 & 14 & 1,580 \\
\hline NAHR-E SARAJ & 615 & KHALIFA SHIREEN & KHALIFA SHIREEN & 300 & 30 & 10 & 9 & 270 \\
\hline NAHR-E SARAJ & 1623 & KHAROTIAN & KHAROTIAN & 870 & 0 & 0 & 0 & 0 \\
\hline NAHR-E SARAJ & 618 & KHODAI RAHIM SHAKH & KHODAI RAHIM & 420 & 90 & 21 & 9 & 810 \\
\hline NAHR-E SARAJ & 619 & KHODAIDAD SHAKH & KHODAIDAD SHAKH & 600 & 60 & 10 & 9 & 540 \\
\hline NAHR-E SARAJ & 624 & KHOGIANI GHUNDY & KHOGANI GHUNDAI & 1,676 & 838 & 50 & 14 & 11,313 \\
\hline NAHR-E SARAJ & 623 & KHOMARI & KHOMARI & 400 & 200 & 50 & 14 & 2,700 \\
\hline NAHR-E SARAJ & 616 & KHUAJA MURAD & KHUAJA MURAD & 600 & 60 & 10 & 22 & 1,320 \\
\hline NAHR-E SARAJ & 621 & KONJAK & KONJAK & 411 & 250 & 61 & 18 & 4,500 \\
\hline NAHR-E SARAJ & 614 & KUNJAK & KUNJAK ABDULSAMAD & 1,117 & 372 & 33 & 9 & 3,348 \\
\hline NAHR-E SARAJ & 681 & LANDI TALE GAWMISHI & LANDI TALE & 1,020 & 600 & 59 & 18 & 10,800 \\
\hline NAHR-E SARAJ & 668 & MADINZAI KELY & MADINZAI & 220 & 73 & 33 & 9 & 657 \\
\hline NAHR-E SARAJ & 1614 & MALAKHY KAREZ & MALKHI KAREZ & 60 & 2 & 3 & 14 & 27 \\
\hline NAHR-E SARAJ & 1619 & MANDA WAZIRAN & WAZIRAN & 900 & 50 & 6 & 9 & 450 \\
\hline NAHR-E SARAJ & 635 & MARY & MARY & 2,000 & 600 & 30 & 14 & 8,100 \\
\hline NAHR-E SARAJ & 629 & MIR MANDAB & WAZIR KHAN & 646 & 250 & 39 & 14 & 3,375 \\
\hline NAHR-E SARAJ & 634 & MIR MANDAB & ABDUL ALI & 600 & 400 & 67 & 18 & 7,200 \\
\hline NAHR-E SARAJ & 628 & MOHD WAZIR & MOHD WAZIR & 300 & 150 & 50 & 18 & 2,700 \\
\hline NAHR-E SARAJ & 573 & MOHD YAQUB & MOHD YAQUB & 1,800 & 600 & 33 & 9 & 5,400 \\
\hline NAHR-E SARAJ & 602 & MOHD ZAI & MOHD ZAI & 1,100 & 700 & 64 & 18 & 12,600 \\
\hline NAHR-E SARAJ & 630 & MOHD ZAI & MOHD ZAI & 450 & 200 & 44 & 18 & 3,600 \\
\hline NAHR-E SARAJ & 1612 & MOHD ZOY & MOHD ZOY & 400 & 120 & 30 & 9 & 1,080 \\
\hline NAHR-E SARAJ & 1400 & MULLAH M. AKHUND & MULLAH M.AKHUND & 1,100 & 50 & 5 & 9 & 450 \\
\hline
\end{tabular}

* I Jerib $=1 / 5 \mathrm{Ha}$

* ${ }^{2}$ Dry Opium $=$ Wet Opium - $30 \%$ moisture
Page $34 \quad 69$
Source: UNDCP Opium Production Survey, 1995 File: HLD3.XLS - 07/08/95 
Opium Production Survey by village in Helmand province, 1995.

\begin{tabular}{|c|c|c|c|c|c|c|c|c|}
\hline \multirow{3}{*}{ District } & \multirow{3}{*}{$\begin{array}{l}\text { Village } \\
\text { Code }\end{array}$} & \multirow{3}{*}{ Area/Main Village } & \multirow{3}{*}{ Village } & \multirow{3}{*}{$\begin{array}{c}\text { Total } \\
\text { Cultivated } \\
\text { land } \\
\end{array}$} & \multirow{2}{*}{\multicolumn{2}{|c|}{$\begin{array}{c}\text { Land Under } \\
\text { Poppy cultivation }\end{array}$}} & \multirow{3}{*}{$\begin{array}{c}\text { Wet Opium }{ }^{* 2} \\
\text { Yield } \\
(\mathrm{kg} / \mathrm{jr} .) \\
\end{array}$} & \multirow{3}{*}{$\begin{array}{c}\text { Wet Opium } \\
\text { Production } \\
(\mathrm{kg}) \\
\end{array}$} \\
\hline & & & & & & & & \\
\hline & & & & & $\left(\right.$ jerib $\left.^{*}\right)$ & $(\%)$ & & \\
\hline \multicolumn{9}{|c|}{ Province: Helmand } \\
\hline NAHR-E SARAJ & 637 & NAHR-E-SARAJ PAYAN & NAHR-E-SARAJ & 100 & 0 & 0 & 0 & 0 \\
\hline NAHR-E SARAJ & 636 & NAQELIN & NAQELIN & 1,400 & 100 & 7 & 14 & 1,350 \\
\hline NAHR-E SARAJ & 639 & NEKZAI & KHUDAI NAZAR & 1,220 & 814 & 67 & 14 & 10,989 \\
\hline NAHR-E SARAJ & 638 & NIKZAY & ABDUL HAMID & 350 & 175 & 50 & 9 & 1,575 \\
\hline NAHR-E SARAJ & 1392 & NOORZO & NOORZO & 90 & 20 & 22 & 14 & 270 \\
\hline NAHR-E SARAJ & 640 & OMAR KHANZI & UMER KHAN ZAY & 250 & 50 & 20 & 9 & 450 \\
\hline NAHR-E SARAJ & 666 & PAIAN KILA & PAIAN KILA & 680 & 250 & 37 & 14 & 3,375 \\
\hline NAHR-E SARAJ & 643 & PASAB 1980 & PASAB 1980 & 1,200 & 50 & 4. & 9 & 450 \\
\hline NAHR-E SARAJ & 644 & PAYA KELA & NASRULLAH & 655 & 300 & 46 & 14 & 4,050 \\
\hline NAHR-E SARAJ & 1402 & PAYAN KALBA & PAYAN KALA & 539 & 269 & 50 & 9 & 2,421 \\
\hline NAHR-E SARAJ & 646 & PAYAN KELA & CHANGEZ-E-BALA & 326 & 50 & 15 & 14 & 675 \\
\hline NAHR-E SARAJ & 641 & POPALZAI & POPALZAI & 2,700 & 50 & 2 & 9 & 450 \\
\hline NAHR-E SARAJ & 626 & QALA GAZ & LAND KAREZ & 120 & 60 & 50 & 14 & 810 \\
\hline NAHR-E SARAJ & 1393 & QAYSARAN MULLAH EZAT & QAYSARA ML.EZAT & 189 & 87. & 46 & 9 & 783 \\
\hline NAHR-E SARAJ & 1403 & SAIDAN DAGIAN & SAYEDIAN DAGIAN & 350 & 200 & 57 & 18 & 3,600 \\
\hline NAHR-E SARAJ & 658 & SAIDANO SHAKH & SAIDANO SHAKH & 1,500 & 30 & 2 & 9 & 270 \\
\hline NAHR-E SARAJ & 653 & SANGCHAL & SANGCHAL & 378 & 70 & 19 & 9 & 630 \\
\hline NAHR-E SARAJ & 1609 & SAR KELA KUNJ & SAYED NAZAR & 1,000 & 400 & 40 & 13 & 5,200 \\
\hline NAHR-E SARAJ & 1415 & SAR KELA SHORKAI & PEER. MOHD & 152 & 30 & 20 & 14 & 405 \\
\hline NAHR-E SARAJ & 1610 & SARKELA & HAJI BAREDAD & 745 & 300 & 40 & 13 & 3,900 \\
\hline NAHR-E SARAJ & 649 & SAYDAN & SAYDAN & 60 & 0 & 0 & 0 & 0 \\
\hline NAHR-E SARAJ & 672 & SAYED TAJDAR WALI & SAYED TAJDAR & 900 & 40 & 4. & 14 & 540 \\
\hline NAHR-E SARAJ & 631 & SAYEDAN-E-MALGEER & SAYEDAN-E-MALGEER & 278 & 92 & 33 & 14 & 1,242 \\
\hline NAHR-E SARAJ & 627 & SEER-E-SORKH & SERI SORKH & 3,288 & 1,500 & 46 & 18 & 27,000 \\
\hline NAHR-E SARAJ & 657 & SHAMAZAI KALAY & SHEMZAI KELY & 320 & 106 & 33 & 9 & 954 \\
\hline NAHR-E SARAJ & 662 & SHORA GAZ & HAJI ZAFARAN & 1,650 & 400 & 24 & 14 & 5,400 \\
\hline NAHR-E SARAJ & 670 & SHORAKAI BARIKZAI & MIAN KELA BARIK & 649 & 324 & 50 & 14 & 4,374 \\
\hline NAHR-E SARAJ & 1412 & SHOURKI BARAKZAI & BARAKZAY & 663 & 331 & 50 & 14 & 4,469 \\
\hline NAHR-E SARAJ & 1414 & SIRKAY SARKELA & SIRKELA SHOUKI & 1,088 & 544 & 50 & 14 & 7,344 \\
\hline
\end{tabular}

* $1 \mathrm{Jerib}=1 / 5 \mathrm{Ha}$

${ }^{*} 2$ Dry Opium $=$ Wet Opium $-30 \%$ moisture 
Opium Production Survey by village in Helmand province, 1995.

\begin{tabular}{|c|c|c|c|c|c|c|c|c|}
\hline \multirow{3}{*}{ District } & \multirow{3}{*}{$\begin{array}{l}\text { Village } \\
\text { Code }\end{array}$} & \multirow{3}{*}{ Area/Main Village } & \multirow{3}{*}{ Village } & \multirow{3}{*}{$\begin{array}{c}\text { Total } \\
\text { Cultivated } \\
\text { land }\end{array}$} & \multirow{2}{*}{\multicolumn{2}{|c|}{$\begin{array}{c}\text { Land Under } \\
\text { Poppy cultivation }\end{array}$}} & \multirow{3}{*}{$\begin{array}{c}\text { Wet }_{\text {Opium }}^{* 2} \\
\text { Yield } \\
(\mathrm{kg} / \mathrm{jr} .)\end{array}$} & \multirow{3}{*}{$\begin{array}{c}\text { Wet Opium } \\
\text { Production } \\
(\mathrm{kg}) \\
\end{array}$} \\
\hline & & & & & & & & \\
\hline & & & & & $\left(\right.$ jerib $\left.^{*}\right)$ & $(\%)$ & & \\
\hline \multicolumn{9}{|c|}{ Province: Helmand } \\
\hline NAHR-E SARAJ & 651 & SOOR SHAKH & SUR SHAKH & 1,650 & 80 & 5 & 9 & 720 \\
\hline NAHR-E SARAJ & 678 & SORANI & SORANI & 696 & 90 & 13 & 9 & 810 \\
\hline NAHR-E SARAJ & 674 & SORKH ABAD KAREZ & SORKH ABADKAREZ & 330 & 60 & 18 & 14 & 810 \\
\hline NAHR-E SARAJ & 585 & TABELA SAFIAN & TABELA SAFIAN & 1,500 & 0 & 0 & 0 & 0 \\
\hline NAHR-E SARAJ & 683 & TAJIKAN\&MOHD ZO KELY & TAJIKAN KELY & 1,200 & 250 & 21 & 14 & 3,375 \\
\hline NAHR-E SARAJ & 676 & TAKHTYAN & TAKHTAIAN & 200 & 0 & 0 & 0 & 0 \\
\hline NAHR-E SARAJ & 1613 & TALE GAWMISHI & MOHD RASOL & 620 & 290 & 47 & 18 & 5,220 \\
\hline NAHR-E SARAJ & 680 & TELE GAWMISHI & HASAN KHAN & 1,000 & 500 & 50 & 18 & 9,000 \\
\hline NAHR-E SARAJ & 675 & TELE ZAHIR & TELE ZAHIR & 600 & 0 & 0 & 0 & 0 \\
\hline NAHR-E SARAJ & 677 & TOGHEE & TOGHEE & 900 & 100 & 11 & 9 & 900 \\
\hline NAHR-E SARAJ & 682 & TORMA & TORMA & 400 & 200 & 50 & 14 & 2,700 \\
\hline NAHR-E SARAJ & 581 & TORMA & CHINA & 200 & 100 & 50 & 14 & 1,350 \\
\hline NAHR-E SARAJ & 1624 & WARDAKAN & WARDAGAN & 250 & 0 & 0 & 0 & 0 \\
\hline NAHR-E SARAJ & 1621 & YAKLENG & YAKLENG & 1,500 & 0 & 0 & 0 & 0 \\
\hline NAHR-E SARAJ & 687 & ZAMBOLE & HAJI G. FAROOQ & 250 & 30 & 12 & 14 & 405 \\
\hline NAHR-E SARAJ & 1611 & ZAMBOLI & KASHMIR & 500 & 75 & 15 & 9 & 675 \\
\hline NAHR-E SARAJ & 686 & ZAMBOLI & HAJI MOHD ALAM & 3,700 & 400 & 11 & 14. & 5,400 \\
\hline NAW ZAD & 6705 & AHANGARAK & AHANGARAK & 80 & 30 & 38 & 10 & 300 \\
\hline NAW ZAD & 230 & AKHTAK & AKHTAK & 80 & 20 & 25 & 10 & 200 \\
\hline NAW ZAD & 1260 & ALI KHAFA & ALI KHAFA & 50 & 40 & 80 & 12. & 480 \\
\hline NAW ZAD & 337 & ALIZAY & ALIZAY & 300 & 200 & 67 & 9 & 1,800 \\
\hline NAW ZAD & 228 & ALTIGAI & ALTIGAI & 200 & 160 & 80 & 12 & 1,920 \\
\hline NAW ZAD & 233 & ANARAK & ANARAK & 220 & 110 & 50 & 11 & 1,210 \\
\hline NAW ZAD & 226 & ANGEEREY & ANGEEREY & 300 & 150 & 50 & 9 & 1,350 \\
\hline NAW ZAD & 232 & ANZIR SHALI & ANZIR SHALI & 2,000 & 136 & 7. & 11 & 1,496 \\
\hline NAW ZAD & 220 & ASPA RASHPA & ASPA RASHPA & 50 & 20 & 40 & 10 & 200 \\
\hline NAW ZAD & 235 & BACHA RABEA & BACHA RABEA & 100 & 80 & 80 & 10 & 800 \\
\hline NAW ZAD & 225 & BAD SHIMA & DARA-E-BRESHPAI & 70 & 20 & 29 & 11 & 220 \\
\hline NAW ZAD & 360 & BADAMAK/CHAHAK & BADAMAK/СНАНАK & 180 & 70 & 39 & 10 & 700 \\
\hline
\end{tabular}

* $1 \mathrm{Jerib}=1 / 5 \mathrm{Ha}$

* 2 Dry Opium $=$ Wet Opium $-30 \%$ moisture
Source: UNDCP Opium Production Survey, 1995 File: HLD3.XLS - 07/08/95 
Opium Production Survey by village in Helmand province, 1995.

\begin{tabular}{|c|c|c|c|c|c|c|c|c|}
\hline \multirow{3}{*}{ District } & \multirow{3}{*}{$\begin{array}{c}\text { Village } \\
\text { Code }\end{array}$} & \multirow{3}{*}{ Area/Main Village } & \multirow{3}{*}{ Village } & \multirow{3}{*}{$\begin{array}{c}\text { Total } \\
\text { Cultivated } \\
\text { land }\end{array}$} & \multirow{2}{*}{\multicolumn{2}{|c|}{$\begin{array}{c}\text { Land Under } \\
\text { Poppy cultivation }\end{array}$}} & \multirow{3}{*}{$\begin{array}{c}\text { Wet Opium *2 } \\
\text { Yield } \\
(\mathrm{kg} / \mathrm{jr} .)\end{array}$} & \multirow{3}{*}{$\begin{array}{c}\text { Wet Opium } \\
\text { Production } \\
(\mathrm{kg})\end{array}$} \\
\hline & & & & & & & & \\
\hline & & & & & $($ jerib *) & $(\%)$ & & \\
\hline \multicolumn{9}{|c|}{ Province: Helmand } \\
\hline NAW ZAD & 244 & BANOSHAK & BANOSHAK & 110 & 70 & 64 & 11 & 770 \\
\hline NAW ZAD & 241 & BAR NAW ZAD ULYA & BAR NAW ZAD ULY & 250 & 170 & 68 & 10 & 1,700 \\
\hline NAW ZAD & 242 & BAR NAWZAD GHARBI & BAR NAWAZGHARBI & 300 & 150 & 50 & 11 & 1,650 \\
\hline NAW ZAD & 240 & BAR NAZAD SOFLA & BAR NAWZAD & 330 & 180 & 55 & 11 & 1,980 \\
\hline NAW ZAD & 237 & BAY SOOFI & BAY SOOFI & 300 & 100 & 33 & 12 & 1,200 \\
\hline NAW ZAD & 239 & BAZE DEH & BAZE DEH & 45 & 20 & 44 & 10 & 200 \\
\hline NAW ZAD & 419 & CHA GALAY & CHA GALAY & 50 & 30 & 60 & 9 & 270 \\
\hline NAW ZAD & 272 & CHANGOLAK & CHANGOLAK & 100 & 80 & 80 & 10 & 800 \\
\hline NAW ZAD & 279 & CHARMANISTAN/CHRBAND & CHARBAND/CHARM. & 150 & 100 & 67 & 9 & 900 \\
\hline NAW ZAD & 277 & CHAWIN & CHAWIN & 127 & 60 & 47 & 10 & 600 \\
\hline NAW ZAD & 289 & DARA & DARA & 100 & 80 & 80 & 9 & 720 \\
\hline NAW ZAD & 338 & DARA MIAN & EIDZY & 600 & 400 & 67 & 12 & 4,800 \\
\hline NAW ZAD & 1275 & DARA MIAN & FEROZI & 600 & 500 & 83 & 13 & 6,500 \\
\hline NAW ZAD & 347 & DARA MIAN & FEROZI & 600 & 500 & 83 & 13 & 6,500 \\
\hline NAW ZAD & 288 & DARA-E- MEYAN & KHANI KHEL & 300 & 200 & 67 & 12 & 2,400 \\
\hline NAW ZAD & 1274 & DARBAND & DARBAND & 200 & 100 & 50 & 10 & 1,000 \\
\hline NAW ZAD & 298 & DARZY & DARZY & 60 & 20 & 33 & 8 & 160 \\
\hline NAW ZAD & 303 & DAUOD ZAI & DAUOD ZAI & 50 & 30 & 60 & 11 & 330 \\
\hline NAW ZAD & 291 & DEH BALOOCH & DEH BALOOCH & 50 & 30 & 60 & 12 & 360 \\
\hline NAW ZAD & 304 & DEH MEYAN & DEH MEYAN & 400 & 300 & 75 & 10 & 3,000 \\
\hline NAW ZAD & 1576 & DEWANAK & DEWANAK & 160 & 90 & 56 & 10 & 900 \\
\hline NAW ZAD & 293 & DO SANG ALI BILAND & DOSANG ALI BELA & 1,000 & 300 & 30 & 10 & 3,000 \\
\hline NAW ZAD & 287 & DOOR BINI & DOOR BINI & 60 & 30 & 50 & 10 & 300 \\
\hline NAW ZAD & 346 & FATEH JANG & FATHE JANG & 150 & 80 & 53 & 10 & 800 \\
\hline NAW ZAD & 381 & GARMA ULYA & GARMA ULYA & 800 & 300 & 38 & 10 & 3,000 \\
\hline NAW ZAD & 283 & GHALAR BINI & GHALAR BINI & 30 & 3 & 10 & 8 & 24 \\
\hline NAW ZAD & 1678 & GHALBAI & GHALBAI & 150 & 50 & 33 & 10 & 500 \\
\hline NAW ZAD & 368 & GINA SUFLA & GINA SUFLA & 200 & 90 & 45 & 10 & 900 \\
\hline NAW ZAD & 296 & GINA ULIA & GINA ULIA & 300 & 120 & 40 & 10 & 1,200 \\
\hline
\end{tabular}

* 1 Jerib $=1 / 5 \mathrm{Ha}$

* ${ }^{2}$ Dry Opium $=$ Wet Opium $-30 \%$ moisture 
Opium Production Survey by village in Helmand province, 1995.

\begin{tabular}{|c|c|c|c|c|c|c|c|c|}
\hline \multirow{3}{*}{ District } & \multirow{3}{*}{$\begin{array}{l}\text { Village } \\
\text { Code }\end{array}$} & \multirow{3}{*}{ Area/Main Village } & \multirow{3}{*}{ Village } & \multirow{3}{*}{$\begin{array}{c}\text { Total } \\
\text { Cultivated } \\
\text { land }\end{array}$} & \multirow{2}{*}{\multicolumn{2}{|c|}{$\begin{array}{c}\text { Land Under } \\
\text { Poppy cultivation }\end{array}$}} & \multirow{3}{*}{$\begin{array}{c}\text { Wet Opium }{ }^{* 2} \\
\text { Yield } \\
(\mathrm{kg} / \mathrm{jr} .) \\
\end{array}$} & \multirow{3}{*}{$\begin{array}{c}\text { Wet Opium } \\
\text { Production } \\
(\mathrm{kg})\end{array}$} \\
\hline & & & & & & & & \\
\hline & & & & & $\left(\right.$ jerib $\left.^{*}\right)$ & $(\%)$ & & \\
\hline \multicolumn{9}{|c|}{ Province: Helmand } \\
\hline NAW ZAD & 344 & GOURDA & GOURDA & 150 & 70 & 47 & 11 & 770 \\
\hline NAW ZAD & 342 & GULBINA & GULBINA & 100 & 30 & 30 & 11 & 330 \\
\hline NAW ZAD & 1259 & GULDAN & GULDAN & 100 & 80 & 80 & 12 & 960 \\
\hline NAW ZAD & 1677 & HATA KO & HATA KO & 150 & 50 & 33 & 10 & 500 \\
\hline NAW ZAD & 229 & IMAM RUBATO & IMAM RUBATO & 200 & 100 & 50 & 10 & 1,000 \\
\hline NAW ZAD & 269 & JAFAR ABAD & JAFAR ABAD & 200 & 100 & 50 & 10 & 1,000 \\
\hline NAW ZAD & 281 & JAK AAB & JAK AAB & 100 & 60 & 60 & 10 & 600 \\
\hline NAW ZAD & 275 & JAZE & JAZE & 300 & 100 & 33 & 10 & 1,000 \\
\hline NAW ZAD & 375 & KANYAN SAYDAN & KANYAN SAYDAN & 95 & 30 & 32 & 11 & 330 \\
\hline NAW ZAD & 1262 & KAREZ AFGHAN & KAREZ AFGHAN & 300 & 200 & 67 & 12 & 2,400 \\
\hline NAW ZAD & 336 & KAREZ ALI ABAD & KAREZ ALI ABAD & 400 & 300 & 75 & 10 & 3,000 \\
\hline NAW ZAD & 236 & KAREZ BAGHAK & KAREZ BAGHAK & 300 & 200 & 67 & 10 & 2,000 \\
\hline NAW ZAD & 245 & KAREZ BARANG & KAREZ BARANG & 300 & 100 & 33 & 11 & 1,100 \\
\hline NAW ZAD & 270 & KAREZ JALAUDEN & KAREZ JALAUDEN & 300 & 100 & 33 & 10 & 1,000 \\
\hline NAW ZAD & 274 & KAREZ JAMSHED & KAREZ JAMSHED & 150 & 80 & 53 & 11 & 880 \\
\hline NAW ZAD & 1681 & KAREZ LANGAR & KAREZ LANGAR & 100 & 60 & 60 & 10 & 600 \\
\hline NAW ZAD & 353 & KAREZ MOHAMMAD SHAH & KAREZ MOHAMMD SHAH & 250 & 100 & 40 & 10 & 1,000 \\
\hline NAW ZAD & 354 & KAREZ MOOSA & KAREZ MOOSA & 200 & 90 & 45 & 10 & 900 \\
\hline NAW ZAD & 401 & KAREZ NAL & KAREZ NAL & 150 & 60 & 40 & 10 & 600 \\
\hline NAW ZAD & 357 & KAREZ SAFED & KAREZ SAFED & 450 & 250 & 56 & 12 & 3,000 \\
\hline NAW ZAD & 331 & KAREZ SHAH MAHMOOD & KAREZ SH.MAHMOD & 100 & 40 & 40 & 11 & 440 \\
\hline NAW ZAD & 314 & KAREZ ZIRAK & KAREZ ZIRAK & 180 & 50 & 28 & 10 & 500 \\
\hline NAW ZAD & 273 & KAREZ-E-NAW & KAREZ-E-NAW & 200 & 100 & 50 & 11 & 1,100 \\
\hline NAW ZAD & 369 & KAREZGAI & KAREZGAI & 50 & 35 & 70 & 10 & 350 \\
\hline NAW ZAD & 370 & KELE SEIA & KELE SEIA & 300 & 100 & 33 & 9 & 900 \\
\hline NAW ZAD & 343 & KHAWAY & KHAWAY & 600 & 100 & 17 & 9 & 900 \\
\hline NAW ZAD & 1264 & KHWAJA JAMAL & KHWAJA JAMAL & 400 & 150 & 38 & 12 & 1,800 \\
\hline NAW ZAD & 367 & KINA ULIA \SUFLA & KINA ULIAISUFLA & 350 & 200 & 57 & 11 & 2,200 \\
\hline NAW ZAD & 356 & KISHK & KISHK & 200 & 90 & 45 & 11 & 990 \\
\hline
\end{tabular}

* 1 Jerib $=1 / 5 \mathrm{Ha}$

* 2 Dry Opium $=$ Wet Opium - $30 \%$ moisture
Page 3873
Source: UNDCP Opium Production Survey, 1995 File: HLD3.XLS - 07/08/95 
Opium Production Survey by village in Helmand province, 1995.

\begin{tabular}{|c|c|c|c|c|c|c|c|c|}
\hline \multirow{3}{*}{ District } & \multirow{3}{*}{$\begin{array}{l}\text { Village } \\
\text { Code }\end{array}$} & \multirow{3}{*}{ Area/Main Village } & \multirow{3}{*}{ Village } & \multirow{3}{*}{$\begin{array}{c}\text { Total } \\
\text { Cultivated } \\
\text { land } \\
\end{array}$} & \multirow{2}{*}{\multicolumn{2}{|c|}{$\begin{array}{c}\text { Land Under } \\
\text { Poppy cultivation }\end{array}$}} & \multirow{3}{*}{$\begin{array}{c}\text { Wet Opium }{ }^{* 2} \\
\text { Yield } \\
(\mathrm{kg} / \mathrm{jr} .) \\
\end{array}$} & \multirow{3}{*}{$\begin{array}{c}\text { Wet Opium } \\
\text { Production } \\
(\mathrm{kg}) \\
\end{array}$} \\
\hline & & & & & & & & \\
\hline & & & & & $\left(\right.$ jerib $\left.^{*}\right)$ & $(\%)$ & & \\
\hline \multicolumn{9}{|c|}{ Province: Helmand } \\
\hline NAW ZAD & 372 & KOJDE KHASHROD & KOJDE KHASHROD & 100 & 20 & 20 & 9 & 180 \\
\hline NAW ZAD & 358 & KORGHAI & KORGHAI & 200 & 90 & 45 & 10 & 900 \\
\hline NAW ZAD & 363 & KUNJAK & KUNJAK & 320 & 220 & 69 & 9 & 1,980 \\
\hline NAW ZAD & 1576 & LAR ULYA & LAR ULYA & 100 & 30 & 30 & 11 & 330 \\
\hline NAW ZAD & 322 & LARI & LARI/SAR LARI & 150 & 80 & 53 & 9 & 720 \\
\hline NAW ZAD & 385 & LAY KHENJAK & LAY KHENJAK & 100 & 80 & 80 & 10 & 800 \\
\hline NAW ZAD & 247 & LOGHAR & LAR GHAR & 80 & 70 & 88 & 11 & 770 \\
\hline NAW ZAD & 384 & LORA MER & LORA MER & 100 & 50 & 50 & 10 & 500 \\
\hline NAW ZAD & 386 & LOWARA ZANGI & LOWARA ZANGI & 200 & 100 & 50 & 10 & 1,000 \\
\hline NAW ZAD & 387 & LOY KHOD & LOY KHOD & 50 & 20 & 40 & 10 & 200 \\
\hline NAW ZAD & 396 & MAMA KAREZ & MAMA KAREZ & 300 & 120 & 40 & 10 & 1,200 \\
\hline NAW ZAD & 393 & MASHIN ZAI & MASHIN ZAI & 300 & 200 & 67 & 10 & 2,000 \\
\hline NAW ZAD & 391 & MASLAHAT & MASLAHAT & 200 & 80 & 40 & 10 & 800 \\
\hline NAW ZAD & 394 & MIAN JOY & MIAN JOY & 600 & 200 & 33 & 9 & 1,800 \\
\hline NAW ZAD & 400 & MISH MAST & MISH MAST & 300 & 100 & 33 & 10 & 1,000 \\
\hline NAW ZAD & 388 & MOHAMMAD ABAD & MOHAMMAD ABAD & 100 & 80 & 80 & 10 & 800 \\
\hline NAW ZAD & 221 & OWR MOWZ & OWR MWOZ & 300 & 100 & 33 & 12 & 1,200 \\
\hline NAW ZAD & 248 & PANDO & PANDO & 150 & 100 & 67 & 10 & 1,000 \\
\hline NAW ZAD & 252 & PATAN & HAJI AZAM KHAN & 400 & 150 & 38 & 11 & 1,650 \\
\hline NAW ZAD & 250 & PATAN & PATAN & 600 & 300 & 50 & 12 & 3,600 \\
\hline NAW ZAD & 362 & QALA BADAM & QALA BADAM & 150 & 100 & 67 & 11 & 1,100 \\
\hline NAW ZAD & 351 & QALA-E ZIR & QALA-E ZIR & 100 & 40 & 40 & 11 & 440 \\
\hline NAW ZAD & 348 & QASEM ABAD & QASEM ABAD & 300 & 180 & 60 & 10 & 1,800 \\
\hline NAW ZAD & 313 & RABAT QAZI & RABAT QAZI & 240 & 100 & 42 & 10 & 1,000 \\
\hline NAW ZAD & 308 & RAZ DAN SUFLA & RAZ DAN SUFLA & 80 & 40 & 50 & 9 & 360 \\
\hline NAW ZAD & 301 & RAZ DAN ULIA & RAZ DAN ULIA & 100 & 50 & 50 & 11 & 550 \\
\hline NAW ZAD & 292 & ROUSHAN ABAD & SOOR KANE & 60 & 40 & 67 & 12 & 480 \\
\hline NAW ZAD & 312 & RUBAT & RUBAT & 350 & 200 & 57 & 12 & 2,400 \\
\hline NAW ZAD & 321 & SADAT KAREZ & SADAT KAREZ & 200 & 120 & 60 & 10 & 1,200 \\
\hline
\end{tabular}

* $1 \mathrm{Jerib}=1 / 5 \mathrm{Ha}$

* 2 Dry Opium $=$ Wet Opium $-30 \%$ moisture
Page 3974
Source: UNDCP Opium Production Survey, 1995 File: HLD3.XLS - 07/08/95 
Opium Production Survey by village in Helmand province, 1995.

\begin{tabular}{|c|c|c|c|c|c|c|c|c|}
\hline \multirow{3}{*}{ District } & \multirow{3}{*}{$\begin{array}{l}\text { Village } \\
\text { Code }\end{array}$} & \multirow{3}{*}{ Area/Main Village } & \multirow{3}{*}{ Village } & \multirow{3}{*}{$\begin{array}{c}\text { Total } \\
\text { Cultivated } \\
\text { land } \\
\end{array}$} & \multirow{2}{*}{\multicolumn{2}{|c|}{$\begin{array}{c}\text { Land Under } \\
\text { Poppy cultivation }\end{array}$}} & \multirow{3}{*}{$\begin{array}{c}\text { Wet Opium }^{* 2} \\
\text { Yield } \\
(\mathrm{kg} / \mathrm{jr} .) \\
\end{array}$} & \multirow{3}{*}{$\begin{array}{l}\text { Wet Opium } \\
\text { Production } \\
(\mathrm{kg}) \\
\end{array}$} \\
\hline & & & & & & & & \\
\hline & & & & & $($ jerib *) & $(\%)$ & & \\
\hline \multicolumn{9}{|l|}{ Province: Helmand } \\
\hline NAW ZAD & 315 & SANGAK & SANGAK & 600 & 200 & 33 & 12. & 2,400 \\
\hline NAW ZAD & 318 & SAR GHAL & SAR GHAL & 52 & 10 & 19 & 9 & 90 \\
\hline NAW ZAD & 317 & SARANG & SARANG & 100 & 50 & 50 & 10 & 500 \\
\hline NAW ZAD & 326 & SHAMSABAD/GHORIAN & SHAMSABAD/GHORI & 300 & 200 & 67 & 10 & 2,000 \\
\hline NAW ZAD & 332 & SHEKH ZAI & SHEKH ZAI & 310 & 250 & 81 & 12 & 3,000 \\
\hline NAW ZAD & 328 & SHEREEN BAZ & SHEREEN BAZ & 150 & 100 & 67. & 12 & 1,200 \\
\hline NAW ZAD & 330 & SHERIN BAZ & ZABET WAZIR & 150 & 100 & 67 & 12 & 1,200 \\
\hline NAW ZAD & 325 & SHOR AAB & SHOR ABB & 180 & 30 & 17 & 10 & 300 \\
\hline NAW ZAD & 266 & SIA KHOLA & SIA KHOLA & 400 & 200 & 50 & 11 & 2,200 \\
\hline NAW ZAD & 414 & SIAPUSHTA & BA NAMAKE & 70 & 30 & 43 & 9 & 270 \\
\hline NAW ZAD & 294 & SOOR KANI & HAJI PACHA & 60 & 50 & 83 & 11 & 550 \\
\hline NAW ZAD & 323 & SPI GHAL & PSI GHAL & 250 & 100 & 40 & 10 & 1,000 \\
\hline NAW ZAD & 267 & TABOT & TABOT & 150 & 70 & 47 & 10 & 700 \\
\hline NAW ZAD & 265 & TANGE ULIA & TANGE ULIA & 120 & 80 & 67 & 11 & 880 \\
\hline NAW ZAD & 264 & TANGI SUFLA & TANGE SUFLA & 600 & 200 & 33 & 10 & 2,000 \\
\hline NAW ZAD & 268 & TARODI DAHANA & TARODI DAHANA & 230 & 80 & 35 & 10 & 800 \\
\hline NAW ZAD & 1268 & TEZMANI & GAMIRZAD & 230 & 100 & 43 & 11 & 1,100 \\
\hline NAW ZAD & 1269 & TEZMANI & GHALBA KALAN & 170 & 100 & 59 & 11 & 1,100 \\
\hline NAW ZAD & 1270 & TEZMANI & BOZURGI WALI & 100 & 50 & 50 & 10 & 500 \\
\hline NAW ZAD & 379 & TEZMANI & GADAZI & 275 & 100 & 36 & 11 & 1,100 \\
\hline NAW ZAD & 1272 & TEZMANI & ALI ZAI & 250 & 180 & 72 & 12 & 2,160 \\
\hline NAW ZAD & 365 & WALI MOHD.KHAN & WALI MOHD.KHAN & 300 & 200 & 67 & 11 & 2,200 \\
\hline NAW ZAD & 404 & WOCHA GHALABA & WOCHA GHALABA & 140 & 100 & 71 & 9 & 900 \\
\hline NAW ZAD & 311 & ZARWIJI & ZARWIJI & 200 & 110 & 55 & 11 & 1,210 \\
\hline NAWA BARAKZAI & 473 & ABDUL ALI & ABDUL ALI & 600 & 130 & 22 & 18 & 2,340 \\
\hline NAWA BARAKZAI & 1560 & ABDUL ALI & ABDUL ALI & 500 & 53. & 11 & 22 & 1,166 \\
\hline NAWA BARAKZAI & 479 & ABDUL AZIZ & ABDUL AZIZ & 500 & 80 & 16 . & 18 & 1,440 \\
\hline NAWA BARAKZAI & 1554 & ABDUL AZIZ & ABDUL AZIZ & 115 & 22 & 19 & 16 & 352 \\
\hline NAWA BARAKZAI & 545 & ABDUL BAQI KHAN KELY & ABDUL BAQI KHAN KELY & 700 & 50 & 7 & 18 & 900 \\
\hline
\end{tabular}

* I Jerib $=1 / 5 \mathrm{Ha}$

* 2 Dry Opium = Wet Opium - $30 \%$ moisture
Page 4075
Source: UNDCP Opium Production Survey, 1995 File: HLD3.XLS - 07/08/95 
Opium Production Survey by village in Helmand province, 1995.

\begin{tabular}{|c|c|c|c|c|c|c|c|c|}
\hline & Village & & & Total & Land & & Wet Opium * 2 & Wet Opium \\
\hline \multirow[t]{2}{*}{ District } & Code & Area/Main Village & Village & Cultivated & \multicolumn{2}{|c|}{ Poppy cultivation } & Yield & Production \\
\hline & & & & land & $\left(\right.$ jerib $\left.^{*}\right)$ & $(\%)$ & $(\mathrm{kg} / \mathrm{jr})$. & $(\mathrm{kg})$ \\
\hline \multicolumn{9}{|l|}{ Province: Helmand } \\
\hline NAWA BARAKZAI & 1293 & ABDUL MAJID KHAN & ABDUL MAJID & 400 & 30 & 8 & 18 & 540 \\
\hline NAWA BARAKZAI & 1305 & ABDUL RAWOF KHAN & ABDUL RAWOF KHAN & 600 & 46 & 8 & 22 & 1,012 \\
\hline NAWA BARAKZAI & 1301 & AJTASI & MEHDIJO & 244 & 25 & 10 & 18 & 450 \\
\hline NAWA BARAKZAI & 512 & AKHOND KHAIL & AKHOND KHAIL & 450 & 100 & 22 & 18 & 1,800 \\
\hline NAWA BARAKZAI & 539 & AKHTAR M. KALAY & & 400 & 30 & 8 & 16 & 480 \\
\hline NAWA BARAKZAI & 1287 & AKHUND KHAIL & ABDUL RASHID & 800 & 150 & 19 & 18 & 2,700 \\
\hline NAWA BARAKZAI & 1309 & ALAH NOOR KHAN & ALAH NOOR KHAN & 200 & 39 & 20 & 18 & 702 \\
\hline NAWA BARAKZAI & 1308 & ALAM GUL KHAN & ALAM GUL KHAN & 290 & 30 & 10 & 18 & 540 \\
\hline NAWA BARAKZAI & 488 & AMINULLAH KHAN & AMINULLAH KHAN & 600 & 35 & 6 & 18 & 630 \\
\hline NAWA BARAKZAI & 492 & ANAR BAGH & ANAR BAGH & 200 & 35 & 18 & 18 & 630 \\
\hline NAWA BARAKZAI & 1563 & AQA GUL & AQA GUL & 300 & 25 & 8 & 22 & 550 \\
\hline NAWA BARAKZAI & 520 & AZAD SHAH KHAN & AZAD SHAH KHAN & 500 & 30 & 6 & 18 & 540 \\
\hline NAWA BARAKZAI & 532 & BADUR KHAN & BADUR KHAN & 1,000 & 40 & 4 & 10 & 400 \\
\hline NAWA BARAKZAI & 486 & BADUR KHAN & BADUR KHAN & 55 & 6 & 11 & 16 & 96 \\
\hline NAWA BARAKZAI & 552 & BARAT KHAN & BARAT KHAN & 500 & 20 & 4 & 18 & 360 \\
\hline NAWA BARAKZAI & 530 & BISMILLAH KHAN & BISMILLAH KHAN & 1,220 & 62 & 5 & 10 & 620 \\
\hline NAWA BARAKZAI & 476 & BLOCK 3 NAQELIN & BLOCK 3 NAQELIN & 300 & 19 & 6 & 18 & 342 \\
\hline NAWA BARAKZAI & 515 & CHANGIZI & CHANGIZI & 400 & 44 & 11 & 18 & 792 \\
\hline NAWA BARAKZAI & 716 & DABAK & DABAK & 120 & 80 & 67 & 19 & 1,480 \\
\hline NAWA BARAKZAI & 503 & DASTAGIR KHAN & DASTAGIR KHAN & 450 & 50 & 11 & 18 & 900 \\
\hline NAWA BARAKZAI & 522 & DR. NAQIBULLAH & DR. NAQIBULLAH & 990 & 70 & 7 & 18 & 1,260 \\
\hline NAWA BARAKZAI & 500 & ESMATULLAH & ESMATULLAH & 500 & 125 & 25 & 18 & 2,250 \\
\hline NAWA BARAKZAI & 484 & ESMATULLAH KHAN & ESMATULLAH KHAN & 200 & 7 & 4 & 16 & 112 \\
\hline NAWA BARAKZAI & 521 & FAIZ MOHD & FAIZ MOHD & 300 & 30 & 10 & 16 & 480 \\
\hline NAWA BARAKZAI & 553 & FAIZO JAN & FAIZO JAN & 90 & 15 & 17 & 22 & 330 \\
\hline NAWA BARAKZAI & 523 & FAQIRAN & FAQIR MAHMOOD & 780 & 155 & 20 & 18 & 2,790 \\
\hline NAWA BARAKZAI & 510 & GHAIBIZI & GHAIBIZI & 700 & 250 & 36 & 18 & 4,500 \\
\hline NAWA BARAKZAI & 504 & GHULAM NABI & GHULAM NABI & 500 & 100 & 20 & 16 & 1,600 \\
\hline NAWA BARAKZAI & 542 & GHULAM NABI & GHULAM NABI & 100 & 5 & 5 & 16 & 80 \\
\hline
\end{tabular}

* 1 Jerib $=1 / 5 \mathrm{Ha}$

* 2 Dry Opium $=$ Wet Opium $-30 \%$ moisture
Page 4176
Source: UNDCP Opium Production Survey, 1995 File: HLD3.XLS - 07/08/95 
Opium Production Survey by village in Helmand province, 1995.

\begin{tabular}{|c|c|c|c|c|c|c|c|c|}
\hline \multirow{3}{*}{ District } & \multirow{3}{*}{$\begin{array}{l}\text { Village } \\
\text { Code }\end{array}$} & \multirow{3}{*}{ Area/Main Village } & \multirow{3}{*}{ Village } & \multirow{3}{*}{$\begin{array}{c}\text { Total } \\
\text { Cultivated } \\
\text { land } \\
\end{array}$} & \multirow{2}{*}{\multicolumn{2}{|c|}{$\begin{array}{c}\text { Land Under } \\
\text { Poppy cultivation }\end{array}$}} & \multirow{3}{*}{$\begin{array}{c}\text { Wet }^{\text {Opium }}{ }^{* 2} \\
\text { Yield } \\
(\mathrm{kg} / \mathrm{jr} .) \\
\end{array}$} & \multirow{3}{*}{$\begin{array}{c}\text { Wet Opium } \\
\text { Production } \\
\text { (kg) }\end{array}$} \\
\hline & & & & & & & & \\
\hline & & & & & $\left(\right.$ jerib $\left.^{*}\right)$ & $(\%)$ & & \\
\hline \multicolumn{9}{|l|}{ Province: Helmand } \\
\hline NAWA BARAKZAI & 493 & GUL AGHA KHAN & GUL AGHA KHAN & 200 & 3 & 2 & 16 & 48 \\
\hline NAWA BARAKZAI & 499 & H. M. WAKIL & H. M. WAKIL & 800 & 200 & 25 & 20 & 4,000 \\
\hline NAWA BARAKZAI & 1303 & HAJI ABDUL ALI KHAN & HAJI ABDUL ALI . & 1,000 & 150 & 15 & 18 & 2,700 \\
\hline NAWA BARAKZAI & 497 & HAJI AKRAM & HAJI AKRAM & 150 & 40 & 27 & 18 & 720 \\
\hline NAWA BARAKZAI & 1277 & HAJI G.ALI KHAN & HAJI G.ALI KHAN & 110 & 25 & 23 & 15 & 375 \\
\hline NAWA BARAKZAI & 1282 & HAJI GHEYAS SAHIB & HAJI GHEYAS SAHIB & 200 & 50 & 25 & 18 & 900 \\
\hline NAWA BARAKZAI & 1299 & HAJI GHULAM BUZ & HAJI ABDULLAH AKA & 600 & 80 & 13 & 18 & 1,440 \\
\hline NAWA BARAKZAI & 1300 & HAJI GHULAM BUZ KHAN & HAJI AHMAD KHAN & 620 & 120 & 19 & 18 & 2,160 \\
\hline NAWA BARAKZAI & 1287 & HAJI GUL MOHD KHAN & HAJI GUL MOHD KHAN & 100 & 25 & 25 & 18 & 450 \\
\hline NAWA BARAKZAI & 525 & HAJI JANGUL & HAJI JANGUL & 1,000 & 200 & 20 & 22 & 4,400 \\
\hline NAWA BARAKZAI & 1279 & HAJI JUMA KHAN & HAJI JUMA KHAN & 150 & 20 & 13 & 19 & 380 \\
\hline NAWA BARAKZAI & 480 & HAJI MIR ALAM & HAJI MIR ALAM & 200 & 45 & 23 & 18 & 810 \\
\hline NAWA BARAKZAI & 519 & HAJI MOHD AMIN & HAJI MOHD AMIN & 1,100 & 20 & 2 & 16 & 320 \\
\hline NAWA BARAKZAI & 1565 & HAJI NAZER M. MALANG & HAJI NAZER M. MALANG & 72 & 10 & 14 & 16 & 160 \\
\hline NAWA BARAKZAI & 1302 & HAJI RASHID KHAN & HAJI RASHID KHAN & 200 & 50 & 25 & 18 & 900 \\
\hline NAWA BARAKZAI & 1306 & HAJI RASHID KHAN & HAJI RASHID KHAN & 250 & 25 & 10 & 18 & 450 \\
\hline NAWA BARAKZAI & 1281 & HAJI SHISTA KHAN & HAJI SHISTA KHAN & 250 & 10 & 4 & 10 & 100 \\
\hline NAWA BARAKZAI & 518 & HAJI TOOR AKA & HAJI TOOR AKA & 1,200 & 180 & 15 & 16 & 2,880 \\
\hline NAWA BARAKZAI & 528 & HJ.JAMAL KHAN & HJ.JAMAL KHAN & 465 & 30 & 6 & 18 & 540 \\
\hline NAWA BARAKZAI & 534 & HJ.MOHAMMAD OMER & HJ.MOHAMMAD OMR & 430 & 38 & 9 & 18 & 684 \\
\hline NAWA BARAKZAI & 527 & HJ.ZARIF SHAH & HJ.ZARIF SHAH & 900 & 50 & 6 & 16 & 800 \\
\hline NAWA BARAKZAI & 481 & JALAT KHAN & JALAT KHAN & 300 & 20 & 7 & 18 & 360 \\
\hline NAWA BARAKZAI & 1557 & JAN MOHD & JAN MOHD & 200 & 20 & 10 & 18 & 360 \\
\hline NAWA BARAKZAI & 1294 & JANGZAI & MATA KHAIL & 100 & 3 & 3 & 18 & 54 \\
\hline NAWA BARAKZAI & 1552 & KAKARAN & KAKARAN & 250 & 50 & 20 & 22 & 1,100 \\
\hline NAWA BARAKZAI & 413 & KATAKYAN & KATAKYAN & 700 & 100 & 14 & 18 & 1,800 \\
\hline NAWA BARAKZAI & 524 & KHAN ALMAS & KHAN ALMAS & 1,287 & 72 & 6 & 16 & 1,152 \\
\hline NAWA BARAKZAI & 1298 & KHAN MOHD KHAN & KHAN MOHD KHAN & 200 & 20 & 10 & 18 & 360 \\
\hline NAWA BARAKZAI & 505 & KHODAI NAZAR & KHODAI NAZAR & 302 & 12 & 4 & 18 & 216 \\
\hline
\end{tabular}

* $1 \mathrm{Jerib}=1 / 5 \mathrm{Ha}$

*2 Dry Opium $=$ Wet Opium - $30 \%$ moisture
Page 4277
Source: UNDCP Opium Production Survey, 1995 File: HLD3.XLS - 07/08/95 
Opium Production Survey by village in Helmand province, 1995.

\begin{tabular}{|c|c|c|c|c|c|c|c|c|}
\hline & \multirow{3}{*}{$\begin{array}{l}\text { Village } \\
\text { Code }\end{array}$} & \multirow{3}{*}{ Area/Main Village } & \multirow{3}{*}{ Village } & \multirow{3}{*}{$\begin{array}{c}\text { Total } \\
\text { Cultivated } \\
\text { land }\end{array}$} & \multirow{2}{*}{\multicolumn{2}{|c|}{$\begin{array}{c}\text { Land Under } \\
\text { Poppy cultivation }\end{array}$}} & \multirow{3}{*}{$\begin{array}{c}{\text { Wet } \text { Opium }^{* 2}}^{2} \\
\text { Yield } \\
(\mathrm{kg} / \mathrm{jr} .)\end{array}$} & \multirow{3}{*}{$\begin{array}{c}\text { Wet Opium } \\
\text { Production } \\
(\mathrm{kg}) \\
\end{array}$} \\
\hline \multirow[t]{2}{*}{ District } & & & & & & & & \\
\hline & & & & & $\left(\right.$ jerib $\left.^{*}\right)$ & $(\%)$ & & \\
\hline \multicolumn{9}{|l|}{ Province: Helmand } \\
\hline NAWA BARAKZAI & 508 & KHOONTAGAY & KHOONTAGAY & 300 & 7 & 2 & 18 & 126 \\
\hline NAWA BARAKZAI & 541 & KULAWAL & KULAWAL & 270 & 30 & 11 & 14 & 420 \\
\hline NAWA BARAKZAI & 1297 & LAR KALA & SHAH WALI KHAN & 400 & 50 & 13 & 14 & 700 \\
\hline NAWA BARAKZAI & 1561 & LOY KALAY & & 800 & 50 & 6 & 18 & 900 \\
\hline NAWA BARAKZAI & 1284 & MAJERYANO & MAJERYANO & 100 & 21 & 21 & 20 & 420 \\
\hline NAWA BARAKZAI & 1310 & MALEK HASHEM KHAN & MALEK HASHEM KHAN & 350 & 82 & 23 & 18 & 1,476 \\
\hline NAWA BARAKZAI & 494 & MALEK KHAN & MALEK KHAN & 500 & 8 & 2 & 18 & 144 \\
\hline NAWA BARAKZAI & 1558 & MALIK RAZ MOHAMMAD & MALIK RAZ MOHAMMAD & 392 & 28 & 7 & 22 & 616 \\
\hline NAWA BARAKZAI & 535 & MALIK ZAHIR & 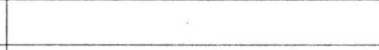 & 290 & 14 & 5 & 18 & 252 \\
\hline NAWA BARAKZAI & 531 & MALKANZAI & & 3,000 & 150 & 5 & 18 & 2,700 \\
\hline NAWA BARAKZAI & 543 & MALZAI & & 200 & 55 & 28 & 18 & 990 \\
\hline NAWA BARAKZAI & 1555 & MAMOOR KATA & MAMOOR KATA & 330 & 12 & 4 & 18 & 216 \\
\hline NAWA BARAKZAI & 509 & MASHOZO & MASHOZO & 1,500 & 300 & 20 & 18 & 5,400 \\
\hline NAWA BARAKZAI & 474 & MASOOM KHAN & & 140 & 7 & 5 & 18 & 126 \\
\hline NAWA BARAKZAI & 537 & MEMBASHAN & MEMBASHAN & 220 & 9 & 4 & 15 & 135 \\
\hline NAWA BARAKZAI & 554 & MERO DO & 42 & 390 & 25 & 6 & 18 & 450 \\
\hline NAWA BARAKZAI & 1556 & MIRAB M. GUL & MIRAB M. GUL & 300 & 10 & 3 & 18 & 180 \\
\hline NAWA BARAKZAI & 485 & MOHAMMAD ALAM KHAN & MOHAMMAD ALAM KHAN & 100 & 26 & 26 & 18 & 468 \\
\hline NAWA BARAKZAI & 477 & MOHAMMAD KARIM & MOHAMMAD KARIM & 550 & 15 & 3 & 18 & 270 \\
\hline NAWA BARAKZAI & 489 & MOHAMMAD RAHIM & MOHAMMAD RAHIM & 800 & 100 & 13 & 16 & 1,600 \\
\hline NAWA BARAKZAI & 1307 & MOHD BAYAN & MOHD BAYAN & 200 & 14 & 7 & 18 & 252 \\
\hline NAWA BARAKZAI & 1290 & MOHD KARIM KHAN & MIR HASHEM & 1,300 & 220 & 17 & 18 & 3,960 \\
\hline NAWA BARAKZAI & 1291 & MOHD KARIM KHAN & MOHD JUMA KHAN & 200 & 15 & 8 & 18 & 270 \\
\hline NAWA BARAKZAI & 529 & MULA SULTAN & MULA SULTAN & 300 & 35 & 12 & 16 & 560 \\
\hline NAWA BARAKZAI & 551 & NAGARE & NAGARE & 450 & 50 & 11 & 22 & 1,100 \\
\hline NAWA BARAKZAI & 549 & NAGARE & NAGARE & 120 & 13 & 11 & 18 & 234 \\
\hline NAWA BARAKZAI & 478 & NAIM KHAN & NAIM KHAN & 70 & 0 & 0 & 0 & 0 \\
\hline NAWA BARAKZAI & 498 & NAQILIN & NAQILIN & 640 & 0 & 0 & 0 & 0 \\
\hline NAWA BARAKZAI & 1564 & NAWZAD KALAY & NAWZAD KALAY & 100 & 0 & 0 & 0 & 0 \\
\hline
\end{tabular}

* $1 \mathrm{Jerib}=1 / 5 \mathrm{Ha}$

* 2 Dry Opium $=$ Wet Opium - $30 \%$ moisture 
Opium Production Survey by village in Helmand province, 1995.

\begin{tabular}{|c|c|c|c|c|c|c|c|c|}
\hline \multirow{3}{*}{ District } & \multirow{3}{*}{$\begin{array}{c}\text { Village } \\
\text { Code } \\
\end{array}$} & \multirow{3}{*}{ Area/Main Village } & \multirow{3}{*}{ Village } & \multirow{3}{*}{$\begin{array}{c}\text { Total } \\
\text { Cultivated } \\
\text { land }\end{array}$} & \multirow{2}{*}{\multicolumn{2}{|c|}{$\begin{array}{c}\text { Land Under } \\
\text { Poppy cultivation }\end{array}$}} & \multirow{3}{*}{$\begin{array}{c}\text { Wet Opium *2 } \\
\text { Yield } \\
(\mathrm{kg} / \mathrm{jr} .)\end{array}$} & \multirow{3}{*}{$\begin{array}{l}\text { Wet Opium } \\
\text { Production } \\
(\mathrm{kg})\end{array}$} \\
\hline & & & & & & & & \\
\hline & & & & & $\left(\right.$ jerib $\left.^{*}\right)$ & $(\%)$ & & \\
\hline \multicolumn{9}{|l|}{ Province: Helmand } \\
\hline NAWA BARAKZAI & 1553 & NET RAQID & NET RAQID & 300 & 12 & 4 & 16 & 192 \\
\hline NAWA BARAKZAI & 490 & NIK MOHD & NIK MOHD & 300 & 21 & 7 & 16 & 336 \\
\hline NAWA BARAKZAI & 1276 & PAIN KALA . & NADER KHAN & 150 & 0 & 0 & 0 & 0 \\
\hline NAWA BARAKZAI & 1295 & PAYEEN KALA & PASTO KHAN & 500 & 10 & 2 & 16 & 160 \\
\hline NAWA BARAKZAI & 1296 & PAYEEN KALA & HAJI WALI MOHD KHAN & 600 & 30 & 5 & 16 & 480 \\
\hline NAWA BARAKZAI & 538 & POPALZAI & POPALZAI & 208 & 27 & 13 & 18 & 486 \\
\hline NAWA BARAKZAI & 1289 & QAZI KHAIL & QAZI KHAIL & 130 & 16 & 12 & 18 & 288 \\
\hline NAWA BARAKZAI & 483 & SADAT & SADAT & 100 & 10 & 10 & 18 & 180 \\
\hline NAWA BARAKZAI & 501 & SADULLAH KHAN & SADULLAH KHAN & 150 & 13 & 9 & 16 & 208 \\
\hline NAWA BARAKZAI & 506 & SAEYD AMAN AGHA & SAEYD AMAN AGHA & 209 & 26 & 12 & 22 & 572 \\
\hline NAWA BARAKZAI & 547 & SAHIB KHAN & SAHIB KHAN & 350 & 60 & 17 & 18 & 1,080 \\
\hline NAWA BARAKZAI & 536 & SAHIB KHAN & SAHIB KHAN & 300 & 12 & 4 & 18 & 207 \\
\hline NAWA BARAKZAI & 540 & SAIDAN & SAIDAN & 100 & 17 & 17 & 18 & 306 \\
\hline NAWA BARAKZAI & 502 & SARWAR KHAN & SARWAR KHAN & 400 & 70 & 18 & 20 & 1,400 \\
\hline NAWA BARAKZAI & 1550 & SAYED ABDUL AHMAD & SAYED ABDUL AHMAD & 72 & 6 & 8 & 18 & 108 \\
\hline NAWA BARAKZAI & 1283 & SAYED MIR AMZA & SAYED MIR AMZA & 80 & 16 & 20 & 18 & 288 \\
\hline NAWA BARAKZAI & 495 & SAYED SEKANDAR SHAH & SAYED SEKANDAR SHAH & 120 & 10 & 8 & 18 & 180 \\
\hline NAWA BARAKZAI & 471 & SAYED SHIR MOHD & SAYED SHIR MOHD & 300 & 28 & 9 & 18 & 504 \\
\hline NAWA BARAKZAI & 555 & SHABIDAN ZABER & WAKIL SHIR JAN & 700 & 80 & 11 & 18 & 1,440 \\
\hline NAWA BARAKZAI & 511 & SHABOZAI & SHABOZAI & 800 & 300 & 38 & 18 & 5,400 \\
\hline NAWA BARAKZAI & 546 & SHAH PESAND & SHAH PESAND & 90 & 14 & 16 & 16 & 224 \\
\hline NAWA BARAKZAI & 516 & SHAHZADA & SHAHZADA & 400 & 30 & 8 & 16 & 480 \\
\hline NAWA BARAKZAI & 1286 & SHAR KALA SOZI & SHAR KALA SOZI & 400 & 60 & 15 & 18 & 1,080 \\
\hline NAWA BARAKZAI & 496 & SHAR PAZHI & SHAR PAZHI & 480 & 35 & 7 & 18 & 630 \\
\hline NAWA BARAKZAI & 533 & SHARPABRAI & SHARPABRAI & 600 & 36 & 6 & 18 & 648 \\
\hline NAWA BARAKZAI & 556 & SIR BOZAY & SIR BOZAY & 320 & 30 & 9 & 16 & 480 \\
\hline NAWA BARAKZAI & 544 & SOOR GUL KHAN & SOOR GUL KHAN & 600 & 30 & 5 & 18 & 540 \\
\hline NAWA BARAKZAI & 482 & SRA KALA & & 200 & 5 & 2 & 16 & 72 \\
\hline NAWA BARAKZAI & 1280 & SULTAN MOHAMMAD & SULTAN MOHAMMAD & 500 & 39 & 8 & 18 & 702 \\
\hline
\end{tabular}

* I Jerib $=1 / 5 \mathrm{Ha}$

* 2 Dry Opium $=$ Wet Opium - $30 \%$ moisture 
Opium Production Survey by village in Helmand province, 1995.

\begin{tabular}{|c|c|c|c|c|c|c|c|c|}
\hline & Village & & & Total & Land & & Wet Opium *2 & Wet Opium \\
\hline \multirow[t]{2}{*}{ District } & \multirow[t]{2}{*}{ Code } & \multirow[t]{2}{*}{ Area/Main Village } & \multirow[t]{2}{*}{ Village } & \multirow{2}{*}{$\begin{array}{c}\text { Cultivated } \\
\text { land }\end{array}$} & \multicolumn{2}{|c|}{ Poppy cultivation } & \multirow{2}{*}{$\begin{array}{c}\text { Yield } \\
(\mathrm{kg} / \mathrm{jr} .) \\
\end{array}$} & \multirow{2}{*}{$\begin{array}{c}\text { Production } \\
(\mathrm{kg})\end{array}$} \\
\hline & & & & & $\left(\right.$ jerib $\left.^{*}\right)$ & $(\%)$ & & \\
\hline \multicolumn{9}{|l|}{ Province: Helmand } \\
\hline NAWA BARAKZAI & 517 & SULTANZAI & SULTANZAI & 800 & 50 & 6 & 18 & 900 \\
\hline NAWA BARAKZAI & 548 & TAAJ MOHAMMAD & TAAJ MOHAMMAD & 400 & 30 & 8 & 16 & 480 \\
\hline NAWA BARAKZAI & 1285 & TOOR KHAN & TOOR KHAN & 200 & 60 & 30 & 18 & 1,080 \\
\hline NAWA BARAKZAI & 1562 & TORA GAZ & TORA GAZ & 309 & 37 & 12 & 16 & 592 \\
\hline NAWA BARAKZAI & 514 & ZHAMEZAI & ZHAMEZAI & 250 & 50 & 20 & 18 & 900 \\
\hline SARBAN QALA & 1354 & AHMADZI & GARO & 65 & 12 & 18 & 8 & 96 \\
\hline SARBAN QALA & 173 & AHMADZI & AHMADZI & 180 & 85 & 47 & 8 & 680 \\
\hline SARBAN QALA & 171 & AKHUNDZADA KHEL & AKHUNDZADA KHEL & 190 & 80 & 42 & 9 & 720 \\
\hline SARBAN QALA & 175 & BALOZAI & BALOZI, NIM SAR & 400 & 120 & 30 & 12 & 1,440 \\
\hline SARBAN QALA & 178 & BARIKZAI & BARIKZAI & 150 & 100 & 67 & 6 & 600 \\
\hline SARBAN QALA & 174 & BAZAR SANGIN & BAZAR SANGIN & 350 & 190 & 54 & 10 & 1,900 \\
\hline SARBAN QALA & 194 & CHARDEH & CHARDEH & 125 & 80 & 64 & 7 & 560 \\
\hline SARBAN QALA & 181 & CHEDAR KHAN & CHEDAR KAHN & 80 & 40 & 50 & 6 & 240 \\
\hline SARBAN QALA & 182 & CHOGHAK & CHOGHAK & 100 & 40 & 40 & 8 & 320 \\
\hline SARBAN QALA & 186 & FIROZI & FIROZI & 150 & 60 & 40 & 8 & 480 \\
\hline SARBAN QALA & 192 & GANG & GANG & 200 & 50 & 25 & 9 & 450 \\
\hline SARBAN QALA & 187 & GARM AB & GARM AB & 80 & 20 & 25 & 9 & 180 \\
\hline SARBAN QALA & 205 & GHARGAI & GHARGAI & 700 & 300 & 43 & 8 & 2,400 \\
\hline SARBAN QALA & 1353 & GHULBAH & GHULBAH & 80 & 35 & 44 & 12 & 420 \\
\hline SARBAN QALA & 185 & GORA (CHAKA) & GORA (CHAKA) & 270 & 190 & 70 & 8 & 1,520 \\
\hline SARBAN QALA & 188 & HERATYAN & HERATYAN & 300 & 170 & 57 & 8 & 1,360 \\
\hline SARBAN QALA & 190 & JOSHILI & HABIBULLAH & 200 & 80 & 40 & 8 & 640 \\
\hline SARBAN QALA & 189 & JOSHILI & HAJI ABDULLAH & 200 & 100 & 50 & 8 & 800 \\
\hline SARBAN QALA & 191 & JOSHPALI & ABDUL QAYOM & 200 & 80 & 40 & 8 & 640 \\
\hline SARBAN QALA & 1362 & KACHGARD & KACHGARD & 250 & 60 & 24 & 9 & 540 \\
\hline SARBAN QALA & 195 & KATOZI & KATOZI & 350 & 180 & 51 & 8 & 1,440 \\
\hline SARBAN QALA & 135 & KHANANO KILY & KHANANO KILY & 100 & 30 & 30 & 8 & 240 \\
\hline SARBAN QALA & 1363 & LALAZI & LALAZI & 200 & 80 & 40 & 9 & 720 \\
\hline SARBAN QALA & 1359 & MALEK ABDULLAH & MALEK ABDULLAH & 50 & 15 & 30 & 9 & 135 \\
\hline
\end{tabular}


Opium Production Survey by village in Helmand province, 1995.

\begin{tabular}{|c|c|c|c|c|c|c|c|c|}
\hline \multirow{3}{*}{ District } & \multirow{3}{*}{$\begin{array}{c}\text { Village } \\
\text { Code }\end{array}$} & \multirow{3}{*}{ Area/Main Village } & \multirow{3}{*}{ Village } & \multirow{3}{*}{$\begin{array}{c}\text { Total } \\
\text { Cultivated } \\
\text { land } \\
\end{array}$} & \multirow{2}{*}{\multicolumn{2}{|c|}{$\begin{array}{c}\text { Land Under } \\
\text { Poppy cultivation }\end{array}$}} & \multirow{3}{*}{$\begin{array}{c}\text { Wet }^{\text {Opium }}{ }^{* 2} \\
\text { Yield } \\
(\mathrm{kg} / \mathrm{jr} .)\end{array}$} & \multirow{3}{*}{$\begin{array}{c}\text { Wet Opium } \\
\text { Production } \\
(\mathrm{kg}) \\
\end{array}$} \\
\hline & & & & & & & & \\
\hline & & & & & $\left(\right.$ jerib $\left.^{*}\right)$ & $(\%)$ & & \\
\hline \multicolumn{9}{|c|}{ Province: Helmand } \\
\hline SARBAN QALA & 177 & MALIK ABAD & MALIK ABAD & 250 & 100 & 40 & 12 & 1,200 \\
\hline SARBAN QALA & 1356 & MIAN QALA & OMI & 100 & 40 & 40 & 8 & 320 \\
\hline SARBAN QALA . & 1357 & MIAN QALA & KHOJA GADA & 130 & 40 & 31 & 9 & 360 \\
\hline SARBAN QALA & 1358 & MIAN QALA & CHAR DEH & 280 & 170 & 61 & 8 & 1,360 \\
\hline SARBAN QALA & 214 & MIYAN RODI & TORA OBA & 40 & 15 & 38 & 5 & 75 \\
\hline SARBAN QALA & 198 & MIYAN RODI & MOZAK & 60 & 20 & 33 & 5 & 100 \\
\hline SARBAN QALA & 199 & MIYAN RODI & MIYAN RODI & 200 & 80 & 40 & 8 & 640 \\
\hline SARBAN QALA & 202 & MOHAMMADYAN & MOHAMMADYAN & 70 & 35 & 50 & 5 & 175 \\
\hline SARBAN QALA & 1361 & MULLAZI & KESHATA MULLAZI & 300 & 80 & 27 & 8 & 640 \\
\hline SARBAN QALA & 196 & MULLAZI & MULLAZI & 1,700 & 500 & 29 & 6 & 3,000 \\
\hline SARBAN QALA & 1360 & NOKROZI & NOKROZI & 350 & 100 & 29 & 8 & 800 \\
\hline SARBAN QALA & 207 & PAEEN QALA & HAJI A. WAHAB & 700 & 250 & 36 & 9 & 2,250 \\
\hline SARBAN QALA & 206 & PAEEN QALA & H. FATEH MOHD & 400 & 100 & 25 & 10 & 1,000 \\
\hline SARBAN QALA & 208 & POTI & POTI & 220 & 160 & 73 & 8 & 1,280 \\
\hline SARBAN QALA & 176 & PUSHTA JOEY & PUSHTA JOEY & 210 & 45 & 21 & 11 & 495 \\
\hline SARBAN QALA & 193 & QALAWAL & QALAWAL & 130 & 80 & 62 & 10 & 800 \\
\hline SARBAN QALA & 1364 & SAHIBZADA, MALIKZAI & SAHIBZADA & 175 & 85 & 49 & 6 & 510 \\
\hline SARBAN QALA & 179 & SARPOZAH & SARPOZAH & 325 & 70 & 22 & 5 & 350 \\
\hline SARBAN QALA & 1355 & SHAKER SHELAH & SHAKER SHELAH & 0 & 0 & \#DIV/0! & 0 & 0 \\
\hline SARBAN QALA & 1365 & SRAH KILY & SRAH KILY & 120 & 30 & 25 & 8 & 240 \\
\hline SARBAN QALA & 213 & TOGHI & JAMALZAI & 300 & 180 & 60 & 9 & 1,620 \\
\hline SARBAN QALA & 210 & TOGHI & SANG CHAL & 100 & 30 & 30 & 8 & 240 \\
\hline SARBAN QALA & 212 & TOGHI & QALAWAL & 150 & 80 & 53 & 8 & 640 \\
\hline SARBAN QALA & 211 & TOGHI & PAI GAW & 150 & 80 & 53 & 8 & 640 \\
\hline SARBAN QALA & 216 & ZAFRANI & ZAFRANI & 250 & 170 & 68 & 8 & 1,360 \\
\hline SARBAN QALA & 209 & ZAHEDANAN & PANIZAI & 180 & 80 & 44 & 6 & 480 \\
\hline SARBAN QALA & 215 & ZAHEDANAN & ZAHEDANAN & 80 & 20 & 25 & 6 & 120 \\
\hline SARBAN QALA & 217 & ZARD REGI & ZARDREGI & 100 & 30 & 30 & 6 & 180 \\
\hline WASHIR & 408 & AJRAM & AJRAM & 40 & 10 & 25 & 11 & 110 \\
\hline
\end{tabular}


Opium Production Survey by village in Helmand province, 1995.

\begin{tabular}{|c|c|c|c|c|c|c|c|c|}
\hline & Village & & & \multirow{3}{*}{$\begin{array}{c}\text { Total } \\
\text { Cultivated } \\
\text { land }\end{array}$} & \multirow{2}{*}{\multicolumn{2}{|c|}{$\begin{array}{c}\text { Land Under } \\
\text { Poppy cultivation } \\
\end{array}$}} & \multirow{3}{*}{$\begin{array}{c}\text { Wet Opium }^{* 2} \\
\text { Yield } \\
(\mathrm{kg} / \mathrm{jr} .) \\
\end{array}$} & \multirow{3}{*}{$\begin{array}{c}\text { Wet Opium } \\
\text { Production } \\
\text { (kg) }\end{array}$} \\
\hline \multirow[t]{2}{*}{ District } & Code & Area/Main Village & Village & & & & & \\
\hline & & & & & $\left(\right.$ jerib $\left.^{*}\right)$ & $(\%)$ & & \\
\hline \multicolumn{9}{|c|}{ Province: Helmand } \\
\hline WASHIR & 409 & ALAH GHOLI & ALLAH GHALE & 30 & 10 & 33 & 9 & 90 \\
\hline WASHIR & 410 & AMIR MOMTA & AMIR MATA & 40 & 25 & 63 & 22 & 550 \\
\hline WASHIR & 415 & BAKHESH ABAD & BAKHESH ABAD & 60 & 40. & 67 & 10 & 400 \\
\hline WASHIR & 416 & BIABANK & BIABANK & 100 & 50 & 50 & 9 & 450 \\
\hline WASHIR & 417 & CHAK AAB/ZOLAIKHAR & CHAK AAB/ZOLAIKHAR & 150 & 40 & 27 & 25 & 1,000 \\
\hline WASHIR & 30 & CHAY DARVAZA/NAWABAD & CHAY DARVAZA/NAWABAD & 30 & 10 & 33 & 9 & 90 \\
\hline WASHIR & 435 & CHESHMA QASIM & CHESHMA QASIM & 70 & 40 & 57 & 10 & 400 \\
\hline WASHIR & 421 & DELBAR KHAN & DELBAR KHAN & 300 & 220 & 73 & 20 & 4,400 \\
\hline WASHIR & 422 & DIZNO & DIZNO & 100 & 75 & 75 & 11 & 825 \\
\hline WASHIR & 1590 & DOSHAKH GARAK & DOSHAKH GARAK & 60 & 40 & 67 & 11 & 440 \\
\hline WASHIR & 423 & DOZAK-E-PAYAN & DOZDAK & 20 & 8 & 40 & 9 & 72 \\
\hline WASHIR & 424 & FIAZ ABAD & FIAZ ABAD & 40 & 20) & 50 & 9 & 180 \\
\hline WASHIR & 425 & GALABAD & GALABED & 50 & 20 & 40 & 9 & 180 \\
\hline WASHIR & 427 & GANDA CHA & GANDA CHA & 50 & 30 & 60 & 9. & 270 \\
\hline WASHIR & 418 & GANDA MASHAK & GANDA MASHAK & 50 & 30 & 60 & 9 & 270 \\
\hline WASHIR & 428 & GORAZAN & GORAZAN & 200 & 155 & 78 & 11 & 1,705 \\
\hline WASHIR & 430 & JAMAL KAREZ & JAMAL KAREZ & 60 & 30 & 50 & 10 & 300 \\
\hline WASHIR & 442 & KAREZ & KAREZ & 150 & 100 & 67 & 14 & 1,400 \\
\hline WASHIR & 436 & KAREZ SANG & KAREZ SANG & 40 & 30 & 75 & 10 & 300 \\
\hline WASHIR & 1680 & KAREZ SAYED ABAD & KAREZ SAYEDABAD & 40 & 25 & 63 & 12 & 300 \\
\hline WASHIR & 464 & KAREZ SULTAN & KAREZ SULTAN & 60 & 40 & 67 & 10 & 400 \\
\hline WASHIR & 440 & KAREZ ZIARAT & KAREZ ZIARAT & 50 & 30 & 60 & 9 & 270 \\
\hline WASHIR & 441 & KARKAN & KARKAN & 40 & 10 & 25 & 10 & 100 \\
\hline WASHIR & 1350 & KELKAK GESTAN & KELKAK GESTAN & 80 & 38 & 48 & 20 & 760 \\
\hline WASHIR & 431 & KHORASAN & KHORASN & 250 & 200 & 80 & 11 & 2,200 \\
\hline WASHIR & 433 & KHURASAN & KHURASAN & 60 & 36 & 60 & 15 & 540 \\
\hline WASHIR & 432 & KHYRADAY NAYAK & KHERA DA NA YAK & 50 & 20 & 40 & 9. & 180 \\
\hline WASHIR & 438 & LANDAI & LANDAI & 60 & 40 & 67 & 12 & 480 \\
\hline WASHIR & 445 & LANDYI KAREZ & LANDYI KAREZ & 100 & 85 & 85 & 9 & 765 \\
\hline
\end{tabular}

* $1 \mathrm{Jerib}=1 / 5 \mathrm{Ha}$

* 2 Dry Opium $=$ Wet Opium $-30 \%$ moisture
Page $47 \quad 82$
Source: UNDCP Opium Production Survey, 1995 File: HLD3.XLS - 07/08/95 
Opium Production Survey by village in Helmand province, 1995.

\begin{tabular}{|c|c|c|c|c|c|c|c|c|}
\hline & Village & & & & Land $\mathrm{C}$ & & Wet Opium *2 & Wet Opium \\
\hline \multirow[t]{2}{*}{ District } & Code & Area/Main Village & Village & Cultivated & \multicolumn{2}{|c|}{ Poppy cultivation } & Yield & Production \\
\hline & & & & land & $\left(\right.$ jerib $\left.^{*}\right)$ & (\%) & $(\mathrm{kg} / \mathrm{jr})$. & $(\mathrm{kg})$ \\
\hline \multicolumn{9}{|c|}{ Province: Helmand } \\
\hline WASHIR & 444 & LANGAR & LANGAR & 60 & 20 & 33 & 20 & 400 \\
\hline WASHIR & 446 & LAPATU PANATU & LAPATU PANATU & 100 & 30 & 30 & 9 & 270 \\
\hline WASHIR & 448 & MALGHZAR KARE $Z$ & MALGHZAR KAREZ & 40 & 25 & 63 & 11 & 275 \\
\hline WASHIR & 449 & MANZARAKA & MANZARAKA & 50 & 30 & 60 & 10 & 300 \\
\hline WASHIR & 451 & MARGHZAR & MARGHZAR & 100 & 50 & 50 & 22 & 1,100 \\
\hline WASHIR & 457 & MULA ZIAULHAQ & MULA ZIAULHAQ & 200 & 150 & 75 & 11 & 1,650 \\
\hline WASHIR & 452 & NAKHN BIRA & NAKHN BIRA & 100 & 30 & 30 & 10 & 300 \\
\hline WASHIR & 456 & NAWDEH LANDAK & NAWDEH LANDAK & 30 & 20 & 67 & 11 & 220 \\
\hline WASHIR & 411 & OAR BAND & WORBAND & 60 & 40 & 67 & 10 & 400 \\
\hline WASHIR & 412 & OAR QULY & OAR QULY & 100 & 50 & 50 & 9 & 450 \\
\hline WASHIR & 413 & PANGEY/USAIA USHTUR & PANGEY/USAIAUST & 500 & 400 & 80 & 14 & 5,400 \\
\hline WASHIR & 454 & PAYAWAK & PAYAWAK & 300 & 210 & 70 & 13 & 2,730 \\
\hline WASHIR & 1351 & PAYAWAK KHUSH & PAYAWAK KHUSHK & 500 & 320 & 64 & 11 & 3,520 \\
\hline WASHIR & 443 & POZARK & KAREZ NAW & 10 & 5 & 50 & 21 & 105 \\
\hline WASHIR & 1349 & PUZERAK & PUZERAK & 50 & 25 & 50 & 10 & 250 \\
\hline WASHIR & 459 & QALA-E-NAW & QALA-E-NAW & 30 & 20 & 67 & 9 & 180 \\
\hline WASHIR & 455 & QALA-E-NAZERGAH & QALA-E-NAZERGAH & 45 & 20 & 44 & 22 & 440 \\
\hline WASHIR & 465 & SAR FOLAD & SAR FOLAD & 70 & 25 & 36 & 11 & 275 \\
\hline WASHIR & 460 & SHAH MIRZA KAREZ & SHAH MIRZA KAREZ & 125 & 60 & 48 & 9 & 540 \\
\hline WASHIR & 467 & TUT SHAH & TUT SHAH & 30 & 20 & 67 & 15 & 300 \\
\hline WASHIR & 469 & WALGAK & WALGAK & 36 & 30 & 83 & 15 & 450 \\
\hline WASHIR & 468 & WASHIR MARKAZ & WASHIR MARKAZ & 500 & 295 & 59 & 25 & 7,375 \\
\hline
\end{tabular}


Opium Production Survey by village in Kunar province, 1995.

\begin{tabular}{|c|c|c|c|c|c|c|c|c|}
\hline & \multirow{3}{*}{\begin{tabular}{|l|} 
Village \\
Code \\
\end{tabular}} & \multirow{3}{*}{ Areal Main Village } & \multirow{3}{*}{ Village } & \multirow{3}{*}{$\begin{array}{c}\text { Total } \\
\text { Cultivated } \\
\text { Land }\left(\text { Jerib }^{*}\right) \\
\end{array}$} & \multirow{2}{*}{\multicolumn{2}{|c|}{$\begin{array}{c}\text { Land Under } \\
\text { Poppy Cultivation }\end{array}$}} & \multirow{3}{*}{$\begin{array}{c}\text { Wet Opium }^{* 2} \\
\text { Yield } \\
(\mathrm{kg} / \mathrm{jr})\end{array}$} & \multirow{3}{*}{$\begin{array}{c}\text { Wet Opium } \\
\text { Production } \\
(\mathrm{kg})\end{array}$} \\
\hline \multirow[t]{2}{*}{ District } & & & & & & & & \\
\hline & & & & & (jerib) & $(\%)$ & & \\
\hline \multicolumn{9}{|c|}{ Province: Kunar } \\
\hline CHAWKI & 4001 & GARIGIAL & GARIGIAL & 170 & 0 & 0 & 0 & 0 \\
\hline CHAWKI & 4002 & ANDERLACHAK & ANDARLACHAK & 330 & 0 & 0 & 0 & 0 \\
\hline CHAWKI & 4012 & AMREY & AMREY KALAY & 22 & 0 & 0 & 0 & 0 \\
\hline CHAWKI & 4014 & PLAR DARAH & PLAR DARAH & 85 & 0 & 0 & 0 & 0 \\
\hline CHAWKI & 4015 & BADENZI & BADENZO KALAY & 45 & 0 & 0 & 0 & 0 \\
\hline CHAWKI & 4016 & KHARAH & KHARAH & 30 & 0 & 0 & 0 & 0 \\
\hline CHAWKI & 4018 & NASAPA & NASAPA & 100 & 0 & 0 & 0 & 0 \\
\hline CHAWKI & 4021 & GATO QALA & GATO QALA & 200 & 5 & 3 & 10 & 50 \\
\hline CHAWKI & 4024 & KALMANI & QAZI BANDAH & 210 & 3 & 1 & 11 & 33 \\
\hline CHAWKI & 4030 & BAR CHAMBEL & BAR CHAMBEL & 40 & 0 & 0 & 0 & 0 \\
\hline CHAWKI & 4033 & ISLAM KHANA & ISLAM KHANA & 35 & 0 & 0 & 0 & 0 \\
\hline CHAWKI & 4034 & SAFIDAR & SAFIDAR & 90 & 0 & 0 & 0 & 0 \\
\hline CHAWKI & 4035 & KHAKY QALA & KHAKY QALA & 150 & 8 & 5 & 10 & 80 \\
\hline CHAWKI & 4038 & KHADE KHEL & KHADE KHEL & 500 & 6 & 1 & 12 & 72 \\
\hline CHAWKI & 4039 & QALAH & QALA & 29 & 0 & 0 & 0 & 0 \\
\hline CHAWKI & 4042 & KARBOREY & KARBOREY & 60 & 0 & 0 & 0 & 0 \\
\hline CHAWKI & 4043 & BABAR & BABAR & 490 & 0 & 0 & 0 & 0 \\
\hline CHAWKI & 4044 & BABAR & ROGEY & 75 & 0 & 0 & 0 & 0 \\
\hline CHAWKI & 4045 & POHAH SEREY & POHAH SEREY & 100 & 0 & 0 & 0 & 0 \\
\hline CHAWKI & 4229 & BADGOR & BADGOR & 50 & 0 & 0 & 0 & 0 \\
\hline CHAWKI & 4231 & CHALAS/NORASH & NORASH & 41 & 0 & 0 & 0 & 0 \\
\hline CHAWKI & 4232 & CHALAS LOY & CHALAS LOY KALAY & 65 & 0 & 0 & 0 & 0 \\
\hline CHAWKI & 4233 & BADGOR/MORCHEL KHAIL & MORCHEL KHAIL & 11 & 0 & 0 & 0 & 0 \\
\hline CHAWKI & 4234 & CHALAS & SHERGAL & 113 & 0 & 0 & 0 & 0 \\
\hline CHAWKI & 4238 & CHAMBEL & CHAMBEL & 402 & 0 & 0 & 0 & 0 \\
\hline CHAWKI & 4448 & KANDAY & KANDAY & 30 & 0 & 0 & 0 & 0 \\
\hline CHAWKI & 4449 & CHAWKI & CHAWKI & 250 & 3 & 1 & 8 & 24 \\
\hline CHAWKI & 4450 & TARNAB & TARNAB & 30 & 0 & 0 & 0 & 0 \\
\hline CHAWKI & 4451 & KALMANI & MURSALIN & 40 & 3 & 8 & 9 & 27 \\
\hline
\end{tabular}

* Jerib (jr.) $=1 / 5$ ha

* 2 Dry Opium $=$ Wet Opium - $30 \%$ moisture 
Opium Production Survey by village in Kunar province, 1995.

\begin{tabular}{|c|c|c|c|c|c|c|c|c|}
\hline & Village & & & Total & Land $L$ & & Wet Opium * 2 & Wet Opium \\
\hline \multirow[t]{2}{*}{ District } & \multirow[t]{2}{*}{ Code } & \multirow[t]{2}{*}{ Areal Main Village } & \multirow[t]{2}{*}{ Village } & \multirow{2}{*}{$\begin{array}{c}\text { Cultivated } \\
\left.\text { Land (Jerib }{ }^{*}\right)\end{array}$} & \multicolumn{2}{|c|}{ Poppy Cultivation } & \multirow{2}{*}{$\begin{array}{l}\text { Yield } \\
(\mathrm{kg} / \mathrm{jr}) \\
\end{array}$} & \multirow{2}{*}{$\begin{array}{c}\text { Production } \\
(\mathrm{kg})\end{array}$} \\
\hline & & & & & (jerib) & $(\%)$ & & \\
\hline \multicolumn{9}{|c|}{ Province: Kunar } \\
\hline CHAWKI & 4452 & BABAR & KANADAR & 115 & 0 & 0 & 0 & 0 \\
\hline CHAWKI & 4453 & SHULOTAY & SHULOTAY & 470 & 6 & 1 & 12 & 72 \\
\hline CHAWKI & 4454 & KALMANI & ZARA KALMANI & 350 & 15 & 4 & 11 & 165 \\
\hline CHAWKI & 4455 & KALMANAI & ZARONDAY & 170 & 4 & 2 & 12 & 48 \\
\hline CHAWKI & 4456 & KALMANA & KOZA KAMANAI & 110 & 4 & 4 & 12 & 48 \\
\hline CHAWKI & 4457 & KANDAR & KANDAR & 28 & 0 & 0 & 0 & 0 \\
\hline CHAWKI & 4462 & SOLAIMANZAI & SOLAIMANZAI & 40 & 0 & 0 & 0 & 0 \\
\hline CHAWKI & 4475 & DEWARA BETAL & DEWARA & 15 & 0 & 0 & 0 & 0 \\
\hline KHAS KONAR & 4077 & ARAZI & BARA ARAZI & 510 & 20 & 4 & 13 & 260 \\
\hline KHAS KONAR & 4079 & KHAS KONAR & MLAKANO BANDAH & 130 & 10 & 8 & 10 & 100 \\
\hline KHAS KONAR & 4080 & KHAS KONAR & SAHEBJAM & 220 & 15 & 7 & 12 & 180 \\
\hline KHAS KONAR & 4081 & SHALY & DANDKALAY/BAR SHALAY & 200 & 1 & 1 & 8 & 8 \\
\hline KHAS KONAR & 4082 & KAWEER & KAWEER & 280 & 15 & 5 & 11 & 158 \\
\hline KHAS KONAR & 4083 & TANER & BANDA-E- RASOUL & 210 & 8 & 4 & 10 & 80 \\
\hline KHAS KONAR & 4084 & HAKIMABAD & BAR SHAMKAR & 104 & 0 & 0 & 0 & 0 \\
\hline KHAS KONAR & 4085 & KHAS KONAR & BARA CHARKAI & 125 & 9 & 7 & 11 & 95 \\
\hline KHAS KONAR & 4086 & KHAS KONAR & KUZA CHARKAI & 250 & 25 & 10 & 11 & 263 \\
\hline KHAS KONAR & 4087 & KHAS KONAR & MIAGANO KALAY & 140 & 8 & 6 & 12 & 92 \\
\hline KHAS KONAR & 4089 & TANER & PACHAYANO BANDAH & 178 & 10 & 6 & 11 & 110 \\
\hline KHAS KONAR & 4090 & TANER & TANER BANDAH & 74 & 7 & 9 & 11 & 74 \\
\hline KHAS KONAR & 4092 & TANER & TANER & 490 & 35 & 7 & 11 & 385 \\
\hline KHAS KONAR & 4093 & HAKIM ABAD & SARAI & 95 & 5 & 5 & 11 & 55 \\
\hline KHAS KONAR & 4095 & HAKIM ABAD & DAG KALAY/BAR KALAY & 252 & 10 & 4 & 11 & 110 \\
\hline KHAS KONAR & 4096 & HAKIM ABAD & HAKIMABAD/KUZA KALAY & 600 & 8 & 1 & 11 & 88 \\
\hline KHAS KONAR & 4097 & HAKIM ABAD & BANDA-E-HAKIMABAD & 45 & 2 & 4 & 12 & 24 \\
\hline KHAS KONAR & 4098 & HAKIM ABAD & AKHUNDZADAGANO BABDA & 45 & 3 & 7 & 11 & 33 \\
\hline KHAS KONAR & 4099 & ARAZI & KOKI BANDAY & 155 & 6 & 4 & 11 & 66 \\
\hline KHAS KONAR & 4101 & ARAZI & KUZA ARAZI & 600 & 30 & 5 & 12 & 360 \\
\hline KHAS KONAR & 4145 & CHANDRAWEY & NAWHI CHANDRAWEY & 190 & 12 & 6 & 12 & 144 \\
\hline
\end{tabular}

* Jerib (jr.) $=1 / 5$ ha

* 2 Dry Opium $=$ Wet Opium $-30 \%$ moisture 
Opium Production Survey by village in Kunar province, 1995.

\begin{tabular}{|c|c|c|c|c|c|c|c|c|}
\hline \multirow{3}{*}{ District } & \multirow{2}{*}{\begin{tabular}{|l|} 
Village \\
Code
\end{tabular}} & \multirow{3}{*}{ Area/ Main Village } & \multirow{3}{*}{ Village } & \multirow{3}{*}{$\begin{array}{c}\text { Total } \\
\text { Cultivated } \\
\text { Land }\left(\text { Jerib }^{*}\right) \\
\end{array}$} & \multirow{2}{*}{\multicolumn{2}{|c|}{$\begin{array}{c}\text { Land Under } \\
\text { Poppy Cultivation }\end{array}$}} & \multirow{3}{*}{$\begin{array}{c}\text { Wet } \text { Opium }^{* 2} \\
\text { Yield } \\
(\mathrm{kg} / \mathrm{jr})\end{array}$} & \multirow{3}{*}{$\begin{array}{c}\text { Wet Opium } \\
\text { Production } \\
(\mathrm{kg})\end{array}$} \\
\hline & & & & & & & & \\
\hline & & & & & (jerib) & $(\%)$ & & \\
\hline \multicolumn{9}{|c|}{ Province: Kunar } \\
\hline KHAS KONAR & 4146 & CHANDRAWEY & LOWTAN & 180 & 15 & 8 & 12 & 180 \\
\hline KHAS KONAR & 4147 & CHANDRAWEY & KANDAR & 112 & 12 & 11 & 11 & 132 \\
\hline KHAS KONAR & 4148 & CHANDRAWEY & CHAND RAWI KILY. & 130 & 8 & 6 & 12 & 92 \\
\hline KHAS KONAR & 4149 & CHANDRAWEY & JABAH & 150 & 14 & 9 & 12 & 161 \\
\hline KHAS KONAR & 4150 & SHALY & MANOGAI/SHALY KALAY & 80 & 0 & 0 & 0 & 0 \\
\hline KHAS KONAR & 4151 & WALI & KARBADAG/ABOTANGAI & 90 & 1 & 1 & 10 & 10 \\
\hline KHAS KONAR & 4152 & SHALY & ENZERY/YAKHAH CHINAN & 50 & 1 & 2 & 11 & 11 \\
\hline KHAS KONAR & 4155 & CHEMEARY & CHEMEARY & 778 & 40 & 5 & 11 & 420 \\
\hline KHAS KONAR & 4158 & MANGWAL & MANGWAL KALAY & 200 & 17. & 9 & 11 & 187 \\
\hline KHAS KONAR & 4237 & MANGWAL & BELAM & 145 & 14 & 10 & 12 & 168 \\
\hline KHAS KONAR & 4458 & MANGWAL & BAR CHAN CHAND & 50 & 5 & 10 & 12 & 58 \\
\hline KHAS KONAR & 4459 & MANGWAL & KUZ CHAN CHAN & 45 & 3 & 7 & 11 & 32 \\
\hline KHAS KONAR & 4460 & HAKIM ABAD & SAHIBZADAGANO BANDA & 160 & 20 & 13 & 11 & 220 \\
\hline KHAS KONAR & 4461 & HAKIM ABAD & KUZ SHAMKAR & 76 & 2 & 3 & 11 & 22 \\
\hline KHAS KONAR & 4463 & ARAZI & KOKY BANDAH & 73 & 5 & 7 & 12 & 60 \\
\hline KHAS KONAR & 4480 & SERKY & SERKY & 70 & 6 & 9 & 9 & 54 \\
\hline KHAS KONAR & 4481 & SHALY & TANGAI/GHONDY KALAY & 70 & 7 & 10 & 10 & 67 \\
\hline KHAS KONAR & 4482 & WOLI & KALAWOL/TOTKAI & 60 & 1 & 2 & 9 & 9 \\
\hline NARANG & 4105 & KHAIR ABAD & KHAIRABAD & 58 & 0 & 0 & 0 & 0 \\
\hline NARANG & 4106 & SHALMAR & MAMSIDOKHEL & 29 & 1. & 3 & 6 & 6 \\
\hline NARANG & 4107 & SHALMAR & NAJARANOCHAM & 103 & 2 & 1 & 6 & 8 \\
\hline NARANG & 4108 & LAMATAK & LAMATAK & 270 & 5 & 2 & 8 & 40 \\
\hline NARANG & 4109 & KOTAKEY & KOTAKEY & 550 & 15 & 3 & 9 & 135 \\
\hline NARANG & 4122 & DANDONAH & DANDONAH & 285 & 5 & 2 & 6 & 30 \\
\hline NARANG & 4123 & LACHAGALEY & BAR LACHAGALEY & 140 & 0 & 0 & 0 & 0 \\
\hline NARANG & 4124 & LACHAGALEY & KUZ LACHAGALEY & 90 & 0 & 0 & 0 & 0 \\
\hline NARANG & 4126 & KARAMAR & KARAMAR & 220 & 5 & 2 & 9 & 43 \\
\hline NARANG & 4129 & SHALMAR & YARANWAL CHAM & 203 & 1. & 0 & 5 & 5 \\
\hline NARANG & 4130 & KADO & KADO & 150 & 3 & 2 & 8 & 24 \\
\hline
\end{tabular}

* Jerib (jr.) = 1/5 ha

${ }^{*}{ }^{2}$ Dry Opium $=$ Wet Opium $-30 \%$ moisture
Source: UNDCP Opium Production Survey, 1995 File: KNR3.XLS - 07/08/95 
Opium Production Survey by village in Kunar province, 1995.

\begin{tabular}{|c|c|c|c|c|c|c|c|c|}
\hline \multirow{3}{*}{ District } & \multirow{3}{*}{\begin{tabular}{|l|} 
Village \\
Code \\
\end{tabular}} & \multirow{3}{*}{ Area/ Main Village } & \multirow{3}{*}{ Village } & \multirow{3}{*}{\begin{tabular}{|c|} 
Total \\
Cultivated \\
Land (Jerib $\left.{ }^{*}\right)$ \\
\end{tabular}} & \multirow{2}{*}{\multicolumn{2}{|c|}{$\begin{array}{c}\text { Land Under } \\
\text { Poppy Cultivation }\end{array}$}} & \multirow{3}{*}{$\begin{array}{c}\text { Wet Opium }^{* 2} \\
\text { Yield } \\
(\mathrm{kg} / \mathrm{jr}) \\
\end{array}$} & \multirow{3}{*}{$\begin{array}{c}\text { Wet Opium } \\
\text { Production } \\
(\mathrm{kg}) \\
\end{array}$} \\
\hline & & & & & & & & \\
\hline & & & & & (jerib) & $(\%)$ & & \\
\hline \multicolumn{9}{|c|}{ Province: Kunar } \\
\hline NARANG & 4131 & NARANG/GHONDO KALAY & GHONDO KALAY/DAG & 450 & 12 & 3 & 8 & 96 \\
\hline NARANG & 4132 & KUZ NARANG & KUZ NARANG & 322 & 15 & 5 & 9 & 135 \\
\hline NARANG & 4135 & CHARQALA & MOHD AMIN CHAM & 178 & 0 & 0 & 0 & 0 \\
\hline NARANG & 4136 & QALA WONO & QALA WONA & 85 & 5 & 6 & 9 & 45 \\
\hline NARANG & 4141 & NARANG & GHAZAN KORONA & 127 & 4 & 3 & 8 & 32 \\
\hline NARANG & 4194 & LOND NAW & KHORAGASH & 18 & 0 & 0 & 0 & 0 \\
\hline NARANG & 4197 & WOCH NAW & LEWAT & 18 & 0 & 0 & 0 & 0 \\
\hline NARANG & 4198 & WOCH NAW & AHMAD KHAN MENA & 25 & 0 & 0 & 0 & 0 \\
\hline NARANG & 4205 & WOCH NAW & BARO GUL/KHALATAK & 18 & 0 & 0 & 0 & 0 \\
\hline NARANG & 4206 & WOCH NAW & BAJGAL & 33 & 0 & 0 & 0 & 0 \\
\hline NARANG & 4208 & SOBA GHUNDY & GHAZAY KHAN GHWANDAI & 50 & 1 & 2 & 6 & 6 \\
\hline NARANG & 4209 & LOND NAW & BANGO OBAH & 30 & 0 & 0 & 0 & 0 \\
\hline NARANG & 4470 & SHALIMAR & KOJA KHELO KILY & 190 & 0 & 0 & 0 & 0 \\
\hline NARANG & 4471 & SPIN KAR & SPIN KAR & 58 & 0 & 0 & 0 & 0 \\
\hline NARANG & 4472 & LANDI KALAY & KASABGARO QALA & 85 & 0 & 0 & 0 & 0 \\
\hline NARANG & 4473 & SHALAMAR & AKONDZADGAN & 25 & 0 & 0 & 0 & 0 \\
\hline NARANG & 4474 & BADILO CHAM & BADILO CHAM & 160 & 0 & 0 & 0 & 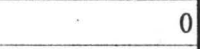 \\
\hline NURGAL & 4159 & KUZ NURGAL & KUZ NURGAL & 300 & 25 & 8 & 14 & 350 \\
\hline NURGAL & 4160 & AREET & MAMA GUL & 49 & 0 & 0 & 0 & 0 \\
\hline NURGAL & 4162 & SHOLAT/MARAWARAH & SHOLIT & 105 & 0 & 0 & 0 & 0 \\
\hline NURGAL & 4163 & AREET & OSHAN KONARAK & 60 & 0 & 0 & 0 & 0 \\
\hline NURGAL & 4164 & AREET & KANDAH KALAY & 24 & 0 & 0 & 0 & 0 \\
\hline NURGAL & 4165 & AREET & AREET & 240 & 0 & 0 & 0 & 0 \\
\hline NURGAL & 4166 & AREET & MANGWAL KHAWAR & 80 & 0 & 0 & 0 & 0 \\
\hline NURGAL & 4167 & AREET & SALAMBAD & 103 & 0 & 0 & 0 & 0 \\
\hline NURGAL & 4168 & GHAZI ABAD & SHOMASH & 130 & 0 & 0 & 0 & 0 \\
\hline NURGAL & 4170 & GHAZI ABAD & WADERO KALAY & 230 & 0 & 0 & 0 & 0 \\
\hline NURGAL & 4171 & GHAZI ABAD & GURBAZ/KASHNI AU QAL & 200 & 0 & 0 & 0 & 0 \\
\hline NURGAL & 4172 & BARIZO & GHONDAY KALAY & 70 & 0 & 0 & 0 & 0 \\
\hline
\end{tabular}

* Jerib (jr.) $=1 / 5 \mathrm{ha}$

* 2 Dry Opium = Wet Opium - $30 \%$ moisture
Page 487
Source: UNDCP Opium Production Survey, 1995 File: KNR3.XLS - 07/08/95 
Opium Production Survey by village in Kunar province, 1995.

\begin{tabular}{|c|c|c|c|c|c|c|c|c|}
\hline & Village & & & Total & Land & & Wet Opium *2 & Wet Opium \\
\hline \multirow[t]{2}{*}{ District } & Code & Areal Main Village & Village & Cultivated & \multicolumn{2}{|c|}{ Poppy Cultivation } & Yield & Production \\
\hline & & & & Land (Jerib *) & (jerib) & $(\%)$ & $(\mathrm{kg} / \mathrm{jr})$ & $(\mathrm{kg})$ \\
\hline \multicolumn{9}{|c|}{ Province: Kunar } \\
\hline NURGAL & 4174 & GHAZI ABAD & SHOMAI KALAY & 220 & 0 & 0 & 0 & 0 \\
\hline NURGAL & 4175 & LOODLAAM & LOODLAAM & 330 & 5 & 2 & 14 & 70 \\
\hline NURGAL & 4177 & KARBORY & KARBORY & 110 & 1 & 1 & 12 & 12 \\
\hline NURGAL & 4178 & KARCHANDO/HUSSAINZO & KARCHANDO & 150 & 5 & 3 & 10 & 50 \\
\hline NURGAL & 4179 & SERAWAR & SERAWAR & 100 & 1 & 1 & 14 & 7 \\
\hline NURGAL & 4180 & BARIZO & BARIZO & 150 & 3 & 2 & 12 & 36 \\
\hline NURGAL & 4181 & SINZO & KHAKHO & 60 & 0 & 0 & 0 & 0 \\
\hline NURGAL & 4182 & MORAWARA & MORAWARA & 170 & 1 & 1 & 12 & 12 \\
\hline NURGAL & 4183 & GORWA/HUSSAINZO & GORWA & 200 & 7 & 4 & 10 & 70 \\
\hline NURGAL & 4184 & HUSSANINZAI/SINZO & SINZO & 65 & 0) & 0 & 0 & 0 \\
\hline NURGAL & 4185 & SINZO & SAIRI & 400 & 2 & 1 & 11 & 22 \\
\hline NURGAL & 4186 & GHAZIABAD/BAR NURGAL & MASOOD KALAY & 210 & 0 & 0 & 0 & 0 \\
\hline NURGAL & 4189 & TIPAR & TIPAR KHAIL & 40 & 2 & 4 & 12 & 18 \\
\hline NURGAL & 4190 & LORLAM/BAR NURGAL & DORKANDAK/MASHIA QAL & 130 & 1 & 1 & 15 & 15 \\
\hline NURGAL & 4191 & TORA TIGA & TORA TIGA & 240 & 1. & 0 & 14 & 14 \\
\hline NURGAL & 4193 & BELLA/KUZ NURGAL & BELLA & 60 & 0 & 0 & 0 & 0 \\
\hline NURGAL & 4240 & BAR NURGAL & BAR NURGAL & 460 & 20 & 4 & 13 & 260 \\
\hline NURGAL & 4241 & FAZULLAH KALAY & FAZULLAH KALAY/ROZI & 90 & 1 & 1 & 10 & 10 \\
\hline NURGAL & 4242 & LOODLAAM & WARINA & 60 & 3 & 5 & 14 & 42 \\
\hline NURGAL & 4443 & BAR NURGAL & JABA & 220 & 0 & 0 & 0 & 0 \\
\hline NURGAL & 4444 & PATTAN & KATTAR & 75 & 0 & 0 & 0 & 0 \\
\hline NURGAL & 4445 & PATTAN & PATTAN & 310 & 2 & 1 & 10 & 20 \\
\hline NURGAL & 4446 & NURGAL & CHOKADA & 100 & 15 & 15 & 12 & 180 \\
\hline NURGAL & 4447 & PATTAN & MIA QALA & 140 & 0 & 0 & 0 & 0 \\
\hline SARKANI & 4046 & PASHAD & BELAH & 430 & 0 & 0 & 0 & 0 \\
\hline SARKANI & 4047 & PASHAD & KHANI BABA/IDAG KALAY & 40 & 0 & 0 & 0 & 0 \\
\hline SARKANI & 4048 & PASHAD & CHENGEY & 340 & 5 & 1 & 12 & 58 \\
\hline SARKANI & 4049 & PASHAD & TANGO & 250 & 0 & 0 & 0 & 0 \\
\hline SARKANI & 4056 & BAHRAH ABAD & SHADALAM & 110 & 4 & 4 & 12 & 46 \\
\hline
\end{tabular}

* Jerib (jr.) = 1/5 ha

* Dry Opium = Wet Opium - $30 \%$ moisture
Page 5
Source: INDCP Opium Production Survey, 1995 File: KNR3.XLS - 07/08/95 
Opium Production Survey by village in Kunar province, 1995.

\begin{tabular}{|c|c|c|c|c|c|c|c|c|}
\hline & \multirow{3}{*}{\begin{tabular}{|l} 
Village \\
Code
\end{tabular}} & \multirow{3}{*}{ Area/ Main Village } & \multirow{3}{*}{ Village } & \multirow{3}{*}{$\begin{array}{c}\text { Total } \\
\text { Cultivated } \\
\text { Land }\left(\text { Jerib }^{*}\right) \\
\end{array}$} & \multirow{2}{*}{\multicolumn{2}{|c|}{$\begin{array}{c}\text { Land Under } \\
\text { Poppy Cultivation }\end{array}$}} & \multirow{3}{*}{$\begin{array}{c}\text { Wet Opium *2 } \\
\text { Yield } \\
(\mathrm{kg} / \mathrm{jr}) \\
\end{array}$} & \multirow{3}{*}{$\begin{array}{c}\text { Wet Opium } \\
\text { Production } \\
(\mathrm{kg})\end{array}$} \\
\hline \multirow[t]{2}{*}{ District } & & & & & & & & \\
\hline & & & & & (jerib) & $(\%)$ & & \\
\hline \multicolumn{9}{|c|}{ Province: Kunar } \\
\hline SARKANI & 4057 & NANGAHSAR & NANGAHSAR & 115 & 0 & 0 & 0 & 0 \\
\hline SARKANI & 4060 & DONAHI & BARCHAM & 450 & 7 & 2 & 13 & 91 \\
\hline SARKANI & 4061 & DARA SHARENGRY & MULAGOREY & 70 & 3 & 4 & 11 & 33 \\
\hline SARKANI & 4062 & DARA SHARENGRY & GHOHTOONKEY & 430 & 6 & 1 & 15 & 90 \\
\hline SARKANI & 4063 & DARA SHONGAREY & WACHAH JAURAH & 42 & 2 & 5 & 11 & 22 \\
\hline SARKANI & 4064 & DARA SHONGAREY & GARGO & 210 & 2 & 1 & 13 & 26 \\
\hline SARKANI & 4065 & DARA SHONGAREY & GARGAREY & 68 & 2 & 3 & 14 & 28 \\
\hline SARKANI & 4066 & DARA SHONGAREY & SECANDARAH/DORDANAI & 65 & 1 & 2 & 15 & 15 \\
\hline SARKANI & 4067 & DARA SHONGAREY & KHAD-E-KHAIL & 130 & 1 & 1 & 15 & 15 \\
\hline SARKANI & 4068 & DARA SHONGAREY & SECANDARAH & 70 & 2 & 3 & 13 & 26 \\
\hline SARKANI & 4069 & DARA SHONGAREY & SOBAGEY & 60 & 2 & 3 & 14 & 28 \\
\hline SARKANI & 4072 & DARA SHONGAREY & MANOGAI & 70 & 1 & 1 & 14 & 14 \\
\hline SARKANI & 4073 & DARA SHONGAREY & BAGH & 40 & 0 & 0 & 0 & 0 \\
\hline SARKANI & 4074 & DARA SHONGAREY & SHANAH JAURAH & 32 & 0 & 0 & 0 & 0 \\
\hline SARKANI & 4075 & DARA SHONGAREY & DEWANAH DEMBEY & 40 & 0 & 0 & 0 & 0 \\
\hline SARKANI & 4076 & DARA SHONGAREY & BAR-QALAUNAH & 33 & 0 & 0 & 0 & 0 \\
\hline SARKANI & 4211 & GULAPARI & GULAPARI & 135 & 1 & 1 & 9 & 9 \\
\hline SARKANI & 4213 & NOLIMATUNAH & NOLIMATUNAH & 50 & 2 & 4 & 10 & 20 \\
\hline SARKANI & 4214 & WARGHALI & WARGHALI & 15 & 0 & 0 & 0 & 0 \\
\hline SARKANI & 4216 & KOCHIYANO BELAH & KOCHIANO BELA & 1,100 & 20 & 2 & 19 & 380 \\
\hline SARKANI & 4217 & SARKANI & SARKANI & 1,400 & 25 & 2 & 17 & 425 \\
\hline SARKANI & 4218 & GANJGAL & DAM DARAH & 200 & 6 & 3 & 10 & 60 \\
\hline SARKANI & 4225 & BAROGI & LANDY KALAY & 100 & 2 & 2 & 9 & 18 \\
\hline SARKANI & 4227 & NAWAH/BABARAH & BABARAH & 48 & 3 & 6 & 10 & 30 \\
\hline SARKANI & 4228 & NAWAH & NAWAH & 52 & 6 & 12 & 11 & 66 \\
\hline SARKANI & 4236 & PASHAD & JIBA & 78 & 0 & 0 & 0 & 0 \\
\hline SARKANI & 4239 & PASHAD & DAMAN & 100 & 1 & 1 & 8 & 8 \\
\hline SARKANI & 4464 & PASHAD & PASHAD & 980 & 0 & 0 & 0 & 0 \\
\hline SARKANI & 4465 & BAHRAHBAD & BAHRAHBAD & 400 & 14 & 4 & 12 & 168 \\
\hline
\end{tabular}


Opium Production Survey by village in Kunar province, 1995.

\begin{tabular}{|c|c|c|c|c|c|c|c|c|}
\hline & \multirow{3}{*}{$\begin{array}{l}\text { Village } \\
\text { Code }\end{array}$} & \multirow{3}{*}{ Area/ Main Village } & \multirow{3}{*}{ Village } & \multirow{3}{*}{$\begin{array}{c}\text { Total } \\
\text { Cultivated } \\
\left.\text { Land (Jerib }{ }^{*}\right) \\
\end{array}$} & \multirow{2}{*}{\multicolumn{2}{|c|}{$\begin{array}{c}\text { Land Under } \\
\text { Poppy Cultivation }\end{array}$}} & \multirow{3}{*}{$\begin{array}{c}\text { Wet Opium *2 } \\
\text { Yield } \\
(\mathrm{kg} / \mathrm{jr}) \\
\end{array}$} & \multirow{3}{*}{$\begin{array}{l}\text { Wet Opium } \\
\text { Production } \\
\quad(\mathrm{kg}) \\
\end{array}$} \\
\hline \multirow[t]{2}{*}{ District } & & & & & & & & \\
\hline & & & & & (jerib) & $(\%)$ & & \\
\hline \multicolumn{9}{|c|}{ Province: Kunar } \\
\hline SARKANI & 4466 & TOOT NAU & TOOTNAU & 100 & 0 & 0 & 0 & 0 \\
\hline SARKANI & 4467 & DARA SHONGAREY & PATASALY & 40 & 2 & 5 & 11 & 22 \\
\hline SARKANI & 4468 & DARA SHONGAREY & SHEKHAL & 140 & 2 & 1 & 15 & 30 \\
\hline SARKANI & 4469 & DARA SHONGAREY & KUZ QALAUNAH & 30 & 0 & 0 & 0 & 0 \\
\hline SARKANI & 4476 & GALAH & GALAH & 70 & 3 & 4 & 10 & 30 \\
\hline SARKANI & 4479 & KOTAKAY & KOTAKAY & 18 & 1 & 6 & 9 & 9 \\
\hline
\end{tabular}


Opium Production Survey by village in Nangarhar province, 1995.

\begin{tabular}{|c|c|c|c|c|c|c|c|c|}
\hline \multirow{3}{*}{ District } & \multirow{3}{*}{$\begin{array}{l}\text { Village } \\
\text { Code }\end{array}$} & \multirow{3}{*}{ Area/ Main Village } & \multirow{3}{*}{ Village } & \multirow{3}{*}{$\begin{array}{c}\text { Total } \\
\text { Cultivated } \\
\text { Land }\left(\text { jerib }{ }^{*}\right) \\
\end{array}$} & \multirow{2}{*}{\multicolumn{2}{|c|}{$\begin{array}{c}\text { Land Under } \\
\text { Poppy Cultivation }\end{array}$}} & \multirow{3}{*}{$\begin{array}{c}\text { Wet Opium }^{* 2} \\
\text { Yield } \\
(\mathrm{kg} / \mathrm{jr} .) \\
\end{array}$} & \multirow{3}{*}{$\begin{array}{c}\text { Wet Opium } \\
\text { Production } \\
(\mathrm{kg})\end{array}$} \\
\hline & & & & & & & & \\
\hline & & & & & (jerib) & $(\%)$ & & \\
\hline \multicolumn{9}{|c|}{ Province: Nangarhar } \\
\hline ACHIN & 3407 & ABDUL KHAIL & CHINI MANZ & 150 & 43 & 29 & 14 & 602 \\
\hline ACHIN & 1141 & ABDUL KHAIL & NAWGAI & 300 & 56 & 19 & 9 & 504 \\
\hline ACHIN & 1085 & ABDUL KHAIL & SURAY GHARA & 94 & 42 & 45 & 9 & 357 \\
\hline ACHIN & 3408 & ABDUL KHAIL & TOGHAN & 30 & 3 & 10 & 8 & 24 \\
\hline ACHIN & 1953 & ABDUL KHAIL & ZHAY KALAY & 100 & 42 & 42 & 15 & 630 \\
\hline ACHIN & 2657 & ABDUL KHALIL & DAMGAL KHOLA & 100 & 38 & 38 & 9 & 342 \\
\hline ACHIN & 3401 & ABDUL KHALIL & MANO CHINA & 50 & 25 & 50 & 25 & 625 \\
\hline ACHIN & 3402 & ABDUL KHALIL/NARGOSA & DAGRAI & 325 & 104 & 32 & 9 & 936 \\
\hline ACHIN & 2661 & ABDUL KHEL & ASH KHEL & 800 & 47 & 6 & 10 & 470 \\
\hline ACHIN & 1075 & ABDUL KHEL & BAGH DARA & 210 & 20 & 10 & 12 & 240 \\
\hline ACHIN & 1951 & ABDUL KHEL & CHINE (MOHMAND KALAY & 200 & 70 & 35 & 11 & 770 \\
\hline ACHIN & 1088 & ABDUL KHEL & DAWLAT KHAIL & 150 & 90 & 60 & 11 & 990 \\
\hline ACHIN & 2658 & ABDUL KHEL & DOMGAL & 150 & 88 & 59 & 8 & 704 \\
\hline ACHIN & 1073 & ABDUL KHEL & GAR KOTO KALA & 50 & 14 & 28 & 14 & 196 \\
\hline ACHIN & 3388 & ABDUL KHEL & KANDO & 50 & 27 & 54 & 13 & 351 \\
\hline ACHIN & 1086 & ABDUL KHEL & KOLALAN & 200 & 100 & 50 & 14 & 1,400 \\
\hline ACHIN & 1087 & ABDUL KHEL & MUZDAKAY & 200 & 120 & 60 & 11 & 1,320 \\
\hline ACHIN & 3405 & ABDUL KHEL & NARGOSH & 200 & 65 & 33 & 10 & 650 \\
\hline ACHIN & 2660 & ABDUL KHEL & SURAY GHARA & 100 & 20 & 20 & 9 & 170 \\
\hline ACHIN & 2659 & ABDUL KHEL(NARGOSIA) & GERDI JOMAT & 100 & 37 & 37 & 8 & 296 \\
\hline ACHIN & 1076 & ABDUL KHEL/BAGHDARA & SAWARU BAND & 31 & 4 & 13 & 8 & 32 \\
\hline ACHIN & 1924 & AKHONDZADAGAN & ASH KHAIL & 100 & 35 & 35 & 8 & 280 \\
\hline ACHIN & 2636 & ASAD KHAIL & KHUM BEK & 600 & 350 & 58 & 10 & 3,500 \\
\hline ACHIN & 1948 & BAHLOL & BAHLOL & 100 & 35 & 35 & 8 & 280 \\
\hline ACHIN & 1929 & BANDAR & BAR ZARI KHOLEH & 80 & 9 & 11 & 9 & 81 \\
\hline ACHIN & 3400 & BANDAR & BARO & 110 & 41 & 37 & 12 & 492 \\
\hline ACHIN & 3392 & BANDAR & JAMAL KHEL & 200 & 104 & 52 & 17 & 1,768 \\
\hline ACHIN & 1077 & BANDAR & KHODAY KHOLEH & 15 & 1 & 7 & 10 & 10 \\
\hline ACHIN & 1931 & BANDAR & KONDI BORJ & 50 & 12 & 24 & 14 & 168 \\
\hline
\end{tabular}

* Jerib (jr.) $=1 / 5 \mathrm{ha}$

* ${ }^{2}$ Dry Opium $=$ Wet Opium $-30 \%$ moisture
Page 1
Source: UNDCP Opium Production Survey, 1995. File: NGR2.XLS - 06/08/9 
Opium Production Survey by village in Nangarhar province, 1995.

\begin{tabular}{|c|c|c|c|c|c|c|c|c|}
\hline \multirow{3}{*}{ District } & \multirow{3}{*}{$\begin{array}{l}\text { Village } \\
\text { Code }\end{array}$} & \multirow{3}{*}{ Area/ Main Village } & \multirow{3}{*}{ Village } & \multirow{3}{*}{$\begin{array}{c}\text { Total } \\
\text { Cultivated } \\
\text { Land }\left(\text { jerib }{ }^{*}\right)\end{array}$} & \multirow{2}{*}{\multicolumn{2}{|c|}{$\begin{array}{c}\text { Land Under } \\
\text { Poppy Cultivation }\end{array}$}} & \multirow{3}{*}{$\begin{array}{c}\text { Wet Opium *2 } \\
\text { Yield } \\
(\mathrm{kg} / \mathrm{jr} .)\end{array}$} & \multirow{3}{*}{$\begin{array}{l}\text { Wet Opium } \\
\text { Production } \\
\text { (kg) }\end{array}$} \\
\hline & & & & & & & & \\
\hline & & & & & (jerib) & $(\%)$ & & \\
\hline \multicolumn{9}{|c|}{ Province: Nangarhar } \\
\hline ACHIN & 1082 & BANDAR & KUZ ZARI KHOLEH & 70 & 5 & 7 & 10 & 50 \\
\hline ACHIN & 1930 & BANDAR & LANDAY & 115 & 22 & 19 & 11 & 242 \\
\hline ACHIN & 1083 & BANDAR & OBI KALAY & 40 & 4 & 10 & 11 & 44 \\
\hline ACHIN & 1942 & BANDAR & ORYA KHOLEH & 20 & 1 & 5 & 9 & 9 \\
\hline ACHIN & 1943 & BANDAR & SHINSHAH-I-KANDAW & 15 & 2 & 13 & 10 & 20 \\
\hline ACHIN & 1081 & BANDAR & SHOLGAR GHUNDAY & 120 & 72 & 60 & 17 & 1,224 \\
\hline ACHIN & 1078 & BANDAR & SIN GOKHA KHOLEH & 55 & 15 & 27 & 14 & 210 \\
\hline ACHIN & 1938 & BANDAR & SPARAWUNE KALAY & 100 & 55 & 55 & 17 & 935 \\
\hline ACHIN & 1079 & BANDAR & SPIN ZHAY KALAY & 100 & 10 & 10 & 9 & 90 \\
\hline ACHIN & 3391 & BANDAR & WACH BANDAR & 10 & 1 & 10 & 8 & 8 \\
\hline ACHIN & 1939 & BANDAR & ZER GHALI & 200 & 72 & 36 & 12 & 864 \\
\hline ACHIN & 2655 & BANDAR KHOLA & BANDAR KHOLA & 150 & 60 & 40 & 16 & 960 \\
\hline ACHIN & 3394 & BANDAR KHOLA & MARZI & 150 & 50 & 33 & 13 & 650 \\
\hline ACHIN & 1916 & BATAN & BATAN & 200 & 137 & 69 & 15 & 2,055 \\
\hline ACHIN & 1069 & CHEHEL GAZI & LOKHAI & 180 & 50 & 28 & 13 & 650 \\
\hline ACHIN & 2645 & CHEHL GAZAI & ENZARAY & 300 & 120 & 40 & 12 & 1,440 \\
\hline ACHIN & 1070 & CHEHL GAZAI & KUZ CHEHL GAZAI & 900 & 444 & 49 & 10 & 4,440 \\
\hline ACHIN & 1932 & CHEHL GAZAI & MANZ CHEHLGAZAI & 450 & 230 & 51 & 13 & 2,990 \\
\hline ACHIN & 1071 & CHEL GAZAI & CHEL GAZAI & 350 & 185 & 53 & 10 & 1,850 \\
\hline ACHIN & 1068 & CHEL GAZAI & HOJUMA QALA & 300 & 180 & 60 & 10 & 1,800 \\
\hline ACHIN & 3411 & CHINA & CHINA & 400 & 155 & 39 & 15 & 2,325 \\
\hline ACHIN & 1946 & CHINA (BABA KILY) & CHINA (BABA KILY) & 80 & 52 & 65 & 8 & 416 \\
\hline ACHIN & 1921 & DEH SARAK & $\mathrm{KACH}$ & 300 & 210 & 70 & 16 & 3,360 \\
\hline ACHIN & 2646 & DEH SARAK & KOHNATER & 520 & 376 & 72 & 16 & 6,016 \\
\hline ACHIN & 2654 & DEH SARAK & PANSAR & 35 & 19 & 54 & 15 & 285 \\
\hline ACHIN & 3409 & DEH SARAK & PIRO KHAIL & 580 & 298 & 51 & 17 & 5,066 \\
\hline ACHIN & 3399 & DESARAK & ASH KHAIL & 70 & 46 & 66 & 13 & 598 \\
\hline ACHIN & 3393 & DESARAK & LALMA & 55 & 21 & 38 & 15 & 315 \\
\hline ACHIN & 1914 & DONI (SARI KOTI) & DONI (SARI KOTI) & 110 & 40 & 36 & 10 & 400 \\
\hline
\end{tabular}

* Jerib (jr.) $=1 / 5$ ha

${ }^{*}$ Dry Opium $=$ Wet Opium $-30 \%$ moisture
Page 292
Source: UNDCP Opium Production Survey, 1995 File: NGR2.XLS - 06/08/95 
Opium Production Survey by village in Nangarhar province, 1995.

\begin{tabular}{|c|c|c|c|c|c|c|c|c|}
\hline \multirow{3}{*}{ District } & \multirow{3}{*}{$\begin{array}{l}\text { Village } \\
\text { Code }\end{array}$} & \multirow{3}{*}{ Area/ Main Village } & \multirow{3}{*}{ Village } & \multirow{3}{*}{$\begin{array}{c}\text { Total } \\
\text { Cultivated } \\
\text { Land (jerib }{ }^{*} \text { ) } \\
\end{array}$} & \multirow{2}{*}{\multicolumn{2}{|c|}{$\begin{array}{c}\text { Land Under } \\
\text { Poppy Cultivation }\end{array}$}} & \multirow{3}{*}{$\begin{array}{c}\text { Wet Opium }{ }^{* 2} \\
\text { Yield } \\
(\mathrm{kg} / \mathrm{jr} .) \\
\end{array}$} & \multirow{3}{*}{$\begin{array}{c}\text { Wet Opium } \\
\text { Production } \\
\text { (kg) }\end{array}$} \\
\hline & & & & & & & & \\
\hline & & & & & (jerib) & $(\%)$ & & \\
\hline \multicolumn{9}{|c|}{ Province: Nangarhar } \\
\hline ACHIN & 2623 & DORAGAY & DORAGAY & 55 & 20 & 36 & 10 & 200 \\
\hline ACHIN & 1906 & GAWA & GAWA & 120 & 70 & 58 & 14 & 980 \\
\hline ACHIN & 1934 & GERDI & GERDI & 600 & 355 & 59 & 11 & 3,905 \\
\hline ACHIN & 2651 & GHANI & GHANI & 200 & 100 & 50 & 13 & 1,300 \\
\hline ACHIN & 2651 & GHANI KHAIL & WARA KHOGA KHEL & 100 & 55 & 55 & 11 & 605 \\
\hline ACHIN & 1950 & GOSHTAL & DAWLAT KHEL & 150 & 65 & 43 & 14 & 910 \\
\hline ACHIN & 3490 & GOSHTAL & GATO & 50 & 20 & 40 & 12 & 240 \\
\hline ACHIN & 1089 & GOSHTAL & HASAN KHEL & 80 & 30 & 38 & 11 & 330 \\
\hline ACHIN & 2650 & GOSHTAL & KHURAM KHEL & 80 & 30 & 38 & 13 & 390 \\
\hline ACHIN & 1090 & GOSHTAL & SANDUQ & 300 & 105 & 35 & 13 & 1,365 \\
\hline ACHIN & 1901 & GUL POR & GUL POR & 25 & 9 & 36 & 9 & 81 \\
\hline ACHIN & 3398 & KAHI & KOCHNAI KAHI & 100 & 55 & 55 & 13 & 715 \\
\hline ACHIN & 1918 & KAMKAI & KAMKAI & 280 & 170 & 61 & 15 & 2,550 \\
\hline ACHIN & 3412 & KANDAR & GAWAH KHOLA & 120 & 45 & 38 & 7 & 315 \\
\hline ACHIN & 3396 & KANDAR & MIAGAN & 160 & 75 & 47 & 8 & 600 \\
\hline ACHIN & 2643 & KARKANAI & KARKANAI & 260 & 100 & 38 & 10 & 1,000 \\
\hline ACHIN & 3395 & KATARTOT & SULTAN & 70 & 30 & 43 & 13 & 390 \\
\hline ACHIN & 2628 & KHAS ACHIN & CHINAR & 25 & 15 & 60 & 10 & 150 \\
\hline ACHIN & 1904 & KHAS ACHIN & DABONA & 120 & 45 & 38 & 10 & 450 \\
\hline $\mathrm{ACHIN}$ & 2629 & KHAS ACHIN & KHAS ACHIN & 500 & 242 & 48 & 12 & 2,904 \\
\hline $\mathrm{ACHIN}$ & 2627 & KHAS ACHIN & KOBZAI & 60 & 40 & 67 & 11 & 440 \\
\hline ACHIN & 2626 & KHAS ACHIN & KONJ AWLIA & 20 & 7 & 35 & 11 & 77 \\
\hline ACHIN & 3491 & KHAS ACHIN & TARILAI & 400 & 195 & 49 & 14 & 2,730 \\
\hline ACHIN & 2653 & $\mathrm{KOHI}$ & KOHI & 200 & 75 & 38 & 17 & 1,275 \\
\hline ACHIN & 2652 & $\mathrm{KOHI}$ & PAKHEL & 500 & 285 & 57. & 11 & 3,135 \\
\hline ACHIN & 1945 & LANDAY BAZAR SRAKMA & LANDAY BAZAR SRAKMA & 400 & 220 & 55 & 12 & 2,640 \\
\hline ACHIN & 2644 & MAIDANAK & ALI KHEL & 490 & 315 & 64 & 9 & 2,835 \\
\hline ACHIN & 1919 & MAIDANAK & BAHRAM KHAIL & 200 & 90 & 45 & 10 & 900 \\
\hline ACHIN & 1920 & MAIDANAK & KAMAR SAR & 300 & 200 & 67 & 9 & 1,800 \\
\hline
\end{tabular}

* Jerib (jr.) $=1 / 5$ ha

${ }^{*}{ }^{2}$ Dry Opium $=$ Wet Opium $-30 \%$ moisture
Page 393
Source: UNDCP Opium Production Survey, 1995 File: NGR2.XLS - 06/08/95 
Opium Production Survey by village in Nangarhar province, 1995.

\begin{tabular}{|c|c|c|c|c|c|c|c|c|}
\hline & \multirow{3}{*}{$\begin{array}{l}\text { Village } \\
\text { Code }\end{array}$} & \multirow{3}{*}{ Area/ Main Village } & \multirow{3}{*}{ Village } & \multirow{3}{*}{$\begin{array}{c}\text { Total } \\
\text { Cultivated } \\
{\text { Land }\left(\text { jerib }^{*}\right)}^{*} \\
\end{array}$} & \multirow{2}{*}{\multicolumn{2}{|c|}{$\begin{array}{c}\text { Land Under } \\
\text { Poppy Cultivation }\end{array}$}} & \multirow{3}{*}{$\begin{array}{c}\text { Wet Opium }{ }^{* 2} \\
\text { Yield } \\
(\mathrm{kg} / \mathrm{jr} .)\end{array}$} & \multirow{3}{*}{$\begin{array}{c}\text { Wet Opium } \\
\text { Production } \\
\text { (kg) }\end{array}$} \\
\hline \multirow[t]{2}{*}{ District } & & & & & & & & \\
\hline & & & & & (jerib) & $(\%)$ & & \\
\hline \multicolumn{9}{|c|}{ Province: Nangarhar } \\
\hline ACHIN & 1935 & MAIDANAK & KHARKHANI KALAY & 500 & 300 & 60 & 9 & 2,700 \\
\hline ACHIN & 1067 & MAIDANAK & SHAHMIR KALAY & 280 & 195 & 70 & 14 & 2,730 \\
\hline ACHIN & 1936 & MAMAND & AMBER MELA & .50 & 7 & 14 & 6 & 42 \\
\hline ACHIN & 1066 & MAMAND & MAKRANAY & 150 & 85 & 57 & 10 & 850 \\
\hline ACHIN & 1933 & MAMAND & MARO CHINA & 620 & 290 & 47 & 10 & 2,900 \\
\hline ACHIN & 1937 & MAMAND & SPINKAI MURGHA & 80 & 15 & 19 & 6 & 90 \\
\hline ACHIN & 1912 & MARGHA & MARGHA & 200 & 100 & 50 & 8 & 800 \\
\hline ACHIN & 1913 & MOMAND & DAB KALAY & 60 & 35 & 58 & 9 & 315 \\
\hline ACHIN & 1908 & MOMAND & KHANAKAY & 100 & 0 & 0 & 0 & 0 \\
\hline ACHIN & 1947 & MOMAND & KHARWAY & 50 & 0 & 0 & 0 & 0 \\
\hline ACHIN & 2635 & MORGHAN GHONDAI & MORGHAN GHONDAI & 250 & 145 & 58 & 14 & 2,030 \\
\hline ACHIN & 3420 & MURGAI & MURGAI & 100 & 45 & 45 & 10 & 450 \\
\hline ACHIN & 1048 & NARGOSA & BABA SAHEB & 200 & 65 & 33 & 9 & 585 \\
\hline ACHIN & 3414. & NARGOSAI & NARGOSAI & 120 & 30 & 25 & 7 & 210 \\
\hline ACHIN & 2631 & PAKHAIL & PAKHAIL & 320 & 200 & 63 & 14 & 2,800 \\
\hline ACHIN & 3404 & PAKHEL & SHIKH MERKHEL & 120 & 55 & 46 & 11 & 605 \\
\hline ACHIN & 2633 & PESHAH & DOKAN KILY & 300 & 123 & 41 & 14 & 1,722 \\
\hline ACHIN & 3389 & PESHAH & GHAR KILY & 140 & 45 & 32 & 12 & 540 \\
\hline ACHIN & 2638 & PESHAH & LAWAR & 620 & 74 & 12 & 12 & 888 \\
\hline ACHIN & 1898 & PESHAH & MIRAN & 200 & 76 & 38 & 12 & 912 \\
\hline ACHIN & 1897 & PESHAH & WOCH ZHAWAR & 50 & 3 & 6 & 9 & 27 \\
\hline ACHIN & 2632 & PINBA & JAWAR GAR & 300 & 176 & 59 & 14 & 2,464 \\
\hline ACHIN & 2630 & PINBA & KANDAR & 150 & 70 & 47 & 14 & 980 \\
\hline ACHIN & 1899 & PINBA & KATARTOT & 150 & 70 & 47 & 15 & 1,050 \\
\hline ACHIN & 2648 & PINBA KHOR & AKHONDZADAGAN & 150 & 80 & 53 & 15 & 1,200 \\
\hline ACHIN & 3410 & PISHA & SAPARAI & 110 & 0 & 0 & 0 & 0 \\
\hline ACHIN & 3390 & PISHA & SHEKHAN KALAY & 20 & 0 & 0 & 0 & 0 \\
\hline ACHIN & 1923 & PISHA KHWAR & KARYAN & 250 & 163 & 65 & 15 & 2,445 \\
\hline ACHIN & 1949 & PISHA KHWAR & KHOGA KHAIL & 200 & 110 & 55 & 14 & 1,540 \\
\hline
\end{tabular}

* Jerib (jr.) $=1 / 5$ ha

* ${ }^{2}$ Dry Opium $=$ Wet Opium - $30 \%$ moisture
Page 494
Source: UNDCP Opium Production Survey, 1995. File: NGR2.XLS - 06/08/95 
Opium Production Survey by village in Nangarhar province, 1995.

\begin{tabular}{|c|c|c|c|c|c|c|c|c|}
\hline & Village & & & Total & Land $L$ & & Wet Opium *2 & Wet Opium \\
\hline \multirow[t]{2}{*}{ District } & \multirow{2}{*}{ Code } & \multirow[t]{2}{*}{ Area/ Main Village } & \multirow[t]{2}{*}{ Village } & \multirow{2}{*}{$\begin{array}{c}\text { Cultivated } \\
\text { Land (jerib }^{*} \text { ) }\end{array}$} & \multicolumn{2}{|c|}{ Poppy Cultivation } & \multirow{2}{*}{$\begin{array}{c}\text { Yield } \\
(\mathrm{kg} / \mathrm{jr} .) \\
\end{array}$} & \multirow{2}{*}{$\begin{array}{c}\text { Production } \\
\text { (kg) }\end{array}$} \\
\hline & & & & & (jerib) & $(\%)$ & & \\
\hline \multicolumn{9}{|c|}{ Province: Nangarhar } \\
\hline ACHIN & 2649 & PISHA KHWAR & MULAYAN/HAJIANO KALA & 50 & 30 & 60 & 13 & 390 \\
\hline ACHIN & 3403 & PISHA KHWAR & WIALA & 250 & 125 & 50 & 13 & 1,625 \\
\hline ACHIN & 3406 & RAGHA & HAJI LALUKI & 80 & 30 & 38 & 11 & 330 \\
\hline ACHIN & 3397 & RAGHA & JABAR & 90 & 32 & 36 & 12 & 384 \\
\hline ACHIN & 2634 & RAGHA & RAGHA & 130 & 48 & 37 & 14 & 672 \\
\hline ACHIN & 3419 & SABINO KHOLEH & SABINO KHOLEH & 60 & 5 & 8 & 10 & 50 \\
\hline ACHIN & 3413 & SEPORI & SEPORI & 90 & 40 & 44 & 8 & 320 \\
\hline ACHIN & 3416 & SHEDOKANI & SHEDOKANI & 200 & 60 & 30 & 8 & 480 \\
\hline ACHIN & 3417 & SHEEN SHUBAI & SHEEN SHUBAI & 10 & 1 & 10 & 6 & 6 \\
\hline ACHIN & 2647 & SHEKHMAN KHAIL & SHEKHMAN KHAIL & 200 & 95 & 48 & 15 & 1,425 \\
\hline ACHIN & 3380 & SHOND KANDA & SHOND KANDA & 230 & 85 & 37. & 8 & 680 \\
\hline ACHIN & 1911 & SPARAI & SPARAI & 70 & 12 & 17 & 10 & 120 \\
\hline ACHIN & 1926 & SRA KALA & SRA KALA & 100 & 40 & 40 & 9 & 360 \\
\hline ACHIN & 1927 & SRAH QALA & LANDAY & 60 & 22 & 37 & 11 & 242 \\
\hline ACHIN & 2625 & SUROBI & SUROBI & 80 & 35 & 44 & 8 & 280 \\
\hline ACHIN & 3418 & SUTKAI & SUTKAI & 35 & 3 & 9 & 9 & 27 \\
\hline ACHIN & 1915 & TANGA KILY & TANGA KILY & 20 & 8 & 40 & 9 & 72 \\
\hline ACHIN & 3415 & WACH ZHAWAR & WACH ZHAWAR & 35 & 0 & 0 & 0 & 0 \\
\hline ACHIN & 3379 & WATCHKOT & SAHIBZADAGAN & 100 & 55 & 55 & 8 & 440 \\
\hline ACHIN & 1903 & WATCHKOT & SANGORAY & 60 & 15 & 25 & 7 & 105 \\
\hline BATI KOT & 3247 & ANBAR KHANA & AKHONZADAGAN & 250 & 120 & 48 & 16 & 1,920 \\
\hline BATI KOT & 3448 & ANBAR KHANA & AOTAKI & 40 & 9 & 23 & 13 & 117 \\
\hline BATI KOT & 3260 & ANBAR KHANA & AYNI KHAN KHAIL & 340 & 126 & 37 & 13 & 1,638 \\
\hline BATI KOT & 3257 & ANBAR KHANA & GARDAB & 150 & 56 & 37 & 12 & 672 \\
\hline BATI KOT & 3255 & BARIKAB & ALI KHAIL & 2,000 & 0 & 0 & 0 & 0 \\
\hline BATI KOT & 3252 & BARIKAB & $\mathrm{KOZ}$ & 1,200 & 0 & 0 & 0 & 0 \\
\hline BATI KOT & 3256 & BARIKAB & PARAW & 100 & 0 & 0 & 0 & 0 \\
\hline BATI KOT & 3261 & BARIKAB & QALA WAAL & 150 & 0 & 0 & 0 & 0 \\
\hline BATI KOT & 3259 & BARIKAB & RAGHA & 100 & 0 & 0 & 0 & 0 \\
\hline
\end{tabular}

* Jerib (jr.) $=1 / 5$ ha

* 2 Dry Opium = Wet Opium - $30 \%$ moisture
Page 5
Source: UNDCP Opium Production Survey, 1995. File: NGR2.XLS - 06/08/95 
Opium Production Survey by village in Nangarhar province, 1995.

\begin{tabular}{|c|c|c|c|c|c|c|c|c|}
\hline & Village & 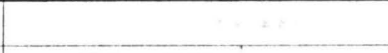 & & Total & Land 1 & & Wet Opium * 2 & Wet Opium \\
\hline \multirow[t]{2}{*}{ District } & \multirow[t]{2}{*}{ Code } & \multirow[t]{2}{*}{ Area/ Main Village } & \multirow[t]{2}{*}{ Village } & \multirow{2}{*}{$\begin{array}{c}\text { Cultivated } \\
\left.\text { Land (jerib }{ }^{*}\right)\end{array}$} & \multicolumn{2}{|c|}{ Poppy Cultivation } & \multirow{2}{*}{$\begin{array}{c}\text { Yield } \\
(\mathrm{kg} / \mathrm{jr} .)\end{array}$} & \multirow{2}{*}{$\begin{array}{c}\text { Production } \\
(\mathrm{kg})\end{array}$} \\
\hline & & & & & (jerib) & $(\%)$ & & \\
\hline \multicolumn{9}{|c|}{ Province: Nangarhar } \\
\hline BATI KOT & 3244 & CHARDEHI & HASAN KHAIL & 100 & 25 & 25 & 13 & 325 \\
\hline BATI KOT & 3258 & CHARDEHI & LAR & 300 & 56 & 19 & 12 & 672 \\
\hline BATI KOT & 3295 & CHARDEHI & MAHIYANO JAMAAT & 900 & 162 & 18 & 12 & 1,944 \\
\hline BATI KOT & 3263 & CHARDEHI & MIRAN & 120 & 22 & 18 & 13 & 286 \\
\hline BATI KOT & 3239 & CHARDEHI & NIKOZAI & 200 & 48 & 24 & 14 & 672 \\
\hline BATI KOT & 3249 & CHARDEHI & OSMAN KHAIL & 250 & 50 & 20 & 14 & 700 \\
\hline BATI KOT & 3240 & CHARDEHI & SARGHANI & 200 & 38 & 19 & 13 & 494 \\
\hline BATI KOT & 3241 & CHARDEHI & SHIRA KHAIL & 100 & 20 & 20 & 12 & 240 \\
\hline BATI KOT & 3298 & DAG & BAKHTAKI & 600 & 60 & 10 & 14 & 840 \\
\hline BATI KOT & 3282 & DAG & NAZAR BEGI & 300 & 55 & 18 & 14 & 770 \\
\hline BATI KOT & 3290 & DAG & TOR KHEL & 250 & 50 & 20 & 15 & 750 \\
\hline BATI KOT & 3275 & DAMAN & GASHIANI & 300 & 10 & 3 & 9 & 90 \\
\hline BATI KOT & 3250 & DAMAN & LEWANYAN & 400 & 34 & 9 & 14 & 476 \\
\hline BATI KOT & 3274 & DAMAN & SARBAN & 200 & 14 & 7 & 10 & 140 \\
\hline BATI KOT & 3287 & GARAI & GARAI & 320 & 105 & 33 & 15 & 1,575 \\
\hline BATI KOT & 3269 & GARDIDAR & MATA KHAN & 150 & 10 & 7 & 12 & 120 \\
\hline BATI KOT & 3294 & GHAZIABAD & GHAZIABAD & 90 & 20 & 22 & 12 & 240 \\
\hline BATI KOT & 3265 & GHUNDAY & GHUNDAY & 200 & 0 & 0 & 0 & 0 \\
\hline BATI KOT & 3291 & GUNDYANI & GUNDYANI & 500 & 100 & 20 & 14 & 1,400 \\
\hline BATI KOT & 1021 & HADA & BANGASH MASJID & 200 & 3 & 2 & 14 & 42 \\
\hline BATI KOT & 3257 & HANBERKHAN & GRAPOVA & 200 & 95 & 48 & 13 & 1,235 \\
\hline BATI KOT & 3245 & HANBERKHANA & ARATONA & 130 & 78 & 60 & 12 & 936 \\
\hline BATI KOT & 3246 & HMBAR KHAN & KUCHNIYONO KELE & 25 & 23 & 92 & 11 & 253 \\
\hline BATI KOT & 3497 & KAREZ-E-AKHONZADAGAN & KAREZ-E-AKHONZADAGAN & 400 & 100 & 25 & 9 & 900 \\
\hline BATI KOT & 3262 & KODAY & KODAY & 500 & 65 & 13 & 12 & 780 \\
\hline BATI KOT & 3242 & LACHAPOR & LACHAPOR & 770 & 0 & 0 & 0 & 0 \\
\hline BATI KOT & 3254 & LACHAPOR & NAGHLO & 200 & 0 & 0 & 0 & 0 \\
\hline BATI KOT & 3267 & LORTI & NASRAT KHAIL & 200 & 5 & 3 & 10 & 50 \\
\hline BATI KOT & 3283 & LOWARTI & ALI KHAIL & 240 & 6 & 3 & 12 & 72 \\
\hline
\end{tabular}

* Jerib (jr.) $=1 / 5$ ha

* 2 Dry Opium = Wet Opium - $30 \%$ moisture
Page 696
Source: UNDCP Opium Production Survey, 1995 File: NGR2.XLS - 06/08/95 
Opium Production Survey by village in Nangarhar province, 1995.

\begin{tabular}{|c|c|c|c|c|c|c|c|c|}
\hline \multirow{3}{*}{ District } & \multirow{3}{*}{$\begin{array}{l}\text { Village } \\
\text { Code }\end{array}$} & \multirow{3}{*}{ Area/ Main Village } & \multirow{3}{*}{ Village } & \multirow{3}{*}{$\begin{array}{c}\text { Total } \\
\text { Cultivated } \\
\text { Land (jerib }{ }^{*} \text { ) } \\
\end{array}$} & \multirow{2}{*}{\multicolumn{2}{|c|}{$\begin{array}{c}\text { Land Under } \\
\text { Poppy Cultivation }\end{array}$}} & \multirow{3}{*}{$\begin{array}{c}\text { Wet Opium *2 } \\
\text { Yield } \\
(\mathrm{kg} / \mathrm{jr} .) \\
\end{array}$} & \multirow{3}{*}{$\begin{array}{c}\text { Wet Opium } \\
\text { Production } \\
(\mathrm{kg}) \\
\end{array}$} \\
\hline & & & & & & & & \\
\hline & & & & & (jerib) & $(\%)$ & & \\
\hline \multicolumn{9}{|c|}{ Province: Nangarhar } \\
\hline BATI KOT & 3278 & LOWARTI & SHAMA KHAIL & 200 & 23 & 12 & 12 & 276 \\
\hline BATI KOT & 3277 & MOSHWANI & $\mathrm{KOZ}$ & 160 & 0 & 0 & 0 & 0 \\
\hline BATI KOT & 3243 & MOSHWANI & MOSHWANI & 300 & 0 & 0 & 0 & 0 \\
\hline BATI KOT & 3251 & NAQILIN & NAQILIN JOY 20 & 200 & 0 & 0 & 0 & 0 \\
\hline BATI KOT & 3293 & NAWAQILI JOY 23 & NAWAQILI JOY 23 & 1,200 & 100 & 8 & 13 & 1,300 \\
\hline BATI KOT & 3253 & SAPAW & SHANDIANI & 500 & 45 & 9 & 10 & 450 \\
\hline BATI KOT & 3276 & SAPAW & ZOR KALAY & 200 & 23 & 12 & 12 & 276 \\
\hline BATI KOT & 3272 & SAPSAW & MOBIN & 50 & 0 & 0 & 0 & 0 \\
\hline BATI KOT & 3449 & SAR PAAY & RANA KHAN KELAY & 250 & 23 & 9 & 12 & 276 \\
\hline BATI KOT & 3273 & SEPSAW & LIWANIANO & 100 & 4 & 4 & 10 & 40 \\
\hline BATI KOT & 3279 & SHABAD BANI & JOOY -E-NAW & 300 & 42 & 14 & 10 & 420 \\
\hline BATI KOT & 3292 & SHABADIYANI & EBRAHIM KHAIL & 200 & 50 & 25 & 12 & 600 \\
\hline BATI KOT & 3280 & SHABADIYANI & GARNI & 180 & 50 & 28 & 12 & 600 \\
\hline BATI KOT & 3268 & SHABADIYANI & JANATIANO KELY & 120 & 23 & 19 & 11 & 253 \\
\hline BATI KOT & 3285 & SHABADIYANI & KELY KHAIL & 100 & 9 & 9 & 11 & 99 \\
\hline BATI KOT & 3271 & SHABDIYANI & CHUNI & 200 & 40 & 20 & 10 & 400 \\
\hline BATI KOT & 3270 & SHABDIYANI & GRAWIDAR & 100 & 18 & 18 & 11 & 198 \\
\hline BATI KOT & 3297 & SHABDIYANI & HAYBAT KHEL & 360 & 90 & 25 & 10 & 900 \\
\hline BATI KOT & 3289 & SHABDIYANI & KHUJA AHMADKHIL & 400 & 80 & 20 & 11 & 880 \\
\hline BATI KOT & 3284 & SHABDIYANI & SHASH KHEL & 200 & 40 & 20 & 13 & 520 \\
\hline BATI KOT & 3300 & SHASH KHAIL & SHASH KHAIL & 800 & 115 & 14 & 10 & 1,150 \\
\hline BATI KOT & 3299 & SHATI KHEL & HAJI BULBUL KEL & 300 & 75 & 25 & 11 & 825 \\
\hline BATI KOT & 3447 & TAKIA & BADLA KHAIL & 45 & 8 & 18 & 15 & 120 \\
\hline BATI KOT & 3296 & TAKIA & JANI KHAIL & 225 & 42 & 19 & 15 & 630 \\
\hline BATI KOT & 3264 & TAKIA & MOSA KHAIL & 380 & 55 & 14 & 14 & 770 \\
\hline BATI KOT & 3288 & TAKIA ALI KHAIL & TAKIA ALI KHAIL & 400 & 108 & 27 & 15 & 1,620 \\
\hline BATI KOT & 3286 & TOURTI & JANI KHEL & 300 & 0 & 0 & 0 & 0 \\
\hline BATI KOT & 3231 & TOURTI & MIRANO KELY & 800 & 15 & 2 & 12 & 180 \\
\hline BEHSUD & 1013 & ABDYAN & ABDYAN & 500 & 0 & 0 & 0 & 0 \\
\hline
\end{tabular}

* Jerib (jr.) $=1 / 5$ ha

*2 Dry Opium = Wet Opium - $30 \%$ moisture
Page $7 \quad 97$
Source: UNDCP Opium Production Survey, 1995. File: NGR2.XLS - 06/08/95 
Opium Production Survey by village in Nangarhar province, 1995.

\begin{tabular}{|c|c|c|c|c|c|c|c|c|}
\hline & Village & & & \multirow{3}{*}{$\begin{array}{c}\text { Total } \\
\text { Cultivated } \\
\text { Land (jerib }^{*} \text { ) } \\
\end{array}$} & \multirow{2}{*}{\multicolumn{2}{|c|}{$\begin{array}{c}\text { Land Under } \\
\text { Poppy Cultivation }\end{array}$}} & \multirow{3}{*}{$\begin{array}{c}\text { Wet Opium }^{* 2} \\
\text { Yield } \\
(\mathrm{kg} / \mathrm{jr} .) \\
\end{array}$} & \multirow{3}{*}{$\begin{array}{c}\text { Wet Opium } \\
\text { Production } \\
(\mathrm{kg})\end{array}$} \\
\hline \multirow[t]{2}{*}{ District } & Code & Area/ Main Village & Village & & & & & \\
\hline & & & & & (jerib) & $(\%)$ & & \\
\hline \multicolumn{9}{|c|}{ Province: Nangarhar } \\
\hline BEHSUD & 3451 & ALI & ALI & 300 & 0 & 0 & 0 & 0 \\
\hline BEHSUD & 3452 & ARABAN & ARABAN & 40 & 3 & 8 & 14 & 42 \\
\hline BEHSUD & 1054 & ARABAN & ARABANO GHONDI & 300 & 0 & 0 & 0 & 0 \\
\hline BEHSUD & 993 & ARABAN & KHURAM KHAIL & 120 & 0 & 0 & 0 & 0 \\
\hline BEHSUD & 992 & ARABAN & ZEKAMEL & 200 & 0 & 0 & 0 & 0 \\
\hline BEHSUD & 996 & ARABAN & ZILFAZEL & 200 & 0 & 0 & 0 & 0 \\
\hline BEHSUD & 998 & BAHRABAD & MIJER ABAD & 280 & 0 & 0 & 0 & 0 \\
\hline BEHSUD & 1005 & BANDAHE QAZYAN & BANDAHE QAZYAN & 200 & 0 & 0 & 0 & 0 \\
\hline BEHSUD & 1014 & BEHSUD-E-KHAS & BEHSUD-E-KHAS & 200 & 0 & 0 & 0 & 0 \\
\hline BEHSUD & 3500 & BELAND GAR & BELAND GAR & 60 & 0 & 0 & 0 & 0 \\
\hline BEHSUD & 1031 & BELAND GHAR & BENNEY MAH & 360 & 0 & 0 & 0 & 0 \\
\hline BEHSUD & 1037 & BELAND GHAR & KHUJA UMAR & 270 & 1 & 0 & 14 & 14 \\
\hline BEHSUD & 1038 & BELAND GHAR & QALA-E-HASAN & 90 & 0 & 0 & 0 & 0 \\
\hline BEHSUD & 1036 & BELAND GHAR & SHIKHALI & 250 & 0 & 0 & 0 & 0 \\
\hline BEHSUD & 1039 & CHAMYAR & CHAMYAR & 200 & 0 & 0 & 0 & 0 \\
\hline BEHSUD & 1002 & CHURAGALAI & VLAYATI & 100 & 0 & 0 & 0 & 0 \\
\hline BEHSUD & 1053 & DAMAN & DAMAN & 150 & 0 & 0 & 0 & 0 \\
\hline BEHSUD & 1015 & DARWAZGAY & DARWAZGAY & 100 & 0 & 0 & 0 & 0 \\
\hline BEHSUD & 1057 & DOBANDI & DOBANDI & 600 & 0 & 0 & 0 & 0 \\
\hline BEHSUD & 1006 & DOBELA & DOBELA & 200 & 0 & 0 & 0 & 0 \\
\hline BEHSUD & 1030 & GERDI KAC & GERDI KAC & 220 & 0 & 0 & 0 & 0 \\
\hline BEHSUD & 1042 & GERDI KAC & ZAKHEL & 150 & 0 & 0 & 0 & 0 \\
\hline BEHSUD & 3453 & GOWMESH BELA & GOWMESH BELA & 200 & 0 & 0 & 0 & 0 \\
\hline BEHSUD & 1008 & HADA & BAR MASJID & 90 & 2 & 2 & 15 & 30 \\
\hline BEHSUD & 1022 & HADA & CHOGHAKI & 270 & 0 & 0 & 0 & 0 \\
\hline BEHSUD & 1033 & HADA & HADA & 130 & 4 & 3 & 16 & 64 \\
\hline BEHSUD & 1032 & HADA & KOZ MASJED & 2,402 & 4 & 0 & 13 & 52 \\
\hline BEHSUD & 1034 & HADA & LOY MASJED & 500 & 25 & 5 & 14 & 350 \\
\hline BEHSUD & 1020 & HADA & MOLA KHEL & 170 & 3 & 2 & 14 & 42 \\
\hline
\end{tabular}


Opium Production Survey by village in Nangarhar province, 1995.

\begin{tabular}{|c|c|c|c|c|c|c|c|c|}
\hline & \multirow{3}{*}{$\begin{array}{l}\text { Village } \\
\text { Code }\end{array}$} & \multirow{3}{*}{ Area/ Main Village } & \multirow{3}{*}{ Village } & Total & \multicolumn{2}{|c|}{ Land Under } & \multirow{3}{*}{$\begin{array}{c}\text { Wet Opium *2 } \\
\text { Yield } \\
(\mathrm{kg} / \mathrm{jr} .) \\
\end{array}$} & \multirow{3}{*}{$\begin{array}{c}\text { Wet Opium } \\
\text { Production } \\
(\mathrm{kg}) \\
\end{array}$} \\
\hline \multirow[t]{2}{*}{ District } & & & & Cultivated & \multicolumn{2}{|c|}{ Poppy Cultivation } & & \\
\hline & & & & Land (jerib *) & (jerib) & $(\%)$ & & \\
\hline \multicolumn{9}{|c|}{ Province: Nangarhar } \\
\hline BEHSUD & 1060 & JAMALI & JAMALI & 600 & 0 & 0 & 0 & 0 \\
\hline BEHSUD & 1001 & JAMALI & KOCHIANO BANDAH & 40 & 0 & 0 & 0 & 0 \\
\hline BEHSUD & 1018 & JAMALI & SALOO & 400 & 0 & 0 & 0. & 0 \\
\hline BEHSUD & 1064 & KAKARYAN & KAKARYAN & 250 & 0 & 0 & 0 & 0 \\
\hline BEHSUD & 1059 & KARIZE KABIR & KARIZE KABIR & 500 & 2 & 0 & 14 & 28 \\
\hline BEHSUD & 1058 & KARIZE NAGHLU & KARIZE NAGHLU & 500 & 0 & 0 & 0 & 0 \\
\hline BEHSUD & 1027 & KHOSH GUNBAD & KHOSH GUNBAD & 350 & 0 & 0 & 0 & 0 \\
\hline BEHSUD & 1092 & KHOSH GUNBAD & QALAA-E- AKHOND & 100 & 0 & 0 & 0 & 0 \\
\hline BEHSUD & 999 & LAJGAR & LAJGAR & 150 & 0 & 0 & 0 & 0 \\
\hline BEHSUD & 1041 & MALEK BELA & MALEK BELA & 200 & 0 & 0 & 0 & 0 \\
\hline BEHSUD & 1028 & MAQAM KHAN & MAQAM KHAN & 500 & 40 & 8 & 14 & 560 \\
\hline BEHSUD & 1062 & MIRAN & MIRAN & 600 & 0 & 0 & 0 & 0 \\
\hline BEHSUD & 1065 & NAGHLU & NAGHLU & 700 & 3 & 0 & 17 & 51 \\
\hline BEHSUD & 1049 & NAHR SHAHI & NAHR SHAHI & 150 & 0 & 0 & 0 & 0 \\
\hline BEHSUD & 1026 & NAHR SHAHI 1 & QALA-I-SHAHI & 150 & 0 & 0 & 0 & 0 \\
\hline BEHSUD & 1024 & NAHR SHAHI 2 & BANDAHE TELA & 140 & 0 & 0 & 0 & 0 \\
\hline BEHSUD & 1047 & NAHR SHAHI 2 & QALA-I-DAWRAN & 80 & 0 & 0 & 0 & 0 \\
\hline BEHSUD & 1043 & NAHR SHAHI 2 & QALAA-E-GULJAN & 250 & 0 & 0 & 0 & 0 \\
\hline BEHSUD & 1023 & NAHR SHAHI 2 & QALAA-E-KHANA & 70 & 0 & 0 & 0 & 0 \\
\hline BEHSUD & 1035 & NAHR SHAHI AWAL & REGEY VILLAGE & 300 & 0 & 0 & 0 & 0 \\
\hline BEHSUD & 1046 & NAHR SHAHI DOWOM & BANDA HUJAT & 80 & 0 & 0 & 0 & 0 \\
\hline BEHSUD & 1025 & NAHR SHAHI DOWOM & BANDA KALAN & 130 & 0 & 0 & 0 & 0 \\
\hline BEHSUD & 1045 & NAHR SHAHI DOWOM & MIRZADA & 300 & 0 & 0 & 0 & 0 \\
\hline BEHSUD & 1044 & NAHR SHAHI DOWOM & QALE DESHET & 100 & 0 & 0 & 0 & 0 \\
\hline BEHSUD & 1051 & NAHR SHAHI DOWOM & QALE GHAIBAN & 200 & 0 & 0 & 0 & 0 \\
\hline BEHSUD & 1050 & NAHR SHAHI DOWOM & QALE ZARGARAN & 70 & 0 & 0 & 0 & 0 \\
\hline BEHSUD & 3560 & NAHR-E-SHAHI 1 & QALA-I-SANGI & 100 & 0 & 0 & 0 & 0 \\
\hline BEHSUD & 3455 & NAQILIE JOY 11 & NAQILIE JOY 11 & 800 & 11 & 1 & 13 & 143 \\
\hline BEHSUD & 3454 & NAQILIEN JOY 10 & NAQILIEN JOY 10 & 700 & 15 & 2 & 13 & 195 \\
\hline
\end{tabular}

* Jerib (jr.) $=1 / 5 \mathrm{ha}$

${ }^{2}$ Dry Opium $=$ Wet Opium $-30 \%$ moisture
Page 999
Source: UNDCP Opium Production Survey, 1995. File: NGR2.XLS - 06/08/95 
Opium Production Survey by village in Nangarhar province, 1995.

\begin{tabular}{|c|c|c|c|c|c|c|c|c|}
\hline \multirow{3}{*}{ District } & Village & \multirow{3}{*}{ Area/ Main Village } & \multirow{3}{*}{ Village } & \multirow{3}{*}{$\begin{array}{c}\text { Total } \\
\text { Cultivated } \\
\text { Land (jerib }^{*} \text { ) }\end{array}$} & \multirow{2}{*}{\multicolumn{2}{|c|}{$\begin{array}{c}\text { Land Under } \\
\text { Poppy Cultivation }\end{array}$}} & \multirow{3}{*}{$\begin{array}{c}\text { Wet Opium *2 } \\
\text { Yield } \\
(\mathrm{kg} / \mathrm{jr} .) \\
\end{array}$} & \multirow{3}{*}{$\begin{array}{c}\text { Wet Opium } \\
\text { Production } \\
(\mathrm{kg})\end{array}$} \\
\hline & Code & & & & & & & \\
\hline & & & & & (jerib) & $(\%)$ & & \\
\hline \multicolumn{9}{|c|}{ Province: Nangarhar } \\
\hline BEHSUD & 1017 & NARMASI & NARMASI & 100 & 0 & 0 & 0 & 0 \\
\hline BEHSUD & 1063 & PERAWAR & PERAWAR & 258 & 0 & 0 & 0 & 0 \\
\hline BEHSUD & 997 & PETAW & PETAW & 120 & 4 & 3. & 15 & 60 \\
\hline BEHSUD & 3384 & PIR SAHIB & PIR SAHIB & 350 & 0 & 0 & 0 & 0 \\
\hline BEHSUD & 1012 & QALA-I-JANAN KHAN & QALA-I-JANAN KHAN & 1,000 & 0 & 0 & 0 & 0 \\
\hline BEHSUD & 1010 & QASEM ABAD & BAR QASEM ABAD & 200 & 0 & 0 & 0 & 0 \\
\hline BEHSUD & 1009 & QASEM ABAD & KUZ QASIM ABAD & 250 & 0 & 0 & 0 & 0 \\
\hline BEHSUD & 1055 & SAMAR KHAIL & SAMAR KHAIL & 300 & 0 & 0 & 0 & 0 \\
\hline BEHSUD & 1056 & SAMAR KHEL & BAR SAMAR KHAIL/REGR & 120 & 0 & 0 & 0 & 0 \\
\hline BEHSUD & 994 & SARACHA & KUZ SARACHA & 300 & 6 & 2 & 16 & 96 \\
\hline BEHSUD & 995 & SARACHA & SARACHA & 200 & 0 & 0 & 0 & 0 \\
\hline BEHSUD & 1040 & SARBAD ALI & SARBAD ALI & 150 & 0 & 0 & 0 & 0 \\
\hline BEHSUD & 1019 & SARESHTADARI & SARESHTADARI & 450 & 0 & 0 & 0 & 0 \\
\hline BEHSUD & 1011 & SHEKHAN & SHEKHAN & 70 & 0 & 0 & 0 & 0 \\
\hline BEHSUD & 1016 & SURGOG & SURGOG & 120 & 0 & 0 & 0 & 0 \\
\hline BEHSUD & 1007 & TAGHAR & TAGHAR & 300 & 0 & 0 & 0 & 0 \\
\hline BEHSUD & 1061 & TANGI TOKHCHI & TANGI TOKHCHI & 400 & 2 & 0 & 14 & 21 \\
\hline BEHSUD & 1000 & VELAYATE & ASHQ MOHD & 100 & 0 & 0 & 0 & 0 \\
\hline BEHSUD & 1004 & VELAYATE & TAMIRAT & 0 & 0 & \#DIV/0! & 0 & 0 \\
\hline BEHSUD & 1003 & VELAYATE & VELAYATE & 300 & 0 & 0 & 0 & 0 \\
\hline BEHSUD & 1052 & ZANGOI & ZANGOI & 500 & 30 & 6 & 14 & 420 \\
\hline CHAPARHAR & 3494 & ABDUL RAHMAN & ABDUL RAHMAN & 80 & 40 & 50 & 14 & 560 \\
\hline CHAPARHAR & 128 & AHMADI & AHMADI & 60 & 20 & 33 & 10 & 200 \\
\hline CHAPARHAR & 106 & AKHON KALAY & AKHON KALAY & 350 & 100 & 29 & 12 & 1,200 \\
\hline CHAPARHAR & 149 & ASHAR KHAIL & ASHAR KHAIL & 130 & 65 & 50 & 14 & 910 \\
\hline CHAPARHAR & 118 & BANDA & BANDA & 300 & 160 & 53 & 10 & 1,600 \\
\hline CHAPARHAR & 123 & BAQI & BAQI & 80 & 60 & 75 & 10 & 600 \\
\hline CHAPARHAR & 165 & BAR LAWANGPOR & BAR LAWANGPOR & 60 & 40 & 67 & 10 & 400 \\
\hline CHAPARHAR & 145 & BARA GULDARA & BARA GULDARA & 100 & 75 & 75 & 7 & 525 \\
\hline
\end{tabular}

* Jerib (jr.) $=1 / 5$ ha

* 2 Dry Opium = Wet Opium - $30 \%$ moisture
Page 10

100
Source: UNDCP Opium Production Survey, 1995. File: NGR2.XLS - 06/08/95 
Opium Production Survey by village in Nangarhar province, 1995.

\begin{tabular}{|c|c|c|c|c|c|c|c|c|}
\hline \multirow{3}{*}{ District } & \multirow{3}{*}{$\begin{array}{c}\text { Village } \\
\text { Code }\end{array}$} & \multirow{3}{*}{ Areal Main Village } & \multirow{3}{*}{ Village } & \multirow{3}{*}{\begin{tabular}{|c|} 
Total \\
Cultivated \\
Land (jerib ${ }^{*}$ ) \\
\end{tabular}} & \multirow{2}{*}{\multicolumn{2}{|c|}{$\begin{array}{c}\text { Land Under } \\
\text { Poppy Cultivation }\end{array}$}} & \multirow{3}{*}{$\begin{array}{c}\text { Wet Opium *2 } \\
\text { Yield } \\
(\mathrm{kg} / \mathrm{jr} .) \\
\end{array}$} & \multirow{3}{*}{$\begin{array}{c}\text { Wet Opium } \\
\text { Production } \\
\text { (kg) }\end{array}$} \\
\hline & & & & & & & & \\
\hline & & & & & (jerib) & $(\%)$ & & \\
\hline \multicolumn{9}{|c|}{ Province: Nangarhar } \\
\hline CHAPARHAR & 120 & BARLO NEGIOR & BARLO NEGIOR & 60 & 40 & 67 & 19 & 760 \\
\hline CHAPARHAR & 111 & BAWIL & BAWIL & 300 & 150 & 50 & 14 & 2,100 \\
\hline CHAPARHAR & 154 & CHAR QALA & CHAR QALA & 80 & 40 & 50 & 14 & 560 \\
\hline CHAPARHAR & 170 & CHINA DAWLATZAI & BAR KALA (ACHIN) & 70 & 35 & 50 & 10 & 350 \\
\hline CHAPARHAR & 170 & DAWLAT ZAI & CHINA & 100 & 50 & 50 & 14 & 700 \\
\hline CHAPARHAR & 175 & DAWLAT ZAYEE & AKHONZADAGAN & 117 & 58 & 50 & 14 & 812 \\
\hline CHAPARHAR & 177 & DAWLAT ZAYEE & DAWLAT ZAYEE & 500 & 300 & 60 & 14 & 4,200 \\
\hline CHAPARHAR & 178 & DAWLAT ZAYEE & SHOAIB KALAY & 90 & 45 & 50 & 12 & 540 \\
\hline CHAPARHAR & 172 & DOULAT ZAI & BEBE MAHRO & 150 & 75 & 50 & 14 & 1,050 \\
\hline CHAPARHAR & 110 & GAMBEL & GAMBEL & 80 & 50 & 63 & 14 & 700 \\
\hline CHAPARHAR & 125 & GARATAK & GARATAK & 120 & 80 & 67 & 10 & 800 \\
\hline CHAPARHAR & 159 & GHAZO KALAY & MANDO ZAI & 550 & 200 & 36 & 14 & 2,800 \\
\hline CHAPARHAR & 158 & GHAZO KALAY & MOHD.KHAIL & 600 & 200 & 33 & 14 & 2,800 \\
\hline CHAPARHAR & 179 & GHOR ABAD & GHOR ABAD & 150 & 75 & 50 & 12 & 900 \\
\hline CHAPARHAR & 146 & GULDARA & MANZ GULDARA & 100 & 60 & 60 & 10 & 600 \\
\hline CHAPARHAR & 142 & GULSHAH & GULSHAH & 200 & 150 & 75 & 14 & 2,100 \\
\hline CHAPARHAR & 152 & GULSHER & GULSHER & 60 & 30 & 50 & 14 & 420 \\
\hline CHAPARHAR & 151 & HADIA KHEL & MIAGANO KALAY & 30 & 15 & 50 & 10 & 150 \\
\hline CHAPARHAR & 115 & HAFEZAN & HAFEZAN & 300 & 100 & 33 & 10 & 1,000 \\
\hline CHAPARHAR & 183 & HAJYAN & HAJYAN & 200 & 150 & 75 & 14 & 2,100 \\
\hline CHAPARHAR & 148 & HALIM & HALIM & 160 & 65 & 41 & 14 & 910 \\
\hline CHAPARHAR & 108 & KHALIFA KHAIL & KHALIFA KHAIL & 100 & 50 & 50 & 14 & 700 \\
\hline CHAPARHAR & 3428 & KHAN & KHAN & 60 & 40 & 67 & 17 & 680 \\
\hline CHAPARHAR & 153 & KHANANO KALAY & KHANANO KALAY & 150 & 100 & 67 & 14 & 1,400 \\
\hline CHAPARHAR & 161 & KHOJA GAN & KHOJA GAN & 70 & 35 & 50 & 14 & 490 \\
\hline CHAPARHAR & 117 & KOZ LAWANGPOR & KOZ LAWANGPOR & 100 & 70 & 70 & 12 & 840 \\
\hline CHAPARHAR & 147 & KUZ GULDARA & KUZ GULDARA & 150 & 80 & 53 & 8 & 640 \\
\hline CHAPARHAR & 144 & LALA & LALA & 25 & 20 & 80 & 10 & 200 \\
\hline CHAPARHAR & 133 & LALM & HAROON KHAIL & 80 & 5 & 6 & 7 & 35 \\
\hline
\end{tabular}

* Jerib (jr.) $=1 / 5$ ha

${ }^{2}$ Dry Opium $=$ Wet Opium - $30 \%$ moisture
Page $11 \quad 107$
Source: UNDCP Opium Production Survey, 1995. File: NGR2.XLS - 06/08/95 
Opium Production Survey by village in Nangarhar province, 1995.

\begin{tabular}{|c|c|c|c|c|c|c|c|c|}
\hline & Village & & & Total & Land 1 & & Wet Opium * 2 & Wet Opium \\
\hline \multirow[t]{2}{*}{ District } & \multirow[t]{2}{*}{ Code } & \multirow[t]{2}{*}{ Areal Main Village } & \multirow[t]{2}{*}{ Village } & \multirow{2}{*}{$\begin{array}{c}\text { Cultivated } \\
{\text { Land }\left(\text { jerib }^{*} \text { ) }\right.}^{\text {L }}\end{array}$} & \multicolumn{2}{|c|}{ Poppy Cultivation } & \multirow{2}{*}{$\begin{array}{c}\text { Yield } \\
(\mathrm{kg} / \mathrm{jr} .) \\
\end{array}$} & \multirow{2}{*}{$\begin{array}{c}\text { Production } \\
(\mathrm{kg}) \\
\end{array}$} \\
\hline & & & & & (jerib) & $(\%)$ & & \\
\hline \multicolumn{9}{|c|}{ Province: Nangarhar } \\
\hline CHAPARHAR & 135 & LALM & LALM & 900 & 200 & 22 & 10 & 2,000 \\
\hline CHAPARHAR & 3466 & LAMZARI & LAMZARI & 250 & 125 & 50 & 10 & 1,250 \\
\hline CHAPARHAR & 113 & LANDAN & LANDAN & 400 & 200 & 50 & 14 & 2,800 \\
\hline CHAPARHAR & 139 & LOY & LOY & 60 & 0 & 0 & 0 & 0 \\
\hline CHAPARHAR & 143 & LOY & LOY & 500 & 300 & 60 & 10 & 3,000 \\
\hline CHAPARHAR & 3493 & MAJBOOR ABAD & MAJBOOR ABAD & 200 & 3 & 2 & 14 & 42 \\
\hline CHAPARHAR & 138 & MALEKANO KALAY & MALEKANO KALAY & 90 & 45 & 50 & 14 & 630 \\
\hline CHAPARHAR & 124 & MANDA KHAIL & MANDA KHAIL & 400 & 300 & 75 & 10 & 3,000 \\
\hline CHAPARHAR & 166 & MANO & LOY & 250 & 150 & 60 & 10 & 1,500 \\
\hline CHAPARHAR & 127 & MANO & MALEKAN & 520 & 260 & 50 & 12 & 3,120 \\
\hline CHAPARHAR & 155 & MANZ LAWANGPUR & TRILAY & 1,000 & 300 & 30 & 12 & 3,600 \\
\hline CHAPARHAR & 150 & MASTER KHAIL & MASTER KHAIL & 120 & 80 & 67 & 14 & 1,120 \\
\hline CHAPARHAR & 119 & MIAGAN & MIAGAN & 150 & 100 & 67 & 12 & 1,200 \\
\hline CHAPARHAR & 112 & MIRZA TAWAKAL & MIRZA TAWAKAL & 100 & 60 & 60 & 21 & 1,260 \\
\hline CHAPARHAR & 114 & MONDA KORAK & MONDA KORAK & 140 & 70 & 50 & 21 & 1,470 \\
\hline CHAPARHAR & 126 & MORA & MORA & 90 & 65 & 72 & 10 & 650 \\
\hline CHAPARHAR & 109 & QALA WAL & QALA WAL & 400 & 200 & 50 & 14 & 2,800 \\
\hline CHAPARHAR & 156 & QALA-E ASLAM & QALA-E ASLAM & 130 & 65 & 50 & 14 & 910 \\
\hline CHAPARHAR & 180 & QALA-E SHAIKH & KHOJAGAN & 500 & 250 & 50 & 10 & 2,500 \\
\hline CHAPARHAR & 181 & QALA-E SHAIKH KHALIF & QALA-E SHAIKH KHALIF & 100 & 40 & 40 & 12 & 480 \\
\hline CHAPARHAR & 130 & QALA-E SURKH & QALA-E SURKH & 400 & 200 & 50 & 10 & 2,000 \\
\hline CHAPARHAR & 140 & SALO ZAI & LOKHI & 50 & 20 & 40 & 10 & 200 \\
\hline CHAPARHAR & 132 & SALO ZAI KALAY & KARO KHEL & 150 & 100 & 67 & 12 & 1,200 \\
\hline CHAPARHAR & 141 & SALOZAI & KAMBO & 300 & 200 & 67 & 14 & 2,800 \\
\hline CHAPARHAR & 3495 & SANGINA & SANGINA & 200 & 150 & 75 & 10 & 1,500 \\
\hline CHAPARHAR & 157 & SHIRKHIL-SHIKHZARIN & TATAR KHEL & 100 & 60 & 60 & 18 & 1,050 \\
\hline CHAPARHAR & 107 & SPARI & SPARI & 140 & 70 & 50 & 14 & 980 \\
\hline CHAPARHAR & 116 & SPIN JAMAT & SPIN JAMAT & 50 & 25 & 50 & 12 & 300 \\
\hline CHAPARHAR & 162 & TARAKI & MOLA KALAY & 200 & 60 & 30 & 10 & 600 \\
\hline
\end{tabular}


Opium Production Survey by village in Nangarhar province, 1995.

\begin{tabular}{|c|c|c|c|c|c|c|c|c|}
\hline \multirow{3}{*}{ District } & \multirow{3}{*}{$\begin{array}{l}\text { Village } \\
\text { Code }\end{array}$} & \multirow{3}{*}{ Area/ Main Village } & \multirow{3}{*}{ Village } & \multirow{3}{*}{$\begin{array}{c}\text { Total } \\
\text { Cultivated } \\
\text { Land (jerib }^{*} \text { ) } \\
\end{array}$} & \multirow{2}{*}{\multicolumn{2}{|c|}{$\begin{array}{c}\text { Land Under } \\
\text { Poppy Cultivation }\end{array}$}} & \multirow{3}{*}{$\begin{array}{c}\text { Wet }^{\text {Opium }}{ }^{* 2} \\
\text { Yield } \\
(\mathrm{kg} / \mathrm{jr} .) \\
\end{array}$} & \multirow{3}{*}{$\begin{array}{c}\text { Wet Opium } \\
\text { Production } \\
(\mathrm{kg})\end{array}$} \\
\hline & & & & & & & & \\
\hline & & & & & (jerib) & $(\%)$ & & \\
\hline \multicolumn{9}{|c|}{ Province: Nangarhar } \\
\hline CHAPARHAR & 163 & TARAKI & TARAKI & 500 & 120 & 24 & 10 & 1,200 \\
\hline CHAPARHAR & 164 & TARELY & PORE.KHALO.TARE & 300 & 100 & 33 & 14 & 1,400 \\
\hline CHAPARHAR & 137 & ZARGARAN & ZARGARAN & 80 & 40 & 50 & 12. & 480 \\
\hline DARA-E NUR & 981 & AMLA & AKBAR KHAN KALAY & 0 & 0 & \#DIV/0! & 0 & 0 \\
\hline DARA-E NUR & 966 & AMLA & BAR KALAY & 150 & 85 & 57 & 10 & 850 \\
\hline DARA-E NUR & 970 & AMLA & DAWOD KALAY & 70 & 38 & 54 & 13 & 494 \\
\hline DARA-E NUR & 987 & AMLA & DIWA & 50 & 35 & 70 & 12 & 420 \\
\hline DARA-E NUR & 986 & AMLA & FAQIR QALE & 100 & 80 & 80 & 16 & 1,280 \\
\hline DARA-E NUR & 982 & AMLA & MOHAMMAD NOOR & 150 & 60 & 40 & 13 & 780 \\
\hline DARA-E NUR & 971 & AMLA & OBAR & 60 & 20 & 33 & 12 & 240 \\
\hline DARA-E NUR & 984 & AMLA & PUCHA KHAIL & 50 & 35 & 70 & 9 & 315 \\
\hline DARA-E NUR & 985 & AMLA & QARAL DEH & 220 & 100 & 45 & 10 & 1,000 \\
\hline DARA-E NUR & 983 & AMLA & TATANG & 80 & 50 & 63 & 14 & 700 \\
\hline DARA-E NUR & 1874 & BAR KANDAK & BAR KANDAK & 50 & 0 & 0 & 0 & 0 \\
\hline DARA-E NUR & 1895 & CHIWAL & CHIWAL & 40 & 15 & 38 & 12 & 180 \\
\hline DARA-E NUR & 989 & DO DARAK & DO DARAK & 300 & 23 & 8 & 7 & 161 \\
\hline DARA-E NUR & 967 & DORALAK & DORALAK & 70 & 0 & 0 & 0 & 0 \\
\hline DARA-E NUR & 990 & JANSHE GAL & JANSHE GAL & 100 & 0 & 0 & 0 & 0 \\
\hline DARA-E NUR & 976 & KASHMON QALA & KASHMANA QALA & 300 & 20 & 7 & 8 & 160 \\
\hline DARA-E NUR & 1873 & KOZ KANDAK & KOZ KANDAK & 100 & 0 & 0 & 0 & 0 \\
\hline DARA-E NUR & 1864 & LAMATEK & LAMATEK & 120 & 60 & 50 & 13 & 780 \\
\hline DARA-E NUR & 980 & LOY KALAY AMLA & LOY KALAY AMLA & 100 & 50 & 50 & 14 & 700 \\
\hline DARA-E NUR & 1891 & MACHGANDOL & BONTA MACHGANDO & 150 & 75 & 50 & 13 & 975 \\
\hline DARA-E NUR & 1890 & MACHGANDOL & DURATA(MACHGANDOL) & 150 & 60 & 40 & 13 & 780 \\
\hline DARA-E NUR & 1894 & MACHGANDOL & MACHGANDOL & 100 & 40 & 40 & 12 & 480 \\
\hline DARA-E NUR & 1887 & OTRAN & OTRAN & 210 & 40 & 19 & 8 & 320 \\
\hline DARA-E NUR & 969 & QALA SHAHI & BARA NANGRA & 25 & 10 & 40 & 15 & 150 \\
\hline DARA-E NUR & 1877 & QALA SHAHI & BUNIAIKOZ TAPA & 110 & 30 & 27 & 12 & 360 \\
\hline DARA-E NUR & 1872 & QALA SHAHI & JANJA POR & 400 & 80 & 20 & 14 & 1,120 \\
\hline
\end{tabular}

$*$ Jerib (jr.) $=1 / 5$ ha

* 2 Dry Opium = Wet Opium - 30\% moisture
Page 13103
Source: UNDCP Opium Production Survey, 1995. File: NGR2.XLS - 06/08/95 
Opium Production Survey by village in Nangarhar province, 1995.

\begin{tabular}{|c|c|c|c|c|c|c|c|c|}
\hline \multirow{3}{*}{ District } & \multirow{3}{*}{$\begin{array}{c}\text { Village } \\
\text { Code }\end{array}$} & \multirow{3}{*}{ Area/ Main Village } & & Total & Land & & Wet Opium * 2 & Wet Opium \\
\hline & & & \multirow[t]{2}{*}{ Village } & \multirow{2}{*}{$\begin{array}{c}\text { Cultivated } \\
\text { Land (jerib }{ }^{*} \text { ) }\end{array}$} & \multicolumn{2}{|c|}{ Poppy Cultivation } & \multirow{2}{*}{$\begin{array}{l}\text { Yield } \\
(\mathrm{kg} / \mathrm{jr} \text {. })\end{array}$} & \multirow{2}{*}{$\frac{\text { Production }}{(\mathrm{kg})}$} \\
\hline & & & & & (jerib) & $(\%)$ & & \\
\hline \multicolumn{9}{|c|}{ Province: Nangarhar } \\
\hline DARA-E NUR & 1876 & QALA SHAHI & JANMIR & 400 & 100 & 25 & 10 & 1,000 \\
\hline DARA-E NUR & 988 & QALA SHAHI & KOTGA & 140 & 30 & 21 & 12 & 360 \\
\hline DARA-E NUR & 968 & QALA SHAHI & KOZ NANGRA & 95 & 15 & 16 & 13 & 195 \\
\hline DARA-E NUR & 1875 & QALA-I-SHAHI & QALA-I-SHAHI & 300 & 160 & 53 & 8 & 1,280 \\
\hline DARA-E NUR & 3382 & SAFAR QALA & KALATEK & 100 & 0 & 0 & 0 & 0 \\
\hline DARA-E NUR & 973 & SAFAR QALA & LAM KANDA & 70 & 0 & 0 & 0 & 0 \\
\hline DARA-E NUR & 962 & SAFAR QALA & SAFAR QALA & 500 & 0 & 0 & 0 & 0 \\
\hline DARA-E NUR & 975 & SAROR & AKHOND & 10 & 0 & 0 & 0 & 0 \\
\hline DARA-E NUR & 974 & SAROR & SAROR & 20 & 0 & 0 & 0 & 0 \\
\hline DARA-E NUR & 1884 & SHAKYALI & BAMBA KOT & 250 & 85 & 34 & 12 & 1,020 \\
\hline DARA-E NUR & 1866 & SHAKYALI & OMER & 70 & 35 & 50 & 10 & 350 \\
\hline DARA-E NUR & 1871 & SHAKYALI & PANJO QALA & 50 & 10 & 20 & 12 & 120 \\
\hline DARA-E NUR & 1870 & SHAKYALI & SEGAIY & 42 & 15 & 36 & 12 & 180 \\
\hline DARA-E NUR & 1867 & SHAKYALI & SHAKYALI & 120 & 28 & 23 & 10 & 280 \\
\hline DARA-E NUR & 1868 & SHAKYALI & SHEROM & 180 & 60 & 33 & 14 & 840 \\
\hline DARA-E NUR & 1892 & SHIMAL & KUZ CHAM & 150 & 0 & 0 & 0 & 0 \\
\hline DARA-E NUR & 1893 & SHIMAL & SHIMAL & 200 & 0 & 0 & 0 & 0 \\
\hline DARA-E NUR & 1883 & SOTAN & KOZ CHAM & 150 & 75 & 50 & 10 & 750 \\
\hline DARA-E NUR & 1882 & SOTAN ABDUL RAHMAN K & ABDUL RAHMAN KELY & 60 & 35 & 58 & 12 & 420 \\
\hline DARA-E NUR & 1878 & STAN & BABA LAMTAK & 90 & 40 & 44 & 14 & 560 \\
\hline DARA-E NUR & 1889 & STAN & GOR KHAL & 50 & 25 & 50 & 10 & 250 \\
\hline DARA-E NUR & 1880 & STAN & GUL MASJED & 50 & 25 & 50 & 12 & 300 \\
\hline DARA-E NUR & 1865 & STAN & LAGEK & 60 & 30 & 50 & 12 & 360 \\
\hline DARA-E NUR & 1881 & STAN & MANZ KOCHA & 50 & 20 & 40 & 12. & 240 \\
\hline DARA-E NUR & 1869 & STAN & MESKIN BANDA & 65 & 30 & 46 & 14 & 420 \\
\hline DARA-E NUR & 1879 & Stan & SIR GELACK & 40 & 25 & 63 & 11 & 275 \\
\hline DARA-E NUR & 1888 & STAN & WARA MASJED & 75 & 30 & 40 & 12 & 360 \\
\hline DARA-E NUR & 978 & WIGAL & BADRAK & 200 & 0 & 0 & 0 & 0 \\
\hline DARA-E NUR & 965 & WIGAL & BARCHAM & 190 & 0 & 0 & 0 & 0 \\
\hline
\end{tabular}


Opium Production Survey by village in Nangarhar province, 1995.

\begin{tabular}{|c|c|c|c|c|c|c|c|c|}
\hline & \multirow{3}{*}{$\begin{array}{l}\text { Village } \\
\text { Code }\end{array}$} & \multirow{3}{*}{ Areal Main Village } & \multirow{3}{*}{ Village } & \multirow{3}{*}{\begin{tabular}{|c|} 
Total \\
Cultivated \\
Land (jerib $\left.{ }^{*}\right)$ \\
\end{tabular}} & \multirow{2}{*}{\multicolumn{2}{|c|}{$\begin{array}{c}\text { Land Under } \\
\text { Poppy Cultivation }\end{array}$}} & \multirow{3}{*}{$\begin{array}{c}\text { Wet Opium }{ }^{* 2} \\
\text { Yield } \\
(\mathrm{kg} / \mathrm{jr} .)\end{array}$} & \multirow{3}{*}{$\begin{array}{c}\text { Wet Opium } \\
\text { Production } \\
(\mathrm{kg})\end{array}$} \\
\hline \multirow[t]{2}{*}{ District } & & & & & & & & \\
\hline & & & & & (jerib) & $(\%)$ & & \\
\hline \multicolumn{9}{|c|}{ Province: Nangarhar } \\
\hline DARA-E NUR & 977 & WIGAL & CHIGONO KALAY & 170 & 0 & 0 & 0 & 0 \\
\hline DARA-E NUR & 965 & WIGAL & WIGAL & 150 & 80 & 53 & 12 & 960 \\
\hline DARA-E NUR & 961 & WIGAL & WIGAL & 240 & 0 & 0 & 0 & 0 \\
\hline DARA-E NUR & 963 & WIGAL & ZABAN MALEK & 150 & 0 & 0 & 0 & 0 \\
\hline DEH BALA & 243 & AKA KHIL & AKA KHIL & 380 & 58 & 15 & 14 & 812 \\
\hline DEH BALA & 3472 & AKAKHEL & BABA KALAY & 360 & 65 & 18 & 12 & 780 \\
\hline DEH BALA & 1112 & ANGOR KALA & ANGOR KALA & 25 & 10 & 40 & 15 & 150 \\
\hline DEH BALA & 1111 & ANGOR KALA & SHEKHA & 40 & 18 & 45 & 14 & 252 \\
\hline DEH BALA & 3350 & BADAR KHEL & MOHAMMAD YAR & 25 & 13 & 52 & 17 & 221 \\
\hline DEH BALA & 1117 & BADER KHEL & BADER KHEL & 35 & 15 & 43 & 15 & 225 \\
\hline DEH BALA & 3357 & BADER KHEL & RAJI KHAIL & 45 & 20 & 44 & 17 & 340 \\
\hline DEH BALA & 3373 & CHARWAZAI & CHARWAZAI & 160 & 60 & 38 & 15 & 900 \\
\hline DEH BALA & 244 & DEH BALA & CHINA & 120 & 50 & 42 & 15 & 750 \\
\hline DEH BALA & 3486 & DEH BALA & DAWLAT KHEL & 170 & 120 & 71 & 14 & 1,680 \\
\hline DEH BALA & 241 & DEH BALA & GHAR KHALE & 150 & 75 & 50 & 14 & 1,050 \\
\hline DEH BALA & 3476 & DEH BALA & JAWDARA & 200 & 80 & 40 & 14 & 1,120 \\
\hline DEH BALA & 1107 & GAGRAH & KHANANO KELY & 120 & 30 & 25 & 16 & 480 \\
\hline DEH BALA & 1106 & GAGRAH & LIAS KHEL & 70 & 25 & 36 & 14 & 350 \\
\hline DEH BALA & 1105 & GAGRAH & MADE KHEL & 100 & 20 & 20 & 12 & 240 \\
\hline DEH BALA & 3353 & GAGRAH & SURKAY MANZAY & 125 & 35 & 28 & 15 & 525 \\
\hline DEH BALA & 3374 & GAJANA & DANDER GOSH & 55 & 20 & 36 & 17. & 340 \\
\hline DEH BALA & 3351 & GAJANA & MALEKANO KALAY & 145 & 50 & 34 & 14 & 700 \\
\hline DEH BALA & 1103 & GAJNA & GAJNA & 115 & 40 & 35 & 16 & 640 \\
\hline DEH BALA & 3363 & GHOSHTARAH & GHOSHTARAH & 80 & 25 & 31 & 12 & 300 \\
\hline DEH BALA & 3359 & GORGORAI & GODAR KALAY & 190 & 69 & 36 & 14 & 966 \\
\hline DEH BALA & 3366 & GORGORAI & MIAGANO \& PACHANO KE & 100 & 25 & 25 & 14 & 350 \\
\hline DEH BALA & 3479 & GORGORI & RAGHA KALAY & 110 & 50 & 45 & 14 & 700 \\
\hline DEH BALA & 1114 & GOSHTARA & ABA KHEL & 110 & 35 & 32 & 12 & 420 \\
\hline DEH BALA & 3361 & HASKA MENA & HASKA MENA & 150 & 45 & 30 & 15 & 675 \\
\hline
\end{tabular}

* Jerib (jr.) $=1 / 5$ ha

* 2 Dry Opium = Wet Opium - $30 \%$ moisture
Page 15205
Source: UNDCP Opium Production Survey, 1995. File: NGR2.XLS - 06/08/95 
Opium Production Survey by village in Nangarhar province, 1995.

\begin{tabular}{|c|c|c|c|c|c|c|c|c|}
\hline & Village & 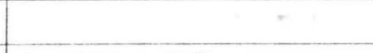 & & Total & Land 1 & & Wet Opium * 2 & Wet Opium \\
\hline \multirow[t]{2}{*}{ District } & Code & Area/ Main Village & Village & Cultivated & \multicolumn{2}{|c|}{ Poppy Cultivation } & Yield & Production \\
\hline & & & & Land (jerib $\left.{ }^{*}\right)$ & (jerib) & $(\%)$ & $(\mathrm{kg} / \mathrm{jr})$. & $(\mathrm{kg})$ \\
\hline \multicolumn{9}{|c|}{ Province: Nangarhar } \\
\hline DEH BALA & 3474 & HASKA MINA & KOZ AHMAD KHEL & 100 & 15 & 15 & 13 & 195 \\
\hline DEH BALA & 1954 & JAMAL KHEL & JAMAL KHEL & 40 & 15 & 38 & 16 & 240 \\
\hline DEH BALA & 3365 & JOLAGAN & JOLAGAN & 25 & 10 & 40 & 15 & 150 \\
\hline DEH BALA & 3367 & KAM PAPIN & KAM PAPIN & 200 & 60 & 30 & 14 & 840 \\
\hline DEH BALA & 3481 & KASO & KASO & 115 & 30 & 26 & 13 & 390 \\
\hline DEH BALA & 3480 & KATAYAN & KATAYAN & 90 & 28 & 31 & 13 & 364 \\
\hline DEH BALA & 242 & KAZHAY (OGHZ) & KAZHAY & 125 & 60 & 48 & 12 & 720 \\
\hline DEH BALA & 1110 & KHARKAY & KHARKAY & 110 & 30 & 27 & 13 & 390 \\
\hline DEH BALA & 1125 & KHARKI KALAY & KHARKI KALAY & 120 & 50 & 42 & 13 & 650 \\
\hline DEH BALA & 1124 & KOTWAL & KOTWAL & 300 & 100 & 33 & 16 & 1,600 \\
\hline DEH BALA & 1123 & KOTWAL & SHARMAKHAY & 100 & 27 & 27 & 14 & 378 \\
\hline DEH BALA & 3475 & KOTWAL & SONA KHEL & 200 & 85 & 43 & 14 & 1,190 \\
\hline DEH BALA & 3348 & LANDY KALAY KAGARAY & LANDY KALAY KAGARAY & 140 & 60 & 43 & 16 & 960 \\
\hline DEH BALA & 3368 & LOY PAPIN & LOY PAPIN & 100 & 20 & 20 & 17 & 340 \\
\hline DEH BALA & 3354 & MAIN KAC & MAIN KAC & 140 & 40 & 29 & 13 & 520 \\
\hline DEH BALA & 3369 & MANZ GORGORY & MANZ GORGORY & 180 & 50 & 28 & 13 & 650 \\
\hline DEH BALA & 1961 & MARIZ & SUNGOTA KALAY & 150 & 60 & 40 & 16 & 960 \\
\hline DEH BALA & 3372 & MARIZ \BARIZ & MARIZ \BARIZ & 220 & 25 & 11 & 14 & 350 \\
\hline DEH BALA & 3477 & MATAR KHAN BABA & DAGO & 90 & 30 & 33 & 12 & 360 \\
\hline DEH BALA & 3478 & MATAR KHAN BABA & CHINGAI & 60 & 20 & 33 & 13 & 260 \\
\hline DEH BALA & 3503 & MIN KAC & GHALNI & 150 & 55. & 37. & 14 & 770 \\
\hline DEH BALA & 3370 & MITER KHAN BABA & MITER KHAN BABA & 80 & 35 & 44 & 15 & 525 \\
\hline DEH BALA & 1119 & MOLA AW KOCHIAN & MOLA AW KOCHIAN & 18 & 7. & 39 & 15 & 105 \\
\hline DEH BALA & 1122 & $\mathrm{OGHZ}$ & KAC & 65 & 20 & 31 & 14 & 280 \\
\hline DEH BALA & 3485 & OGHZ & KOTAGAI & 80 & 0 & 0 & 0 & 0 \\
\hline DEH BALA & 1121 & $\mathrm{OGHZ}$ & MOHD. MUSA KALAY & 130 & 60 & 46 & 14 & 840 \\
\hline DEH BALA & 3473 & QUDRAT & MORGAI & 150 & 40 & 27 & 14 & 560 \\
\hline DEH BALA & 3358 & QUDRAT & QUDRAT & 140 & 60 & 43 & 14 & 840 \\
\hline DEH BALA & 1118 & QUDRAT KALAY & GOZARA & 500 & 110 & 22 & 15 & 1,650 \\
\hline
\end{tabular}

$*$ Jerib (jr.) $=1 / 5$ ha

* 2 Dry Opium $=$ Wet Opium $-30 \%$ moisture
Page $16 \quad 206$
Source: UNDCP Opium Production Survey, 1995. File: NGR2,XLS - 06/08/95 
Opium Production Survey by village in Nangarhar province, 1995.

\begin{tabular}{|c|c|c|c|c|c|c|c|c|}
\hline & \multirow{3}{*}{$\begin{array}{l}\text { Village } \\
\text { Code }\end{array}$} & \multirow{3}{*}{ Area/ Main Village } & \multirow{3}{*}{ Village } & \multirow{3}{*}{ 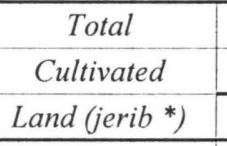 } & \multirow{2}{*}{\multicolumn{2}{|c|}{$\begin{array}{c}\text { Land Under } \\
\text { Poppy Cultivation }\end{array}$}} & \multirow{3}{*}{$\begin{array}{c}\text { Wet Opium }{ }^{* 2} \\
\text { Yield } \\
(\mathrm{kg} / \mathrm{jr} .) \\
\end{array}$} & \multirow{3}{*}{$\begin{array}{c}\text { Wet Opium } \\
\text { Production } \\
(\mathrm{kg})\end{array}$} \\
\hline \multirow[t]{2}{*}{ District } & & & & & & & & \\
\hline & & & & & (jerib) & (\%) & & \\
\hline \multicolumn{9}{|c|}{ Province: Nangarhar } \\
\hline DEH BALA & 1957 & RAWGAI & KHARLOZY KALAY & 95 & 35 & 37 & 15 & 525 \\
\hline DEH BALA & 3352 & ROD KHANA & ROD KHANA & 95 & 45 & 47 & 13 & 585 \\
\hline DEH BALA & 1959 & RWAGAY KALAY & RWAGAY KALAY & 250 & 80 & 32 & 15 & 1,200 \\
\hline DEH BALA & 3482 & SALA KOS & SALA KAS & 70 & 25 & 36 & 14 & 350 \\
\hline DEH BALA & 1956 & SERA MIRAN & SERA MIRAN & 130 & 60 & 46 & 15 & 900 \\
\hline DEH BALA & 1104 & SHABI & BAR LAKHTY & 230 & 65 & 28 & 16 & 1,040 \\
\hline DEH BALA & 1120 & SHABI KHEL & SHABI KHEL & 230 & 80 & 35 & 16 & 1,280 \\
\hline DEH BALA & 3349 & SHAH TORY & CHAGHARIAN & 9 & 2 & 22 & 17 & 34 \\
\hline DEH BALA & 1115 & SHAHZADA KAC & SHAZADA KAC & 60 & 20 & 33 & 13 & 260 \\
\hline DEH BALA & 1958 & SHATORY KALAY & SHATORY KALAY & 40 & 8 & 20 & 16 & 128 \\
\hline DEH BALA & 3355 & SHEKH MAIDAN & SHEKH MAIDAN & 70 & 35 & 50 & 11 & 385 \\
\hline DEH BALA & 1960 & SHPOLA & KHAM KALAY & 300 & 90 & 30 & 15 & 1,350 \\
\hline DEH BALA & 1113 & SHPOLA & LAKARAY & 50 & 20 & 40 & 18 & 360 \\
\hline DEH BALA & 3375 & SHPOLA & RAYES KHEL & 140 & 60 & 43 & 16 & 960 \\
\hline DEH BALA & 1116 & SHPOLA KALAY & SHPOLA KALAY & 150 & 70 & 47 & 15 & 1,050 \\
\hline DEH BALA & 3358 & SHPOLAY & KOCHIANO KALAY & 90 & 15 & 17 & 15 & 225 \\
\hline DEH BALA & 3484 & SRA KALA & SRA KALA & 150 & 40 & 27 & 14 & 560 \\
\hline DEH BALA & 3364 & SRA MORGA & SRA MORGA & 160 & 47 & 29 & 13 & 611 \\
\hline DEH BALA & 1962 & TANGAI & TANGAI & 282 & 82 & 29 & 15 & 1,230 \\
\hline DEH BALA & 1955 & TERNAW & TERNAW & 35 & 15 & 43 & 15 & 225 \\
\hline DEH BALA & 3358 & YAGHI BAND & YAGHI BAND & 180 & 80 & 44 & 14 & 1,120 \\
\hline DURBABA & 1815 & ANZAR & ANZAR & 60 & 20 & 33 & 9 & 180 \\
\hline DURBABA & 1812 & BAR GODKAI & BAR GODKAI & 12 & 0 & 0 & 0 & 0 \\
\hline DURBABA & 1811 & BEBRAI MINA & BEBRAI MINA & 10 & 2 & 20 & 6 & 12 \\
\hline DURBABA & 1817 & CHINAR & CHINAR & 10 & 7 & 70 & 8 & 56 \\
\hline DURBABA & 1818 & DARBAND & DARBAND & 0 & 0 & \#DIV/0! & 0 & 0 \\
\hline DURBABA & 1834 & DURBABA & ABDUL WAHAB & 40 & 22 & 55 & 6 & 132 \\
\hline DURBABA & 1813 & JAM GORA & JAM GORA & 30 & 0 & 0 & 0 & 0 \\
\hline DURBABA & 1832 & KHASIRI & KHASIRI & 40 & 10 & 25 & 8 & 80 \\
\hline
\end{tabular}

* Jerib (jr.) $=1 / 5 \mathrm{ha}$

${ }^{*}{ }_{2}$ Dry Opium $=$ Wet Opium $-30 \%$ moisture
Page $17 \quad 207$
Source: UNDCP Opium Production Survey, 1995 File: NGR2.XLS - 06/08/95 
Opium Production Survey by village in Nangarhar province, 1995.

\begin{tabular}{|c|c|c|c|c|c|c|c|c|}
\hline \multirow{3}{*}{ District } & \multirow{3}{*}{$\begin{array}{l}\text { Village } \\
\text { Code }\end{array}$} & \multirow{3}{*}{ Areal Main Village } & \multirow{3}{*}{ Village } & \multirow{3}{*}{\begin{tabular}{|c|} 
Total \\
Cultivated \\
${\text { Land }\left(\text { jerib }^{*}\right)}^{*}$ ) \\
\end{tabular}} & \multirow{2}{*}{\multicolumn{2}{|c|}{$\begin{array}{c}\text { Land Under } \\
\text { Poppy Cultivation }\end{array}$}} & \multirow{3}{*}{$\begin{array}{c}\text { Wet Opium *2 } \\
\text { Yield } \\
(\mathrm{kg} / \mathrm{jr} .) \\
\end{array}$} & \multirow{3}{*}{$\begin{array}{l}\text { Wet Opium } \\
\text { Production } \\
(\mathrm{kg})\end{array}$} \\
\hline & & & & & & & & \\
\hline & & & & & (jerib) & $(\%)$ & & \\
\hline \multicolumn{9}{|c|}{ Province: Nangarhar } \\
\hline DURBABA & 1835 & KHUSA CHINGAI & KHUSA CHINGAI & 0 & 0 & \#DIV/0! & 0 & 0 \\
\hline DURBABA & 3487 & KHWAR MINA & KHWAR MINA & 5 & 0 & 0 & 0 & 0 \\
\hline DURBABA & 1809 & KOZ GODKAI & KOZ GODKAI & 10 & 0 & 0 & 0 & 0 \\
\hline DURBABA & 1808 & NARGOSI & NARGOSI & 26 & 20 & 77 & 9 & 180 \\
\hline DURBABA & 1824 & OBLEEN & OBLEEN & 11 & 8 & 73 & 7 & 56 \\
\hline DURBABA & 1829 & OMAR KHAIL & OMAR KHAIL & 20 & 8 & 40 & 6 & 48 \\
\hline DURBABA & 1816 & PAKHA MINA & PAKHA MINA & 18 & 8 & 44 & 6 & 48 \\
\hline DURBABA & 1814 & QASABA & QASABA & 20 & 5 & 25 & 5 & 25 \\
\hline DURBABA & 1836 & SHINKAI GERDI & SHINKAI GERDI & 30 & 5 & 17 & 6 & 30 \\
\hline DURBABA & 1825 & SHOLGAR & KAMARSAR & 40 & 30 & 75 & 8 & 240 \\
\hline DURBABA & 1819 & SHOLGAR & SANDALI & 10 & 5 & 50 & 9 & 45 \\
\hline DURBABA & 1837 & SHOLGAR & SENZELI & 10 & 3 & 30 & 8 & 24 \\
\hline DURBABA & 1827 & SHOLGAR & SHALIMAN & 18 & 6 & 33 & 7 & 42 \\
\hline DURBABA & 1826 & SHOLGAR & SHOLGAR & 60 & 40 & 67 & 9 & 360 \\
\hline DURBABA & 1828 & SHOLGAR & SREH GHARA & 50 & 30 & 60 & 10 & 300 \\
\hline DURBABA & 1833 & SORKANDO & SORKANDO & 100 & 30 & 30 & 6 & 180 \\
\hline DURBABA & 1838 & TABI GHASHI & TABI GHASHI & 0 & 0 & \#DIV/0! & 0 & 0 \\
\hline DURBABA & 1831 & TORI & MOLA GORI & 16 & 8 & 50 & 7 & 56 \\
\hline DURBABA & 1830 & TORI & TORI & 40 & 30 & 75 & 8 & 240 \\
\hline DURBABA & 1807 & TORLALA & TORLALA & 30 & 3 & 10 & 5 & 15 \\
\hline DURBABA & 1820 & UGHUZGAI & SOOR & 100 & 20 & 20 & 7 & 140 \\
\hline DURBABA & 1821 & UGHUZGAI & UGHUZGAI & 20 & 10 & 50 & 7 & 70 \\
\hline DURBABA & 3488 & WOCHA LEGADA & WOCHA LEGADA & 25 & 5 & 20 & 8 & 40 \\
\hline DURBABA & 1810 & ZAZOBI & ZAZOBI & 40 & 20 & 50 & 8 & 160 \\
\hline DURBABA & 1822 & ZIARAT & GUL & 50 & 25 & 50 & 9 & 225 \\
\hline DURBABA & 1823 & ZIARAT & ZIARAT & 20 & 10 & 50 & 8 & 80 \\
\hline GOSHTA & 1736 & ARKHI & AFRIDO KALAY & 190 & 120 & 63 & 16 & 1,920 \\
\hline GOSHTA & 1735 & ARKHI & ARKHI & 140 & 66 & 47 & 17 & 1,122 \\
\hline GOSHTA & 1732 & ARKHI & KODA KHEL & 95 & 53 & 56 & 16 & 848 \\
\hline
\end{tabular}


Opium Production Survey by village in Nangarhar province, 1995.

\begin{tabular}{|c|c|c|c|c|c|c|c|c|}
\hline \multirow{3}{*}{ District } & \multirow{3}{*}{$\begin{array}{l}\text { Village } \\
\text { Code }\end{array}$} & \multirow{3}{*}{ Area/ Main Village } & \multirow{3}{*}{ Village } & \multirow{3}{*}{\begin{tabular}{|c|} 
Total \\
Cultivated \\
Land (jerib $\left.{ }^{*}\right)$ \\
\end{tabular}} & \multirow{2}{*}{\multicolumn{2}{|c|}{$\begin{array}{c}\text { Land Under } \\
\text { Poppy Cultivation }\end{array}$}} & \multirow{3}{*}{$\begin{array}{c}\text { Wet Opium }{ }^{* 2} \\
\text { Yield } \\
(\mathrm{kg} / \mathrm{jr} .) \\
\end{array}$} & \multirow{3}{*}{$\begin{array}{c}\text { Wet Opium } \\
\text { Production } \\
\text { (kg) }\end{array}$} \\
\hline & & & & & & & & \\
\hline & & & & & (jerib) & $(\%)$ & & \\
\hline \multicolumn{9}{|c|}{ Province: Nangarhar } \\
\hline GOSHTA & 1863 & BADY & BADY & 40 & 26 & 65 & 9 & 234 \\
\hline GOSHTA & 1859 & BAR SAPARAY & BAR SAPARAY & 60 & 32 & 53 & 10 & 320 \\
\hline GOSHTA & 1856 & BARA OGARAY & BARA OGARAY & 120 & 23 & 19 & 9 & 207 \\
\hline GOSHTA & 1723 & BELA & BARA BELA & 160 & 45 & 28 & 11 & 495 \\
\hline GOSHTA & 1851 & DAG KALAY & DAG KALAY & 90 & 28 & 31 & 8 & 224 \\
\hline GOSHTA & 1849 & DRABO JAWAR & DRABO JAWAR & 40 & 22 & 55 & 9 & 198 \\
\hline GOSHTA & 1848 & GHULAMO KAC & GHULAMO KAC & 100 & 24 & 24 & 9 & 216 \\
\hline GOSHTA & 1840 & GHUSHTA & GHUSHTA & 1,000 & 430 & 43 & 18 & 7,740 \\
\hline GOSHTA & 1724 & GOSHTA & BANDA & 300 & 163 & 54 & 18 & 2,934 \\
\hline GOSHTA & 1861 & KATO KALAY & KATO KALAY & 100 & 35 & 35 & 10 & 350 \\
\hline GOSHTA & 1730 & KHOZI & AHMAD KHEL & 230 & 21 & 9 & 17 & 357 \\
\hline GOSHTA & 1839 & KHOZI & CHUR KHEL & 200 & 40 & 20 & 15 & 600 \\
\hline GOSHTA & 1734 & KHOZI & MERA KHEL & 240 & 35 & 15 & 16 & 560 \\
\hline GOSHTA & 1728 & KHOZI & PESHO KHEL & 100 & 22 & 22 & 16 & 352 \\
\hline GOSHTA & 1725 & KHOZI & SURA KHEL & 170 & 35 & 21 & 13 & 455 \\
\hline GOSHTA & 1727 & KHOZI & YAQUB KHEL & 220 & 53 & 24 & 11 & 583 \\
\hline GOSHTA & 1733 & KHUIZO & AKAH KHEL & 130 & 30 & 23 & 11 & 330 \\
\hline GOSHTA & 1731 & KHYR BANDAY & KHYR BANDAY & 200 & 121 & 61 & 18 & 2,178 \\
\hline GOSHTA & 1862 & KUZ SPARAY & KUZ SPARAY & 75 & 40 & 53 & 10 & 400 \\
\hline GOSHTA & 3376 & KUZA BELA & BARA BAILA & 260 & 95 & 37 & 12 & 1,140 \\
\hline GOSHTA & 1852 & KUZA OGARAY & KUZA OGARAY & 50 & 10 & 20 & 8 & 80 \\
\hline GOSHTA & 3378 & LALOW & LALOW & 80 & 29 & 36 & 10 & 290 \\
\hline GOSHTA & 1846 & LOSHORA & LOSHORA & 70 & 19 & 27 & 9 & 171 \\
\hline GOSHTA & 1843 & PADSHAHI GHUNDAY & PADSHAHI GHUNDAY & 60 & 18 & 30 & 11 & 198 \\
\hline GOSHTA & 1726 & RAGHA & RAGHA & 120 & 47 & 39 & 14 & 658 \\
\hline GOSHTA & 1857 & SALALA & SALALA & 200 & 80 & 40 & 10 & 800 \\
\hline GOSHTA & 1841 & SARBAND & BAR SARBAND & 200 & 75 & 38 & 17 & 1,275 \\
\hline GOSHTA & 1842 & SARBAND & SARBAND & 240 & 88 & 37 & 15 & 1,320 \\
\hline GOSHTA & 1844 & SORANGI & SORANGI & 70 & 10 & 14 & 8 & 80 \\
\hline
\end{tabular}

* Jerib (jr.) $=1 / 5$ ha

${ }^{*}{ }^{2}$ Dry Opium $=$ Wet Opium $-30 \%$ moisture
Page $19 \quad 109$
Source: UNDCP Opium Production Survey, 1995. File: NGR2.XLS - 06/08/95 
Opium Production Survey by village in Nangarhar province, 1995.

\begin{tabular}{|c|c|c|c|c|c|c|c|c|}
\hline \multirow{3}{*}{ District } & \multirow{3}{*}{$\begin{array}{l}\text { Village } \\
\text { Code }\end{array}$} & \multirow{3}{*}{ Area/ Main Village } & \multirow{3}{*}{ Village } & \multirow{3}{*}{$\begin{array}{c}\text { Total } \\
\text { Cultivated } \\
\text { Land (jerib }{ }^{*} \text { ) }\end{array}$} & \multirow{2}{*}{\multicolumn{2}{|c|}{$\begin{array}{c}\text { Land Under } \\
\text { Poppy Cultivation }\end{array}$}} & \multirow{3}{*}{$\begin{array}{c}\text { Wet Opium }{ }^{* 2} \\
\text { Yield } \\
(\mathrm{kg} / \mathrm{jr} .)\end{array}$} & \multirow{3}{*}{$\begin{array}{c}\text { Wet Opium } \\
\text { Production } \\
(\mathrm{kg}) \\
\end{array}$} \\
\hline & & & & & & & & \\
\hline & & & & & (jerib) & $(\%)$ & & \\
\hline \multicolumn{9}{|c|}{ Province: Nangarhar } \\
\hline GOSHTA & 1850 & SPERA & SPERA & 150 & 20 & 13 & 8 & 160 \\
\hline GOSHTA & 1858 & SPERA & SPERA & 95 & 30 & 32 & 7 & 210 \\
\hline GOSHTA & 1853 & SUFI SAHEB KALAY & SUFI SAHEB KALAY & 60 & 8 & 13 & 7 & 56 \\
\hline GOSHTA & 1722 & SUR DAG & SUR DAG & 110 & 58 & 53 & 17 & 986 \\
\hline GOSHTA & 1847 & TOR RAGHA & TOR RAGHA & 80 & 16 & 20 & 8 & 128 \\
\hline GOSHTA & 1845 & TURKANI & TURKANI & 200 & 30 & 15 & 8 & 240 \\
\hline GOSHTA & 1721 & WARSAK & WARSAK & 300 & 56 & 19 & 13 & 728 \\
\hline GOSHTA & 1854 & ZERAY MAMA KHEL & ZERAY & 140 & 34 & 24 & 8 & 272 \\
\hline GOSHTA & 3371 & ZIARAT DAG & KUZ KALAY & 90 & 30 & 33 & 10 & 300 \\
\hline GOSHTA & 1860 & ZIARAT DAG & ZIARAT DAG & 100 & 46 & 46 & 10 & 460 \\
\hline GOSHTA & 3377 & ZOR KALAY & ZOR KALAY & 160 & 71 & 44 & 10 & 710 \\
\hline HISARAK & 1636 & AHMADZO KALAY & AHMADZO KALAY & 0 & 0 & \#DIV/0! & 0 & 0 \\
\hline HISARAK & 1638 & ALAM KAC & ALAM KAC & 120 & 60 & 50 & 12 & 720 \\
\hline HISARAK & 1671 & ALAM KHEL & ALAM KHEL & 90 & 0 & 0 & 0 & 0 \\
\hline HISARAK & 1633 & ALI KHEL & ALI KHEL & 140 & 40 & 29 & 8 & 320 \\
\hline HISARAK & 1630 & ARAB KHEL & ARAB KHEL & 200 & 100 & 50 & 11 & 1,100 \\
\hline HISARAK & 1658 & ASHPAN & SHIKHAN & 300 & 50 & 17 & 14 & 700 \\
\hline HISARAK & 42 & BADI NAW & BADI NAW & 25 & 0 & 0 & 0 & 0 \\
\hline HISARAK & 1655 & BAHAWLAY & BAHAWLAY & 60 & 40 & 67 & 15 & 600 \\
\hline HISARAK & 1645 & BARA KOLALA & BARA KOLALA & 30 & 13 & 43 & 11 & 143 \\
\hline HISARAK & 1674 & CHARTUT & CHARTUT & 400 & 100 & 25 & 10 & 1,000 \\
\hline HISARAK & 1648 & CHINZAI & CHINZAI & 60 & 10 & 17 & 10 & 100 \\
\hline HISARAK & 20 & CHORCHANG(ORCHANG) & CHORCHANG(ORCHANG) & 200 & 100 & 50 & 11 & 1,100 \\
\hline HISARAK & 32 & DAGYAN & DAGYAN & 100 & 20 & 20 & 10 & 200 \\
\hline HISARAK & 24 & DARALIKHEL & DARALIKHEL & 150 & 60 & 40 & 13 & 780 \\
\hline HISARAK & 13 & DOAW & DOAW & 80 & 0 & 0 & 0 & 0 \\
\hline HISARAK & 8 & GANJA KHEL & GANJA KHEL & 160 & 40 & 25 & 10 & 400 \\
\hline HISARAK & 1 & GARAY & GARAY & 25 & 5 & 20 & 9 & 45 \\
\hline HISARAK & 1668 & GARI (PAS GARI) & GARI (PAS GARI) & 100 & 0 & 0 & 0 & 0 \\
\hline
\end{tabular}

* Jerib (jr.) $=1 / 5$ ha

${ }^{* 2}$ Dry Opium $=$ Wet Opium $-30 \%$ moisture
Page 20
Source: UNDCP Opium Production Survey, 1995. File: NGR2.XLS - 06/08/95 
Opium Production Survey by village in Nangarhar province, 1995.

\begin{tabular}{|c|c|c|c|c|c|c|c|c|}
\hline \multirow{3}{*}{ District } & \multirow{3}{*}{$\begin{array}{l}\text { Village } \\
\text { Code }\end{array}$} & \multirow{3}{*}{ Area/ Main Village } & \multirow{3}{*}{ Village } & \multirow{3}{*}{\begin{tabular}{c|} 
Total \\
Cultivated \\
Land (jerib ${ }^{*}$ ) \\
\end{tabular}} & \multirow{2}{*}{\multicolumn{2}{|c|}{$\begin{array}{c}\text { Land Under } \\
\text { Poppy Cultivation }\end{array}$}} & \multirow{3}{*}{$\begin{array}{c}\text { Wet Opium *2 } \\
\text { Yield } \\
(\mathrm{kg} / \mathrm{jr} .) \\
\end{array}$} & \multirow{3}{*}{$\begin{array}{l}\text { Wet Opium } \\
\text { Production } \\
(\mathrm{kg})\end{array}$} \\
\hline & & & & & & & & \\
\hline & & & & & (jerib) & $(\%)$ & & \\
\hline \multicolumn{9}{|c|}{ Province: Nangarhar } \\
\hline HISARAK & 4 & GAZAK & GAZAK & 80 & 50 & 63 & 12 & 600 \\
\hline HISARAK & 1631 & GHAZI KHEL & GHAZI KHEL & 100 & 35 & 35 & 15 & 525 \\
\hline HISARAK & 1660 & GHOGEZ & KARIM KHIL & 50 & 0 & 0 & 0 & . \\
\hline HISARAK & 1632 & GHOGEZ & KHENGAY & 100 & 0 & 0 & 0 & 0 \\
\hline HISARAK & 1637 & GHWAGIZA & GHWAGIZA & 120 & 0 & 0 & 0 & 0 \\
\hline HISARAK & 1650 & GODARAH & GODARAH & 60 & 0 & 0 & 0 & 0 \\
\hline HISARAK & 1672 & GOLA KHEL & GOLA KHEL & 200 & 50 & 25 & 10 & 500 \\
\hline HISARAK & 1672 & GOLA KHEL & GOLA KHEL & 0 & 0 & \#DIV/0! & 0 & 0 \\
\hline HISARAK & 1667 & HASAN KHEL & HASAN KHEL & 0 & 0 & \#DIV/0! & 0 & 0 \\
\hline HISARAK & 1651 & JABAY KALAY & JABAY KALAY & 100 & 0 & 0 & 0 & 0 \\
\hline HISARAK & 1662 & JAMAL KHEL & JAMAL KHEL & 0 & 0 & \#DIV/0! & 0 & 0 \\
\hline HISARAK & 40 & KADAY & KADAY & 50 & 20 & 40 & 10 & 200 \\
\hline HISARAK & 1665 & KAFAR KHEL & KAFAR KHEL & 0 & 0 & \#DIV/0! & 0 & 0 \\
\hline HISARAK & 1663 & KAMI KHEL & KAMI KHEL & 0 & 0 & \#DIV/0! & 0 & 0 \\
\hline HISARAK & 21 & KAMRAKI & KAMRAKI & 80 & 40 & 50 & 12 & 480 \\
\hline HISARAK & 26 & KHAIR ABAD & KHAIR ABAD & 100 & 50 & 50 & 11 & 550 \\
\hline HISARAK & 2 & KHANA & KHANA & 40 & 0 & 0 & 0 & 0 \\
\hline HISARAK & 16 & KHANANO KOTE & KHANANO KOTE & 20 & 10 & 50 & 14 & 140 \\
\hline HISARAK & 1653 & KHANJAR & KHANJAR & 200 & 20 & 10 & 10 & 200 \\
\hline HISARAK & 1642 & KHAROTI & KHAROTI & 25 & 0 & 0 & 0 & 0 \\
\hline HISARAK & 6 & KHOMAR KHEL & KHOMAR KHEL & 180 & 10 & 6 & 12 & 120 \\
\hline HISARAK & 1649 & KHOMAR KHEL & KHOMAR KHEL & 0 & 0 & \#DIV/0! & 0 & 0 \\
\hline HISARAK & 1652 & KHYALO KAC & KHYALO KAC & 110 & 0 & 0 & 0 & 0 \\
\hline HISARAK & 5 & KOLALA & KOLALA & 170 & 130 & 76 & 14 & 1,820 \\
\hline HISARAK & 11 & KOZ GARAY & KOZ GARAY & 60 & 0 & 0 & 0 & 0 \\
\hline HISARAK & 3 & LAJGAR & GHUNDAY KALAY & 180 & 25 & 14 & 10 & 250 \\
\hline HISARAK & 1643 & LAJGAR & LAJGAR & 400 & 100 & 25 & 10 & 1,000 \\
\hline HISARAK & 1644 & LAJGAR & POORA KELT & 180 & 80 & 44 & 10 & 800 \\
\hline HISARAK & 7 & LANGAR KHEL & LANGAR KHEL & 80 & 30 & 38 & 10 & 300 \\
\hline
\end{tabular}

* Jerib (jr.) $=1 / 5$ ha

*2 Dry Opium $=$ Wet Opium $-30 \%$ moisture
Page $21 \quad 1 / /$
Source: UNDCP Opium Production Survey, 1995. File: NGR2.XLS - 06/08/9 
Opium Production Survey by village in Nangarhar province, 1995.

\begin{tabular}{|c|c|c|c|c|c|c|c|c|}
\hline \multirow{3}{*}{ District } & \multirow{3}{*}{$\begin{array}{l}\text { Village } \\
\text { Code }\end{array}$} & \multirow{3}{*}{ Area/ Main Village } & \multirow{3}{*}{ Village } & \multirow{3}{*}{\begin{tabular}{|c|} 
Total \\
Cultivated \\
Land (jerib ${ }^{*}$ ) \\
\end{tabular}} & \multirow{2}{*}{\multicolumn{2}{|c|}{$\begin{array}{c}\text { Land Under } \\
\text { Poppy Cultivation }\end{array}$}} & \multirow{3}{*}{$\begin{array}{c}\text { Wet Opium }^{* 2} \\
\text { Yield } \\
(\mathrm{kg} / \mathrm{jr} .) \\
\end{array}$} & \multirow{3}{*}{$\begin{array}{c}\text { Wet Opium } \\
\text { Production } \\
(\mathrm{kg}) \\
\end{array}$} \\
\hline & & & & & & & & \\
\hline & & & & & (jerib) & (\%) & & \\
\hline \multicolumn{9}{|c|}{ Province: Nangarhar } \\
\hline HISARAK & 9 & MANI & MANI & 0 & 0 & \#DIV/0! & 0 & 0 \\
\hline HISARAK & 10 & MANSUR KALAY & MANSUR KALAY & 160 & 30 & 19 & 10 & 300 \\
\hline HISARAK & 3493 & MARKEZ HISARAK & MARKEZ HISARAK & 400 & 180 & 45 & 12 & 2,160 \\
\hline HISARAK & 3388 & MAYAN KHEL & MAYAN KHEL & 150 & 20 & 13 & 12 & 240 \\
\hline HISARAK & 1647 & MIYA SAHIB KILY & MIYA SAHIB KILY & 200 & 0 & 0 & 0 & 0 \\
\hline HISARAK & 28 & MOHAMMAD KALA & MOHAMMAD KALA & 210 & 20 & 10 & 10 & 200 \\
\hline HISARAK & 1670 & MOHAMMADI & MOHAMMADI & 400 & 50 & 13 & 8 & 400 \\
\hline HISARAK & 3423 & MYAGAN & MYAGAN & 150 & 0 & 0 & 0 & 0 \\
\hline HISARAK & 36 & MYASAHEB KALAY & MYASAHEB KALAY & 100 & 20 & 20 & 8 & 160 \\
\hline HISARAK & 19 & NARGOSAY & NARGOSAY & 200 & 100 & 50 & 10 & 1,000 \\
\hline HISARAK & 41 & NASER KHEL & NASER KHEL & 160 & 60 & 38 & 12 & 720 \\
\hline HISARAK & 37 & PARJINA & PARJINA & 150 & 50 & 33 & 9 & 450 \\
\hline HISARAK & 1661 & QADAR KHEL & QADAR KHEL & 0 & 0 & \#DIV/0! & 0 & 0 \\
\hline HISARAK & 3421 & RAGHA & BOSTAN KHEL & 100 & 10 & 10 & 12 & 120 \\
\hline HISARAK & 39 & RAGHA & RAGHA & 400 & 130 & 33 & 10 & 1,300 \\
\hline HISARAK & 43 & SAHAKAN & SAHAKAN & 40 & 20 & 50 & 15 & 300 \\
\hline HISARAK & 15 & SARAW (SAHRA) & SARAW (SAHRA) & 0 & 0 & \#DIV/0! & 0 & 0 \\
\hline HISARAK & 23 & SARWAR BAGH & SARWAR BAGH & 0 & 0 & \#DIV/0! & 0 & 0 \\
\hline HISARAK & 1654 & SEHAW PAYAN & SEHAW PAYAN & 100 & 15 & 15 & 9 & 135 \\
\hline HISARAK & 14 & SENA KHEL & SENA KHEL & 20 & 0 & 0 & 0 & 0 \\
\hline HISARAK & 31 & SEYAW BALA & SEYAW BALA & 400 & 40 & 10 & 12 & 480 \\
\hline HISARAK & 1640 & SHAN KHAN & SHAN KHAN & 40 & 20 & 50 & 12 & 240 \\
\hline HISARAK & 1635 & SHAN SHOBAY & SHAN SHOBAY & 20 & 0 & 0 & 0 & 0 \\
\hline HISARAK & 12 & SHARIF KHEL & SHARIF KHEL & 0 & 0 & \#DIV/0! & 0 & 0 \\
\hline HISARAK & 3422 & SHIN PAN & SHIN PAN & 50 & 10 & 20 & 9 & 90 \\
\hline HISARAK & 1641 & SHINWARI & SHINWARI & 30 & 20 & 67 & 12 & 240 \\
\hline HISARAK & 25 & SHINWARI & SHINWARI & 0 & 0 & \#DIV/0! & 0 & 0 \\
\hline HISARAK & 45 & SORONDU KALAY & SORONDU KALAY & 150 & 20 & 13 & 9 & 180 \\
\hline HISARAK & 44 & SRAW & SRAW & 0 & 0 & \#DIV/0! & 0 & 0 \\
\hline
\end{tabular}


Opium Production Survey by village in Nangarhar province, 1995.

\begin{tabular}{|c|c|c|c|c|c|c|c|c|}
\hline & \multirow{3}{*}{ Code } & & & Total & Land 1 & & Wet Opium *2 & Wet Opium \\
\hline \multirow[t]{2}{*}{ District } & & \multirow[t]{2}{*}{ Area/ Main Village } & \multirow[t]{2}{*}{ Village } & \multirow{2}{*}{$\begin{array}{c}\text { Cultivated } \\
\text { Land (jerib }{ }^{*} \text { ) }\end{array}$} & \multicolumn{2}{|c|}{ Poppy Cultivation } & \multirow{2}{*}{$\begin{array}{c}\text { Yield } \\
(\mathrm{kg} / \mathrm{jr} .) \\
\end{array}$} & \multirow{2}{*}{$\begin{array}{c}\text { Production } \\
\text { (kg) }\end{array}$} \\
\hline & & & & & (jerib) & (\%) & & \\
\hline \multicolumn{9}{|c|}{ Province: Nangarhar } \\
\hline HISARAK & 22 & TERAY & TERAY & 25 & 0 & 0 & 0 & 0 \\
\hline HISARAK & 1673 & TORI KHEL & TORI KHEL & 100 & 50 & 50 & 11 & 550 \\
\hline HISARAK & 3424 & TOWDA CHINA & TOWDA CHINA & 40 & 30 & 75 & 13 & 390 \\
\hline HISARAK & 1634 & WUDI HESAR & WUDI HESAR & 250 & 20 & 8 & 12 & 240 \\
\hline HISARAK & 1669 & YAGHI BAND & YAGHI BAND & 140 & 10 & 7 & 12 & 120 \\
\hline HISARAK & 38 & ZARIF KHEL & ZARIF KHEL & 300 & 50 & 17 & 14 & 700 \\
\hline HISARAK & 1639 & ZEYARAT GARI & ZEYARAT GARI & 80 & 0 & 0 & 0 & 0 \\
\hline KAMA & 3338 & ANARGAR SAHIBZADAGAN & ANARGAR SAHIBZADAGAN & 340 & 0 & 0 & 0 & 0 \\
\hline KAMA & 1701 & ARAB KHEL & ARAB KHEL & 1,200 & 0 & 0 & 0 & 0 \\
\hline KAMA & 1684 & ARBABAN & BARA ARBABAN & 570 & 0 & 0 & 0 & 0 \\
\hline KAMA & 1709 & ARBABAN & KUZ ARBABAN & 450 & 0 & 0 & 0 & 0 \\
\hline KAMA & 1681 & BANJANI & BANJANI & 1,000 & 0 & 0 & 0 & 0 \\
\hline KAMA & 1694 & BAZID KHEL & BAZID KHEL & 600 & 0 & 0 & 0 & 0 \\
\hline KAMA & 1702 & BELA & BELA & 400 & 0 & 0 & 0 & 0 \\
\hline KAMA & 1678 & CHONA GACH & CHONA GACH & 0 & 0 & \#DIV/0! & 0 & 0 \\
\hline KAMA & 1676 & DAG KALAY & DAG KALAY & 0 & 0 & \#DIV/0! & 0 & 0 \\
\hline KAMA & 1687 & DAR BANG & DAR BANG & 600 & 0 & 0 & 0 & 0 \\
\hline KAMA & 1706 & DARGALAY & DARGALAY & 500 & 0 & 0 & 0 & 0 \\
\hline KAMA & 1679 & DEH GHAZI & DEH GHAZI & 400 & 0 & 0 & 0 & 0 \\
\hline KAMA & 1693 & DEH GHAZI & GARAY & 200 & 0 & 0 & 0 & 0 \\
\hline KAMA & 1714 & DEH TAHER (GH.RASOL) & DEH TAHER (GH.RASOL) & 540 & 0 & 0 & 0 & 0 \\
\hline KAMA & 1717 & DEH TAR & DEH TAR & 500 & 0 & 0 & 0 & 0 \\
\hline KAMA & 1680 & GAHAK & GAHAK & 1,000 & 0 & 0 & 0 & 0 \\
\hline KAMA & 3343 & GARDAB & GARDAB & 1,000 & 40 & 4 & 10 & 400 \\
\hline KAMA & 1689 & GOJ & GOJ & 0 & 0 & \#DIV/0! & 0 & 0 \\
\hline KAMA & 1699 & JAMALI & PIR BAGH.PAS.BA & 600 & 0 & 0 & 0 & 0 \\
\hline KAMA & 1685 & KAGUL & BAJAWARI & 500 & 0 & 0 & 0 & 0 \\
\hline KAMA & 1710 & KAKUL & KAKUL & 800 & 0 & 0 & 0 & 0 \\
\hline KAMA & 1691 & KANDAHARI & KANDAHARI & 300 & 0 & 0 & 0 & 0 \\
\hline
\end{tabular}

$*^{*}$ Jerib (jr.) $=1 / 5$ ha

* 2 Dry Opium = Wet Opium - $30 \%$ moisture 
Opium Production Survey by village in Nangarhar province, 1995.

\begin{tabular}{|c|c|c|c|c|c|c|c|c|}
\hline \multirow{3}{*}{ District } & \multirow{3}{*}{$\begin{array}{c}\text { Village } \\
\text { Code }\end{array}$} & \multirow{3}{*}{ Area/ Main Village } & \multirow{3}{*}{ Village } & \multirow{3}{*}{$\begin{array}{c}\text { Total } \\
\text { Cultivated } \\
\text { Land (jerib }{ }^{*} \text { ) } \\
\end{array}$} & \multirow{2}{*}{\multicolumn{2}{|c|}{$\begin{array}{c}\text { Land Under } \\
\text { Poppy Cultivation }\end{array}$}} & \multirow{3}{*}{$\begin{array}{c}\text { Wet Opium }{ }^{* 2} \\
\text { Yield } \\
(\mathrm{kg} / \mathrm{jr} .) \\
\end{array}$} & \multirow{3}{*}{$\begin{array}{c}\text { Wet Opium } \\
\text { Production } \\
(\mathrm{kg}) \\
\end{array}$} \\
\hline & & & & & & & & \\
\hline & & & & & (jerib) & $(\%)$ & & \\
\hline \multicolumn{9}{|c|}{ Province: Nangarhar } \\
\hline KAMA & 1719 & KANDI MIR KHUAJA & KANDI MIR KHUAJA & 600 & 0 & 0 & 0 & 0 \\
\hline KAMA & 1703 & KANDIHA & KANDI & 600 & 0 & 0 & 0 & 0 \\
\hline KAMA & 1700 & KANDIHA & KANDI MIR LALA & 100 & 0 & 0 & 0 & 0 \\
\hline KAMA & 3341 & KHAS KAMA ANAR BAGH & KHAS KAMA ANAR BAGH & 700 & 0 & 0 & 0 & 0 \\
\hline KAMA & 3342 & LANDA BOCH & LANDA BOCH & 1,300 & 0 & 0 & 0 & 0 \\
\hline KAMA & 1720 & LANDA BOCH & LANDA BOCH.MALIKANA & 200 & 0 & 0 & 0 & 0 \\
\hline KAMA & 1695 & MAMA KHEL & MAMA KHEL & 1,200 & 0 & 0 & 0 & 0 \\
\hline KAMA & 1692 & MASTALI & MASTALI & 1,200 & 0 & 0 & 0 & 0 \\
\hline KAMA & 1696 & MATA KHEL & MATA KHEL & 50 & 18 & 36 & 10 & 180 \\
\hline KAMA & 3344 & MIRZA KHEL & MIRZA KHEL & 0 & 0 & \#DIV/0! & 0 & 0 \\
\hline KAMA & 3339 & NARAY OBAH & NARAY OBAH & 40 & 17 & 43 & 11 & 187 \\
\hline KAMA & 1704 & NAW ABAD & NAW ABAD & 0 & 0 & \#DIV/0! & 0 & 0 \\
\hline KAMA & 1715 & PIRZAI & PIRZAI & 1,200 & 0 & 0 & 0 & 0 \\
\hline KAMA & 1690 & QALA-I-AKHUND & QALA-I-AKHUND & 0 & 0 & \#DIV/0! & 0 & 0 \\
\hline KAMA & 1683 & QALA-I-WUDUD & QALA-I-WUDUD & 600 & 0 & 0 & 0 & 0 \\
\hline KAMA & 1712 & SADA & SADA ABDULLAH & 150 & 0 & 0 & 0 & 0 \\
\hline KAMA & 3347 & SADA-I-SHER ALI & SADA-I- SHER ALI & 480 & 0 & 0 & 0 & 0 \\
\hline KAMA & 3340 & SAFDARI & SAFDARI & 900 & 0 & 0 & 0 & 0 \\
\hline KAMA & 3346 & SANGAR & SANGAR & 200 & 0 & 0 & 0 & 0 \\
\hline KAMA & 1716 & SAPARI & SAPARI & 40 & 15 & 38 & 10 & 150 \\
\hline KAMA & 3337 & SARAY & SARAY & 200 & 0 & 0 & 0 & 0 \\
\hline KAMA & 1688 & SHADEL QALA & SHADEL QALA & 200 & 0 & 0 & 0 & 0 \\
\hline KAMA & 3345 & SHEKHAI & SHEKHAI & 400 & 0 & 0 & 0 & 0 \\
\hline KAMA & 1698 & SHELAM KHANA & SHELAM KHANA & 750 & 0 & 0 & 0 & 0 \\
\hline KAMA & 1698 & SHELAM KHANA ROSHNA & SHELAM KHAN & 500 & 0 & 0 & 0 & 0 \\
\hline KAMA & 1707 & SHER GAR & KHAR GANI & 900 & 0 & 0 & 0 & 0 \\
\hline KAMA & 1708 & SHERGAR & SHERGAR & 1,000 & 0 & 0 & 0 & 0 \\
\hline KAMA & 1686 & SHIR GAR & KODO KALAY & 500 & 0 & 0 & 0 & 0 \\
\hline KAMA & 1705 & SHODA & SHODA & 500 & 0 & 0 & 0 & 0 \\
\hline
\end{tabular}


Opium Production Survey by village in Nangarhar province, 1995.

\begin{tabular}{|c|c|c|c|c|c|c|c|c|}
\hline & \multirow{3}{*}{$\begin{array}{l}\text { Village } \\
\text { Code }\end{array}$} & \multirow{3}{*}{ Area/ Main Village } & \multirow{3}{*}{ Village } & \multirow{3}{*}{$\begin{array}{c}\text { Total } \\
\text { Cultivated } \\
\left.\text { Land (jerib }{ }^{*}\right) \\
\end{array}$} & \multirow{2}{*}{\multicolumn{2}{|c|}{$\begin{array}{c}\text { Land Under } \\
\text { Poppy Cultivation }\end{array}$}} & \multirow{3}{*}{$\begin{array}{c}\text { Wet Opium *2 } \\
\text { Yield } \\
(\mathrm{kg} / \mathrm{jr} .) \\
\end{array}$} & \multirow{3}{*}{$\begin{array}{c}\text { Wet Opium } \\
\text { Production } \\
(\mathrm{kg})\end{array}$} \\
\hline \multirow[t]{2}{*}{ District } & & & & & & & & \\
\hline & & & & & (jerib) & $(\%)$ & & \\
\hline \multicolumn{9}{|c|}{ Province: Nangarhar } \\
\hline KAMA & 1718 & ZAKHEL & ZAKHEL & 2,000 & 0 & 0 & 0 & 0 \\
\hline KAMA & 1682 & ZARSHOI & AFRIDO KALAY & 800 & 0 & 0 & 0 & 0 \\
\hline KAMA & 1711 & ZARSHOI & ZARSHOI & . $\quad 1,500$ & 0 & 0 & 0 & 0 \\
\hline KHOGIANI & 2596 & ADAD KHEL & DADA KHEL & 145 & 70 & 48 & 14 & 980 \\
\hline KHOGIANI & 2568 & ADOR & ADOR & 800 & 500 & 63 & 14 & 7,000 \\
\hline KHOGIANI & 2565 & AHMAD KHAIL & SHERO KHAIL & 270 & 100 & 37 & 12 & 1,200 \\
\hline KHOGIANI & 2622 & AHMAD KHEL & AHMAD KHEL & 370 & 170 & 46 & 10 & 1,700 \\
\hline KHOGIANI & 3440 & AHMAD KHEL & MERZA KHEL & 350 & 170 & 49 & 17 & 2,890 \\
\hline KHOGIANI & 3441 & AHMAD KHEL & PER GHANDAY & 250 & 100 & 40 & 16 & 1,600 \\
\hline KHOGIANI & 2588 & ASMAN KALAY & ASMAN KALAY & 155 & 75 & 48 & 10 & 750 \\
\hline KHOGIANI & 2569 & AWDAY & AWDAY & 400 & 200 & 50 & 12 & 2,400 \\
\hline KHOGIANI & 2551 & BABAKER KHEL & BABAKER KHEL & 340 & 160 & 47 & 9 & 1,440 \\
\hline KHOGIANI & 2604 & BAGHICHA & BAGHICHA & 400 & 200 & 50 & 14 & 2,800 \\
\hline KHOGIANI & 2621 & BAR ARGHACH & BAR ARGHACH & 1,300 & 650 & 50 & 13. & 8,450 \\
\hline KHOGIANI & 2603 & BAR KALA KHEL & BAR KALA KHEL & 380 & 180 & 47 & 15 & 2,700 \\
\hline KHOGIANI & 2554 & BAR KELAY & BAR KELAY & 200 & 40 & 20 & 12 & 480 \\
\hline KHOGIANI & 2591 & BAZID KHEL & BAZID KHEL & 550 & 200 & 36 & 10 & 2,000 \\
\hline KHOGIANI & 2614 & BUTKHAKAY & BUTKHAKAY & 250 & 120 & 48 & 15 & 1,800 \\
\hline KHOGIANI & 2560 & CHINO MYAGAN & CHINO MYAGAN & 90 & 40 & 44 & 11 & 440 \\
\hline KHOGIANI & 2613 & DARABGAY & DARABGAY & 260 & 110 & 42 & 13 & 1,430 \\
\hline KHOGIANI & 2586 & DARGA & DARGA & 190 & 90 & 47 & 10 & 900 \\
\hline KHOGIANI & 2552 & DEGAN & DEGAN & 500 & 270 & 54 & 16 & 4,320 \\
\hline KHOGIANI & 3436 & DOABA TATANG & DO AB & 230 & 100 & 43 & 13 & 1,300 \\
\hline KHOGIANI & 2556 & DOAWA & DOAWA & 75 & 0 & 0 & 0 & 0 \\
\hline KHOGIANI & 2566 & EBRAHIM & EBRAHIM & 70 & 0 & 0 & 0 & 0 \\
\hline KHOGIANI & 2549 & EBRAHIM KHAIL & GORA CHINA(CHAKAW) & 150 & 80 & 53 & 11 & 880 \\
\hline KHOGIANI & 2615 & EBRAHIM KHEL & CHINARGAI & 210 & 100 & 48 & 13 & 1,300 \\
\hline KHOGIANI & 2589 & EBRAHIM KHEL & GODALI & 175 & 70 & 40 & 10 & 700 \\
\hline KHOGIANI & 2562 & EBRAHIM KHEL & LOYA KALAY & 700 & 300 & 43 & 12 & 3,600 \\
\hline
\end{tabular}

* Jerib (jr.) $=1 / 5$ ha

${ }^{*}{ }^{2}$ Dry Opium $=$ Wet Opium $-30 \%$ moisture
Source: UNDCP Opium Production Survey, 1995. File: NGR2.XLS - 06/08/95 
Opium Production Survey by village in Nangarhar province, 1995.

\begin{tabular}{|c|c|c|c|c|c|c|c|c|}
\hline \multirow{3}{*}{ District } & \multirow{3}{*}{$\begin{array}{l}\text { Village } \\
\text { Code }\end{array}$} & \multirow{3}{*}{ Area/ Main Village } & \multirow{3}{*}{ Village } & \multirow{3}{*}{\begin{tabular}{c|} 
Total \\
Cultivated \\
Land (jerib *) \\
\end{tabular}} & \multirow{2}{*}{\multicolumn{2}{|c|}{$\begin{array}{c}\text { Land Under } \\
\text { Poppy Cultivation }\end{array}$}} & \multirow{3}{*}{$\begin{array}{c}\text { Wet Opium }^{* 2} \\
\text { Yield } \\
(\mathrm{kg} / \mathrm{jr} .)\end{array}$} & \multirow{3}{*}{$\begin{array}{c}\text { Wet Opium } \\
\text { Production } \\
\text { (kg) }\end{array}$} \\
\hline & & & & & & & & \\
\hline & & & & & (jerib) & $(\%)$ & & \\
\hline \multicolumn{9}{|c|}{ Province: Nangarhar } \\
\hline KHOGIANI & 2616 & EBRAHIM KHEL & SRAH GHUNDAI & 190 & 90 & 47 & 10 & 900 \\
\hline KHOGIANI & 2599 & FATEH KHERKAY & FATEH KHERKAY & 100 & 50 & 50 & 14 & 700 \\
\hline KHOGIANI & 2580 & HAKIM ABAD & HAKIM ABAD & 750 & 350 & 47 & 14 & 4,900 \\
\hline KHOGIANI & 2548 & HAMZA KHIL & BAR HMZA KHEL & 80 & 30 & 38 & 11 & 330 \\
\hline KHOGIANI & 2612 & HAMZA KHIL & KOZ HAMZA KHEL & 500 & 250 & 50 & 10 & 2,500 \\
\hline KHOGIANI & 2576 & IBRAHIM KHAIL & MALEK KALA & 360 & 150 & 42 & 10 & 1,500 \\
\hline KHOGIANI & 2547 & IRODERAN & IRODERAN & 140 & 70 & 50 & 12 & 840 \\
\hline KHOGIANI & 2571 & JAN BAZ KHEL & JAN BAZ KHEL & 240 & 120 & 50 & 13 & 1,560 \\
\hline KHOGIANI & 2555 & JANGAL KALAY & JANGAL KALAY & 110 & 0 & 0 & 0 & 0 \\
\hline KHOGIANI & 2593 & JAWARA & JAWARA & 260 & 110 & 42 & 16 & 1,760 \\
\hline KHOGIANI & 2545 & KABULYAN & KABULYAN & 160 & 100 & 63 & 16 & 1,600 \\
\hline KHOGIANI & 2620 & KAJA & KHAN KHEL & 900 & 500 & 56 & 12 & 6,000 \\
\hline KHOGIANI & 3438 & KALMA & KALMA & 75 & 40 & 53 & 10 & 400 \\
\hline KHOGIANI & 2578 & KAMBO & KAMBO & 500 & 230 & 46 & 16 & 3,680 \\
\hline KHOGIANI & 3443 & KANDWALY & KANDWALY & 280 & 100 & 36 & 11 & 1,100 \\
\hline KHOGIANI & 2619 & KHADAR KHEL & KHADAR KHEL & 550 & 150 & 27 & 11 & 1,650 \\
\hline KHOGIANI & 2611 & KHARABA & KHARABA & 150 & 70 & 47 & 10 & 700 \\
\hline KHOGIANI & 2570 & KHAWARAN & KHAWARAN & 500 & 240 & 48 & 12 & 2,880 \\
\hline KHOGIANI & 2598 & KHERMANA & KHERMANA & 500 & 240 & 48 & 16 & 3,840 \\
\hline KHOGIANI & 2600 & KHOGAY & KHOGAY & 700 & 300 & 43 & 19 & 5,700 \\
\hline KHOGIANI & 3434 & KHOZA KHEL & KHOZA KHEL & 540 & 240 & 44 & 18 & 4,320 \\
\hline KHOGIANI & 2581 & KUNWALE & KUNWALE & 0 & 0 & \#DIV/0! & 0 & 0 \\
\hline KHOGIANI & 2561 & KUZ ARGHACH & KUZ ARGHACH & 500 & 250 & 50 & 14 & 3,500 \\
\hline KHOGIANI & 2546 & KUZ KALA KHEL & KOZ KALLA KHIL & 150 & 80 & 53 & 15 & 1,200 \\
\hline KHOGIANI & 2582 & LANDAY KALAY & LANDAY KALAY & 160 & 80 & 50 & 14 & 1,120 \\
\hline KHOGIANI & 3437 & LANDY KALAY & LANDY KALAY & 200 & 100 & 50 & 10 & 1,000 \\
\hline KHOGIANI & 2592 & LOGH DARA & LOGH DARA & 570 & 240 & 42 & 13 & 3,120 \\
\hline KHOGIANI & 2573 & LOKHAY & LOKHAY & 420 & 169 & 40 & 16 & 2,704 \\
\hline KHOGIANI & 2618 & LOY MEMLA & LOY MEMLA & 200 & 100 & 50 & 18 & 1,800 \\
\hline
\end{tabular}

$*$ Jerib (jr.) $=1 / 5$ ha

* 2 Dry Opium = Wet Opium - $30 \%$ moisture
Page $26 \quad 1 / 6$
Source: UNDCP Opium Production Survey, 1995. File: NGR2.XLS - 06/08/95 
Opium Production Survey by village in Nangarhar province, 1995.

\begin{tabular}{|c|c|c|c|c|c|c|c|c|}
\hline & Village & \multirow{3}{*}{ Area/ Main Village } & \multirow{3}{*}{ Village } & \multirow{3}{*}{$\begin{array}{c}\text { Total } \\
\text { Cultivated } \\
\text { Land (jerib }{ }^{*} \text { ) } \\
\end{array}$} & \multirow{2}{*}{\multicolumn{2}{|c|}{$\begin{array}{c}\text { Land Under } \\
\text { Poppy Cultivation }\end{array}$}} & \multirow{3}{*}{$\begin{array}{c}\text { Wet Opium }^{* 2} \\
\text { Yield } \\
(\mathrm{kg} / \mathrm{jr} .)\end{array}$} & \multirow{3}{*}{$\begin{array}{c}\text { Wet Opium } \\
\text { Production } \\
(\mathrm{kg})\end{array}$} \\
\hline \multirow[t]{2}{*}{ District } & \multirow{2}{*}{ Code } & & & & & & & \\
\hline & & & & & (jerib) & (\%) & & \\
\hline \multicolumn{9}{|c|}{ Province: Nangarhar } \\
\hline KHOGIANI & 2574 & MALEK ASHEQ & MALEK ASHEQ & 120 & 70 & 58 & 14 & 980 \\
\hline KHOGIANI & 2585 & MANKAY & MANKAY & 230 & 110 & 48 & 13 & 1,430 \\
\hline KHOGIANI . & 2575 & MAYDAN KAMAI & MAYDAN K.AMAI & 230 & 100 & 43 & 13 & 1,300 \\
\hline KHOGIANI & 2587 & MELA & QAYOM KHEL & 280 & 150 & 54 & 13 & 1,950 \\
\hline KHOGIANI & 2595 & MEMLA & ARABAN & 270 & 130 & 48 & 11 & 1,430 \\
\hline KHOGIANI & 2564 & MEMLA & DARWAZAGAY & 250 & 120 & 48 & 11 & 1,320 \\
\hline KHOGIANI & 2563 & MONDA CHINAR & MONDA CHINAR & 245 & 130 & 53 & 12 & 1,560 \\
\hline KHOGIANI & 2558 & MYAGAN & MYAGAN & 100 & 0 & 0 & 0 & 0 \\
\hline KHOGIANI & 2617 & NEWAI BAZAAR & NEWAI BAZAAR & 200 & 100 & 50 & 10 & 1,000 \\
\hline KHOGIANI & 2567 & OMAR KHEL & OMAR KHEL & 700 & 300 & 43 & 16 & 4,800 \\
\hline KHOGIANI & 2572 & PACHA KALAY & PACHA KALAY & 200 & 100 & 50 & 20 & 2,000 \\
\hline KHOGIANI & 3439 & PAYRA KHEL & ANGORA & 160 & 75 & 47 & 12 & 900 \\
\hline KHOGIANI & 3444 & PAYRA KHEL & MORAGI & 340 & 140 & 41 & 11 & 1,540 \\
\hline KHOGIANI & 2553 & PIRA KHEL & PIRA KHEL & 400 & 200 & 50 & 16 & 3,200 \\
\hline KHOGIANI & 2601 & QADERKHAN BANDEH & QADERKHAN BANDEH & 360 & 170 & 47 & 12 & 2,040 \\
\hline KHOGIANI & 2550 & QAILAGHU & QAILAGHU & 1,100 & 500 & 45 & 11 & 5,500 \\
\hline KHOGIANI & 2606 & SAMOKAY & SAMOKAY & 130 & 0 & 0 & 0 & 0 \\
\hline KHOGIANI & 3435 & SAQAWA & SAQAWA & 750 & 300 & 40 & 12 & 3,600 \\
\hline KHOGIANI & 25943 & SEKANDARA & SEKANDARA & 80 & 0 & 0 & 0 & 0 \\
\hline KHOGIANI & 2610 & SEMASAY & SEMASAY & 290 & 140 & 48 & 10 & 1,400 \\
\hline KHOGIANI & 2559 & SENGANI & BAR SENGANI & 350 & 150 & 43 & 20 & 3,000 \\
\hline KHOGIANI & 2584 & SEWAY ZANGAL & SEWAY ZANGAL & 550 & 200 & 36 & 10 & 2,000 \\
\hline KHOGIANI & 3442 & SHANGINA/TANTAG & SHENGTAH & 220 & 100 & 45 & 11 & 1,100 \\
\hline KHOGIANI & 2577 & SHAQOL KHEL & SHAQOL KHEL & 250 & 125 & 50 & 10 & 1,250 \\
\hline KHOGIANI & 2608 & SHEGAY & SHEGAY & 650 & 300 & 46 & 10 & 3,000 \\
\hline KHOGIANI & 2590 & SHERGAR & SHERGAR & 160 & 100 & 63 & 17 & 1,700 \\
\hline KHOGIANI & 2597 & SORDAG & SORDAG & 180 & 100 & 56 & 13 & 1,300 \\
\hline KHOGIANI & 2607 & SURAY TIGA & SURAY TIGA & 0 & 0 & \#DIV/0! & 0 & 0 \\
\hline KHOGIANI & 2579 & SURMAY & SURMAY & 330 & 175 & 53 & 19 & 3,325 \\
\hline
\end{tabular}


Opium Production Survey by village in Nangarhar province, 1995.

\begin{tabular}{|c|c|c|c|c|c|c|c|c|}
\hline \multirow{3}{*}{ District } & \multirow{3}{*}{$\begin{array}{l}\text { Village } \\
\text { Code }\end{array}$} & \multirow{3}{*}{ Area/ Main Village } & \multirow{3}{*}{ Village } & \multirow{3}{*}{$\begin{array}{c}\text { Total } \\
\text { Cultivated } \\
\text { Land (jerib }{ }^{*} \text { ) } \\
\end{array}$} & \multirow{2}{*}{\multicolumn{2}{|c|}{$\begin{array}{c}\text { Land Under } \\
\text { Poppy Cultivation }\end{array}$}} & \multirow{3}{*}{$\begin{array}{c}\text { Wet Opium *2 } \\
\text { Yield } \\
(\mathrm{kg} / \mathrm{jr} .)\end{array}$} & \multirow{3}{*}{$\begin{array}{c}\text { Wet Opium } \\
\text { Production } \\
\quad(\mathrm{kg}) \\
\end{array}$} \\
\hline & & & & & & & & \\
\hline & & & & & (jerib) & (\%) & & \\
\hline \multicolumn{9}{|c|}{ Province: Nangarhar } \\
\hline KHOGIANI & 2583 & TATANG & TATANG & 400 & 200 & 50 & 10 & 2,000 \\
\hline KHOGIANI & 2557 & TORA CHINA & TORA CHINA & 90 & 40 & 44 & 10 & 400 \\
\hline KHOGIANI & 2602 & ZOR BAZAR & ZOR BAZAR & 14 & 6 & 43 & 11 & 66 \\
\hline KHOGIANI & 2605 & ZUR HOKUMATI & ZUR HOKUMATI & 35 & 10 & 29 & 10 & 100 \\
\hline KUZ KONAR & 2417 & ABDUL KHEL & ABDUL KHEL & 250 & 6 & 2 & 12 & 72 \\
\hline KUZ KONAR & 2436 & AHMAD BIG & AHMAD BIG & 100 & 20 & 20 & 12 & 240 \\
\hline KUZ KONAR & 2476 & ATAWOR & ATAWOR & 400 & 20 & 5 & 9 & 180 \\
\hline KUZ KONAR & 2427 & BADYALAY & NAGREZAN & 600 & 100 & 17 & 10 & 1,000 \\
\hline KUZ KONAR & 2425 & BADYALEE & BAR QALE & 200 & 40 & 20 & 14 & 560 \\
\hline KUZ KONAR & 2426 & BADYALI & BADYALI & 200 & 40 & 20 & 10 & 400 \\
\hline KUZ KONAR & 2402 & BANGAW & BANGAW & 600 & 20 & 3 & 7 & 140 \\
\hline KUZ KONAR & 2408 & BAR KASHKOT & GHOUNDAI & 250 & 8 & 3 & 14 & 112 \\
\hline KUZ KONAR & 2448 & BAR SHAD KHAN & BAR SHAD KHAN & 80 & 4 & 5 & 11 & 44 \\
\hline KUZ KONAR & 2461 & BARKASHKOT & BARKASHKOT & 350 & 35 & 10 & 9 & 315 \\
\hline KUZ KONAR & 2430 & BAZARAK & BAZARAK & 400 & 20 & 5 & 10 & 200 \\
\hline KUZ KONAR & 2414 & DARBA KHAIL & KOZ DARBA KHAIL & 70 & 3 & 4 & 10 & 30 \\
\hline KUZ KONAR & 2412 & DARBA KHAIL & SHEKHAN & 50 & 4 & 8 & 10 & 40 \\
\hline KUZ KONAR & 2411 & DURBA KHEL & BAR DURBA KHEL & 150 & 11 & 7 & 7 & 77 \\
\hline KUZ KONAR & 2435 & GOREK & BANDA & 0 & 0 & \#DIV/0! & 0 & 0 \\
\hline KUZ KONAR & 2469 & GOREK & GOLDER & 0 & 0 & \#DIV/0! & 0 & 0 \\
\hline KUZ KONAR & 2434 & GOREK & GOREK & 300 & 0 & 0 & 0 & 0 \\
\hline KUZ KONAR & 2432 & GOREK & WADO KANDER & 0 & 0 & \#DIV/0! & 0 & 0 \\
\hline KUZ KONAR & 2470 & HOUSE BAGH & HOUSE BAGH & 800 & 15 & 2 & 13 & 195 \\
\hline KUZ KONAR & 2464 & HOUSE BAGH & TANGI & 0 & 0 & \#DIV/0! & 0 & 0 \\
\hline KUZ KONAR & 2433 & KACHARA & KACHARA & 20 & 0 & 0 & 0 & 0 \\
\hline KUZ KONAR & 2446 & KAMTAK & KAMTAK & 100 & 10 & 10 & 11 & 110 \\
\hline KUZ KONAR & 2421 & KANDAY & KANDAY & 0 & 0 & \#DIV/0! & 0 & 0 \\
\hline KUZ KONAR & 3559 & KANDRO & KANDRO & 100 & 15 & 15 & 9 & 135 \\
\hline KUZ KONAR & 2404 & KASHKOT & ANARO TANGAI & 0 & 0 & \#DIV/0! & 0 & 0 \\
\hline
\end{tabular}

* Jerib (jr.) $=1 / 5$ ha

* 2 Dry Opium $=$ Wet Opium - $30 \%$ moisture
Page $28 \quad 118$
Source: UNDCP Opium Production Survey, 1995 File: NGR2.XLS - 06/08/95 
Opium Production Survey by village in Nangarhar province, 1995.

\begin{tabular}{|c|c|c|c|c|c|c|c|c|}
\hline \multirow{3}{*}{ District } & \multirow{3}{*}{$\begin{array}{l}\text { Village } \\
\text { Code }\end{array}$} & \multirow{3}{*}{ Area/ Main Village } & \multirow{3}{*}{ Village } & \multirow{3}{*}{\begin{tabular}{|c|} 
Total \\
Cultivated \\
Land $\left(j e r i b^{*}\right.$ ) \\
\end{tabular}} & \multirow{2}{*}{\multicolumn{2}{|c|}{$\begin{array}{c}\text { Land Under } \\
\text { Poppy Cultivation }\end{array}$}} & \multirow{3}{*}{$\begin{array}{c}\text { Wet Opium *2 } \\
\text { Yield } \\
(\mathrm{kg} / \mathrm{jr} .) \\
\end{array}$} & \multirow{3}{*}{$\begin{array}{c}\text { Wet Opium } \\
\text { Production } \\
(\mathrm{kg}) \\
\end{array}$} \\
\hline & & & & & & & & \\
\hline & & & & & (jerib) & $(\%)$ & & \\
\hline \multicolumn{9}{|c|}{ Province: Nangarhar } \\
\hline KUZ KONAR & 2467 & KHASHKI & KHASHKI & 250 & 10 & 4 & 10 & 100 \\
\hline KUZ KONAR & 2466 & KHORAM & KHORAM & 250 & 50 & 20 & 9 & 450 \\
\hline KUZ KONAR & 2462 & KOHNA DEH . & KOHNA DEH & 350 & 50 & 14 & 11 & 550 \\
\hline KUZ KONAR & 2428 & KOHNA DEH & ORYA KHAIL & 140 & 10 & 7 & 5 & 50 \\
\hline KUZ KONAR & 2455 & KOTAY & KASAI & 50 & 5 & 10 & 11 & 55 \\
\hline KUZ KONAR & 2407 & KOTAY & KOTAY & 380 & 50 & 13 & 10 & 500 \\
\hline KUZ KONAR & 2463 & KOZ KASHKOT & KOZ KASHKOT & 300 & 30 & 10 & 6 & 180 \\
\hline KUZ KONAR & 2457 & KOZ SHAD KHAN & KOZ SHAD KHAN & 100 & 6 & 6 & 11 & 66 \\
\hline KUZ KONAR & 2423 & LORI & LORI & 550 & 120 & 22 & 10 & 1,200 \\
\hline KUZ KONAR & 2401 & MALEKZAI & MALEKZAI & 300 & 5 & 2 & 7 & 35 \\
\hline KUZ KONAR & 2413 & MYAGAN & MYAGAN & 500 & 10 & 2 & 13 & 130 \\
\hline KUZ KONAR & 2450 & NAWJOY & NAWJOY & 150 & 5 & 3 & 11 & 55 \\
\hline KUZ KONAR & 2419 & PACHAHI QALA & PACHAHI QALA & 100 & 15 & 15 & 9 & 135 \\
\hline KUZ KONAR & 3381 & QALAGAI & QALAGAI & 360 & 12 & 3 & 13 & 156 \\
\hline KUZ KONAR & 2445 & QALGHATAK & CHAR QALA & 180 & 10 & 6 & 11 & 110 \\
\hline KUZ KONAR & 2465 & QALGHATAK & QALGHATAK & 1,000 & 20 & 2 & 8 & 160 \\
\hline KUZ KONAR & 2441 & SALAM PUR & SALAM PUR & 0 & 0 & \#DIV/0! & 0 & 0 \\
\hline KUZ KONAR & 2403 & SARBAND & SARBAND & 300 & 10 & 3 & 9 & 90 \\
\hline KUZ KONAR & 2468 & SARJAL & SARJAL & 240 & 15 & 6 & 11 & 165 \\
\hline KUZ KONAR & 2439 & SETORA & SETORA & 500 & 100 & 20 & 10 & 1,000 \\
\hline KUZ KONAR & 2429 & SHAMAL & SHAMAL & 400 & 10 & 3 & 7 & 70 \\
\hline KUZ KONAR & 2453 & SHEGA & ABARZAI & 200 & 10 & 5 & 8 & 80 \\
\hline KUZ KONAR & 2447 & SHEGA & CHARIKAR & 250 & 6 & 2 & 11 & 66 \\
\hline KUZ KONAR & 2409 & SHEGA & GIDARE & 200 & 5 & 3 & 10 & 50 \\
\hline KUZ KONAR & 2415 & SHEGA & PALWARI & 100 & 4 & 4 & 11 & 44 \\
\hline KUZ KONAR & 2410 & SHEGA & SAYED KHAIL & 1,000 & 20 & 2 & 12 & 240 \\
\hline KUZ KONAR & 2451 & SHEGAY & LAMATEK & 250 & 10 & 4 & 12 & 120 \\
\hline KUZ KONAR & 2422 & SHEGAY & SHEGAY & 400 & 15 & 4 & 7 & 105 \\
\hline KUZ KONAR & 2440 & SHETLAW & SHETLAW & 0 & 0 & \#DIV/0! & 0 & 0 \\
\hline
\end{tabular}

* Jerib (jr.) $=1 / 5$ ha

* ${ }^{2}$ Dry Opium = Wet Opium - $30 \%$ moisture
Page $29 \quad 119$
Source: UNDCP Opium Production Survey, 1995 File: NGR2.XLS - 06/08/95 
Opium Production Survey by village in Nangarhar province, 1995.

\begin{tabular}{|c|c|c|c|c|c|c|c|c|}
\hline & \multirow{3}{*}{$\begin{array}{l}\text { Village } \\
\text { Code }\end{array}$} & \multirow{3}{*}{ Area/ Main Village } & \multirow{3}{*}{ Village } & \multirow{3}{*}{$\begin{array}{c}\text { Total } \\
\text { Cultivated } \\
\text { Land (jerib }^{*} \text { ) } \\
\end{array}$} & \multirow{2}{*}{\multicolumn{2}{|c|}{$\begin{array}{c}\text { Land Under } \\
\text { Poppy Cultivation }\end{array}$}} & \multirow{3}{*}{$\begin{array}{c}\text { Wet Opium }^{* 2} \\
\text { Yield } \\
(\mathrm{kg} / \mathrm{jr} .) \\
\end{array}$} & \multirow{3}{*}{$\begin{array}{c}\text { Wet Opium } \\
\text { Production } \\
(\mathrm{kg})\end{array}$} \\
\hline \multirow[t]{2}{*}{ District } & & & & & & & & \\
\hline & & & & & (jerib) & $(\%)$ & & \\
\hline \multicolumn{9}{|c|}{ Province: Nangarhar } \\
\hline KUZ KONAR & 2458 & SHIR GAR & SHIR GAR & 120 & 0 & 0 & 0 & 0 \\
\hline KUZ KONAR & 2418 & SHIWA & DAGYAN & 60 & 4 & 7 & 8 & 32 \\
\hline KUZ KONAR & 2443 & SHIWA & GHUNDAI & 50 & 3 & 6 & 9 & 27 \\
\hline KUZ KONAR & 2444 & SHIWA & SHIWA & 150 & 20 & 13 & 10 & 200 \\
\hline KUZ KONAR & 2424 & SORACH & SORACH & 200 & 95 & 48 & 14 & 1,330 \\
\hline KUZ KONAR & 2459 & TARAN & KATA TANG & 0 & 0 & \#DIV/0! & 0 & 0 \\
\hline KUZ KONAR & 2406 & TARAN & TARAN & 400 & 15 & 4 & 13 & 195 \\
\hline KUZ KONAR & 2438 & WARA KAKAY & WARA KALAY & 250 & 40 & 16 & 14 & 560 \\
\hline KUZ KONAR & 2449 & ZARGARAN & ZARGARAN & 100 & 5 & 5 & 9 & 45 \\
\hline LALPUR & 3323 & AHANGARANO KALAY & SINDGHARI KALAY & 50 & 28 & 56 & 16 & 448 \\
\hline LALPUR & 3311 & AHENGARANO KALAY & AHANGARANO KALAY & 60 & 30 & 50 & 17 & 510 \\
\hline LALPUR & 3304 & BEHDARA & ABDUL WAHAB & 70 & 20 & 29 & 15 & 300 \\
\hline LALPUR & 3335 & BEHDARA & BEHDARA & 100 & 40 & 40 & 13 & 520 \\
\hline LALPUR & 3328 & BEHDARA & M. AZAM & 90 & 30 & 33 & 16 & 480 \\
\hline LALPUR & 3303 & CHANKNOR & CHAWNI & 80 & 30 & 38 & 15 & 450 \\
\hline LALPUR & 3315 & DAG KALAY & DAG KALAY & 90 & 40 & 44 & 16 & 640 \\
\hline LALPUR & 3334 & DEGANANO MAHALA & ALIMZO KALAY & 90 & 25 & 28 & 18 & 450 \\
\hline LALPUR & 3333 & DEHGANANO KALAY & DEHGANANO KALAY & 140 & 60 & 43 & 20 & 1,200 \\
\hline LALPUR & 3308 & GARDAWE & TOTY KALAY & 100 & 50 & 50 & 13 & 650 \\
\hline LALPUR & 3332 & GERDAWI & GERDAWI & 180 & 80 & 44 & 16 & 1,280 \\
\hline LALPUR & 3307 & GULDAG & GULDAG & 200 & 90 & 45 & 15 & 1,350 \\
\hline LALPUR & 3325 & GULDAG & SULAIMANZAI & 160 & 80 & 50 & 15 & 1,200 \\
\hline LALPUR & 3492 & JOLAGAN & JOLAGAN & 360 & 70 & 19 & 16 & 1,120 \\
\hline LALPUR & 3327 & KHEZO & KHEZO & 120 & 0 & 0 & 0 & 0 \\
\hline LALPUR & 3305 & KHEZO KALAY & JANDOL & 60 & 0 & 0 & 0 & 0 \\
\hline LALPUR & 3314 & MADAD KALAY & MADAD KALAY & 40 & 18 & 45 & 18 & 324 \\
\hline LALPUR & 3309 & MARSANGI & MARSANGI & 200 & 70 & 35 & 15 & 1,050 \\
\hline LALPUR & 3329 & MEHRABANO KALAY & MEHRABANO KALAY & 190 & 70 & 37 & 15 & 1,050 \\
\hline LALPUR & 3313 & MORCHA KHAIL & MORCHA KHAIL & 50 & 20 & 40 & 20 & 400 \\
\hline
\end{tabular}

* Jerib (jr.) = 1/5 ha

${ }^{*}$ Dry Opium $=$ Wet Opium - $30 \%$ moisture
Page 30120
Source: UNDCP Opium Production Survey, 1995. File: NGR2.XLS - 06/08/9 
Opium Production Survey by village in Nangarhar province, 1995.

\begin{tabular}{|c|c|c|c|c|c|c|c|c|}
\hline \multirow{3}{*}{ District } & \multirow{3}{*}{$\begin{array}{c}\text { Village } \\
\text { Code }\end{array}$} & \multirow{3}{*}{ Area/ Main Village } & \multirow{3}{*}{ Village } & \multirow{3}{*}{\begin{tabular}{c|} 
Total \\
Cultivated \\
Land (jerib ${ }^{*}$ ) \\
\end{tabular}} & \multirow{2}{*}{\multicolumn{2}{|c|}{$\begin{array}{c}\text { Land Under } \\
\text { Poppy Cultivation }\end{array}$}} & \multirow{3}{*}{\begin{tabular}{c|} 
Wet Opium ${ }^{* 2}$ \\
Yield \\
$(\mathrm{kg} / \mathrm{jr})$.
\end{tabular}} & \multirow{3}{*}{$\begin{array}{c}\text { Wet Opium } \\
\text { Production } \\
(\mathrm{kg})\end{array}$} \\
\hline & & & & & & & & \\
\hline & & & & & (jerib) & $(\%)$ & & \\
\hline \multicolumn{9}{|c|}{ Province: Nangarhar } \\
\hline LALPUR & 3324 & MULAYANO KALAY & BAZAR KALAY & 40 & 0 & 0 & 0 & 0 \\
\hline LALPUR & 3318 & MULAYANO MAHALAH & TURABAZ KHAN KALAY & 50 & 0 & 0 & 0 & 0 \\
\hline LALPUR & 3316 & NAWY MASJID KALAY & NAWY MASJID KALAY & 40 & 25 & 63 & 15 & 375 \\
\hline LALPUR & 3317 & NAYANO KALAY & NAYANO KALAY & 40 & 15 & 38 & 17 & 255 \\
\hline LALPUR & 3312 & NILLAW KALAY & NILLAW KALAY & 70 & 30 & 43 & 15 & 450 \\
\hline LALPUR & 3320 & PAS SARBAND & PAS SARBAND & 150 & 0 & 0 & 0 & 0 \\
\hline LALPUR & 3331 & SADA & SADA & 180 & 60 & 33 & 15 & 900 \\
\hline LALPUR & 3310 & SADAH & MIA M.AMIN KELE & 170 & 70 & 41 & 14 & 980 \\
\hline LALPUR & 3302 & SARBAND & KUZ SARBAND & 120 & 0 & 0 & 0 & 0 \\
\hline LALPUR & 3319 & SEMACY & SEMACY & 50 & 20 & 40 & 13 & 260 \\
\hline LALPUR & 3326 & SHAH KOTI & NEWE KALAY & 160 & 70 & 44 & 14 & 980 \\
\hline LALPUR & 3306 & SHAH KOTI & SHAH KOTI & 230 & 150 & 65 & 16 & 2,400 \\
\hline LALPUR & 3322 & SPINA BELA & SPINA BELA & 150 & 5 & 3 & 16 & 80 \\
\hline LALPUR & 3330 & WARA LALPURA & WARA LALPURA & 150 & 40 & 27 & 16 & 640 \\
\hline MOHMAND DARA & 861 & AWAL KHAN & AWAL KHAN & 130 & 0 & 0 & 0 & 0 \\
\hline MOHMAND DARA & 1611 & BAWAR GHONDAY & BAWAR GHONDAY & 120 & 0 & 0 & 0 & 0 \\
\hline MOHMAND DARA & 845 & BAZID KHEL & BAZID KHEL & 220 & 0 & 0 & 0 & 0 \\
\hline MOHMAND DARA & 840 & DAKA & KHAT & 60 & 0 & 0 & 0 & 0 \\
\hline MOHMAND DARA & 1602 & DAKA & LOYA DAKA & 125 & 0 & 0 & 0 & 0 \\
\hline MOHMAND DARA & 1620 & DAKA & MAIN KHAN KALAY & 140 & 0 & 0 & 0 & 0 \\
\hline MOHMAND DARA & 1621 & DAKA & MALIK MIR ALAM KHAN & 250 & 0 & 0 & 0 & 0 \\
\hline MOHMAND DARA & 870 & DAKA & UTMANZAI & 300 & 0 & 0 & 0 & 0 \\
\hline MOHMAND DARA & 846 & DAMAN & ALI KHAN KHEL & 100 & 0 & 0 & 0 & 0 \\
\hline MOHMAND DARA & 852 & GHAZGAI & ANI KHAIL & 180 & 0 & 0 & 0 & 0 \\
\hline MOHMAND DARA & 1627 & GHAZGAI & KANDO KALAY & 210 & 0 & 0 & 0 & 0 \\
\hline MOHMAND DARA & 853 & GHAZGAI & MUSA KHAIL & 100 & 0 & 0 & 0 & 0 \\
\hline MOHMAND DARA & 1626 & GHAZGAI & SAKA KHAIL & 95 & 0 & 0 & 0 & 0 \\
\hline MOHMAND DARA & 855 & GIRDI GHAWS & ALIZAI & 310 & 0 & 0 & 0 & 0 \\
\hline MOHMAND DARA & 1622 & GIRDI GHAWS & BAMBO KHEL & 250 & 0 & 0 & 0 & 0 \\
\hline
\end{tabular}

* Jerib (jr.) $=1 / 5$ ha

* 2 Dry Opium $=$ Wet Opium $-30 \%$ moisture 
Opium Production Survey by village in Nangarhar province, 1995.

\begin{tabular}{|c|c|c|c|c|c|c|c|c|}
\hline & \multirow{3}{*}{$\begin{array}{l}\text { Village } \\
\text { Code }\end{array}$} & & & Total & Land & & Wet Opium *2 & Wet Opium \\
\hline \multirow[t]{2}{*}{ District } & & \multirow{2}{*}{ Area/ Main Village } & \multirow[t]{2}{*}{ Village } & \multirow{2}{*}{$\begin{array}{c}\text { Cultivated } \\
\text { Land (jerib }{ }^{*} \text { ) }\end{array}$} & \multicolumn{2}{|c|}{ Poppy Cultivation } & \multirow{2}{*}{$\begin{array}{c}\text { Yield } \\
(\mathrm{kg} / \mathrm{jr} .)\end{array}$} & \multirow{2}{*}{$\begin{array}{c}\text { Production } \\
(\mathrm{kg})\end{array}$} \\
\hline & & & & & (jerib) & $(\%)$ & & \\
\hline \multicolumn{9}{|l|}{ Province: Nangarhar } \\
\hline MOHMAND DARA & 854 & GIRDI GHAWS & HASAN KHEL & 225 & 0 & 0 & 0 & 0 \\
\hline MOHMAND DARA & 856 & GIRDI GHAWS & KHWAJAZIA & 400 & 0 & 0 & 0 & 0 \\
\hline MOHMAND DARA & 857 & GIRDI GHAWS & KUZ MYAGAN & 215 & 0 & 0 & 0 & 0 \\
\hline MOHMAND DARA & 1628 & GIRDI GHAWS & MARUF KHEL & 200 & 0 & 0 & 0 & 0 \\
\hline MOHMAND DARA & 3385 & GIRDI GHAWS & NAZER KHEL & 230 & 0 & 0 & 0 & 0 \\
\hline MOHMAND DARA & 839 & GIRDI GHAWS & PAS MYAGAN & 450 & 0 & 0 & 0 & 0 \\
\hline MOHMAND DARA & 1629 & GIRDI GHAWS & SADIZAI & 235 & 0 & 0 & 0 & 0 \\
\hline MOHMAND DARA & 1624 & GIRDI GHAWS & SHAKAR KHEL & 350 & 0 & 0 & 0 & 0 \\
\hline MOHMAND DARA & 1625 & HADIRO KALAY & HADIRO KALAY & 245 & 0 & 0 & 0 & 0 \\
\hline MOHMAND DARA & 841 & HAJI MIR ALI SHAH & HAJI MIR ALI SHAH & 110 & 0 & 0 & 0 & 0 \\
\hline MOHMAND DARA & 860 & HAJI RASUL KHAN KALY & HAJI RASUL KHAN KALY & 220 & 0 & 0 & 0 & 0 \\
\hline MOHMAND DARA & 1606 & HAJI WAKIL KALAY & HAJI WAKIL KALAY & 250 & 0 & 0 & 0 & 0 \\
\hline MOHMAND DARA & 1618 & HAZAR NAW & ABDRRL RAHMAN KHEL & 200 & 0 & 0 & 0 & 0 \\
\hline MOHMAND DARA & 848 & HAZAR NAW & ADIZY & 240 & 0 & 0 & 0 & 0 \\
\hline MOHMAND DARA & 1616 & HAZAR NAW & GHAT KATEZI & 230 & 0 & 0 & 0 & 0 \\
\hline MOHMAND DARA & 1617 & HAZAR NAW & HAJIANO KALAY & 250 & 0 & 0 & 0 & 0 \\
\hline MOHMAND DARA & 871 & HAZAR NAW & JAR KALAY & 170 & 0 & 0 & 0 & 0 \\
\hline MOHMAND DARA & 849 & HAZAR NAW & KHAN KHEL & 250 & 0 & 0 & 0 & 0 \\
\hline MOHMAND DARA & 1619 & HAZAR NAW & KOTI KILY & 160 & 0 & 0 & 0 & 0 \\
\hline MOHMAND DARA & 847 & HAZAR NAW & KUZ KANDAR & 150 & 0 & 0 & 0 & 0 \\
\hline MOHMAND DARA & 1613 & HAZAR NAW & MALIK BAGH KALAY & 210 & 0 & 0 & 0 & 0 \\
\hline MOHMAND DARA & 850 & HAZAR NAW & NARIZY KALAY & 120 & 0 & 0 & 0 & 0 \\
\hline MOHMAND DARA & 1614 & HAZAR NAW & PAS KALAY(PACHAKHEL) & 110 & 0 & 0 & 0 & 0 \\
\hline MOHMAND DARA & 869 & HAZAR NAW & PAS KANDAR & 170 & 0 & 0 & 0 & 0 \\
\hline MOHMAND DARA & 851 & HAZAR NAW & WARA KATEZI & 270 & 0 & 0 & 0 & 0 \\
\hline MOHMAND DARA & 1601 & KAMA DAKA & KAMA DAKA & 350 & 0 & 0 & 0 & 0 \\
\hline MOHMAND DARA & 868 & KHARKANAY & KHARKANAY & 200 & 0 & 0 & 0 & 0 \\
\hline MOHMAND DARA & 859 & KUZA BELA & KUZA BELA & 300 & 0 & 0 & 0 & 0 \\
\hline MOHMAND DARA & 3446 & KUZA LANDY & KUZA LANDY & 240 & 0 & 0 & 0 & 0 \\
\hline
\end{tabular}

* Jerib (jr.) $=1 / 5$ ha

* 2 Dry Opium $=$ Wet Opium $-30 \%$ moisture
Page 32
Source: UNDCP Opium Production Survey, 1995. File: NGR2.XLS - 06/08/95 
Opium Production Survey by village in Nangarhar province, 1995.

\begin{tabular}{|c|c|c|c|c|c|c|c|c|}
\hline & Village & & & Total & Land & & Wet Opium * 2 & Wet Opium \\
\hline \multirow[t]{2}{*}{ District } & Code & Area/ Main Village & Village & Cultivated & \multicolumn{2}{|c|}{ Poppy Cultivation } & Yield & Production \\
\hline & & & & Land (jerib *) & (jerib) & $(\%)$ & $(\mathrm{kg} / \mathrm{jr})$. & $(\mathrm{kg})$ \\
\hline \multicolumn{9}{|l|}{ Province: Nangarhar } \\
\hline MOHMAND DARA & 1604 & MALEK SHER AHMAD & MALEK SHER AHMAD & 200 & 0 & 0 & 0 & 0 \\
\hline MOHMAND DARA & 1605 & MALEK SHINWARI KALAY & MALEK SHINWARI KALAY & 160 & 0 & 0 & 0 & 0 \\
\hline MOHMAND DARA & 1603 & ML.SAYF ULRAHMAN & ML.SAYF ULRAHMAN & 250 & 0 & 0 & 0 & 0 \\
\hline MOHMAND DARA & 842 & PAS BASAWOL & DARO KASH & 230 & 0 & 0 & 0 & 0 \\
\hline MOHMAND DARA & 1607 & PAS BASAWOL & MAMA KHEL & 220 & 0 & 0 & 0 & 0 \\
\hline MOHMAND DARA & 1608 & PAS BASAWOL & MOSA KHEL & 260 & 0 & 0 & 0 & 0 \\
\hline MOHMAND DARA & 1609 & PAS BASAWOL & SULTAN KHEL & 300 & 0 & 0 & 0 & 0 \\
\hline MOHMAND DARA & 843 & PAS BASAWUL & ARZU KHEL & 325 & 0 & 0 & 0 & 0 \\
\hline MOHMAND DARA & 858 & PAS BELA & PAS BELA & 320 & 0 & 0 & 0 & 0 \\
\hline MOHMAND DARA & 3445 & PAS LANDY & PAS LANDY & 180 & 0 & 0 & 0 & 0 \\
\hline MOHMAND DARA & 844 & PIRA KHEL & PIRA KHEL & 240 & 0 & 0 & 0 & 0 \\
\hline MOHMAND DARA & 862 & RAZA KHAN KELAY & RAZA KHAN KELAY & 120 & 0 & 0 & 0 & 0 \\
\hline MOHMAND DARA & 863 & SARDAR KHAN KELAY & SARDAR KHAN KELAY & 100 & 0 & 0 & 0 & 0 \\
\hline MOHMAND DARA & 1610 & ZALA SULTAN KHEL & ZALA SULTAN KHEL & 260 & 0 & 0 & 0 & 0 \\
\hline MOHMAND DARA & 1612 & ZARA WOLUSWALI & MALEK MIR ALI SHAH & 165 & 0 & 0 & 0 & 0 \\
\hline MOHMAND DARA & 1615 & ZOR KALAY & ZOR KALAY & 120 & 0 & 0 & 0 & 0 \\
\hline NAZIAN & 950 & ABDAL KHOLEH & ABDAL KHOLEH & 15 & 12 & 80 & 10 & 120 \\
\hline NAZIAN & 955 & ALIKOH & ALIKOH & 10 & 6 & 60 & 8 & 48 \\
\hline NAZIAN & 954 & BADIN KALAY & BADIN KALAY & 20 & 15 & 75 & 8 & 120 \\
\hline NAZIAN & 960 & BARA KHEL & BARA KHEL & 30 & 25 & 83 & 8 & 200 \\
\hline NAZIAN & 957 & BARU KHOLEH & BARU KHOLEH & 20 & 15 & 75 & 9 & 135 \\
\hline NAZIAN & 935 & DWA NAZIANO KHOLEH & DWA NAZIANO KHOLEH & 15 & 12 & 80 & 10 & 120 \\
\hline NAZIAN & 934 & GHARA KALAY & GHARA KALAY & 14 & 11 & 79 & 9 & 99 \\
\hline NAZIAN & 947 & KAS KALAY & KAS KALAY & 25 & 19 & 76 & 8 & 152 \\
\hline NAZIAN & 938 & KAZHO & KAZHO & 10 & 6 & 60 & 9 & 54 \\
\hline NAZIAN & 942 & KHORAGAI & KHORAGAI & 25 & 18 & 72 & 9 & 162 \\
\hline NAZIAN & 943 & LAKRAI & LAKRAI & 30 & 20 & 67 & 8 & 160 \\
\hline NAZIAN & 959 & LALMANDI & LALMANDAY & 15 & 10 & 67 & 10 & 100 \\
\hline NAZIAN & 958 & LALMAY & LALMAY & 60 & 50 & 83 & 8 & 400 \\
\hline
\end{tabular}

* Jerib (jr.) $=1 / 5$ ha

${ }^{*}$ Dry Opium $=$ Wet Opium $-30 \%$ moisture
Page 33123
Source: UNDCP Opium Production Survey, 1995 File: NGR2.XLS - 06/08/95 
Opium Production Survey by village in Nangarhar province, 1995.

\begin{tabular}{|c|c|c|c|c|c|c|c|c|}
\hline \multirow{3}{*}{ District } & \multirow{3}{*}{$\begin{array}{l}\text { Village } \\
\text { Code }\end{array}$} & \multirow{3}{*}{ Areal Main Village } & \multirow{3}{*}{ Village } & \multirow{3}{*}{\begin{tabular}{|c|} 
Total \\
Cultivated \\
Land.(jerib $\left.{ }^{*}\right)$ \\
\end{tabular}} & \multirow{2}{*}{\multicolumn{2}{|c|}{$\begin{array}{c}\text { Land Under } \\
\text { Poppy Cultivation }\end{array}$}} & \multirow{3}{*}{$\begin{array}{c}\text { Wet Opium *2 } \\
\text { Yield } \\
(\mathrm{kg} / \mathrm{jr} .) \\
\end{array}$} & \multirow{3}{*}{$\begin{array}{c}\text { Wet Opium } \\
\text { Production } \\
(\mathrm{kg}) \\
\end{array}$} \\
\hline & & & & & & & & \\
\hline & & & & & (jerib) & $(\%)$ & & \\
\hline \multicolumn{9}{|l|}{ Province: Nangarhar } \\
\hline NAZIAN & 949 & LANDAI & GADO KHAIL & 250 & 150 & 60 & 9 & 1,350 \\
\hline NAZIAN & 936 & LANDAY MORGA & LANDAY MORGA & 35 & 25 & 71 & 10 & 250 \\
\hline NAZIAN & 953 & MANDATI & MANDATI & 0 & 0 & \#DIV/0! & 0 & . \\
\hline NAZIAN & 933 & MAZID KHEL & MAZID KHEL & 26 & 18 & 69 & 8 & 144 \\
\hline NAZIAN & 951 & MELO KHOLEH & MELO KHOLEH & 18 & 14 & 78 & 9 & 126 \\
\hline NAZIAN & 946 & MORCHAL & MORCHAL & 55 & 45 & 82 & 8 & 360 \\
\hline NAZIAN & 939 & NAKHTAR NAW & NAKHTAR NAW & 0 & 0 & \#DIV/0! & 0 & 0 \\
\hline NAZIAN & 952 & NAKI & NAKI & 0 & 0 & \#DIV/0! & 0 & 0 \\
\hline NAZIAN & 937 & NAKTA & NAKTA & 0 & 0 & \#DIV/0! & 0 & 0 \\
\hline NAZIAN & 941 & SAROBI & KONJ KALAY & 20 & 15 & 75 & 8 & 120 \\
\hline NAZIAN & 944 & SAROBI & MULLAH KHAIL & 60 & 50 & 83 & 9 & 450 \\
\hline NAZIAN & 948 & SAROBI & SAROBI & 28 & 20 & 71 & 9 & 180 \\
\hline NAZIAN & 932 & SAROBI & WACH KHWAR & 30 & 22 & 73 & 10 & 220 \\
\hline NAZIAN & 956 & SPINA ZHAY & SPINA ZHAY & 17 & 12 & 71 & 8 & 96 \\
\hline NAZIAN & 945 & TORKHI & TORKHI & 25 & 20 & 80 & 8 & 160 \\
\hline NAZIAN & 940 & ZARA LANDAI & ZARA LANDAI & 130 & 80 & 62 & 9 & 720 \\
\hline PACHIR WA AGAM & 1796 & BAMO KHEL & KAGI KALAY & 240 & 85 & 35 & 16 & 1,360 \\
\hline PACHIR WA AGAM & 1741 & BAMO KHEL & LAR KALAY & 140 & 30 & 21 & 14 & 420 \\
\hline PACHIR WA AGAM & 1742 & BAMO KHEL & NAZAR KHEL & 70 & 10 & 14 & 12 & 120 \\
\hline PACHIR WA AGAM & 1797 & BAMO KHEL & SUDLA KHEL & 160 & 20 & 13 & 14 & 280 \\
\hline PACHIR WA AGAM & 1738 & GARI KHEL & BARO & 70 & 25 & 36 & 13 & 325 \\
\hline PACHIR WA AGAM & 1740 & GARI KHEL & KHAN KALAY & 70 & 28 & 40 & 12 & 336 \\
\hline PACHIR WA AGAM & 1799 & GARI KHEL & KUNJ KALAY & 50 & 20 & 40 & 13 & 260 \\
\hline PACHIR WA AGAM & 1800 & GARI KHEL & MARSANDIAN & 30 & 6 & 20 & 12 & 72 \\
\hline PACHIR WA AGAM & 1739 & GARI KHEL & SHORO KHEL & 100 & 22 & 22 & 12 & 264 \\
\hline PACHIR WA AGAM & 1737 & GARI KHEL & SULTAN KHEL & 75 & 15 & 20 & 14 & 210 \\
\hline PACHIR WA AGAM & 3497 & KAREZ AKHUNDZADAGAN & KAREZ AKHUNDZADAGAN & 400 & 100 & 25 & 9 & 900 \\
\hline PACHIR WA AGAM & 1795 & LANDA KHAIL & LAR KALAY & 120 & 40 & 33 & 10 & 400 \\
\hline PACHIR WA AGAM & 1752 & LANDI PACHIR & ALIF KHAN KHAIL & 80 & 20 & 25 & 14 & 280 \\
\hline
\end{tabular}

* Jerib (jr.) $=1 / 5$ ha

${ }^{*}$ Dry Opium $=$ Wet Opium $-30 \%$ moisture 
Opium Production Survey by village in Nangarhar province, 1995.

\begin{tabular}{|c|c|c|c|c|c|c|c|c|}
\hline & Village & & & Total & Land $L$ & & Wet Opium *2 & Wet Opium \\
\hline \multirow[t]{2}{*}{ District } & \multirow[t]{2}{*}{ Code } & \multirow[t]{2}{*}{ Area/ Main Village } & \multirow[t]{2}{*}{ Village } & \multirow{2}{*}{$\begin{array}{c}\text { Cultivated } \\
\text { Land (jerib }^{*} \text { ) }\end{array}$} & \multicolumn{2}{|c|}{ Poppy Cultivation } & \multirow{2}{*}{$\begin{array}{c}\text { Yield } \\
(\mathrm{kg} / \mathrm{jr} .) \\
\end{array}$} & \multirow{2}{*}{$\begin{array}{c}\text { Production } \\
\text { (kg) }\end{array}$} \\
\hline & & & & & (jerib) & $(\%)$ & & \\
\hline \multicolumn{9}{|l|}{ Province: Nangarhar } \\
\hline PACHIR WA AGAM & 1753 & LANDI PACHIR & BADIN KHAIL & 210 & 70 & 33 & 13 & 910 \\
\hline PACHIR WA AGAM & 1751 & LANDI PACHIR & ESA KHAN KHAIL & 210 & 60 & 29 & 16 & 960 \\
\hline PACHIR WA AGAM & 1761 & LANDI PACHIR & GHARA KHAIL & 200 & 80 & 40 & 12 & 960 \\
\hline PACHIR WA AGAM & 1774 & LANDI PACHIR & GHASH & 130 & 40 & 31 & 14 & 560 \\
\hline PACHIR WA AGAM & 1776 & LANDI PACHIR & JANDA KHAIL & 220 & 60 & 27 & 14 & 840 \\
\hline PACHIR WA AGAM & 1755 & LANDI PACHIR & KATA KHAIL & 130 & 40 & 31 & 12 & 480 \\
\hline PACHIR WA AGAM & 1760 & LANDI PACHIR & LOLO KHAIL & 110 & 43 & 39 & 13 & 559 \\
\hline PACHIR WA AGAM & 1773 & LANDI PACHIR & MASWAL SADKHAIL & 70 & 18 & 26 & 12 & 216 \\
\hline PACHIR WA AGAM & 1775 & LANDI PACHIR & UBA KHAIL & 230 & 50 & 22 & 12 & 600 \\
\hline PACHIR WA AGAM & 1743 & LANDY KHEL & LANDY KHEL & 210 & 80 & 38 & 12 & 960 \\
\hline PACHIR WA AGAM & 1744 & LANDY KHEL & ZHAWARA & 130 & 45 & 35 & 12 & 540 \\
\hline PACHIR WA AGAM & 1754 & LANDY PACHIR & BARBARO MIYAGAN & 90 & 20 & 22 & 16 & 320 \\
\hline PACHIR WA AGAM & 1806 & LANDY PACHIR & RAHMAT KHEL & 110 & 38 & 35 & 14 & 532 \\
\hline PACHIR WA AGAM & 1805 & LANDY PACHIR & SHIKHAN & 40 & 10 & 25 & 16 & 160 \\
\hline PACHIR WA AGAM & 1798 & MORGAI & BANDYAN & 70 & 20 & 29 & 12 & 240 \\
\hline PACHIR WA AGAM & 1802 & MORGAI & BARA MORGAI & 120 & 35 & 29 & 18 & 630 \\
\hline PACHIR WA AGAM & 1757 & MORGAI & KUZA MORGAI & 200 & 55 & 28 & 12 & 660 \\
\hline PACHIR WA AGAM & 1782 & PAS PACHIR & AZAL KHAIL & 90 & 30 & 33 & 14 & 420 \\
\hline PACHIR WA AGAM & 1779 & PAS PACHIR & AZI KHAIL & 110 & 40 & 36 & 10 & 400 \\
\hline PACHIR WA AGAM & 1770 & PAS PACHIR & BANGARAM & 160 & 30 & 19 & 10 & 300 \\
\hline PACHIR WA AGAM & 1781 & PAS PACHIR & BILAM KHAIL & 90 & 25 & 28 & 12 & 300 \\
\hline PACHIR WA AGAM & 1763 & PAS PACHIR & CHANGAR & 80 & 20 & 25 & 10 & 200 \\
\hline PACHIR WA AGAM & 1765 & PAS PACHIR & GHALI KHAIL & 100 & 35 & 35 & 14 & 490 \\
\hline PACHIR WA AGAM & 1777 & PAS PACHIR & GHANDI KHAIL & 35 & 10 & 29 & 12 & 120 \\
\hline PACHIR WA AGAM & 1767 & PAS PACHIR & IBRAHIM KHAIL & 110 & 40 & 36 & 12 & 480 \\
\hline PACHIR WA AGAM & 1784 & PAS PACHIR & KARIGAR & 40 & 10 & 25 & 14 & 140 \\
\hline PACHIR WA AGAM & 1783 & PAS PACHIR & MUGHUL KHAIL & 70 & 25 & 36 & 12 & 300 \\
\hline PACHIR WA AGAM & 1778 & PAS PACHIR & MUSA KHAIL & 60 & 22 & 37. & 14 & 308 \\
\hline PACHIR WA AGAM & 1780 & PAS PACHIR & PAINDA KHAIL & 120 & 30 & 25 & 14 & 420 \\
\hline
\end{tabular}

* Jerib (jr.) $=1 / 5$ ha

* 2 Dry Opium $=$ Wet Opium - $30 \%$ moisture 
Opium Production Survey by village in Nangarhar province, 1995.

\begin{tabular}{|c|c|c|c|c|c|c|c|c|}
\hline \multirow{3}{*}{ District } & \multirow{3}{*}{\begin{tabular}{|c|} 
Village \\
Code
\end{tabular}} & \multirow{3}{*}{ Area/ Main Village } & \multirow{3}{*}{ Village } & \multirow{3}{*}{$\begin{array}{c}\text { Total } \\
\text { Cultivated } \\
\text { Land (jerib }^{*} \text { ) } \\
\end{array}$} & \multirow{2}{*}{\multicolumn{2}{|c|}{$\begin{array}{c}\text { Land Under } \\
\text { Poppy Cultivation }\end{array}$}} & \multirow{3}{*}{$\begin{array}{c}\text { Wet Opium }^{* 2} \\
\text { Yield } \\
(\mathrm{kg} / \mathrm{jr} .) \\
\end{array}$} & \multirow{3}{*}{$\begin{array}{l}\text { Wet Opium } \\
\text { Production } \\
(\mathrm{kg}) \\
\end{array}$} \\
\hline & & & & & & & & \\
\hline & & & & & (jerib) & (\%) & & \\
\hline \multicolumn{9}{|l|}{ Province: Nangarhar } \\
\hline PACHIR WA AGAM & 1764 & PAS PACHIR & QALANDAR KHEL & 90 & 28 & 31 & 14 & 392 \\
\hline PACHIR WA AGAM & 1766 & PAS PACHIR & SHAHI KHAIL & 90 & 30 & 33 & 11 & 330 \\
\hline PACHIR WA AGAM & 1762 & PAS PACHIR & TANGI KHAIL & 50 & 18 & . 36 & 14 & 252 \\
\hline PACHIR WA AGAM & 1768 & PAS PACHIR & TAWKAL KHAIL & 50 & 15 & 30 & 12. & 180 \\
\hline PACHIR WA AGAM & 1785 & PATANG ZAI & KHARKI DODYAR & 75 & 20 & 27 & 12 & 240 \\
\hline PACHIR WA AGAM & 1769 & PATANG ZAI & MARGHA & 40 & 12 & 30 & 14 & 168 \\
\hline PACHIR WA AGAM & 1787 & SABR SOFLA & SARA QAZLA & 100 & 36 & 36 & 5 & 180 \\
\hline PACHIR WA AGAM & 1788 & SABR SOFLA & SHAMS & 150 & 50 & 33. & 9 & 450 \\
\hline PACHIR WA AGAM & 1749 & SABR SUFLA & KHARKI KALA & 110 & 50 & 45 & 14 & 700 \\
\hline PACHIR WA AGAM & 1748 & SABR SUFLA & SHAKERIAN & 70 & 30 & 43 & 4 & 120 \\
\hline PACHIR WA AGAM & 1791 & SABR ULYA & KATA KHEL & 290 & 120 & 41 & 10 & 1,200 \\
\hline PACHIR WA AGAM & 1745 & SABR ULYA & LAKAY KALA & 90 & 30 & 33 & 14 & 420 \\
\hline PACHIR WA AGAM & 3383 & SABR ULYA & SUDUR KHEL & 140 & 60 & 43 & 12 & 720 \\
\hline PACHIR WA AGAM & 1750 & SABRE SUFLA & FAZEL KHEL & 270 & 110 & 41 & 12 & 1,320 \\
\hline PACHIR WA AGAM & 1746 & SABRE SUFLA & GANDBARI & 200 & 50 & 25 & 10 & 500 \\
\hline PACHIR WA AGAM & 1790 & SABRE SUFLA & KHANANO KALAY & 120 & 50 & 42 & 12 & 600 \\
\hline PACHIR WA AGAM & 1786 & SABRE SUFLA & YAGHI BAND & 150 & 70 & 47 & 6 & 420 \\
\hline PACHIR WA AGAM & 1789 & SABRE SUFLA & ZARA KALAY & 85 & 45 & 53 & 14 & 630 \\
\hline PACHIR WA AGAM & 1793 & SABRE ULYA & MUSA KHAIL & 240 & 75 & 31 & 10 & 750 \\
\hline PACHIR WA AGAM & 131 & SALO ZAI & PATRE & 350 & 200 & 57 & 14 & 2,800 \\
\hline PACHIR WA AGAM & 1747 & SAR SUFLA & DARA & 90 & 35 & 39 & 12 & 420 \\
\hline PACHIR WA AGAM & 1772 & SILAIMAN KHIL & ALIF KHIL & 18 & 0 & 0 & 0 & 0 \\
\hline PACHIR WA AGAM & 1801 & SOLAIMAN KHIL & MARKHALI & 37 & 0 & 0 & 0 & 0 \\
\hline PACHIR WA AGAM & 1756 & SOLAIMAN KHIL & MORIDNI & 17 & 0 & 0 & 0 & 0 \\
\hline PACHIR WA AGAM & 1771 & SOLAIMAN KHIL & TORA LORA & 15 & 0 & 0 & 0 & 0 \\
\hline PACHIR WA AGAM & 3266 & TOORTY & HAJIANO KALAY & 300 & 33 & 11 & 13 & 429 \\
\hline PACHIR WA AGAM & 1759 & ZAMAR KHAIL & ZARA KHAIL & 130 & 45 & 35 & 14 & 630 \\
\hline PACHIR WA AGAM & 1804 & ZEMAR KHEL & KALO KHEL & 110 & 40 & 36 & 12 & 480 \\
\hline PACHIR WA AGAM & 1758 & ZEMAR KHEL & MAREE QALA & 180 & 55 & 31 & 12 & 660 \\
\hline
\end{tabular}

* Jerib (jr.) $=1 / 5 \mathrm{ha}$

* 2 Dry Opium $=$ Wet Opium - $30 \%$ moisture
Page 36126
Source: UNDCP Opium Production Survey, 1995. File: NGR2.XLS - 06/08/95 
Opium Production Survey by village in Nangarhar province, 1995.

\begin{tabular}{|c|c|c|c|c|c|c|c|c|}
\hline \multirow{3}{*}{ District } & \multirow{3}{*}{$\begin{array}{l}\text { Village } \\
\text { Code }\end{array}$} & \multirow{3}{*}{ Area/ Main Village } & \multirow{3}{*}{ Village } & \multirow{3}{*}{\begin{tabular}{|c|} 
Total \\
Cultivated \\
Land (jerib $\left.{ }^{*}\right)$ \\
\end{tabular}} & \multirow{2}{*}{\multicolumn{2}{|c|}{$\begin{array}{c}\text { Land Under } \\
\text { Poppy Cultivation }\end{array}$}} & \multirow{3}{*}{\begin{tabular}{c|} 
Wet Opium $^{* 2}$ \\
Yield \\
$(\mathrm{kg} / \mathrm{jr})$. \\
\end{tabular}} & \multirow{3}{*}{$\begin{array}{c}\text { Wet Opium } \\
\text { Production } \\
(\mathrm{kg})\end{array}$} \\
\hline & & & & & & & & \\
\hline & & & & & (jerib) & $(\%)$ & & \\
\hline \multicolumn{9}{|l|}{ Province: Nangarhar } \\
\hline PACHIR WA AGAM & 1803 & ZEMAR KHEL & SRA QALA & 200 & 45 & 23 & 12 & 540 \\
\hline RODAT & 891 & AKHUND KALAY & AKHUND KALAY & 220 & 100 & 45 & 11 & 1,100 \\
\hline RODAT & 908 & AKHUNDZADA GANO KALY & AKHUNDZADA GANO KALY & 850 & .350 & 41 & 14 & 4,900 \\
\hline RODAT & 895 & AKHUNDZADA KALAY & MASHIN KALAY & 500 & 120 & 24 & 16 & 1,920 \\
\hline RODAT & 914 & AKHUNDZADAGAN & QAZI QALA & 170 & 46 & 27 & 14 & 644 \\
\hline RODAT & 875 & AMLOKY KALAY & AMLOKY KALAY & 153 & 40 & 26 & 11 & 440 \\
\hline RODAT & 887 & ANAR KHAIL & ANAR KHAIL & 140 & 14 & 10 & 10 & 140 \\
\hline RODAT & 881 & ATA KHAN & SENDWANI & 200 & 120 & 60 & 14 & 1,680 \\
\hline RODAT & 930 & ATA KHAN KALAY & ATA KHAN KALAY & 120 & 39 & 33 & 10 & 390 \\
\hline RODAT & 55 & ATA KHAN KALAY & CHAGHANO KALAY & 165 & 50 & 30 & 12 & 600 \\
\hline RODAT & 86 & BANDA & AUTMANZOY QALE & 114 & 40 & 35 & 10 & 400 \\
\hline RODAT & 900 & BANDA & MOCHIANO KALAY & 112 & 35 & 31 & 12 & 420 \\
\hline RODAT & 63 & BANDA & WEZO KALAY & 85 & 40 & 47 & 12 & 480 \\
\hline RODAT & 75 & BANDA & ZARGARANU QELE & 150 & 40 & 27 & 10 & 400 \\
\hline RODAT & 46 & BANDUK & BANDUK & 300 & 140 & 47 & 11 & 1,540 \\
\hline RODAT & 76 & BARO & BABA KHIL KELAY & 50 & 36 & 72 & 14 & 504 \\
\hline RODAT & 873 & BARO & BAR KELAY YASHEGE & 150 & 75 & 50 & 15 & 1,125 \\
\hline RODAT & 917 & BARO & MALEKANO KELAY & 140 & 60 & 43 & 10 & 600 \\
\hline RODAT & 80 & BARO & RASAL KALY & 150 & 73 & 49 & 11 & 803 \\
\hline RODAT & 916 & BARO LOY KALAY & BARO LOY KALAY & 500 & 150 & 30 & 14 & 2,100 \\
\hline RODAT & 74 & CHAGHARAY & CHAGHARAY & 400 & 130 & 33 & 13 & 1,690 \\
\hline RODAT & 50 & CHAH CHI QALA & CHAH CHI QALA & 300 & 130 & 43 & 13 & 1,690 \\
\hline RODAT & 61 & DAWANI & ABAT KHAIL & 235 & 90 & 38 & 14 & 1,260 \\
\hline RODAT & 874 & DAWANI & DAWANI & 140 & 55 & 39 & 15 & 825 \\
\hline RODAT & 886 & DO SHAH GEZWANEY & DO SHAH GEZWANEY & 100 & 50 & 50 & 14 & 700 \\
\hline RODAT & 53 & DOSHAH & KHATWANI & 80 & 35 & 44 & 12 & 420 \\
\hline RODAT & 70 & EASARAK & MOHD. QAYUM & 280 & 88 & 31 & 13 & 1,144 \\
\hline RODAT & 892 & GHALYAN KOCHIAN & GHALYAN KOCHIAN & 130 & 32 & 25 & 12 & 384 \\
\hline RODAT & 48 & HANDRANEY & SHEKHANO KALAY & 250 & 70 & 28 & 15 & 1,050 \\
\hline
\end{tabular}

- Jerib (jr.) $=1 / 5$ ha

${ }^{2}$ Dry Opium $=$ Wet Opium - $30 \%$ moisture
Page $37 \quad 127$
Source: UNDCP Opium Production Survey, 1995 File: NGR2.XLS - 06/08/95 
Opium Production Survey by village in Nangarhar province, 1995.

\begin{tabular}{|c|c|c|c|c|c|c|c|c|}
\hline & Village & & & Total & \multirow{2}{*}{\multicolumn{2}{|c|}{$\begin{array}{c}\text { Land Under } \\
\text { Poppy Cültivation }\end{array}$}} & \multirow{3}{*}{ 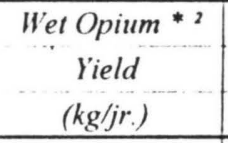 } & \multirow{3}{*}{$\frac{\text { Wet Opium }}{\text { Production }}$} \\
\hline \multirow[t]{2}{*}{ District } & \multirow[t]{2}{*}{ Code } & \multirow{2}{*}{ Area/ Main Village } & \multirow{2}{*}{ Village } & \multirow{2}{*}{$\begin{array}{c}\text { Cultivated } \\
\left.\text { Land (jerib }{ }^{*}\right)\end{array}$} & & & & \\
\hline & & & & & \multicolumn{2}{|c|}{\begin{tabular}{l|l} 
(jerib) & $(\%)$ \\
\end{tabular}} & & \\
\hline \multicolumn{9}{|c|}{ Province: Nangarhar } \\
\hline RODAT & 894 & HASAR SHAHI & MALAKANO & 60 & 22 & 37 & 15 & 330 \\
\hline RODAT & 906 & HESAR SHAHI & BARNAW & 163 & 42 & 26 & 14 & 588 \\
\hline RODAT & 903 & HINDRANI & HINDRANI & 500 & 120 & 24 & 10 & 1,200 \\
\hline RODAT & 69 & HISAR SHAHEE & DIWANO KALAY & 190 & 24 & 13 & 13 & 312 \\
\hline RODAT & 84 & HISAR SHAHEE & KULALANO KALAY & 85 & 30 & 35 & 14 & 420 \\
\hline RODAT & 103 & HISAR SHAHEE & SHAH WALI KALAY & 50 & 13 & 26 & 14 & 182 \\
\hline RODAT & 85 & HISAR SHAHI & CHINAR KALAY & 160 & 40 & 25 & 14 & 560 \\
\hline RODAT & 924 & HISAR SHAHI & JORA MIRANO KAL & 290 & 60 & 21 & 14 & 840 \\
\hline RODAT & 894 & HISAR SHAHI & MALIKANU QELE & 60 & 22 & 37 & 15 & 330 \\
\hline RODAT & 92 & HISAR SHAHI & MYA BANDAH & 50 & 24 & 48 & 13 & 312 \\
\hline RODAT & 907 & HISAR SHAHI & PIAWARO QALA & 250 & 142 & 57 & 14 & 1,988 \\
\hline RODAT & 885 & HISAR SHAHI & TATANG KALAY & 300 & 75 & 25 & 14 & 1,050 \\
\hline RODAT & 66 & HISARAK & MALIK MAZDOR KA & 300 & 100 & 33 & 12 & 1,200 \\
\hline RODAT & 923 & ISARAK & S.GUL PACHA & 300 & 150 & 50 & 10 & 1,500 \\
\hline RODAT & 67 & ISARAK & ZAMARAI & 300 & 85 & 28 & 14 & 1,190 \\
\hline RODAT & 904 & ISARAK LOY KALAY & ISARAK LOY KALAY & 420 & 220 & 52 & 12 & 2,640 \\
\hline RODAT & 897 & JABA & BARBARA KHIL KELAY & 90 & 23 & 26 & 8 & 184 \\
\hline RODAT & 71 & KADAI & CHINAR GAI KALY & 300 & 100 & 33 & 10 & 1,000 \\
\hline RODAT & 902 & KADAY LOY KALAY & KADAY LOY KALAY & 600 & 200 & 33 & 10 & 2,000 \\
\hline RODAT & 96 & KADE & MALIKANO KELAY & 330 & 120 & 36 & 9 & 1,080 \\
\hline RODAT & 929 & KAHAN & BAR KALAY & 300 & 130 & 43 & 13 & 1,690 \\
\hline RODAT & 880 & KAHAN & DEKAN ZEYARAT & 280 & 120 & 43 & 12 & 1,440 \\
\hline RODAT & 82 & KAHAN & MOMMAND SARKAN & 580 & 230 & 40 & 12 & 2,760 \\
\hline RODAT & 913 & KAHAN DEGAN & MURGHANU QALE & 160 & 70 & 44 & 13 & 910 \\
\hline RODAT & 927 & KAHAN DEGAN & SHEIAGUNU QELE & 150 & 80 & 53 & 14 & 1,120 \\
\hline RODAT & 3468 & KAHAN KOZ DAWLAT KHL & KAHAN KOZ DAWL.AT KHL & 900 & 200 & 22 & 11 & 2,200 \\
\hline RODAT & 81 & KAHAN MOMMAND & ZAFAR KHEL & 300 & 70) & 23 & 12 & 840 \\
\hline RODAT & 79 & KAHAN MUHMAND & NAWAI KALAY & 300 & 120 & 40 & 13 & 1.560 \\
\hline RODAT & 872 & KAMBARO & KAMBARO & 60 & 24 & 40 & 10 & 240 \\
\hline
\end{tabular}

- Jerib (jr.) = 1/5 ha

* Dry Opium = Wet Opium - $30 \%$ moisture
Page 38
Source: UNDCP Opium Production Survey, 1995 File: NGR2.XLS - 06/08/95 
Opium Production Survey by village in Nangarhar province, 1995.

\begin{tabular}{|c|c|c|c|c|c|c|c|c|}
\hline & Village & & & Total & Land & & Wet Opium * 2 & Wet Opium \\
\hline \multirow[t]{2}{*}{ District } & \multirow[t]{2}{*}{ Code } & \multirow[t]{2}{*}{ Area/ Main Village } & \multirow[t]{2}{*}{ Village } & \multirow{2}{*}{$\begin{array}{c}\text { Cultivated } \\
\text { Land (jerib }{ }^{*} \text { ) }\end{array}$} & \multicolumn{2}{|c|}{ Poppy Cultivation } & \multirow{2}{*}{$\begin{array}{l}\text { Yield } \\
(\mathrm{kg} / \mathrm{jr} .) \\
\end{array}$} & \multirow{2}{*}{$\frac{\text { Production }}{\text { (kg) }}$} \\
\hline & & & & & (jerih) & $(\%)$ & & \\
\hline \multicolumn{9}{|c|}{ Province: Nangarhar } \\
\hline RODAT & 52 & KHADAR KHANI & KHADAR KHANI & 1,400 & 320 & 23 & 11 & 3,520 \\
\hline RODAT & 910 & KHADAR KHANI & KHAN KHAIL & 480 & 170 & 35 & 10 & 1,700 \\
\hline RODAT & 78 & KOT - JABA & JABA & 300 & 150 & 50 & 14 & 2,100 \\
\hline RODAT & 47 & LAFERCHI & SHASH KHAIL & 420 & 176 & 42 & 12. & 2,112 \\
\hline RODAT & 59 & LAGHAR GI JOSHI & LAGHAR GI JOSHI & 500 & 215 & 43 & 11 & 2,365 \\
\hline RODAT & 77 & LANDY BARO & LANDY BARO & 50 & 16 & 32 & 10 & 160 \\
\hline RODAT & 918 & LOY KALAY BANDA & GHUNDAY LOY KALAY & 400 & 160 & 40 & 15 & 2,400 \\
\hline RODAT & 62 & MAKH SHOY & BARO KHIL & 100 & 18 & 18 & 10 & 180 \\
\hline RODAT & 899 & MANZANAI & MANZANAI & 140 & 22 & 16 & 14 & 308 \\
\hline RODAT & 909 & MAZINA & AKHUNDZADA QELE & 115 & 55 & 48 & 16 & 880 \\
\hline RODAT & 88 & MAZINA & BADEH JALIL PACHA & 110 & 50 & 45 & 14 & 700 \\
\hline RODAT & 911 & MAZINA & COWTOW QELAE & 70 & 20 & 29 & 16 & 320 \\
\hline RODAT & 87 & MAZINA & DEGANU QELE & 65 & 30 & 46 & 13 & 390 \\
\hline RODAT & 64 & MAZINA & GHULAMI & 60 & 25 & 42 & 18 & 450 \\
\hline RODAT & 65 & MAZINA & LAL SAYED PACHA & 100 & 40 & 40 & 14 & 560 \\
\hline RODAT & 99 & MAZINA & MAZINA LOY KELY & 170 & 45 & 26 & 12 & 540 \\
\hline RODAT & 928 & MAZINA & RANGRIZANO KALAY & 80 & 30 & 38 & 17 & 510 \\
\hline RODAT & 931 & MAZINA & SAYED MOHAMMAD SHAH & 100 & 30 & 30 & 10 & 300 \\
\hline RODAT & 920 & METRANI & METRANI & 900 & 350 & 39 & 14 & 4,900 \\
\hline RODAT & 89 & MIR HAI & KHAIR ABAD & 300 & 85 & 28 & 12 & 1,020 \\
\hline RODAT & 98 & MIR JEE & BAR KELY & 90 & 28 & 31 & 14 & 392 \\
\hline RODAT & 877 & MIR JEE & NAGHARY KELY & 120 & 24 & 20 & 16 & 384 \\
\hline RODAT & 3336 & MIRANO KALAY & MIRANO KALAY & 105 & 20 & 19 & 8 & 160 \\
\hline RODAT & 100 & MIRJI & JABO KALAY & 100 & 40 & 40 & 15 & 600 \\
\hline RODAT & 922 & PARSA KHAIL & PARSA KHAIL & 240 & 70 & 29 & 14 & 980 \\
\hline RODAT & 51 & QALAJAT & QALAJAT & 100 & 35 & 35 & 14 & 490 \\
\hline RODAT & 97 & QATAR GHAY & ZORAND KHAIL & 130 & 50 & 38 & 14 & 700 \\
\hline RODAT & 97 & RODAT & RODAT & 230 & 75 & 33 & 16 & 1,200 \\
\hline RODAT & 68 & ROGHANI & JAHANGER KALAY & 230 & 60 & 26 & 7 & 420 \\
\hline
\end{tabular}

* Jerib (jr.) $=1 / 5$ ha

* 2 Dry Opium $=$ Wet Opium - $30 \%$ moisture 
Opium Production Survey by village in Nangarhar province, 1995.

\begin{tabular}{|c|c|c|c|c|c|c|c|c|}
\hline & Village & & & \multirow{3}{*}{$\begin{array}{c}\text { Total } \\
\text { Cultivated } \\
\left.\text { Land (jerib }{ }^{*}\right) \\
\end{array}$} & \multirow{2}{*}{\multicolumn{2}{|c|}{$\begin{array}{c}\text { Land Under } \\
\text { Poppy Cultivation }\end{array}$}} & \multirow{3}{*}{$\begin{array}{c}\text { Wet Opium *2 } \\
\text { Yield } \\
(\mathrm{kg} / \mathrm{jr} .)\end{array}$} & \multirow{3}{*}{$\begin{array}{c}\text { Wet Opium } \\
\text { Production } \\
(\mathrm{kg})\end{array}$} \\
\hline \multirow[t]{2}{*}{ District } & \multirow[t]{2}{*}{ Code } & \multirow[t]{2}{*}{ Areal Main Village } & \multirow[t]{2}{*}{ Village } & & & & & \\
\hline & & & & & (jerih) & $(\%)$ & & \\
\hline \multicolumn{9}{|c|}{ Province: Nangarhar } \\
\hline RODAT & 882 & ROGHANI & QALA-I-MIRGI(MANZ ) & 230 & 60 & 26 & 9 & 540 \\
\hline RODAT & 901 & ROGHANI & ROGHANI & 420 & 175 & 42 & 13 & 2,275 \\
\hline RODAT & 93 & ROGHANI & SAR QALA & 220 & 50 & 23 & 14 & 700 \\
\hline RODAT & 926 & S.AHMAD KHAIL & UMRA KALAY & 150 & 40 & 27 & 11 & 440 \\
\hline RODAT & 56 & SADATMAND & SADATMAND & 250 & 66 & 26 & 13 & 858 \\
\hline RODAT & 889 & SADR KALAY & SADR KALAY & 600 & 200 & 33 & 14 & 2,800 \\
\hline RODAT & 72 & SANGAR GAREY & SANGAR GAREY & 200 & 53 & 27 & 13 & 689 \\
\hline RODAT & 896 & SAY BAY & SADER KHAIL & 240 & 96 & 40 & 13 & 1,248 \\
\hline RODAT & 919 & SAYED AHMAD KHEL & SAYED AHMAD KHEL & 250 & 90 & 36 & 10 & 900 \\
\hline RODAT & 896 & SEY PAI & SEY PAI & 200 & 120 & 60 & 9 & 1,080 \\
\hline RODAT & 91 & SHAHI COOT & KUZ QALA & 230 & 78 & 34 & 15 & 1,170 \\
\hline RODAT & 54 & SHAHI COT & SHAHI COT & 135 & 45 & 33 & 9 & 405 \\
\hline RODAT & 57 & SHAMA KHEL & SHAMA KHEL & 100 & 35 & 35 & 13 & 455 \\
\hline RODAT & 888 & SHAS KHEL & SARO KHEL & 35 & 15 & 43 & 12 & 180 \\
\hline RODAT & 90 & SHAS KHEL & SHAS KHEL & 400 & 130 & 33 & 14 & 1,820 \\
\hline RODAT & 884 & SHEKH AHMAD KALAY & SHEKH AHMAD KALAY & 150 & 45 & 30 & 12 & 540 \\
\hline RODAT & 3467 & SRA QALA & SRA QALA & 280 & 50 & 18 & 13 & 650 \\
\hline RODAT & 925 & TARILAY & TARILAY & 1,500 & 680 & 45 & 11 & 7,480 \\
\hline RODAT & 49 & TAWOS KHAIL & TAWOS KHAIL & 900 & 250 & 28 & 11 & 2,750 \\
\hline RODAT & 94 & ZAKHAIL & EQBAL SHAH KELY & 60 & 20 & 33 & 13 & 260 \\
\hline RODAT & 878 & ZAKHEL & MULLAH M.AYUB & 80 & 30 & 38 & 14 & 420 \\
\hline RODAT & 95 & ZAKHEL & SAFI KALAY & 45 & 15 & 33 & 10 & 150 \\
\hline RODAT & 102 & ZAKHEL LOY KALAY & ZAKHEL LOY KALAY & 140 & 56 & 40 & 13 & 728 \\
\hline RODAT & 883 & ZANGO KAS & ZANGO KAS & 350 & 136 & 39 & 14 & 1,904 \\
\hline RODAT & 915 & ZAR BACHA & ZAR BACHA & 1,000 & 250 & 25 & 9 . & 2,250 \\
\hline RODAT & 893 & ZAR KHEL & MIRZA GUL QELE & 60 & 20 & 33 & 14 & 280 \\
\hline RODAT & 3504 & ZINO KALAY & ZINO KALAY & 200 & 70 & 35 & 15 & 1,050 \\
\hline SHERZAD & 228 & AKRAM KHEL & AKRAM KHEL & 180 & 50 & 28 & 12 & 600 \\
\hline SHERZAD & 234 & ALI KHEL & ALI KHEL & 300 & 200 & 67 & 13 & 2,600 \\
\hline
\end{tabular}


Opium Production Survey by village in Nangarhar province, 1995.

\begin{tabular}{|c|c|c|c|c|c|c|c|c|}
\hline & Village & & & Total & Land & & Wet Opium *2 & Wet Opium \\
\hline \multirow[t]{2}{*}{ District } & \multirow[t]{2}{*}{ Code } & \multirow[t]{2}{*}{ Area/ Main Village } & \multirow[t]{2}{*}{ Village } & \multirow{2}{*}{$\begin{array}{c}\text { Cultivated } \\
\text { Land (jerib }{ }^{*} \text { ) }\end{array}$} & \multicolumn{2}{|c|}{ Poppy Cultivation } & \multirow{2}{*}{$\begin{array}{c}\text { Yield } \\
(\mathrm{kg} / \mathrm{jr} .) \\
\end{array}$} & \multirow{2}{*}{$\begin{array}{c}\text { Production } \\
(\mathrm{kg}) \\
\end{array}$} \\
\hline & & & & & (jerib) & $(\%)$ & & \\
\hline \multicolumn{9}{|c|}{ Province: Nangarhar } \\
\hline SHERZAD & 3430 & AMAN KHEL & MALEK KHEL & 200 & 120 & 60 & 12 & 1,440 \\
\hline SHERZAD & 220 & AMAN KHIL & AMAN KHIL & 500 & 210 & 42 & 14 & 2,940 \\
\hline SHERZAD & 33 & ANGURTAK & SHAZKHEL & 55 & 35 & 64 & 14 & 490 \\
\hline SHERZAD & 3499 & ASHPAN & SHAZ KHEL & 100 & 50 & 50 & 11 & 550 \\
\hline SHERZAD & 221 & ASHPAN & SURDAK & 480 & 280 & 58 & 15 & 4,200 \\
\hline SHERZAD & 1095 & BAHZID KHEL & BAHZID KHEL & 170 & 80 & 47 & 14 & 1,120 \\
\hline SHERZAD & 1657 & BATLO & PATLA & 400 & 200 & 50 & 11 & 2,200 \\
\hline SHERZAD & 216 & BELAL KHEL & BELAL KHEL & 230 & 140 & 61 & 12 & 1,680 \\
\hline SHERZAD & 236 & CELA & CELA & 45 & 20 & 44 & 11 & 220 \\
\hline SHERZAD & 203 & FATO KAC & FATO KAC & 180 & 85 & 47 & 12 & 1,020 \\
\hline SHERZAD & 229 & GANDOMAK & QAZYAN & 300 & 140 & 47 & 12 & 1,680 \\
\hline SHERZAD & 3432 & GANDUMAK & BASHI KHEL & 750 & 400 & 53 & 12 & 4,800 \\
\hline SHERZAD & 217 & GHARA & GHARA & 200 & 100 & 50 & 14 & 1,400 \\
\hline SHERZAD & 212 & GHUNDAY & GHUNDAY & 200 & 100 & 50 & 13 & 1,300 \\
\hline SHERZAD & 188 & GODAR & GODAR & 250 & 150 & 60 & 13 & 1,950 \\
\hline SHERZAD & 198 & HASHEM KHEL & HASHEM KHEL & 300 & 125 & 42 & 12 & 1,500 \\
\hline SHERZAD & 201 & JANGALYAN & JANGALYAN & 300 & 100 & 33 & 12 & 1,200 \\
\hline SHERZAD & 209 & KACHARA/YABE KASS & KACHARA/YABE KASS & 200 & 120 & 60 & 14 & 1,680 \\
\hline SHERZAD & 209 & KACHRA & KACHARA & 90 & 40 & 44 & 12 & 480 \\
\hline SHERZAD & 1092 & KALA KHEL & KALA KHEL & 200 & 80 & 40 & 13 & 1,040 \\
\hline SHERZAD & 233 & KAMA TERMAY & KAMA TERMAY & 200 & 85 & 43 & 13 & 1,105 \\
\hline SHERZAD & 1098 & KANDI & ALEKOZAI & 300 & 220 & 73 & 11 & 2,420 \\
\hline SHERZAD & 193 & KANDI KHEL & KANDI KHEL & 220 & 120 & 55 & 13 & 1,560 \\
\hline SHERZAD & 215 & KARM GHUNDAY & KARM GHUNDAY & 100 & 40 & 40 & 14 & 560 \\
\hline SHERZAD & 3425 & KESHRAN & KESHRAN & 200 & 70 & 35 & 13 & 910 \\
\hline SHERZAD & 224 & KHAROT & KHAROT & 120 & 50 & 42 & 14 & 700 \\
\hline SHERZAD & 222 & KHATE & KHATE & 50 & 20 & 40 & 10 & 200 \\
\hline SHERZAD & 18 & KHOSHAL KHIL & KHOSHAL KHIL & 0 & 0 & \#DIV/0! & 0 & 0 \\
\hline SHERZAD & 208 & KHWAZI KHEL & KHWAZI KHEL & 400 & 200 & 50 & 12 & 2,400 \\
\hline
\end{tabular}


Opium Production Survey by village in Nangarhar province, 1995.

\begin{tabular}{|c|c|c|c|c|c|c|c|c|}
\hline \multirow{3}{*}{ District } & \multirow{3}{*}{$\begin{array}{l}\text { Village } \\
\text { Code }\end{array}$} & \multirow{3}{*}{ Area/ Main Village } & \multirow{3}{*}{ Village } & \multirow{3}{*}{$\begin{array}{c}\text { Total } \\
\text { Cultivated } \\
\text { Land (jerib }{ }^{*} \text { ) } \\
\end{array}$} & \multirow{2}{*}{\multicolumn{2}{|c|}{$\begin{array}{c}\text { Land Under } \\
\text { Poppy Cultivation }\end{array}$}} & \multirow{3}{*}{$\begin{array}{c}\text { Wet Opium }{ }^{* 2} \\
\text { Yield } \\
(\mathrm{kg} / \mathrm{jr} .) \\
\end{array}$} & \multirow{3}{*}{$\begin{array}{c}\text { Wet Opium } \\
\text { Production } \\
(\mathrm{kg}) \\
\end{array}$} \\
\hline & & & & & & & & \\
\hline & & & & & (jerib) & $(\%)$ & & \\
\hline \multicolumn{9}{|c|}{ Province: Nangarhar } \\
\hline SHERZAD & 190 & KODI KHEL & SHORLA KHEL & 300 & 180 & 60 & 14 & 2,520 \\
\hline SHERZAD & 1103 & KOTAGAY & KOTAGAY & 250 & 150 & 60 & 14 & 2,100 \\
\hline SHERZAD & 240 & KOTGAY & KOTGAY & 300 & 130 & 43 & 13 & 1,690 \\
\hline SHERZAD & 3489 & KOZ KHADE KHEL & KUZ KHADE KHEL & 300 & 210 & 70 & 14 & 2,940 \\
\hline SHERZAD & 1097 & KUDI KHEL & KUDI KHEL & 140 & 40 & 29 & 13 & 520 \\
\hline SHERZAD & 3431 & KUZA GHARA & KUZA GHARA & 350 & 200 & 57 & 14 & 2,800 \\
\hline SHERZAD & 1094 & LADA BAGH & LADA BAGH & 25 & 0 & 0 & 0 & 0 \\
\hline SHERZAD & 231 & LANGAR KHEL & LANGAR KHEL & 200 & 90 & 45 & 13 & 1,170 \\
\hline SHERZAD & 3427 & LESHAKA & LESHAKA & 200 & 100 & 50 & 13 & 1,300 \\
\hline SHERZAD & 211 & LOYA TERMAY & LOYA TERMAY & 320 & 180 & 56 & 12 & 2,160 \\
\hline SHERZAD & 207 & MALIK QYAS & MALIK QYAS & 800 & 550 & 69 & 14 & 7,700 \\
\hline SHERZAD & 235 & MAMA KHEL & MIRAK KHEL & 250 & 150 & 60 & 14 & 2,100 \\
\hline SHERZAD & 239 & MAMA KHEL & SHIR ZAD & 280 & 160 & 57 & 13 & 2,080 \\
\hline SHERZAD & 1093 & MAZDAKY & MAZDAKY & 150 & 100 & 67 & 13 & 1,300 \\
\hline SHERZAD & 235 & MERKE KHEL & MERKE KHEL & 300 & 150 & 50 & 15 & 2,250 \\
\hline SHERZAD & 3387 & MOMAND & MOMAND & 130 & 50 & 38 & 14 & 700 \\
\hline SHERZAD & 238 & MORGA & MORGAN & 550 & 300 & 55 & 14 & 4,200 \\
\hline SHERZAD & 200 & NARAY JABAH & NARAY JABAH & 100 & 50 & 50 & 13 & 650 \\
\hline SHERZAD & 232 & NOKAR KHEL & NORAK KHEL & 800 & 450 & 56 & 15 & 6,750 \\
\hline SHERZAD & 187 & PANACAZI & PANAGAZI & 200 & 50 & 25 & 14 & 700 \\
\hline SHERZAD & 187 & PANAGAZI & PANAGAZI & 40 & 30 & 75 & 13 & 390 \\
\hline SHERZAD & 225 & PANZYAN & PANZYAN & 200 & 120 & 60 & 14 & 1,680 \\
\hline SHERZAD & 204 & PAS KALA & PAS KALA & 300 & 170 & 57 & 13 & 2,210 \\
\hline SHERZAD & 1675 & PETLA & PETLA & 300 & 100 & 33 & 10 & 1,000 \\
\hline SHERZAD & 199 & QALA-I- HAJI CORONA & QALA-I- HAJI CORONA & 550 & 250 & 45 & 13 & 3,250 \\
\hline SHERZAD & 3556 & ROD & DARGIAN & 180 & 80 & 44 & 14 & 1,120 \\
\hline SHERZAD & 210 & RODIHESAR & RODIHESAR & 100 & 50 & 50 & 12 & 600 \\
\hline SHERZAD & 1091 & SADA KAC & SADA KAC & 200 & 125 & 63 & 13 & 1,625 \\
\hline SHERZAD & 186 & SAMAR KHEL & SAMAR KHEL & 500 & 300 & 60 & 12 & 3,600 \\
\hline
\end{tabular}

$*$ Jerib (jr.) $=1 / 5$ ha

* 2 Dry Opium $=$ Wet Opium - $30 \%$ moisture
Page $42 \quad 132$
Source: UNDCP Opium Production Survey, 1995 File: NGR2.XLS - 06/08/95 
Opium Production Survey by village in Nangarhar province, 1995.

\begin{tabular}{|c|c|c|c|c|c|c|c|c|}
\hline & Village & & & \multirow{3}{*}{$\begin{array}{c}\text { Total } \\
\text { Cultivated } \\
\left.\text { Land (jerib }^{*}\right)\end{array}$} & Land & & \multirow{3}{*}{$\begin{array}{c}\text { Wet Opium }{ }^{* 2} \\
\text { Yield } \\
(\mathrm{kg} / \mathrm{jr} .) \\
\end{array}$} & \multirow{3}{*}{$\begin{array}{c}\text { Wet Opium } \\
\text { Production } \\
(\mathrm{kg})\end{array}$} \\
\hline \multirow[t]{2}{*}{ District } & \multirow{2}{*}{ Code } & \multirow[t]{2}{*}{ Area/ Main Village } & \multirow[t]{2}{*}{ Village } & & \multicolumn{2}{|c|}{ Poppy Cultivation } & & \\
\hline & & & & & (jerib) & $(\%)$ & & \\
\hline \multicolumn{9}{|c|}{ Province: Nangarhar } \\
\hline SHERZAD & 227 & SANGARAY & SANGARAY & 500 & 259 & 52 & 14 & 3,626 \\
\hline SHERZAD & 1099 & SANGOZI(SANGAI) & SANGOZI(SANGAI) & 180 & 60 & 33 & 12 & 720 \\
\hline SHERZAD & 205 & SAWATYAN & SAWATYAN & 400 & 210 & 53 & 12 & 2,520 \\
\hline SHERZAD & 196 & SHADI KHEL & SHADI KHEL & 400 & 200 & 50 & 14 & 2,800 \\
\hline SHERZAD & 237 & SHANAWAR & SHANAWAR & 400 & 200 & 50 & 12 & 2,400 \\
\hline SHERZAD & 219 & SHAWE & SHAWE & 600 & 300 & 50 & 15 & 4,500 \\
\hline SHERZAD & 223 & SHEKHAN/GANDUMAK & SHAIKHAN & 200 & 120 & 60 & 14 & 1,680 \\
\hline SHERZAD & 213 & SHORLA KHEL & SHORLA KHEL & 20 & 10 & 50 & 10 & 100 \\
\hline SHERZAD & 206 & SORAB KHEL & SORAB KHEL & 300 & 170 & 57 & 14 & 2,380 \\
\hline SHERZAD & 3555 & SPIN KALAY & SPIN KALAY & 160 & 60 & 38 & 10 & 600 \\
\hline SHERZAD & 218 & SRE KHAWRE & SRE KHAWRE & 100 & 60 & 60 & 12 & 720 \\
\hline SHERZAD & 3501 & SURKH PUL & SURKH PUL & 230 & 60) & 26 & 14 & 840 \\
\hline SHERZAD & 1646 & SURKHAB & SURKHAB & 80 & 40 & 50 & 13 & 520 \\
\hline SHERZAD & 3429 & SURKOT & SURKOT & 200 & 120 & 60 & 14 & 1,680 \\
\hline SHERZAD & 3426 & TOTO & BAR KHADI KHEL & 400 & 250 & 63 & 12 & 3,000 \\
\hline SHERZAD & 1101 & TOTO & GAR KHEL & 500 & 300 & 60 & 12 & 3,600 \\
\hline SHERZAD & 3551 & Tото & KHADAKHEL & 200 & 60 & 30 & 13 & 780 \\
\hline SHERZAD & 197 & Tото & MALEKANO KALA & 600 & 350 & 58 & 12 & 4,200 \\
\hline SHERZAD & 194 & Tото & PESHDARA & 250 & 150 & 60 & 13 & 1,950 \\
\hline SHERZAD & 195 & TOTO & RAMEL KHEL & 400 & 200 & 50 & 12 & 2,400 \\
\hline SHERZAD & 1100 & TOTO & SAYED KH. NAZAR KHEL & 200 & 130 & 65 & 13 & 1,690 \\
\hline SHERZAD & 3550 & TOTO/SUR PAL & KHADA KHEL & 230 & 60 & 26 & 14 & 840 \\
\hline SHERZAD & 214 & WATAY & WATAY & 60 & 20 & 33 & 12 & 240 \\
\hline SHERZAD & 191 & WECH LAGADA & WECH LAGADA & 20 & 0 & 0 & 0 & 0 \\
\hline SHERZAD & 226 & YOSUF KAC & YOSUF KAC & 500 & 200 & 40 & 12 & 2,400 \\
\hline SHERZAD & 3386 & ZAZI QALA & ZAZI QALA & 50 & 20 & 40 & 13 & 260 \\
\hline SHINWAR & 2505 & AKA LEMASY & AKA LEMASY & 440 & 280 & 64 & 9 & 2,520 \\
\hline SHINWAR & 2506 & AKHONDZADAGAN & AKHONDZADAGAN & 120 & 80 & 67 & 8 & 640 \\
\hline SHINWAR & 2541 & AMIN KHAN & AMIN KHAN & 135 & 120 & 89 & 14 & 1,680 \\
\hline
\end{tabular}


Opium Production Survey by village in Nangarhar province, 1995.

\begin{tabular}{|c|c|c|c|c|c|c|c|c|}
\hline & Village & & & Total & Land 1 & & Wet Opium *2 & Wet Opium \\
\hline \multirow[t]{2}{*}{ District } & \multirow[t]{2}{*}{ Code } & \multirow[t]{2}{*}{ Area/ Main Village } & \multirow[t]{2}{*}{ Village } & \multirow{2}{*}{$\begin{array}{c}\text { Cultivated } \\
\left.\text { Land (jerib }{ }^{*}\right) \\
\end{array}$} & \multicolumn{2}{|c|}{ Poppy Cultivation } & \multirow{2}{*}{$\begin{array}{c}\text { Yield } \\
(\mathrm{kg} / \mathrm{jr} .) \\
\end{array}$} & \multirow{2}{*}{$\begin{array}{c}\text { Production } \\
(\mathrm{kg})\end{array}$} \\
\hline & & & & & (jerib) & $(\%)$ & & \\
\hline \multicolumn{9}{|c|}{ Province: Nangarhar } \\
\hline SHINWAR & 2531 & AMINGUL KALAY & AMINGUL KALAY & 180 & 90 & 50 & 8 & 720 \\
\hline SHINWAR & 2520 & ANAR BAGH & ANAR BAGH & 580 & 400 & 69 & 10 & 4,000 \\
\hline SHINWAR & 2537 & ARBAB & ARBAB & 180 & 150 & 83 & 9 & 1,350 \\
\hline SHINWAR & 2500 & AYOB KHAN KALAY & AYOB KHAN KALAY & 110 & 80 & 73 & 9 & 720 \\
\hline SHINWAR & 2540 & BAR MIRANO KALAY & BAR MIRANO KALAY & 300 & 200 & 67 & 12 & 2,400 \\
\hline SHINWAR & 2496 & DABA & DABA & 130 & 85 & 65 & 9 & 765 \\
\hline SHINWAR & 2498 & DABAGAR & DABAGAR & 90 & 60 & 67 & 10 & 600 \\
\hline SHINWAR & 2486 & DAG & DAG & 200 & 150 & 75 & 11 & 1,650 \\
\hline SHINWAR & 2515 & DOWAKI & DOWAKI & 150 & 120 & 80 & 12 & 1,440 \\
\hline SHINWAR & 2530 & DUBA KUZHA & DUBA KUZHA & 55 & 40 & 73 & 10 & 400 \\
\hline SHINWAR & 2516 & GULAHI & GULAHI & 80 & 70 & 88 & 14 & 980 \\
\hline SHINWAR & 2532 & HAJI MOHD.ILIAS KELY & HAJI ILIAS KELY & 130 & 85 & 65 & 9 & 765 \\
\hline SHINWAR & 2534 & JIBAGAI & JIBAGAI & 65 & 58 & 89 & 11 & 638 \\
\hline SHINWAR & 2542 & KANGALAN KALAY & KANGALAN KALAY & 300 & 250 & 83 & 11 & 2,750 \\
\hline SHINWAR & 2478 & KHANO KHEL SHER GAR & KHANO KHEL SHER GAR & 100 & 70 & 70 & 9 & 630 \\
\hline SHINWAR & 365 & KHANU KELE & KHANU KELE & 180 & 100 & 56 & 9 & 900 \\
\hline SHINWAR & 2485 & KHYRBANA & KHYRBANA & 60 & 40 & 67 & 9 & 360 \\
\hline SHINWAR & 2482 & KOCHIANO & KOCHIANO & 0 & 0 & \#DIV/0! & 0 & 0 \\
\hline SHINWAR & 2503 & KOOZ MIRAN & KATILAY & 105 & 85 & 81 & 9 & 765 \\
\hline SHINWAR & 2499 & KUNDIANO KALAY & KUNDIANO KALAY & 100 & 70 & 70 & 12 & 840 \\
\hline SHINWAR & 2501 & LOKHAY & LOKHAY & 450 & 300 & 67 & 10 & 3,000 \\
\hline SHINWAR & 2502 & LOY KALAY & LOY KALAY & 120 & 80 & 67 & 11 & 880 \\
\hline SHINWAR & 2481 & MALAK AFSAR & MALAK AFSAR & 50 & 40 & 80 & 13 & 520 \\
\hline SHINWAR & 2484 & MALEK ISMAIL KELY & MALEK ISMAIL KELY & 150 & 50 & 33 & 13 & 650 \\
\hline SHINWAR & 2543 & MARJAN KHEL & MARJAN KHEL & 200 & 150 & 75 & 12 & 1,800 \\
\hline SHINWAR & 2521 & MASHANGAH & MASHANGAH & 350 & 250 & 71 & 9 & 2,250 \\
\hline SHINWAR & 2539 & MIR KALAY & MIR KALAY & 95 & 80 & 84 & 12 & 960 \\
\hline SHINWAR & 2508 & MOHAMMAD ALI KHAN & MOHAMMAD ALI KHAN & 120 & 80 & 67 & 9 & 720 \\
\hline SHINWAR & 2509 & MORCHA KHAIL & MORCHA KHAIL & 200 & 130 & 65 & 10 & 1,300 \\
\hline
\end{tabular}

* Jerib (jr.) $=1 / 5$ ha

* ${ }^{2}$ Dry Opium = Wet Opium - $30 \%$ moisture
Page $44 \quad 134$
Source: UNDCP Opium Production Survey, 1995. File: NGR2.XLS - 06/08/95 
Opium Production Survey by village in Nangarhar province, 1995.

\begin{tabular}{|c|c|c|c|c|c|c|c|c|}
\hline \multirow{3}{*}{ District } & \multirow{3}{*}{\begin{tabular}{|c|} 
Village \\
Code \\
\end{tabular}} & \multirow{3}{*}{ Area/ Main Village } & \multirow{3}{*}{ Village } & \multirow{3}{*}{\begin{tabular}{|c|} 
Total \\
Cultivated \\
Land $\left(\right.$ jerib $\left.{ }^{*}\right)$ \\
\end{tabular}} & \multirow{2}{*}{\multicolumn{2}{|c|}{$\begin{array}{c}\text { Land Under } \\
\text { Poppy Cultivation }\end{array}$}} & \multirow{3}{*}{$\begin{array}{c}\text { Wet Opium }^{* 2} \\
\text { Yield } \\
(\mathrm{kg} / \mathrm{jr} .)\end{array}$} & \multirow{3}{*}{$\begin{array}{c}\text { Wet Opium } \\
\text { Production } \\
(\mathrm{kg}) \\
\end{array}$} \\
\hline & & & & & & & & \\
\hline & & & & & (jerib) & $(\%)$ & & \\
\hline \multicolumn{9}{|c|}{ Province: Nangarhar } \\
\hline SHINWAR & 2535 & NABI JAN KALAY & NABI JAN KALAY & 200 & 160 & 80 & 10 & 1,600 \\
\hline SHINWAR & 2533 & QALANDAR KALAY & QALANDAR KALAY & 110 & 100 & 91 & 10 & 1,000 \\
\hline SHINWAR & 2477 & RAHMATULLA KHAN KALA & RAHMATULLA KHAN KALA & 300 & 50 & 17 & 10 & 500 \\
\hline SHINWAR & 2538 & RAJA KALAY & RAJA KALAY & 200 & 180 & 90 & 12 & 2,160 \\
\hline SHINWAR & 2504 & SAYDRUD KALAY & SAYDRUD KALAY & 125 & 80 & 64 & 10 & 800 \\
\hline SHINWAR & 2536 & SHAH AHMAD KALAY & SHAH AHMAD KALAY & 450 & 300 & 67 & 10 & 3,000 \\
\hline SHINWAR & 2529 & SHARAF KALA & SHARAF KALA & 90 & 70 & 78 & 9 & 630 \\
\hline SHINWAR & 2493 & SHERGAR & SHERGAR & 60 & 55 & 92 & 12 & 660 \\
\hline SHINWAR & 2544 & SHIEKHANO KALAY & SHIEKHANO KALAY & 90 & 75 & 83 & 9 & 675 \\
\hline SHINWAR & 2518 & SHIN SARAW & SHIN SARAW & 80 & 70 & 88 & 8 & 560 \\
\hline SHINWAR & 2528 & SYA CHOB & SYA CHOB & 1,000 & 700 & 70 & 11 & 7,700 \\
\hline SHINWAR & 2495 & TAWDA CHINA & TAWDA CHINA & 80 & 40 & 50 & 7 & 280 \\
\hline SHINWAR & 2513 & TILIANO & TILIANO & 70 & 50 & 71 & 9 & 450 \\
\hline SHINWAR & 2490 & WIALA 25 & GHRA GHARIKORON & 500 & 0 & 0 & 0 & 0 \\
\hline SHINWAR & 2479 & WIALA 25 & KOZ KORONA & 450 & 0 & 0 & 0 & 0 \\
\hline SHINWAR & 2489 & WIALA 25 & MARKO & 300 & 0 & 0 & 0 & 0 \\
\hline SHINWAR & 2524 & WIALA 25 & NAHER GHARI KORONA & 400 & 0 & 0 & 0 & 0 \\
\hline SHINWAR & 2497 & WIALA 26 & KHARI FARAM & 480 & 0 & 0 & 0 & 0 \\
\hline SHINWAR & 2512 & WIALA 26 & SHAGI FARAM & 540 & 0 & 0 & 0 & 0 \\
\hline SHINWAR & 2494 & WIALA 26 & ZARGHON KORONA & 350 & 0 & 0 & 0 & 0 \\
\hline SHINWAR & 2526 & WIALA 26 BACHI & GULAHI TAMIR & 350 & 0 & 0 & 0 & 0 \\
\hline SHINWAR & 2519 & WIALA 27 & HAJI HAIAT & 200 & 0 & 0 & 0 & 0 \\
\hline SHINWAR & 2492 & WIALA 27 & KOZA DAGA & 385 & 0 & 0 & 0 & 0 \\
\hline SHINWAR & 2488 & WIALA 27 & MANZ KORONA & 400 & 0 & 0 & 0 & 0 \\
\hline SHINWAR & 2510 & WIALA 27 & RAGHA PATI & 300 & 0 & 0 & 0 & 0 \\
\hline SHINWAR & 2517 & WIALA 27 & SARAK GHARIKORO & 560 & 0 & 0 & 0 & 0 \\
\hline SHINWAR & 2523 & WIALA 28 & JAME JOMAT & 800 & 0 & 0 & 0 & 0 \\
\hline SHINWAR & 2491 & WIALA 28 & KOCHIANO KORONA & 150 & 0 & 0 & 0 & 0 \\
\hline SHINWAR & 2525 & WIALA 28 & MANZ KORONA & 760 & 0 & 0 & 0 & 0 \\
\hline
\end{tabular}


Opium Production Survey by village in Nangarhar province, 1995.

\begin{tabular}{|c|c|c|c|c|c|c|c|c|}
\hline \multirow{3}{*}{ District } & \multirow{3}{*}{$\begin{array}{l}\text { Village } \\
\text { Code }\end{array}$} & \multirow{3}{*}{ Area/ Main Village } & \multirow{3}{*}{ Village } & \multirow{3}{*}{\begin{tabular}{|c|} 
Total \\
Cultivated \\
Land (jerib ${ }^{*}$ ) \\
\end{tabular}} & \multirow{2}{*}{\multicolumn{2}{|c|}{$\begin{array}{c}\text { Land Under } \\
\text { Poppy Cultivation }\end{array}$}} & \multirow{3}{*}{$\begin{array}{c}\text { Wet }^{\text {Opium }}{ }^{* 2} \\
\text { Yield } \\
(\mathrm{kg} / \mathrm{jr} .) \\
\end{array}$} & \multirow{3}{*}{$\begin{array}{c}\text { Wet Opium } \\
\text { Production } \\
(\mathrm{kg}) \\
\end{array}$} \\
\hline & & & & & & & & \\
\hline & & & & & (jerib) & $(\%)$ & & \\
\hline \multicolumn{9}{|c|}{ Province: Nangarhar } \\
\hline SHINWAR & 2514 & WIALA 29 & WIALA 29 & 400 & 0 & 0 & 0 & 0 \\
\hline SHINWAR & 2480 & WIALA 30 & WIALA 30 & 0 & 0 & \#DIV/0! & 0 & 0 \\
\hline SHINWAR & 2487 & WIALA $30 \mathrm{BACHI}$ & WIALA 30 BACHI & 0 & 0 & \#DIV/0! & 0 & 0 \\
\hline SHINWAR & 2527 & ZAKHAIL & ZAKHAIL & 450 & 380 & 84 & 10 & 3,800 \\
\hline SHINWAR & 2511 & ZANANO KELE & ZANANO KELE & 180 & 100 & 56 & 9 & 900 \\
\hline SHINWAR & 2507 & ZARA DAGAH & ZARA DAGAH & 400 & 70 & 18 & 9 & 630 \\
\hline SURKH ROD & 828 & ADAM KHAIL & ADAM KHEL & 1,650 & 0 & 0 & 0 & 0 \\
\hline SURKH ROD & 802 & AHMADZAI & AHMADZAI & 1,150 & 0 & 0 & 0 & 0 \\
\hline SURKH ROD & 815 & AMAR KHAIL & AMAR KHAIL & 630 & 0 & 0 & 0 & 0 \\
\hline SURKH ROD & 3231 & BAGHBANI & BAGHBANI & 500 & 7. & 1 & 11 & 77 \\
\hline SURKH ROD & 805 & BAGRAMI & BAGRAMI & 590 & 0 & 0 & 0 & 0 \\
\hline SURKH ROD & 3205 & BAKHTAN & BAKHTAN & 430 & 0 & 0 & 0 & 0 \\
\hline SURKH ROD & 803 & BALA BAGH & BALA BAGH & 1,900 & 10 & 1 & 10 & 100 \\
\hline SURKH ROD & 3461 & BALA BAGH & DARYAB & 350 & 0 & 0 & 0 & 0 \\
\hline SURKH ROD & 3216 & BALADEH & BALADEH & 200 & 0 & 0 & 0 & 0 \\
\hline SURKH ROD & 821 & BALOCH KELY & BALOCH KELY & 190 & 0 & 0 & 0 & 0 \\
\hline SURKH ROD & 817 & BANDA HAJI MIR ALAM & BANDA HAJI MIR ALAM & 350 & 0 & 0 & 0 & 0 \\
\hline SURKH ROD & 3206 & BANDAH BEMARAN & BANDAH BEMARAN & 580 & 0 & 0 & 0 & 0 \\
\hline SURKH ROD & 293 & BAR ABAD & BAR ABAD & 290 & 0 & 0 & 0 & 0 \\
\hline SURKH ROD & 3234 & BAR BANGAH SHER & BAR BANGAH SHER & 80 & 20 & 25 & 13 & 260 \\
\hline SURKH ROD & 3464 & BAR KAKALAK & BAR KAKALAK & 1,500 & 70 & 5 & 14 & 980 \\
\hline SURKH ROD & 822 & BAR KAKO KHEL & BAR KAKO KHEL & 320 & 0 & 0 & 0 & 0 \\
\hline SURKH ROD & 3238 & BARENAH & BARENAH & 350 & 0 & 0 & 0 & 0 \\
\hline SURKH ROD & 3457 & BAWLI & BAWLI & 350 & 4 & 1 & 12 & 48 \\
\hline SURKH ROD & 3233 & BAZID KHIL & BAZID KHIL & 500 & 0 & 0 & 0 & 0 \\
\hline SURKH ROD & 3218 & CHAKNORI & CHAKNORI & 400 & 0 & 0 & 0 & 0 \\
\hline SURKH ROD & 833 & CHAR BAGH SAFA & CHAR BAGH SAFA & 2,400 & 0 & 0 & 0 & 0 \\
\hline SURKH ROD & 3237 & CHAR BAGH SAFA & QALA HAZRATAN & 1,900 & 0 & 0 & 0 & 0 \\
\hline SURKH ROD & 3202 & DAND & DAND & 200 & 0 & 0 & 0 & 0 \\
\hline
\end{tabular}

* Jerib (jr.) $=1 / 5$ ha

*2 Dry Opium = Wet Opium - $30 \%$ moisture
Page $46 \quad 136$
Source: UNDCP Opium Production Survey, 1995 File: NGR2.XLS - 06/08/95 
Opium Production Survey by village in Nangarhar province, 1995.

\begin{tabular}{|c|c|c|c|c|c|c|c|c|}
\hline & \multirow{3}{*}{$\begin{array}{l}\text { Village } \\
\text { Code }\end{array}$} & \multirow{3}{*}{ Area/ Main Village } & \multirow{3}{*}{ Village } & \multirow{3}{*}{$\begin{array}{c}\text { Total } \\
\text { Cultivated } \\
\text { Land (jerib }^{*} \text { ) } \\
\end{array}$} & \multirow{2}{*}{\multicolumn{2}{|c|}{$\begin{array}{c}\text { Land Under } \\
\text { Poppy Cultivation }\end{array}$}} & \multirow{3}{*}{$\begin{array}{c}\text { Wet Opium }{ }^{* 2} \\
\text { Yield } \\
(\mathrm{kg} / \mathrm{jr} .)\end{array}$} & \multirow{3}{*}{$\begin{array}{c}\text { Wet Opium } \\
\text { Production } \\
(\mathrm{kg})\end{array}$} \\
\hline \multirow[t]{2}{*}{ District } & & & & & & & & \\
\hline & & & & & (jerib) & $(\%)$ & & \\
\hline \multicolumn{9}{|c|}{ Province: Nangarhar } \\
\hline SURKH ROD & 3553 & DARONTA & DARONTA & 100 & 0 & 0 & 0 & 0 \\
\hline SURKH ROD & 3458 & DEH BALA & DEH BALA & 400 & 0 & 0 & 0 & 0 \\
\hline SURKH ROD & 3224 & DEH WALID & DEH WALID & 7.50 & 0 & 0 & 0 & .0 \\
\hline SURKH ROD & 3554 & DUAB & DUAB & 350 & 100 & 29 & 14 & 1,400 \\
\hline SURKH ROD & 3211 & FATEH ABAD & DEH DABAN & 900 & 40 & 4 & 14 & 560 \\
\hline SURKH ROD & 3235 & FATEH ABAD & FATEH ABAD & 2,500 & 70 & 3 & 14 & 980 \\
\hline SURKH ROD & 3204 & FATEH ABAD & QALE SALIM & 500 & 40 & 8 & 15 & 600 \\
\hline SURKH ROD & 3203 & FATEH ABAD & QOROOTAK & 550 & 30 & 5 & 13 & 390 \\
\hline SURKH ROD & 3215 & GANDAH CHESHMAH & GANDAH CHESHMAH & 200 & 6 & 3 & 12 & 72 \\
\hline SURKH ROD & 3217 & GAZAK & GAZAK & 150 & 10 & 7 & 15 & 150 \\
\hline SURKH ROD & 3212 & GHAWCHAK & GHAWCHAK & 1,200 & 0 & 0 & 0 & 0 \\
\hline SURKH ROD & 3460 & GHUNDI QALA & GHUNDI QALA & 200 & 0 & 0 & 0 & 0 \\
\hline SURKH ROD & 3469 & KACHARA & KACHARA & 300 & 0 & 0 & 0 & 0 \\
\hline SURKH ROD & 838 & KACHRAH & KACHRAH & 200 & 20 & 10 & 15 & 300 \\
\hline SURKH ROD & 3463 & KALATA & KALATA & 95 & 0 & 0 & 0 & 0 \\
\hline SURKH ROD & 824 & KATA PUR & KATA PUR & 500 & 0 & 0 & 0 & 0 \\
\hline SURKH ROD & 3462 & KATA PUR & QALAA-E-PATAN & 400 & 0 & 0 & 0 & 0 \\
\hline SURKH ROD & 831 & KHAIR ABAD & KHAIR ABAD & 2,000 & 6 & 0 & 11 & 66 \\
\hline SURKH ROD & 819 & KOCHYANO KELY & KOCHYANO KELY & 200 & 4 & 2 & 10 & 40 \\
\hline SURKH ROD & 826 & KOROM & KOROM & 40 & 20 & 50 & 15 & 300 \\
\hline SURKH ROD & 813 & KOZ KAKO KHIL & KOZ KAKO KHIL & 350 & 3 & 1 & 15 & 45 \\
\hline SURKH ROD & 3229 & KUSHKAK & KUSHKAK & 1,200 & 0 & 0 & 0 & 0 \\
\hline SURKH ROD & 3219 & KUZ BANGAH SHER & KUZ BANGAH SHER & 170 & 10 & 6 & 12 & 120 \\
\hline SURKH ROD & 3221 & LALA QALA & LALA QALA & 780 & 0 & 0 & 0 & 0 \\
\hline SURKH ROD & 801 & MANSOOR KELY & MANSOOR KELY & 200 & 14 & 7 & 12 & 168 \\
\hline SURKH ROD & 3230 & MIRAN & MIRAN & 200 & 0 & 0 & 0 & 0 \\
\hline SURKH ROD & 3459 & MIRZA ALI ABAD & MIRZA ALI ABAD & 230 & 0 & 0 & 0 & 0 \\
\hline SURKH ROD & 3495 & MIRZAYAN & MIRZAYAN & 500 & 0 & 0 & 0 & 0 \\
\hline SURKH ROD & 836 & MOGHUL KHAIL & MOGHUL KHAIL & 300 & 0 & 0 & 0 & 0 \\
\hline
\end{tabular}


Opium Production Survey by village in Nangarhar province, 1995.

\begin{tabular}{|c|c|c|c|c|c|c|c|c|}
\hline & Village & & & Total & Land & & Wet Opium *2 & Wet Opium \\
\hline \multirow[t]{2}{*}{ District } & \multirow[t]{2}{*}{ Code } & \multirow[t]{2}{*}{ Area/ Main Village } & \multirow[t]{2}{*}{ Village } & \multirow{2}{*}{$\begin{array}{c}\text { Cultivated } \\
\text { Land (jerib }{ }^{*} \text { ) }\end{array}$} & \multicolumn{2}{|c|}{ Poppy Cultivation } & \multirow{2}{*}{$\begin{array}{c}\text { Yield } \\
(\mathrm{kg} / \mathrm{jr} .) \\
\end{array}$} & \multirow{2}{*}{$\begin{array}{c}\text { Production } \\
(\mathrm{kg})\end{array}$} \\
\hline & & & & & (jerib) & $(\%)$ & & \\
\hline \multicolumn{9}{|c|}{ Province: Nangarhar } \\
\hline SURKH ROD & 3210 & MULAYEMPOR & MULAYEMPOR & 260 & 0 & 0 & 0 & 0 \\
\hline SURKH ROD & 812 & MUSTI KHEL & MUSTI KHEL & 570 & 0 & 0 & 0 & 0 \\
\hline SURKH ROD & 3220 & NABO KALAY & NABO KALAY & 200 & 8 & 4 & 13 & 104 \\
\hline SURKH ROD & 810 & NAGHRAK & NAGHRAK & 1,400 & 0 & 0 & 0 & 0 \\
\hline SURKH ROD & 804 & NAZAR ABAD & BITNI & 200 & 0 & 0 & 0 & 0 \\
\hline SURKH ROD & 804 & NAZAR ABAD & NAZAR ABAD & 800 & 0 & 0 & 0 & 0 \\
\hline SURKH ROD & 816 & QALAI GHAWCHAK & QALA MALIK & 1,450 & 0 & 0 & 0 & 0 \\
\hline SURKH ROD & 808 & QALAI HJ.DAWLAT & QALAI HJ. KHAN & 150 & 0 & 0 & 0 & 0 \\
\hline SURKH ROD & 3552 & QALAI JAMIDAD & QALAI JAMIDAD & 280 & 0 & 0 & 0 & 0 \\
\hline SURKH ROD & 837 & QALAI ABDULLAH & QALAI ABDULLAH & 400 & 0 & 0 & 0 & 0 \\
\hline SURKH ROD & 3228 & QALAI AFANDI & QALAI AFANDI & 240 & 0 & 0 & 0 & 0 \\
\hline SURKH ROD & 3207 & QALAI DASHT & QALAI DASHT & 75 & 0 & 0 & 0 & 0 \\
\hline SURKH ROD & 3470 & QALAI HAJI SAHEB & QALAI HAJI SAHEB & 295 & 0 & 0 & 0 & 0 \\
\hline SURKH ROD & 811 & QALAI KHAN & QALAI KHAN & 190 & 0 & 0 & 0 & 0 \\
\hline SURKH ROD & 809 & QALAI MAROUF & QALAI MAROUF & 245 & 0 & 0 & 0 & 0 \\
\hline SURKH ROD & 3225 & QALAI MIRZA & QALAI MIRZA & 300 & 0 & 0 & 0 & 0 \\
\hline SURKH ROD & 3465 & QALAI NAW & QALAI NAW & 160 & 0 & 0 & 0 & 0 \\
\hline SURKH ROD & 3201 & QALAI NOOR KHAN & QALAI NOOR KHAN & 530 & 0 & 0 & 0 & 0 \\
\hline SURKH ROD & 830 & SABZ ABAD & SABZ ABAD & 950 & 0 & 0 & 0 & 0 \\
\hline SURKH ROD & 835 & SAWATI & SAWATI & 190 & 0 & 0 & 0 & 0 \\
\hline SURKH ROD & 820 & SAYEDAN ARABI & SAYEDAN ARABI & 1,300 & 0 & 0 & 0 & 0 \\
\hline SURKH ROD & 3456 & SAYEDAN FOLADI & SAYEDAN FOLADI & 500 & 0 & 0 & 0 & 0 \\
\hline SURKH ROD & 806 & SHAMSHAPUR BALA & SHAMSHAPUR BALA & 2,000 & 0 & 0 & 0 & 0 \\
\hline SURKH ROD & 3223 & SHAMSHAPUR ULYA & TATANG NAWAB JABAR & 360 & 0 & 0 & 0 & 0 \\
\hline SURKH ROD & 3209 & SHAMSHOR PAYAN & SHAMSHOR PAYAN & 130 & 0 & 0 & 0 & 0 \\
\hline SURKH ROD & 814 & SHAMSPOOR & KOHA KI HA & 180 & 0 & 0 & 0 & 0 \\
\hline SURKH ROD & 827 & SHEKH MESRI & SHEKH MESRI & 47 & 0 & 0 & 0 & 0 \\
\hline SURKH ROD & 823 & SPEEN JAMAAT & SPEEN JAMAAT & 350 & 40 & 11 & 13 & 520 \\
\hline SURKH ROD & 818 & SULTANPUR & SULTANPUR & 15,003 & 0 & 0 & 0 & 0 \\
\hline
\end{tabular}


Opium Production Survey by village in Nangarhar province, 1995.

\begin{tabular}{|c|c|c|c|c|c|c|c|c|}
\hline & Village & & & Total & Land $L$ & & Wet Opium *2 & Wet Opium \\
\hline \multirow[t]{2}{*}{ District } & \multirow[t]{2}{*}{ Code } & \multirow[t]{2}{*}{ Area/ Main Village } & \multirow[t]{2}{*}{ Village } & \multirow{2}{*}{$\begin{array}{c}\text { Cultivated } \\
\text { Land (jerib }^{*} \text { ) }\end{array}$} & \multicolumn{2}{|c|}{ Poppy Cultivation } & \multirow{2}{*}{$\begin{array}{c}\text { Yield } \\
(\mathrm{kg} / \mathrm{jr} .)\end{array}$} & \multirow{2}{*}{$\begin{array}{c}\text { Production } \\
\text { (kg) }\end{array}$} \\
\hline & & & & & (jerib) & $(\%)$ & & \\
\hline \multicolumn{9}{|c|}{ Province: Nangarhar } \\
\hline SURKH ROD & 832 & SULTANPUR OLYA & DOST KHAIL & 500 & 0 & 0 & 0 & 0 \\
\hline SURKH ROD & 3227 & SULTANPUR SUFLA & BANDA AZIM KHAN & 230 & 0 & 0 & 0 & 0 \\
\hline SURKH ROD & 3232 & SULTANPUR SUFLA & SULTANPUR SUFLA & 1,600 & 0 & 0 & 0 & 0 \\
\hline SURKH ROD & 3222 & SULTANPUR ULYA & QALA ALAH NAZAR & 400 & 0 & 0 & 0 & 0 \\
\hline SURKH ROD & 3214 & WATAH POR & WATAH POR & 450 & 0 & 0 & 0 & 0 \\
\hline SURKH ROD & 829 & ZERANI & ZERANI & 490 & 0 & 0 & 0 & 0 \\
\hline SURKH ROD & 3226 & ZULM ABAD & ZULM ABAD & 300 & 0 & 0 & 0 & 0 \\
\hline
\end{tabular}


Opium Production Survey by village in Nimroz province, 1995.

\begin{tabular}{|c|c|c|c|c|c|c|c|c|}
\hline \multirow{3}{*}{ District } & \multirow{3}{*}{$\begin{array}{c}\text { Village } \\
\text { Code }\end{array}$} & \multirow{3}{*}{ Area/ Main Village } & \multirow{3}{*}{ Village } & \multirow{3}{*}{$\begin{array}{c}\text { Total } \\
\text { Cultivated } \\
\text { Land }\left(\text { Jerib }^{*}\right) \\
\end{array}$} & \multirow{2}{*}{\multicolumn{2}{|c|}{$\begin{array}{c}\text { Land Under } \\
\text { Poppy Cultivation }\end{array}$}} & \multirow{3}{*}{$\begin{array}{c}\text { Wet Opium }{ }^{* 2} \\
\text { Yield } \\
(\mathrm{kg} / \mathrm{jr} .)\end{array}$} & \multirow{3}{*}{$\begin{array}{c}\text { Wet Opium } \\
\text { Production } \\
(\mathrm{kg}) \\
\end{array}$} \\
\hline & & & & & & & & \\
\hline & & & & & (jerib) & $(\%)$ & & \\
\hline \multicolumn{9}{|c|}{ Province: Nimroz } \\
\hline KANG & 1155 & ABDUL BAQI & ABDUL BAQI & 0 & 0 & 0 & 0 & 0 \\
\hline KANG & 104 & ABDUL QADER & ABDUL QADER & 0 & 0 & 0 & 0 & 0 \\
\hline KANG & 99 & ABDUL RASHID & ABDUL RASHID & 0 & 0 & 0 & 0 & . \\
\hline KANG & 121 & AQA MOHAMMAD & AQA MOHAMMAD & 0 & 0 & 0 & 0 & 0 \\
\hline KANG & 113 & AZIZULLAH & AZIZULLAH & 0 & 0 & 0 & 0 & 0 \\
\hline KANG & 1164 & BALOCH & BALOCH & 0 & 0 & 0 & 0 & 0 \\
\hline KANG & 1182 & BARICHI & BARICHI & 0 & 0 & 0 & 0 & 0 \\
\hline KANG & 1172 & DARWISH & DARWISH & 0 & 0 & 0 & 0 & 0 \\
\hline KANG & 1162 & GUL MOHD & GUL MOHD & 0 & 0 & 0 & 0 & 0 \\
\hline KANG & 1168 & HABIBULLAH & HABIBULLAH & 0 & 0 & 0 & 0 & 0 \\
\hline KANG & 1178 & HAJI AMIR MOHD & HAJI AMIR MOHD & 0 & 0 & 0 & 0 & 0 \\
\hline KANG & 98 & HAJI HAKIM & HAJI HAKIM & 0 & 0 & 0 & 0 & 0 \\
\hline KANG & 105 & HAJI KHODA BAKHSH & HAJI KHODA BAKHSH & 0 & 0 & 0 & 0 & 0 \\
\hline KANG & 1174 & HAJI MOHD ALAM & HAJI MOHD ALAM & 250 & 3 & 1 & 0 & 0 \\
\hline KANG & 1180 & HAJI MOHD RAFIQ & HAJI MOHD RAFIQ & 500 & 2 & 0 & 0 & 0 \\
\hline KANG & 118 & HAJI MOHD SEDIQ & NABI JAN & 200 & 2 & 1 & 0 & 0 \\
\hline KANG & 94 & HAJI MULA SHERDEL & HAJI MULA SHERDEL & 0 & 0 & 0 & 0 & 0 \\
\hline KANG & 1179 & HAJI NAMWAR & HAJI NAMWAR & 0 & 0 & 0 & 0 & 0 \\
\hline KANG & 1175 & HAJI YAR MOHD KHAN & HAJI YAR MOHD KHAN & 100 & 1 & 1 & 0 & 0 \\
\hline KANG & 125 & ISMAIL & ISMAIL & 0 & 0 & 0 & 0 & 0 \\
\hline KANG & 1173 & KANG MOQIM & KANG MOQIM & 0 & 0 & 0 & 0 & 0 \\
\hline KANG & 1165 & KHAIR MOHAMMAD & KHAIR MOHAMMAD & 0 & 0 & 0 & 0 & 0 \\
\hline KANG & 1171 & KHAROT & KHAROT & 0 & 0 & 0 & 0 & 0 \\
\hline KANG & 108 & M. AKBAR & M. AKBAR & 0 & 0 & 0 & 0 & 0 \\
\hline KANG & 1177 & MAGULI & MAGULI & 0 & 0 & 0 & 0 & 0 \\
\hline KANG & 107 & MAHAJER ABAD & MAHAJER ABAD & 0 & 0 & 0 & 0 & 0 \\
\hline KANG & 1168 & MALEM JAHA & MALEM JAHA & 0 & 0 & 0 & 0 & 0 \\
\hline KANG & 1181 & MOHD RASOL ZORI & MOHD RASOL ZORI & 0 & 0 & 0 & 0 & 0 \\
\hline KANG & 1159 & MOLA MIRZA & MOLA MIRZA & 0 & 0 & 0 & 0 & 0 \\
\hline
\end{tabular}


Opium Production Survey by village in Nimroz province, 1995

\begin{tabular}{|c|c|c|c|c|c|c|c|c|}
\hline & Village & & & Total & Land & & Wet Opium * 2 & Wet Opium \\
\hline \multirow[t]{2}{*}{ District } & Code & Area/ Main Village & Village & Cultivated & \multicolumn{2}{|c|}{ Poppy Cultivation } & Yield & Production \\
\hline & & & & Land (Jerib *) & (jerib) & $(\%)$ & $(\mathrm{kg} / \mathrm{jr})$. & $(\mathrm{kg})$ \\
\hline \multicolumn{9}{|c|}{ Province: Nimroz } \\
\hline KANG & 120 & PIR MOHAMMAD SAFAR & PIR MOHAMMAD SAFAR & 0 & 0 & 0 & 0 & 0 \\
\hline KANG & 111 & RAHMATULLAH & RAHMATULLAH & 0 & 0 & 0 & 0 & 0 \\
\hline KANG & 1163 & ROD SHAHI & ROD SHAHI & 0 & 0 & 0 & 0 & 0 \\
\hline KANG & 117 & SAID ZEE & SAID ZEE & 0 & 0 & 0 & 0 & 0 \\
\hline KANG & 1170 & SAMAD KHAN & SAMAD KHAN & 0 & 0 & 0 & 0 & 0 \\
\hline KANG & 1153 & SAYED NOOR & SAYED NOOR & 0 & 0 & 0 & 0 & 0 \\
\hline KANG & 1161 & SHAH GUL & SHAH GUL & 1,600 & 1 & 0 & 0 & 0 \\
\hline KHASH RUD & 76 & BORJ & BORJ & 700 & 28 & 4 & 0 & 0 \\
\hline KHASH RUD & 90 & DASTAK & DASTAK & 1,800 & 35 & 2 & 0 & 0 \\
\hline KHASH RUD & 71 & DEHMAZANG & DEHMAZANG & 700 & 50 & 7 & 0 & 0 \\
\hline KHASH RUD & 78 & DEWALAK & DEWALAK & 700 & 50 & 7 & 0 & 0 \\
\hline KHASH RUD & 79 & GARO & GARO & 2,500 & 80 & 3. & 0 & 0 \\
\hline KHASH RUD & 77 & KESHT & KESHT & 1,000 & 30 & 3 & 0 & 0 \\
\hline KHASH RUD & 88 & KHASH & KHASH & 2,500 & 5 & 0 & 0 & 0 \\
\hline KHASH RUD & 89 & KHONYA & KHONYA & 0 & 0 & 0 & 0 & 0 \\
\hline KHASH RUD & 72 & KHWAJA MARD & KHWAJA MARD & 780 & 48 & 6 & 0 & 0 \\
\hline KHASH RUD & 81 & KOTKAK & KOTKAK & 1,200 & 48 & 4 & 0 & 0 \\
\hline KHASH RUD & 75 & LANDAI & LANDAI & 800 & 36 & 5 & 0 & 0 \\
\hline KHASH RUD & 91 & LOKHI & LOKHI & 800 & 12 & 2 & 0 & 0 \\
\hline KHASH RUD & 82 & LOY RAZI AFGHANI & LOY RAZI AFGHANI & 0 & 0 & 0 & 0 & 0 \\
\hline KHASH RUD & 86 & MAZAD QALA NAW & MAZAD QALA NAW & 0 & 0 & 0 & 0 & 0 \\
\hline KHASH RUD & 74 & MONAR & MONAR & 900 & 35 & 4 & 0 & 0 \\
\hline KHASH RUD & 80 & PUSHTA HASAN & PUSHTA HASAN & 1,400 & 50 & 4 & 0 & 0 \\
\hline KHASH RUD & 87 & QALA NAW & QALA NAW & 0 & 0 & 0 & 0 & 0 \\
\hline KHASH RUD & 70 & RAKIN & RAKIN & 800 & 60 & 8 & 0 & 0 \\
\hline KHASH RUD & 83 & RAZI BALOSI & RAZI BALOSI & 350 & 0 & 0 & 0 & 0 \\
\hline KHASH RUD & 85 & SHISAW & SHISAW & 0 & 0 & 0 & 0 & 0 \\
\hline KHASH RUD & 84 & SHORI & SHORI & 0 & 0 & 0 & 0 & 0 \\
\hline KHASH RUD & 73 & SITOY & SITOY & 380 & 20 & 5 & 0 & 0 \\
\hline
\end{tabular}


Opium Production Survey by village in Oruzgan province, 1995.

\begin{tabular}{|c|c|c|c|c|c|c|c|c|}
\hline \multirow{3}{*}{ District } & \multirow{3}{*}{$\begin{array}{l}\text { Village } \\
\text { Code }\end{array}$} & \multirow{3}{*}{ Area/ Main Village } & \multirow{3}{*}{ Village } & \multirow{3}{*}{$\begin{array}{c}\text { Total } \\
\text { Cultivated } \\
{\text { Land }\left(\text { Jerib }^{*}\right)}^{*} \\
\end{array}$} & \multirow{2}{*}{\multicolumn{2}{|c|}{$\begin{array}{c}\text { Land Under } \\
\text { Poppy Cultivation }\end{array}$}} & \multirow{3}{*}{$\begin{array}{c}\text { Wet Opium }{ }^{* 2} \\
\text { Yield } \\
(\mathrm{kg} / \mathrm{jr} .) \\
\end{array}$} & \multirow{3}{*}{$\begin{array}{c}\text { Wet Opium } \\
\text { Production } \\
(\mathrm{kg}) \\
\end{array}$} \\
\hline & & & & & & & & \\
\hline & & & & & (jerib) & $(\%)$ & & \\
\hline \multicolumn{9}{|l|}{ Province: Oruzgan } \\
\hline CHAR CHASHMA & 1722 & SHOORDAG & SHOORDAG & 110 & 58 & 53 & 17 & 986 \\
\hline CHORA & 48 & ABAS ALI & ABAS ALI & 1,010 & 305 & 30 & 12 & 3,660 \\
\hline CHORA & 70 & ALEKOZO KELAI & ALEKOZO KELAI & 100 & 15 & 15 & 11 & 165 \\
\hline CHORA & 64 & BADAMAK & BADAMAK & 37 & 4 & 11 & 10 & 40 \\
\hline CHORA & 59 & BALAGH & BALAGH & 45 & 12 & 27 & 11 & 132 \\
\hline CHORA & 71 & BARIKOB & BARIKOB & 30 & 3 & 10 & 9 & 27 \\
\hline CHORA & 63 & BARQOLI & BARQOLI & 39 & 7 & 18 & 11 & 77 \\
\hline CHORA & 69 & BAYANZOKELAI & BAYANZOKELAI & 54 & 12 & 22 & 11 & 132 \\
\hline CHORA & 26 & BOCHI & BOCHI & 0 & 0 & 0 & 0 & 0 \\
\hline CHORA & 33 & BUME PATAN & BUME PATAN & 50 & 5 & 10 & 9 & 45 \\
\hline CHORA & 91 & BURGANA & BURGANA & 140 & 30 & 21 & 11 & 330 \\
\hline CHORA & 89 & CHAR PELING & CHAR PELING & 85 & 17 & 20 & 10 & 170 \\
\hline CHORA & 68 & CHINA KALAY & CHINA KALAY & 30 & 2 & 7 & 9 & 18 \\
\hline CHORA & 25 & CHINARTO & CHINARTO & 850 & 17 & 2 & 8 & 136 \\
\hline CHORA & 66 & DAB KALAY & DAB KALAY & 95 & 18 & 19 & 11 & 198 \\
\hline CHORA & 34 & DALQANA & DALQANA BALA & 75 & 4 & 5 & 10 & 40 \\
\hline CHORA & 56 & DEWALAK & DEWALAK & 0 & 0 & 0 & 0 & 0 \\
\hline CHORA & 65 & FAISAL & DEBA KALAY & 400 & 60 & 15 & 11 & 660 \\
\hline CHORA & 52 & GHOYANA & GHOYANA & 125 & 25 & 20 & 11 & 275 \\
\hline CHORA & 43 & GURJIZAI NAWA & GURJIZAI NAWA & 200 & 48 & 24 & 12 & 576 \\
\hline CHORA & 30 & KARAK TOGHI & KARAK TOGHI & 85 & 12 & 14 & 10 & 120 \\
\hline CHORA & 32 & KARMISTAN & KARMISTAN & 640 & 90 & 14 & 10 & 900 \\
\hline CHORA & 54 & KAROLAGH KARIZ & KAROLAGH KARIZ & 40 & 8 & 20 & 11 & 88 \\
\hline CHORA & 53 & KESHATA KARIZ & KESHATA KARIZ & 90 & 20 & 22 & 10 & 200 \\
\hline CHORA & 49 & KHALILEA & KHALILEA & 58 & 14 & 24 & 10 & 140 \\
\hline CHORA & 61 & KHOSHKSHARAK & KHOSHKSHARAK & 48 & 7 & 15 & 12 & 84 \\
\hline CHORA & 51 & KHOWAJA KHADIR & KHOWAJA KHADIR & 130 & 22 & 17 & 10 & 220 \\
\hline CHORA & 55 & KOCHAGIR KARIZ & KOCHAGIR KARIZ & 27 & 4 & 15 & 10 & 40 \\
\hline CHORA & 27 & KOHNA QALA & KOHNA QALA & 0 & 0 & 0 & 0 & 0 \\
\hline
\end{tabular}

${ }^{*}$ Jerib (jr.) $=1 / 5$ ha

* 2 Dry Opium $=$ Wet Opium - $30 \%$ moisture
Page 1142

Source: UNDCP Opium Production Survey, 199 File: URZ3.XLS - 07/08/95 
Opium Production Survey by village in Oruzgan province, 1995.

\begin{tabular}{|c|c|c|c|c|c|c|c|c|}
\hline & Village & \multirow{3}{*}{ Area/ Main Village } & \multirow{3}{*}{ Village } & \multirow{3}{*}{$\begin{array}{c}\text { Total } \\
\text { Cultivated } \\
\text { Land }\left(\text { Jerib }{ }^{*}\right) \\
\end{array}$} & \multirow{2}{*}{\multicolumn{2}{|c|}{$\begin{array}{c}\text { Land Under } \\
\text { Poppy Cultivation }\end{array}$}} & \multirow{3}{*}{$\begin{array}{c}\text { Wet Opium }{ }^{* 2} \\
\text { Yield } \\
(\mathrm{kg} / \mathrm{jr} .)\end{array}$} & \multirow{3}{*}{$\begin{array}{c}\text { Wet Opium } \\
\text { Production } \\
(\mathrm{kg})\end{array}$} \\
\hline \multirow[t]{2}{*}{ District } & Code & & & & & & & \\
\hline & & & & & (jerib) & $(\%)$ & & \\
\hline \multicolumn{9}{|c|}{ Province: Oruzgan } \\
\hline CHORA & 35 & KUCHKEL & KUCHKEL & 65 & 15 & 23 & 10 & 150 \\
\hline CHORA & 31 & LANDI WALEH & LANDI WALEH & 54 & 11 & 20 & 9 & 99 \\
\hline CHORA & 41 & MANIGHAR & MANIGHAR & 200 & 15 & 8 & 10 & 150 \\
\hline CHORA & 29 & MASUD KALAY & MASUD KALAY & 60 & 9 & 15 & 10 & 90 \\
\hline CHORA & 38 & NAWAB KHAN & NAWAB KHAN & 65 & 10 & 15 & 10 & 100 \\
\hline CHORA & 45 & NIAZI & NIAZI & 810 & 227 & 28 & 12 & 2,724 \\
\hline CHORA & 40 & NURI & NURI & 90 & 10 & 11 & 9 & 90 \\
\hline CHORA & 47 & QALA NOW & QALA NOW & 930 & 290 & 31 & 12 & 3,480 \\
\hline CHORA & 46 & QALA QALA & QALA QALA & 1,050 & 304 & 29 & 12 & 3,648 \\
\hline CHORA & 44 & QALA RAGH & QALA RAGH & 700 & 210 & 30 & 13 & 2,730 \\
\hline CHORA & 57 & QALA-E-SORKH & QALA-E-SORKH & 54 & 9 & 17 & 11 & 99 \\
\hline CHORA & 37 & SARAB & SARAB & 480 & 90 & 19 & 11 & 990 \\
\hline CHORA & 60 & SARKI NAWA & SARKI NAWA & 100 & 20 & 20 & 11 & 220 \\
\hline CHORA & 36 & SHAH GHASI & SHAH GHASI & 70 & 18 & 26 & 11 & 198 \\
\hline CHORA & 58 & SHINA & SHINA & 24 & 5 & 21 & 11 & 55 \\
\hline CHORA & 28 & SHIPLIGH & SHIPLIGH & 150 & 20 & 13 & 10 & 200 \\
\hline CHORA & 42 & TALABA & TALABA & 55 & 5 & 9 & 10 & 50 \\
\hline CHORA & 67 & TANGY & TANGY & 27 & 3 & 11 & 9 & 27 \\
\hline CHORA & 90 & TANO KARIZ & TANO KARIZ & 110 & 21 & 19 & 10 & 210 \\
\hline CHORA & 62 & WALANG & WALANG & 36 & 6 & 17 & 11 & 66 \\
\hline CHORA & 86 & WALGAI KARIZ & WALGAI KARIZ & 200 & 39 & 20 & 12 & 468 \\
\hline CHORA & 39 & ZARDEGY & ZARDEGY & 150 & 20 & 13 & 11 & 220 \\
\hline DEH RAWUD & 56 & ABDULRAZAQ MASJ & ABDULRAZAQ MASJ & 120 & 32 & 27 & 6 & 192 \\
\hline DEH RAWUD & 75 & ADUZI KARIZ & ADUZI KARIZ & 430 & 50 & 12 & 7 & 350 \\
\hline DEH RAWUD & 80 & AGAR & JETKA & 370 & 80 & 22 & 6 & 480 \\
\hline DEH RAWUD & 78 & ALARI & MASHEANZO KAREZ & 1,350 & 220 & 16 & 6 & 1,320 \\
\hline DEH RAWUD & 12 & AMRANZI & HJ.ALADAD.MASJI & 160 & 45 & 28 & 7 & 315 \\
\hline DEH RAWUD & 39 & ANARJOY & WAZIR AGA .M & 125 & 20 & 16 & 6 & 120 \\
\hline DEH RAWUD & 15 & BABOZI & BABOZI MASJID & 200 & 35 & 18 & 6 & 210 \\
\hline
\end{tabular}


Opium Production Survey by village in Oruzgan province, 1995.

\begin{tabular}{|c|c|c|c|c|c|c|c|c|}
\hline \multirow{3}{*}{ District } & \multirow{3}{*}{$\begin{array}{c}\text { Village } \\
\text { Code }\end{array}$} & \multirow{3}{*}{ Area/ Main Village } & \multirow{3}{*}{ Village } & \multirow{3}{*}{\begin{tabular}{c|} 
Total \\
Cultivated \\
Land (Jerib $\left.{ }^{*}\right)$ \\
\end{tabular}} & \multirow{2}{*}{\multicolumn{2}{|c|}{$\begin{array}{c}\text { Land Under } \\
\text { Poppy Cultivation }\end{array}$}} & \multirow{3}{*}{$\begin{array}{c}\text { Wet Opium * } 2 \\
\text { Yield } \\
(\mathrm{kg} / \mathrm{jr} .)\end{array}$} & \multirow{3}{*}{$\begin{array}{c}\text { Wet Opium } \\
\text { Production } \\
(\mathrm{kg})\end{array}$} \\
\hline & & & & & & & & \\
\hline & & & & & (jerib) & $(\%)$ & & \\
\hline \multicolumn{9}{|c|}{ Province: Oruzgan } \\
\hline DEH RAWUD & 76 & BAZ GIR & BAHGAL & 1,400 & 180 & 13 & 6 & 1,080 \\
\hline DEH RAWUD & 211 & BILA & KHAN GUL MASJID & 400 & 60 & 15 & 7 & 420 \\
\hline DEH RAWUD & 60 & CHALGA & KARIZ KALAY & 400 & 64 & 16 & 6 & 384 \\
\hline DEH RAWUD & 52 & CHARAKIAN & HAJI MODRAD .M & 240 & 25 & 10 & 6 & 150 \\
\hline DEH RAWUD & 199 & CHARKH & HJ.A.WAHAB MASJ & 220 & 45 & 20 & 8 & 360 \\
\hline DEH RAWUD & 222 & CHARKH & KHODOY RAHM.MAS & 300 & 37 & 12 & 7 & 259 \\
\hline DEH RAWUD & 200 & CHARKH & KHUDAYDAD MASJI & 350 & 48 & 14 & 8 & 384 \\
\hline DEH RAWUD & 48 & CHENAR & HABIB AGA .M & 75 & 15 & 20 & 7 & 105 \\
\hline DEH RAWUD & 24 & CHENEYAN & KANDA & 1,200 & 300 & 25 & 7 & 2,100 \\
\hline DEH RAWUD & 84 & CHERAGH & CHERAGH & 80 & 20 & 25 & 6 & 120 \\
\hline DEH RAWUD & 47 & DARAZJOY & TUTAK & 57 & 10 & 18 & 6 & 60 \\
\hline DEH RAWUD & 54 & DASTAGEIR .M & DASTAGEIR .M & 75 & 10 & 13 & 6 & 60 \\
\hline DEH RAWUD & 38 & DERAYAT & NOORZAI.M & 85 & 11 & 13 & 6 & 66 \\
\hline DEH RAWUD & 36 & DERO & HAJI TOR AGA & 100 & 15 & 15 & 6 & 90 \\
\hline DEH RAWUD & 35 & DEW SANG & NOOR AGA MASJID & 300 & 70 & 23 & 6 & 420 \\
\hline DEH RAWUD & 32 & DEWANA WARKH & HAJI NADER AGA MASJD & 183 & 23 & 13 & 7 & 161 \\
\hline DEH RAWUD & 31 & DEWANA WARKH & SAYD AMIR LALA & 230 & 35 & 15 & 6 & 210 \\
\hline DEH RAWUD & 33 & DEWANA WARKH & SULTAN MASJID & 100 & 13 & 13 & 7 & 91 \\
\hline DEH RAWUD & 61 & DIZAK BALA & REGAK & 700 & 90 & 13 & 6 & 540 \\
\hline DEH RAWUD & 63 & DIZAK-E BALA & GERGAN & 400 & 45 & 11 & 6 & 270 \\
\hline DEH RAWUD & 59 & DIZAK-E PAYAN & BAHRAM KARIZ & 50 & 15 & 30 & 6 & 90 \\
\hline DEH RAWUD & 62 & DIZAK-E PAYAN & BAZGIR & 1,000 & 220 & 22 & 7 & 1,540 \\
\hline DEH RAWUD & 69 & DIZAK-E PAYAN & GANDOM SHAHR & 650 & 100 & 15 & 6 & 600 \\
\hline DEH RAWUD & 65 & DIZAK-E PAYAN & GARY BAZAR & 350 & 36 & 10 & 6 & 216 \\
\hline DEH RAWUD & 70 & DIZAK-E PAYAN & KALAY ZANBORY & 1,750 & 350 & 20 & 6 & 2,100 \\
\hline DEH RAWUD & 71 & DIZAK-E PAYAN & SACHO KAREZ & 700 & 130 & 19 & 7 & 910 \\
\hline DEH RAWUD & 64 & DIZAK-E PAYAN & SHENGAN & 230 & 34 & 15 & 7 & 238 \\
\hline DEH RAWUD & 74 & DIZAK-E PAYAN & SHORJI & 650 & 185 & 28 & 6 & 1,110 \\
\hline DEH RAWUD & 50 & DURJI & MOHD.LALA .M & 235 & 27 & 11 & 6 & 162 \\
\hline
\end{tabular}

* Jerib (jr.) $=1 / 5$ ha

* 2 Dry Opium $=$ Wet Opium $-30 \%$ moisture 
Opium Production Survey by village in Oruzgan province, 1995.

\begin{tabular}{|c|c|c|c|c|c|c|c|c|}
\hline \multirow{3}{*}{ District } & \multirow{3}{*}{$\begin{array}{c}\text { Village } \\
\text { Code }\end{array}$} & \multirow{3}{*}{ Area/ Main Village } & \multirow{3}{*}{ Village } & \multirow{3}{*}{$\begin{array}{c}\text { Total } \\
\text { Cultivated } \\
\left.\text { Land (Jerib }{ }^{*}\right)\end{array}$} & \multirow{2}{*}{\multicolumn{2}{|c|}{$\begin{array}{c}\text { Land Under } \\
\text { Poppy Cultivation }\end{array}$}} & \multirow{3}{*}{$\begin{array}{c}\text { Wet Opium }^{* 2} \\
\text { Yield } \\
(\mathrm{kg} / \mathrm{jr} .)\end{array}$} & \multirow{3}{*}{$\begin{array}{c}\text { Wet Opium } \\
\text { Production } \\
(\mathrm{kg}) \\
\end{array}$} \\
\hline & & & & & & & & \\
\hline & & & & & (jerib) & $(\%)$ & & \\
\hline \multicolumn{9}{|c|}{ Province: Oruzgan } \\
\hline DEH RAWUD & 42 & ESHAQZI & TOKHI & 50 & 10 & 20 & 7 & 70 \\
\hline DEH RAWUD & 44 & GANGAL & GAZAK & 80 & 12 & 15 & 6 & 72 \\
\hline DEH RAWUD & 27 & GARA & HJ.MESKIEN MASJ & 110 & 20 & 18 & 6 & 120 \\
\hline DEH RAWUD & 57 & GARGAK & GAZ KHALIFA MAS & 550 & 100 & 18 & 7 & 700 \\
\hline DEH RAWUD & 34 & GARI & MALEK BAIDAD M & 130 & 25 & 19 & 6 & 150 \\
\hline DEH RAWUD & 7 & GHARAM & HJ.MALANG.M & 62 & 14 & 23 & 6 & 84 \\
\hline DEH RAWUD & 53 & HAJI QAZI .M & HAJI QAZI .M & 140 & 30 & 21 & 6 & 180 \\
\hline DEH RAWUD & 166 & HJ.AGHA MASJID & HJ.AGHA MASJID & 350 & 66 & 19 & 7 & 462 \\
\hline DEH RAWUD & 14 & HJ.JANDAD MASJID & HJ.JANDAD MASJID & 157 & 34 & 22 & 6 & 204 \\
\hline DEH RAWUD & 10 & HJ.SAFDER MASJID & HJ.SAFDER MASJID & 74 & 10 & 14 & 6 & 60 \\
\hline DEH RAWUD & 30 & HJ.WAKIL MASJID & HJ.WAKIL MASJID & 180 & 25 & 14 & 6 & 150 \\
\hline DEH RAWUD & 82 & JAGHDAR KAREZ & LALA JAN KAREZ & 1,650 & 120 & 7 & 6 & 720 \\
\hline DEH RAWUD & 23 & JANDA & SADOZAI MASJID & 1,120 & 250 & 22 & 7 & 1,750 \\
\hline DEH RAWUD & 43 & JOY NAW & REGAK & 64 & 15 & 23 & 7 & 105 \\
\hline DEH RAWUD & 41 & KAKARAK & ABDUL RAHMAN.M & 170 & 25 & 15 & 6 & 150 \\
\hline DEH RAWUD & 4 & KAKARAK BALA & AKHUNDZADA MASJ & 250 & 45 & 18 & 8 & 360 \\
\hline DEH RAWUD & 13 & KAKRAK & HJ.MULA MASJID & 125 & 26 & 21 & 6 & 156 \\
\hline DEH RAWUD & 2 & KAKRAK BALA & SHAGHA KHEL & 350 & 65 & 19 & 7 & 455 \\
\hline DEH RAWUD & 5 & KAKRAK-E PAYAN & SHAGHA KHEL & 230 & 45 & 20 & 8 & 360 \\
\hline DEH RAWUD & 81 & KAM COCH & NAZIR KAREZ & 530 & 90 & 17 & 7 & 630 \\
\hline DEH RAWUD & 73 & KANAI SANG SURA & KANAI SANG SURA & 350 & 80 & 23 & 7 & 560 \\
\hline DEH RAWUD & 177 & KAZWALA & MULA.M.AKHUND.M & 170 & 37. & 22 & 6 & 222 \\
\hline DEH RAWUD & 51 & KHOGYANO MASJID & KHOGYANO MASJID & 135 & 25 & 19 & 6 & 150 \\
\hline DEH RAWUD & 77 & KOWRAK & KOWRAK & 180 & 35 & 19 & 6 & 210 \\
\hline DEH RAWUD & 28 & MAKTAB & BAQI LALA MASJID & 80 & 10 & 13 & 6 & 60 \\
\hline DEH RAWUD & 26 & MAKTAB & LOI AKHONZADA MASJID & 170 & 33 & 19 & 8 & 264 \\
\hline DEH RAWUD & 188 & MAKTAB & MALEK DASTAGIR & 80 & 10 & 13 & 8 & 80 \\
\hline DEH RAWUD & 25 & MAKTAB & MAWLADAD MASJID & 230 & 43 & 19 & 7 & 301 \\
\hline DEH RAWUD & 83 & MATINA & MATINA & 180 & 40 & 22 & 7 & 260 \\
\hline
\end{tabular}


Opium Production Survey by village in Oruzgan province, 1995.

\begin{tabular}{|c|c|c|c|c|c|c|c|c|}
\hline \multirow{3}{*}{ District } & \multirow{3}{*}{$\begin{array}{l}\text { Village } \\
\text { Code }\end{array}$} & \multirow{3}{*}{ Area/ Main Village } & \multirow{3}{*}{ Village } & \multirow{3}{*}{$\begin{array}{c}\text { Total } \\
\text { Cultivated } \\
\text { Land }\left(\text { Jerib }^{*}\right) \\
\end{array}$} & \multirow{2}{*}{\multicolumn{2}{|c|}{$\begin{array}{c}\text { Land Under } \\
\text { Poppy Cultivation }\end{array}$}} & \multirow{3}{*}{$\begin{array}{c}\text { Wet Opium }{ }^{* 2} \\
\text { Yield } \\
(\mathrm{kg} / \mathrm{jr} .) \\
\end{array}$} & \multirow{3}{*}{$\begin{array}{c}\text { Wet Opium } \\
\text { Production } \\
(\mathrm{kg}) \\
\end{array}$} \\
\hline & & & & & & & & \\
\hline & & & & & (jerib) & $(\%)$ & & \\
\hline \multicolumn{9}{|c|}{ Province: Oruzgan } \\
\hline DEH RAWUD & 11 & MIAMDO & DAHAN AB & 87 & 14 & 16 & 6 & 84 \\
\hline DEH RAWUD & 45 & MORCH & TANDOR & 60 & 10 & 17 & 6 & 60 \\
\hline DEH RAWUD & 55 & MULA RASHID.M & MULA RASHID.M & 85 & 11 & 13 & 7 & 77 \\
\hline DEH RAWUD & 72 & NAWA & GANGAL & 700 & 75 & 11 & 6 & 450 \\
\hline DEH RAWUD & 66 & PAY DIZAK & AMRANZAI & 30 & 45 & 150 & 7 & 315 \\
\hline DEH RAWUD & 68 & PAY DIZAK & CHEGHAK KAREZ & 180 & 26 & 14 & 6 & 156 \\
\hline DEH RAWUD & 67 & PAY DIZAK & OWDAM & 200 & 30 & 15 & 6 & 180 \\
\hline DEH RAWUD & 6 & PIR AKAH MASJED & PIR AKAH MASJED & 53 & 9 & 17 & 6 & 54 \\
\hline DEH RAWUD & 40 & PUTEA & HAJI ALADAD.M & 135 & 22 & 16 & 6 & 132 \\
\hline DEH RAWUD & 29 & SAHIB AGHA & MALANG.MASJID & 250 & 34 & 14 & 7 & 238 \\
\hline DEH RAWUD & 67 & SAKIVA KARIZ & SAKIVA KARIZ & 19 & 4 & 21 & 12 & 48 \\
\hline DEH RAWUD & 1 & SAR KAKRAK & AKHUNDZADA MASJED & 250 & 45 & 18 & 8 & 360 \\
\hline DEH RAWUD & 3 & SAR KAKRAK & SAR KAKRAK & 300 & 70 & 23 & 7 & 490 \\
\hline DEH RAWUD & 58 & SARAW & SHAGHA MASJID & 450 & 75 & 17 & 7 & 488 \\
\hline DEH RAWUD & 79 & SAWABAT KAREZ & CHENA KAREZ & 520 & 80 & 15 & 7 & 520 \\
\hline DEH RAWUD & 46 & SHOKHAK & SADOZI.M & 60 & 4 & 7 & 7 & 28 \\
\hline DEH RAWUD & 8 & SHUKARYANO MASJ & SHUKARYANO MASJ & 47 & 8 & 17 & 6 & 48 \\
\hline DEH RAWUD & 9 & YAKHGAN & GUL AGHA MASJID & 84 & 13 & 15 & 6 & 78 \\
\hline DEH RAWUD & 49 & ZAYARAT & HAJI BADAR .M & 180 & 37 & 21 & 6 & 222 \\
\hline DEH RAWUD & 37 & ZYARAT & PIR JAN AGA. M & 270 & 45 & 17 & 6 & 270 \\
\hline GEZAB & 11 & ADA NEKO ZAI & ALLAH DAD ZAI & 300 & 0 & 0 & 0 & 0 \\
\hline GEZAB & 7 & AOMAR FAROOQ.M & AOMAR FAROOQ.M & 210 & 6 & 3 & 6 & 36 \\
\hline GEZAB & 6 & CHASHA & NOOR ZAI MASJID & 320 & 9 & 3 & 5 & 45 \\
\hline GEZAB & 8 & CHENA & NAWAB MASJID & 230 & 8 & 3 & 5 & 40 \\
\hline GEZAB & 5 & DAHAN AB & NOOR ZI MASJID & 220 & 7 & 3 & 7 & 49 \\
\hline GEZAB & 12 & DASHT MALIZI & CHAR BAGH & 85 & 0 & 0 & 0 & 0 \\
\hline GEZAB & 14 & GHAR SHIN KELAY & GHAR SHIN KELAY & 300 & 0 & 0 & 0 & 0 \\
\hline GEZAB & 20 & HAJI QASIM KALY & HAJI QASIM KALY & 85 & 0 & 0 & 0 & 0 \\
\hline GEZAB & 18 & HAJI TAJ M.KELY & HAJI TAJ M.KELY & 340 & 0 & 0 & 0 & 0 \\
\hline
\end{tabular}

* Jerib (jr.) $=1 / 5$ ha

* 2 Dry Opium $=$ Wet Opium $-30 \%$ moisture 
Opium Production Survey by village in Oruzgan province, 1995.

\begin{tabular}{|c|c|c|c|c|c|c|c|c|}
\hline & \multirow{3}{*}{$\begin{array}{l}\text { Codle } \\
\text { Code }\end{array}$} & \multirow{3}{*}{ Area/ Main Village } & \multirow{3}{*}{ Village } & \multirow{3}{*}{$\begin{array}{c}\text { Total } \\
\text { Cultivated } \\
{\text { Land }\left(\text { Jerib }^{*}\right)}^{*} \\
\end{array}$} & \multirow{2}{*}{\multicolumn{2}{|c|}{$\begin{array}{c}\text { Land Under } \\
\text { Poppy Cultivation }\end{array}$}} & \multirow{3}{*}{$\begin{array}{c}\text { Wet }^{\text {Opium }}{ }^{* 2} \\
\text { Yield } \\
(\mathrm{kg} / \mathrm{jr} .)\end{array}$} & \multirow{3}{*}{$\begin{array}{c}\text { Wet Opium } \\
\text { Production } \\
(\mathrm{kg}) \\
\end{array}$} \\
\hline \multirow[t]{2}{*}{ District } & & & & & & & & \\
\hline & & & & & (jerib) & $(\%)$ & & \\
\hline \multicolumn{9}{|l|}{ Province: Oruzgan } \\
\hline GEZAB & 16 & HELMAND KELAY & DASHT KELAY & 250 & 0 & 0 & 0 & 0 \\
\hline GEZAB & 13 & JALIZI & JALIZI & 400 & 0 & 0 & 0 & 0 \\
\hline GEZAB & 15 & KHACHOZI & NOOR ZAI KELAY & 570 & 0 & 0 & 0 & 0 \\
\hline GEZAB & 22 & NOOR ZAI KELAY & MAKA ZAI & 0 & 0 & 0 & 0 & 0 \\
\hline GEZAB & 19 & NOORZAI KELAY & NOORZAI KELAY & 370 & 0 & 0 & 0 & 0 \\
\hline GEZAB & 4 & PAY KALAJ & ABDUL WAHID KHAN.M & 850 & 9 & 1 & 7 & 63 \\
\hline GEZAB & 9 & QUDDUS KELAY & AKBAR KHAN MASJID & 200 & 9 & 5 & 7 & 63 \\
\hline GEZAB & 21 & RABAT KELAY & RABAT KELAY & 400 & 0 & 0 & 0 & 0 \\
\hline GEZAB & 10 & SANG SHEKH & PALTAN KELAY & 300 & 0 & 0 & 0 & 0 \\
\hline GEZAB & 3 & SAR KALAJ & BOSTANZI & 600 & 9 & 2 & 7 & 63 \\
\hline GEZAB & 17 & SAR KALY & ANOK KALY & 590 & 0 & 0 & 0 & 0 \\
\hline GEZAB & 1 & SAYEADANO KELAY & HAJI FIROZ.M & 750 & 10 & 1 & 7 & 70 \\
\hline GEZAB & 2 & SHDI ZI & WATA & 800 & 12 & 2 & 7 & 84 \\
\hline KHAS ORUZGAN & 22 & ANA KALAY & ANA KALAY & 0 & 0 & 0 & 0 & 0 \\
\hline KHAS ORUZGAN & 14 & CHAKA JOW & CHAKA JOW & 0 & 0 & 0 & 0 & 0 \\
\hline KHAS ORUZGAN & 15 & FEROZAI & FEROZAI & 0 & 0 & 0 & 0 & 0 \\
\hline KHAS ORUZGAN & 23 & GARMABE & GARMABE & 0 & 0 & 0 & 0 & 0 \\
\hline KHAS ORUZGAN & 15 & HAJI KHUDYDAD & HAJI KHUDYDAD & 0 & 0 & 0 & 0 & 0 \\
\hline KHAS ORUZGAN & 21 & KAKHTAWA & KAKHTAWA & 0 & 0 & 0 & 0 & 0 \\
\hline KHAS ORUZGAN & 50 & KHOSHK HADIR & KHOSHK HADIR & 124 & 20 & 16 & 11 & 220 \\
\hline KHAS ORUZGAN & 17 & MALIK ALI MOHD & MALIK ALI MOHD & 0 & 0 & 0 & 0 & 0 \\
\hline KHAS ORUZGAN & 16 & MOHD KABIR KHAN & MOHD KABIR KHAN & 0 & 0 & 0 & 0 & 0 \\
\hline KHAS ORUZGAN & 19 & SALEH ZAI & SALEH ZAI & 0 & 0 & 0 & 0 & 0 \\
\hline KHAS ORUZGAN & 20 & SHIEKHAN & SHIEKHAN & 0 & 0 & 0 & 0 & 0 \\
\hline KHAS ORUZGAN & 18 & TOSHAKAN & TOSHAKAN & 0 & 0 & 0 & 0 & 0 \\
\hline TIRIN KOT & 85 & ABDUL WAHID KAREZ & ABDUL WAHID KAREZ & 75 & 0 & 0 & 0 & 0 \\
\hline TIRIN KOT & 81 & ABEBARDA & ABEBARDA & 450 & 110 & 24 & 13 & 1,430 \\
\hline TIRIN KOT & 32 & ALI ZAHID & ALI ZAHID & 220 & 40 & 18 & 12 & 480 \\
\hline TIRIN KOT & 34 & AWARI & AWARI & 180 & 30 & 17 & 11 & 330 \\
\hline
\end{tabular}

* Jerib (jr.) $=1 / 5$ ha

${ }^{*}$ Dry Opium $=$ Wet Opium $-30 \%$ moisture
Page 6147
Source: UNDCP Opium Production Survey, 1995 File: URZ3.XLS - 07/08/95 
Opium Production Survey by village in Oruzgan province, 1995.

\begin{tabular}{|c|c|c|c|c|c|c|c|c|}
\hline \multirow{3}{*}{ District } & \multirow{3}{*}{\begin{tabular}{|c|} 
Village \\
Code \\
\end{tabular}} & \multirow{3}{*}{ Area/ Main Village } & \multirow{3}{*}{ Village } & \multirow{3}{*}{$\begin{array}{c}\text { Total } \\
\text { Cultivated } \\
\text { Land }\left(\text { Jerib }{ }^{*}\right) \\
\end{array}$} & \multirow{2}{*}{\multicolumn{2}{|c|}{$\begin{array}{c}\text { Land Under } \\
\text { Poppy Cultivation }\end{array}$}} & \multirow{3}{*}{$\begin{array}{c}\text { Wet Opium }^{* 2} \\
\text { Yield } \\
(\mathrm{kg} / \mathrm{jr} .) \\
\end{array}$} & \multirow{3}{*}{$\begin{array}{c}\text { Wet Opium } \\
\text { Production } \\
(\mathrm{kg})\end{array}$} \\
\hline & & & & & & & & \\
\hline & & & & & (jerib) & $(\%)$ & & \\
\hline \multicolumn{9}{|c|}{ Province: Oruzgan } \\
\hline TIRIN KOT & 57 & AWLIYA KARIZ & AWLIYA KARIZ & 10 & 2 & 20. & 11 & 22 \\
\hline TIRIN KOT & 80 & BABUR QULAY & BABUR QULAY & 55 & 15 & 27 & 11 & 165 \\
\hline TIRIN KOT & 66 & BAGHIR KARIZ & BAGHIR KARIZ & 20 & 4 & 20 & 12 & 48 \\
\hline TIRIN KOT & 65 & BAGHNI KARIZ & BAGHNI KARIZ & 28 & 6 & 21 & 12 & 72 \\
\hline TIRIN KOT & 25 & BALAGH & BALAGH & 1,000 & 270 & 27 & 13 & 3,510 \\
\hline TIRIN KOT & 21 & BALAGH & BOKA KAREZ & 12 & 4 & 33 & 12 & 48 \\
\hline TIRIN KOT & 31 & BALOSI & BALOSI & 200 & 24 & 12 & 12 & 288 \\
\hline TIRIN KOT & 68 & BIDUK KARIZ & BIDUK KARIZ & 80 & 11 & 14 & 14 & 154 \\
\hline TIRIN KOT & 91 & BURGANA & BURGANA & 30 & 11 & 37 & 9 & 99 \\
\hline TIRIN KOT & 55 & CAHRMGAR & CHAMARK KARIZ & 160 & 27 & 17 & 11 & 297 \\
\hline TIRIN KOT & 1 & CENTER OF TRINKOT & TORI & 4,000 & 1,100 & 28 & 14 & 15,400 \\
\hline TIRIN KOT & 89 & CHAR PALANG & CHAR PALANG & 85 & 17 & 20 & 10 & 170 \\
\hline TIRIN KOT & 54 & CHARMGAR & SAFAR KARIZ & 22 & 6 & 27 & 11 & 66 \\
\hline TIRIN KOT & 44 & CHESHMA MIRAN & CHESHMA MIRAN & 40 & 11 & 28 & 13 & 143 \\
\hline TIRIN KOT & 3 & CHINA KAREZ & CHINA KAREZ & 110 & 28 & 25 & 10 & 280 \\
\hline TIRIN KOT & 86 & CHINARAK & N.2 & 70 & 15 & 21 & 11 & 165 \\
\hline TIRIN KOT & 87 & CHINARAK & NO.1 & 140 & 30 & 21 & 10 & 300 \\
\hline TIRIN KOT & 56 & CHINARK KARIZ & KARIZ NOW & 25 & 6 & 24 & 12 & 72 \\
\hline TIRIN KOT & 8 & DAM KHORAK & DAM KHORAK & 65 & 17 & 26 & 11 & 187 \\
\hline TIRIN KOT & 94 & DARAZ JOY & DARAZ JOY & 80 & 20 & 25 & 11 & 220 \\
\hline TIRIN KOT & 41 & DEH JAZ BARAKZAI & DEH JAZ BARAKZAI & 310 & 80 & 26 & 12 & 960 \\
\hline TIRIN KOT & 47 & DEH JAZ BARAKZI & DEH JAZ BARAKZI & 310 & 80 & 26 & 12 & 960 \\
\hline TIRIN KOT & 39 & DEH JAZ HASSANZAI & DEH JAZ HASSANZAI & 600 & 210 & 35 & 12 & 2,520 \\
\hline TIRIN KOT & 6 & DEHAK LALOBIG & ALIZOY DEHAK & 35 & 12 & 34 & 13 & 156 \\
\hline TIRIN KOT & 7 & DEHAK LALOBIG & DEHAK LALOBIG & 155 & 50 & 32 & 11 & 550 \\
\hline TIRIN KOT & 10 & DEHAK SARKANI & DEHAK SARKANI & 160 & 45 & 28 & 12 & 540 \\
\hline TIRIN KOT & 13 & DEHAK SARKANI & SAHEB ZADA KHEL & 25 & 8 & 32 & 11 & 88 \\
\hline TIRIN KOT & 12 & DEHAK SARKANI & TALA KAREZ & 35 & 12 & 34 & 11 & 132 \\
\hline TIRIN KOT & 27 & DEWALAK & DEWALAK & 170 & 45 & 26 & 13 & 585 \\
\hline
\end{tabular}


Opium Production Survey by village in Oruzgan province, 1995.

\begin{tabular}{|c|c|c|c|c|c|c|c|c|}
\hline \multirow{3}{*}{ District } & \multirow{3}{*}{$\begin{array}{l}\text { Village } \\
\text { Code }\end{array}$} & \multirow{3}{*}{ Area/ Main Village } & \multirow{3}{*}{ Village } & \multirow{3}{*}{$\begin{array}{c}\text { Total } \\
\text { Cultivated } \\
\text { Land }(\text { Jerib } \\
\end{array}$} & \multirow{2}{*}{\multicolumn{2}{|c|}{$\begin{array}{c}\text { Land Under } \\
\text { Poppy Cultivation }\end{array}$}} & \multirow{3}{*}{$\begin{array}{c}\text { Wet Opium *2 } \\
\text { Yield } \\
(\mathrm{kg} / \mathrm{jr} .)\end{array}$} & \multirow{3}{*}{$\begin{array}{c}\text { Wet Opium } \\
\text { Production } \\
(\mathrm{kg})\end{array}$} \\
\hline & & & & & & & & \\
\hline & & & & & (jerib) & $(\%)$ & & \\
\hline \multicolumn{9}{|c|}{ Province: Oruzgan } \\
\hline TIRIN KOT & 96 & DUR GUL KHAN KELAY & DUR GUL KHAN KELAY & 40 & 6 & 15 & 11 & 66 \\
\hline TIRIN KOT & 11 & GANDAB & GANDAB & 100 & 30 & 30 & 12 & 360 \\
\hline TIRIN KOT & 97 & GARMABE & GARMABE & 190 & 40 & 21 & 12 & 480 \\
\hline TIRIN KOT & 15 & GARNI KAREZ & GARNI KAREZ & 60 & 17 & 28 & 11 & 187 \\
\hline TIRIN KOT & 83 & GHANIGY KAREZ & GHANIGY KAREZ & 245 & 42 & 17 & 11 & 462 \\
\hline TIRIN KOT & 4 & GHULAM AKA KAREZ & GHULAM AKA KAREZ & 60 & 10 & 17 & 11 & 110 \\
\hline TIRIN KOT & 35 & HAJI MULA & DEHBA & 50 & 12 & 24 & 12 & 144 \\
\hline TIRIN KOT & 48 & HAZARAGAI & HAZARAGAI & 22 & 6 & 27 & 12 & 72 \\
\hline TIRIN KOT & 77 & HINDOGAG & HINDOGAG & 60 & 14 & 23 & 12 & 168 \\
\hline TIRIN KOT & 93 & HOJRA & HOJRA & 110 & 30 & 27 & 13 & 390 \\
\hline TIRIN KOT & 38 & KAKARAK & KHUSHKHARAB & 130 & 20 & 15 & 12 & 240 \\
\hline TIRIN KOT & 37 & KAKRAK & KAKRAK & 100 & 30 & 30 & 12. & 360 \\
\hline TIRIN KOT & 72 & KALAN KARIZ & KALAN KARIZ & 15 & 5 & 33 & 13 & 65 \\
\hline TIRIN KOT & 22 & KANDA KAREZ & KANDA KAREZ & 230 & 45 & 20 & 12 & 540 \\
\hline TIRIN KOT & 28 & KARAM KAREZ & KARAM KAREZ & 65 & 20 & 31 & 12 & 240 \\
\hline TIRIN KOT & 16 & KHAIR ABAD & KAREZ NAWABAD & 25 & 6 & 24 & 11 & 66 \\
\hline TIRIN KOT & 71 & KHALIZAR KARIZ & KHALIZAR KARIZ & 20 & 5 & 25 & 12 & 60 \\
\hline TIRIN KOT & 79 & KHAR KOL KAREZ & KHAR KOL KAREZ & 15 & 3 & 20 & 12 & 36 \\
\hline TIRIN KOT & 2 & KHARO KAREZ & KHARO KAREZ & 120 & 30 & 25 & 14 & 420 \\
\hline TIRIN KOT & 62 & KHIRABAD & KHIRABAD & 150 & 48 & 32 & 13 & 624 \\
\hline TIRIN KOT & 40 & KHORMA & KHORMA & 440 & 112 & 25 & 13 & 1,456 \\
\hline TIRIN KOT & 69 & KHUSHKHARAB & KHUSHKHARAB & 35 & 7 & 20 & 11 & 77 \\
\hline TIRIN KOT & 29 & KHWAJA AHMAD & KHWAJA AHMAD & 200 & 50 & 25 & 11 & 550 \\
\hline TIRIN KOT & 50 & KOCHKIN & KOCHKIN & 80 & 16 & 20 & 12 & 192 \\
\hline TIRIN KOT & 26 & KOSH KHONJAK & KOSH KHONJAK & 300 & 105 & 35 & 12 & 1,260 \\
\hline TIRIN KOT & 45 & KOT WAL & KOT WAL & 65 & 20 & 31 & 12 & 240 \\
\hline TIRIN KOT & 82 & MALIZAI & MALIZAI & 110 & 40 & 36 & 12 & 480 \\
\hline TIRIN KOT & 73 & MHMOD KARIZ & MHMOD KARIZ & 25 & 3. & 12 & 13 & 39 \\
\hline TIRIN KOT & 52 & MIRABAD NAWA & MIRABAD & 700 & 115 & 16 & 13 & 1,495 \\
\hline
\end{tabular}

* Jerib (jr.) $=1 / 5$ ha

* 2 Dry Opium $=$ Wet Opium $-30 \%$ moisture 
Opium Production Survey by village in Oruzgan province, 1995.

\begin{tabular}{|c|c|c|c|c|c|c|c|c|}
\hline \multirow{3}{*}{ District } & Village & \multirow{3}{*}{ Area/ Main Village } & \multirow{3}{*}{ Village } & \multirow{3}{*}{$\begin{array}{c}\text { Total } \\
\text { Cultivated } \\
\text { Land }\left(\text { Jerib }{ }^{*}\right) \\
\end{array}$} & \multirow{2}{*}{\multicolumn{2}{|c|}{$\begin{array}{c}\text { Land Under } \\
\text { Poppy Cultivation }\end{array}$}} & \multirow{3}{*}{$\begin{array}{c}\text { Wet Opium }^{* 2} \\
\text { Yield } \\
(\mathrm{kg} / \mathrm{jr} .) \\
\end{array}$} & \multirow{3}{*}{$\begin{array}{c}\text { Wet Opium } \\
\text { Production } \\
(\mathrm{kg})\end{array}$} \\
\hline & \multirow[t]{2}{*}{ Code } & & & & & & & \\
\hline & & & & & (jerib) & $(\%)$ & & \\
\hline \multicolumn{9}{|c|}{ Province: Oruzgan } \\
\hline TIRIN KOT & 49 & NACHIN & NACHIN & 700 & 150 & 21 & 12 & 1,800 \\
\hline TIRIN KOT & 95 & NAJOY & NAJOY & 48 & 10 & 21 & 11 & 110 \\
\hline TIRIN KOT & 14 & PATI KAREZ & PATI KAREZ & 15 & 5 & 33 & 11 & 55 \\
\hline TIRIN KOT & 9 & QALAM TALA & QALAM TALA & 150 & 50 & 33 & 11 & 550 \\
\hline TIRIN KOT & 76 & QANAT GANG & GANGI KAREZ & 16 & 3 & 19 & 13 & 39 \\
\hline TIRIN KOT & 84 & QASIM KAREZ & QASIM KAREZ & 130 & 25 & 19 & 11 & 275 \\
\hline TIRIN KOT & 64 & SABZABAD KARIZ & SABZABAD KARIZ & 45 & 10 & 22 & 13 & 130 \\
\hline TIRIN KOT & 42 & SABZAK & SABZAK & 180 & 45 & 25 & 11 & 495 \\
\hline TIRIN KOT & 51 & SADMARDEH & SADMARDEH & 45 & 95 & 211 & 12 & 1,140 \\
\hline TIRIN KOT & 30 & SAJAWAL & SAJAWAL & 55 & 10 & 18 & 12 & 120 \\
\hline TIRIN KOT & 67 & SAKIRA KARIZ & SAKIRA KARIZ & 19 & 4 & 21 & 12 & 48 \\
\hline TIRIN KOT & 53 & SAR CHOKHLI & SAR CHOKHLI & 290 & 68 & 23 & 11 & 748 \\
\hline TIRIN KOT & 24 & SARABE & SARABE & 75 & 15 & 20 & 11 & 165 \\
\hline TIRIN KOT & 18 & SARKHOM & SARKHOM & 350 & 110 & 31 & 12 & 1,320 \\
\hline TIRIN KOT & 19 & SARKHOM OLYA & SARKHOM OLYA & 220 & 60 & 27 & 11 & 660 \\
\hline TIRIN KOT & 43 & SARTOR BABA & SARTOR BABA & 80 & 24 & 30 & 11 & 264 \\
\hline TIRIN KOT & 47 & SHAH MANSOR & SHAH MANSOR & 110 & 22 & 20 & 12 & 264 \\
\hline TIRIN KOT & 61 & SHAH WALI KARIZ & SHAH WALI KARIZ & 110 & 22 & 20 & 12 & 264 \\
\hline TIRIN KOT & 33 & SHAH ZAFAR & SHAH ZAFAR & 310 & 60 & 19 & 11 & 660 \\
\hline TIRIN KOT & 23 & SHORABE & SHORABE & 80 & 20 & 25 & 12 & 240 \\
\hline TIRIN KOT & 58 & SIRA QALA & KUCHNI KARIZ & 65 & 15 & 23 & 11 & 165 \\
\hline TIRIN KOT & 92 & SOLAYEE & SOLAYEE & 150 & 30 & 20 & 13 & 390 \\
\hline TIRIN KOT & 20 & SPIN KICHA & SPIN KICHA & 40 & 12 & 30 & 12 & 144 \\
\hline TIRIN KOT & 60 & SUR KARIZ & SUR KARIZ & 95 & 22 & 23 & 11 & 242 \\
\hline TIRIN KOT & 70 & SUR SANIK & SUR SANIK & 25 & 30 & 120 & 13 & 390 \\
\hline TIRIN KOT & 36 & SURKH MURGHAB & SURKH MURGHAB & 200 & 44 & 22 & 12 & 528 \\
\hline TIRIN KOT & 75 & SYAH CHOW KAREZ & SYAH CHOW KAREZ & 9 & 3 & 33 & 12 & 36 \\
\hline TIRIN KOT & 59 & SYEDAN KARIZ & SYEDAN KARIZ & 130 & 28 & 22 & 11 & 308 \\
\hline TIRIN KOT & 5 & TALANI & TALANI & 3,300 & 1,100 & 33 & 13 & 14,300 \\
\hline
\end{tabular}

* Jerib (jr.) $=1 / 5$ ha

* 2 Dry Opium $=$ Wet Opium $-30 \%$ moisture
Page 9 in 0
Source: UNDCP Opium Production Survey, 1995 File: URZ3.XLS - 07/08/95 
Opium Production Survey by village in Oruzgan province, 1995.

\begin{tabular}{|c|c|c|c|c|c|c|c|c|}
\hline & Village & & & Total & Land L & & Wet Opium $^{* 2}$ & Wet Opium \\
\hline \multirow[t]{2}{*}{ District } & \multirow{2}{*}{ Code } & \multirow[t]{2}{*}{ Area/ Main Village } & \multirow[t]{2}{*}{ Village } & Cultivated & \multicolumn{2}{|c|}{ Poppy Cultivation } & Yield & Production \\
\hline & & & & Land $\left(\right.$ Jerib $\left.{ }^{*}\right)$ & (jerib) & $(\%)$ & $(\mathrm{kg} / \mathrm{jr})$. & $(\mathrm{kg})$ \\
\hline \multicolumn{9}{|c|}{ Province: Oruzgan } \\
\hline TIRIN KOT & 90 & TANO KARIZ & TANO KARIZ & 110 & 21 & 19 & 10 & 210 \\
\hline TIRIN KOT & 86 & WALGAI KAREZ & WALGAI KAREZ & 200 & 39 & 20 & 12 & 468 \\
\hline TIRIN KOT & 46 & WANI & WANI & 160 & 27 & 17 & 12 & 324 \\
\hline TIRIN KOT & 63 & WICH KARIZ & WICH KARIZ & 160 & 32 & 20 & 12 & 384 \\
\hline TIRIN KOT & 74 & YAK LENGA KAREZ & YAK LENGA KAREZ & 15 & 4 & 27 & 13 & 52 \\
\hline TIRIN KOT & 17 & YAKLINGA & YAKLINGA & 1,400 & 440 & 31 & 13 & 5,720 \\
\hline TIRIN KOT & 78 & ZAW KAREZ & ZAW KAREZ & 55 & 10 & 18 & 11 & 110 \\
\hline
\end{tabular}


Opium Production Survey by village in Qandahar province, 1995.

\begin{tabular}{|c|c|c|c|c|c|c|c|c|}
\hline . & Village & & . & Total & Land $L$ & & Wet Opium *2 & Wet Opium \\
\hline \multirow[t]{2}{*}{ District } & \multirow[t]{2}{*}{ Code } & \multirow[t]{2}{*}{ Area/ Main Village } & \multirow[t]{2}{*}{ Village } & \multirow{2}{*}{$\begin{array}{c}\text { Cultivated } \\
\left.\text { Land (Jerib }{ }^{*}\right) \\
\end{array}$} & \multicolumn{2}{|c|}{ Poppy Cultivation } & \multirow{2}{*}{$\begin{array}{c}\text { Yield } \\
(\mathrm{kg} / \mathrm{jr}) \\
\end{array}$} & \multirow{2}{*}{$\begin{array}{c}\text { Production } \\
(\mathrm{kg})\end{array}$} \\
\hline & & & & & (jerib) & $(\%)$ & & \\
\hline \multicolumn{9}{|c|}{ Province: Qandahar } \\
\hline ARGHANDAB & 9914 & ABDUL SHUKOOR MASJID & ABDUL SHUKOOR MASJID & 185 & 0 & 0 & 0 & 0 \\
\hline ARGHANDAB & 9132 & ALAKZO MASJID & ALAKZO MASJID & 100 & 0 & 0 & 0 & 0 \\
\hline ARGHANDAB & 9915 & ALI KALAY & ALI KALAY & 500 & 10 & 2 & 8 & 80 \\
\hline ARGHANDAB & 9128 & AQAI GUL & AQAI GUL & 190 & 0) & 0 & 0 & 0 \\
\hline ARGHANDAB & 9892 & BABA WALI SAHIB & BABA WALI SAHIB & 200 & 0 & 0 & 0 & 0 \\
\hline ARGHANDAB & 9262 & BABE KALAY & BABER KALY & 1,500 & 10 & 1 & 9 & 90 \\
\hline ARGHANDAB & 9901 & BALOCHANO MASJID & BALOCHANO MASJID & 190 & 0 & 0 & 0 & 0 \\
\hline ARGHANDAB & 9884 & BARAT QALACHA & BARAT QALACHA & 165 & 0 & 0 & 0 & 0 \\
\hline ARGHANDAB & 9135 & BAZAR & BAZAR & 200 & 0 & 0 & 0 & 0 \\
\hline ARGHANDAB & 9908 & BILKHIRO MASJED & BILKHIRO MASJED & 670 & 0 & 0 & 0 & 0 \\
\hline ARGHANDAB & 9097 & CHANGUL & CHUNGUL & 120 & 0 & 0 & 0 & 0 \\
\hline ARGHANDAB & 9880 & CHAR BAGH & CHAR BAGH & 550 & 10 & 2 & 22 & 220 \\
\hline ARGHANDAB & 9879 & CHAR QULBA ULYA & CHAR QALBA ULYA & 350 & 0 & 0 & 0 & 0 \\
\hline ARGHANDAB & 9882 & CHAR QULBAI SUFLA & CHAR QULBAI SUFLA & 415 & 10 & 2 & 15 & 150 \\
\hline ARGHANDAB & 9141 & DEH KOCHI & GHARB DARIA & 350 & 30 & 9 & 22 & 660 \\
\hline ARGHANDAB & 9144 & DEH SABZY & DEH SABZY & 218 & 4 & 2 & 15 & 60 \\
\hline ARGHANDAB & 9103 & DILAWAR KHAN QALACHA & DILAWAR KHAN QALACHA & 550 & 0 & 0 & 0 & 0 \\
\hline ARGHANDAB & 9140 & FAQIRAN SHIRMARD & FAQIRAN SHIRMARD & 320 & 6 & 2 & 20 & 120 \\
\hline ARGHANDAB & 9106 & GHONDAY & GHONDAY & 122 & 5 & 4 & 15 & 75 \\
\hline ARGHANDAB & 9124 & GUL MASJED & GUL MASJED & 219 & 7 & 3 & 15 & 105 \\
\hline ARGHANDAB & 9102 & GUL QALACHA & GUL QALACHA & 500 & 0 & 0 & 0 & 0 \\
\hline ARGHANDAB & 9897 & H. AMANULLAH MASJED & H. AMANULLAH MASJED & 370 & 7 & 2 & 18 & 117 \\
\hline ARGHANDAB & 9898 & H. GH. QADER MASJED & H. GH. QADER MASJED & 1,500 & 14 & 1 & 20 & 280 \\
\hline ARGHANDAB & 9869 & H. GULAN MASJED & H. GULAN MASJED & 120 & 2 & 2 & 15 & 30 \\
\hline ARGHANDAB & 9905 & H. SANGIN MASJED & H. SANGIN MASJED & 415 & 4. & 1 & 0 & 0 \\
\hline ARGHANDAB & 9129 & HAJI AHAD MASJED & HAJI AHAD MASJED & 232 & 0 & 0 & 0 & 0 \\
\hline ARGHANDAB & 9126 & HAJI MALOOK MASJID & HAJI MALOOK MASJID & 192 & 5 & 3 & 15 & 75 \\
\hline ARGHANDAB & 9902 & HAJI NABAT MASJED & HAJI NABAT MASJED & 560 & 4 & 1 & 12 & 48 \\
\hline ARGHANDAB & 9130 & HAJI PAHLAWAN & HAJI PAHLAWAN & 193 & 0 & 0 & 0 & 0 \\
\hline
\end{tabular}

* Jerib (jr.) $=1 / 5$ ha

* ${ }^{2}$ Dry Opium = Wet Opium - $30 \%$ moisture

Page 1 
Opium Production Survey by village in Qandahar province, 1995.

\begin{tabular}{|c|c|c|c|c|c|c|c|c|}
\hline \multirow{3}{*}{ District } & \multirow{3}{*}{\begin{tabular}{|l|} 
Village \\
Code
\end{tabular}} & \multirow{3}{*}{ Areal Main Village } & \multirow{3}{*}{ Village } & \multirow{3}{*}{$\begin{array}{c}\text { Total } \\
\text { Cultivated } \\
\text { Land }\left(\text { Jerib }{ }^{*}\right)\end{array}$} & \multirow{2}{*}{\multicolumn{2}{|c|}{$\begin{array}{c}\text { Land Under } \\
\text { Poppy Cultivation }\end{array}$}} & \multirow{3}{*}{$\begin{array}{c}\text { Wet Opium *2 } \\
\text { Yield } \\
(\mathrm{kg} / \mathrm{jr})\end{array}$} & \multirow{3}{*}{$\begin{array}{l}\text { Wet Opium } \\
\text { Production } \\
(\mathrm{kg})\end{array}$} \\
\hline & & & & & & & & \\
\hline & & & & & (jerib) & $(\%)$ & & \\
\hline \multicolumn{9}{|c|}{ Province: Qandahar } \\
\hline ARGHANDAB & 9913 & HAJI PIR DOST MASJED & HAJI PIR DOST MASJED & 170 & 0 & 0 & 0 & 0 \\
\hline ARGHANDAB & 9138 & HAJI QADUS MASJED & HAJI QADUS MASJED & 150 & 0 & 0 & 0 & 0 \\
\hline ARGHANDAB & 9121 & HAJI SAID MOHD QALA & HAJI SAID MOHD QALA & 90 & 0 & 0 & 0 & 0 \\
\hline ARGHANDAB & 9906 & HAJI SHAESTA GUL & SHAESTA GUL & 260 & 0 & 0 & 0 & 0 \\
\hline ARGHANDAB & 9127 & HAJI TOOR MASJID & HAJI TOOR MASJID & 173 & 0 & 0 & 0 & 0 \\
\hline ARGHANDAB & 9100 & HAJI TOOR QALACHA & HAJI ROOR QALACHA & 300 & 0 & 0 & 0 & 0 \\
\hline ARGHANDAB & 9891 & JALMIRAN & PELE KALACHA & 108 & 0 & 0 & 0 & 0 \\
\hline ARGHANDAB & 9886 & JAZA & JAZA & 850 & 2 & 0 & 25 & 50 \\
\hline ARGHANDAB & 9894 & JCBDAR & JABDAR & 200 & 15 & 8 & 11 & 165 \\
\hline ARGHANDAB & 2883 & JILAHOR & JILAHOR & 420 & 0 & 0 & 0 & 0 \\
\hline ARGHANDAB & 9911 & KANDYALI & KANDYALI & 150 & 0 & 0 & 0 & 0 \\
\hline ARGHANDAB & 9145 & KHALSHAK & KHALSHAK & 218 & 0 & 0 & 0 & 0 \\
\hline ARGHANDAB & 9881 & KHANA GIRDAB & KHANA GIRDAB & 180 & 20 & 11 & 25 & 500 \\
\hline ARGHANDAB & 9797 & KHAROTO KAS & KHAROTO KAS & 90 & 0 & 0 & 0 & 0 \\
\hline ARGHANDAB & 9899 & KHESRAW SUFLA & HAJI MOHD. HUSAIN & 480 & 2 & 0 & 20 & 30 \\
\hline ARGHANDAB & 9093 & KHISHKI & KHISHKI & 500 & 0 & 0 & 0 & 0 \\
\hline ARGHANDAB & 9139 & KOCHI & KOCHI & 450 & 110 & 24 & 20 & 2,200 \\
\hline ARGHANDAB & 9904 & KOH-E-NEGAR & MIRZA MOHD KHAN QALA & 650 & 5 & 1 & 25 & 125 \\
\hline ARGHANDAB & 9146 & KOHAK & KOHAK & 265 & 0 & 0 & 0 & 0 \\
\hline ARGHANDAB & 9867 & LANGAR & LANGAR & 0 & 0 & \#DIV/0! & 0 & 0 \\
\hline ARGHANDAB & 9099 & M. YAQOOB QALACHA & M. YAQOOB QALACHA & 300 & 2 & 1 & 10 & 20 \\
\hline ARGHANDAB & 9092 & MAFRA-E-NOW & MAFRA-E-NOW & 800 & 20 & 3 & 9 & 180 \\
\hline ARGHANDAB & 9112 & MAKTAB KALAY & MAKTAB KALAY & 140 & 0 & 0 & 0 & 0 \\
\hline ARGHANDAB & 9887 & MANSOR ABAD & MANSOR ABAD & 2,500 & 5 & 0 & 20 & 100 \\
\hline ARGHANDAB & 11172 & MARANJAN & SHINGHAZI MASJD & 300 & 0 & 0 & 0 & 0 \\
\hline ARGHANDAB & 9110 & MASHKORA & MASHKORA & 255 & 0 & 0 & 0 & 0 \\
\hline ARGHANDAB & 9115 & MASJED H. A. RAZAQ & MASJED H. A. RAZAQ & 275 & 1 & 0 & 9 & 9 \\
\hline ARGHANDAB & 9095 & MAZRA-E-KOHNA & MAZRA-E-KOHNA & 190 & 0 & 0 & 0 & 0 \\
\hline ARGHANDAB & 9890 & MENJOI & MENJOI & 400 & 20 & 5 & 11 & 220 \\
\hline
\end{tabular}

* Jerib (jr.) = $1 / 5$ ha

* 2 Dry Opium $=$ Wet Opium $-30 \%$ moisture 
Opium Production Survey by village in Qandahar province, 1995.

\begin{tabular}{|c|c|c|c|c|c|c|c|c|}
\hline \multirow{3}{*}{ District } & \multirow{3}{*}{\begin{tabular}{|l|} 
Village \\
Code \\
\end{tabular}} & \multirow{3}{*}{ Area/ Main Village } & \multirow{3}{*}{ Village } & \multirow{3}{*}{$\begin{array}{c}\text { Total } \\
\text { Cultivated } \\
\text { Land }\left(\text { Jerib }^{*}\right) \\
\end{array}$} & \multirow{2}{*}{\multicolumn{2}{|c|}{$\begin{array}{c}\text { Land Under } \\
\text { Poppy Cultivation }\end{array}$}} & \multirow{3}{*}{$\begin{array}{c}\text { Wet Opium }{ }^{* 2} \\
\text { Yield } \\
(\mathrm{kg} / \mathrm{jr}) \\
\end{array}$} & \multirow{3}{*}{$\begin{array}{c}\text { Wet. Opium } \\
\text { Production } \\
(\mathrm{kg}) \\
\end{array}$} \\
\hline & & & & & & & & \\
\hline & & & & & (jerib) & $(\%)$ & & \\
\hline \multicolumn{9}{|c|}{ Province: Qandahar } \\
\hline ARGHANDAB & 9094 & MHERA KHORA & MHERA KHORA & 350 & 0 & 0 & 0 & 0 \\
\hline ARGHANDAB & 9120 & MINARA-E-KALAN & ABDULLAH MASJED & 95 & 0 & 0 & 0 & 0 \\
\hline ARGHANDAB & 9119 & MIRGYANO MASJID & MIRGYANO MASJID & 160 & 0 & 0 & 0 & 0 \\
\hline ARGHANDAB & 9261 & MIRZA.M.KHAN QALACHA & MIRZA M.KHAN QALACHA & 580 & 0 & 0 & 0 & 0 \\
\hline ARGHANDAB & 9101 & MOHD NOOR QALAHA & MOHD NOOR QALAHA & 117 & 0 & 0 & 0 & 0 \\
\hline ARGHANDAB & 9117 & MULAZO MASJID & MULAZO MASJID & 122 & 0 & 0 & 0 & 0 \\
\hline ARGHANDAB & 9123 & NASRUDIN MASJID & NASRUDIN MASJID & 215 & 0 & 0 & 0 & 0 \\
\hline ARGHANDAB & 9116 & NOORZO MASJID & NOORZO MASJID & 135 & 0 & 0 & 0. & 0 \\
\hline ARGHANDAB & 9111 & PALEZI & PALEZI & 145 & 0 & 0 & 0 & 0 \\
\hline ARGHANDAB & 9108 & PETAWAY & PETAWAY & 220 & 6 & 3. & 15 & 90 \\
\hline ARGHANDAB & 9893 & PIR PAIEMAL & PIR PAIEMAL & 630 & 2 & 0 & 20 & 40 \\
\hline ARGHANDAB & 9131 & POPALZO MASJED & POPALZO MASJED & 100 & 0 & 0 & 0 & 0 \\
\hline ARGHANDAB & 9143 & SAEYDANO QALACHA & ALAKZO MASJED & 37 & 0 & 0 & 0 & 0 \\
\hline ARGHANDAB & 9909 & SALIMANZO MASJID & SALIMANZO MASJID & 280 & 5 & 2 & 12 & 60 \\
\hline ARGHANDAB & 9877 & SAMI QALACHA & SAMIE QALACHA & 115 & 0 & 0 & 0 & 0 \\
\hline ARGHANDAB & 9903 & SANZARI & SAYED ANWAR MASJID & 170 & 4 & 2 & 14 & 56 \\
\hline ARGHANDAB & 9114 & SARA KALAN & TANZO MASJID & 95 & 0 & 0 & 0 & 0 \\
\hline ARGHANDAB & 9104 & SARDE ULYA & SARDE ULYA & 650 & 0 & 0 & 0 & 0 \\
\hline ARGHANDAB & 9137 & SAYEDANO MASJED & SAYEDANO MASJED & 60 & 0 & 0 & 0 & 0 \\
\hline ARGHANDAB & 9133 & SAYEDANO QALACHA & SAYEDANO QALACHA & 1,200 & 6 & 1 & 0 & 0 \\
\hline ARGHANDAB & 9118 & SHAH MOHD MASJID & SHAH MOHD MASJID & 155 & 0 & 0 & 0 & 0 \\
\hline ARGHANDAB & 9866 & SHAH TOOR & SHAH TOOR & 250 & 20 & 8 & 22 & 440 \\
\hline ARGHANDAB & 9889 & SHAHIN ULYA & BAQI JAN QALACHA & 1,650 & 10 & 1 & 22 & 220 \\
\hline ARGHANDAB & 9896 & SHAHIN WASAT & SHAHIN WASAT & 800 & 30 & 4 & 15 & 450 \\
\hline ARGHANDAB & 11207 & SHARDEH SUFLA & SHARDEH SUFLA & 400 & 0 & 0 & 0 & 0 \\
\hline ARGHANDAB & 9895 & SHIAEN & SHIAEN & 175 & 7 & 4 & 15 & 105 \\
\hline ARGHANDAB & 9096 & SIEKH CHALA SUFLA & SIEKH CHALA SUFLA & 228 & 5 & 2 & 9. & 45 \\
\hline ARGHANDAB & 9098 & SIEKH CHALA ULYA & SIEKH CHALA ULYA & 480 & 0 & 0 & 0 & 0 \\
\hline ARGHANDAB & 9125 & SULTAN M.MASJED & SULTAN M.MASJED & 186 & 0 & 0 & 0 & 0 \\
\hline
\end{tabular}

* Jerib (jr.) = $1 / 5$ ha

* 2 Dry Opium = Wet Opium - $30 \%$ moisture

Page 3154

Source: UNDCP Opium Production Survey, 1995 File: KDR3.XLS - 07/08/95 
Opium Production Survey by village in Qandahar province, 1995.

\begin{tabular}{|c|c|c|c|c|c|c|c|c|}
\hline & Village & & . & Total & Land $L$ & & Wet.Opium $* 2$ & Wet Opium \\
\hline \multirow[t]{2}{*}{ District } & \multirow[t]{2}{*}{ Code } & \multirow[t]{2}{*}{ Area/ Main Village } & \multirow[t]{2}{*}{ Village } & \multirow{2}{*}{$\begin{array}{c}\text { Cultivated } \\
\left.\text { Land (Jerib }{ }^{*}\right)\end{array}$} & \multicolumn{2}{|c|}{ Poppy Cultivation } & \multirow{2}{*}{$\begin{array}{l}\text { Yield } \\
(\mathrm{kg} / \mathrm{jr})\end{array}$} & \multirow{2}{*}{$\begin{array}{c}\text { Production } \\
(\mathrm{kg})\end{array}$} \\
\hline & & & & & (jerib) & $(\%)$ & & \\
\hline \multicolumn{9}{|c|}{ Province: Qandahar } \\
\hline ARGHANDAB & 9107 & TA MAHALA & TA MAHALA & 280 & 5 & 2 & 0 & 0 \\
\hline ARGHANDAB & 9868 & TABIN & BABRIANO MUSJID & 93 & 3 & 3 & 17 & 51 \\
\hline ARGHANDAB & 9871 & TABIN & MULA BAQI MUSJID & 75 & 0 & 0 & 9 & 0 \\
\hline ARGHANDAB & 9878 & TABIN SUFLA & GHAFAR SHAH AGHA & 80 & 2 & 3 & 20 & 40 \\
\hline ARGHANDAB & 9869 & TABIN SUFLA & HAJI GHULAM MASJID & 120 & 2 & 2 & 15 & 30 \\
\hline ARGHANDAB & 9875 & TABIN ULYA & GHANI MASJED & 130 & 0 & 0 & 0 & 0 \\
\hline ARGHANDAB & 9872 & TABIN ULYA & HAJI ABDULLAH MASJED & 110 & 0 & 0 & 0 & 0 \\
\hline ARGHANDAB & 9876 & TABIN ULYA & WALO MASJED & 135 & 0 & 0 & 0 & 0 \\
\hline ARGHANDAB & 9907 & TOORDEH & TOORDEH & 320 & 0 & 0 & 0 & 0 \\
\hline ARGHANDAB & 9874 & YATIMAK ULYA & LOWAR YATIMAK & 1,300 & 0 & 0 & 0 & 0 \\
\hline ARGHANDAB & 9873 & YATIMAK-E- SUFLA & KESHATA YATIMAK & 800 & 0 & 0 & 0 & 0 \\
\hline ARGHANDAB & 9910 & ZORMANDA & ZORMANDA & 0 & 0 & \#DIV/0! & 0 & 0 \\
\hline ARGHISTAN & 12011 & A.RAHIM KHAN KALAY & A.RAHIM KHAN KALAY & 100 & 0 & 0 & 0 & 0 \\
\hline ARGHISTAN & 9866 & ABOZANA & ABOZANA & 180 & 0 & 0 & 0 & 0 \\
\hline ARGHISTAN & 9287 & AHMADZAI & SHILAGAI & 80 & 0 & 0 & 0 & 0 \\
\hline ARGHISTAN & 9824 & AKHOND KALAY & AKHOND KALAY & 180 & 0 & 0 & 0 & 0 \\
\hline ARGHISTAN & 9833 & AKHOND KALAY & AKHTAR M.KALAY & 70 & 0 & 0 & 0 & 0 \\
\hline ARGHISTAN & 9329 & ALI KHANZI KALAY & ALI KHANZI KALAY & 560 & 0 & 0 & 0 & 0 \\
\hline ARGHISTAN & 9813 & ALI M.KAS & ALI M.KAS & 75 & 0 & 0 & 0 & 0 \\
\hline ARGHISTAN & 9851 & ALSHIRZAI & ALSHIRZAI & 288 & 0 & 0 & 0 & 0 \\
\hline ARGHISTAN & 9324 & AMARAT & AMARAT & 480 & 0 & 0 & 0 & 0 \\
\hline ARGHISTAN & 9275 & AMBAR KALAY & AMBAR KALAY & 840 & 0 & 0 & 0 & 0 \\
\hline ARGHISTAN & 9293 & AMIN QALA & AMIN QALA & 1,780 & 0 & 0 & 0 & 0 \\
\hline ARGHISTAN & 12188 & ANZERGI CHOGI & NARI MANDA & 260 & 0 & 0 & 0 & 0 \\
\hline ARGHISTAN & 9273 & AOTMANZI & AOTMANZI & 270 & 0 & 0 & 0 & 0 \\
\hline ARGHISTAN & 9808 & ARAMZAI CHOKAZAI & NASIR KALAY & 400 & 0 & 0 & 0 & 0 \\
\hline ARGHISTAN & 9827 & ARGHAM CHI & TRE DAM & 180 & 0 & 0 & 0 & 0 \\
\hline ARGHISTAN & 9299 & AWARYAZI/YOSUF KHAIL & YOUSUF KHAIL & 380 & 0 & 0 & 0 & 0 \\
\hline ARGHISTAN & 9297 & BABARAY & BABARAY & 488 & 0 & 0 & 0 & 0 \\
\hline
\end{tabular}


Opium Production Survey by village in Qandahar province, 1995.

\begin{tabular}{|c|c|c|c|c|c|c|c|c|}
\hline . & Village & & . & Total & Land & & Wet Opium *2 & Wet Opium \\
\hline \multirow[t]{2}{*}{ District } & \multirow[t]{2}{*}{ Code } & \multirow[t]{2}{*}{ Area/ Main Village } & \multirow[t]{2}{*}{ Village } & \multirow{2}{*}{$\begin{array}{c}\text { Cultivated } \\
\left.\text { Land (Jerib }{ }^{*}\right) \\
\end{array}$} & \multicolumn{2}{|c|}{ Poppy Cultivation } & \multirow{2}{*}{$\begin{array}{l}\text { Yield } \\
(\mathrm{kg} / \mathrm{jr}) \\
\end{array}$} & \multirow{2}{*}{$\begin{array}{c}\text { Production } \\
\text { (kg) }\end{array}$} \\
\hline & & & & & (jerib) & $(\%)$ & & \\
\hline \multicolumn{9}{|c|}{ Province: Qandahar } \\
\hline ARGHISTAN & 9289 & BABIZAI & BABIZAI & 220 & 0 & 0 & 0 & 0 \\
\hline ARGHISTAN & 9805 & BADIZAI & BADIZAI & 700 & 0 & 0 & 0 & 0 \\
\hline ARGHISTAN & 9846 & BAGH & DARGAI,ZAWARA,SHURAN & 100 & 0 & 0 & 0 & 0 \\
\hline ARGHISTAN & 9272 & BAGH KALAY & BAGH KALAY & 120 & 0 & 0 & 0 & 0 \\
\hline ARGHISTAN & 9295 & BAGH KAS,TAZI GHABAG & HAJI HABIB KHAN & 480 & 0 & 0 & 0 & 0 \\
\hline ARGHISTAN & 9856 & BALA ZHARA & LAGHAR & 450 & 0 & 0 & 0 & 0 \\
\hline ARGHISTAN & 12170 & BALA ZHARA & PADA & 180 & 0 & 0 & 0 & 0 \\
\hline ARGHISTAN & 12171 & BALA ZHARA & SALEH MOHD & 190 & 0 & 0 & 0 & 0 \\
\hline ARGHISTAN & 12189 & BALA ZHARA & SROKANI/PATAWAY & 400 & 0 & 0 & 0 & 0 \\
\hline ARGHISTAN & 12174 & BALA ZHARA & WALA & 180 & 0 & 0 & 0 & 0 \\
\hline ARGHISTAN & 9806 & BALAWAY & NASIR KALAY & 300 & 0 & 0 & 0 & 0 \\
\hline ARGHISTAN & 9300 & BALAWAY & TARNAWA,ASEF KHEL & 300 & 0 & 0 & 0 & 0 \\
\hline ARGHISTAN & 9353 & BALAZHAR,AMANULLAH & (LANDI,PABANDY)KALAY & 200 & 0 & 0 & 0 & 0 \\
\hline ARGHISTAN & 9285 & BALOZAI & BALA JARA & 320 & 0 & 0 & 0 & 0 \\
\hline ARGHISTAN & 12187 & BANO KHEL & NASER KALAY & 370 & 0 & 0 & 0 & 0 \\
\hline ARGHISTAN & 9304 & BAQUL ZAI & BAQUL ZAI & 236 & 0 & 0 & 0 & 0 \\
\hline ARGHISTAN & 9828 & BAROK KHEL,SAROK KHE & AKHTAR M.KALAY & 200 & 0 & 0 & 0 & 0 \\
\hline ARGHISTAN & 9346 & BOKA & BOKA & 230 & 0 & 0 & 0 & 0 \\
\hline ARGHISTAN & 12009 & BOLAN & GARA,KADO KAREZ & 1,000 & 0 & 0 & 0 & 0 \\
\hline ARGHISTAN & 9274 & BREZE & BREZE & 280 & 0 & 0 & 0 & 0 \\
\hline ARGHISTAN & 11184 & BURJ & BURJ & 240 & 0 & 0 & 0 & 0 \\
\hline ARGHISTAN & 9862 & CHINO KALAY & CHINO & 200 & 0 & 0 & 0 & 0 \\
\hline ARGHISTAN & 9831 & CHOGI AKHUND & CHOGI AKHUND & 160 & 0 & 0 & 0 & 0 \\
\hline ARGHISTAN & 9853 & CHOGI LALAK & CHOGI LALAK & 120 & 0 & 0 & 0 & 0 \\
\hline ARGHISTAN & 9850 & CHOWKAZI & MALIK SALIH M.JAN & 220 & 0 & 0 & 0 & 0 \\
\hline ARGHISTAN & 12007 & DAB KAREZ & TARAKI TANGI,MULA GH & 1,620 & 0 & 0 & 0 & 0 \\
\hline ARGHISTAN & 9265 & DERGA KALAY & DERGA KALAY & 180 & 0 & 0 & 0 & 0 \\
\hline ARGHISTAN & 9294 & FAIZ MOHAMMAD KHAN & ABDUL KHALIQ & 2,120 & 0 & 0 & 0 & 0 \\
\hline ARGHISTAN & 9847 & FOLAD/HAJI MASTI & SPINKI,SURKI,DAWLAT. & 260 & 0 & 0 & 0 & 0 \\
\hline
\end{tabular}

* Jerib (jr.) $=1 / 5$ ha

* ${ }^{2}$ Dry Opium $=$ Wet Opium $-30 \%$ moisture

Page 5156

Source: UNDCP Opium Production Survey, 1995 File: KDR3.XLS - 07/08/95 
Opium Production Survey by village in Qandahar province, 1995.

\begin{tabular}{|c|c|c|c|c|c|c|c|c|}
\hline \multirow{3}{*}{ District } & \multirow{3}{*}{$\begin{array}{l}\text { Village } \\
\text { Code }\end{array}$} & \multirow{3}{*}{ Areal Main Village } & \multirow{3}{*}{ Village } & \multirow{3}{*}{$\begin{array}{c}\text { Total } \\
\text { Cultivated } \\
\left.\text { Land (.Jerib }{ }^{*}\right) \\
\end{array}$} & \multirow{2}{*}{\multicolumn{2}{|c|}{$\begin{array}{c}\text { Land Under } \\
\text { Poppy Cultivation }\end{array}$}} & \multirow{3}{*}{$\begin{array}{c}\text { Wet Opium *2 } \\
\text { Yield } \\
(\mathrm{kg} / \mathrm{jr})\end{array}$} & \multirow{3}{*}{$\begin{array}{c}\text { Wet Opium } \\
\text { Production } \\
\text { (kg) }\end{array}$} \\
\hline & & & & & & & & \\
\hline & & & & & (jerib) & $(\%)$ & & \\
\hline \multicolumn{9}{|c|}{ Province: Qandahar } \\
\hline ARGHISTAN & 9840 & GAWARI/WAZIR KHAIL & WAZIR KHEL,SARMENT K & 280 & 0 & 0 & 0 & 0 \\
\hline ARGHISTAN & 9331 & GHABARGA & GHABARGA & 340 & 0 & 0 & 0 & 0 \\
\hline ARGHISTAN & 9842 & H. MULLAH SALEH MOHD & H. MULLAH SALEH MOHD & 170 & 0 & 0 & 0 & 0 \\
\hline ARGHISTAN & 9364 & HAJI ABDULRAHMAN & HAJI ABDULRAHMAN & 180 & 0 & 0 & 0 & 0 \\
\hline ARGHISTAN & 9354 & HAJI FAIZ MOHD & HAJI FAIZ MOHD & 420 & 0 & 0 & 0 & 0 \\
\hline ARGHISTAN & 12002 & HAJI MOHD YOUSIF & HAJI MOHD YOUSIF & 120 & 0 & 0 & 0 & 0 \\
\hline ARGHISTAN & 12012 & HAJI.MOHD.YOSOF AGHA & HAJI.MOHD.YOSOF AGHA & 100 & 0 & 0 & 0 & 0 \\
\hline ARGHISTAN & 9338 & INZARGAI NARAI MANDA & INZARGAI NARAI MANDA & 130 & 0 & 0 & 0 & 0 \\
\hline ARGHISTAN & 9262 & ISHAQ ZAI & ISHAQ ZAI & 140 & 0 & 0 & 0 & 0 \\
\hline ARGHISTAN & 9844 & JABAR KAREZ & KOKHI,DANIKA,MALTANI & 240 & 0 & 0 & 0 & 0 \\
\hline ARGHISTAN & 9810 & JALAL KHEL & MULA JUMA KHEL & 175 & 0 & 0 & 0 & 0 \\
\hline ARGHISTAN & 9865 & JAMALI & JALAT & 410 & 0 & 0 & 0 & 0 \\
\hline ARGHISTAN & 9290 & JARARGZI & SHILAGAI & 260 & 0 & 0 & 0 & 0 \\
\hline ARGHISTAN & 9829 & KAHI/LALAKZAI & LALAK ZAI,KHWAGI,BEZ & 130 & 0 & 0 & 0 & 0 \\
\hline ARGHISTAN & 9857 & KAKARAN & PANZI KALAY & 300 & 0 & 0 & 0 & 0 \\
\hline ARGHISTAN & 9284 & KAMALZI & LANDI KALAY & 160 & 0 & 0 & 0 & 0 \\
\hline ARGHISTAN & 9233 & KARAM KHAIL & KARAM KHAIL & 320 & 0 & 0 & 0 & 0 \\
\hline ARGHISTAN & 9825 & KAREZGAI,AESA ZAI & BOA ASHAQ ZAI,TOROZI & 120 & 0 & 0 & 0 & 0 \\
\hline ARGHISTAN & 9841 & KAREZWAL & KAREZWAL & 145 & 0 & 0 & 0 & 0 \\
\hline ARGHISTAN & 9260 & KARWAN KASI & KARWAN KASI & 160 & 0 & 0 & 0 & o \\
\hline ARGHISTAN & 9832 & KAS KALAY & KAS KALAY & 160 & 0 & 0 & 0 & 0 \\
\hline ARGHISTAN & 9830 & KEAMATA & KEAMATA & 100 & 0 & 0 & 0 & 0 \\
\hline ARGHISTAN & 9259 & KEYASO & KEYASO & 260 & 0 & 0 & 0 & 0 \\
\hline ARGHISTAN & 9330 & KHALO KHEL KALAY & DARYAB KALAY & 400 & 0 & 0 & 0 & 0 \\
\hline ARGHISTAN & 9845 & KHAN GUL KHEL & KHAN GUL KHEL & 108 & 0 & 0 & 0 & 0 \\
\hline ARGHISTAN & 9822 & KHASTA & LOWARA KHESHTA,ADAGZ & 200 & 0 & 0 & 0 & 0 \\
\hline ARGHISTAN & 9363 & KHATAKI KALAY & KOTEYAN KALAY & 160 & 0 & 0 & 0 & 0 \\
\hline ARGHISTAN & 9263 & KHOGYANI & SHAGHMAZY,SHAFZAI & 340 & 0 & 0 & 0 & 0 \\
\hline ARGHISTAN & 9821 & KHONDERA & SHORAN & 85 & 0 & 0 & 0 & 0 \\
\hline
\end{tabular}

* Jerib (jr.) = 1/5 ha

*2 Dry Opium $=$ Wet Opium - $30 \%$ moisture 
Opium Production Survey by village in Qandahar province, 1995.

\begin{tabular}{|c|c|c|c|c|c|c|c|c|}
\hline & Village & . & & Total & Land & & Wet Opium ${ }^{* 2}$ & Wet Opium \\
\hline \multirow[t]{2}{*}{ District } & \multirow[t]{2}{*}{ Code } & \multirow[t]{2}{*}{ Area/ Main Village } & \multirow[t]{2}{*}{ Village } & \multirow{2}{*}{$\begin{array}{c}\text { Cultivated } \\
\left.\text { Land (Jerib }{ }^{*}\right)\end{array}$} & \multicolumn{2}{|c|}{ Poppy Cultivation } & \multirow{2}{*}{$\begin{array}{l}\text { Yield } \\
(\mathrm{kg} / \mathrm{jr})\end{array}$} & \multirow{2}{*}{$\begin{array}{c}\text { Production } \\
(\mathrm{kg})\end{array}$} \\
\hline & & & & & (jerih) & $(\%)$ & & \\
\hline \multicolumn{9}{|c|}{ Province: Qandahar } \\
\hline ARGHISTAN & 9796 & KHUDIDAD KHEL & KHUDIDAD KHEL & 75 & 0 & 0 & 0 & 0 \\
\hline ARGHISTAN & 9323 & KOGHI & NARWA & 800 & 0 & 0 & 0 & 0 \\
\hline ARGHISTAN & 12004 & KOTAZAI & KOTAZAI & 80 & 0 & 0 & 0 & 0 \\
\hline ARGHISTAN & 12175 & LAWARA & SUR GAZ KALAY & 500 & 0 & 0 & 0 & 0 \\
\hline ARGHISTAN & 12001 & LAYWANIANO & LAYWANIANO & 60 & 0 & 0 & 0 & 0 \\
\hline ARGHISTAN & 9339 & LOWAR SHAMIZI & SHIRIZI & 150 & 0 & 0 & 0 & 0 \\
\hline ARGHISTAN & 12190 & LOWARA & BADEZO CHAMAN & 400 & 0 & 0 & 0 & 0 \\
\hline ARGHISTAN & 12173 & LOWERA & JAMALZI & 900 & 0 & 0 & 0 & 0 \\
\hline ARGHISTAN & 9292 & LOY DEH & ABDULLAH JAN KALAY & 1,600 & 0 & 0 & 0 & 0 \\
\hline ARGHISTAN & 9838 & LOY DEH & KAZKAI,TORA GARA & 128 & 0 & 0 & 0 & 0 \\
\hline ARGHISTAN & 9328 & LOY KALAY & LOY KALAY & 800 & 0 & 0 & 0 & 0 \\
\hline ARGHISTAN & 9345 & M.UMAR,SALAM JAN & M.UMAR,SALAM JAN & 420 & 0 & 0 & 0 & 0 \\
\hline ARGHISTAN & 3807 & MAIO & BANDAGEE & 220 & 0 & 0 & 0 & 0 \\
\hline ARGHISTAN & 9818 & MALAK ABDULLAH JAN & MALAK ABDULLAH JAN & 60 & 0 & 0 & 0 & 0 \\
\hline ARGHISTAN & 9856 & MALEK ZAI & MALEK ZAI & 850 & 0 & 0 & 0 & 0 \\
\hline ARGHISTAN & 9282 & MALIK ZAI & JAWARZAI & 80 & 0 & 0 & 0 & 0 \\
\hline ARGHISTAN & 9848 & MAMO KHEL & MAMO KHEL & 182 & 0 & 0 & 0 & 0 \\
\hline ARGHISTAN & 9823 & MAMON ZAI & MAMON ZAI & 360 & 0 & 0 & 0 & 0 \\
\hline ARGHISTAN & 9817 & MARDAN KHEL & MARDAN KHEL & 140 & 0 & 0 & 0 & 0 \\
\hline ARGHISTAN & 9341 & MEHRABAN KALAY & MEHRABAN KALAY & 580 & 0 & 0 & 0 & 0 \\
\hline ARGHISTAN & 9807 & MIAN KAREZ & BANDAKAI & 200 & 0 & 0 & 0 & 0 \\
\hline ARGHISTAN & 9343 & MOHD KHAN & MOHD KHAN & 520 & 0 & 0 & 0 & 0 \\
\hline ARGHISTAN & 11180 & MUHKAMA/SHELGAI & SHILAGAI & 380 & 0 & 0 & 0 & 0 \\
\hline ARGHISTAN & 9336 & NAHR ZAHID & DAGALAN KHONI & 380 & 0 & 0 & 0 & 0 \\
\hline ARGHISTAN & 9321 & NARGHAL & NARGHAL & 500 & 0 & 0 & 0 & 0 \\
\hline ARGHISTAN & 9266 & NEGAHAN & NEGAHAN & 140 & 0 & 0 & 0 & 0 \\
\hline ARGHISTAN & 9359 & NOOR DEN & NOOR DEN & 150 & 0 & 0 & 0 & 0 \\
\hline ARGHISTAN & 11181 & NOWABAD & NARGARGY/SPIAN & 300 & 0 & 0 & 0 & 0 \\
\hline ARGHISTAN & 9276 & PABANDA KHEL & PABANDA KHEL & 200 & 0 & 0 & 0 & 0 \\
\hline
\end{tabular}

${ }^{*} \operatorname{Jerib}(j \mathrm{r}$.) $=1 / 5 \mathrm{ha}$

*2 Dry Opium = Wet Opium - $30 \%$ moisture

Page 7158

Source: UNDCP Opium Production Survey, 1995 File: KDR3.XLS - 07/08/95 
Opium Production Survey by village in Qandahar province, 1995.

\begin{tabular}{|c|c|c|c|c|c|c|c|c|}
\hline & Village & & . & Total & Land & & Wet Opium *2 & Wet Opium \\
\hline \multirow[t]{2}{*}{ District } & \multirow[t]{2}{*}{ Code } & \multirow[t]{2}{*}{ Area/ Main Village } & \multirow[t]{2}{*}{ Village } & \multirow{2}{*}{$\begin{array}{c}\text { Cultivated } \\
\left.\text { Land (Jerib }{ }^{*}\right)\end{array}$} & \multicolumn{2}{|c|}{ Poppy Cultivation } & \multirow{2}{*}{$\begin{array}{c}\text { Yield } \\
(\mathrm{kg} / \mathrm{jr})\end{array}$} & \multirow{2}{*}{$\frac{\text { Production }}{(\mathrm{kg})}$} \\
\hline & & & & & (jerib) & $(\%)$ & & \\
\hline \multicolumn{9}{|c|}{ Province: Qandahar } \\
\hline ARGHISTAN & 9268 & PAI KAREZ & SAYALY & 120 & 0 & 0 & 0 & 0 \\
\hline ARGHISTAN & 9327 & PARWAR ZAI & PARWAR ZAI & 1,080 & 0 & 0 & 0 & 0 \\
\hline ARGHISTAN & 9334 & PATAW & PATAW & 160 & 0 & 0 & 0 & 0 \\
\hline ARGHISTAN & 11178 & PETAW & DERGAI,SPIN MASJID & 200 & 0 & 0 & 0 & 0 \\
\hline ARGHISTAN & 11179 & PETAW & KOCHNAI ISHAQZAI,ZHA & 120 & 0 & 0 & 0 & 0 \\
\hline ARGHISTAN & 9854 & PETAW QALAGAI & SHAGI,QIAD KHEL & 160 & 0 & 0 & 0 & 0 \\
\hline ARGHISTAN & 9811 & PONGI KAS & SRA KAN TOR KHODAIDA & 65 & 0 & 0 & 0 & 0 \\
\hline ARGHISTAN & 9351 & POPALZAI & POPALZAI & 140 & 0 & 0 & 0 & 0 \\
\hline ARGHISTAN & 9271 & POSI KALAY & POSI KALAY & 320 & 0 & 0 & 0 & 0 \\
\hline ARGHISTAN & 9277 & QADIR KHEL & QADIR KHEL & 200 & 0 & 0 & 0 & 0 \\
\hline ARGHISTAN & 12010 & ROKHAN KHAIL & NEK KHAIL & 100 & 0 & 0 & 0 & 0 \\
\hline ARGHISTAN & 9843 & ROSTAM KAREZ & ROSTAM KAREZ & 280 & 0 & 0 & 0 & 0 \\
\hline ARGHISTAN & 9332 & SALAKTO & GUNBADI & 180 & 0 & 0 & 0 & 0 \\
\hline ARGHISTAN & 9352 & SALAM KHAN & SALAM KHAN & 460 & 0 & 0 & 0 & 0 \\
\hline ARGHISTAN & 12003 & SALGARY & SALGARY & 120 & 0 & 0 & 0 & 0 \\
\hline ARGHISTAN & 9819 & SALO KHEL & SALO KHEL & 60 & 0 & 0 & 0 & 0 \\
\hline ARGHISTAN & 9350 & SAMAKI WALA & NAKOOT TOZNA & 260 & 0 & 0 & 0 & 0 \\
\hline ARGHISTAN & 9270 & SAR KALAY & SAR KALAY & 1,820 & 0 & 0 & 0 & 0 \\
\hline ARGHISTAN & 9269 & SAR KAREZ & SAR KAREZ & 280 & 0 & 0 & 0 & 0 \\
\hline ARGHISTAN & 9794 & SAR KOMANDI & PAI KOMANDI & 200 & 0 & 0 & 0 & 0 \\
\hline ARGHISTAN & 12006 & SARCHI & KABAD KHEL & 160 & 0 & 0 & 0 & 0 \\
\hline ARGHISTAN & 9834 & SARDARA & SARDARA & 200 & 0 & 0 & 0 & 0 \\
\hline ARGHISTAN & 9836 & SARDARKAI & SARDARKAI & 480 & 0 & 0 & 0 & 0 \\
\hline ARGHISTAN & 9326 & SARMAST & SARMAST & 144 & 0 & 0 & 0 & 0 \\
\hline ARGHISTAN & 9281 & SARTEB MOHAMAD ZAI & LANDI KAREZ & 4,000 & 0 & 0 & 0 & 0 \\
\hline ARGHISTAN & 9340 & SARWAN & SARABAN & 430 & 0 & 0 & 0 & 0 \\
\hline ARGHISTAN & 9301 & SHABDIN ZAI & KOTIZAI & 60 & 0 & 0 & 0 & 0 \\
\hline ARGHISTAN & 12005 & SHALGI KALAY & SHALGI KALAY & 120 & 0 & 0 & 0 & 0 \\
\hline ARGHISTAN & 12192 & SHAMALI CHIGHI & JONOBI CHIGHI & 1,400 & 0 & 0 & 0 & 0 \\
\hline
\end{tabular}

* Jerib (jr.) = 1/5 ha

* 2 Dry Opium $=$ Wet Opium $-30 \%$ moisture 
Opium Production Survey by village in Qandahar province, 1995.

\begin{tabular}{|c|c|c|c|c|c|c|c|c|}
\hline . & Village & & . & \multirow{3}{*}{$\begin{array}{c}\text { Total } \\
\text { Cultivated } \\
\text { Land }\left(\text { Jerib }{ }^{*}\right) \\
\end{array}$} & \multirow{2}{*}{\multicolumn{2}{|c|}{$\begin{array}{c}\text { Land Under: } \\
\text { Poppy Cultivation }\end{array}$}} & \multirow{3}{*}{$\begin{array}{c}\text { Wet Opium * }{ }^{2} \\
\text { Yield } \\
(\mathrm{kg} / \mathrm{jr}) \\
\end{array}$} & \multirow{3}{*}{$\begin{array}{c}\text { Wet Opium } \\
\text { Production } \\
(\mathrm{kg})\end{array}$} \\
\hline \multirow[t]{2}{*}{ District } & Code & Area/ Main Village & Village & & & & & \\
\hline & & & & & (jerib) & $(\%)$ & & \\
\hline \multicolumn{9}{|c|}{ Province: Qandahar } \\
\hline ARGHISTAN & 9296 & SHANAKHTA & SHANAKHTA & 1,600 & 0 & 0 & 0 & 0 \\
\hline ARGHISTAN & 9347 & SHERZAI & SHERZAI & 420 & 0 & 0 & 0 & 0 \\
\hline ARGHISTAN & 9809 & SHIKHAN & DOMANDI,BOLAN,RAHIM & 280 & 0 & 0 & 0 & 0 \\
\hline ARGHISTAN & 12008 & SHIN CHAH & HEYJEEM & 160 & 0 & 0 & 0 & 0 \\
\hline ARGHISTAN & 9337 & SHIRA BOLAK & SHAMIZI & 900 & 0 & 0 & 0 & 0 \\
\hline ARGHISTAN & 9820 & SHOLGAR & SHOLGAR & 125 & 0 & 0 & 0 & 0 \\
\hline ARGHISTAN & 9261 & SHORLAN & SHORLAN & 576 & 0 & 0 & 0 & 0 \\
\hline ARGHISTAN & 9283 & SOPANZAI & SOPANZAI & 320 & 0 & 0 & 0 & 0 \\
\hline ARGHISTAN & 9837 & SORKI & SORKI & 246 & 0 & 0 & 0 & 0 \\
\hline ARGHISTAN & 11182 & SPIN GARA & KAZHAKY & 128 & 0 & 0 & 0 & 0 \\
\hline ARGHISTAN & 11177 & SPIN GARA & NASHR KH.,H.M.OMAR & 240 & 0 & 0 & 0 & 0 \\
\hline ARGHISTAN & 9349 & SPIN NARAY SINZALA & SPIN NARAY SINZALA & 280 & 0 & 0 & 0 & 0 \\
\hline ARGHISTAN & 9852 & SPINA & SPINA & 360 & 0 & 0 & 0 & 0 \\
\hline ARGHISTAN & 11186 & SPINA GARA & MANARAS(ISHAQZAI) & 94 & 0 & 0 & 0 & 0 \\
\hline ARGHISTAN & 11187 & SPINA GARA & SHIR QALA,AQA KHEL & 180 & 0 & 0 & 0 & 0 \\
\hline ARGHISTAN & 9839 & SPINA GARA & ZOR KALAY,TAK KALAY & 80 & 0 & 0 & 0 & 0 \\
\hline ARGHISTAN & 11199 & SPINA GARA YABONI & NOWABAD,JALA KHEL & 220 & 0 & 0 & 0 & 0 \\
\hline ARGHISTAN & 11185 & SPINA GARA/BOYANI & TARW PAKHEL,SHAILAGI & 180 & 0 & 0 & 0 & 0 \\
\hline ARGHISTAN & 11188 & SPINA YAYAWATY & TAROZI,ISAZAY & 220 & 0 & 0 & 0 & 0 \\
\hline ARGHISTAN & 9325 & SURPAN & DAWATY,JARGI & 442 & 0 & 0 & 0 & 0 \\
\hline ARGHISTAN & 9303 & TAHSILDAR QALA & ZAREN ZAI & 1,690 & 0 & 0 & 0 & 0 \\
\hline ARGHISTAN & 9267 & TAJAW & TAJAW & 600 & 0 & 0 & 0 & 0 \\
\hline ARGHISTAN & 9826 & TAKRE & SALEHGAI & 300 & 0 & 0 & 0 & 0 \\
\hline ARGHISTAN & 9358 & TARGHAK & HAJI RAHIM & 160 & () & 0 & 0 & 0 \\
\hline ARGHISTAN & 9362 & TARGHAK & HAJI SHINKAI KALAY & 170 & 0 & 0 & 0 & 0 \\
\hline ARGHISTAN & 9361 & TARGHAK & MULA ABDUL SAMAD & 180 & 0 & 0 & 0 & 0 \\
\hline ARGHISTAN & 9360 & TARGHAK & MULA DARO KHAN & 180 & 0 & 0 & 0 & 0 \\
\hline ARGHISTAN & 9364 & TARGHAT & BAGHAKA,H.A.RAHMAN & 160 & 0 & 0 & 0 & 0 \\
\hline ARGHISTAN & 9279 & TARWOKAI & YASEN,QABUL,WOROKAI & 300 & 0 & 0 & 0 & 0 \\
\hline
\end{tabular}

* Jerib (jr.) $=1 / 5$ ha

* 2 Dry Opium $=$ Wet Opium $-30 \%$ moisture 
Opium Production Survey by village in Qandahar province, 1995.

\begin{tabular}{|c|c|c|c|c|c|c|c|c|}
\hline & Village & $\cdot$ & & Total & Land & & Wet Opium *2 & Wet Opium \\
\hline \multirow[t]{2}{*}{ District } & \multirow[t]{2}{*}{ Code } & \multirow[t]{2}{*}{ Area/ Main Village } & \multirow[t]{2}{*}{ Village } & \multirow{2}{*}{$\begin{array}{c}\text { Cultivated } \\
\left.\text { Land (Jerib }{ }^{*}\right)\end{array}$} & \multicolumn{2}{|c|}{ Poppy Cultivation } & \multirow{2}{*}{$\begin{array}{c}\text { Yield } \\
(\mathrm{kg} / \mathrm{jr})\end{array}$} & \multirow{2}{*}{$\begin{array}{l}\text { Production } \\
\qquad(\mathrm{kg})\end{array}$} \\
\hline & & & & & (jerib) & $(\%)$ & & \\
\hline \multicolumn{9}{|c|}{ Province: Qandahar } \\
\hline ARGHISTAN & 12191 & TESON & WORSAKI & 450 & 0 & 0 & 0 & 0 \\
\hline ARGHISTAN & 9264 & TORAGARA & TORAGARA & 252 & 0 & 0 & 0 & 0 \\
\hline ARGHISTAN & 12172 & TORWA NAWA & SURKANI & 150 & 0 & 0 & 0 & 0 \\
\hline ARGHISTAN & 11176 & WACH GHABARG & ALISHER & 120 & 0 & 0 & 0 & 0 \\
\hline ARGHISTAN & 11175 & WACH GHABARG & AQILZI & 100 & 0 & 0 & 0 & 0 \\
\hline ARGHISTAN & 11183 & WACH GHABARG & SAIFULLAH KHEL KORCH & 150 & 0 & 0 & 0 & 0 \\
\hline ARGHISTAN & 12176 & WAM & MAIWAND KAR & 280 & 0 & 0 & 0 & 0 \\
\hline ARGHISTAN & 9855 & WOLGI & HAJI ALI M.,LADI & 430 & 0 & 0 & 0 & 0 \\
\hline ARGHISTAN & 9335 & WORSHAKI & WORSHAKI & 150 & 0 & 0 & 0 & 0 \\
\hline ARGHISTAN & 9858 & YARZI & H.KHUDAIDOST KHAN & 350 & 0 & 0 & 0 & 0 \\
\hline ARGHISTAN & 9860 & ZAHID & ZAHID & 200 & 0 & 0 & 0 & 0 \\
\hline ARGHISTAN & 9302 & ZAREN ZAI & ZAREN ZAI & 630 & 0 & 0 & 0 & 0 \\
\hline ARGHISTAN & 9863 & ZARGBANY & ZAHID & 160 & 0 & 0 & 0 & 0 \\
\hline ARGHISTAN & 9849 & ZEYARAT & YARI, CJUKAZI KALAY & 1,060 & 0 & 0 & 0 & 0 \\
\hline ARGHISTAN & 9344 & ZEYARAT & ZEYARAT & 500 & 0 & 0 & 0 & 0 \\
\hline DAMAN & 9251 & AENZAR GAI KAREZ & MESHE KAREZ & 190 & 0 & 0 & 0 & 0 \\
\hline DAMAN & 1289 & AINO KAREZ & AINO KAREZ & 400 & 0 & 0 & 0 & 0 \\
\hline DAMAN & 3249 & ALI ABAD & KHAMZORI & 240 & 0 & 0 & 0 & 0 \\
\hline DAMAN & 11149 & ARGISTAN & ALIZI & 180 & 0 & 0 & 0 & 0 \\
\hline DAMAN & 11166 & ARGISTAN & DAI & 540 & 0 & 0 & 0 & 0 \\
\hline DAMAN & 11148 & ARGISTAN & H.BASHAR & 310 & 0 & 0 & 0 & 0 \\
\hline DAMAN & 11165 & ARGISTAN & H.DEH & 340 & 0 & 0 & 0 & 0 \\
\hline DAMAN & 9252 & AZAM QALA & KALACHA & 2,400 & 0 & 0 & 0 & 0 \\
\hline DAMAN & 9226 & BAGOCHA & BAGOCHA & 300 & 0 & 0 & 0 & 0 \\
\hline DAMAN & 11108 & BERGE KALAY & BERGE KALAY & 260 & 0 & 0 & 0 & 0 \\
\hline DAMAN & 9238 & BOSTAN KAREZ & KHERATY KAREZ & 240 & 0 & 0 & 0 & 0 \\
\hline DAMAN & 11118 & CHAHAR BAND & CHAHAR BAND & 72 & 0 & 0 & 0 & 0 \\
\hline DAMAN & 11153 & CHRA KALAY & H.MANAN KALAY & 100 & 0 & 0 & 0 & 0 \\
\hline DAMAN & 11145 & CHRA KALAY & TOOR ZARIF & 160 & 0 & 0 & 0 & 0 \\
\hline
\end{tabular}


Opium Production Survey by village in Qandahar province, 1995.

\begin{tabular}{|c|c|c|c|c|c|c|c|c|}
\hline & Village & . & & Total & Land & & Wet Opium *2 & Wet Opium \\
\hline \multirow[t]{2}{*}{ District } & \multirow[t]{2}{*}{ Code } & \multirow[t]{2}{*}{ Area/ Main Village } & \multirow[t]{2}{*}{ Village } & \multirow{2}{*}{$\begin{array}{c}\text { Cultivated } \\
\left.\text { Land (Jerib }{ }^{*}\right)\end{array}$} & \multicolumn{2}{|c|}{ Poppy Cultivation } & \multirow{2}{*}{$\begin{array}{l}\text { Yield } \\
(\mathrm{kg} / \mathrm{jr}) \\
\end{array}$} & \multirow{2}{*}{$\begin{array}{l}\text { Production } \\
\text { (kg) }\end{array}$} \\
\hline & & & & & (jerib) & $(\%)$ & & \\
\hline \multicolumn{9}{|c|}{ Province: Qandahar } \\
\hline DAMAN & 11160 & FADA GUL MASJID & FADA GUL MASJID & 200 & 0 & 0 & 0 & 0 \\
\hline DAMAN & 9255 & GARY KAREZ & GARY KAREZ & 170 & 0 & 0 & 0 & 0 \\
\hline DAMAN & 11150 & GHRA KALAY & H.ABDUL MASJID & 200 & 0 & 0 & 0 & 0 \\
\hline DAMAN & 11146 & GHRA KALAY & H.AQA MASJID & 200 & 0 & 0 & 0 & 0 \\
\hline DAMAN & 11156 & GHRA KALAY & H.DARO KHAN & 200 & 0 & 0 & 0 & 0 \\
\hline DAMAN & 11151 & GHRA KALAY & H.FAZEL MASJID & 200 & 0 & 0 & 0 & 0 \\
\hline DAMAN & 11157 & GHRA KALAY & H.M.AKBAR MASJID & 200 & 0 & 0 & 0 & 0 \\
\hline DAMAN & 11163 & GHRA KALAY & H.M.SHAH AQA MASJID & 100 & 0 & 0 & 0 & 0 \\
\hline DAMAN & 11152 & GHRA KALAY & H.NAZAR JAN MASJID & 300 & 0 & 0 & 0 & 0 \\
\hline DAMAN & 11154 & GHRA KALAY & H.NIAZ M. MASJID & 180 & 0 & 0 & 0 & 0 \\
\hline DAMAN & 11155 & GHRA KALAY & H.NIDA M. & 100 & 0 & 0 & 0 & 0 \\
\hline DAMAN & 9997 & GHRA KALAY & WAKIL KHANDAR MASJID & 400 & 0 & 0 & 0 & 0 \\
\hline DAMAN & 11248 & GUL MIR KALACHA & GUL MIR KALACHA & 300 & 0 & 0 & 0 & 0 \\
\hline DAMAN & 11254 & H. AGHA JAN MASJID & H. AGHA JAN MASJID & 250 & 0 & 0 & 0 & 0 \\
\hline DAMAN & 11247 & H. ALI MOHAMMAD & H. ALI MOHAMMAD & 120 & 0 & 0 & 0 & 0 \\
\hline DAMAN & 11246 & H. KHUDAY RAHIM & H. KHUDAY RAHIM & 300 & 0 & 0 & 0 & 0 \\
\hline DAMAN & 11164 & H.KHAIR M.MASJID & H.KHAIR M.MASJID & 250 & 0 & 0 & 0 & 0 \\
\hline DAMAN & 11147 & HAJI KHUDAI DAD QALA & HAJI KHUDAI DAD QALA & 380 & 0 & 0 & 0 & 0 \\
\hline DAMAN & 9254 & HAJI LAL BAIG & HAJI LAL BAIG & 280 & 0 & 0 & 0 & 0 \\
\hline DAMAN & 9247 & HAMAI KALAY & AJRAN & 240 & 0 & 0 & 0 & 0 \\
\hline DAMAN & 21 & HEJRAN & HEJRAN & 200 & 0 & 0 & 0 & 0 \\
\hline DAMAN & 9236 & HINDO KAREZ & AMBAR KALAY S.ABAD & 120 & 0 & 0 & 0 & 0 \\
\hline DAMAN & 9237 & KHALIQDAD KAREZ & KHALIQDAD KAREZ & 100 & 0 & 0 & 0 & 0 \\
\hline DAMAN & 11109 & KHAN MOHD KAREZ & KUBANDI KAREZ,M.AYUB & 240 & 0 & 0 & 0 & 0 \\
\hline DAMAN & 11250 & LANDAI & LANDAI & 300 & 0 & 0 & 0 & 0 \\
\hline DAMAN & 11105 & LANDAY KAREZ & LANDAY KAREZ & 110 & 0 & 0 & 0 & 0 \\
\hline DAMAN & 9230 & LWAR MASJED/NAJOY UL & NAJOY ULYA & 2,000 & 0 & 0 & 0 & 0 \\
\hline DAMAN & 9983 & M. SEDDIQUE KAREZ & MULLAH MASHAK KAREZ & 300 & 0 & 0 & 0 & 0 \\
\hline DAMAN & 11107 & M.RABAT & BUR M.MASJID & 7,200 & 0 & 0 & 0 & 0 \\
\hline
\end{tabular}


Opium Production Survey by village in Qandahar province, 1995.

\begin{tabular}{|c|c|c|c|c|c|c|c|c|}
\hline & Village & & . & Total & Land L & & Wet.Opium *2 & Wet Opium \\
\hline \multirow[t]{2}{*}{ District } & Code & Area/ Main Village & Village & Cultivated & \multicolumn{2}{|c|}{ Poppy Cultivation } & Yield & Production \\
\hline & & & & Land (Jerib $\left.{ }^{*}\right)$ & (jerib) & $(\%)$ & $(\mathrm{kg} / \mathrm{jr})$ & $(\mathrm{kg})$ \\
\hline \multicolumn{9}{|c|}{ Province: Qandahar } \\
\hline DAMAN & 11106 & M.RABAT & H.MUSA MASJID & 2,400 & 0 & 0 & 0 & 0 \\
\hline DAMAN & 11116 & M.RABAT & JOHN M.MASJID & 300 & 0 & 0 & 0 & 0 \\
\hline DAMAN & 9235 & MABEN JAKAN & SAYEED KAREZ,AQA KAR & 720 & 0 & 0 & 0 & 0 \\
\hline DAMAN & 9985 & MADZO KALAY & SAFLA & 600 & 0 & 0 & 0 & 0 \\
\hline DAMAN & 11113 & MAKIAN BARGHANA & MAKIAN BARGHANA & 680 & 0 & 0 & 0 & 0 \\
\hline DAMAN & 9241 & MALANG KAREZ & I.ANDAI KAREZ & 220 & 0 & 0 & 0 & 0 \\
\hline DAMAN & 10003 & MAND HESAR & ABDULLAH AKA MASJID & 400 & 0 & 0 & 0 & 0 \\
\hline DAMAN & 10001 & MAND HESAR & MLK.S.M.KAKA MASJID & 240 & 0 & 0 & 0 & 0 \\
\hline DAMAN & 10002 & MAND HESAR & MOHD.YOUSAF & 120 & 0 & 0 & 0 & 0 \\
\hline DAMAN & 9999 & MANDI HESAR & HAJI JANAN MASJID & 900 & 0 & 0 & 0 & 0 \\
\hline DAMAN & 11158 & MANDISAR & AKTHAR M.KAKA(A) & 100 & 0 & 0 & 0 & 0 \\
\hline DAMAN & 11159 & MANDISAR & ALI M. MASJID & 200 & 0 & 0 & 0 & 0 \\
\hline DAMAN & 11161 & MANDISAR & H.TAJ M. & 300 & 0 & 0 & 0 & 0 \\
\hline DAMAN & 11162 & MANDISAR & MAJED KAKA & 120 & 0 & 0 & 0 & 0 \\
\hline DAMAN & 1 & MANJA & KOCHNAI MASJID & 300 & 0 & 0 & 0 & 0 \\
\hline DAMAN & 9228 & MANJA & SAR MASJID & 500 & 0 & 0 & 0 & 0 \\
\hline DAMAN & 9966 & MASJED FAROQ & MASJED FAROQ & 2,600 & 0 & 0 & 0 & 0 \\
\hline DAMAN & 36 & MASJID MALEK M. NABI & MASJID H. TOR AKA & 200 & 0 & 0 & 0 & 0 \\
\hline DAMAN & 9231 & MASJID NOOR M. NAJOY & NAJOY SAFLA & 420 & 0 & 0 & 0 & 0 \\
\hline DAMAN & 9960 & MIAN MASJED YAR MOHD & MIAN MASJED YAR MOHD & 200 & 0 & 0 & 0 & 0 \\
\hline DAMAN & 2 & MIAN MASJID & MIAN MASJID & 800 & 0 & 0 & 0 & 0 \\
\hline DAMAN & 11255 & MIRO GUL & MIRO GUL & 460 & 0 & 0 & 0 & 0 \\
\hline DAMAN & 9987 & MIRSINO KALAY & MIRSINO KALAY & 1,220 & 0 & 0 & 0 & 0 \\
\hline DAMAN & 11111 & MOMAND & H.A.LATIF & 7,300 & 0 & 0 & 0 & 0 \\
\hline DAMAN & 11117 & MOMAND & M.QASIM QALACHA & 60 & 0 & 0 & 0 & 0 \\
\hline DAMAN & 9245 & MOMAND RABAT WALA & H.MOHD & 300 & 0 & 0 & 0 & 0 \\
\hline DAMAN & 9246 & MOMAND RABAT WALA & H.MUSA KHAN MASJID & 4,860 & 0 & 0 & 0 & 0 \\
\hline DAMAN & 9232 & MOMAND RABAT WALA & QADER JAN MASJID & 830 & 0 & 0 & 0 & 0 \\
\hline DAMAN & 9233 & MOMAND RABAT WALA & SOFI KARIM MASJID & 4.800 & 0 & 0 & 0 & 0 \\
\hline
\end{tabular}

* Jerib (jr.) = 1/5 ha

*2 Dry Opium $=$ Wet Opium - $30 \%$ moisture 
Opium Production Survey by village in Qandahar province, 1995.

\begin{tabular}{|c|c|c|c|c|c|c|c|c|}
\hline . & Village & & . & Total & Land & der. & Wet Opium *2 & Wet Opium \\
\hline \multirow[t]{2}{*}{ District } & Code & Area/ Main Village & Village & Cultivated & \multicolumn{2}{|c|}{ Poppy Cultivation } & Yield & Production \\
\hline & & & & Land $\left(\right.$ Jerib $\left.^{*}\right)$ & (jerib) & $(\%)$ & $(\mathrm{kg} / \mathrm{jr})$ & $(\mathrm{kg})$ \\
\hline \multicolumn{9}{|c|}{ Province: Qandahar } \\
\hline DAMAN & 11245 & MULLAH MUSHK KAREZ & MULLAH MUSHK KAREZ & 400 & 0 & 0 & 0 & 0 \\
\hline DAMAN & 9981 & MURGHAN KAICHA & SAFLA/BABA KREZ MASJ & 300 & 0 & 0 & 0 & 0 \\
\hline DAMAN & 11253 & NAWAY DEH & NAWAY DEH & 1,200 & 0 & 0 & 0 & 0 \\
\hline DAMAN & 9225 & NAZAR QALA KAREZ & SHAHDAD KAREZ & 190 & 0 & 0 & 0 & 0 \\
\hline DAMAN & 11249 & NEMATULLAH & NEMATULLAH & 200 & 0 & 0 & 0 & 0 \\
\hline DAMAN & 9240 & PIRDAWS KAREZ & HAJI AZIZ KAREZ & 30 & 0 & 0 & 0 & 0 \\
\hline DAMAN & 9933 & PONGAI & PONGAI & 2,000 & 0 & 0 & 0 & 0 \\
\hline DAMAN & 11104 & QALACHA KAREZ & WACHKA KAY KAREZ & 240 & 0 & 0 & 0 & 0 \\
\hline DAMAN & 9998 & QANAT QAZI & QANAT QAZI & 1,680 & 0 & 0 & 0 & 0 \\
\hline DAMAN & 11112 & SAHIBZADA QALACHA & NARKY KAREZ & 320 & 0 & 0 & 0 & 0 \\
\hline DAMAN & 5 & SAID ABAD & SAID ABAD & 720 & 0 & 0 & 0 & 0 \\
\hline DAMAN & 11114 & SAIDAN QALACHA & SAIDAN QALACHA & 4,000 & 0 & 0 & 0 & 0 \\
\hline DAMAN & 9258 & SALAM QALACHA & LANDI KAREZ & 140 & 0 & 0 & 0 & 0 \\
\hline DAMAN & 9234 & SAR JAKAN & SULTAN \& SHAH KALAY & 320 & 0 & 0 & 0 & 0 \\
\hline DAMAN & 9244 & SAYED HAB KAREZ & H.MUSA JAN MASJID & 300 & 0 & 0 & 0 & 0 \\
\hline DAMAN & 9242 & SEMALZAY & SEMALZAY & 300 & 0 & 0 & 0 & 0 \\
\hline DAMAN & 11103 & SERA QALA H.HABIB KA & SERA QALA H.HABIB KA & 4,800 & 0 & 0 & 0 & 0 \\
\hline DAMAN & 9982 & SERA QALA,KHAAN MOHA & SHER M.KAREZ,QALACH. & 480 & 0 & 0 & 0 & 0 \\
\hline DAMAN & 11110 & SHAHDAD & BARKY KALAY & 280 & 0 & 0 & 0 & 0 \\
\hline DAMAN & 11115 & SHKER GANJ & SHKER GANJ & 100 & 0 & 0 & 0 & 0 \\
\hline DAMAN & 11251 & SHORANDAM & SHORANDAM & 400 & 0 & 0 & 0 & 0 \\
\hline DAMAN & 9996 & SHORANDAM & SHORANDAM & 300 & 0 & 0 & 0 & 0 \\
\hline DAMAN & 11252 & SHUKUR KAREZ & SHUKUR KAREZ & 600 & 0 & 0 & 0 & 0 \\
\hline DAMAN & 11244 & SOKHTA & SOKHTA & 420 & 0 & 0 & 0 & 0 \\
\hline DAMAN & 9248 & SOR KAREZ & CHAHAR BAND & 550 & 0 & 0 & 0 & 0 \\
\hline DAMAN & 9257 & SULTAN M.KHAN KALAY & BORJ KALAY & 700 & 0 & 0 & 0 & 0 \\
\hline DAMAN & 9243 & TREO KAREZ & PAINDI/SAHIBZADA QAL & 420 & 0 & 0 & 0 & 0 \\
\hline DAMAN & 9984 & WAHAB KHAIL MASJED & NIAZ MOHAMMAD & 420 & 0 & 0 & 0 & 0 \\
\hline DAMAN & 9227 & WAKIL SAHEB JAKAN & WAKIL SAHEB JAKAN & 700 & 0 & 0 & 0 & 0 \\
\hline
\end{tabular}

* Jerib (jr.) $=1 / 5$ ha

*2 Dry Opium $=$ Wet Opium $-30 \%$ moisture 
Opium Production Survey by village in Qandahar province, 1995.

\begin{tabular}{|c|c|c|c|c|c|c|c|c|}
\hline & Village & . & & Total & Land 1 & & Wet Opium *2 & Wet Opium \\
\hline \multirow[t]{2}{*}{ District } & \multirow[t]{2}{*}{ Code } & \multirow[t]{2}{*}{ Areal Main Village } & \multirow[t]{2}{*}{ Village } & \multirow{2}{*}{$\begin{array}{c}\text { Cultivated } \\
\left.\text { Land (Jerib }{ }^{*}\right)\end{array}$} & \multicolumn{2}{|c|}{ Poppy Cultivation } & \multirow{2}{*}{$\begin{array}{l}\text { Yield } \\
(\mathrm{kg} / \mathrm{jr})\end{array}$} & \multirow{2}{*}{$\begin{array}{c}\text { Production } \\
\text { (kg) }\end{array}$} \\
\hline & & & & & (jerih) & $(\%)$. & & \\
\hline \multicolumn{9}{|c|}{ Province: Qandahar } \\
\hline DAMAN & 9256 & WALI KALAY & HAMZA KALAY & 350 & () & 0 & 0 & 0 \\
\hline DAMAN & 9250 & ZAN ABAD & MESHI KAREZ & 680 & () & 0 & 0 & 0 \\
\hline DAND & 9187 & AEWAZ KAI.AY & AEWAZ KALAY & 187 & 0 & 0 & 0 & 0 \\
\hline DAND & 9167 & AIMARAT & AIMARAT & 2.090 & () & 0 & 0 & 0 \\
\hline DAND & 12189 & AKHONDAZAGANO KALAY & AKHONDAZAGANO KALAY & 400 & () & 0 & 0 & 0 \\
\hline DAND & 9936 & ANGORIAN & ANGORIAN & 2,000 & 0 & 0 & 0 & 0 \\
\hline DAND & 9959 & ARAZI & ARAZI & 500 & 0 & 0 & 0 & 0 \\
\hline DAND & 9174 & ASEA CHAF & ASEA CHAF & 650 & 0 & 0 & 0 & 0 \\
\hline DAND & 9179 & ATMANZAI & ATMANZAI & 30 & ()) & 0 & 0 & 0 \\
\hline DAND & 12110 & BADIZO & BADIZO & 0 & ()) & HI)IV/0! & 0 & 0 \\
\hline DAND & 9175 & BAKHTYAR & BAKHTYAR & 600 & () & 0 & 0 & 0 \\
\hline DAND & 9969 & BALADEH SUFLA & BALADEH SUFLA & 30 & 0 & 0 & 0 & 0 \\
\hline DAND & 9968 & BAL.ADEH UI.YA & BAL,ADEH UI,YA & 60 & 0) & 0 & 0 & 0 \\
\hline DAND & 9974 & BALADI & MASJID FEROZ KIIAN & 30 & ()) & 0 & 0 & 0 \\
\hline DAND & 9971 & BALADI & SAYEDANO QALACHA & 60 & () & 0 & 0 & 0 \\
\hline DAND & 12113 & BALUCHANO ACHAKZO QA & BALUCHANO ACHAKZO QA & 200 & 0 & 0 & 0 & 0 \\
\hline DAND & 9184 & BAMBLI KAREZ & NOORZY & 320 & 0 & 0 & 0 & 0 \\
\hline DAND & 9960 & BAZARCHA & BAZARCHA & 1,000 & 0 & 0 & 0 & 0 \\
\hline DAND & 12121 & BURZAI & BURZAI & 80 & 0 & 0 & 0 & 0 \\
\hline DAND & 9149 & CHAPLANI & CHAPLANI & 500 & 0 & 0 & 0 & 0 \\
\hline DAND & 9922 & CHARDIWAL & CHARDIWAL & 200 & 20 & 10 & 14 & 280 \\
\hline DAND & 9937 & DEH GHULAMAN & DEH GHULAMAN & 650 & 0 & 0 & 0 & 0 \\
\hline DAND & 9955 & DEH KHATAI & DEH KHATAI & 400 & 0 & 0 & 0 & 0 \\
\hline DAND & 11128 & DEH KOCHI & DEH KOCHI & 300 & 20 & 7 & 14 & 280 \\
\hline DAND & 9150 & DEH RAJAB & DEH RAJAB & 570 & 0 & 0 & 0 & 0 \\
\hline DAND & 12108 & FAZAI KARIM KOCHI KA & FAZAL KARIM KOCHI KA & 50 & 0 & 0 & 0 & 0 \\
\hline DAND & 9962 & GHRA KALAY & BIBI QALACIIA MASJID & 50 & 5 & 10 & 14 & 70 \\
\hline DAND & 9964 & GHRA KALAY & H.DIN M.,H.GUL M.MAJ & 0 & 0 & $\# \mid) \mid V / 0$ ! & 0 & 0 \\
\hline DAND & 9963 & GHRA KALAY & KHANDAQ MASJID,DAMBE & 0 & 0 & \#I)|V/0! & 0 & 0 \\
\hline
\end{tabular}

- Jerib (jr.) = I/S ha

- 2 Dry Opium $=$ Wet Opium $-30 \%$ moisture 
Opium Production Survey by village in Qandahar province, 1995.

\begin{tabular}{|c|c|c|c|c|c|c|c|c|}
\hline \multirow{3}{*}{ District } & \multirow{3}{*}{\begin{tabular}{|l|} 
Village \\
Code \\
\end{tabular}} & \multirow{3}{*}{ Area/ Main Village } & \multirow{3}{*}{ Village } & \multirow{3}{*}{$\begin{array}{c}\text { Total } \\
\text { Cultivated } \\
\text { Land }\left(\text { Jerib }^{*}\right)\end{array}$} & \multirow{2}{*}{\multicolumn{2}{|c|}{$\begin{array}{c}\text { Land Under } \\
\text { Poppy Cultivation }\end{array}$}} & \multirow{3}{*}{$\begin{array}{c}\text { Wet Opium *2 } \\
\text { Yield } \\
(\mathrm{kg} / \mathrm{jr}) \\
\end{array}$} & \multirow{3}{*}{$\begin{array}{c}\text { Wet Opium } \\
\text { Production } \\
\quad(\mathrm{kg})\end{array}$} \\
\hline & & & & & & & & \\
\hline & & & & & (jerib) & $(\%)$ & & \\
\hline \multicolumn{9}{|c|}{ Province: Qandahar } \\
\hline DAND & 11129 & GHRAH KALAY & MASJED MOSA & 0 & 0 & \#DIV/0! & 0 & 0 \\
\hline DAND & 9165 & GORGAN/ATA MOHD QALA & GORGAN/ATA MOHD QALA & 668 & 0 & 0 & 0 & 0 \\
\hline DAND & 11131 & GOSHKHANA & GOSHKHANA & 190 & 3 & 2 & 14 & 42 \\
\hline DAND & 12114 & GOSHKHANA & GOSHKHANA & 200 & 0 & 0 & 0 & 0 \\
\hline DAND & 12117 & GOSHKHANA ALOKOZAI & GOSHKHANA ALOKOZAI & 60 & 0 & 0 & 0 & 0 \\
\hline DAND & 9949 & HAJI ARAB & HAJI ARAB & 300 & 0 & 0 & 0 & 0 \\
\hline DAND & 11242 & HAJI MURAD KALAY & HAJI MURAD KALAY & 200 & 0 & 0 & 0 & 0 \\
\hline DAND & 12120 & HAJI NAZAR MOHAMMAD & HAJI NAZAR MOHAMMAD & 176 & 0 & 0 & 0 & 0 \\
\hline DAND & 11236 & HAKIM JAN KAREZ & HAKIM JAN KAREZ & 120 & 0 & 0 & 0 & 0 \\
\hline DAND & 11234 & HAQ DAD KAREZ & HAQ DAD KAREZ & 120 & 0 & 0 & 0 & 0 \\
\hline DAND & 12111 & HIJARO CHINA & HIJARO CHINA & 200 & 0 & 0 & 0 & 0 \\
\hline DAND & 9148 & HINDO BAGH & HINDO BAGH & 300 & 0 & 0 & 0 & 0 \\
\hline DAND & 9176 & JAMRANI & JAMRANI & 200 & 2 & 1 & 14 & 28 \\
\hline DAND & 9954 & KALACH ABAD & H.BAZ M.,GHANI QALAC & 500 & 0 & 0 & 0 & 0 \\
\hline DAND & 9956 & KALACH ABAD & H.RAZ M. LOWAR HAMAL & 300 & 0 & 0 & 0 & 0 \\
\hline DAND & 9934 & KALACH ABAD & H.SHER MASJID & 400 & 0 & 0 & 0 & 0 \\
\hline DAND & 12157 & KALANTAR & KALANTAR & 500 & 0 & 0 & 0 & 0 \\
\hline DAND & 9932 & KAREZAK KHURD & KAREZAK KHURD & 30 & 0 & 0 & 0 & 0 \\
\hline DAND & 9939 & KARZ & AKHTER AGHA MASJID & 560 & 0 & 0 & 0 & 0 \\
\hline DAND & 9947 & KARZ & ANAR MASJID,DESERANO & 300 & 3 & 1 & 14 & 42 \\
\hline DAND & 9946 & KARZ & H.M.KARIM MASJID & 310 & 0 & 0 & 0 & 0 \\
\hline DAND & 9942 & KARZ & H.RAHIM MASJID/KHANA & 350 & 0 & 0 & 0 & 0 \\
\hline DAND & 9178 & KHADANI & KHADANI & 240 & 0 & 0 & 0 & 0 \\
\hline DAND & 9967 & KHANANO MASJID & KHANANO MASJID & 500 & 0 & 0 & 0 & 0 \\
\hline DAND & 9945 & KHAWJA ALI BABA & KHAWJA ALI BABA & 696 & 0 & 0 & 0 & 0 \\
\hline DAND & 11241 & KHUSHAB & KHUSHAB & 2,000 & 0 & 0 & 0 & 0 \\
\hline DAND & 12109 & KISHANI & KISHANI & 200 & 0 & 0 & 0 & 0 \\
\hline DAND & 9172 & KOCHI & MULLA NAZAR KALAY & 80 & 0 & 0 & 0 & 0 \\
\hline DAND & 9154 & KOCHNAI BALA KARZ & KOCHNAI BALA KARZ & 1,350 & 0 & 0 & 0 & 0 \\
\hline
\end{tabular}


Opium Production Survey by village in Qandahar province, 1995.

\begin{tabular}{|c|c|c|c|c|c|c|c|c|}
\hline \multirow{3}{*}{ District } & \multirow{3}{*}{\begin{tabular}{|l|} 
Village \\
Code
\end{tabular}} & \multirow{3}{*}{ Areal Main Village } & \multirow{3}{*}{ Village } & \multirow{3}{*}{$\begin{array}{c}\text { Total } \\
\text { Cultivated } \\
\text { Land }\left(\text { Jerib }{ }^{*}\right) \\
\end{array}$} & \multirow{2}{*}{\multicolumn{2}{|c|}{$\begin{array}{c}\text { Land Under } \\
\text { Poppy Cultivation }\end{array}$}} & \multirow{3}{*}{$\begin{array}{c}\text { Wet Opium *2 } \\
\text { Yield } \\
(\mathrm{kg} / \mathrm{jr})\end{array}$} & \multirow{3}{*}{$\begin{array}{c}\text { Wet Opium } \\
\text { Production } \\
(\mathrm{kg})\end{array}$} \\
\hline & & & & & & & & \\
\hline & & & & & (jerib) & $(\%)$ & & \\
\hline \multicolumn{9}{|c|}{ Province: Qandahar } \\
\hline DAND & 12187 & KOCHNAI LANDAI & KOCHNAI LANDAI & 40 & 0 & 0 & 0 & 0 \\
\hline DAND & 9164 & KOHKARAN & KOHKARAN & 500 & 15 & 3 & 14 & 210 \\
\hline DAND & 12158 & KORY & KORY & 200 & 3 & 2 & 14 & 42 \\
\hline DAND & 11127 & KUBI & KUBI & 490 & 30 & 6 & 14 & 420 \\
\hline DAND & 11239 & LANDAI KAREZ DAMAN & LANDAI KAREZ DAMAN & 0 & 0 & \#DIV/0! & 0 & 0 \\
\hline DAND & 9161 & LOY BALAKAREZ & KHANANO MASJED & 500 & 0 & 0 & 0 & 0 \\
\hline DAND & 9160 & LOY BALAKAREZ & MIR AGHA MASJID & 250 & 0 & 0 & 0 & 0 \\
\hline DAND & 9162 & LOY BALAKAREZ & SPIN MASJID & 350 & 0 & 0 & 0 & 0 \\
\hline DAND & 9159 & LOY BALAKAREZ & TARZO MASJID & 670 & 0 & 0 & 0 & 0 \\
\hline DAND & 11238 & LOY KAREZ & LOY KAREZ & 0 & 0 & \#DIV/0! & 0 & 0 \\
\hline DAND & 9933 & LOY KAREZAK & LOY KAREZAK & 380 & 0 & 0 & 0 & 0 \\
\hline DAND & 11230 & MACHO & MACHO & 320 & 0 & 0 & 0 & 0 \\
\hline DAND & 11130 & MAHALAJAT & QLACHA KHAN AQA & 700 & 3 & 0 & 14 & 42 \\
\hline DAND & 9958 & MAHI & MAHI & 300 & 6 & 2 & 14 & 84 \\
\hline DAND & 9975 & MAHSHOOR & MAHSHOOR & 666 & 0 & 0 & 0 & 0 \\
\hline DAND & 9977 & MAHSHOOR KOHNA & H.JALALUDIN MASJID & 800 & 0 & 0 & 0 & 0 \\
\hline DAND & 9976 & MAHSHOOR NAW & ABDULLAH JAN & 2,000 & 0 & 0 & 0 & 0 \\
\hline DAND & 9169 & MANSOOR GHUNDAY & MANSOOR GHUNDAY & 600 & 0 & 0 & 0 & 0 \\
\hline DAND & 9153 & MAQAM/NAHR DEHNAW & MAQAM/NAHR DEHNAW & 3,000 & 0 & 0 & 0 & 0 \\
\hline DAND & 9921 & MARD QALA & AB.BAQI,AB.WADOOD(MA & 670 & 0 & 0 & 0 & 0 \\
\hline DAND & 9923 & MARD QALA & H.M.RASUL,H.GUL MASJ & 660 & 0 & 0 & 0 & 0 \\
\hline DAND & 9166 & MAROOF KAREZ & MAROOF KAREZ & 140 & 0 & 0 & 0 & 0 \\
\hline DAND & 12122 & MASJED A. KARIM & MASJED A. KARIM & 300 & 3 & 1 & 8 & 24 \\
\hline DAND & 11126 & MASJID-E-GHAZI & SABZIKAR & 850 & 0 & 0 & 0 & 0 \\
\hline DAND & 9931 & MIAN JOY & MIAN JOY & 200 & 10 & 5 & 14 & 140 \\
\hline DAND & 11231 & MIAN JOY MOHAMMADZO & MIAN JOY MOHAMMADZO & 260 & 0 & 0 & 0 & 0 \\
\hline DAND & 9973 & MINARA MIA SAHEB & MINARA MIA SAHEB & 200 & 0 & 0 & 0 & 0 \\
\hline DAND & 9152 & MIR BAZAR & SYED GULA JAN MASJID & 230 & 10 & 4 & 14 & 140 \\
\hline DAND & 9151 & MIR BAZAR/YAR MOHD & HAJI YAR MOHD & 200 & 10 & 5 & 14 & 140 \\
\hline
\end{tabular}


Opium Production Survey by village in Qandahar province, 1995.

\begin{tabular}{|c|c|c|c|c|c|c|c|c|}
\hline \multirow{3}{*}{ District } & \multirow{3}{*}{$\begin{array}{l}\text { Village } \\
\text { Code }\end{array}$} & \multirow{3}{*}{ Area/ Main Village } & \multirow{3}{*}{ Village } & \multirow{3}{*}{\begin{tabular}{|c|} 
Total \\
Cultivated \\
Land $\left(\right.$ Jerib $\left.^{*}\right)$ \\
\end{tabular}} & \multirow{2}{*}{\multicolumn{2}{|c|}{$\begin{array}{c}\text { Land Under } \\
\text { Poppy Cultivation }\end{array}$}} & \multirow{3}{*}{$\begin{array}{c}\text { Wet Opium }^{* 2} \\
\text { Yield } \\
(\mathrm{kg} / \mathrm{jr}) \\
\end{array}$} & \multirow{3}{*}{$\begin{array}{c}\text { Wet Opium } \\
\text { Production } \\
(\mathrm{kg}) \\
\end{array}$} \\
\hline & & & & & & & & \\
\hline & & & & & (jerib) & $(\%)$ & & \\
\hline \multicolumn{9}{|c|}{ Province: Qandahar } \\
\hline DAND & 9170 & MORGHAN & MORGHAN & 300 & 3 & 1 & 14 & 42 \\
\hline DAND & 12123 & MULADAD QALA & MAWLADAD QALA & 0 & 0 & \#DIV/0! & 0 & 0 \\
\hline DAND & 9938 & MULLA KOCHI & MULLA KOCHI & 500 & 0 & 0 & 0 & 0 \\
\hline DAND & 11240 & MULLAH ABDULLAH & MULLAH ABDULLAH & 800 & 0 & 0 & 0 & 0 \\
\hline DAND & 9930 & MUSHKIZI & MUSHKIZI & 0 & 0 & \#DIV/0! & 0 & 0 \\
\hline DAND & 9163 & NAKODAK & NAKODAK & 2,066 & 0 & 0 & 0 & 0 \\
\hline DAND & 9180 & NASERAN & NASERAN & 800 & 0 & 0 & 0 & 0 \\
\hline DAND & 9948 & NAW DEH & NAW DEH & 200 & 17 & 9 & 14 & 238 \\
\hline DAND & 12190 & NAWAY QALACHA & NAWAY QALACHA & 100 & 0 & 0 & 0 & 0 \\
\hline DAND & 9917 & NAWAY QALACHA,KARAM & NAWAY QALACHA,KARAM & 1,008 & 0 & 0 & 0 & 0 \\
\hline DAND & 9957 & NEKO KAREZ & NEKO KAREZ & 80 & 0 & 0 & 0 & 0 \\
\hline DAND & 9156 & PERU QALACHA & MASJID H.GUL & 300 & 0 & 0 & 0 & 0 \\
\hline DAND & 9155 & PERU QALACHA & MASJID H.RAMAZAN & 800 & 0 & 0 & 0 & 0 \\
\hline DAND & 9158 & PERU QALACHA & PERU MASJID & 400 & 0 & 0 & 0 & 0 \\
\hline DAND & 9935 & QALACHA REGI & QALACHA BELO,QALACHA & 200 & 20 & 10 & 14 & 280 \\
\hline DAND & 11237 & QANAT ISMAIL KAREZ & QANAT ISMAIL KAREZ & 300 & 0 & 0 & 0 & 0 \\
\hline DAND & 9943 & RAMBASI & H.MULA KHUDAIDAD. MAS & 1,180 & 0 & 0 & 0 & 0 \\
\hline DAND & 9944 & RAMBASI/TAZOKO MASJI & YAR M. MASJID & 900 & 20 & 2 & 14 & 280 \\
\hline DAND & 9920 & RAWANI & H.DAUD MASJID & 450 & 0 & 0 & 0 & 0 \\
\hline DAND & 9918 & RAWANI & LOY,ZOOK(MASJID) & 920 & 0 & 0 & 0 & 0 \\
\hline DAND & 9919 & RAWANI & QALACHA MASJID,GAR M & 500 & 0 & 0 & 0 & 0 \\
\hline DAND & 9926 & ROHRABAD & ALI M.MASJID & 1,200 & 0 & 0 & 0 & 0 \\
\hline DAND & 9927 & ROHRABAD & H.SATTAR MASJID & 1,290 & 0 & 0 & 0 & 0 \\
\hline DAND & 9924 & ROHRABAD & KHAWAJA M.MASJID & 1,120 & 0 & 0 & 0 & 0 \\
\hline DAND & 9929 & ROHRABAD & M.RASSUL MASJID & 1,620 & 0 & 0 & 0 & 0 \\
\hline DAND & 9978 & SABZIKAR & SABZIKAR & 850 & 0 & 0 & 0 & 0 \\
\hline DAND & 12112 & SADOZO CHINA & SADOZO CHINA & 10 & 0 & 0 & 0 & 0 \\
\hline DAND & 11232 & SALO KAREZ & SALO KAREZ & 130 & 0 & 0 & 0 & 0 \\
\hline DAND & 9966 & SAR BULAND QALACHA & SAR BULAND QALACHA & 200 & 0 & 0 & 0 & 0 \\
\hline
\end{tabular}

* Jerib (jr.) $=1 / 5$ ha

${ }^{*}$ Dry Opium $=$ Wet Opium - $30 \%$ moisture 
Opium Production Survey by village in Qandahar province, 1995.

\begin{tabular}{|c|c|c|c|c|c|c|c|c|}
\hline & Village & $\therefore$ & & Total & Land $l$ & & Wet Opium *2 & Wet Opium \\
\hline \multirow[t]{2}{*}{ District } & \multirow[t]{2}{*}{ Code } & \multirow[t]{2}{*}{ Area/ Main Village } & \multirow[t]{2}{*}{ Village } & \multirow{2}{*}{$\begin{array}{c}\text { Cultivated } \\
\left.\text { Land (Jerih }^{*}\right)\end{array}$} & \multicolumn{2}{|c|}{ Poppy Cultivation } & \multirow{2}{*}{$\begin{array}{l}\text { Yield } \\
(\mathrm{kg} / \mathrm{jr}) \\
\end{array}$} & \multirow{2}{*}{$\begin{array}{c}\text { Production } \\
\text { (kg) }\end{array}$} \\
\hline & & & & & (jerih) & (\%) & & \\
\hline \multicolumn{9}{|c|}{ Province: Qandahar } \\
\hline DAND & 9950 & SARPOZA & SARPOZA & 50 & 0 & 0 & 0 & 0 \\
\hline DAND & 9916 & SHAH MANSOOR & SHAH MANSOOR & 100 & 0 & 0 & 0 & 0 \\
\hline DAND & 9970 & SHAIKH MOHAMADI & SHAIKH MOHAMADI & 30 & 0 & 0 & 0 & 0 \\
\hline DAND & 9182 & SHAMSHER KAREZ & SHAMSHER KAREZ & 3,000 & 0 & 0 & 0 & 0 \\
\hline DAND & 12188 & SHEFAH & SHEFAH & 360 & 0 & 0 & 0 & 0 \\
\hline DAND & 12116 & SHER SURKH & SHER SURKH & 450 & 0) & 0 & 0 & 0 \\
\hline DAND & 12115 & SHER SURKH MASJID AI. & SHER SURKHI MASJID AL, & 450 & 0 & 0 & 0 & 0 \\
\hline DAND & 9173 & SOOP & $S(O)$ & 700 & 40 & 6 & 14 & 560 \\
\hline DAND & 9961 & SPIN ZEYARAT & SPIN ZEYARAT & 300 & 0 & 0 & 0 & 0 \\
\hline DAND & 9183 & TEMOR KAREZ/YAROQ & TEMOR KAREZ/YAROQ & 0 & () & HI)|V/0! & 0 & 0 \\
\hline DAND & 9940 & TEMORIAN & AB.GHAFAR MASJID & 350 & 0 & 0 & 0 & 0 \\
\hline DAND & 9941 & TEMORIAN & LOY MASJID & 550 & 0 & 0 & 0 & 0 \\
\hline DAND & 9965 & TOORA PAKHOLA & TOORA PAKHOLA & 0 & 0 & \#I)IV/0! & 0 & 0 \\
\hline DAND & 12156 & TOORGUL AGHA & TOORGUL AGHA & 30 & 0 & 0 & 0 & 0 \\
\hline DAND & 12119 & WALAKAN & WALAKAN & 220 & 0 & 0 & 0 & 0 \\
\hline DAND & 12118 & WAZIRO KALAY & WAZIRO KALAY & 350 & 20 & 6 & 14 & 280 \\
\hline DAND & 9928 & YAKH KAREZ & YAKH KAREZ & 150 & 4 & 3 & 14 & 56 \\
\hline DAND & 9953 & ZAKIR SHARIF & H.NIK,H.MALIK(MASJID & 230 & 0 & 0 & 0 & 0 \\
\hline DAND & 9951 & ZAKIR SHARIF & M.SHAH,H.JUMA KH.MAS & 860 & 0 & 0 & 0 & 0 \\
\hline DAND & 9952 & ZAKIR SHARIF & MALIK WASAY,SAHIBJAN & 390 & 0 & 0 & 0 & 0 \\
\hline GHORAK & 12177 & AGHA CHENA & AGHA CHENA & 100 & 25 & 25 & 8 & 200 \\
\hline GHORAK & 9603 & AKAZI SAR KANDA & WASHA KUNDA & 150 & 20) & 13 & 9 & 180 \\
\hline GHORAK & 9550 & ANGIRAK & ANGIRAK & 150 & 80 & 53. & 4 & 320 \\
\hline GHORAK & 39550 & ANJIRAK & ANJIRAK & 150 & 80 & 53 & 4 & 320 \\
\hline GHORAK & 9596 & ATALAI AOW GALAK & WAGALAK KAREZONA & 400 & 20 & 5 & 9 & 180 \\
\hline GHORAK & 9610 & AWKHANA & AWKHANA & 400 & 80 & 20 & 9 & 720 \\
\hline GHORAK & 9595 & AWMAKI MADKHANI & MADKHANI KAREZ & 400 & 25 & 6 & 8 & 200 \\
\hline GHORAK & 9577 & $B \wedge D \wedge K$ & MARK, SHAKARY KARI:Z, & 300 & 150 & 50 & 8 & 1.200 \\
\hline GHORAK & 9564 & BAGH MERAB KARFZ & BAGH MERAB & 200 & 25 & 13 & 20 & 500 \\
\hline
\end{tabular}


Opium Production Survey by village in Qandahar province, 1995.

\begin{tabular}{|c|c|c|c|c|c|c|c|c|}
\hline & Village & . & & & land $l$ & & Wet Opium *2 & Wet Opium \\
\hline \multirow[t]{2}{*}{ District } & \multirow[t]{2}{*}{ Code } & \multirow{2}{*}{ Areal Main Village } & \multirow[t]{2}{*}{ Village } & \multirow{2}{*}{$\begin{array}{c}\text { Cultivated } \\
\text { Land (Jerib *) }\end{array}$} & \multicolumn{2}{|c|}{ Poppy Cultivation } & \multirow{2}{*}{$\begin{array}{l}\text { Yield } \\
(\mathrm{kg} / \mathrm{jr})\end{array}$} & \multirow{2}{*}{$\begin{array}{c}\text { Production } \\
\text { (kg) }\end{array}$} \\
\hline & & & & & (jerib) & $(n / n)$ & & \\
\hline \multicolumn{9}{|c|}{ Province: Qandahar } \\
\hline GHORAK & 9576 & BAHRAM & JAHPARY KAREZONA & 750 & 80 & 11 & 8 & 640 \\
\hline GHORAK & 9557 & BAI KHOSH KAREZ & BAI KHOSH KAREZ & 200 & 50 & 25 & 18 & 900 \\
\hline GHORAK & 9614 & BALA HASSAN & TOOR/BALA HASSAN & 160 & 0 & 0 & 0 & 0 \\
\hline GHORAK & 9597 & BALGHA & SIA SANG KAREZONA & 200 & 20 & 10 & 8 & 160 \\
\hline GHORAK & 9598 & BAR KAREZ & BAR KAREZ & 200 & 25 & 13 & 7 & 175 \\
\hline GHORAK & 9599 & BARAKZO KAREZ & BARAKZO KAREZ & 200 & 16 & 8 & 8 & 128 \\
\hline GHORAK & 9604 & BARGHATO & BARGHATO & 100 & 10 & 10 & 8 & 80 \\
\hline GHORAK & 12195 & BIADAK KAREZ & BIADAK KAREZ & 50 & 30 & 60 & 8 & 240 \\
\hline GHORAK & 9618 & CHAH BABAR & CHAH BABAR & 30 & 30 & 100 & 4 & 120 \\
\hline GHORAK & 9566 & CHAH GAAK KAREZ & CHAH GAAK KAREZ & 100 & 10 & 10 & 7 & 70 \\
\hline GHORAK & 9578 & CHAHAR PAEO & AHMAD ABAD KAREZONA & 150 & 50 & 33 & 8 & 400 \\
\hline GHORAK & 9584 & CHALAK & ZRD REGY KAREZONA & 200 & 20 & 10 & 10 & 200 \\
\hline GHORAK & 9616 & CHAR SOKHTA & CHAR SOKHTA & 25 & 12 & 48 & 5 & 54 \\
\hline GHORAK & 9556 & CHIGHAK KAREZ & CHIGHAK & 40 & 10 & 25 & 16 & 160 \\
\hline GHORAK & 9572 & CHINA CHOGEEN KAREZ & CHOGEEN KAREZ & 380 & 50 & 13 & 8 & 400 \\
\hline GHORAK & 9565 & CHINAR KAREZ & CHINAR & 200 & 50 & 25 & 12 & 600 \\
\hline GHORAK & 9625 & CHOGAI & CHOGAI & 125 & 20 & 16 & 9 & 180 \\
\hline GHORAK & 9594 & CHONI KAREZ & CHONI & 200 & 25 & 13 & 8 & 200 \\
\hline GHORAK & 11143 & CHORCHORK/NAR KAREZ & NAR KAREZ & 600 & 500 & 83 & $9]$ & 4,500 \\
\hline GHORAK & 9581 & ENZER KAREZ & ENZER KAREZ & 500 & 50 & 10 & 8 & 400 \\
\hline GHORAK & 9567 & GERKAK KAREZ & GERKAK KAREZ & 100 & 20 & 20 & 8 & 160 \\
\hline GHORAK & 9619 & GHORAK & BADAK & 50 & 45 & 90 & 9 & 405 \\
\hline GHORAK & 9549 & GHORAK & CHA HAIDAR & 50 & 8 & 16 & 12 & 96 \\
\hline GHORAK & 9622 & GHORAK & TERAWI ROBA & 120 & 25 & 21 & 6 & 150 \\
\hline GHORAK & 9570 & GHORAK KAREZ & GHORAK KAREZ & 800 & 120 & 15 & 16 & 1,920 \\
\hline GHORAK & 9558 & GHOW KHUR KAREZ & GUL KHANA & 400 & 60 & 15 & 18 & 1,080 \\
\hline GHORAK & 12194 & GUL ABAD & SHODAB KAREZONA & 25 & 20 & 80 & 9 & 180 \\
\hline GHORAK & 9623 & HAJI PIR MOHD & HAJI PIR MOHD & 25 & 9 & 36 & 9 & 81 \\
\hline GHORAK & 9609 & HAJI TOOP & HAJI TOOP & 200 & 30 & 15 & 10 & 300 \\
\hline
\end{tabular}

* Jerib (jr.) = I/5 ha

${ }^{*}$ Dry Opium $=$ Wet Opium $-30 \%$ moisture 
Opium Production Survey by village in Qandahar province, 1995.

\begin{tabular}{|c|c|c|c|c|c|c|c|c|}
\hline & \multirow{3}{*}{$\begin{array}{l}\text { Village } \\
\text { Code }\end{array}$} & & . & Total & Land $L$ & & Wet Opium *2 & Wet Opium \\
\hline \multirow[t]{2}{*}{ District } & & \multirow[t]{2}{*}{ Areal Main Village } & \multirow[t]{2}{*}{ Village } & \multirow{2}{*}{$\begin{array}{c}\text { Cultivated } \\
\left.\text { Land (Jerib }{ }^{*}\right)\end{array}$} & \multicolumn{2}{|c|}{ Poppy Cultivation } & \multirow{2}{*}{$\begin{array}{c}\text { Yield } \\
(\mathrm{kg} / \mathrm{jr}) \\
\end{array}$} & \multirow{2}{*}{$\begin{array}{c}\text { Production } \\
(\mathrm{kg}) \\
\end{array}$} \\
\hline & & & & & (jerib) & $(\%)$ & & \\
\hline \multicolumn{9}{|c|}{ Province: Qandahar } \\
\hline GHORAK & 9563 & HASSAN ABAD & HASSAN ABAD & 300 & 80 & 27 & 9 & 720 \\
\hline GHORAK & 9606 & JALAT KHAN KALAY & JALAT KHAN KALAY & 120 & 20 & 17 & 9 & 180 \\
\hline GHORAK & 9568 & JALO & JALO & 50 & 5 & 10 & 8 & 40 \\
\hline GHORAK & 9562 & JAN ABAD KAREZ & JAN ABAD KAREZ & 400 & 100 & 25 & 9 & 900 \\
\hline GHORAK & 9571 & KAIKAK & KAIKAK & 550 & 100 & 18 & 10 & 1,000 \\
\hline GHORAK & 9593 & KAKAR KAREZ & KAKAR KAREZ & 200 & 20 & 10 & 8 & 160 \\
\hline GHORAK & 9582 & KARKOSHAM KAREZ & KARKOSHAM KAREZ & 250 & 60 & 24 & 8 & 480 \\
\hline GHORAK & 9555 & KOCHONAI KAREZ & KOCHONAI KAREZ & 25 & 10 & 40 & 9 & 90 \\
\hline GHORAK & 12178 & KOCHONAI KAREZ/ALL.M & KOCHONAI KAREZ/ALL.M & 10 & 10 & 100 & 4. & 40 \\
\hline GHORAK & 9573 & KOHNA KAREZ & KOHNA KAREZ & 100 & 25 & 25 & 8. & 200 \\
\hline GHORAK & 9707 & LAKHSHAK & LAKHSHAK & 400 & 25 & 6 & 8 & 200 \\
\hline GHORAK & 9607 & LAKHSHAK & LAKHSHAK & 400 & 25 & 6 & 8 & 200 \\
\hline GHORAK & 9586 & LAM KAREZ & LAM KAREZ & 600 & 30 & 5 & 9. & 270 \\
\hline GHORAK & 11145 & LASHKARY-JAFARY & LASHKARY-JAFARY & 100 & 30 & 30 & 8 & 240 \\
\hline GHORAK & 9575 & LOWARSHAH KAREZ & LOWARSHAH KAREZ & 300 & 60 & 20 & 8 & 480 \\
\hline GHORAK & 9613 & MALMAND & MALMAND & 1,500 & 400 & 27 & 9 & 3,600 \\
\hline GHORAK & 9585 & MANGAL/MALOK KAREZ & MALOK KAREZ & 200 & 15 & 8 & 8 & 120 \\
\hline GHORAK & 9591 & MOHABAT KAREZ & MOHABAT KAREZ & 300 & 40 & 13 & 8 & 320 \\
\hline GHORAK & 9605 & MOHD AMIN KALAY & MOHD AMIN KALAY & 200 & 10 & 5 & 8 & 80 \\
\hline GHORAK & 9615 & NALA KAREZ & NALA & 200 & 50 & 25 & 16 & 800 \\
\hline GHORAK & 9559 & OWESHAN & KHAT MALIK KAREZ & 900 & 150 & 17 & 9 & 1,350 \\
\hline GHORAK & 9574 & PER QADEM & DEH SEZI KAREZONA & 400 & 25 & 6 & 8 & 200 \\
\hline GHORAK & 9554 & QANAT LATIF KHAN & QANAT LATIF KHAN & 100 & 25 & 25 & 16 & 400 \\
\hline GHORAK & 9602 & QATA KAREZ SANG & QATA KAREZ SANG & 400 & 30 & 8 & 10 & 300 \\
\hline GHORAK & 9592 & RORABAT KAREZ & RORABAT & 400 & 20 & 5 & 8 & 160 \\
\hline GHORAK & 9621 & SAM ANZAR & SAM ANZAR & 100 & 50 & 50 & 8 & 400 \\
\hline GHORAK & 9579 & SANGINAK & SANGINAK & 200 & 20 & 10 & 8 & 160 \\
\hline GHORAK & 9590 & SARI DAGH KAREZONA & SARI DAGH KAREZONA & 200 & 20 & 10 & 8 & 160 \\
\hline GHORAK & 9561 & SEKANDAR KAREZ & SEKANDAR & 400 & 200 & 50 & 18 & 3,600 \\
\hline
\end{tabular}

* Jerib (jr.) $=1 / 5$ ha

* 2Dry Opium $=$ Wet Opium - 30\% moisture 
Opium Production Survey by village in Qandahar province, 1995.

\begin{tabular}{|c|c|c|c|c|c|c|c|c|}
\hline \multirow{3}{*}{ District } & \multirow{3}{*}{\begin{tabular}{|l|} 
Village \\
Code \\
\end{tabular}} & \multirow{3}{*}{ Area/ Main Village } & \multirow{3}{*}{ Village } & \multirow{3}{*}{$\begin{array}{c}\text { Total } \\
\text { Cultivated } \\
\text { Land }\left(\text { Jerib }^{*}\right) \\
\end{array}$} & \multirow{2}{*}{\multicolumn{2}{|c|}{$\begin{array}{c}\text { Land Under } \\
\text { Poppy Cultivation }\end{array}$}} & \multirow{3}{*}{$\begin{array}{c}\text { Wet Opium }{ }^{* 2} \\
\text { Yield } \\
(\mathrm{kg} / \mathrm{jr}) \\
\end{array}$} & \multirow{3}{*}{$\begin{array}{c}\text { Wet Opium } \\
\text { Production } \\
(\mathrm{kg})\end{array}$} \\
\hline & & & & & & & & \\
\hline & & & & & (jerib) & $(\%)$ & & \\
\hline \multicolumn{9}{|c|}{ Province: Qandahar } \\
\hline GHORAK & 9553 & SHORAB & SHORAB & 40 & 10 & 25 & 9 & 90 \\
\hline GHORAK & 9549 & SORAT KHAN KAREZ & CHAH HIDAR KAREZ & 200 & 60 & 30 & 9 & 540 \\
\hline GHORAK & 9580 & SORKH ABAD & SORKH ABAD & 500 & 80 & 16 & 8 & 640 \\
\hline GHORAK & 9589 & SORPOTI MULLA GHUBAR & MULLA GHUBAR CHINA & 200 & 20 & 10 & 8 & 160 \\
\hline GHORAK & 9551 & SPENKAI & SPENKAI & 125 & 40 & 32 & 4 & 160 \\
\hline GHORAK & 9588 & SUFILAM KAREZ & SUFILAM KAREZ & 370 & 25 & 7 & 8 & 200 \\
\hline GHORAK & 12193 & SURKH BEAD & KAKOZAI & 30 & 30 & 100 & 9 & 270 \\
\hline GHORAK & 11142 & WANY & WANY & 250 & 50 & 20 & 8 & 400 \\
\hline GHORAK & 11144 & YAKHTOL & YAKHTOL & 600 & 100 & 17 & 9 & 900 \\
\hline GHORAK & 9552 & ZAR ALI & SAM ENZAR KAREZ & 50 & 25 & 50 & 18 & 450 \\
\hline GHORAK & 9560 & ZARD REGAI & ZARD REGAI & 200 & 20 & 10 & 8 & 160 \\
\hline KHAKREZ & 9412 & ALAM KAREZ & ALAM KAREZ & 300 & 45 & 15 & 8 & 360 \\
\hline KHAKREZ & 9415 & ALI KAREZ & ALI KAREZ & 450 & 30 & 7 & 9 & 270 \\
\hline KHAKREZ & 9383 & AROOQ & BAHMAT KAREZ & 300 & 8 & 3 & 9 & 72 \\
\hline KHAKREZ & 9628 & ASAD KAREZ & ASAD KAREZ & 750 & 30 & 4 & 9 & 270 \\
\hline KHAKREZ & 10006 & AZMAT KAREZ & AZMAT KAREZ & 30 & 0 & 0 & 0 & 0 \\
\hline KHAKREZ & 9630 & BAGHAK,KESHATA BAGHA & BAGHAK,KESHATA BAGHA & 1,250 & 30 & 2 & 8 & 240 \\
\hline KHAKREZ & 9629 & BAGHAKA & BARAN KHELO KAREZ & 1,700 & 20 & 1 & 8 & 160 \\
\hline KHAKREZ & 11205 & BAGHAKA & CHINA & 650 & 20 & 3 & 8 & 160 \\
\hline KHAKREZ & 10009 & BAREKEY & ANARAK/BEKH & 100 & 8 & 8 & 5 & 40 \\
\hline KHAKREZ & 10012 & BAZHANDOKAI & FETAWAY KAREZUN & 30 & 0 & 0 & 0 & 0 \\
\hline KHAKREZ & 10005 & BE BE GAWHARA & BEBE GAWHARA/KA & 30 & 0 & 0 & 0 & 0 \\
\hline KHAKREZ & 9620 & BOOM & H.M.USMAN KALAY & 50 & 0 & 0 & 0 & 0 \\
\hline KHAKREZ & 9626 & BORA GANA & BORA GANA & 50 & 5 & 10 & 8 & 40 \\
\hline KHAKREZ & 10010 & CHAGAI & CHAGAI & 260 & 20 & 8 & 9 & 180 \\
\hline KHAKREZ & 9406 & CHAR SANG KALAN & CHAR SANG KALAN & 700 & 30 & 4 & 8 & 240 \\
\hline KHAKREZ & 11206 & CHARBAND & CHARBAND & 500 & 20 & 4 & 8 & 160 \\
\hline KHAKREZ & 9405 & CHARSANG KHURD & CHARSANG KHURD & 500 & 50 & 10 & 9 & 450 \\
\hline KHAKREZ & 9623 & CHINAGAY,ZIARAT SHAH & CHINAGAY,ZIARAT SHAH & 20 & 0 & 0 & 0 & 0 \\
\hline
\end{tabular}

* Jerib (jr.) $=1 / 5$ ha

* 2 Dry Opium $=$ Wet Opium $-30 \%$ moisture 
Opium Production Survey by village in Qandahar province, 1995.

\begin{tabular}{|c|c|c|c|c|c|c|c|c|}
\hline & Village & . & & Total & Land & & Wet Opium *2 & Wet Opium \\
\hline \multirow[t]{2}{*}{ District } & Code & Area/ Main Village & Village & Cultivated & \multicolumn{2}{|c|}{ Poppy Cultivation } & Yield & Production \\
\hline & & & & Land $\left(\right.$ Jerib $\left.^{*}\right)$ & (jerib) & (\%) & $(\mathrm{kg} / \mathrm{jr})$ & $(\mathrm{kg})$ \\
\hline \multicolumn{9}{|c|}{ Province: Qandahar } \\
\hline KHAKREZ & 10007 & CHINAR & ZAHIR CHINA & 150 & 10 & 7 & 9 & 90 \\
\hline KHAKREZ & 9397 & CHINAR KALAY & CHINAR KALAY & 150 & 15 & 10 & 8 & 120 \\
\hline KHAKREZ & 11120 & DAB KHAKREZ & HAJI KAL & 200 & 30 & 15 & 9 & 270 \\
\hline KHAKREZ & 11119 & DAB KHAKREZ/KHOSHAI & KHOHAI & 300 & 50 & 17 & 9 & 450 \\
\hline KHAKREZ & 9400 & DARWISHAN & KAREZAK/ANARAK & 800 & 30 & 4 & 8 & 240 \\
\hline KHAKREZ & 9381 & DOKHANA & DOKHANA & 2,000 & 20 & 1 & 7 & 140 \\
\hline KHAKREZ & 9220 & EAHAQ ZAI & LOY KALAY & 30 & 0 & 0 & 0 & 0 \\
\hline KHAKREZ & 10011 & GHABARGAI & GHABARGAI & 10 & 0 & 0 & 0 & 0 \\
\hline KHAKREZ & 9392 & GHULAMANO KAREZ & GHULAMANO KAREZ & 200 & 50 & 25 & 6 & 300 \\
\hline KHAKREZ & 9411 & KESHATA LOET SHARQA & KESHATA LOET SHARQA & 300 & 40 & 13 & 9 . & 360 \\
\hline KHAKREZ & 9384 & KHUSHDAD & KHUSHDAD & 300 & 50 & 17 & 7. & 350 \\
\hline KHAKREZ & 9414 & KIDO KAREZ & KIDO KAREZ & 1,100 & 60 & 5 & 9 & 540 \\
\hline KHAKREZ & 9380 & LETY & JUSHAN KAREZ & 600 & 100 & 17 & 9. & 900 \\
\hline KHAKREZ & 9390 & MER ABAD & MER ABAD & 300 & 50 & 17 & 9 . & 450 \\
\hline KHAKREZ & 10014 & NASAR KAREZ & NASAR KAREZ & 600 & 20 & 3 & 9. & 180 \\
\hline KHAKREZ & 9391 & NAW ABAD & NAW ABAD & 400 & 60 & 15 & 9 & 540 \\
\hline KHAKREZ & 9385 & SAFED KHAK & SAFED KHAK & 200 & 10 & 5 & 9 & 90 \\
\hline KHAKREZ & 9624 & SAIDOM & SAIDOM & 60 & 5 & 8 & 8 & 40 \\
\hline KHAKREZ & 9379 & SANG REZ/TANG REZ & SANG REZ/TANG REZ & 150 & 5 & 3 & 5 & 25 \\
\hline KHAKREZ & 9622 & SARI KHAK & SARI KHAK & 60 & 10 & 17 & 8 & 80 \\
\hline KHAKREZ & 9377 & SARKANAY KAREZ & SARKANAY KAREZ & 410 & 10 & 2 & 5 & 50 \\
\hline KHAKREZ & 9401 & SARPOSH & SARPOSH & 400 & 100 & 25 & 8 & 800 \\
\hline KHAKREZ & 9393 & SHAHO KHAN KAREZ & SHAHO KHAN KAREZ & 300 & 50 & 17 & 9 & 450 \\
\hline KHAKREZ & 9409 & SHERUDIN & SHERUDIN & 120 & 40 & 33 & 9 & 360 \\
\hline KHAKREZ & 9627 & SHUTUR GARDAN & SHUTUR GARDAN & 120 & 10 & 8 & 8 & 80 \\
\hline KHAKREZ & 9387 & SIAD KAREZ KALAY & SIAD KAREZ KALAY & 300 & 30 & 10 & 8 & 240 \\
\hline KHAKREZ & 9376 & SOZAL KAREZ & SALAMAK & 250 & 50 & 20 & 9 & 450 \\
\hline KHAKREZ & 9386 & TAIQ KAREZ & TAIQ KAREZ & 700 & 100 & 14 & 9 & 900 \\
\hline KHAKREZ & 10004 & TAMBIL CHINA & TAMBIL CHINA & 300 & 10 & 3 & 8 & 80 \\
\hline
\end{tabular}


Opium Production Survey by village in Qandahar province, 1995.

\begin{tabular}{|c|c|c|c|c|c|c|c|c|}
\hline \multirow{3}{*}{ District } & \multirow{3}{*}{\begin{tabular}{|l|} 
Village \\
Code \\
\end{tabular}} & \multirow{3}{*}{ Area/ Main Village } & \multirow{3}{*}{ Village } & \multirow{3}{*}{$\begin{array}{c}\text { Total } \\
\text { Cultivated } \\
\text { Land }\left(\text { Jerib }^{*}\right) \\
\end{array}$} & \multirow{2}{*}{\multicolumn{2}{|c|}{$\begin{array}{c}\text { Land Under } \\
\text { Poppy Cultivation }\end{array}$}} & \multirow{3}{*}{$\begin{array}{c}\text { Wet Opium *2 } \\
\text { Yield } \\
(\mathrm{kg} / \mathrm{jr})\end{array}$} & \multirow{3}{*}{$\begin{array}{c}\text { Wet Opium } \\
\text { Production } \\
(\mathrm{kg})\end{array}$} \\
\hline & & & & & & & & \\
\hline & & & & & (jerib) & $(\%)$ & & \\
\hline \multicolumn{9}{|c|}{ Province: Qandahar } \\
\hline KHAKREZ & 9407 & ZAIL ABAD & ZAIL ABAD & 500 & 40 & 8 & 9 & 360 \\
\hline MAIWAND & 9021 & AKHTARE & AKHTARE & 30 & 0 & 0 & 0 & 0 \\
\hline MAIWAND & 9467 & AZAM JAN KAREZ & AZAM JAN KAREZ & 250 & 20 & 8 & 9 & 180 \\
\hline MAIWAND & 9306 & BAND-E-TEMOR & AGHA KAREZ,KHAN KHEL & 250 & 40 & 16 & 9 & 360 \\
\hline MAIWAND & 9311 & BAND-E-TEMOR & BALOOLZAI & 400 & 0 & 0 & 0 & 0 \\
\hline MAIWAND & 9310 & BAND-E-TEMOR & H.KHEL & 60 & 10 & 17 & 8 & 80 \\
\hline MAIWAND & 9441 & BAND-E-TEMOR & LAL M. KAREZ,BARANG & 180 & 16 & 9 & 8 & 128 \\
\hline MAIWAND & 9018 & BAND-E-TEMOR & LALAZI & 30 & 0 & 0 & 0 & 0 \\
\hline MAIWAND & 9440 & BAND-E-TEMOR & LANDI & 300 & 0 & 0 & 0 & 0 \\
\hline MAIWAND & 9307 & BAND-E-TEMOR & MALI KHEL & 100 & 0 & 0 & 0 & 0 \\
\hline MAIWAND & 9309 & BAND-E-TEMOR & NOKAR KHEL & 200 & 0 & 0 & 0 & 0 \\
\hline MAIWAND & 9319 & BAND-E-TEMOR & SHALI & 1,000 & 0 & 0 & 0 & 0 \\
\hline MAIWAND & 9305 & BAND-E-TEMOR & ZAR ZADA, KAIKAK & 400 & 0 & 0 & 0 & 0 \\
\hline MAIWAND & 9312 & BAND-E-TIMOR & MAHMOOD KHEL & 200 & 70 & 35 & 7 & 490 \\
\hline MAIWAND & 9487 & BARAKZAI & BARAKZAI & 300 & 0 & 0 & 0 & 0 \\
\hline MAIWAND & 9011 & BAZAR CHIL GUDY & BAZAR CHIHIL GAZAI & 300 & 8 & 3 & 11 & 88 \\
\hline MAIWAND & 9253 & BULAGH & LANDI & 180 & 10 & 6 & 10 & 100 \\
\hline MAIWAND & 9469 & CHAR SHAKHA & CHAR SHAKHA & 400 & 17 & 4 & 10 & 170 \\
\hline MAIWAND & 9316 & CHASHMA & WALIWAL,ACHAKZAI & 115 & 0 & 0 & 0 & 0 \\
\hline MAIWAND & 9315 & CHASHMA/BABEYAN & KAKARAN,KHAROTAY KAR & 130 & 0 & 0 & 0 & 0 \\
\hline MAIWAND & 9314 & CHASHMA/BABRAN & ABDULAH,M.HASHIM NAI & 100 & 0 & 0 & 0 & 0 \\
\hline MAIWAND & 9011 & CHEL GAZI & CHEL GAZI & 300 & 8 & 3 & 9 & 72 \\
\hline MAIWAND & 9452 & CHINA GARMABAK & MASJID TIPA & 420 & 24 & 6 & 10 & 240 \\
\hline MAIWAND & 9459 & CHOSHMA/BAYZI HAMID & MAMA KAREZ,BAYZI HA. & 70 & 0 & 0 & 0 & 0 \\
\hline MAIWAND & 9320 & DARWEZA & MANSOOR & 130 & 0 & 0 & 0 & 0 \\
\hline MAIWAND & 9489 & DIWAR & DIWAR & 200 & 20. & 10 & 8 & 160 \\
\hline MAIWAND & 9475 & $\mathrm{DOO} \mathrm{AB}$ & KANZI & 140 & 0 & 0 & 0 & 0 \\
\hline MAIWAND & 9449 & GARMABAK & DOKA & 240 & 14 & 6 & 10 & 140 \\
\hline MAIWAND & 9447 & GARMABAK & IRAN ZANGITAN & 288 & 18 & 6 & 10 & 180 \\
\hline
\end{tabular}

* Jerib (jr.) $=1 / 5$ ha

* 2 Dry Opium $=$ Wet Opium $-30 \%$ moisture 
Opium Production Survey by village in Qandahar province, 1995.

\begin{tabular}{|c|c|c|c|c|c|c|c|c|}
\hline \multirow{3}{*}{ District } & \multirow{3}{*}{\begin{tabular}{|l|} 
Village \\
Code
\end{tabular}} & \multirow{3}{*}{ Area/ Main Village } & \multirow{3}{*}{ Village } & \multirow{3}{*}{$\begin{array}{c}\text { Total } \\
\text { Cultivated } \\
\text { Land }\left(\text { Jerib }^{*}\right) \\
\end{array}$} & \multirow{2}{*}{\multicolumn{2}{|c|}{$\begin{array}{c}\text { Land Under } \\
\text { Poppy Cultivation }\end{array}$}} & \multirow{3}{*}{$\begin{array}{c}\text { Wet. Opium }^{* 2} \\
\text { Yield } \\
(\mathrm{kg} / \mathrm{jr}) \\
\end{array}$} & \multirow{3}{*}{$\begin{array}{c}\text { Wet Opium } \\
\text { Production } \\
(\mathrm{kg}) \\
\end{array}$} \\
\hline & & & & & & & & \\
\hline & & & & & (jerib) & $(\%)$ & & \\
\hline \multicolumn{9}{|c|}{ Province: Qandahar } \\
\hline MAIWAND & 3445 & GARMABAK & KORKING ALIABAD & 450 & 10 & 2 & 9 & 90 \\
\hline MAIWAND & 9450 & GARMABAK & ZAREKHAR,NEZADA,EKHT & 286 & 17 & 6 & 10 & 170 \\
\hline MAIWAND & 9442 & GARMAWAK & M.RAREEM,MULA NAIM & 720 & 40 & 6 & 11 & 440 \\
\hline MAIWAND & 9460 & HJ.HINO KASA & NAWI KASA ATTA .M. & 300 & 0 & 0 & 0 & 0 \\
\hline MAIWAND & 9461 & JAW GARM & ABDUL RAHMAN & 600 & 100 & 17 & 9 & 900 \\
\hline MAIWAND & 9443 & KALA KHAN KALAY & SHAH M.KAREZ & 90 & 18 & 20 & 10 & 180 \\
\hline MAIWAND & 9317 & KALAWAL,CHASHMA & SHAH KAREZ,DARY KARE & 150 & 0 & 0 & 0 & 0 \\
\hline MAIWAND & 9476 & KHAMERI KISHATA SOBA & KHAMERI KISHATA SOBA & 240 & 0 & 0 & 0 & 0 \\
\hline MAIWAND & 9444 & KHANABAD, NAWABAD & OMAR KAREZ & 144 & 9 & 6 & 10 & 90 \\
\hline MAIWAND & 9488 & KHASHTA BALOOCHAN & HAJI HASHEM & 600 & 40 & 7 & 9 & 360 \\
\hline MAIWAND & 9008 & KISHK-E-NAKHOD & AZIZ ABAD & 200 & 20 & 10 & 10 & 200 \\
\hline MAIWAND & 9022 & KISHK-E-NAKHOD & BIA BANAK,MAKO & 100 & 0 & 0 & 0 & 0 \\
\hline MAIWAND & 9007 & KISHK-E-NAKHOD & ESHQ ABAD & 200 & 7 & 4 & 12 & 84 \\
\hline MAIWAND & 9027 & KISHK-E-NAKHOD & HASSAN ABAD & 280 & 12 & 4 & 10 & 120 \\
\hline MAIWAND & 9004 & KISHK-E-NAKHOD & KHAWJA AHMAD MAIWAND & 480 & 28 & 6 & 11 & 308 \\
\hline MAIWAND & 9005 & KISHK-E-NAKHOD & KHIG MANDABAD & 270 & 17 & 6 & 11 & 187 \\
\hline MAIWAND & 9024 & KISHK-E-NAKHOD & KHUDAIDAD NAWAB & 100 & 0 & 0 & 0 & 0 \\
\hline MAIWAND & 9002 & KISHK-E-NAKHOD & LALAK & 270 & 16 & 6 & 9 & 144 \\
\hline MAIWAND & 9028 & KISHK-E-NAKHOD & LOY KAREZ & 100 & 0 & 0 & 0 & 0 \\
\hline MAIWAND & 9012 & KISHK-E-NAKHOD & M.MOSEH & 400 & 0 & 0 & 0 & 0 \\
\hline MAIWAND & 9003 & KISHK-E-NAKHOD & MAIWAND KAREZ & 32 & 23 & 72 & 10 & 230 \\
\hline MAIWAND & 11102 & KISHK-E-NAKHOD & MASJID SHAH MOHD & 1,000 & 20 & 2 & 10 & 200 \\
\hline MAIWAND & 9010 & KISHK-E-NAKHOD & MAZREAA & 360 & 13 & 4 & 10 & 130 \\
\hline MAIWAND & 9006 & KISHK-E-NAKHOD & MOSHAK,KAREZAK & 160 & 16 & 10 & 11 & 176 \\
\hline MAIWAND & 9014 & KISHK-E-NAKHOD & NASO GACH KAREZ & 380 & 40 & 11 & 7 & 280 \\
\hline MAIWAND & 9025 & KISHK-E-NAKHOD & PERZADA & 280 & 16 & 6 & 9 & 144 \\
\hline MAIWAND & 9013 & KISHK-E-NAKHOD & SAFOZAI & 200 & 0 & 0 & 0 & 0 \\
\hline MAIWAND & 9029 & KISHK-E-NAKHOD & SARWAR KAREZ & 230 & 0 & 0 & 0 & 0 \\
\hline MAIWAND & 9023 & KISHK-E-NAKHOD & SHAKOOR, LANDAY & 150 & 0 & 0 & 0 & 0 \\
\hline
\end{tabular}

* Jerib (jr.) $=1 / 5$ ha

* 2 Dry Opium $=$ Wet Opium - $30 \%$ moisture 
Opium Production Survey by village in Qandahar province, 1995.

\begin{tabular}{|c|c|c|c|c|c|c|c|c|}
\hline \multirow{3}{*}{ District } & \multirow{3}{*}{$\begin{array}{l}\text { Village } \\
\text { Code }\end{array}$} & \multirow{3}{*}{ Area/ Main Village } & \multirow{3}{*}{ Village } & \multirow{3}{*}{$\begin{array}{c}\text { Total } \\
\text { Cultivated } \\
\text { Land }\left(\text { Jerib }^{*}\right) \\
\end{array}$} & \multirow{2}{*}{\multicolumn{2}{|c|}{$\begin{array}{c}\text { Land Under } \\
\text { Poppy Cultivation }\end{array}$}} & \multirow{3}{*}{$\begin{array}{c}\text { Wet Opium }^{* 2} \\
\text { Yield } \\
(\mathrm{kg} / \mathrm{jr}) \\
\end{array}$} & \multirow{3}{*}{$\begin{array}{c}\text { Wet Opium } \\
\text { Production } \\
(\mathrm{kg}) \\
\end{array}$} \\
\hline & & & & & & & & \\
\hline & & & & & (jerib) & $(\%)$ & & \\
\hline \multicolumn{9}{|c|}{ Province: Qandahar } \\
\hline MAIWAND & 9001 & KISHK-E-NAKHOD & ZARAK & 200 & 25 & 13 & 9 & 225 \\
\hline MAIWAND & 11101 & KISHK-E-NAKHOD & ZEBANKA & 120 & 12 & 10 & 11 & 132 \\
\hline MAIWAND & $9026^{\circ}$ & KISHKI-E-NAKHOD & KHAWJA KHALIQ & 540 & 17 & 3 & 11 & 187 \\
\hline MAIWAND & 9009 & KISHKI-E-NAKHOD & MORCHA & 240 & 12 & 5 & 10 & 120 \\
\hline MAIWAND & 9028 & LOY KAREZ & LOY KAREZ & 360 & 13 & 4 & 10 & 130 \\
\hline MAIWAND & 9474 & MANDOZI & MAGAT & 450 & 0 & 0 & 0 & 0 \\
\hline MAIWAND & 9451 & MASHJID ABAD & YASIN & 320 & 6 & 2 & 11 & 66 \\
\hline MAIWAND & 9458 & MASOOM KAREZ,SANGBOR & SHAH RAG, BAIDAK & 141 & 14 & 10 & 9 & 126 \\
\hline MAIWAND & 9445 & MERA KHOR & MERA KHOR & 600 & 10 & 2 & 8 & 80 \\
\hline MAIWAND & 9480 & MIR KHANZI & MIR KHANZI & 1,000 & 100 & 10 & 10 & 1,000 \\
\hline MAIWAND & 9017 & MIS KAREZ & GHANICHI & 60 & 20 & 33 & 8 & 160 \\
\hline MAIWAND & 9015 & MIS KAREZ & KHOGYANI,NASRULLAH J & 150 & 18 & 12 & 8 & 144 \\
\hline MAIWAND & 9016 & MIS KAREZ & QAZI KAREZ & 466 & 14 & 3 & 10 & 140 \\
\hline MAIWAND & 9478 & NAHR KAREZ & AOTOMANZI & 350 & 0 & 0 & 0 & 0 \\
\hline MAIWAND & 9479 & NAHR KAREZ & ASGARE,ACHAKZAI & 400 & 0 & 0 & 0 & 0 \\
\hline MAIWAND & 9306 & OSMAN KHAIL & OSMAN KHAIL & 100 & 10 & 10 & 8 & 80 \\
\hline MAIWAND & 9494 & PATINZAI/SARTAK & PANIZAI & 200 & 20 & 10 & 8 & 160 \\
\hline MAIWAND & 9463 & QALA SHAH MEER & JAW KARI & 500 & 50 & 10 & 7 & 350 \\
\hline MAIWAND & 9462 & QALA SHAH MEER & PAI KEELA & 400 & 50 & 13 & 8 & 400 \\
\hline MAIWAND & 9465 & QALA SHAH MEER & SHALGHAMI & 600 & 100 & 17 & 7 & 700 \\
\hline MAIWAND & 9464 & QALA SHAH MEER & SHYKHANZAI & 600 & 20 & 3 & 9 & 180 \\
\hline MAIWAND & 11174 & QALAI SHAH MEER & SPEEN KANY & 300 & 30 & 10 & 7 & 210 \\
\hline MAIWAND & 9468 & RANGREZAN & KAREZ SALEEM & 200 & 16 & 8 & 7 & 112 \\
\hline MAIWAND & 9481 & SANG HESAR & GHONDI & 1,300 & 130 & 10 & 8 & 1,040 \\
\hline MAIWAND & 9484 & SANG HESAR & LAKO KHAIL & 3,600 & 0 & 0 & 0 & 0 \\
\hline MAIWAND & 9483 & SANG HESAR & LOWAR BALOCHAN & 160 & 0 & 0 & 0 & 0 \\
\hline MAIWAND & 9486 & SANG HESAR & MISIZI & 450 & 0 & 0 & 0 & 0 \\
\hline MAIWAND & 9485 & SANG HESAR & MOHAJEREEN & 200 & 0 & 0 & 0 & 0 \\
\hline MAIWAND & 9482 & SANG HESAR & QALACHA & 730 & 0 & 0 & 0 & 0 \\
\hline
\end{tabular}

* Jerib (jr.) $=1 / 5$ ha

${ }^{*}{ }^{2}$ Dry Opium $=$ Wet Opium $-30 \%$ moisture 
Opium Production Survey by village in Qandahar province, 1995.

\begin{tabular}{|c|c|c|c|c|c|c|c|c|}
\hline \multirow{3}{*}{ District } & \multirow{3}{*}{$\begin{array}{l}\text { Village } \\
\text { Code }\end{array}$} & \multirow{3}{*}{ Area/ Main Village } & \multirow{3}{*}{ Village } & \multirow{3}{*}{$\begin{array}{c}\text { Total } \\
\text { Cultivated } \\
\left.\text { Land (Jerib }{ }^{*}\right)\end{array}$} & \multirow{2}{*}{\multicolumn{2}{|c|}{$\begin{array}{c}\text { land l/nder } \\
\text { Poppy ('ultivation }\end{array}$}} & \multirow{3}{*}{$\begin{array}{c}\text { Wet Opium *? } \\
\text { Yield } \\
(\mathrm{kg} / \mathrm{jr}) \\
\end{array}$} & \multirow{3}{*}{$\begin{array}{l}\text { Wet Opium } \\
\text { Production } \\
\text { (kg) }\end{array}$} \\
\hline & & & & & & & & \\
\hline & & & & & (jerih) & $(\%)$ & & \\
\hline \multicolumn{9}{|c|}{ Province: Qandahar } \\
\hline MAIWAND & 9456 & SANGBOR & AMANAT BABA,KOLPAK & 293 & 20 & 7 & 15 & 300 \\
\hline MAIWAND & 9454 & SANGBOR & KHALIZADA,GARM AAB & 140 & 7 & 5 & 12 & 84 \\
\hline MAIWAND & 9455 & SANGBOR & PADA,TORA SHAH & 64 & 7 & 10 & 11 & 72 \\
\hline MAIWAND & 9457 & SANGBOR & SANGBOR & 140 & 0 & 0 & 0 & 0 \\
\hline MAIWAND & 9489 & SANGHISAR & DEWAR & 600 & 13 & 2 & 8 & 104 \\
\hline MAIWAND & 9495 & SARTAK & ADOZI & 400 & 30 & 8 & 9 & 270 \\
\hline MAIWAND & 9497 & SARTAK & ALIF KHAIL & 300 & 0 & 0 & 0 & 0 \\
\hline MAIWAND & 9493 & SARTAK & ARDOZI & 300 & 60 & 20 & 9 & 540 \\
\hline MAIWAND & 9499 & SARTAK & BABIYAN & 300 & 0 & 0 & 0 & 0 \\
\hline MAIWAND & 9501 & SARTAK & GULANO QALACHA & 330 & 0 & 0 & 0 & 0 \\
\hline MAIWAND & 9498 & SARTAK & HANIF & 600 & 0 & 0 & 0 & 0 \\
\hline MAIWAND & 9496 & SARTAK & HAQ DAR & 160 & 0 & 0 & 0 & 0 \\
\hline MAIWAND & 9491 & SARTAK & KASHANI & 270 & 30 & 11 & 8 & 240 \\
\hline MAIWAND & 9502 & SARTAK & KHUDAY NAZAR KALAY & 370 & 0 & 0 & 0 & 0 \\
\hline MAIWAND & 9492 & SARTAK & MALANGIAN & 50 & 0 & 0 & 0 & 0 \\
\hline MAIWAND & 9500 & SARTAK & NAWY QALACHA & 300 & 0 & 0 & 0 & 0 \\
\hline MAIWAND & 9490 & SARTAK & ZARIF KHEL & 300 & 0 & 0 & 0 & 0 \\
\hline MAIWAND & 9471 & SHAHI KAREZ & SHAHI KAREZ & 150 & 0 & 0 & 0 & 0 \\
\hline MAIWAND & 9472 & SHIR ABAD & POPAL ZAI & 80 & 0 & 0 & 0 & 0 \\
\hline MAIWAND & 9466 & SHIR ALI KAREZ & SHIR ALI KAREZ & 900 & 40 & 4 & 10 & 400 \\
\hline MAIWAND & 9473 & $\mathrm{SO}(\mathrm{IIAT}$ & CIIIORCIIIORI: & 100 & 0 & 0 & 0 & 0 \\
\hline MAIWAND & 9446 & TOLAK & TOLAK & 168 & 12 & 7 & 10 & 120 \\
\hline MAIWAND & 9313 & TOOR NAIKA & TOOR NIKA KAREZ,NOOR & 80 & 0 & 0 & 0 & 0 \\
\hline MAIWAND & 9504 & WAZIRAN & PAS AOB & 500 & 0 & 0 & 0 & 0 \\
\hline MAIWAND & 9503 & WAZIRAN & WAZIRAN & 520 & 0 & 0 & 0 & $?$ \\
\hline MAIWAND & 9448 & ZARD ALOO & ZARD ALOO & 720 & 20 & 3 & 11 & 220 \\
\hline MARUF & 9775 & AB.BAQI KHAN KALAY & AB.BAQI KHAN KALAY & 250 & 4 & 2 & 8 & 32 \\
\hline MARUF & 11204 & AHO CHESHMA & MIRO KHEL & 1,400 & 0 & 0 & 0 & 0 \\
\hline MARUF & 9777 & AKHTO KHEL & AKHTO KHEL & 80 & 4 & 4 & 9 & 32 \\
\hline
\end{tabular}


Opium Production Survey by village in Qandahar province, 1995.

\begin{tabular}{|c|c|c|c|c|c|c|c|c|}
\hline & Village & . & & Total & Land 1 & & Wet Opium *2 & Wet Opium \\
\hline \multirow[t]{2}{*}{ District } & Code & Area/ Main Village & Village & Cultivated & \multicolumn{2}{|c|}{ Poppy Cultivation } & Yield & Production \\
\hline & & & & Land (Jerib $\left.{ }^{*}\right)$ & (jerib) & $(\%)$ & $(\mathrm{kg} / \mathrm{jr})$ & $(\mathrm{kg})$ \\
\hline \multicolumn{9}{|c|}{ Province: Qandahar } \\
\hline MARUF & 9789 & ALI SHER & ALI SHER & 100 & 0 & 0 & 0 & 0 \\
\hline MARUF & 11203 & ALI ZAI & AZIM KAREZ & 100 & 0 & 0 & 0 & 0 \\
\hline MARUF & 9219 & ANA KHEL & SAIDAN & 500 & $\dot{0}$ & 0 & 0 & 0 \\
\hline MARUF & 11200 & ANGIR & YOLAR/TORA & 300 & 0 & 0 & 0 & 0 \\
\hline MARUF & 9781 & ANGO KHEL & KHALARTI, ANGO KHEL & 80 & 5 & 6 & 7 & 35 \\
\hline MARUF & 9214 & ARDO ZAI & GALANG & 200 & 0 & 0 & 0 & 0 \\
\hline MARUF & 11201 & AZIZ KHEL & DARA KHEL & 200 & 0 & 0 & 0 & 0 \\
\hline MARUF & 9779 & BAHLOL ZAI & SULAIMAN ZAI CHINA & 200 & 2 & 1 & 6 & 12 \\
\hline MARUF & 11189 & BAHLOL ZAI & TANGI & 400 & 0 & 0 & 0 & 0 \\
\hline MARUF & 9195 & BALKHERZAI & SAYEDAN & 2,000 & 5 & 0 & 8 & 40 \\
\hline MARUF & 9773 & BANOZAI & SAHRA PAMOZAI & 350 & 0 & 0 & 0 & 0 \\
\hline MARUF & 11191 & BARA KHEL & BATI/NEKOWALA/GHOTA. & 680 & 0 & 0 & 0 & 0 \\
\hline MARUF & 11202 & BARY & BARY & 600 & 0 & 0 & 0 & 0 \\
\hline MARUF & 9210 & BAZ MOHD KHAN & BAZ MOHD KHAN & 500 & 0 & 0 & 0 & 0 \\
\hline MARUF & 11193 & BESMELA & KHAROKI-M.ZAI SILIA & 70 & 0 & 0 & 0 & 0 \\
\hline MARUF & 9764 & BIANZI & BIANZI & 200 & 0 & 0 & 0 & 0 \\
\hline MARUF & 9192 & CHORMAI & ZIARAT TANGI & 1,250 & 15 & 1 & 5 & 68 \\
\hline MARUF & 9206 & DAAG & GHONDI & 1,800 & 5 & 0 & 6 & 30 \\
\hline MARUF & 9194 & DAGAI & BALANZAI & 450 & 0 & 0 & 0 & 0 \\
\hline MARUF & 9196 & DANGAR KAREZ & ALI SHER & 1,200 & 0 & 0 & 0 & 0 \\
\hline MARUF & 9211 & GADY & GADY & 250 & 0 & 0 & 0 & 0 \\
\hline MARUF & 9782 & GHARIB & GUL.M.WALGAI & 350 & 0 & 0 & 0 & 0 \\
\hline MARUF & 9761 & GIRDI & GIRDI & 160 & 0 & 0 & 0 & 0 \\
\hline MARUF & 9791 & GOJAN & GOJAN & 80 & 0 & 0 & 0 & 0 \\
\hline MARUF & 9205 & GORJIZAI & ZIRAK & 680 & 0 & 0 & 0 & 0 \\
\hline MARUF & 9223 & GOVEDA & MIRMAN KHEL & 250 & 0 & 0 & 0 & 0 \\
\hline MARUF & 9203 & HADO QALA & NAZAR KHAIL & 1,500 & 0 & 0 & 0 & 0 \\
\hline MARUF & 9202 & JAMROD & KHOSHBOYENA & 400 & 0 & 0 & 0 & 0 \\
\hline MARUF & 9784 & JUMMA KHEL & KHOJANZAI & 150 & 4 & 3 & 8 & 32 \\
\hline
\end{tabular}

* Jerib (jr.) $=1 / 5$ ha

* 2 Dry Opium $=$ Wet Opium $-30 \%$ moisture 
Opium Production Survey by village in Qandahar province, 1995.

\begin{tabular}{|c|c|c|c|c|c|c|c|c|}
\hline & Village & & . & Total & Land & & Wet Opium * 2 & Wet Opium \\
\hline \multirow[t]{2}{*}{ District } & \multirow[t]{2}{*}{ Code } & \multirow[t]{2}{*}{ Area/ Main Village } & \multirow[t]{2}{*}{ Village } & \multirow{2}{*}{$\begin{array}{c}\text { Cultivated } \\
\left.\text { Land (Jerib }{ }^{*}\right)\end{array}$} & \multicolumn{2}{|c|}{ Poppy Cultivation } & \multirow{2}{*}{$\begin{array}{c}\text { Yield } \\
(\mathrm{kg} / \mathrm{jr}) \\
\end{array}$} & \multirow{2}{*}{$\begin{array}{c}\text { Production } \\
\text { (kg) }\end{array}$} \\
\hline & & & & & (jerib) & (\%) & & \\
\hline \multicolumn{9}{|c|}{ Province: Qandahar } \\
\hline MARUF & 9778 & $\mathrm{KACH}$ & $\mathrm{KACH}$ & 250 & 0 & 0 & 0 & 0 \\
\hline MARUF & 9222 & KAZHKAI & MIGAI & 90 & 0 & 0 & 0 & 0 \\
\hline MARUF & 9783 & KAZHLICH & TAKHAM, RORWAM & 270 & 0 & 0 & 0 & 0 \\
\hline MARUF & 9772 & KHADER KHEL & KHADER KHEL & 300 & 0 & 0 & 0 & 0 \\
\hline MARUF & 9208 & KHOGYANI & KHOGYANI & 400 & 0 & 0 & 0 & 0 \\
\hline MARUF & 9207 & KHONDARA & LANDAI & 120 & 0 & 0 & 0 & 0 \\
\hline MARUF & $9217^{\circ}$ & KSHATA SHEKHZAI & TAKHAEE & 900 & 15 & 2 & 5 & 75 \\
\hline MARUF & 9212 & LALI KALAY & LALI KALAY & 80 & 0 & 0 & 0 & 0 \\
\hline MARUF & 9762 & LANDY & LANDY & 480 & 0 & 0 & 0 & 0 \\
\hline MARUF & 9213 & LOR SHEKHZAI & LOR SHEKHZAI & 300 & 0 & 0 & 0 & 0 \\
\hline MARUF & 9216 & LOWR MALAY & LOWR MALAY & 200 & 0 & 0 & 0 & 0 \\
\hline MARUF & 9188 & LOY & LOY & 700 & 0 & 0 & 0 & 0 \\
\hline MARUF & 9221 & LOY KINGER & KOCHNAI KINGAR & 400 & 0 & 0 & 0 & 0 \\
\hline MARUF & 9769 & MARGHAKAI KAREZ & MARGHAKAI KAREZ & 370 & 0 & 0 & 0 & 0 \\
\hline MARUF & 9774 & MASHOZAI & MASHOZAI & 1,200 & 0 & 0 & 0 & 0 \\
\hline MARUF & 9187 & MIAN & YAN & 200 & 0 & 0 & 0 & 0 \\
\hline MARUF & 11195 & MOCHUSHILA & BARY KAS & 300 & 3 & 1 & 8 & 24 \\
\hline MARUF & 9186 & MOHD ZAI SAR KHAIL & MOHD ZAI SAR KHAIL & 750 & 0 & 0 & 0 & 0 \\
\hline MARUF & 11194 & NARAY & NARY KALAY & 150 & 3 & 2 & 9 & 27 \\
\hline MARUF & 11190 & ODOZI & ODOZI & 200 & 1 & 0 & 9 & 5 \\
\hline MARUF & 9755 & PAYAND KHAN KALAY & NAKERZAI/BANIZI & 1,900 & 0 & 0 & 0 & 0 \\
\hline MARUF & 11198 & SADULLAH KHEL KALAY & SADULLAH KHAIL & 300 & 0 & 0 & 0 & 0 \\
\hline MARUF & 9224 & SAIFALZAI & SAIFALZAI & 320 & 3 & 1 & 6 & 18 \\
\hline MARUF & 9766 & SALAMAT TANA & SALAMAT TANA & 0 & 0 & \#DIV/0! & 0 & 0 \\
\hline MARUF & 9215 & SAMCHAK & CHINGARI & 150 & 2 & 1 & 8 & 16 \\
\hline MARUF & 9190 & SAYEDAN & BASAN KACH & 50 & 0 & 0 & 0 & 0 \\
\hline MARUF & 9189 & SEKANDAR ZAI & SOGNAI & 50 & 2 & 4 & 5 & 9 \\
\hline MARUF & 9780 & SERI & SERI & 60 & 0 & 0 & 0 & 0 \\
\hline MARUF & 9765 & SHADO TANGAI & SHADO TANGAI & 150 & 0 & 0 & 0 & 0 \\
\hline
\end{tabular}


Opium Production Survey by village in Qandahar province, 1995.

\begin{tabular}{|c|c|c|c|c|c|c|c|c|}
\hline . & Village & & . & Total & \multirow{2}{*}{\multicolumn{2}{|c|}{$\begin{array}{c}\text { Land Under } \\
\text { Poppy Cultivation }\end{array}$}} & \multirow{3}{*}{$\begin{array}{c}\text { Wet Opium }{ }^{* 2} \\
\text { Yield } \\
(\mathrm{kg} / \mathrm{jr})\end{array}$} & \multirow{3}{*}{$\begin{array}{c}\text { Wet Opium } \\
\text { Production } \\
(\mathrm{kg})\end{array}$} \\
\hline \multirow[t]{2}{*}{ District } & Code & Area/ Main Village & Village & Cultivated & & & & \\
\hline & & & & Land $\left(\right.$ Jerib $\left.^{*}\right)$ & (jerib) & $(\%)$ & & \\
\hline \multicolumn{9}{|c|}{ Province: Qandahar } \\
\hline MARUF & 9204 & SHAHI KHAIL & SHAHI KHAIL & 720 & 0 & 0 & 0 & 0 \\
\hline MARUF & 9776 & SHAKHGAI & SHAKHGAI & 200 & 2 & 1 & 8 & 16 \\
\hline MARUF & $11192^{\circ}$ & SHIN KHEL & SANDERZAI & 300 & 4 & 1 & 9 & 36 \\
\hline MARUF & 11197 & SINZELA & SRA KALAY-MULA MURGH & 200 & 0 & 0 & 0 & 0 \\
\hline MARUF & 9200 & SORWAM & ZALIRGAI/SORWAM & 200 & 0 & 0 & 0 & 0 \\
\hline MARUF & 11196 & TANG KAREZ & TANG KAREZ & 250 & 0 & 0 & 0 & 0 \\
\hline MARUF & 9771 & TOORKAN & TOORKAN & 160 & 0 & 0 & 0 & 0 \\
\hline MARUF & 9197 & WALGI PORTA GAR & WALGI PORTA GAR & 280 & 0 & 0 & 0 & 0 \\
\hline MARUF & 9757 & ZAGOMI & KALAGI & 400 & 0 & 0 & 0 & 0 \\
\hline MARUF & 9759 & ZERA GHBARGAI & ZERA GHBARGAI & 120 & 0 & 0 & 0 & 0 \\
\hline NESH & 12183 & ADO KALAY & ADO KALAY & 250 & 25 & 10 & 5 & 125 \\
\hline $\mathrm{NESH}$ & 9081 & BAGH AGHA & BAGH AGHA & 350 & 55 & 16 & 5 & 248 \\
\hline NESH & 9402 & BAGH KHALIL & BAGH KHALIL & 150 & 15 & 10 & 8 & 120 \\
\hline NESH & 9038 & BAHLOOL & NAW ABAD,WACH KAREZ & 105 & 10 & 10 & 5 & 50 \\
\hline NESH & 9030 & BALAGH GHABARGAI KAR & SHIN CHOSHMA,SARA CH & 223 & 5 & 2 & 4 & 18 \\
\hline NESH & 9046 & BAR KUNDI & BAR KUNDI & 550 & 40 & 7 & 6 & 220 \\
\hline NESH & 9067 & BARGHANA & BARGHANA & 225 & 15 & 7 & 5 & 68 \\
\hline $\mathrm{NESH}$ & 12180 & BARIKA & BARIKA & 470 & 10 & 2 & 6 & 55 \\
\hline NESH & 9091 & BAZAK & BAZAK & 172 & 40 & 23 & 6 & 240 \\
\hline $\mathrm{NESH}$ & 12182 & CHARGUMBAD & CHARGUMBAD & 350 & 60 & 17 & 6 & 360 \\
\hline NESH & 9066 & CHINARAK & CHINARAK & 215 & 20 & 9 & 4 & 70 \\
\hline $\mathrm{NESH}$ & 9053 & CHOPAN & CHOPAN & 560 & 60 & 11 & 6 & 330 \\
\hline $\mathrm{NESH}$ & 9083 & DARA NOOR & DARA NOOR & 960 & 140 & 15 & 6 & 840 \\
\hline NESH & 12181 & DARAH & SPIN JAR & 240 & 15 & 6 & 5 & 68 \\
\hline NESH & 9055 & DENAR KHEL & DENAR KHEL & 250 & 25 & 10 & 6 & 138 \\
\hline NESH & 9060 & GANDA KAREZ & GANDA KAREZ & 750 & 60 & 8 & 5 & 300 \\
\hline NESH & 9044 & GANDACHA & MUHYEDIN KAREZ,JALAT & 200 & 40 & 20 & 6 & 240 \\
\hline NESH & 9063 & GHANI KALAY ZARNI & GHANI KALAY ZARNI & 750 & 0 & 0 & 0 & 0 \\
\hline NESH & 9050 & GHOIMATAN & KANAT MULLA SHER & 360 & 0 & 0 & 0 & 0 \\
\hline
\end{tabular}

* Jerib (jr.) $=1 / 5$ ha

${ }^{*}$ Dry Opium $=$ Wet Opium $-30 \%$ moisture 
Opium Production Survey by village in Qandahar province, 1995.

\begin{tabular}{|c|c|c|c|c|c|c|c|c|}
\hline & Village & . & & Total & \multirow{2}{*}{\multicolumn{2}{|c|}{$\begin{array}{c}\text { Land Under } \\
\text { Poppy Cultivation }\end{array}$}} & \multirow{3}{*}{$\begin{array}{c}\text { Wet Opium *2 } \\
\text { Yield } \\
(\mathrm{kg} / \mathrm{jr})\end{array}$} & \multirow{3}{*}{$\begin{array}{c}\text { Wet Opium } \\
\text { Production } \\
(\mathrm{kg})\end{array}$} \\
\hline \multirow[t]{2}{*}{ District } & Code & Area/ Main Village & Village & Cultivated & & & & \\
\hline & & & & Land (Jerib *) & (jerib) & $(\%)$ & & \\
\hline \multicolumn{9}{|c|}{ Province: Qandahar } \\
\hline NESH & 9075 & HAJI ALAM & KAREZ KHOSHAK & 410 & 30 & 7 & 6 & 165 \\
\hline NESH & 9069 & JAILAN KAREZ & CHAR PALNAK/KAREZAK & 132 & 4 & 3 & 5 & 20 \\
\hline NESH & 9045 & JAMAK & JAMAK & 300 & 35 & 12 & 6 & 210 \\
\hline NESH & 9037 & KARAM KAREZ & DEWALAK, GHULAM M. & 105 & 17 & 16 & 5 & 77 \\
\hline NESH & 12184 & KAREZ GAI & KAREZ GAI & 50 & 6 & 12 & 5 & 30 \\
\hline NESH & 9048 & KAREZ-E- KHORD & GHURDANG & 630 & 30 & 5 & 6 & 165 \\
\hline $\mathrm{NESH}$ & 9032 & KAREZ-E-NAW & SUR KAREZ & 170 & 20 & 12 & 5 & 100 \\
\hline NESH & 9080 & KAREZAK/KAREZGAI & KAREZAK/KAREZGAI & 185 & 22 & 12 & 4 & 88 \\
\hline NESH & 12179 & KARM KAREZ & KARM KAREZ & 180 & 30 & 17 & 6 & 180 \\
\hline NESH & 9033 & KASHTKHANA,KASHKHANM & NAWA & 165 & 15 & 9 & 5 & 68 \\
\hline NESH & 9031 & KHAR TOOT & KHAR TOOT & 540 & 30 & 6 & 5 & 150 \\
\hline $\mathrm{NESH}$ & 9089 & KHINJAKAK & KHINJAKAK & 400 & 45 & 11 & 6 & 270 \\
\hline NESH & 9051 & KHORDEZI KAREZ & KHORDEZI KAREZ & 100 & 10 & 10 & 5 & 45 \\
\hline $\mathrm{NESH}$ & 9064 & KOOR BAZAK & KOOR BAZAK & 430 & 0 & 0 & 0 & 0 \\
\hline NESH & 9084 & LAWARGAI & LAWARGAI & 450 & 15 & 3 & 5 & 68 \\
\hline NESH & 9071 & LOWAR SABZAL & MULA HABIBULLAH & 150 & 30 & 20 & 6 & 180 \\
\hline $\mathrm{NESH}$ & 9034 & MEYAN KASHKHANMAK & NAWA & 310 & 24 & 8 & 5 & 120 \\
\hline $\mathrm{NESH}$ & 9072 & MEYEN SABZAL NAWA & MOHD KHAN KALAY & 110 & 45 & 41 & 5 & 225 \\
\hline NESH & 9058 & NAW KAREZ & MIR SADAAT & 240 & 30 & 13 & 4 & 120 \\
\hline $\mathrm{NESH}$ & 9040 & NAWA & KHINJAK MAGUL/NAMAKI & 100 & 20 & 20 & 6 & 120 \\
\hline NESH & 9043 & NAWA DASHT & GAMIT PETAW KALAY & 490 & 20 & 4 & 5 & 100 \\
\hline $\mathrm{NESH}$ & 9042 & NAWA DENAW MANDA & GERDI CHINA & 115 & 25 & 22 & 6 & 138 \\
\hline $\mathrm{NESH}$ & 9041 & NAWA GRAM AB & TERI KAREZ & 50 & 20 & 40 & 5 & 100 \\
\hline $\mathrm{NESH}$ & 9039 & NAWA JALI & KAREZGAI,KHUSHK KARE & 194 & 20 & 10 & 5 & 100 \\
\hline $\mathrm{NESH}$ & 9036 & NAWA KANDAK CHINA & TOWDA CHINA,KOCHNAI & 120 & 30 & 25 & 6 & 180 \\
\hline $\mathrm{NESH}$ & 12159 & NAWAY KAREZ & NAWAY KAREZ & 500 & 0 & 0 & 0 & 0 \\
\hline $\mathrm{NESH}$ & 9056 & PETAW KAREZ & WOKLA & 210 & 20 & 10 & 5 & 90 \\
\hline $\mathrm{NESH}$ & 9065 & QASIM KAREZ & QASIM KAREZ & 700 & 35 & 5 & 4 & 140 \\
\hline NESH & 9090 & SAFI & SAFI & 470 & 35 & 7 & 5 & 175 \\
\hline
\end{tabular}

* Jerib (jr.) $=1 / 5$ ha

* ${ }^{2}$ Dry Opium $=$ Wet Opium - $30 \%$ moisture

Source: UNDCP Opium Production Survey, 199 File: KDR3.XLS - 07/08/95 
Opium Production Survey by village in Qandahar province, 1995.

\begin{tabular}{|c|c|c|c|c|c|c|c|c|}
\hline & Village & & & . Total & Land & & Wet Opium *2 & Wet Opium \\
\hline \multirow[t]{2}{*}{ District } & \multirow[t]{2}{*}{ Code } & \multirow[t]{2}{*}{ Areal Main Village } & \multirow[t]{2}{*}{ Village } & \multirow{2}{*}{$\begin{array}{c}\text { Cultivated } \\
\text { Land }\left(\text { Jerib }^{*}\right) \\
\end{array}$} & \multicolumn{2}{|c|}{ Poppy Cultivation } & \multirow{2}{*}{$\begin{array}{l}\text { Yield } \\
(\mathrm{kg} / \mathrm{jr})\end{array}$} & \multirow{2}{*}{$\begin{array}{c}\text { Production } \\
\text { (kg) }\end{array}$} \\
\hline & & & & & (jerib) & $(\%)$ & & \\
\hline \multicolumn{9}{|c|}{ Province: Qandahar } \\
\hline NESH & 9062 & SAR-E-AB & DREE WATER PUMP & 55 & 20 & 36 & 5 & 90 \\
\hline NESH & 9049 & SARDAGH & SARDAGH & 180 & 40 & 22 & 6 & 220 \\
\hline NESH & 9057 & SARPOSHAN & SARPOSHAN & 230 & 10 & 4 & 5 & 45 \\
\hline NESH & 9047 & SAYED KAREZ & CHAHAR GUMBAT & 275 & 25 & 9 & 6 & 138 \\
\hline NESH & 9078 & SEORY & DA KALA KAREZ & 450 & 40 & 9 & 6 & 240 \\
\hline NESH & 9076 & SHARAK & WASIL & 400 & 15 & 4 & 6 & 83 \\
\hline NESH & 9082 & SHAWEZ & SHAWEZ & 466 & 20 & 4 & 6 & 110 \\
\hline $\mathrm{NESH}$ & 12185 & SHENTA & SHENTA & 255 & 16 & 6 & 5 & 80 \\
\hline $\mathrm{NESH}$ & 9077 & SHIN KAREZ & ANZARGAI ENZA CHINA & 610 & 40 & 7 & 6 & 240 \\
\hline NESH & 9061 & SHORAW & MEYANKO & 432 & 12 & 3 & 5 & 60 \\
\hline $\mathrm{NESH}$ & 9068 & SURKH BED & SURKH BED & 440 & 20 & 5 & 5 & 100 \\
\hline NESH & 9086 & TAKHMAK & TAKHMAK & 555 & 16 & 3 & 6 & 96 \\
\hline NESH & 9079 & TATAR & TATAR & 370 & 40 & 11 & 6 & 220 \\
\hline NESH & 9052 & TOORKAMAN & TOOKHOON & 450 & 0 & 0 & 0 & 0 \\
\hline NESH & 9054 & WARJANI & KAREZ-E-KHOKHAK & 360 & 5 & 1 & 6 & 28 \\
\hline NESH & 12186 & WOLGI & WOLGI & 80 & 0 & 0 & 0 & 0 \\
\hline NESH & 9059 & YAKH CHINA & KAREZ CHITA & 350 & 54 & 15 & 6 & 297 \\
\hline PANJWAI & 12167 & ALOKOZO & ALOKOZO & 1,200 & 30 & 3 & 9 & 270 \\
\hline PANJWAI & 9425 & ARMARAH/QALACHA & ARMARAH/QALACHA & 800 & 14 & 2 & 10 & 140 \\
\hline PANJWAI & 9429 & ASHRAF & ASHRAF & 400 & 0 & 0 & 0 & 0 \\
\hline PANJWAI & 12165 & BABAGHDI & BABAGHDI & 700 & 15 & 2 & 9 & 135 \\
\hline PANJWAI & 9517 & BADWAN & BADWAN & 100 & 10 & 10 & 10 & 100 \\
\hline PANJWAI & 11135 & BAHADUR QALA & BAHADUR QALA & 400 & 0 & 0 & 0 & 0 \\
\hline PANJWAI & 9538 & BALMAI & BALMAI & 1,920 & 2 & 0 & 9 & 18 \\
\hline PANJWAI & 11137 & BELANDI & BELANDI & 400 & 0 & 0 & 0 & 0 \\
\hline PANJWAI & 9511 & CHAR KOCHA & CHAR KOCHA & 600 & 30 & 5 & 10 & 300 \\
\hline PANJWAI & 11168 & DEHDAR & DEHDAR & 1,400 & 60 & 4 & 9 & 540 \\
\hline PANJWAI & 9537 & GEERANDY & GEERANDY & 1,360 & 5 & 0 & 9 & 45 \\
\hline PANJWAI & 9424 & HAJI MOHD AKARAM & HAJI MOHD AKARAM & 1,200 & 15 & 1 & 10 & 150 \\
\hline
\end{tabular}


Opium Production Survey by village in Qandahar province, 1995.

\begin{tabular}{|c|c|c|c|c|c|c|c|c|}
\hline \multirow{3}{*}{ District } & \multirow{3}{*}{$\begin{array}{l}\text { Village } \\
\text { Code }\end{array}$} & \multirow{3}{*}{ Area/ Main Village } & \multirow{3}{*}{ Village } & \multirow{3}{*}{$\begin{array}{c}\text { Total } \\
\text { Cultivated } \\
\text { Land }\left(\text { Jerib }{ }^{*}\right)\end{array}$} & \multirow{2}{*}{\multicolumn{2}{|c|}{$\begin{array}{c}\text { Land Under } \\
\text { Poppy Cultivation }\end{array}$}} & \multirow{3}{*}{$\begin{array}{c}\text { Wet Opium }{ }^{* 2} \\
\text { Yield } \\
(\mathrm{kg} / \mathrm{jr})\end{array}$} & \multirow{3}{*}{$\begin{array}{c}\text { Wet Opium } \\
\text { Production } \\
(\mathrm{kg})\end{array}$} \\
\hline & & & & & & & & \\
\hline & & & & & (jerib) & $(\%)$ & & \\
\hline \multicolumn{9}{|c|}{ Province: Qandahar } \\
\hline PANJWAI & 11134 & HAJIANO KALAY & HAJIANO KALAY & 0 & 0 & \#DIV/0! & 0 & 0 \\
\hline PANJWAI & 9989 & KADHAL & KADHAL & 1,700 & 12 & 1 & 9 & 108 \\
\hline PANJWAI & 9543 & KAKARANO ASTGHA & KAKARANO ASTGHA & 1,000 & 30 & 3 & 9 & 270 \\
\hline PANJWAI & 9540 & KESHATA DEHMRASY & KESHATA DEHMRASY & 100 & 0 & 0 & 0 & 0 \\
\hline PANJWAI & 9536 & KHANDAQ & SAYEDAN & 300 & 4 & 1 & 9 & 36 \\
\hline PANJWAI & 9535 & KHANJAKAK & ADAMZAI/NAWROZI & 340 & 0 & 0 & 0 & 0 \\
\hline PANJWAI & 9512 & KHANJAKAK & WAZIR KHAN KALAY & 60 & 0 & 0 & 0 & 0 \\
\hline PANJWAI & 9512 & KHANJKAK & WAZIR KHAN NAWROZI & 120 & 0 & 0 & 0 & 0 \\
\hline PANJWAI & 9533 & KHAROTI & KHAROTI & 100 & 0 & 0 & 0 & 0 \\
\hline PANJWAI & 9516 & KOCHNAI CHALGHOR & KOCHNAI CHALGHOR & 280 & 10 & 4 & 10 & 100 \\
\hline PANJWAI & 9524 & KOLK & HAJI PEERDAL & 2,000 & 80 & 4 & 10 & 800 \\
\hline PANJWAI & 9525 & KOLK & SADULLA QALA,HASANZI & 1,800 & 50 & 3 & 10 & 500 \\
\hline PANJWAI & 9541 & KOSHKAK & KOSHKAK & 1,120 & 80 & 7 & 10 & 800 \\
\hline PANJWAI & 11141 & LAKARY & LAKARY & 1,000 & 0 & 0 & 0 & 0 \\
\hline PANJWAI & 9995 & LAL KHAN QALAH & LAL KHAN QALAH & 560 & 0 & 0 & 0 & 0 \\
\hline PANJWAI & 9507 & LOWAR DAHMRASY & LOWAR DAHMRASY & 120 & 7 & 6 & 9 & 63 \\
\hline PANJWAI & 9516 & LOY CHALGHOR & LOY CHALGHOR & 1,120 & 12 & 1 & 9 & 108 \\
\hline PANJWAI & 9422 & MAHAJEREEN & MAHAJEREEN & 150 & 0 & 0 & 0 & 0 \\
\hline PANJWAI & 9513 & MARKAZ H.ABDULA.QALA & MARKAZ H.ABDULA.QALA & 1,000 & 10 & 1 & 10 & 100 \\
\hline PANJWAI & 11140 & MAZANGAN & MAZANGAN & 300 & 0 & 0 & 0 & 0 \\
\hline PANJWAI & 9547 & MERWALIAN & MERWALIAN & 300 & 5 & 2 & 8 & 40 \\
\hline PANJWAI & 11136 & MOSA KALIMKAREZ & MOSA KALIMKAREZ & 300 & 0 & 0 & 0 & 0 \\
\hline PANJWAI & 9531 & MOSHAN & MOSHAN & 3,400 & 20 & 1 & 9 & 180 \\
\hline PANJWAI & 11133 & MULA DOST & MULA DOST & 1,000 & 0 & 0 & 0 & 0 \\
\hline PANJWAI & 9528 & NAKHONI & FAQIR ZAI/H.G.HAIDER & 500 & 0 & 0 & 0 & 0 \\
\hline PANJWAI & 11132 & NAKHONI & HAJI LOY LALA & 260 & 0 & 0 & 0 & 0 \\
\hline PANJWAI & 9434 & NAKHONI/H.M. KALACHA & TOR KALACHA & 600 & 0 & 0 & 0 & 0 \\
\hline PANJWAI & 11172 & NAWROZ & NAWROZ & 640 & 0 & 0 & 0 & 0 \\
\hline PANJWAI & 12164 & NEAHRE KAREZ & NASRAT KHEL & 800 & 15 & 2 & 9 & 135 \\
\hline
\end{tabular}

* Jerib (jr.) $=1 / 5$ ha

${ }^{*}{ }^{2}$ Dry Opium $=$ Wet Opium $-30 \%$ moisture

Page $32 \quad 183$

Source: UNDCP Opium Production Survey, 1995 File: KDR3.XLS - 07/08/95 
Opium Production Survey by village in Qandahar province, 1995.

\begin{tabular}{|c|c|c|c|c|c|c|c|c|}
\hline \multirow{3}{*}{ District } & \multirow{3}{*}{\begin{tabular}{|l|} 
Village \\
Code \\
\end{tabular}} & \multirow{3}{*}{ Area/ Main Village } & \multirow{3}{*}{ Village } & \multirow{3}{*}{\begin{tabular}{|c|} 
Total \\
Cultivated \\
Land $\left(\right.$ Jerib $\left.^{*}\right)$ \\
\end{tabular}} & \multirow{2}{*}{\multicolumn{2}{|c|}{$\begin{array}{c}\text { Land Under } \\
\text { Poppy Cultivation }\end{array}$}} & \multirow{3}{*}{$\begin{array}{c}\text { Wet Opium }^{* 2} \\
\text { Yield } \\
(\mathrm{kg} / \mathrm{jr}) \\
\end{array}$} & \multirow{3}{*}{$\begin{array}{c}\text { Wet Opium } \\
\text { Production } \\
(\mathrm{kg})\end{array}$} \\
\hline & & & & & & & & \\
\hline & & & & & (jerib) & $(\%)$ & & \\
\hline \multicolumn{9}{|c|}{ Province: Qandahar } \\
\hline PANJWAI & 9543 & NEWI SALIJAN & KAKARANO ASHOGHA & 1,000 & 30 & 3 & 9 & 270 \\
\hline PANJWAI & 9521 & NILGHAM & BOGHANJI & 1,600 & 60 & 4 & 10 & 600 \\
\hline PANJWAI & $9510^{\circ}$ & NILGHAM/TOL & KHAN MOHD QALAH & 800 & 20 & 3 & 9 & 180 \\
\hline PANJWAI & 9522 & NILGHAM/TOL & SAIDAN & 560 & 15 & 3 & 9 & 135 \\
\hline PANJWAI & 9534 & NOWROZI & NOWROZI & 70 & 7 & 10 & 10 & 70 \\
\hline PANJWAI & 9430 & PAI MALOK & PAI MALOK & 5,000 & 60 & 1 & 10 & 600 \\
\hline PANJWAI & 9428 & PANJWAI MARKAZ & PANJWAI BAZAR & 800 & 15 & 2 & 9 & 135 \\
\hline PANJWAI & 9435 & PANJWAI MARKEZ & SARDARAN & 1,000 & 30 & 3 & 10 & 300 \\
\hline PANJWAI & 9419 & PASHMOL & HAJI MOHD AEWAZ & 1,000 & 90 & 9 & 9 & 810 \\
\hline PANJWAI & 9432 & PASHMOL & HAJI MOSA KHAN & 1,500 & 40 & 3 & 10 & 400 \\
\hline PANJWAI & 9420 & PASHMOL & HAJI MUSA KHAN SALAM & 1,500 & 50 & 3 & 10 & 500 \\
\hline PANJWAI & 9506 & PASHMOL & MAKOMEYAN JOY & 900 & 100 & 11 & 9 & 900 \\
\hline PANJWAI & 11167 & PASHMOL & MAKWAN & 480 & 80 & 17 & 9 & 720 \\
\hline PANJWAI & 9436 & PASHMOL & PAINDY & 1,500 & 50 & 3 & 10 & 500 \\
\hline PANJWAI & 9505 & PASHMOL & QALACHA & 200 & 30 & 15 & 10 & 300 \\
\hline PANJWAI & 12168 & POPALZO SHAH NOHD & POPALZO SHAH MOHD & 800 & 20 & 3 & 9 & 180 \\
\hline PANJWAI & 9421 & QALA FATEHULLAH & QALA FATEHULLAH & 40 & 0 & 0 & 0 & 0 \\
\hline PANJWAI & 9426 & QASEM RABAT & QASEM RABAT & 800 & 5 & 1 & 9 & 45 \\
\hline PANJWAI & 9427 & REGEY & REGEY & 650 & 0 & 0 & 0 & 0 \\
\hline PANJWAI & 9514 & REGWA & ABDUL KHALEQ & 900 & 0 & 0 & 0 & 0 \\
\hline PANJWAI & 9527 & REGWA BALOSAN KALACH & BALOCHANO KALACHA & 200 & 6 & 3 & 10 & 60 \\
\hline PANJWAI & 9546 & SABALGHI & SPEEN PEER & 1,500 & 50 & 3 & 10 & 500 \\
\hline PANJWAI & 9519 & SABLAGHI & MULAYAN & 1,500 & 80 & 5 & 10 & 800 \\
\hline PANJWAI & 9532 & SAEYDAN KALAY & AHMAD JAN QALA & 120 & 10 & 8 & 9 & 90 \\
\hline PANJWAI & 9423 & SAID QALA & SAID QALA & 300 & 7 & 2 & 10 & 70 \\
\hline PANJWAI & 11139 & SALAWAT & SALAWAT & 1,200 & 0 & 0 & 0 & 0 \\
\hline PANJWAI & 9526 & SALEHAN & ZOR SALEN & $\because 800$ & 15 & 2 & 10 & 150 \\
\hline PANJWAI & 9515 & SAT MALOK & SA'T MALOK & 3,000 & 30 & 1 & 10 & 300 \\
\hline PANJWAI & 9544 & SEYA CHOY & HAJI ABDUL HAMID & 800 & 60 & 8 & 9 & 540 \\
\hline
\end{tabular}

* Jerib (jr.) = 1/5 ha

* ${ }^{2}$ Dry Opium $=$ Wet Opium - $30 \%$ moisture

Page 33184

Source: UNDCP Opium Production Survey, 1995 File: KDR3.XLS - 07/08/95 
Opium Production Survey by village in Qandahar province, 1995.

\begin{tabular}{|c|c|c|c|c|c|c|c|c|}
\hline \multirow{3}{*}{ District } & \multirow{3}{*}{$\begin{array}{l}\text { Village } \\
\text { Code } \\
\end{array}$} & \multirow{3}{*}{ Area/ Main Village } & \multirow{3}{*}{ Village } & \multirow{3}{*}{$\begin{array}{c}\text { Total } \\
\text { Cultivated } \\
\text { Land }\left(\text { Jerib }^{*}\right) \\
\end{array}$} & \multirow{2}{*}{\multicolumn{2}{|c|}{$\begin{array}{c}\text { Land Under } \\
\text { Poppy Cultivation }\end{array}$}} & \multirow{3}{*}{$\begin{array}{c}\text { Wet Opium *2 } \\
\text { Yield } \\
(\mathrm{kg} / \mathrm{jr})\end{array}$} & \multirow{3}{*}{$\begin{array}{c}\text { Wet Opium } \\
\text { Production } \\
(\mathrm{kg}) \\
\end{array}$} \\
\hline & & & & & & & & \\
\hline & & & & & (jerib) & $(\%)$ & & \\
\hline Province: Qandahar & & $\therefore$ & & & & & & \\
\hline PANJWAI & 9523 & SEYA CHOY & MANIZI & 480 & 40 & 8 & 10 & 400 \\
\hline PANJWAI & 11138 & SHARA KHAN & SHARA KHAN & 500 & 0 & 0 & 0 & 0 \\
\hline PANJWAI & 9994 & SHKELCHI & SHKELCHI & 450 & 12 & $\dot{3}$ & 10 & 120 \\
\hline PANJWAI & 9992 & SPERWAN & HAJI QUDOS & 1,100 & 0 & 0 & 0 & 0 \\
\hline PANJWAI & 9518 & SPERWAN & HAJI TORAK & 900 & 0 & 0 & 0 & 0 \\
\hline PANJWAI & 9990 & SPERWAN & MULA ABDUL RAHMAN & 1,000 & 0 & 0 & 0 & 0 \\
\hline PANJWAI & 9417 & TOLKAN & HAJI MUDER SAHIB & 3,000 & 20 & 1 & 10 & 200 \\
\hline PANJWAI & 9539 & TOLKAN & MALEK QAYOM KALAY & 1,500 & 10 & 1 & 10 & 100 \\
\hline PANJWAI & 9529 & TOLKAN & TAHIR AKHOND KALAY & 1,500 & 0 & 0 & 0 & 0 \\
\hline PANJWAI & 9509 & YAKLANG/NILGHAM & ACHAKZAI & 560 & 15 & 3 & 10 & 150 \\
\hline PANJWAI & 9508 & YAKLANG/NILGHAM & H.ABDULLAH,KHAN.M & 800 & 40 & 5 & 10 & 400 \\
\hline PANJWAI & 9433 & ZAFARAN ZAI & ZAFARAN ZAI & 80 & 0 & 0 & 0 & 0 \\
\hline PANJWAI & 12166 & ZAHDANAN & ZAHDANAN & 560 & 100 & 18 & 10 & 1,000 \\
\hline PANJWAI & 12163 & ZANGI ABAD & LALKANI & 600 & 15 & 3 & 10 & 150 \\
\hline PANJWAI & 11171 & ZANGI ABAD & SAIDAN KALAY & 500 & 45 & 9 & 9 & 405 \\
\hline PANJWAI & 11170 & ZANGI ABAD & SHAH BOZI & 1,100 & 0 & 0 & 0 & 0 \\
\hline PANJWAI & 11169 & ZANGI ABAD & SULTAN MOHD GARAJ & 1,500 & 10 & 1 & 9 & 90 \\
\hline SHAH WALI KOT & 9705 & ABDUL WAHED,A.BAQI & ABDUL WAHED,A.BAQI & 150 & 15 & 10 & 4 & 60 \\
\hline SHAH WALI KOT & 10015 & ALOKOZO,AHIKZO KALAY & ALOKOZO,AHIKZO KALAY & 300 & 15 & 5 & 4 & 60 \\
\hline SHAH WALI KOT & 9709 & AMIN KHAN KAREZ & BAGH DESHRA & 50 & 4 & 8 & 5 & 20 \\
\hline SHAH WALI KOT & 10027 & ANGORAK,LOAR BAGHTO & ANGORAK,LOAR BAGHTO & 80 & 5 & 6 & 4 & 20 \\
\hline SHAH WALI KOT & 9736 & ARGAD/ANAGI & ASHOQZAI & 150 & 6 & 4 & 5 & 30 \\
\hline SHAH WALI KOT & 9676 & BABA SAHIB & BABA SAHIB & 40 & 5 & 13 & 4 & 20 \\
\hline SHAH WALI KOT & 9690 & BELO SANG & SHAZAMEEN & 100 & 10 & 10 & 5 & 50 \\
\hline SHAH WALI KOT & 9675 & BORGENA(DOLANAH) & BORGENA(DOLANAH) & 80 & 4 & 5 & 5 & 20 \\
\hline SHAH WALI KOT & 9664 & CHAGHNI & SURKH SANG & 80 & 3 & 4 & 5 & 15 \\
\hline SHAH WALI KOT & 9632 & CHAMAN & DARAGI TANGI & 70 & 9 & 13 & 5 & 45 \\
\hline SHAH WALI KOT & 9070 & CHEHEL GAZI & NAWA KASHKHANAMAK & 120 & 6 & 5 & 5 & 30 \\
\hline SHAH WALI KOT & 9667 & CHINA & LWARA WALA(BARKHANZI & 150 & 5 & 3 & 5 & 25 \\
\hline
\end{tabular}

* Jerib (jr.) = 1/5 ha

${ }^{*}$ Dry Opium $=$ Wet Opium $-30 \%$ moisture

Page $34 \quad 185$

Source: UNDCP Opium Production Survey, 1995 File: KDR3.XLS - 07/08/95 
Opium Production Survey by village in Qandahar province, 1995.

\begin{tabular}{|c|c|c|c|c|c|c|c|c|}
\hline \multirow{3}{*}{ District } & \multirow{3}{*}{\begin{tabular}{|l|} 
Village \\
Code
\end{tabular}} & \multirow{3}{*}{ Area/ Main Village } & \multirow{3}{*}{ Village } & \multirow{3}{*}{$\begin{array}{c}\text { Total } \\
\text { Cultivated } \\
\text { Land }(\text { Jerib } *) \\
\end{array}$} & \multirow{2}{*}{\multicolumn{2}{|c|}{$\begin{array}{c}\text { Land Under } \\
\text { Poppy Cultivation }\end{array}$}} & \multirow{3}{*}{$\begin{array}{c}\text { Wet Opium }{ }^{* 2} \\
\text { Yield } \\
(\mathrm{kg} / \mathrm{jr})\end{array}$} & \multirow{3}{*}{$\begin{array}{c}\text { Wet Opium } \\
\text { Production } \\
(\mathrm{kg}) \\
\end{array}$} \\
\hline & & & & & & & & \\
\hline & & & & & (jerib) & $(\%)$ & & \\
\hline \multicolumn{9}{|l|}{ Province: Qandahar } \\
\hline SHAH WALI KOT & 9749 & CHINAR & CHINAR : & 150 & 5 & 3 & 4 & 20 \\
\hline SHAH WALI KOT & 9658 & CHINARTO & CHINARTO & 150 & 10 & 7 & 5 & 50 \\
\hline SHAH WALI KOT & 9372 & DAMANA & QILA KAREZ MAMANA & 80 & 0 & 0 & 0 & 0 \\
\hline SHAH WALI KOT & 11124 & DARBANAN & DARBANAN & 80 & 0 & 0 & 0 & 0 \\
\hline SHAH WALI KOT & 9693 & DEBA (BARAKI) & URDOKAS & 70 & 0 & 0 & 0 & 0 \\
\hline SHAH WALI KOT & 9662 & DELAK & DELAK & 80 & 6 & 8 & 5 & 30 \\
\hline SHAH WALI KOT & 9663 & DEPAL & DEPAL & 80 & 3 & 4 & 5 & 15 \\
\hline SHAH WALI KOT & 9671 & DIMOSHAK & DIMOSHAK & 100 & 7 & 7 & 5 & 35 \\
\hline SHAH WALI KOT & 9744 & DOAB TANGAI & PRAH/TANGA & 150 & 6 & 4 & 4 & 24 \\
\hline SHAH WALI KOT & 9702 & FAQEERAN KHAR TOOT & GADONA CHARSANG & 80 & 8 & 10 & 5 & 40 \\
\hline SHAH WALI KOT & 10028 & GANA SHING/KONJAI & TANAO CHI & 80 & 5 & 6 & 5 & 25 \\
\hline SHAH WALI KOT & 9719 & GHASHEEN & GHASHEEN & 70 & 5 & 7 & 5 & 25 \\
\hline SHAH WALI KOT & 9712 & GONBAD & SAHLEZAR & 150 & 7 & 5 & 5 & 35 \\
\hline SHAH WALI KOT & 9716 & GONBAD KONDALAN & GONBAD KONDALAN & 300 & 8 & 3 & 4 & 32 \\
\hline SHAH WALI KOT & 9703 & GORGEEN & PARO KHAN/MULA FADAL & 70 & 6 & 9 & 4 & 24 \\
\hline SHAH WALI KOT & 9679 & GORGEEN & PARO KHAN/MULLAH PAD & 300 & 15 & 5 & 4 & 60 \\
\hline SHAH WALI KOT & 9696 & GUNBAD & GUNBAD & 70 & 5 & 7 & 4 & 20 \\
\hline SHAH WALI KOT & 9374 & HAJI PAYO & HAJI PAYO & 100 & 2 & 2 & 4 & 8 \\
\hline SHAH WALI KOT & 9650 & HAJIAN AW TOOR BAGH & HAJIAN AW TOOR BAGH & 80 & 4 & 5 & 4 & 16 \\
\hline SHAH WALI KOT & 9741 & HANAWI REZAI & HASSANZAY & 80 & 4 & 5 & 5 & 20 \\
\hline SHAH WALI KOT & 10023 & HOTAK, SHAH JOY & ARAB & 70 & 7 & 10 & 4 & 28 \\
\hline SHAH WALI KOT & 9371 & JAHAN & JAHAN & 100 & 6 & 6 & 5 & 30 \\
\hline SHAH WALI KOT & 9669 & KABULEAN & KABULEAN & 80 & 4 & 5 & 4 & 16 \\
\hline SHAH WALI KOT & 9699 & KAIGHTO & RAIKHI & 100 & 5 & 5 & 4 & 20 \\
\hline SHAH WALI KOT & 10029 & KAKOL SHAKOT WALA & KALACH-GANIHAY & 110 & 7 & 6 & 5 & 35 \\
\hline SHAH WALI KOT & $9357-$ & KAMPANY & KAMPANY & 60 & 0 & 0 & 0 & 0 \\
\hline SHAH WALI KOT & 9684 & KAREZGI & GORLANGI & 80 & 6 & 8 & 4 & 24 \\
\hline SHAH WALI KOT & 9725 & KEEM & KHOJA ZARKAI & 200 & 15 & 8 & 4 & 60 \\
\hline SHAH WALI KOT & 9742 & KHAL BELAGH & KASHPOAL & 200 & 7. & 4 & 4 & 28 \\
\hline
\end{tabular}

* Jerib (jr.) = 1/5 ha 
Opium Production Survey by village in Qandahar province, 1995.

\begin{tabular}{|c|c|c|c|c|c|c|c|c|}
\hline \multirow{3}{*}{ District } & \multirow{3}{*}{\begin{tabular}{|l|} 
Village \\
Code \\
\end{tabular}} & \multirow{3}{*}{ Area/ Main Village } & \multirow{3}{*}{ Village } & \multirow{3}{*}{$\begin{array}{c}\text { Total } \\
\text { Cultivated } \\
\text { Land }\left(\text { Jerib }^{*}\right) \\
\end{array}$} & \multirow{2}{*}{\multicolumn{2}{|c|}{$\begin{array}{c}\text { Land Under } \\
\text { Poppy Cultivation }\end{array}$}} & \multirow{3}{*}{$\begin{array}{c}{\text { Wet } \text { Opium }^{* 2}}^{\text {Yield }} \\
(\mathrm{kg} / \mathrm{jr})\end{array}$} & \multirow{3}{*}{$\begin{array}{c}\text { Wet Opium } \\
\text { Production } \\
(\mathrm{kg})\end{array}$} \\
\hline & & & & & & & & \\
\hline & & & & & (jerib) & $(\%)$ & & \\
\hline \multicolumn{9}{|l|}{ Province: Qandahar } \\
\hline SHAH WALI KOT & 9677 & KHAWJA ZARKI & KARIM DAD TAMBAH & & & \#DIV/0! & & 0 \\
\hline SHAH WALI KOT & 9733 & KULA KAREZ & LAL JAN/DARGAI & 70 & 5 & 7 & 5 & 25 \\
\hline SHAH WALI KOT & 10025 & LOAR DA PAY CHAGHNI & LOAR DA PAY CHAGHNI & 70 & 2 & 3 & 5 & 10 \\
\hline SHAH WALI KOT & 9692 & LOWAR GANDALAN & KACHKOL & 200 & 8 & 4 & 4 & 32 \\
\hline SHAH WALI KOT & 9642 & LOY KAREZ & MALMAND/S.RAHIM & 200 & 8 & 4 & 5 & 40 \\
\hline SHAH WALI KOT & 9640 & LOY KAREZ & NIMA KEY & 70 & 3 & 4 & 5 & 15 \\
\hline SHAH WALI KOT & 9678 & LWAR TAKHT & LWAR TAKHT & 100 & 6 & 6 & 5 & 30 \\
\hline SHAH WALI KOT & 12169 & M.BIG ABTO AGA KAREZ & MIR AGA KAREZ & 60 & 5 & 8 & 5 & 25 \\
\hline SHAH WALI KOT & 9365 & MALIZO KAREZ & MALIZO KAREZ & 100 & 0 & 0 & 0 & 0 \\
\hline SHAH WALI KOT & 9745 & MANO & TAMBAKOL & 150 & 6 & 4 & 5 & 30 \\
\hline SHAH WALI KOT & 9375 & MARDAN ZARKI & MARDAN ZARKI & 200 & 0 & 0 & 0 & 0 \\
\hline SHAH WALI KOT & 9645 & MAREAM/KOCHNAY KAREZ & MAREAM/HAZAMANI & 150 & 7 & 5 & 4 & 28 \\
\hline SHAH WALI KOT & 9734 & MASTI LORGAI,MINAKAR & MINA KAREZ/CHOKAR & 120 & 8 & 7 & 5 & 40 \\
\hline SHAH WALI KOT & 11122 & MERKAZ SHAH WALIKOT & MERKAZ SHAH WALIKOT & 150 & 0 & 0 & 0 & 0 \\
\hline SHAH WALI KOT & 9708 & MOHD ALI SORKH SARNG & NANGA - NARAI & 70 & 5 & 7 & 5 & 25 \\
\hline SHAH WALI KOT & 9651 & MULAEAN(TATREN) & MULAEAN(TATREN) & 60 & 4 & 7 & 5 & 20 \\
\hline SHAH WALI KOT & 9657 & NAHAR S.BAHODDIN AGA & ALBAK/AKHUNDZAD & 60 & 3 & 5 & 4 & 12 \\
\hline SHAH WALI KOT & 9656 & NAJMUDDIN & NAJMUDDIN & 50 & 3 & 6 & 5 & 15 \\
\hline SHAH WALI KOT & 9681 & NAR KHAR & NAR KHAR & 100 & 6 & 6 & 5 & 30 \\
\hline SHAH WALI KOT & 9074 & NAWA SABZAL & NAWA SHIZI & 180 & 8 & 4 & 5 & 40 \\
\hline SHAH WALI KOT & 9740 & NAWA SHARQI & GAWHAR KHANA & 80 & 8 & 10 & 5 & 40 \\
\hline SHAH WALI KOT & 9644 & NAWAH & KADELAK & 100 & 4 & 4 & 4. & 16 \\
\hline SHAH WALI KOT & 9731 & NAWZARKI/ LANDI & QUDOOS-SHOKAL & 80 & 8 & 10 & 5 & 40 \\
\hline SHAH WALI KOT & 10022 & OTAK-QALAT KAREZ & GANUZAY-SHALI A & 120 & 10 & 8 & 5 & 50 \\
\hline SHAH WALI KOT & 11123 & QOLA KALAY & QOLA KALAY & 80 & 0 & 0 & 0 & 0 \\
\hline SHAH WALI KOT & 9700 & SARKHOSHAK(KHAKOCHI) & SARYAB & 80 & 8 & 10 & 5 & 40 \\
\hline SHAH WALI KOT & 9720 & SAYED HESAMUDIN(NASR & SANBAN & 70 & 5 & 7 & 5 & 25 \\
\hline SHAH WALI KOT & 9728 & SEDOM-SAYED SHAH & SEDOM-SAYED SHAH & 90 & 10 & 11 & 5 & 50 \\
\hline SHAH WALI KOT & 9652 & SHA KAREZ & SHA KAREZ & 90 & 10 & 11 & 5 & 50 \\
\hline
\end{tabular}

* Jerib (jr.) = 1/5 ha

* ${ }^{2}$ Dry Opium $=$ Wet Opium $-30 \%$ moisture 
Opium Production Survey by village in Qandahar province, 1995.

\begin{tabular}{|c|c|c|c|c|c|c|c|c|}
\hline . & Village & & . & Total & Land 1 & Under & Wet Opium *2 & Wet Opium \\
\hline \multirow[t]{2}{*}{ District } & Code & Area/ Main Village & Village & Cultivated & \multicolumn{2}{|c|}{ Poppy Cultivation } & Yield & Production \\
\hline & & & & Land $\left(\right.$ Jerib $\left.^{*}\right)$ & (jerib) & $(\%)$ & $(\mathrm{kg} / \mathrm{jr})$ & $(\mathrm{kg})$ \\
\hline \multicolumn{9}{|l|}{ Province: Qandahar } \\
\hline SHAH WALI KOT & 9613 & SHADA & BARLAS & 300 & 8 & 3 & 4 & 32 \\
\hline SHAH WALI KOT & 11121 & SHAH AQA KELY & SHAH AQA KELY & 70 & 0 & 0 & 0 & 0 \\
\hline SHAH WALI KOT & 9652 & SHAH KAREZ & SHAH KAREZ & 50 & 2 & 4 & 5 & 10 \\
\hline SHAH WALI KOT & 9743 & SHARQI NAWA-SHATOOT & SHARQI NAWA-SHATOOT & 100 & 6 & 6 & 5 & 30 \\
\hline SHAH WALI KOT & 9646 & SHIR JAN/SIASANG & HAJI PAIO & 100 & 5 & 5 & 4 & 20 \\
\hline SHAH WALI KOT & 9710 & SORAB CHASHMA & SORGH MANGALI & 70 & 8 & 11 & 5 & 40 \\
\hline SHAH WALI KOT & 10021 & SOZANI MEANKAL & SOZANI MEANKAL & 300 & 10 & 3 & 4 & 40 \\
\hline SHAH WALI KOT & 9723 & TAHSILDAR & TAM TAK & 100 & 10 & 10 & 4 & 40 \\
\hline SHAH WALI KOT & 9369 & TAKATO & ZARTALA & 150 & 0 & 0 & 0 & 0 \\
\hline SHAH WALI KOT & 9649 & TANGI & TANGI & 150 & 0 & 0 & 0 & 0 \\
\hline SHAH WALI KOT & 9659 & TEZAK & SPEEN KASHAH & 100 & 5 & 5 & 5 & 25 \\
\hline SHAH WALI KOT & 9748 & TOGHRAK & AWOLYA & 80 & 5 & 6 & 4 & 20 \\
\hline SHAH WALI KOT & 9633 & YARDAD & ZAIDANA AW KAKONEKA & 80 & 5 & 6 & 4 & 20 \\
\hline SHAH WALI KOT & 9697 & ZANGITAN & GOBI KOL & 150 & 6 & 4 & 5 & 30 \\
\hline SHAH WALI KOT & 9695 & ZANTO & ZANTO & 300 & 7 & 2 & 5 & 35 \\
\hline SHAH WALI KOT & 10016 & ZINDOR LANGAR KAREZ & ZINDOR LANGAR KAREZ & 100 & 8 & 8 & 5 & 40 \\
\hline SPIN BOLDAK & 11078 & AKHTAR MOHD & AKHTAR MOHD & 420 & 0 & 0 & 0 & 0 \\
\hline SPIN BOLDAK & 11164 & AKHTER KELE & AKHTER KELE & 240 & 8 & 3 & 9 & 72 \\
\hline SPIN BOLDAK & 11044 & ALAM KHAN ZAI & MULAYANO KELY & 750 & 20 & 3 & 5 & 90 \\
\hline SPIN BOLDAK & 11182 & ALIZAY & ALIZAY & 670 & 0 & 0 & 0 & 0 \\
\hline SPIN BOLDAK & 11074 & AMAN KOT SHAGAI & AMAN KOT SHAGAI & 15,000 & 0 & 0 & 0 & 0 \\
\hline SPIN BOLDAK & 11045 & ARNAWAY KAREZ & AZIM ZAI KAREZ & 450 & 0 & 0 & 0 & 0 \\
\hline SPIN BOLDAK & 11069 & BABAK SAHEB KAREZ & BABAK SAHEB KAREZ & 1,500 & 0 & 0 & 0 & 0 \\
\hline SPIN BOLDAK & 11065 & BAGO RAHMAN KAHOL & BAGO RAHMAN KAHOL & 1,000 & 0 & 0 & 0 & 0 \\
\hline SPIN BOLDAK & 11173 & BISA & LOUALA BISA & 400 & 20 & 5 & 5 & 90 \\
\hline SPIN BOLDAK & 11187 & $\ldots \cdots$ & ABDULLAH JOHN & 400 & 0 & 0 & 0 & 0 \\
\hline SPIN BOLDAK & 11059 & DADZAI, METAZAI & DADZAI, METAZAI & 200 & 0 & $\cdots 0$ & 0 & 0 \\
\hline SPIN BOLDAK & 11160 & DE NIKAY KAHOL & DE NIKAY KAHOL & 5,000 & 60 & 1 & 14 & 810 \\
\hline SPIN BOLDAK & 11193 & DE SHAHGAZO KELE $\ldots \ldots$ & KALAGAY & 460 & $\begin{array}{r}-0 \\
\end{array}$ & $\therefore-0$ & 0 & 0 \\
\hline
\end{tabular}

* Jerib (jr.) $=1 / 5$ ha

* 2 Dry Opium $=$ Wet Opium $-30 \%$ moisture

Page $37 \quad 188$

Source: UNDCP Opium Production Survey, 1995 File: KDR3.XLS - 07/08/95 
Opium Production Survey by village in Qandahar province, 1995.

\begin{tabular}{|c|c|c|c|c|c|c|c|c|}
\hline \multirow{3}{*}{ District } & \multirow{3}{*}{\begin{tabular}{|l|} 
Village \\
Code \\
\end{tabular}} & \multirow{3}{*}{ Area/ Main Village } & \multirow{3}{*}{ Village } & \multirow{3}{*}{\begin{tabular}{|c|} 
Total \\
Cultivated \\
Land $\left(\right.$ Jerib $\left.{ }^{*}\right)$ \\
\end{tabular}} & \multicolumn{2}{|c|}{ Land Under } & \multirow{3}{*}{$\begin{array}{c}\text { Wet Opium }^{* 2} \\
\text { Yield } \\
(\mathrm{kg} / \mathrm{jr}) \\
\end{array}$} & \multirow{3}{*}{$\begin{array}{c}\text { Wet Opium } \\
\text { Production } \\
(\mathrm{kg}) \\
\end{array}$} \\
\hline & & & & & Poppy $\mathrm{Cl}$ & Itivation & & \\
\hline & & & & & (jerib) & $(\%)$ & & \\
\hline Province: Qandahar & $\cdots$ & 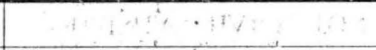 & $+x^{2}+4 \quad b$ & & & & & \\
\hline SPIN BOLDAK & 11165 & DIWARY & 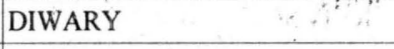 & 700 & 15 & 2 & 9 & 135 \\
\hline SPIN BOLDAK & 11147 & FAKHRAN - PATAN KHEL & FAKHRAN - PATAN KHEL & 240 & : & $\therefore$ & 5 & 30 \\
\hline SPIN BOLDAK & 11192 & FEELANI & SOOR KAREZ & 900 & 25 & 3 & 9 & 225 \\
\hline SPIN BOLDAK & 11170 & GALANDI & BANI DAD KELE & 600 & 0 & 0 & 0 & 0 \\
\hline SPIN BOLDAK & 11086 & GUL KILY & HAJI MOHD SAFAR & 200 & $\therefore$ & 0 & 0 & 0 \\
\hline SPIN BOLDAK & 11167 & GULUZAY & KULA SANGI & 420 & 0 & 0 & 0 & 0 \\
\hline SPIN BOLDAK & 11064 & H. A. QAYOUM H. NIAZ & H. A. QAYOUM H. NIAZ & 800 & 0 & 0 & 0 & 0 \\
\hline SPIN BOLDAK & 11063 & H. ZAHER RAMAZAN & H. ZAHER RAMAZAN & 1,000 & 0 & 0 & 0 & 0 \\
\hline SPIN BOLDAK & 11089 & HAJI ABDUL WAHID & HAJI MIRZA & 800 & 0 & 0 & 0 & 0 \\
\hline SPIN BOLDAK & 11066 & HAJI BAQI HAQDAD & HAJI BAQI HAQDAD & 700 & 0 & 0 & 0 & 0 \\
\hline SPIN BOLDAK & 11087 & HAJI GHULAM HAIDAR & HAJI GHULAM HAIDAR & 200 & 0 & 0 & 0 & 0 \\
\hline SPIN BOLDAK & 11081 & HAJI JANDO & HAJI JANDO & 50 & 0 & 0 & 0 & 0 \\
\hline SPIN BOLDAK & 11151 & HAJI KOTAN KILY & HAJI KOTAN KILY & 300 & 0 & 0 & 0 & 0 \\
\hline SPIN BOLDAK & 11186 & HAJI MHOD. KHAN KELY & ADORZAI HOTAD.K & 400 & 0 & 0 & 0 & 0 \\
\hline SPIN BOLDAK & 11079 & HAJI MULA MOHD OMAR & HAJI MULA MOHD OMAR & 500 & 0 & 0 & 0 & 0 \\
\hline SPIN BOLDAK & 11180 & HAMID ZO KELY & HAMID ZO KELY & 800 & 0 & 0 & 0 & 0 \\
\hline SPIN BOLDAK & 11184 & HANAFIA KELY & HANAFIA KELY & 400 & 0 & 0 & 0 & 0 \\
\hline SPIN BOLDAK & 11172 & HUSSAIN ZAY & PARO KELE & 480 & 0 & 0 & 0 & 0 \\
\hline SPIN BOLDAK & 11149 & IMRANZAI & IMRANZAI & 180 & 0 & 0 & 0 & 0 \\
\hline SPIN BOLDAK & 11171 & IQBAL KHAN KELY & IQBAL KHAN KELY & 400 & 0 & 0 & 0 & 0 \\
\hline SPIN BOLDAK & 11073 & JANGAL & ALI JAN KORONA & 22,400 & 0 & 0 & 0 & 0 \\
\hline SPIN BOLDAK & 11188 & KHANAGAY & KAJAKE : & 1,200 & 0 & 0 & 0 & 0 \\
\hline SPIN BOLDAK & 11072 & KHIRAN KALKAI & KHIRAN KALKAI & 21,600 & 0 & 0 & 0 & 0 \\
\hline SPIN BOLDAK & 11152 & KISATA MESHINGZY & KISATA MESHINGZY : " & 900 & 0 & 0 & 0 & 0 \\
\hline SPIN BOLDAK & 11161 & KISHATA AKHTER ZAY & KISHATA AKHTER ZAY & 680 & 80 & 12 & 14 & 1,080 \\
\hline SPIN BOLDAK & 11181 & KISHATA KANI ZAY & KHIRMOHD QALA & 540 & 10 & 2 & 9 & 90 \\
\hline SPIN BOLDAK & 11067 & KSHATA KHOZHA BANA & H. ABDULLAH & $\quad 600$ & 0 & 0 & 0 & 0 \\
\hline SPIN BOLDAK & 11178 & KUSI & KUSI & 3,300 & 0 & 0 & 0 & 0 \\
\hline SPIN BOLDAK & 11196 & LANDAY & PASHAWAY & 160 & 0 & 0 & $\begin{array}{r}\quad \\
\end{array}$ & 0 \\
\hline
\end{tabular}

* Jerib (jr.) $=1 / 5 \mathrm{ha}$

* ${ }^{2}$ Dry Opium $=$ Wet Opium $-30 \%$ moisture 


\begin{tabular}{|c|c|c|c|c|c|c|c|c|c|}
\hline+3 & Village & . & $\cdots$ & $\because$ & Total & Land $L$ & & Wet Opium *2 & Wet Opium \\
\hline District & Code & Areal Main Village & & Village & Cultivated & Poppy $\mathrm{Cu}$ & & Yield & Production \\
\hline $2 \%$ & & & & & Land (Jerib $\left.{ }^{*}\right)$ & (jerib) & $(\%)$ & $(\mathrm{kg} / \mathrm{jr})$ & $(\mathrm{kg})$ \\
\hline Province: Qandahar & & & (: & 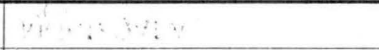 & $\therefore$ & & & & \\
\hline SPIN BOLDAK & 11174 & LANDI KAREZ & $\therefore$ & SLIMANZAY & 500 & 10 & 2. & 9 & 90 \\
\hline SPIN BOLDAK & 11043 & LOUAL AKHTAR ZAI & $\therefore$ & LOUAL AKHTAR ZAI & 700 & 10 & 1 & 9 & 90 \\
\hline SPIN BOLDAK & 11194 & LOWAL HUSSAIN ZAY & $\therefore$ & MASHINGZAY & 320 & $\dot{0}$ & 0 & 0 & 0 \\
\hline SPIN BOLDAK & 11166 & LOWAL KALE & it & LOWAL KALE & 700 & 12 & 2 & 9 & 108 \\
\hline SPIN BOLDAK & 11158 & LOWAL MUJSHUNG ZAI & & LOWAL MUJSHUNG ZAI & 300 & 0 & 0 & 0 & 0 \\
\hline SPIN BOLDAK & 11068 & LOY KALAY KOCHNAI & 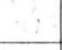 & LOY KALAY KOCHNAI & 500 & 0 & 0 & 0 & 0 \\
\hline SPIN BOLDAK & 11159 & MADIAN/SHAGAY/HUSIAN & & MADIAN/SHAGAY/HUSIAN & 20 & 0 & 0 & 0 & 0 \\
\hline SPIN BOLDAK & 11076 & MALEK ZARIN & & MALEK ZARIN & 540 & 0 & 0 & 0 & 0 \\
\hline SPIN BOLDAK & 11088 & MALIK ZAREEN & & MAWLADAD & 200 & 0 & 0 & 0 & 0 \\
\hline SPIN BOLDAK & 11168 & MARSEEN ZAI & & MARSEEN ZAI & $\quad 700$ & 0 & 0 & 0 & 0 \\
\hline SPIN BOLDAK & 11146 & MARSEENZAI & 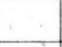 & MARSEENZAI & 1,000 & 0 & 0 & 0 & 0 \\
\hline SPIN BOLDAK & 11051 & MASHANGZI & & MASHANGZI & 2,000 & 0 & 0 & 0 & 0 \\
\hline SPIN BOLDAK & 11162 & MATGAZAY & 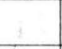 & MATGAZAY & 1,400 & 0 & $\therefore 0$ & 0 & 0 \\
\hline SPIN BOLDAK & 11183 & MHOD. MIR KELY & & MHOD. MIR KELY & 600 & 0 & 0 & 0 & 0 \\
\hline SPIN BOLDAK & 11077 & MIRGI HAJI AHMAD JAN & & MIRGI HAJI AHMAD JAN & 600 & 0 & 0 & 0 & 0 \\
\hline SPIN BOLDAK & 11075 & MIRGIYAN & $\because$ & MIRGIYAN & 3,000 & 0 & 0 & 0 & 0 \\
\hline SPIN BOLDAK & 11150 & MOHD KHAN KILY & & MOHD KHAN KILY & 800 & $\because 10$ & 1 & 5 & 50 \\
\hline SPIN BOLDAK & 11080 & MOSA KHAN/HJ. DANGAR & & MOSA KHAN/HJ. DANGAR & 400 & 0 & 0 & 0 & 0 \\
\hline SPIN BOLDAK & 11082 & NARAI GHEBARGA & & NARAI GHEBARGA & $\quad 900$ & 0 & 0 & 0 & 0 \\
\hline SPIN BOLDAK & 11185 & NIAZ MOHD KHAN & & HAJI HABIB & 400 & 0 & 0 & 0 & 0 \\
\hline SPIN BOLDAK & 11169 & NIKO KELY & & HAJI FAZEL.MOHD & 2,500 & " 0 & $\therefore 0$ & 0 & 0 \\
\hline SPIN BOLDAK & 11144 & PAIEZAI & & PAIEZAI & 1,800 & 0 & 0 & 0 & 0 \\
\hline SPIN BOLDAK & 11145 & PAIEZAI & & PAIEZAI & $\therefore 900$ & 0 & 0 & 0 & 0 \\
\hline SPIN BOLDAK & 11090 & QARARGAH & & MALIK KAKA & $\therefore 1,000$ & 0 & 0 & 0 & 0 \\
\hline SPIN BOLDAK & 11153 & QAZIAYAN/YANDKAHOL & & QAZIAYAN/YANDKAHOL & 800 & 0 & 0 & 0 & 0 \\
\hline SPIN BOLDAK & $-11143 \ldots$ & QURISHAN- HAJI SATON - & - & QURISHAN- HAJI SATON & 200 & 0 & 0 & $-\quad 0$ & 0 \\
\hline SPIN BOLDAK & 11071 & RIGI LAGLAK & & RIGI LAGLAK & 30,000 & 0 & 0 & 0 & 0 \\
\hline SPIN BOLDAK & 11084 & SALEHZAI & 11 & MOLA KHOSHAL & $\because+1$ & 0 & 0 & $\therefore$ & 0 \\
\hline SPIN BOLDAK & $11057 \ldots$ & SAR BURJ, MIYAN BURJ & $\ldots$ & SAR BURJ, MIYAN BURJ & 200 & 0 & 0 & 0 & 0 \\
\hline
\end{tabular}

* Jerib (jr.) $=1 / 5$ ha

*2 Dry Opium $=$ Wet Opium $-30 \%$ moisture
Page $39 \quad 190$
Source: UNDCP Opium Production Survey, 1995 File: KDR3.XLS - 07/08/95 
Opium Production Survey by village in Qandahar province, 1995.

\begin{tabular}{|c|c|c|c|c|c|c|c|c|}
\hline \multirow{3}{*}{ District } & \multirow{3}{*}{\begin{tabular}{|l|} 
Village \\
Code \\
\end{tabular}} & \multirow{3}{*}{ Area/ Main Village } & \multirow{3}{*}{ Village } & \multirow{3}{*}{$\begin{array}{c}\text { Total } \\
\text { Cultivated } \\
{\text { Land }\left(\text { Jerib }^{*}\right)}^{*} \\
\end{array}$} & \multirow{2}{*}{\multicolumn{2}{|c|}{$\begin{array}{c}\text { Land Under } \\
\text { Poppy Cultivation }\end{array}$}} & \multirow{3}{*}{$\begin{array}{c}\text { Wet. Opium }^{* 2} \\
\text { Yield } \\
(\mathrm{kg} / \mathrm{jr}) \\
\end{array}$} & \multirow{3}{*}{$\begin{array}{c}\text { Wet Opium } \\
\text { Production } \\
(\mathrm{kg})\end{array}$} \\
\hline & & & & & & & & \\
\hline & & & & & (jerib) & $(\%)$ & & \\
\hline \multicolumn{9}{|c|}{ Province: Qandahar } \\
\hline SPIN BOLDAK & 11046 & SHAF SHAMIZI & SHAF SHAMIZI & 1,100 & 20 & 2 & 5 & 90 \\
\hline SPIN BOLDAK & 11163 & SHALGAY & FATARY & 500 & 15 & 3 & 9 & 135 \\
\hline SPIN BOLDAK & 11176 & SIRCAR WALA & SIRCAR WALA & $1,400^{\circ}$ & 15 & 1 & 5 & 68 \\
\hline SPIN BOLDAK & 11155 & SIRDAY/FAQIR KELE & SIRDAY/FAQIR KELE & 800 & 0 & 0 & 0 & 0 \\
\hline SPIN BOLDAK & 11177 & SOURKY LEZE & SOURKI LEZE & 7,000 & 80 & 1 & 5 & 360 \\
\hline SPIN BOLDAK & 11083 & SPIN BOLDAK BAZAR & BAZAR & 3,000 & 0 & 0 & 0 & 0 \\
\hline SPIN BOLDAK & 11195 & SPIN KAREZ & SPIN KAREZ & 400 & 30 & 8 & 14 & 405 \\
\hline SPIN BOLDAK & 11197 & SPINKAY & SPINKAY & 650 & 0 & 0 & 0 & 0 \\
\hline SPIN BOLDAK & 11175 & SULTAN ZAY & SULTAN ZAY & 3,200 & 30 & 1 & 14 & 405 \\
\hline SPIN BOLDAK & 11154 & TARGAZI/LANDAY & TARGAZI/LANDAY & 800 & 0 & 0 & 0 & 0 \\
\hline SPIN BOLDAK & 11190 & WALI KAREZ & LANDI/WANGLA & 620 & 5 & 1 & 5 & 23 \\
\hline SPIN BOLDAK & 11189 & WANKA & KSHATA WANKA & 350 & 14 & 4 & 2 & 28 \\
\hline SPIN BOLDAK & 11179 & WAZIR KAHOL & WAZIR KAHOL & 4,000 & 40 & 1 & 14 & 540 \\
\hline SPIN BOLDAK & 11061 & YAKA TOOT, POPLAZAI & YAKA TOOT, POPLAZAI & 400 & 0 & 0 & 0 & 0 \\
\hline SPIN BOLDAK & 11148 & YARO KAREZ & YARO KAREZ & 400 & 0 & 0 & 0 & 0 \\
\hline SPIN BOLDAK & 11156 & ZARO KELY & ZARO KELY & 480 & 0 & 0 & 0 & 0 \\
\hline SPIN BOLDAK & 11085 & ZEREH QALA & BAQI KORONA & 1,000 & 0 & 0 & 0 & 0 \\
\hline SPIN BOLDAK & 11157 & ZHARANDA-SPINZY & ZHARANDA-SPINZY & 400 & 0 & 0 & 0 & 0 \\
\hline SPIN BOLDAK & 11070 & ZHARI HAKIM & MULLAH ZIAUDIN & 1,200 & 0 & 0 & 0 & 0 \\
\hline SPIN BOLDAK & 11191 & ZLA GAGARE & NAWAY GAGARE & 720 & 0 & 0 & 0 & 0 \\
\hline
\end{tabular}

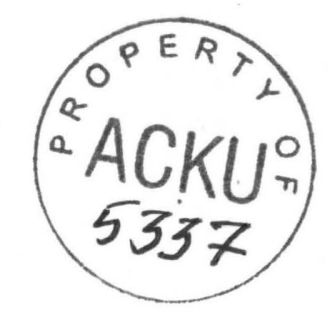

